九州大学学術情報リポジトリ

Kyushu University Institutional Repository

\title{
CHRYSOMELIDAE (COLEOPTERA) OF THAILAND, CAMBODIA, LAOS AND VIETNAM. IV. GALERUCINAE
}

Kimoto, Shinsaku

https://doi.org/10.5109/2511

出版情報 : ESAKIA. 27，pp.1-241，1989-01-25. Entomological Laboratory，Faculty of Agriculture， Kyushu University

バージョン :

権利関係 : 


\title{
CHRYSOMELIDAE (COLEOPTERA) OF THAILAND, CAMBODIA, LAOS AND VIETNAM. IV. GALERUCINAE*
}

\author{
ShINSAKU Kimoto \\ Biological Laboratory, Department of General Education, School of Medicine, \\ Kurume University, Kurume 830, Japan
}

\begin{abstract}
This fourth paper of a series treats the subfamily Galerucinae covering 382 species in 97 genera. Keys are presented to genera and species. Relevant synonymies are presented for genera and species as well as general and local distribution of species. Six genera and 103 species are described as new and a number of species are newly recorded from the area or from individual countries.
\end{abstract}

This paper is the fourth in series attempting to cover the chrysomelid beetles from the Thai-IndoChina area. This series represents a sequel to "Chrysomelidae (Coleoptera) of China and Korea" (Gressitt \& Kimoto, 1961 \& 1963), and should be used in conjunction with that monograph. This installment treats the subfamily Galerucinae covering 97 genera and 382 species, including 6 new genera and 103 new species, and there are many new synonymies and new combinations.

Keys are presented to genera and species, including a few occurring just outside the area of treatment. Pertinent synonymies are presented, and that references under genera included in Gressitt \& Kimoto (1963) are not repeated other than original references and some monographs covering the area and its nearby areas. All known geographical records within the area, as well as general distribution for all the species treated, are also presented. A number of species are newly recorded from this general region, as well as a number of new records to individual countries.

The sources of material are essentially the same as for the preceding installment. The main sources are Bishop Museum collection including the large J. A. Rondon collection from Laos (Gressitt et al., 1970) ; collections from various Japan-United States scientific cooperation projects funded by the U. S. National Science Foundation, the Japan Society for the Promotion of Sciences, and the Ministry of Education of Japan; results of the rice stem borer studies under the direction of the late Prof. K. Yasumatsu and Prof. Y. Hirashima ; collections from Kasetsart University and the Thai Department of Agriculture, Bangkhen ; collections of Zoologische Staatsammlung, München;Naturhistorisches Museum, Basel, and Entomological Laboratory, Ehime University ; as well as private collection made by Dr. Kintaro Baba and others. For further details refer to the introduction in

* Part I, Kimoto, S. \& J. L. Gressitt, 1979, Pac. Ins. 20 (2-3) : 191-256 ; Part II, Kimoto, S. \& J. L. Gressitt, 1981, ibid., 23 (3-4) : 289-391 ; Part III, Kimoto, S. \& J. L. Gressitt, 1982, Esakia, Kyushu Univ., (18) : 1-141. 
Kimoto \& Gressitt (1979, 1981, 1982).

For general information on the principal Laos survey and for locations of collecting localities, see introductory section, map and list of localities of Gressitt et al. (1970).

The following abbreviations are used herein for depository museums.

$\begin{array}{ll}\text { BANGKHEN } & \text { Kasetsart University and Agriculture Department, Bangkhen, Thailand. } \\ \text { BASEL } & \text { Naturhistorishes Museum, Basel. } \\ \text { BISHOP } & \text { Bishop Museum, Honolulu. } \\ \text { BM } & \text { British Museum (Natural History), London. } \\ \text { BRUXELLES } & \text { Institute royale des Sciences Naturelles, Bruxelles. } \\ \text { CAS } & \text { California Academy of Sciences, San Francisco. } \\ \text { CHUJO } & \text { Dr. Michio Chûjô collection, Fukuoka. } \\ \text { EHIME } & \text { Ehime University, Matsuyama. } \\ \text { FREY } & \text { G. Frey Museum, Tutzing bei Mtinchen. } \\ \text { GENOVA } & \text { Museo Civico di Storia Naturale, Genova. } \\ \text { HAMBURG } & \text { Universitat Hamburg, Zoologisches Institute und Zoologisches Museum, } \\ \text { KIMOTO } & \text { Hamburg. } \\ \text { KU } & \text { S. Kimoto collection, Kurume. } \\ \text { MCZ } & \text { Entomological Laboratory, Kyushu University, Fukuoka. } \\ & \text { Museum of Comparative Zoology, Harvard University, Cambridge, Massa- } \\ \text { MUNCHEN } & \text { chusetts. } \\ \text { ZMNH } & \text { Ooologische Staatsammlung, München. } \\ \text { PARIS } & \text { Musa Museum of Natural History, Osaka. } \\ \text { RU } & \text { Entomological Laboratory, University of the Ryukyus, Okinawa. } \\ \text { STOCKHOLM } & \text { Naturhistoriska Riksmuseum, Stockholm. } \\ \text { TAR1 } & \text { Taiwan Agriculture Research Institute and Taiwan University, Taipei. } \\ \text { ZMB } & \text { Zoologisches Museum der Humboldt-Universität zu Berlin. }\end{array}$

The abbreviation "Umgeb." in geographical records refers to the German "Umgebung", meaning "Environs of".

In the course of the study I am grateful to the authorities and staff members at Bishop Museum, Honolulu ; British Museum (Natural History), London; Museum National d'Histoire Naturelle, Paris ; Universitat Hamburg, Zoologisches Institute und Zoologisches Museum, Hamburg ; Institute royal des Sciences Naturelles de Belgique, Bruxelles ; Museo Civico di Storia Naturale, Genova ; Zoologische Staatsammlung, Mtinchen ; Naturhistorisches Museum, Base1 ; Naturhistoriska Liks museum, Stockholm ; Kasetsart University and Agriculture Department, Bangkhen ; Kyushu University, Fukuoka ; and Ehime University, Matsuyama.

Also, I am especially obliged to Drs. R. D. Pope, M. T. Tompson, S. L. Shute, P. Gilbert, Nicole Berti, H. Stümpel, G. Scherer, R. L. A. Damoiseau, R. Poggi, W. Wittmer, M. Brancucci, P. I. Persson, the late J. L. Gressitt, G. A. Samuelson, Y. Hirashima, K. Morimoto, S. Asahina, T. Ishihara, S. Azuma, Y. Miyatake, K. Baba and the late J. A. Rondon. 


\section{Key to Genera of Galerucinae}

1 Antennal insertions generally close, at level of anterior margins of eyes or further anterior ; vertex and pronotum generally deeply punctate ; in male last abdominal segment with a triangular or rounded depression with posterior border often emarginate, but never trilobed

Antenna1 insertions generally separated, situated near, but behind, anterior margins of eyes; when separated or placed further forward, vertex and pronotum not heavily punctate; last abdominal segment of male trilobed, with median lobe always distinct

2(1) Mesosternum free, horizontal or inclined, not covered by a process of metasternum

Mesosternum with largely covered by an anterior process of metasternum .............. 94

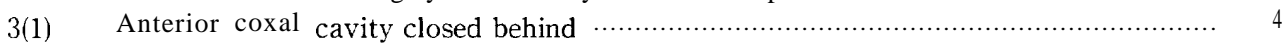

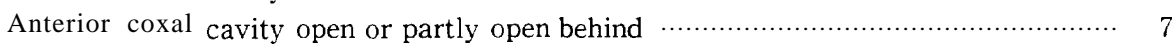

4(3) Lateral border of pronotum rounded, widest near middle ; elytron hardly wider than prothorax basally

Lateral border of pronotum squarish; elytron distinctly wider than prothorax

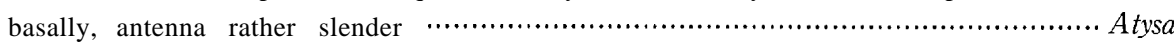

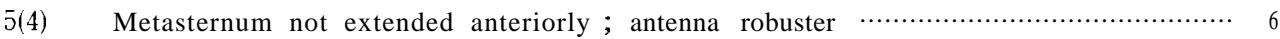

Metasternum extended anteriorly into high triangular process, dorsal surfaces

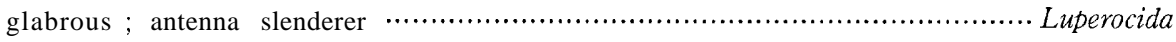

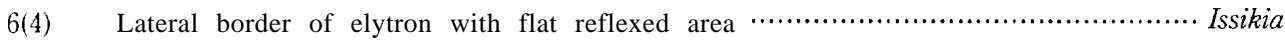

Lateral border of elytron without flat reflexed area $\ldots \ldots \ldots \ldots \ldots \ldots \ldots \ldots \ldots \ldots \ldots \ldots$ Menippus

7(3) Primary setigerous pore on anterior part of lateral margin of pronotum ................ 8

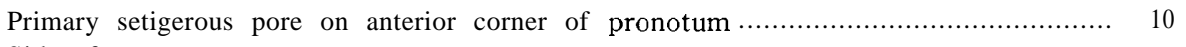

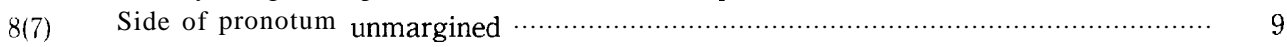
Side of pronotum distinctly margined ; claws bifid..................... Falsoplatyxantha

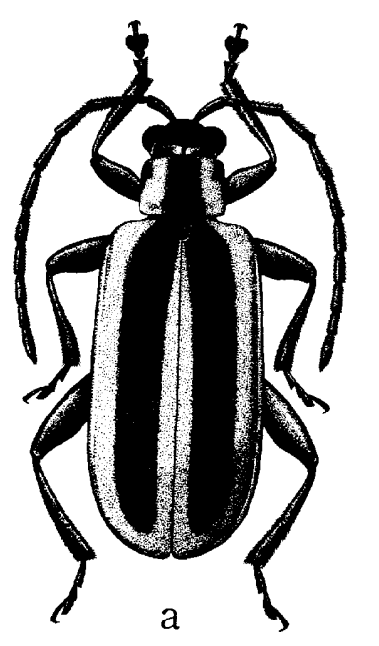

Fig. 1. a, Atysa marginata

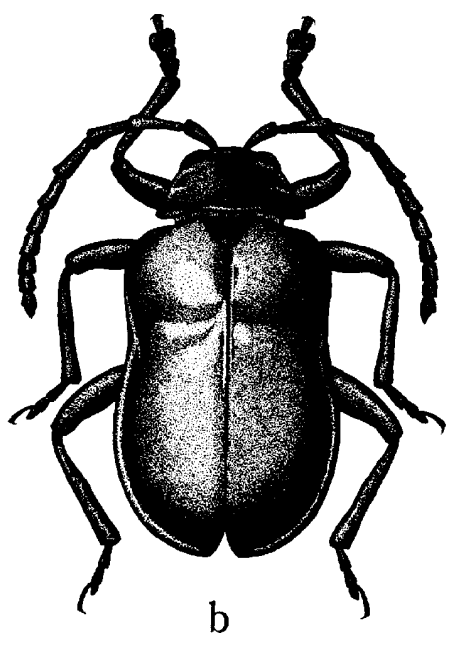

(Hope) ; b, Periclitena cyanea (Clark) ; c, Oides duporti Laboissière. 
9(8) Claws bifid in male, appendiculate in female ; elytron without costa along lateral margin

Claws bifid ; elytron with a costa running closely along elytral margin until at the apex

10(7) Lateral margin of pronotum entire

Lateral margin of pronotum disappearing towards anterior $1 / 3$; dorsal surfaces glabrous, densely and grossly punctured ; anterior border of pronotum unmar gined

Pseudadimonia

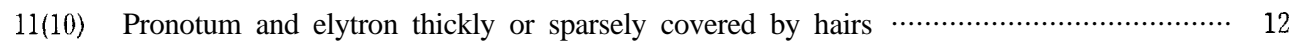

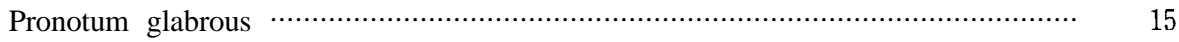

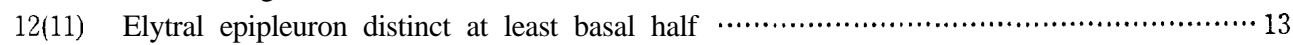

Elytral epipleuron distinct only on basal $1 / 4$ or so ……................................. 14

13(12) Disc of pronotum entirely covered by hairs, but in some cases anterior and lateral margins glabrous …….................................................................... Pyrrhalto

Disc of pronotum with a large glabrous space at middle

Galerucella

14(12) Antenna slender, in preapical segments three times as long as wide or much

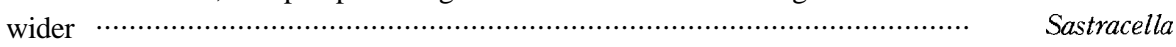

Antenna robust, in preapical segments twice as wide as long or more wider ..........

Clitena

15(11) Mesosternum free, horizontal or inclined, not covered by a process of metaster-

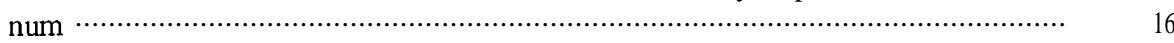

Mesosternum largely covered by an anterior process of metasternum ................. Doryxena

16(15) Elytral epipleuron distinct only on basal $1 / 4$; elytron thickly or sparsely covered by

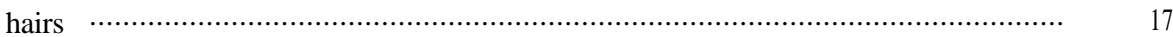

Elytral epipleuron distinct at least to apical $1 / 4$; elytron glabrous .................... Clitenella

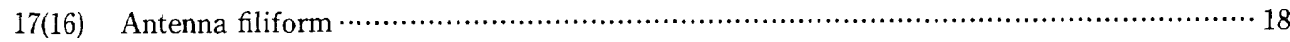

Antenna robust, especially apical four segments shorter than those of mid-

dle …............................................................................... Periclitena

18(17) First segment of posterior tarsus subequal to following segments combined ; third segment of antenna not longer than fourth .......................................... Sastroides

First segment of posterior tarsus longer than the following segments combined ; third segment of antenna at least $11 / 4$ times longer than fourth (Baly, 1865 ; type :

Sastra placida Baly: Mysol) ….......................................................... Sastra*

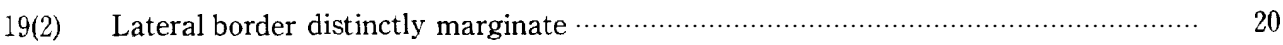

Lateral, anterior and posterior borders not marginate ; tarsal claws appen-

diculate …............................................................................... Meristoides

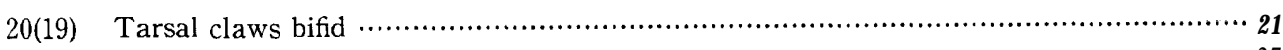

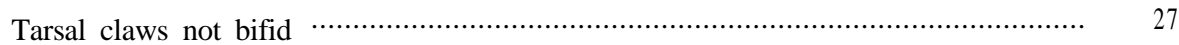

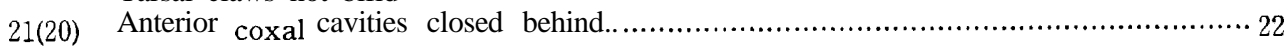

Anterior coxal cavities opened behind ……................................................. 23

22(21 Elytron glabrous ; anterior and posterior borders of pronotum not margined or hairy (Strand, 1935 ; = Merista Chapuis, 1875 ; type: Galleruca sexmaculata Kollar \& Redtenbacher ; Caschmir) …............................................................ Meristata ${ }^{*}$

Elytron pubescent ; pronotum with some fine hairs on anterior and posterior margins ; anterior and posterior borders of pronotum distinctly margined ............ Anadimonia

23(21) Pronotum with a transverse depression, sometimes interrupted at middle .................... 24 Pronotum without a transverse depression ; disc subevenly convex ; body broadly 
ovate ; elytral epipleuron wide, interior, recurved basally .............................. Oides

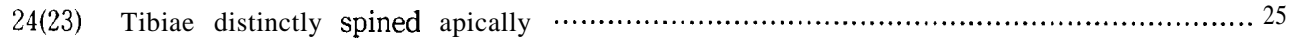

Tibiae not distinctly spined apically ; elytral epipleuron narrow but distinct almost

to apex

Hoplasoma

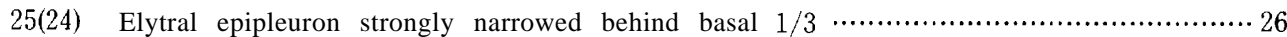

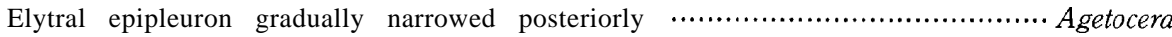

26(25) Elytral epipleuron continued to apex …............................................ Pseudocophora

Elytral epipleuron distinctly abbreviated behind middle ......................... Aulacophora

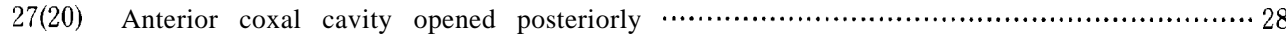

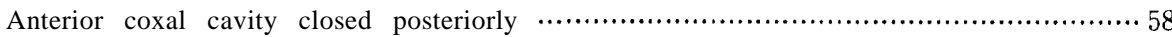

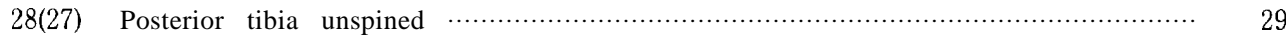

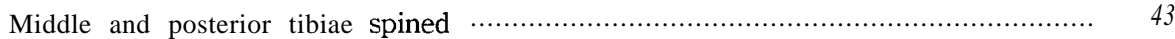

29(28) Anterior and posterior borders of pronotum unmargined .............................. 30

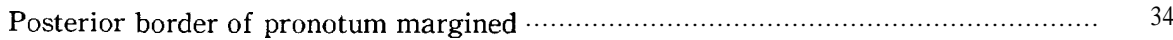

30(29) Pronotum with a distinct depression on each side or with a transverse depres-

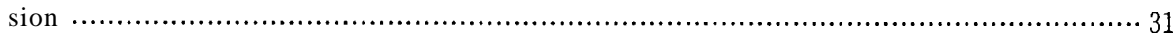

Pronotum convex, without any distinct depression.....$\ldots \ldots \ldots \ldots \ldots \ldots \ldots \ldots \ldots \ldots \ldots$ Kanarella

31(30) Posterior border of pronotum rectangularly emarginate near angle, which is dis-

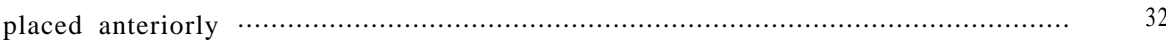

Posterior border of pronotum without rectangular emargination near angle, some-

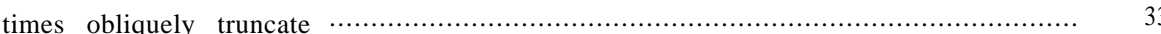

32(31) Elytron with two carinae behind humerus ...................................... Japonitata

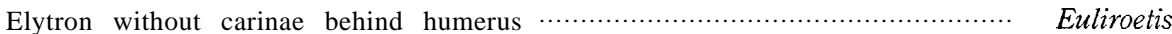

33(31) Pronotum not broader than long, with a longitudinal depression on each side ; fourth to eighth antennal segments each less than three times as long as broad; body

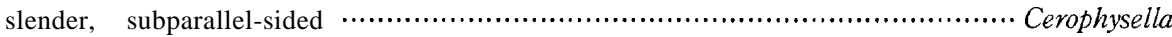

Pronotum at least $1 \frac{1}{2} 2$ times as broad as long, with a transverse groove behind middle which reaches side ; antennal segments beyond third at least three times as

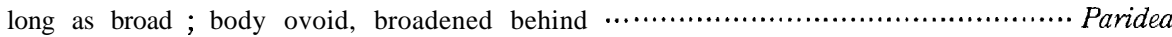

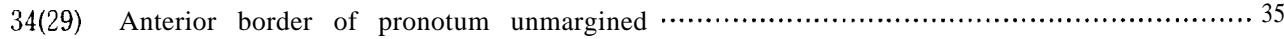

Anterior border of pronotum margined ; elytron more or less glabrous ................ 39

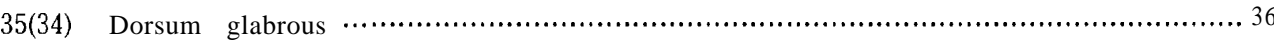

Dorsum with erect hairs ; body slender, parallel-sided .......................... Trichomimastra

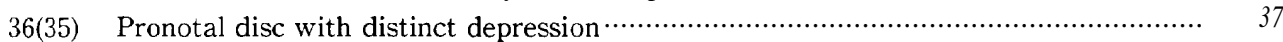

Pronotal disc convex, without depression ; body laıge, elongate oval ; epipleuron

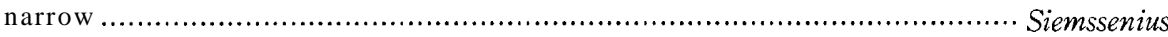

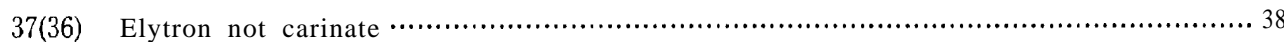
Elytron longitudinally carinate behind humerus, generally with a groove separating two carinae posteriorly ; gena short

Haplosomoides

38(37) Gena not shorter than smallest diameter of eye ; groove behind frontal tubercle complete; frons of male with a large deep cavity and with a prominent process near antennal insertions

Fleutiauxia

Gena much shorter than depth of eye; groove behind frontal tubercle disappearing

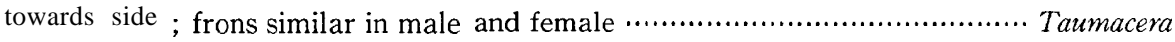

39(34) Mesosternum narrow and not connected with metasternum .............................. 40

Mesosternum wider and distinctly connected with metastemum …............. Cneoranidea

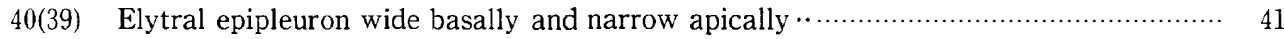



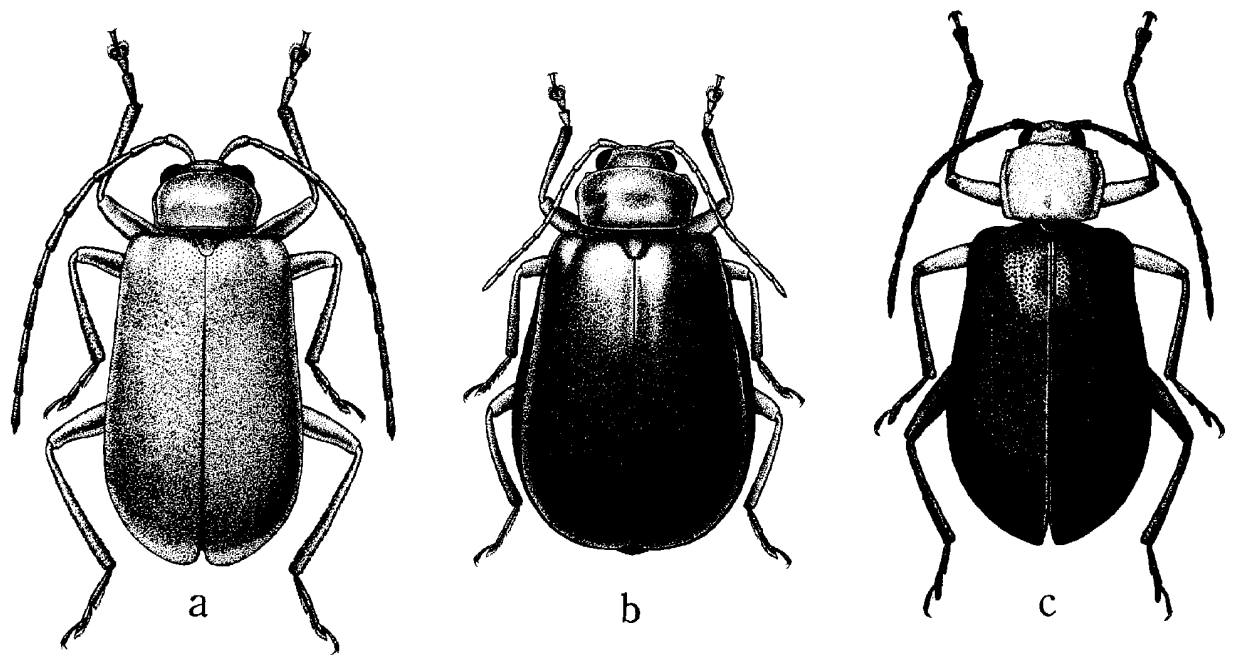

Fig. 2. a, Trichomimastra hirsuta(Jacoby); b, Pseudocophora flaveola Baly ; c, Cneorane femoralis Jacoby.

Elytral epipleuron narrow basally

Liroetis

41(40) Pronotum convex, lateral margin rounded, without any distinct transverse depression dorsally

Pronotum subquadrate, with distinct transverse depression dorsally

Mimastra

42(41) Labrum with numerous irregularly arranged setigerous pores ; pronotum less transverse, less than twice as wide as long

Cneorane

Labrum with a row of six to eight setigerous pores ; pronotum transverse, more than twice as wide a long

Cneorella

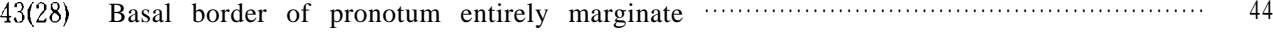
Basal border of pronotum not marginate except near side …..................... Bangprella

44(43) First segment of posterior tarsus distinctly shorter than remainder com-

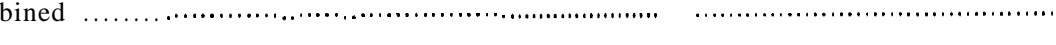

First segment of posterior tarsus as long as, or longer than, remainder combined

45(44) Preapical segments of maxillary palpus large, rounded, fourth much smaller than third and somewhat cubical

Preapical segments of maxillary palpus not so large, slender, fourth not much smaller than third and not cubical

46(45) Antenna filiform but not very slender ; elytral epipleuron distinct only before middle

Morphosphaera

In male second and third antennal segments minute and fourth to tenth with fairly long projections endoapically; in female second antennal segment minute, third twice as long as second, and fifth to eleventh each somewhat flattened and fifth to tenth slightly produced endoapically ; elytral epipleuron distinct until apex

Miltina

47(45) Elytron without any reflexed area on suture

Elytron with posterior portion of suture reflexed upwards and forming a narrow 
smooth space; disc distinctly convex basally, then transversely impressed ; epipleuron broad postmedially, narrowed apically

Parexosoma

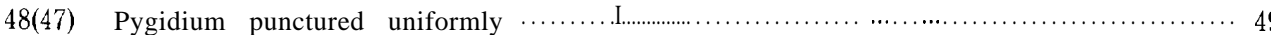

Basal half of pygidium shiny, glabrous, finely shagreened, apical half densely punctured, pubescent ; lateral margin of elytron visible from above ; body ovoid, dilated posteriorly; tibiae carinate and finely gooved above (Chevrolat, 1837 ; type :

Chrysomela alni Linnaeus ; Europe)

Agelastica *

49(48) Third antennal segment almost, or more than, twice as long as second …..................50

Third antennal segment as long as, or $11 / 2$ times as long as second $\ldots \ldots \ldots \ldots \ldots \ldots \ldots \ldots . . . . . . . . . .51$

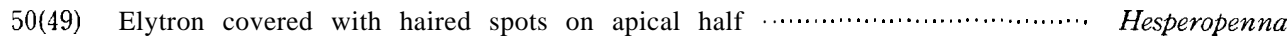

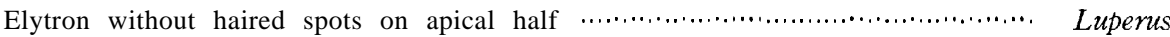

51(49) Prosternal process narrow and not elevated between coxal cavities and not separating them

Calomicrus

Prosternal process elevated between coxal cavities, separating them, at least anteriorly (Jacoby, 1903 ; type : Chrysomela lusitanica Linnaeus ; Europe) …............. Exosoma*

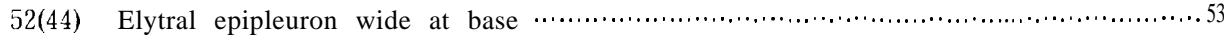

Elytral epipleuron narrow, feebly and gradually narrowed from base to apex ; pronotum transversely rectangular with straight sides and with distinct sublateral depression ; hind femur slightly thickened (Ogloblin, 1936 ; type :Luperus potanini Weise ; W. China)

Stenoluperus*

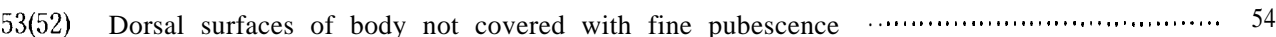
Dorsal surfaces of body covered with fine pubescence; body oval …........... Trichosepharia

54(53) Anterior margin of labrum entire ; frontal tubercles not widely separated by frons ; frons not depressed but with a longitudinal interantennal ridge $\ldots \ldots \ldots \ldots \ldots \ldots \ldots \ldots \ldots \ldots . \ldots . \ldots \ldots$ Anterior margin of labrum emarginate; frontal tubercles widely separated by frons ; frons broad, depressed in middle, without a longitudinal inter-antennal ridge

Sinoluperus

55(54) Head with frontal tubercle elongate-triangular with anterior angle acute, inserted

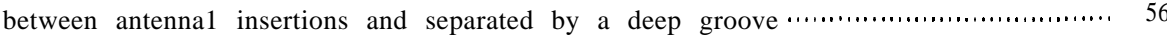
Head with frontal tubercle transverse, with anterior angle short and its apex inserted between these angles or confluent with them

56(55) Antenna robuster, in preapical segments less than three times as wide as long ; pronotum as long as or slightly wider than long

Medythia Antenna slenderer, in preapical segments more than three times as long as wide ; pronotum transverse, much wider than long

Liroetiella

57(55) Antenna robuster, in preapical segments less than three times as long as wide Desbordesius Antenna slenderer, in preapical segments more than three times as wide as

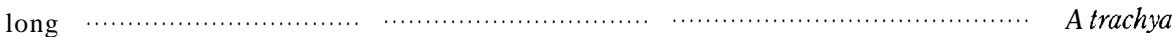

$58(27)$ First segment of posterior tarsus much longer than remainder combined ; tibia with

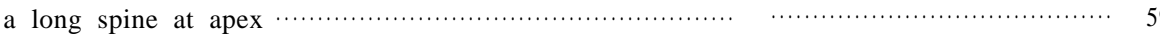
First segment of posterior tarsus usually shorter than, or sometimes subequal to, remainder combined

59(58) Elytral epipleuron gradually narrowed behind, and wider at middle than $1 / 2$ width

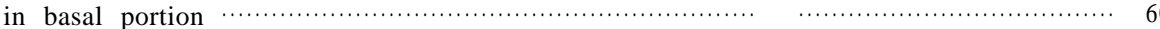
Elytral epipleuron suddenly narrowed at end of basal $1 / 3$ and distinctly narrower at middle than $1 / 2$ width in basal portion Monolepta

60(59) Apex of elytron not truncate but rounded 

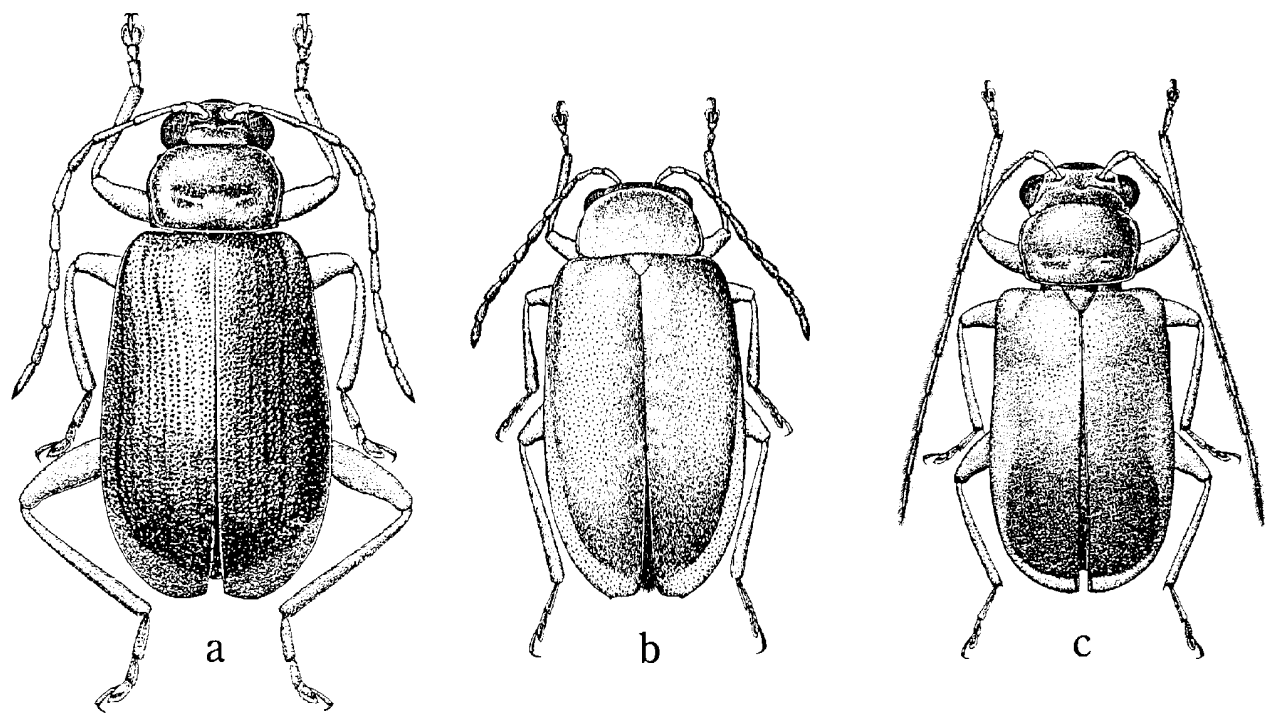

Fig. 3. a, Platyxantha indica (Jacoby); b, Pseudoides pectoralis (Laboissière); c, Palpoxena coeruleipennis (Baly).

Apex of elytron truncate

61(60) Pronotum with basal margin entirely marginate; interocular space much narrower than transverse diameter of eye ; elytron with lateral margin subparallelsided Liroetoides

Pronotum with basal margin marginate only on near side ; interocular space much wider than transverse diameter of eye ; elytron with lateral margin rounded .... Sermyloides

62(60) Elytron subparallel-sided or feebly rounded, truncated apically; elytral epipleuron not unusually wide 63

Elytron strongly widened and rounded laterally; elytral epipleuron abnormally wide (Laboissière, 1936 ; type : Sepharia dilatipennis Fairmaire ; W. China) Pseudosepharia*

63(62) Head without any excavation in male; in many species elytron with a pair of depression subbasally Paleosepharia Head with excavation in male ; elytron without any depression subbasally (Baly,

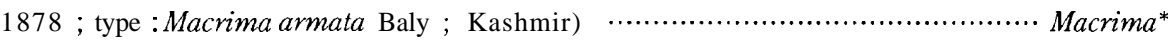

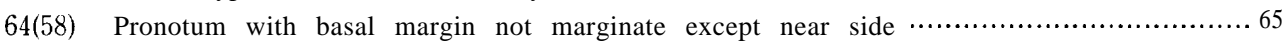

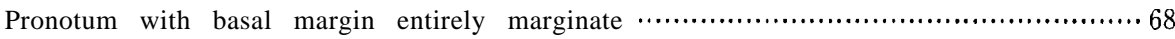

65(64) Pronotum with posterior comer rounded, subobtuse, and transverse furrow or

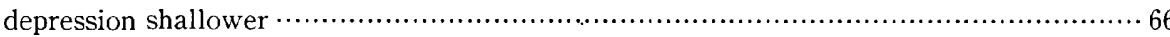

Pronotum with posterior corner distinctly angulated and transverse furrow deepAplosonyx

66(65) Antenna robust, second and third segments subequal 67 Antenna slender, third segment twice as long as second Leptarthra

67(66) Pronotum with a large and deep fovea on each side of middle (Reitter, 1912 ; type :

Chrysomela halensis Linnaeus ; Europe) Seymylassa* Pronotum without a distinct fovea laterally Sphenoraia 
68(64) Pronotum with a pair of short longitudinal furrows which start from basal margin $\ldots . . .69$

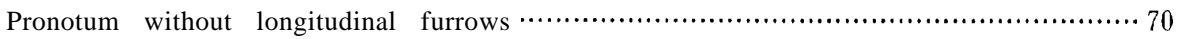

69(68) Body suboval, moderately convex ….......................................... Cassena

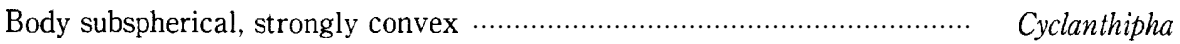

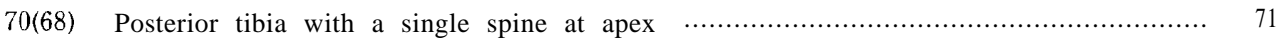

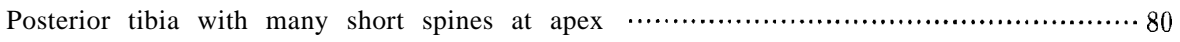

71(70) Pronotum without any depression laterally ; antenna longer than half as long as

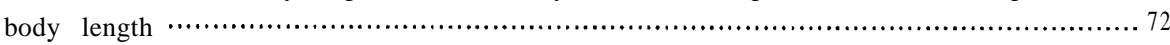

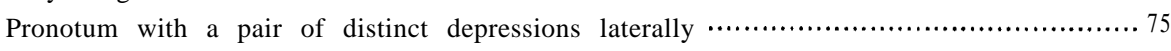

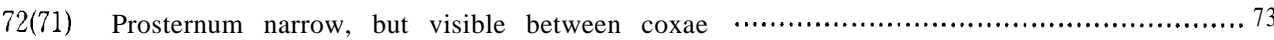

Prosternum not extending between coxae (Weise, 1886 ; type : Crioceris xanthopus

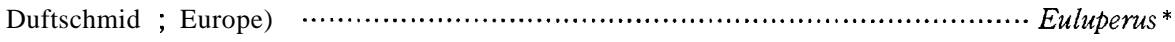

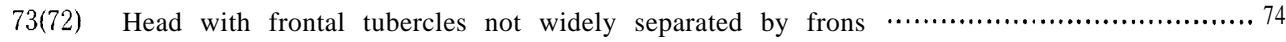

Head with frontal tubercles widely separated by frons, elytron with more or less irregularly arranged in paired rows of punctures, and their interstices slightly

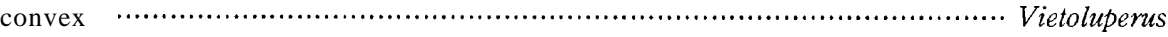

74(73) Antenna slender, fifth to eighth segments more than twice as long as wide ...... Pseudoides Antenna robust, fifth to eighth segments nearly $11 / 2$ times as long as wide ..... Eumelepta

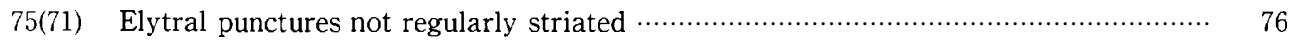

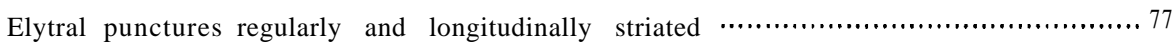

76(75) Head with frontal tubercles not widely separated by frons, anterior margin of

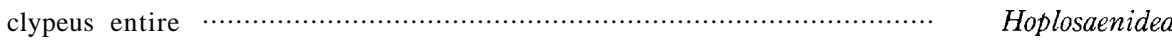

Head with frontal tuberbles widely separated by frons, anterior margin of clypeus

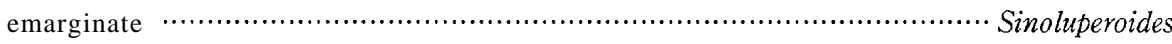

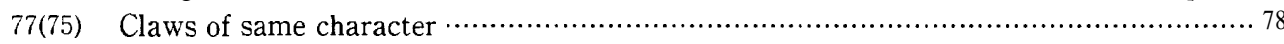

Claws not of same character; anterior and middle claws normal, appendiculate, posterior claw long, curved; dorsal surfaces sparsely covered with fine

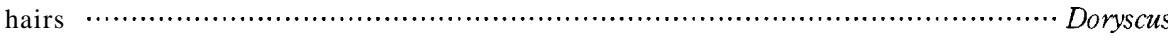

78(77) Each elytron with double rows of punctures with an indistinct short scutellar row,

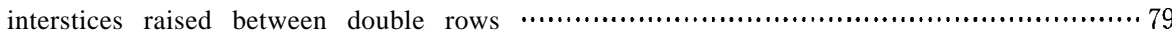

Each elytron with eleven longitudinal rows of punctures including a short scutellar row, the rows equidistant ; pronotum without distinct depressions ................. Strobiderus

79(78) Dorsal surfaces of elytron thickly covered by hairs .................................. Trichobalya

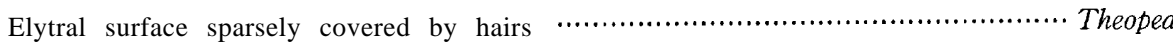

80(70) Pronotum not very short, with or without a pair of depressions ; elytron without rugosities

Pronotum very short, with a large depression which covers much of surface ; elytral epipleuron narrow and disc with rugosities ............................................. Paraplotes

81(80) Eye rather small ; gena subequal to, or deeper than, half transverse diameter of an

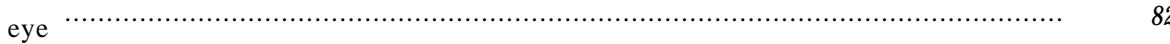

Eye rather large ; gena shallower than half transverse diameter of an eye $\cdots . . . \cdots \cdots \cdot \cdots \cdot \cdots \cdot 84$

82(81) Elytron without any trace of costae, sparsely and confusedly impressed by fine

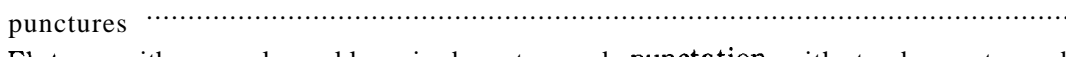

Elytron with several weakly raised costae and punctation with tendency toward

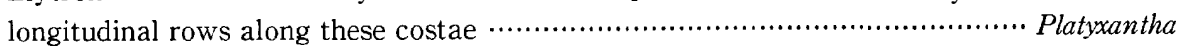

83(82) First segment of posterior tarsus subequal to following segments combined ; pronotum with a pair of lateral depressions ; elytral surface with transverse depres- 

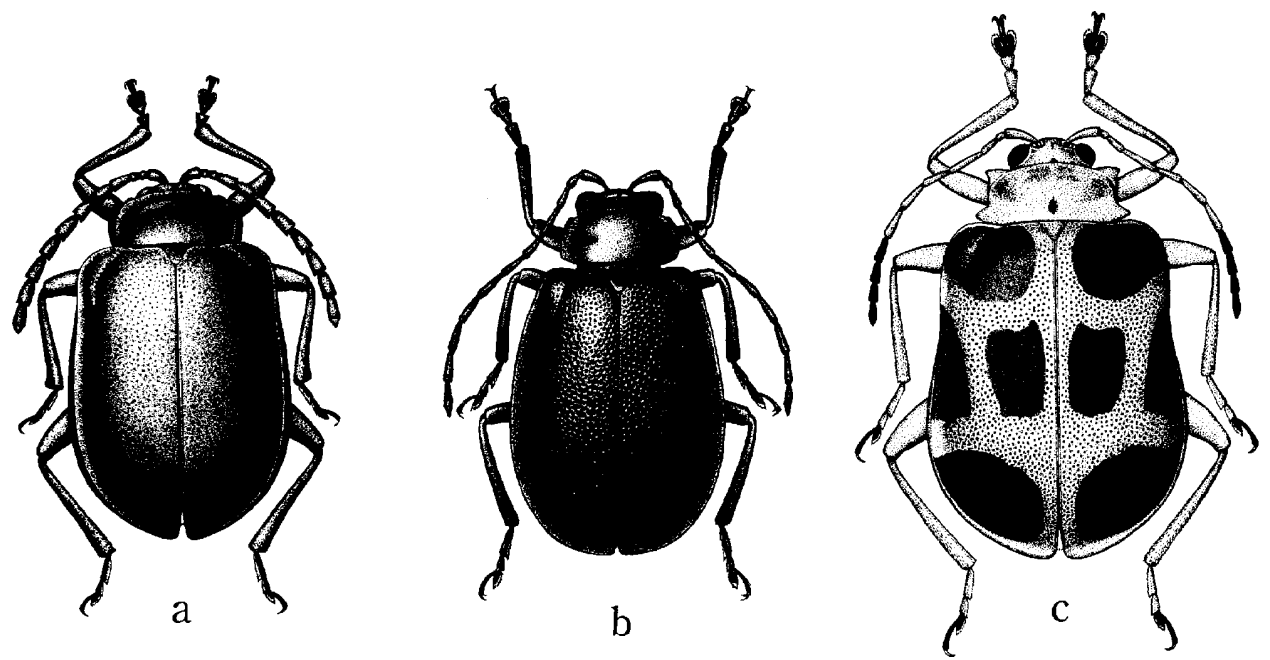

Fig. 4. a, Sphenoraia (Sphenoraioides) micans (Fairmaire); b, Cneorella suisapana (Gressitt \& Kimoto) ; c, Laphris sexplagiata Laboissière.

sion behind subbasal area

Palpoxena

First segment of posterior tarsus distinctly shorter than following segments com-

bined ; pronotum convex side to side, without any trace of depression laterally ;

elytron without any trace of transverse depression behind subbasal area ........ Laosixantha

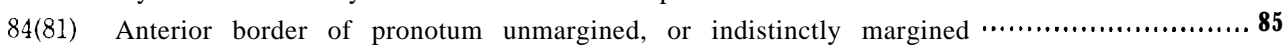

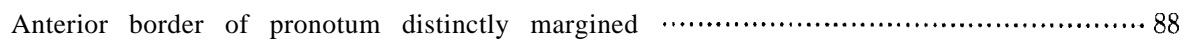

85(84) First tarsal segment of posterior leg slender, subequal to the length of remainder combined

First tarsal segment of posterior leg robust, distinctly shorter than or subequal to the length of remainder combined; depression of pronotum feebly impressed 87

86(85) Pronotum impressed by a pair of deep depressions laterally ...................... Hyphaenia Pronotum convex side to side without any distinct depression laterally...... Cassenoides

87(85) Gena $2 / 5$ to $1 / 2$ as deep as transverse diameter of eye ; antenna with fourth to tenth segments flattened above ; frons of male pitted or carinate

Acroxena

Gena about $1 / 4$ as deep as transverse diameter of eye ; antenna with fourth to tenth segments cylindrical ; frons of male triangulalr, weakly convex (Gressitt \& Kimoto, 1963 ; type : Epaenidea subviridis Gressitt \& Kimoto ; Hainan Is.)

Epaenidea*

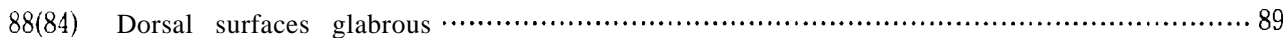

Dorsal surfaces closely covered with fine erect hairs ........................... Pseudeustetha

89(88) Gena distinctly narrower than $1 / 3$ of transverse diameter of an eye $\cdots \cdots \cdots \cdots \cdots \cdots \cdots \cdots \cdots \cdots \cdots \cdots \cdots$

Gena wider than, or subequal to, $1 / 3$ transverse diameter of an eye ; in male fourth antennal segment more than three times as long as length of second and third combined ; pronotum subquadrate (Weise, 1889 ; type: Proegmena pallidipennis Weise ; S. China) …............................................................ Proegmena

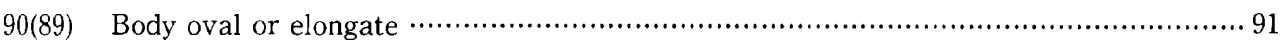

Body semispherical, pronotum convex side to side, in male third antennal segment 


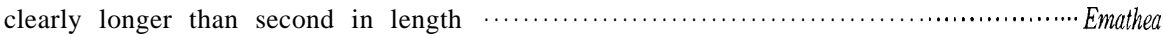

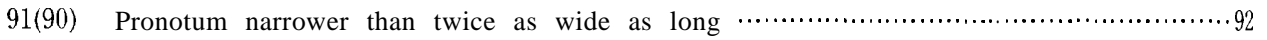

Pronotum nearly twice as wide as long or more wider, and its lateral margin

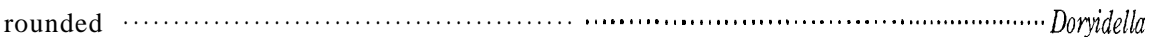

92(91) Pronotum with lateral margin rounded, narrowed anteriorly and posteriorly, and its surface convex from side to side, without any distinct depression laterally $\ldots . . . \ldots \ldots \ldots . . . .93$ Pronotum with lateral margin almost straight, widest almost at anterior margin and narrowed posteriorly, and its surfaces impressed by a pair of shallow depressions laterally; in male third antennal segment longer than second in length ….... Dercetisoma

93(92) Third antenna1 segment shorter than or subequal to second in length in male $\cdots$ Arthrotus Third antennal segment clearly longer than second in length in male .............. Dercetina

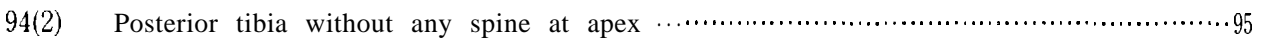

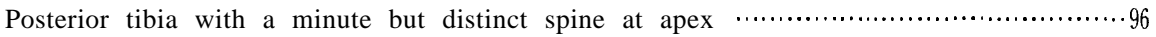

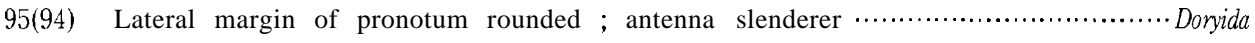
Lateral margin of pronotum almost straight and subparallel-sided ; antenna robus-

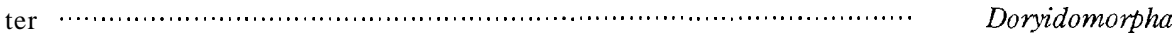

96(94) Third antennal segment four times as long as second ................................. Laphris Third antennal segment subequal to, or twice as long as second............... Gallerucida

\section{Genus Atysa Baly}

Atysa Baly, 1864, Trans. Ent Soc. London, ser. 3, $2: 238$ (type : Atysa terminalis Baly ; New Guinea).

Triaplatarthris Fairmaire, 1878, Ann. Soc. Ent. France, ser. 5, $8: 138$ (type :Triaplatarthris pyrochroides Fairmaire ; China).-Gressitt \& Kimoto, 1963, Pac. Ins. Mon., 1B: 391, 408.

Formosogalerucella Pic, 1928, Mél. Exot. Ent., 61 : 32 (type : Formosogalerucella brevithorax Pic; Formosa).

\section{Atysa marginata (Hope) Fig. la}

Auchenia marginata Hope, 1831, in Gray, Zool. Misc. : 29 (Nepal ; BM).

Atysa marginata : Maulik, 1936, Fauna India, Galeruc.: 245 (Nepal, W. Himalaya, Assam, Burma). -Kimoto\& Takizawa, 1972, Kontyû, Tokyo, 40(4): 216 (Nepal).

Atysa mureana Maulik, 1936, Fauna India, Galeruc. : 247 (Punjub ; BM).-Kimoto, 1982, Ent. Rev. Japan, 37(1):8 (= marginata).

Atysa sudiyana Maulik, 1936, Fauna India, Galeruc. : 248 (Assam ; BM).-Kimoto, 1982, Ent. Rev. Japan, 37(1): 8 (=marginata).

Triaplatarthirs collaris Gressitt \& Kimoto, 1963, Pac. Ins. Mon., 1B: 408 (S. China ; BISHOP). -Kimoto, 1982, Ent. Rev. Japan, 37(1):8 (= marginata).

Triaplatarthrismarginata Gressitt \& Kimoto, 1982, Pac. Ins. Mon., 1B: 408, 409, fig. (SE China ; BISHOP).-Kimoto, 1982, Ent. Rev. Japan, 37(1):8 (=Auchenia marginata Hope).

Distribution: India, Nepal, Burma, Vietnam, S. China.

Oblong, pronotum subquadrate, elytron subparallel-sided, dorsal surfaces entirely covered with fine hairs ; head, antenna, legs and ventral surfaces blackish, dorsal surfaces generally blackish with sutural and lateral margins of elytron reddish brown, in most dark colored specimen entirely blackish ; length $5.0-7.5 \mathrm{~mm}$.

Material examined. VIETNAM : Fyan, 1 ex., 11. vii.-9. viii. 1961, N. R. Spencer (BISHOP). 


\section{Genus Luperocida Medvedev \& Dang Dap}

Luperocida Medvedev \& Dang Dap, 1981, Rev, d'Ent.l'USSR,60(3) : 634 (type: Luperocida kabakovi Medvedev \& Dang Dap ; Vietnam).

According to the original description, this genus should be included in Galerucini in having the head and pronotum densely covered by punctures. This genus somewhat resembles Doryxena, in having the metasternum extended anteriorly, but is separable in having the anterior coxal cavity closed posteriorly and all tibiae with spine, long on posterior tibia.

\section{Luperocida kabakovi Medvedev \& Dang Dap}

Luperocida kabakovi Medvedev \& Dang Dap, 1981, Rev. d'Ent.l'USSR,60(3) : 640 (Vietnam). Distribution : Vietnam.

Body elongate-oval, weakly tapering anteriorly. Uniformly bright yellow, only apical segments of antenna slightly darkened at tip. Clypeus and front shining, with solitary punctures, vertex dull, shagreened, with dense fine punctation. Pronotum shagreened, with dense, smoothed-over shagreening and dense uniform punctation, somewhat larger than on pronotum. Scutellum and epipleuron smooth and shining. Length $10 \mathrm{~mm}$ (after translation by Entomological Rev., Wash. : 114).

No material was examined.

\section{Genus Issikia Chûjô}

Issikia Châjô, 1961, Pub. Ent. Lab Univ. Osaka Pref., 6 : 87 (type : Issikia issikii Châjô; Ryukyu Is.).-Kimoto, 1966, J. Fac. Agr Kyushu Univ., 13(4) : 669 (Isshikia; error).

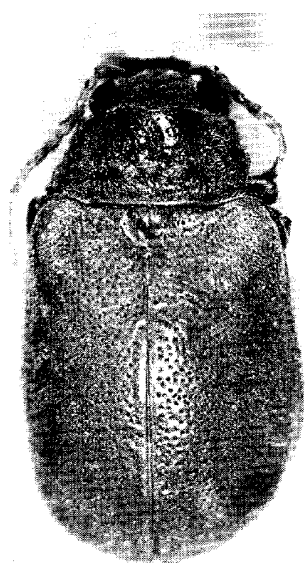

a

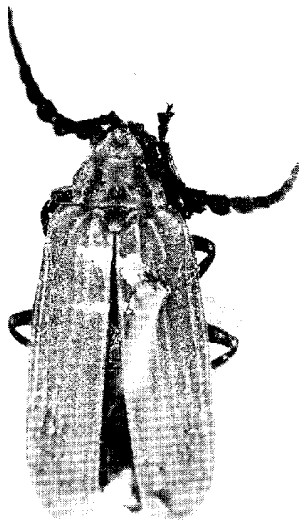

b

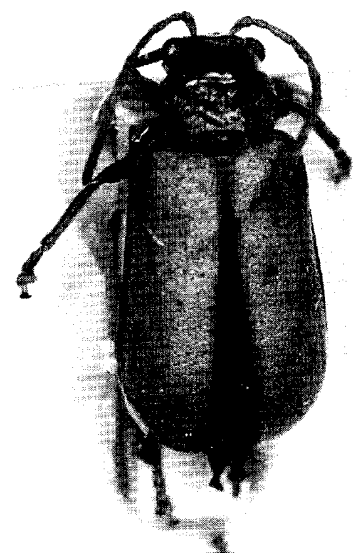

C

Fig. 5. a, Menippus cervinus (Hope) ; b, Falsoplatyxantha aurantiaca $\mathrm{Pic} \mathrm{;} \mathrm{c,} \mathrm{Poneridia} \mathrm{costata}$ (Allard). 


\section{Issikia dimidiaticornis (Jacoby), new combination Fig. 6a}

Menippus dimidiaticornis Jac., 1889, Ann. Mus. Civ. Genova, 27 : 218 (Burma ; BM).-Maulik, 1936, Fauna India, Galeruc. : 243 (Burma).

Galerucella sericea Weise, 1889, Horae Soc. Ent. Ross., 23 : 569, 622 (China ; ZMB).

Galerotella garoana Maulik, 1936, Fauna India, Galeruc.: 271 (Assam ; BM).-Kimoto, 1979, Ent. Basiliensia, $4: 464$ (= dimidiaticomis).

Pyrrhalta sericea: Gressitt \& Kimoto, 1963, Pac. Ins. Mon., 1B: 442, 464, fig. (China).-Kimoto, 1969, Esakia, Kyushu Univ., $7: 24$ (Taiwan) ; 1979, Ent. Basiliensia, $4: 464$ (= dimidiaticomis).

Pyrrhalta dimidiaticomis : Kimoto, 1979, Ent. Basiliensia, $4: 464$ (India).

Distribution : India, Burma, Thailand, Laos, Vietnam, China, Taiwan.

Suboval, slightly depressed, dorsal surfaces closely convered by fine hairs; generally yellowish brown, with apex of each antennal segment blackish ; length $6.8-8.3 \mathrm{~mm}$.

Material examined. THAILAND: Phet Buri, 10 exs., 13. i. 1963 (BANGKHEN) ; Banna, Chawang nr. Nabon, 70 m, 1 ex., 5. ix. 1958, J. L. Gressitt (BISHOP). LAOS : Vientiane Prov., Ban Van Eue, 2 exs., 30. i. 1966, 1 ex., 31, v. 1966, Native collr ; Khammouane Prov., Phon Tiou, 1 ex., 30. iv. 1965, Native collr (BISHOP) ; Umgeb. Vientiane, 2 exs., iii.-vi. 1963 (MUNCHEN). VIETNAM : Ban Me Thuot, 500 m, 2 exs., 16-18. v. 1960, S. Quate \& L. Quate ; Dak Song, 76 km SE of Ban Me Thuot, 870 m, 1 ex., 19-21. v. 1960, S. Quate \& L. Quate ; Mt. Lang, Bian, 1,500-2,000 m, 1 ex., 19. v.8 vi. 1961, N. R. Spencer ; Dilinh (Djiring), 1,200 m, 1 ex., 22-28. iv. 1960, S. Quate (BISHOP).

\section{Genus M enippus Clark}

Menippus Clark, 1864, J. Ent., 2 : 257 (type : Menippus cynicus Clark ; Queensland).-Chapuis, 1875, Gen. Col., $11: 220$, 222.-Maulik, 1936, Fauna India, Galeruc. :241.-Gressitt \& Kimoto, 1963, Pac. Ins. Mon., 1B: 411.

\section{M enippus cervinus (Hope) Fig. 5a}

Galleluca cervina Hope, 1831, in Gray, Zool. Miscel. : 29 (Nepal ; BM).

Menippus canellinus Fairm., 1888, Ann. Soc. Ent. France, ser. 6, $8: 374$ (Tonkin; PARIS). -Gressitt \& Kimoto, 1963, Pac. Ins. Mon., 1B: 411 (China, Vietnam). New synonym.

Menippus cervinus: Maulik, 1936, Fauna India, Galeruc. : 242 (Nepal, United Provinces, Burma). -Kimoto \& Takizawa, 1972, Kontyû, Tokyo, 40(4) : 215 (Nepal).

Distribution : India, Nepal, Burma, Thailand, Laos, Vietnam, China, Hainan.

Suboval, strongly convex ; dorsal surfaces thickly covered by adpressed fine hairs; generally yellowish brown, ventral surfaces and legs mostly pitchy brown; length 6.9-8.7 mm.

Material examined. THAILAND : Chiengmai, 1,100-1,500 m, 3 exs., 1966, J. Sedlacek (BISHOP). LAOS : Vientiane Prov., Vientiane, 1 ex., iii. 1966, J. Sedlacek (BISHOP).

\section{Genus Falsoplatyxan t ha Pic}

Falsoplatyxantha Pic, 1927, Mél. Exot. Ent., 49 : 23 (type : Falsoplatyxan tha auran tiaca Pic ; Vietnam). 


\section{Falsoplatyxantha aurantiaca Pic Fig. 5b}

Falsoplatyxantha aurantiaca Pic, 1927, Mél. Exot. Ent., 49 : 23 (Tonkin; PARIS).

Distribution : Vietnam.

No additional material was examined beside the type series.

\section{Genus Apophylia Thomson}

Apophylia Thomson, 1858, Arch. Ent., 2 : 221 (type: Apophylia chloroptera Thomson ; Gabon). -Maulik, 1936, Fauna India, Galeruc. : 78.-Ogloblin, 1936, Fauna USSR, 26, 1 : 138, 369. -Châjô, 1962, Phil. J. Sci., 91 (1-2) :18.--Gressitt \& Kimoto, 1963, Pac. Ins. Mon., 1B; 391, 426.

-Kimoto, 1965, J. Fac. Agr. Kyushu Univ., $13: 291$.

Malaxia Fairmaire, 1878, Ann. Soc. Ent. France, ser. 5, $8: 139$.

Glyptolus Jacoby, 1884, Notes Leyden Mus., $6: 62$.

Galerucesthis Weise, 1896, Dtsche Ent. Z., 1896 : 296.

\section{KeY TO SPECIES OF Apophylia}

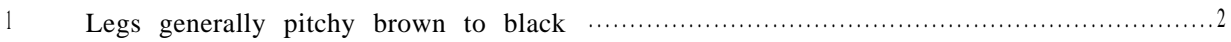

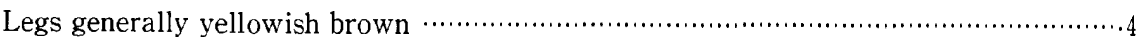

2(I) Pronotum generally with deep excavation or depression dorsally $\ldots \ldots \ldots \ldots \ldots \ldots \ldots \ldots \ldots \ldots$ Pronotum without deep excavation or depression dorsally, mostly dark brown ; generally pitchy black to brown, clypeus yellowish brown, with tibiae and tarsi brownish, elytron brownish green ; in male posterior femur expanded subapically and bearing a tuft of hairs in middle of posterior femur, and antenna more closely covered by long hairs ; length $4.0-5.3 \mathrm{~mm}$.......................................................... purpurea

3(2) Pronotum entirely blackish or bluish; generally black, clypeus and labrum reddish to yellowish brown, elytron dark blue ; length $5.0-5.5 \mathrm{~mm} \ldots \ldots \ldots \ldots \ldots \ldots \ldots \ldots . . .$. 'cyanipennis Pronotum yellowish brown with a large blackish marking on middle ; generally pitchy black, head black with clypeus and labrum reddish to yellowish brown; length 8-9 $\mathrm{mm}$

epipleuralis

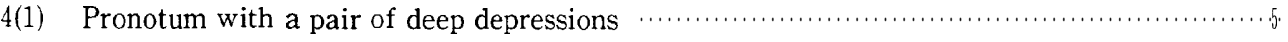
Pronotum without deep depression

5(4) Pronotum entirely black; generally black, with clypeus and legs entirely yellowish brown, antenna pitchy black, elytron greenish ; length $4.5-5.2 \mathrm{~mm} \ldots \ldots \ldots \ldots \ldots \ldots \ldots$ pallipes Pronotum yellowish brown with a distinct blackish marking on middle, in pale colored specimen entirely brownish; generally black, clypeus pitchy brown, legs yellowish brown, in most specimens ventral surfaces of femora and tibiae and entire tarsi pitchy

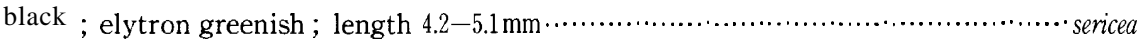

6(4) In male metathorax with distinct tubular projection; ventral surfaces partly brown-

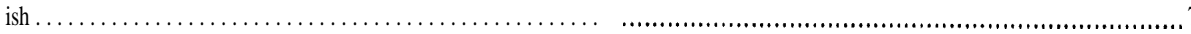

In male metathorax without any remarkable tubular projection; ventral surfaces generally pitchy black; head yellowish brown with vertex black; pronotum entirely yellowish brown, legs entirely yellowish brown, elytron greenish ; length 4.5-4.8

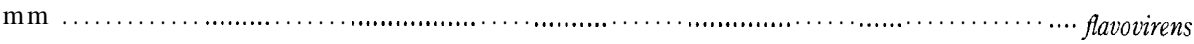

7(6) In male metastemum armed with a robust tubular projection posteriorly and markedly bifurcated apically, and anterior tibia markedly expanded medially ; ventral surfaces 
generally pitchy black, in some specimen abdomen partly or entirely brownish ; head yellowish brown with vertex black, pronotum and legs yellowish brown, elytron greenish ; length $5.0-5.8 \mathrm{~mm}$ fulcigera

In male metasternum strongly prominent into a peculiar beak-like projection apically; ventral surfaces pitchy brown, with abdomen largely yellowish brown ; head yellowish brown with vertex black, pronotum and legs yellowish brown, elytron greenish; length $5.0-5.3 \mathrm{~mm}$ securigera

\section{Apophylia cyanipennis Laboissière}

A pophylia cyanipennis Lab., 1927, Ann. Soc. Ent. France, $96: 59$ (Tonkin HAMBURG). Distribution : Vietnam.

No additional material was examined beside the type series.

\section{Apophylia epipleuralis Laboissière Fig. 6b}

A pophylia epipleuralis Lab., 1927, Ann. Soc. Ent. France, 96 : 60 (Tonkin: Ha-Giang ; Yunnan ; HAMBURG, PARIS).-Gressitt \& Kimoto, 1963, Pac. Ins. Mon., 1B: 427, 428 (China, Hainan). A pophylia geniculataPic, 1931, Mel. Exot. Ent., 57 : 21 (China ; PARIS).-Gressitt \& Kimoto, 1963, Pac. Ins. Mon., 1B: 428 (= epipleuralis).

Distribution : Thailand, Vietnam, S. China, Hainan.

Material examined. THAILAND : Chiengmai Prov., Doi Suthep, 1,278 m, 1 ex., 29. iii.-4. v. 1958, T. C. Maa (BISHOP).

\section{Apophylia flavovirens (Fairmaire) Fig. 6c}

Malaxia flavovirens Fair-m., 1878, Ann. Soc. Ent. France, ser. 5, 8 : 139 (C. China ; PARIS).

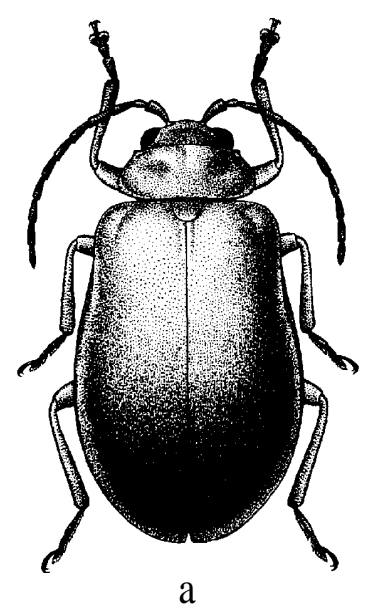

a

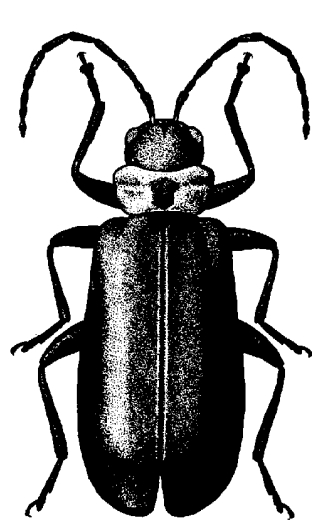

b

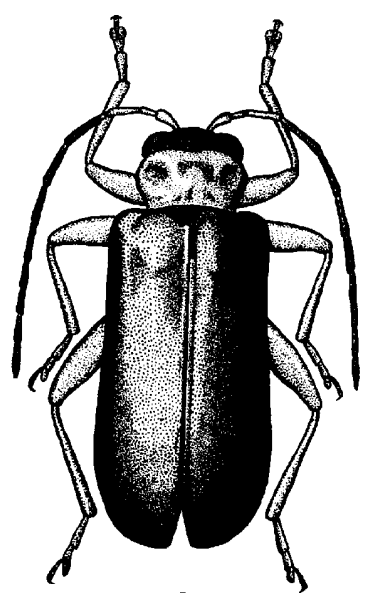

Fig. 6. a, Issikia dimidiaticornis (Jacoby'); b, Apophylia epipleuralis Laboissière ; c, $\cdot A$. flavovirens (Fairmaire). 
A pophylia pectoralis Pic, 1927, Bull. Soc. Zool. France, 52 : 189 (Laos ; PARIS). New synonym.

A pophylia flavovirens: Ogloblin, 1936, Fauna USSR, 26, 1: 141,393 (Tonkin, Annam).—Gressitt \& Kimoto, 1963, Pac. Ins. Mon., 1B: 427, 428 (China, Hainan).-Kimoto, 1965, Ann. Naturhist. Mus. Wien, $68: 489$ (Taiwan).

Distribution : Thailand, Laos, Vietnam, China, Hainan, Taiwan.

Material examined. THAILAND : Chiengmai Prov, Fang, 1 ex., 14. vi. 1965, K. Morimoto (KU). LAOS : Vientiane Prov., Ban Van Eue, 1 ex., 31. i. 1967., Native collr ; Luang Prabang, 300 m, 5 exs., 11-12. vi. 1965, light trap, S. Quate \& L. Quate ; Ban Theuong, $18 \mathrm{~km} \mathrm{NW}$ of Xieng Khouang, 1,035 m, 1 ex., 2-6. viii. 1960, R. E. Leech (BISHOP) ; Umgeb. Paklay, 1 ex., 1963 (MUNCHEN). VIETNAM : Ban Me Thuot, 500 m, 2 exs., 16-18. v. 1960, L. W. Quate ; 31 km S. of Dilinh (Djiring), 1,050 m, 1 ex., 29. 1960, R. E. Leech (BISHOP).

\section{Apophylia fulcigera Chûjô}

A pophylia fulcigera Châjô, 1962, Nature and Life in SE Asia, Kyoto, 2 : 103, figs. (Thailand ; CHUJO) ; 1954, ibid., $3: 284$ (Thailand).

Distribution : Thailand, Laos, Vietnam.

Material examined. THAILAND : Khao Yai Nat. Park, 2 exs., 6. vi. 1965, Y. Miyatake (KU). LAOS : Borikhane Prov., Pakkading, 8 exs., 13. vii. 1965, Native collr (BISHOP) ; Umgeb. Paklay, 1 ex., 1963 (MUNCHEN). VIETNAM : Pleiku, 700 m, 1 ex., 8-14. v. 1960, L. W. Quate ; 20 km N. of Pleiku, 650 m, 6 exs., 9. v. 1960, S. Quate ; 34 km N. Phan Rang, 3 exs., 9. xi. 1960, C. M. Yoshimoto (BISHOP).

It is rather difficult to separate this species from flavovirens in female. The followings are all female specimens and here identified as fulcigera with some question. THAILAND : Khon Kaen, 1 ex., 13. x. 1972, M. Sato (EHIME). LAOS : Vientiane Prov., Gi Sion Vill., de Tha Ngone, 1 ex., 2. i. 1966, Native collr ; Sedone Prov, Pakse, 1 ex., 2. viii. 1965, Native collr ; Sedone Prov., Paksong, 1 ex., 5. vii. 1965, Native collr; Muong Sing, NW of Luang Prabang, 650 m, 1 ex., 6-10. vi. 1960, S. Quate \& L. Quate (BISHOP). VIETNAM : Nha Ho, 14 km N. Phan Rang, 2 exs., 15. xi. 1960, C. M. Yoshimoto (BISHOP).

\section{A pophylia pallipes (Jacoby)}

Malaxia pallipes Jacoby, 1892, Ann. Mus. Civ. Genova, 32 : 969 (Burma ; GENOVA).

A pophylia pallipes: Maulik, 1936, Fauna India, Galeruc. : 86 (Burma, Assam).-Chajô, 1961, Nature and Life in SE Asia, Kyoto, 1 : 349 (Thailand) ; 1964, ibid., $3: 284$ (Thailand).-Kimoto, 1977, Ent. Basiliensia, 2 : 353 (Bhutan).

Distribution : India, Burma, Bhutan, Thailand.

Material examined. THAILAND : Chiengmai Prov., Doi Suthep, 1,300 m, 1 ex., 8. vi. 1965, P. D. Ashlock; Chiengmai Prov., Doi Pui, 1,685 m, 1 ex. 17. vi. 1965, P. D. Ashlock; Trang Prov., Khaophappha Khaochong, 200 m, 11 exs., 9-15. i. 1964, G. A. Samuelson ; Banna, Chawang, nr. Nabon, 70 m, 2 exs., 4-5. ix. 1958, J. L. Gressitt (BISHOP).

\section{A pophylia purpurea (Allard)}

Malaxia purpurea All., 1888 (1889), Ann. Soc. Ent. France, ser. 6, $8: 332$ (China ; HAMBURG) ; 1889, ibid., ser 6, $9: 312$ (Saigon) ; 1889, C. R. Soc. Ent. Belg., 33 : 81 (China).

A pophylia saigonensis Pic, 1927, Mel. Exot. Ent., $50: 2$ (Saigon ; PARIS). New synonym. 
Apophylia purpurea: Gressitt \& Kimoto, 1963, Pac. Ins. Mon., 1B: 427, 431 (China).

Distribution : Cambodia, Laos, Vietnam, China.

Material examined. CAMBODIA : Ph. Chisau, $40 \mathrm{~km} \mathrm{~S}$. of P. Penh, $20 \mathrm{~m}, 1$ ex., 29. iv. 1961, N. R. Spencer (BISHOP). LAOS : Muong Sing, NW of Luang Prabang, 650 m, 8 exs., 6-10. vi. 1960, S. Quate \& L. Quate (BISHOP).

\section{Apophylia securigera Chûjô}

A pophylia securigera Chajô, 1962, Nature and Life in SE Asia, Kyoto, 2 : 105, fig. (Thailand ; CHUJO) ; 1964, ibid., 3 : 285 (Thailand).

Distribution : Thailand, Laos.

Material examined. THAILAND : Chiengmai Prov., Fang, 500 m, 2 exs., 12. iv. 1958, 1 ex., 19. iv. 1958, T. C. Maa (BISHOP). LAOS : Luang Prabang, 1 ex., 11-12. vi. 1960, light trap, S. Quate \& L. Quate (BISHOP) ; Umgeb. Vientiane, 1 ex., iii-vi. 1963 (MUNCHEN).

\section{Apophylia sericea (Fabricius)}

Cantharis sericea Fabr., 1798, Suppl. Ent. Syst. : 69 (Tranquebariae).

Galleruca sericea: Fabr., 1801, Syst. El., 1 : 485 (India orientali).

Apophylia sericea: Maulik, 1936, Fauna India, Galeruc. : 84 (India, Eastern Himalayas).-Kimoto, 1977, Ent. Basiliensia, 2 : 354 (Bhutan).

Distribution : India, Bhutan, Thailand.

Material examined. THAILAND : Trang Prov., Khaophappha Khaochang, 200-400 m, 28 exs., 1-13. i. 1964, G. A. Samuelson ; Nakhon Nayok Prov., Khao Yai Nat. Park, 2 exs., 5-6. vi. 1965, P. D. Ashlock (BISHOP) ; Chieng Dao, 1 ex., 15. vi. 1965, K. Morimoto (KU).

\section{Genus Poneridia Weise}

Peneridia Weise, 1908, Nova Guinea, Zool., 5 : 323 (type: Galleruca australis Boheman ; Nova Hollandia).

\section{Poneridia costata (Allard) Fig. $5 c$}

Galerucella costata All., 1889, Ann. Soc. Ent. France, 58 : 312 (Annam; PARIS).

Poneridia costata : Laboissiere, 1935, Ann. Ass. Nat. Levallois-Perret, 21 : 144 (Indo-China).

Distribution : Vietnam.

No additional material was examined beside the type series.

\section{Genus Pseudadimonia Duvivier}

Pseudadimonia Duviv., 1891, C. R. Soc. Ent. Belg., 35 : 46 (type: Colaspis variolosa Hope ; Nepal). -Maulik, 1936, Fauna India, Galeruc .:92.-Ogloblin, 1936, Fauna URSS, 26, 1:68, 368, -Gressitt \& Kimoto, 1963, Pac. Ins. Mon., 1B : 391, 417.

\section{Pseudadimonia variolosa (Hope) Fig. $7 \mathrm{a}$}

Colaspis variolosa Hope, 1831, in Gray, Zool. Misc., 30 (Napal ; BM). 
Pseudadimonia variolosa: Duvivier, 1891, C. R. Soc. Ent. Belg., $35: 47$ (Ostindien u. Birma). -Maulik, 1936, Fauna India, Galeruc. : 94, figs. (India, Nepal, Burma, Yunnan, Siam).-Gressitt \& Kimoto, 1963, Pac. Ins. Mon., 1B: 417 (Yunnan).-Châjô, 1964, Nature and Life in SE Asia, Kyoto, 3 : 298 (Thailand).-Kimoto, 1972, Tijdschr. Ent., 93 (4) : 145 (Annam).-Kimoto \& Takizawa, 1972, Kontyû, Tokyo, 40(4): 215 (Nepal) ; 1981, Ent. Rev. Japan, 35 (1-2) : 58 (Nepal) ; 1983, Bull. Natn. Sci. Mus., Tokyo, ser. A, 9(2): 86 (Nepal).

Distribution : India, Nepal, Burma, Thailand, Laos, Vietnam, China.

Suboval, convex, dorsal surfaces entirely glabrous, closely impressed by strong punctures, and their interstices distinctly raised and much narrower than average diameter of punctures ; generally black, in some specimen posterior $1 / 3$ of femora yellowish brown ; length 8.0-11.3 mm.

Material examined. THAILAND : Chieng Mai Prov., Doi Suthep, 1,000 m, 1 ex., 12. v. 1965, K. Morimoto (KU) ; Musa, nr. Fang, 1 ex., 22. xi. 1968, K. Hatta (EHIME) ; Kanchanaburi, 2 exs., 31. v. 1962 (BANGKHEN) ; Banna, Nakhon, 108 m, 1 ex., 5-10. v. 1958, T. C. Maa (BISHOP). LAOS : Umgeb. Vientiane, 2 exs., iii-vi. 1963 (MUNCHEN) ; Vientiane Prov., Ban Van Eue, 1 ex., 1-15. v. 1965, J. A. Rondon, 4 exs., 30. x. 1965, 4 exs., 15-30. iii. 1967, 1 ex., 20. xi. 1966, Native collr ; Tonpheng, 1 ex., 15. v. 1966, Native collr (BISHOP). VIETNAM : Dilinh (Djiring), 920 m, 1 ex., 22-28. iv. 1960, L. W. Quate (BISHOP).

\section{Genus Pyrrhalta Joannis}

Pyrrhalta Joannis, 1866, Abeille, $3: 82$ (type :Galeruca viburni Paykull ; Europe).-Gressitt \& Kimoto, 1963, Pac. Ins. Mon., 1B : 392, 438.-Kimoto, 1964, J. Fac. Agr. Kyushu Univ., 13(2) : 294.

Decoomunius Laboissiere, 1927, Ann. Soc. Ent. France, 96: 55 (type: Decoomanius Iimbatus Laboissiere ; Tonkin; monobasic). New synonym.

Chapalia Laboissike, 1929, Ann. Soc. Ent. France, 98 : 269 (type :Chapalia jeanvoinei Laboissière; Vietnam ; monobasic). New synonym.

Tricholochmaea Laboissiere, 1932, Bull. Mus. Natn. Hist. Nat., Paris, ser. 2, 4 : 963 (type : Lochumaea (Tricholochmaea) indica Laboissiere ; India ; as a subgenus of Lochmaea).

Xanthogaleruca Laboissière, 1934, Ann. Soc. Ent. France, 103 : 67 (type :Chrysomela luteola Müller; Europe).

Galerucella: Maulik, 1936, Fauna India, Galeruc. : 214.-Ogloblin, 1936, Fauna USSR, 26, 1: 112, 387.-Chajô, 1962, Phil. J. Sci., 91 : 33.

Galerucella (Pyrrhalta): Ogloblin, 1936, Fauna USSR, 26, 1 : 97.

Galerucella (Tricholochmaea): Ogloblin, 1936, ibid. : 91.

Galerucella (Xanthogaleruca) : Ogloblin, 1936, ibid. : 92.

Neogalerucella Chûjô, 1962, Phil. J. Sci., 91 : 38 (type :Chrysomela tenella Linnaeus ; Europe ; as a subgenus of Galerucella).

\section{Key to SPecies of Pyrrhalta}

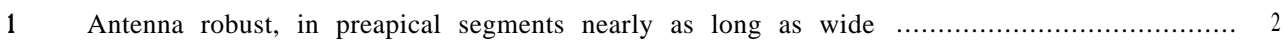

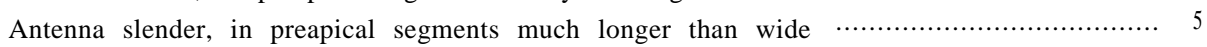

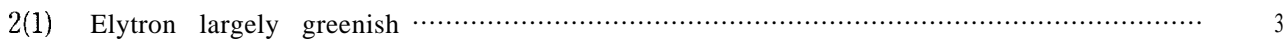

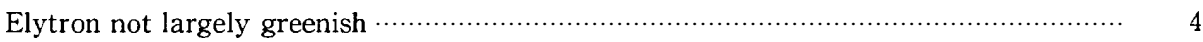

3(2) Elytron greenish with lateral margin including epipleuron brownish ; generally yellowish brown; length $9.0 \mathrm{~mm}$ laboissieri 


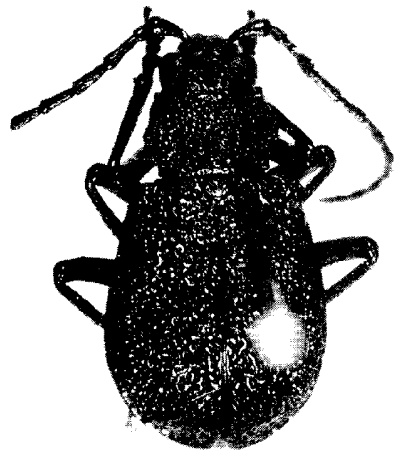

a

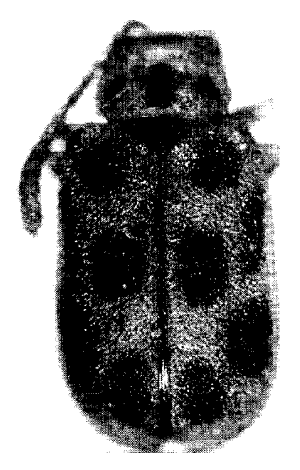

$\mathrm{b}$

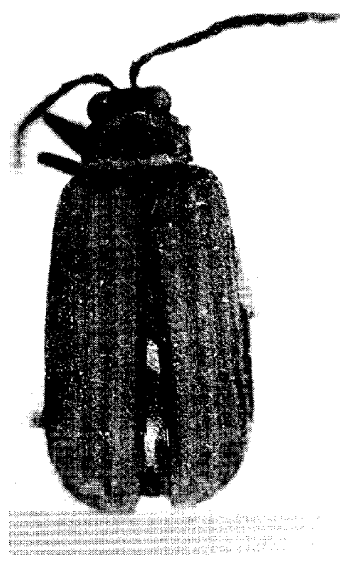

C

Fig. 7. a, Pseudadimonia vasiolosa (Hope) ; b, Pyrrhalta maculata Gressitt \& Kimoto ; c, Pyrrhalta multicostata $(\mathrm{Pic})$.

Generally greenish ; abdomen brownish ; length 3.5-6.5 mm

limbata

4(2) Elytron reddish brown with interior $1 / 3$ blackish ; generally black, clypeus brownish ; length $5.0 \mathrm{~mm}$. rubromarginata

Elytron reddish brown with five spots blackish; generally reddish brown, with middle of vertex, three spots across middle of pronotum, scutellum and metasternum black ; length 4.0-4.5 $\mathrm{mm}$ maculata

5(I) Elytron largely brownish

Elytron greenish with marginal area brownish ; generally yellowish brown, antenna blackish; length $4.5 \mathrm{~mm}$

jeanowine'

6(5) Elytron with four longitudinal costae dorsally ; generally reddish brown with middle of vertex, a longitudinal median stripe, scutellum and sutural margin of elytron blackish ; antenna, legs and ventral surfaces blackish; length $4.8-5.0 \mathrm{~mm} \cdots \cdots . . .$. multicostata Elytron with a longitudinal costa starting from humerus ; entirely yellowish brown; elytral epipleuron distinct only on basal $1 / 2$; length $5.5-6.2 \mathrm{~mm}$ unicostata

\section{Pyrrhalta jeanuoinei (Laboissière), new combination}

Chapalia jeanvoinei Lab., 1929, Ann. Soc. Ent. France, 98 : 270, fig. (Tonkin; BRUXELLES).

Distribution : Vietnam.

No additional material was examined beside the type series.

\section{Pyrrhalta laboissieri, new name}

Periclitena limbata Lab., 1929, Ann. Soc. Ent. France, 98 : 268 (nec Decoomanius limbatus Laboissière, 1927) (Tonkin; BRUXELLES).

Distribution : Vietnam.

No additional material was examined beside the type series. 


\section{Pyrrhalta Zimbata (Laboissière), new combintion Fig. 9a}

Decoomanius limbatus Lab., 1927, Ann. Soc. Ent. France, 96 : 56, fig. (Tonkin; PARIS).

Distribution : Vietnam.

No additional material was examined beside the type series.

\section{Pyrrhalta maculata Gressitt \& Kimoto Fig. 7b}

Pyrrhalta maculata Gressitt \& Kimoto, 1963, Pac. Ins. Mon. 1B : 439, 456, fig. (SE China, Taiwan ; BISHOP).

Distribution : Thailand, Vietnam, SE China, Taiwan.

Material examined. THAILAND : Chiengmai Prov., Doi Suthep, 1 ex., 1-8. iv. 1958, T. C. Maa (BISHOP). VIETNAM : Dalat, 6 km S., 1,400-1,500 m, 1 ex., 9. vi.-7. vii. 1961, N. R. Spencer ; Fyan, 900-1,000 m, 1 ex., 11. vii.-9. viii. 1961, N. R. Spencer (BISHOP).

\section{Pyrrhalta multicostata (Pic), new combination Fig. 7c}

Galerucellamulticostata Pic, 1928, Mél. Exot. Ent., 51 : 32 (Tonkin ; PARIS).

Galerucellaaludela Maulik, 1936, Fauna India, Galeruc. : 220 (Burma ; BM). New synonym.

Distribution : Burma, Thailand, Vietnam.

Material examined. THAILAND : Chieng Mai Prov., Doi Pui, 1,300 m, 1 ex., 8. vi. 1965, Y. Miyatake (KU).

\section{Pyrrhalta ru bromargina ta (Laboissière), new combination}

Galerucella mubromarginata Lab., 1929, Ann. Soc. Ent. France, 98 : 271 (Tonkin; HAMBURG). Distribution : Vietnam.

No additional material was examined beside the type.

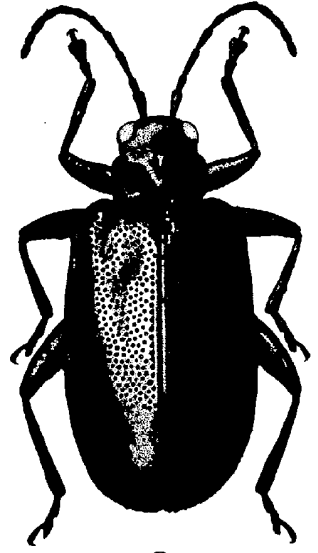

a

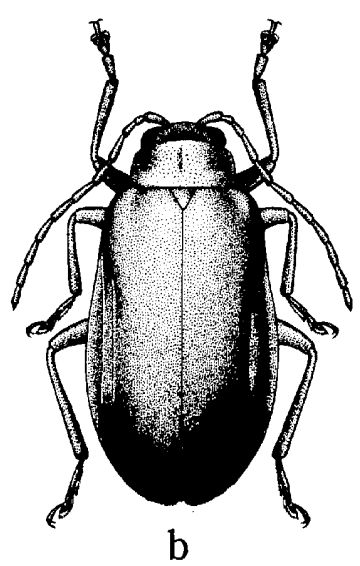

b

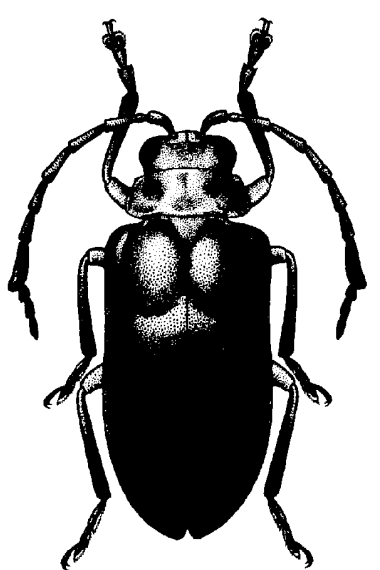

C

Fig. 8. a, Galerucella grisescens (Joannis) , b, Pyrrhalta unicostata (Pic); c, Clitena sinensis (Fairmaire). 


\section{Pyrrhalta unicostata (Pic), new combination Fig. 8b}

Galerucella unicostata Pic, 1937, Mél. Exot. Ent., 69 : 20 (Tonkin ; PARIS).

Pyrrhalta hainanensis Gressitt \& Kimoto, 1963, Pac. Ins. Mon., 1B:440, 450, figs. (Hainan ; CAS).

New synonym.

Distribution : Thailand, Cambodia, Laos, Vietnam, Hainan.

Material examined. THAILAND : Banna, Chawang, nr. Nabon, 70 m, 1 ex., 5. ix. 1958, J. L. Gressitt ; Kao Yai, 1 ex., 11. iv. 1963, Native collr (BISHOP) ; Kao Yai Nat. Park, 7 exs., 6. vi. 1965, K. Morimoto \& Y. Miyatake (KU). CAMBODIA : Kiri Rom, 700 m, 1 ex., 31. iii.-7. iv. 1961, N. R. Spencer (BISHOP). LAOS : Vientiane Prov., Ban Van Eue, 1 ex., 15-31. v. 1965, Native collr ; Khammouane Prov., Phon Tiou, 1 ex., 15-31. v. 1965, Native collr ; Khammouane Prov., Phon Tiou, 1 ex., 15-31. v. 1965, Native collr (BISHOP) ; Umgeb. Vientiane, 2 exs., iii-vi. 1963 ; Umgeb. Vanky, 2 exs., 1963 (MUNCHEN).

\section{Genus Galerucella Crotch}

Galerucella Crotch, 1873, Proc. Acad. Sci. Philad., 1873 : 55 (type : Chrysomela nymphaea Linnaeus ; Europe).-Maulik, 1936, Fauna India, Galeruc. : 214.-Gressitt\& Kimoto, 1963, Pac. Ins. Mon., 1B : 392, 468.-Kimoto, 1964, J. Fac. Agr. Kyushu Univ., 13(2) : 293.

Hydrogaleruca Laboissière, 1922, Rev. Zool. Afr., 10 : 32.

Galerucella (Hydrogaleruca): Ogloblin, 1936, Fauna USSR, 26, 1 :119.—Chûjô, 1962, Phil. J. Sci., 91 $(1-\mathrm{Z})$ : 39.

\section{Galerucella grisescens (Joannis) Fig. 8a}

Galeruca grisescens Joann., 1866, Abeille, 3 : 98 (Sicily).

Galeruca vittaticollis Baly, 1874, Trans. Ent. Soc. London, $1874: 178$ (Nagasaki ;BM).-Chajo \& Kimoto, 1961, Pac. Ins., 3(1): 165 (=grisescens).

Galleruca distincta Baly, 1874, Trans. Ent. Soc. London, 1874 : 178 (Nagasaki ;BM).-Laboissière, 1934, Ann. Soc. Ent. France, $103: 73$ (=grisescens).

Gallenca placida Baly, 1878, Cist. Ent., 2 : 381 (Murree, N. India). New synonym.

Galencella placida: Baly 1878, Second Yorkand Mission : 34 (Jhelam Valley)-Allard, 1889, Ann. Soc. Ent. France, ser. 6, $9: 312$ (Pnom-Penh ; var.).-Maulik, 1936, Fauna India, Galeruc. : 217 (India, Burma).-Kimoto, 1979, Ent. Basiliensia, $4: 466$, fig. (Nepal, India).-Kimoto \& Takizawa, 1983, Bull. Natn. Sci. Mus., Tokyo, ser. A, 9(2): 86 (Nepal).

Galerucellarugosa Jacoby, 1884, Notes Leyden Mus., 6: 65 (Sumatra).-Maulik, 1936, Fauna India, Galeruc. : $218(?=$ placida $)$. New synonym.

Lochmaea (Galerucella) rugosa: Jacoby, 1889, Ann. Mus. Civ. Genova, $27: 216$ (Burma).

Galerucella distincta : Kolbe, 1886, Archiv Naturg., 52(1): 230 (Seoul, Korea).

Galerucella sagitariae var. distincta : Weise, 1889, Horae Soc. Ent. Ross., 23 : 569 (Szetschuan).

Lochmaea rugosa: Duvivier, 1891, C. R. Soc. Ent. Belg., 35 : 150 (Indo-Malaisie).

Galerucella distincta var. jureceki Pic, 1921, Échange, $37: 2$ (China).

Galerucella angulosa Pic, 1928, Mel. Exot. Ent., 52 : 30 (Annam; PARIS). New synonym.

Galerucella (Hydrogaleruca) grisescens :Laboissière, 1934, Ann. Soc. Ent. France, 103 : 69, 73 (Europe, Siberia, Japan).-Ogloblin, 1936, Fauna USSR, 26, 1 : 120 (Europe, Siberia, China, Japan).

Galerucella (Hydrogaleruca) distincta :Châjô, 1941, Trans. Nat. Hist. Soc. Formosa, 31(211): 159 
(Korea) ; 1942, Mushi, Fukuoka, 14(2): 61 (Kwangtung).

Galerucella reducta Chen, 1942, Notes d'Ent. Chinoise, 9 : 19 (Kwangsi ; Yunnan ; Sikang). -Gressitt \& Kimoto, 1961, Pac. Ins. Mon., 1B : 468 (=grisescens).

Hydrogaleruca distincta yakushimana Nakane, 1958, Sci. Rep. Saikyo Univ., 2(5): A308 (Japan : Yakushima ; NSM).

Lochmaea (Tricholochmaea) placida:Bechyné, 1961, Beitr. Naturh. Forsh. S. W. Deutschl., 19(3): 255 (Afghanistan).-Lopatin, 1963, Ann. Hist.-Nat. Mus. Natl. Hungarici, Zool., 55 : 355 (fauna).

Galerucella grisescens distincta :Châjô, 1962, Phil. J. Sci., 91(1-2): 40 (Formosa).

Galerucella grisescens : Gressitt \& Kimoto, 1963, Pac. Ins. Mon., 1B: 468 (Siberia, China, Hainan, Vietnam).-Kimoto, 1964, J. Fac. Agr. Kyushu Univ., 13(2) : 294.

Distribution : Europe, Siberia, Sachalin, Korea, China, Hainan, Taiwan, Korea, Japan, Ryukyu Is., Afghanistan, India, Nepal, Burma, Thailand, Laos, Vietnam, Sumatra, Java.

Suboval, dorsal surface covered by fine hairs and disc of pronotum with a large glabrous space at middle ; reddish brown, in some specimen elytron with an ill-defined submarginal pitchy stripe, which is starting from humerus and running towards apex ; antenna black with basal segments paler ; vertex and meso- and metathorax, in some cases some parts of abdomen also, blackish ; length 3.7$5.2 \mathrm{~mm}$.

Material examined. THAILAND : Chiengmai, Fang, 500 m, 2 exs., 12. iv. 1958, Native collr (BISHOP) ; Fang, 1 ex., 14. vi. 1965, Y. Miyatake ; Mae Klang Water Fall, nr. Chom Thong, 1 ex., 11. vi. 1965, Y. Miyatake (KU). LAOS : Vientiane Prov., Namgueng River, Phou Kou Khouei, 100 m, 4 exs., 10. iv. 1965, J. L. Gressitt ; Vientiane Prov., Ban Van Eue, 2 exs., 15. vii. 1965, Native collr (BISHOP). VIETNAM : Dalat, 6 km S., 1,400-1,500 m, 1 ex., 9. vi.-7. vii. 1961, N. R. Spencer (BISHOP).

\section{Genus Sastracella Jacoby}

Sastracella Jacoby, 1899, Stett. Ent. Ztg., 60 : 294 (type :Sastra fulvicornis Jacoby ; Burma). -Weise, 1902, Tijdschr. Ent., $65: 67$ (as subgenus of Sastra).

\section{KEY TO SPECIES OF Sastracella}

1 Generally bluish, elytron subparallel-sided

$\ldots 2$

Entirely yellowish brown, elytron rounded laterally ; length $8.3-10.0 \mathrm{~mm}$............. laosensis

2(1) Antenna entirely blackish ; violaceous blue, abdomen yellowish brown ; length

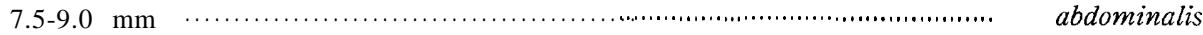
Antenna generally yellowish brown ; dorsal surfaces violaceus blue, ventral surfaces piceous; length 7.4 mm (Jacoby, 1892 ; Sastra; Burma ; GENOVA) …...............fulvicornis

\section{Sastracella abdominalis n. sp. Fig. 9b}

Generally violaceous blue, antenna entirely pitchy black, abdomen yellowish brown.

Head with vertex closely covered by strong punctures and fine pubescence, and with a longitudinal median furrow, slightly depressed at front ; frontal tubercle distinct, finely granulate, subtriangular, contiguous, anterior angle slightly inserted between antennal insertions ; interantennal space distinctly raised. Antenna slender, nearly $3 / 4$ as long as body length, and in preapical segments nearly three times as long as wide ; first segment robust, somewhat club-shaped, second shortest, nearly $2 / 3$ as long as first, third nearly $23 / 4$ times as long as second, fourth $4 / 5$ as long as third, fourth to sixth 


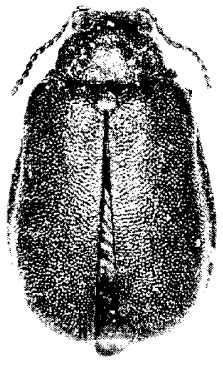

a

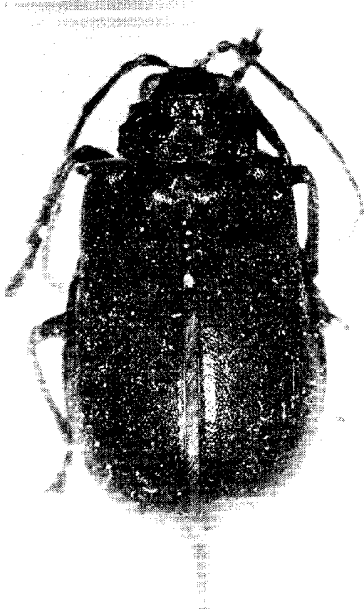

b

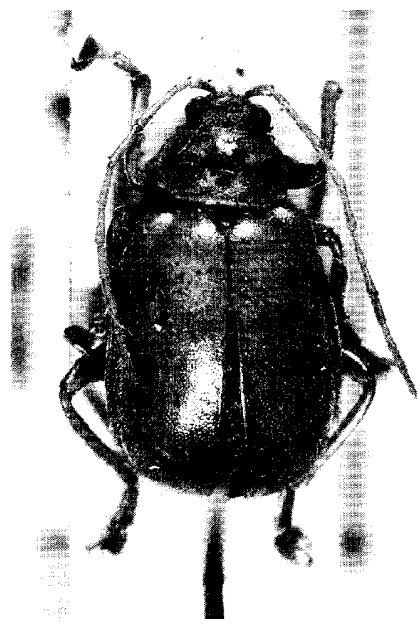

Fig. 9. a, Pyrrhalta limbata (Laboissière); b, Sastracella abdominalis n. sp. ; c, S. laosensis n. sp.

subequal to each other in length and shape, seventh slightly shorter than sixth, eighth subequal to seventh in length and shape, ninth slightly shorter than eighth, tenth subequal to ninth in length and shape, eleventh $12 / 5$ times as long as tenth and its apex pointed. Pronotum transverse, nearly twice as long as wide, anterior margin slightly curved posteriorly, lateral margin rounded, and with a slight projection at $1 / 3$ from anterior margin, and narrowed anteriorly and posteriorly, basal margin widely rounded posteriorly and slightly concaved before scutellum, anterior and posterior corners distinctly produced laterally and each with a setigerous pore; surface closely impressed by strong punctures and covered with fine pubescence, and with distinct an anterior and a posterior median depressions and a pair of deep depressions laterally. Scutellum convex, closely covered by strong punctures and fine pubescence, subtriangular, its apex rounded. Elytron with lateral margin subparallel-sided, rounded at apex, distinctly and closely punctate, and closely covered by fine pubescence.

Length : 7.5-9.0 mm.

Holotype (MUNCHEN) : LAOS : Umgeb. Vientiane, iii-vi. 1963. Paratypes : LAOS : 11 exs., same data as the holotype (MUNCHEN, KIMOTO) ; Ban Van Heue, $20 \mathrm{~km}$ E. of Phou-kow-kuei, 3 exs., 1-15. v. 1965, J. A. Rondon, 1 ex., 15-31. iv. 1965, Native collr (BISHOP). VIETNAM : Kontum, N. of Pleiku, 550 m, 1 ex., 13. v. 1960, S. Quate ; 25 km SW. of Pleiku, 400 m, 1 ex., 12. v. 1960, L. W. Quate ; $45 \mathrm{~km} \mathrm{~W}$. of Dalat, $850 \mathrm{~m}, 1$ ex., L. W. Quate (BISHOP).

This new species closely resembles Sastracella fulvicornis (Jacoby) but is separable in having the antenna entirely blackish.

\section{Sastracella laosensis n. sp. Fig. 9c}

Entirely yellowish brown.

Head with vertex closely covered by strong punctures and fine pubescence, and with a longitudinal median furrow, slightly depressed at front; frontal tubercle subquadrate, contiguous, anterior angle slightly inserted between antennal insertions, distinctly raised, somewhat wrinkled, impressed 
by fine punctures. Antenna slender, nearly $2 / 3$ as long as body length, and in preapical segments nearly three times as long as wide; first segment robust, somewhat club-shaped, second shortest, nearly $2 / 5$ as long as first, third nearly $23 / 4$ times as long as second, fourth nearly $4 / 5$ as long as third, fifth nearly $3 / 4$ as long as fourth, sixth subequal to fifth in length and shape, seventh slightly shorter than sixth, eighth subequal to seventh in length and shape, ninth slightly shorter than eighth, tenth subequal to eighth in length and shape, eleventh subequal to eighth in length and its apex pointed. Pronotum transverse, nearly twice as long as wide, anterior margin slightly curved posteriorly, lateral margin rounded, widest almost at middle, and narrowed anteriorly and posteriorly ; basal margin widely rounded posteriorly, anterior and posterior corners distinctly produced laterally and each with a setigerous pore ; surface closely impressed by strong punctures and fine pubescence, and with a shallow subbasal depression and a pair of deep depressions laterally. Scutellum convex, covered by strong punctures and pubescence, subtriangular, and its apex truncate. Elytron with lateral margin rounded, distinctly and closely punctate, and closely covered by fine pubescence.

Length $8.3-10.0 \mathrm{~mm}$.

Holotype (BISHOP) : LAOS : Sayaboury Prov., Sayaboury, 15. iv. 1965, J. L. Gressitt. Paratopotypes : 11 exs., same data as the holotype (BISHOP, KIMOTO). Paratypes : LAOS : Vientiane Prov., Ban Van Eue, 1 ex., 11. iv. 1965, 1 ex., 13. iv. 1965, J. L. Gressitt, 1 ex., 31. v. 1966, 1 ex., 15. iv. 1966, 1 ex., 15. vi. 1966, 5 exs., 30. iv. 1967, 3 exs., 15. v. 1966, Native collr (BISHOP) ; Umgeb. Vientiane, 27 exs., iii.-vi. 1963 ; Umgeb. Pakse, 1 ex., 1963 (MUNCHEN).

This new species resembles Sastracella harmandi Laboissière, from Sikkim, but differs in having elytron with the lateral margin rounded. Also, this new species somewhat resembles Sastroides indicus Jacoby, but differs in having the pronotum distinctly covered with fine hairs and elytron without any transverse depression near the base.

\section{Genus Clitena Baly}

Clitena Baly, 1864, Trans. Ent. Soc. London, ser. 3, 2 : 229 (type : Clitena limbata Baly ; monobasic ; Siam).-Gressitt \& Kimoto, 1963, Pac. Ins. Mon., 1B : 392, 418.

Mesodonta Baly, 1865, Ent. Monthly Mag., 2 : 99 (type : Clitena Zimbata Baly ; Siam).-Chapuis, 1875, Gen. Col., $11: 198,205$.

\section{Key to SPECIES OF Clitena}

Generally yellowish to reddish brown, elytron entirely violaceous to blackish blue ; tibiae, tarsi and preapical segments of antenna blackish; in male eighth antennal segment enlarged, produced ectoapically, and ninth to eleventh considerably shortened ; length $11.0 \mathrm{~mm}$ (Fairmaire, 1888 ; Agetocera ; China ; PARIS ; Fig. 8c) ….................. sinensis Generally dark yellowish brown, pronotum with a pair of blackish markings, elytron black with margins narrowly brownish; vertex with a blackish marking at middle ; antenna and legs mostly blackish; in male eighth antennal segment not enlarged; length $10.0-12.0 \mathrm{~mm}$

limbata

\section{Clitena limbata Baly Fig. 10a}

Clitena limbata Baly, 1864, Trans. Ent. Soc. London, ser. 3, 2 : 230 (Pachybouri, Siam ; BM). -Maulik, 1936, Fauna India, Galeruc. : 2’29 (Burma, Siam, Tonkin, Laos, Borneo).-Gressitt \& Kimoto, 1963, Pac. Ins. Mon., 1B: 418 (China). 


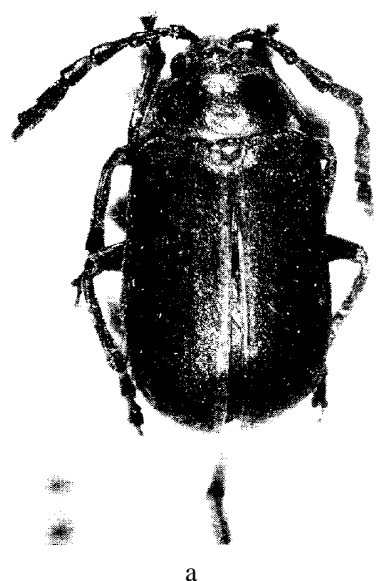

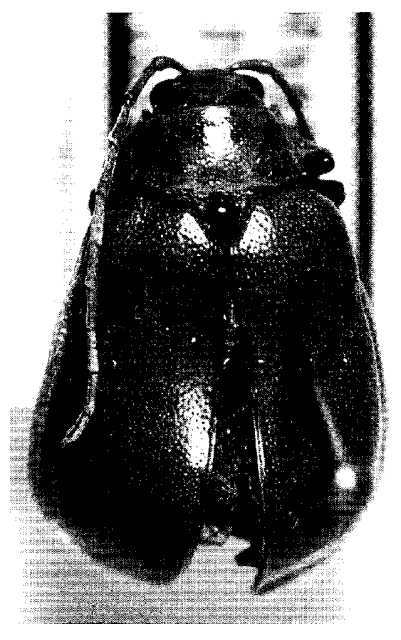

b

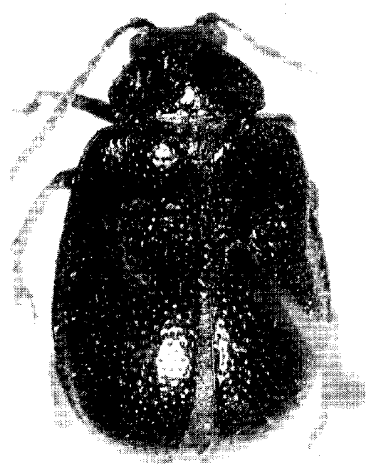

c

Fig. 10. a, Clitena limbata Baly ; b, Doryxena geniculata Baly ; c, Clitenella punctata Laboissière.

Mesodonta limbata : Baly, 1865, Ent. Monthly Mag., 2 : 99 (as a type of Mesodonta).

Hymenesia limbata Jacoby, 1896, Ann. Soc. Ent. Belg., 40 : 297 (Burma).-Maulik, 1936, Fauna India, Galeruc. : 229 (= Clitena Zimbata Baly).

Clitena cincta Laboissière, 1927, Ann. Soc. Ent. France, 96 : 51, fig. (Laos; BRUXELLES). -Maulik, 1936, Fauna India, Galeruc . :229 (=Clitena limbata Baly).-Gressitt \& Kimoto, 1963, Pac. Ins. Mon., 1B:418 (= Clitena limbata Baly).

Distribution : Burma, Thailand, Laos, Vietnam, Borneo.

Material examined. THAILAND: Phu Kae, 1 ex., 15. iv. 1964, 1 ex., 6. vii. 1963, Native collr ; Pakchong, 1 ex., 20. vii. 1963, 1 ex., 21. xi. 1963, Native collr ; Bangkhen, 1 ex., 25. vii. 1968, Native collr ; Songkla, 1 ex., 15. iii. 1963, Native collr ; Srisamrong, 1 ex., 14. x. 1963 ; Khao Yai, 1 ex., 18. viii. 1963, Native collr ; Kanchanaburi, 1 ex., 10. viii. 1963, Native collr (BISHOP) ; Tak, 7 exs., 31. v. 1959, 2 exs., 21. vi. 1959, 2 exs., 25. v. 1959, 1 ex., 29. v. 1959, 2 exs., 27. v. 1959 ; Saraburi, 2 exs., 20. vii. 1956 ; Bangkok, Dhonburi, 1 ex., 8. ix. 1959, 1 ex., 5. ix. 1959, Nakhon Ratchasima, 1 ex., 9. ix. 1959, 1 ex., 4. x. 1959, 2 exs., 6. ix. 1959, Prachuab Khiri Khan, 4 exs., v. 1956 ; Nakhon Ratchasima, 1 ex., 20. vi. 1958, 1 ex., 15. iv. 1958 (BANGKHEN). LAOS : Umgeb. Vientiane, 7 exs., iii-vi. 1963 (MUN. CHEN).

\section{Genus Doryxena Baly}

Doryxena Baly, 1861, J. Ent., 1 : 202 (type : Gallenuca grossa Hope ; Assam)-Chapuis, 1875, Gen. Col., 11 : 197, 207.-Maulik, 1936, Fauna India, Galeruc. : 121.

\section{Doryxena geniculata Baly Fig. 10b}

Doryxena geniculata Baly, 1879, Cist. Ent., 2 : 451 (Assam ; BM).-Maulik, 1936, Fauna India, Galeruc. : 123, fig. (Assam, Manipur).-Laboissière, 1932, Bull. Mus. Hist. Nat., Paris, ser. 2, 4 : 962 (Sikkim). 
Distribution : India, "Indo-China".

Suboval, dorsal surfaces glabrous, strongly and rather closely punctate, lateral and apical margins of elytron broad, anterior process of metasternum produced anteriorly ; yellowish to dark reddish brown, scutellum and apex of femora and base of tibiae blackish ; length $16.0 \mathrm{~mm}$.

Material examined. "Indo-China", Hanglang, Pakka, 2 exs. (BASEL).

\section{Genus Clitenella Laboissière}

Callopistria Chevrolat, 1837, in Dejean, Cat. Col., ed. 3: 402 (nec Huebner, 1821) (type: Galeruca fulminans Faldermann ; China ; monobasic).-Hincks, 1949, Ann. Mag. Nat. Hist., ser. 12, 2 : 619.

Clitenella Laboissière, 1927, Ann. Soc. Ent. France, 96 : 53 (type :Galeruca fulminans Faldermann ; China).-Ogloblin, 1936, Fauna USSR, 26, 1 : 135, 392.-Gressitt \& Kimoto, 1963, Pac. Ins. Mon., 1B : 419.

\section{Key to spectes of Clitenella}

1 Antenna and legs generally bluish black; generally golden green, elytron with basal and postmedian markings reddish, and abdomen reddish to yellowish brown ; length 6.8-8.7

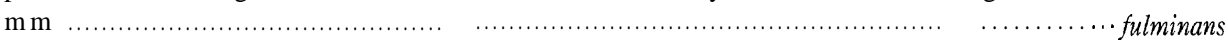
Antenna and legs generally yellowish brown, in some specimen femora bluish black ; violaceous blue ; abdomen reddish to yellowish brown; $6.5 \mathrm{~mm}$

punctata

\section{Clitenella fulminans (Faldermann)}

Galeruca fulminans Fald., 1935, Mếm. Acad. Petersb., 2 : 438, pi. 5, fig. 8 (China).

Clitena jiilminans : Jacobson, 1911, Käfer Russl., 9, pl. 59, fig. 2.

Clitenella fulminans :Laboissière, 1927, Ann. Soc. Ent. France, 96 : 54 (Tonkin: Hanoi, Chine, Formosa) ; 1929, ibid., 98 : 269 (Tonkin).-Ogloblin, 1936, Fauna USSR, 26, 1 : 136, 393 (China, Tonkin,Formosa).-Châjô, 1962, Phil. J. Sci., 91(1-2): 212 (Formosa)--Gressitt \& Kimoto, 1963, Pac. Ins. Mon., 1B : 419 (China).

Distribution : Vietnam, China, Taiwan.

No additional material was examined.

\section{Clitenella punctata Laboissière Fig. 10c}

Clitenella punctata Lab., 1927, Ann. Soc. Ent. France, 96 : 55 (Tonkin; BRUXELLES).

Distribution : Thailand, Vietnam.

Material examined. THAILAND : Khon Kaen, 1 ex., 24. vii. 1980, 1 ex., 4. viii. 1980, 1 ex., 8. x. 1981, S. Azuma (RU).

\section{Genus Periclitena Weise}

Clitena Clark, 1865, Ann. Mag. Nat. Hist., ser. 3, 16 :257, 259 (necBaly).-Chapuis, 1875, Gen. Col., $11: 198,203$.

Periclitena Weise, 1902, Archiv Naturg., 63 : 157 (type :Galleruca vigorsi Hope ; Nepal).-Maulik, 1936, Fauna India, Galeruc. : 210.-Ogloblin, 1936, Fauna USSR, 26, 1 : 133, 368.-Hincks, 1949, 
Ann. Mag. Nat. Hist., ser. 12, 2 :619.-Gressitt \& Kimoto, 1963, Pac. Ins. Mon., 1B : 392, 420.

\section{Key to SPeCies of Periclitena}

Pronotum metallic .2

Pronotum reddish brown; elytron entirely violaceous to blackish blue; generally reddish brown, most of tibiae and tarsi and preapical antennal segments blackish; length $10.0-13.0 \mathrm{~mm}$.......................................................... tonkinensis

21 In male eighth antennal segment enlarged, produced ectoapically and concaved beneath ; in female ninth to eleventh not greatly shortened and distinctly longer than wide ; generally violaceous blue, elytron with or without basal and subapical markings greenish; length $8.7-9.6 \mathrm{~mm}$. cyanea In male eighth antennal segment normal ; in female ninth to eleventh considerably shortened ; generally greenish, violaceous, bluish, blackish, elytron with or without basal and subapical markings somewhat reddish or greenish ; length $8.7-11.0 \mathrm{~mm} \cdots \cdots$ vigorsi

\section{Periclitena cyanea (Clark) Fig. lb}

Clitena cyanea Clark, 1865, Ann. Mag. Nat. Hist., ser. 3, 16 : 259 (Java).-Jacoby, 1889, Ann. Mus. Civ. Genova, 27 : 208 (Burma).-Allard, 1891, Nourv. Arch. Mus. Paris, ser. 3, 3 : 231 (Luang Prabang) ; 1904, Miss. Pavie Indo-Chine, $3: 160$ (Laos, Luang-Prabang à Theng).

Periclitena cyanea : Maulik, 1936, Fauna India, Galeruc. : 213 (key).-Gressitt \& Kimoto, 1963, Pac. Ins. Mon., 1B: 421 (Hainan).

Distribution : Burma, Thailand, Laos, Hainan, Java.

Material examined. LAOS : Savannakhet Prov., Savannakhet, 2 exs., 5. vii. 1965, Native collr; Borikhane Prov., Pakkading, 1 ex., 15. ix. 1965, J. L. Gressitt ; Khammouane Prov., Phon Tiou, 2 exs., 18. viii. 1965, J. A. Rondon, 1 ex., 30. xi. 1966, Native collr (BISHOP) ; Umgeb. Vientiane, 1 ex., iii.-vi. 1963 ; Umgeb. Pakse, 1 ex., 1963 (MUNCHEN).

\section{Periclitena tonkinensis Laboissière, resurrected from synonymy}

Pwiclitena tankinensis Lab., 1929, Ann. Soc. Ent. France, 98 : 266 (Tonkin, Kweichow ; PARIS). Distribution : Vietnam, S. China.

Gressitt \& Kimoto (1963) treated this species as a synonym of Agetocera sinensis (Fairmaire). The latter is treated by Laboissière (1929) as a member of Periclitena. However, this species should be treated as a member of Clitena in having pronotum sparsely covered with fine hairs.

Material examined. VIETNAM : Annam, 5 exs., 1927 (BASEL).

\section{Periclitena vigorsi (Hope)}

Galleruca vigorsi Hope, 1831, in Gray, 2001. Miscell. : 29 (Nepal ; BM).

Gallenca coerulans Hope, 1831, ibid. : 29 (Nepal ; BM).

Clitea melancholica Baly, 1864, Trans. Ent. Soc. London, ser. 3, 2 : 231 (Siam, Laos; BM). New synonym.

Clitena igneipennis Baly, 1865, Ent. Monthly Mag., 2 : 99 (India ;BM).-Allard, 1891, Nourv. Arch. Mus. Paris, ser. 3, 3 : 231 (Luang-Prabang) ; 1904, Miss. Pavie Indo-Chine, 3 : 160 (Laos, Luang-Prabang à Theng). 
Periclitena vigorsi var. viridissima Weise, 1922, Tijdschr. Ent., $65: 66$ (Bengal).

Periclitena vigorsi var. fulgida Laboissière, 1927, Ann. Soc. Ent. France, $96: 63$ (N. Annam; PARIS).

Periclitena vigorsi : Maulik, 1936, Fauna India, Galeruc. : 211 (India, Sikkim, Assam, Burma, Penang, Tonkin).-Gressitt \& Kimoto, 1963, Pac. Ins. Mon., 1B: 420, 422 (Hainan).-Kimoto, 1970, Spec. Bull. Lep. Soc. Japan, 4 : 170 (Nepal).-Kimoto \& Takizawa, 1972, Kontyâ, Tokyo, 40(4) : 216 (Nepal).-Kimoto, 1978, Ent. Basiliensia, $4: 468$ (Nepal, Meghallaya).

Distribution : India, Burma, Nepal, Thailand, Laos, Vietnam, Malaya, Hainan.

Material examined. THAILAND : Chiengmai Prov., Chiangdao, $450 \mathrm{~m}, 4$ exs., 5-11. iv. 1958, T. C. Maa (BISHOP). LAOS : Sayaboury Prov., Sayaboury, 3 exs., 13. iv. 1966, Native collr (BISHOP) ; Umgeb. Vientiane, 1 ex., iii-vi. 1963 ; Umgeb. Pakse, 1 ex., 1964 ; Umgeb. Vanky, 1 ex., 1963 ; Umgeb. Paklay, 1 ex., 1963 (MUNCHEN).

\section{Genus Sastroides Jacoby}

Sastroides Jac., 1884, Notes Leyd. Mus., 6 : 281 (type :Sastroides bimaculata Jacoby ; Sumatra).

$$
\text { KeY to spectes of Sastroides }
$$

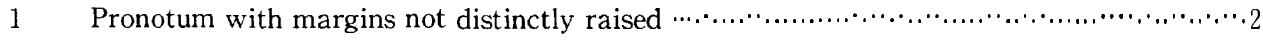
Pronotum with margins in large part strongly raised, surface shiny, feebly punctured ; generally violaceous to greenish blue, antenna and abdomen largely yellowish brown;

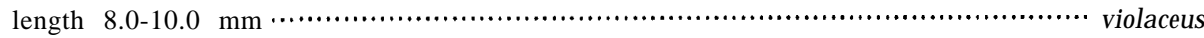

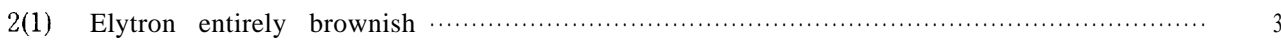

Elytron violaceus blue to blackish blue ; generally yellowish to reddish brown, apical segments of antenna, tarsi and apical half of tibiae blackish ; in dark colored specimen dorsal surfaces and antenna, in some specimens meso- and metathorax also, generally

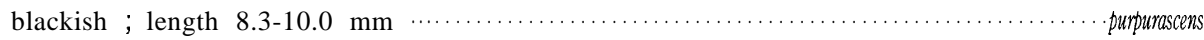

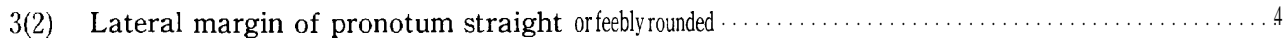

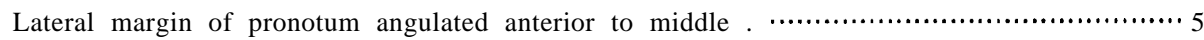

4(3) Prontoum $21 / 4$ times as wide as long, sides nearly straight and slightly narrowed posteriorly, and its surface impressed by a pair of shallow transverse furrows and sparsely impressed by distinct punctures ; entirely yellowish brown ; length 6.8-9.3

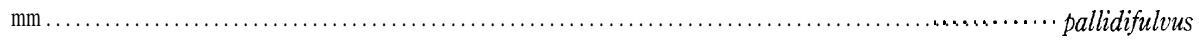

Pronotum $12 / 3$ times as wide as long, sides feebly rounded, widest almost at middle, and its surface impressed by a pair of deep transverse furrows, and each almost united at middle and curved downward laterally, and sparsely impressed by minute punc-

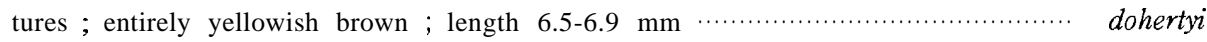

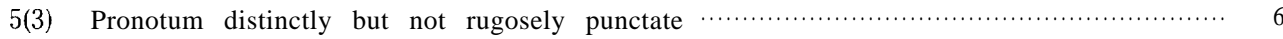
Pronotum distinctly and rugosely punctate ; generally reddish brown, antenna blackish with basal segments brownish, tibiae and tarsi generally blackish ; length $10.0-$

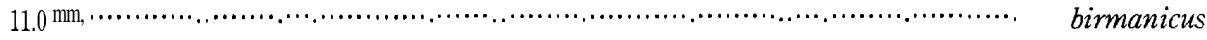

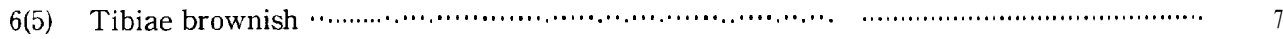
Tibiae and tarsi blackish; generally yellowish to raddish brown, antenna blackish with basal segments brownish ; length 9.0-11.0 $\mathrm{mm}$.................................. tibialis

7(6) Smaller ; elytron without any transverse depression near base ; generally yellowish

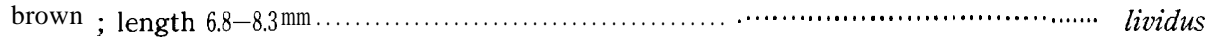


Larger ; elytron with a slight depression near base ; generally yellowish to reddish brown, in many specimens apical segments of antenna and tarsi blackish ; length 9.0$11.0 \mathrm{~mm}$

indicus

\section{Sastroides birmanicus Jacoby Fig. 12b}

Sastroides birmanica Jac., 1889, Ann. Mus. Civ. Genova, 27 : 217 (Burma ; GENOVA).

Sastra birmanica: Maulik, 1936, Fauna India, Galeruc. : 259 (Burma).

Atysa gigantica Maulik, 1936, Fauna India, Galeruc.: 248 (Burma ; BM). New synonym.

Sastroides gigantica: Aslam, 1972, J. Nat. Hist., $6: 501$ (genus).

Distribution : Burma, Laos.

Material examined. LAOS : Umgeb. Vientiane, 1 ex., iii.-vi. 1963 ; Umgeb. Vanky, 1 ex., 1964 (MUNCHEN).

\section{Sastroides dohertyi (Maulik)}

Sastra dohertyi Maulik, 1936, Fauna India, Galeruc. : 265 (Burma ; GENOVA).

Pseudosastra dohertyi: Aslam, 1972, J. Nat. Hist., $6: 501$ (genus).

Distribution : Burma, Laos.

Material examined. LAOS : Vientiane Prov., Ban Van Eue, 1 ex., 1-15. v. 1965, J. A. Rondon, 1 ex., 15-31. v. 1965, Native collr ; Vientiane Prov., Gi Sion Vill., de Tha Ngone, 2 exs., 10-24. x. 1965, 1 ex., 2. i. 1966, Native collr ; Sayaboury Prov., Sayaboury, 1 ex., 15. iv. 1965, J. L. Gressitt (BISHOP).

\section{Sastroides indicus Jacoby}

Sastroides indicus Jac., 1894, Novit. Zool., $1: 315$ (Assam).

Sastra (Sastroides) indica: Weise, 1922, Tijdschr. Ent., $65: 67$ (Tonkin).
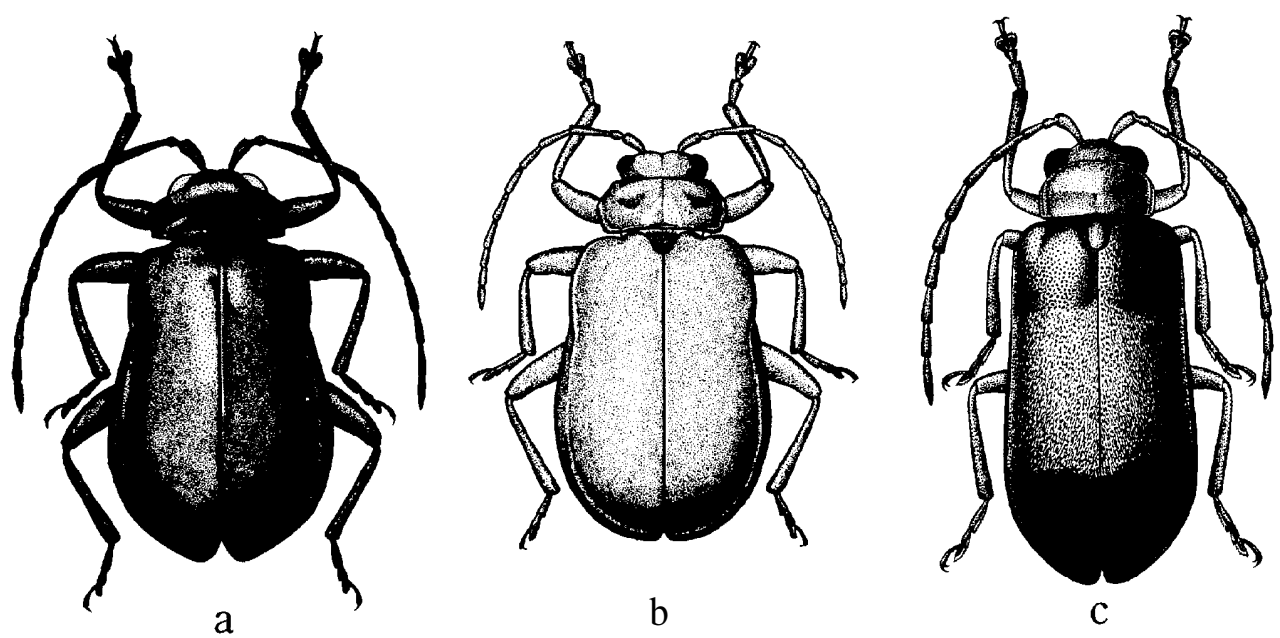

Fig. 11. a, Sastroides violaceus (Weise) ; b, S. lividus (Laboissière); c, Anadimonia latifasciata (Gressitt \& Kimoto). 
Sastra indicus : Maulik, 1936, Fauna India, Galeruc.: 264 (India).-Kimoto, 1979, Ent. Basiliensia, $4: 468$, fig. (Nepal).

Distribution : India, Laos, Vietnam.

I could not trace the type location of this species.

Material examined. LAOS : Vientiane Prov., Ban Van Eue, 2 exs., 12. iv. 1965, 1 ex., 13. iv. 1965, J. L. Gressitt, 1 ex., 15-31. v. 1965, J. A. Rondon, 7 exs., 15-31. iv. 1965, 1 ex., 1-15. v. 1965, 3 exs, $15-$ 31. v. 1965, 1 ex., 15. iv. 1966, 3 exs., 15. v. 1966, 1 ex., 15. vi. 1966, 6 exs., 30. iv. 1967, 9 exs., 15. v. 1967, 1 ex., 31. v. 1967, Native collr ; Borikhane Prov., Pakkading, 1 ex., 15. iv. 1965, J. A. Rondon, 3 exs., 5. iv. 1966, 1 ex., 29. iv. 1966, Native collr; Sayaboury Prov., Sayaboury, 14 exs., 15. iv. 1965, J. L. Gressitt (BISHOP) ; Umgeb. Vientiane, 4 exs., iii-vi. 1963, Umgeb. Pakse, 4 exs., 1963 ; Umgeb. Paklay, 1 ex., 1963 (MUNCHEN).

\section{Sastroides lividus(Laboissière), new combination Fig. 11b}

Galerucella consentanea : Allard, 1889, Ann. Soc. Ent. France, ser. 6, 9 ; 312 (Pnom-Penh) ; 1891, Nouv. Arch. Mus. Paris, ser. 3, 3 : 231 (Luang-Prabang) ; 1904, Miss. Pavie Indo-Chine, 3 : 160 (Cambodge).-Laboissière, 1935, Ann. Ass. Nat. Levaloi-Perret, $2: 143$ (= Sastralivida).

Sastra (Sastroides) livida Laboissière, 1935, Ann. Ass. Nat. Levallois-Perret, 21 : 143 (Cambodia ; PARIS).

Sastra mamaya Maulik, 1936, Fauna India, Galeruc. : 257 (Burma, BM). New synonym.

Mimastracellabrunnea Gressitt \& Kimoto, 1963, Pac. Ins. Mon., 1B: 411, 412, fig. (Hainan Is. ; CAS). New synonym.

Distribution : Burma, Thailand, Cambodia, Laos, Vietnam, Hainan.

Material examined. THAILAND : Chiengmai Prov., Mae Cho, 1 ex., 9. vi. 1965, P. D. Ashlock; Pangmakampon (Pankanpawng), nr. Fang, 450 m, 1 ex., 16. xi. 1957, J. L. Gressitt (BISHOP) ; Phrae, 2 exs., 2. v. 1938, 1 ex., 20. vi. 1938, 1 ex., 29. v. 1938, 5 exs., 2. vi. 1938, 1 ex., 7. vi. 1938, 1 ex., 8. vi. 1938, 7 exs., 18. vi. 1938 (BANGKHEN). LAOS : Vientiane Prov., Ban Van Eue, 3 exs., 15-31. v. 1965,
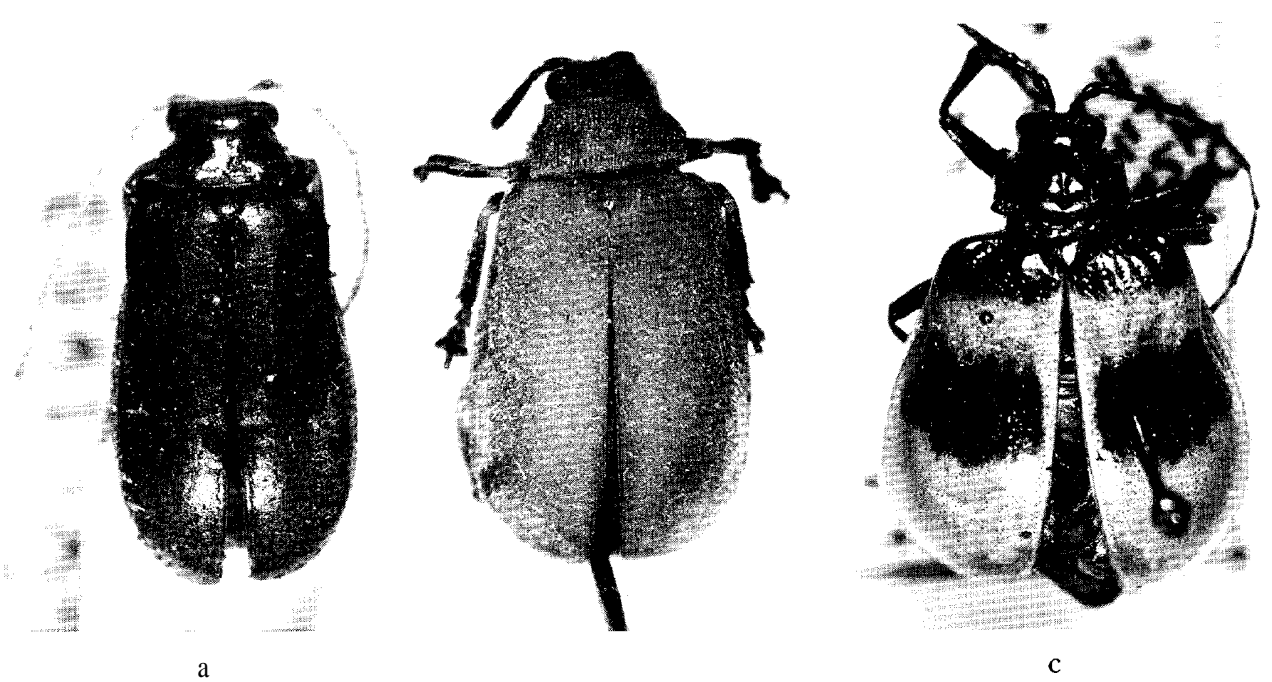

Fig. 12. a, Sastroides pallidifulvus n. sp.; b, S. birmanicus Jacoby ; c, Meristoides keani Laboissière. 
Native collr ; Attopeu Prov., Houei Kong, 1 ex., 31. v. 1965, Native collr ; Khammouane Prov., Phon Tiou, 1 ex., 17. v. 1965, Native collr ; Wapikhamthong Prov., Khong Sedone, 1 ex., '27. ix. 1965, Native collr ; Sayaboury Prov., Sayaboury, 1 ex., 15. iv. 1965, J. L. Gressitt (BISHOP) ; Umgeb. Vientiane, 2 exs., iii-vi. 1963 (MUNCHEN). VIETNAM : Kontum, N. of Pleiku, 550 m, 1 ex., 13. v. 1960, S. Quate (BISHOP).

\section{Sastroides pallidifulvus n. Sp. Fig. 12a}

Generally yellowish brown.

Head with vertex sparsely covered by large punctures and fine pubescence, and with a longitudinal median furrow, slightly depressed at front, frontal tubercle distinct, transverse, subquadrate, contiguous, surface smooth, shining, impunctate. Antenna slender, nearly $2 / 3$ as long as body length, and in preapical segments nearly three times as long as wide ; first segment robust, somewhat clubshaped, second shortest, nearly half as long as first, third $21 / 3$ times as long as second, fourth $11 / 3$ times as long as third, fifth slightly shorter than fourth, sixth subequal to fifth in length and shape, seventh slightly shorter than sixth, eighth subequal to seventh in length and shape, ninth slightly shorter than eighth, tenth subequal to ninth in length and shape, eleventh $1 \frac{1}{3} 3$ times as long as tenth and its apex pointed. Pronotum transverse, nearly $21 / 5$ times as long as wide, anterior margin slightly curved posteriorly, lateral margin almost. straight, widest almost at base, and slightly narrowed anteriorly, basal margin widely rounded posteriorly, anterior and posterior corners slightly produced laterally and each with a setigerous pore; surface glabrous, rather closely impressed by large punctures and with a shallow longitudinal median furrow and a pair of shallow transverse depressions laterally. Scutellum subtriangular, apex rounded, closely covered by fine pubescence. Elytron with lateral margin subparallel-sided, apex rounded, distinctly and closely punctate, and closely covered by fine pubescence.

Length : 6.8-9.3 $\mathrm{mm}$.

Holotype (Type No. 2696, Kyushu Univ.) : THAILAND : Chiengmai Prov., Doi Suthep, 1,000 m, 19. vi. 1965, K. Morimoto. Paratopotype: 1 ex., same data as the holotype (KU). Paratypes: THAILAND : Chiengmai Prov., Doi Suthep, 1,000 m, 2 exs., 12. vi. 1965, K. Morimoto \& Y. Miyatake ; Doi Suthep, Takeo, 800 m, 1 ex., 10. vi. 1965, K. Morimoto (KU) ; Chiengmai Prov., Doi Suthep, 1,000 m, 2 exs., 12. vi. 1965, P. D. Ashlock (BISHOP). LAOS : Khammouane Prov., Phon Tiou, 1 ex., 17. v. 1965, 1 ex., 11. ix. 1965, Native collr ; Muong Sing, NW of Luang Prabang, 650 m, 3 exs., 6-10. vi. 1960, L. W. Quate (BISHOP) ; Umbeg. Vientiane, 1 ex., iii-vi. 1963 ; Umgeb. Paklay, 2 exs., 1963 (MUNCHEN). VIETNAM : Annam, 5 exs., 1927 (BASEL).

This new species somewhat resembles Sastroides dohertyi (Maulik), but differs in having pronotum much wider and the lateral margin nearly straight.

\section{Sastroides purpurascens (Hope)}

Galleruca purpurascens Hope, 1831, in Gray, 2001. Misc. : 29 (Nepal ; BM).

Haplosonyx sublaevicollis Jacoby, 1889, Ann. Mus Civ. Genova, 27 : 218 (Burma ; BM).-Maulik, 1936, Fauna India, Galeruc. : 267 (=purpurascens).

Sastroides purpurascens: Bryant, 1923, Ann. Mag. Nat. Hist., ser. 9, 12 : 146 (Gastroides; error) ; 1937, ibid., ser. 10, 20 :101.-Kimoto, 1979, Ent. Basiliensia, 4 : 469 (Nepal).-Shute, 1983, Bull. Br. Mus. Nat. Hist. (Ent.), 46(3): 256 (India).

Sastra acutipennis Laboissière, 1932, Bull. Mus. Hist. Nat., Paris, ser. 2. 4 : 960 (Sikkim; PARIS). -Kimoto, 1979, Ent. Basiliensia, 4 : 461 (=purpurascens).-Shute, 1983, Bull. Br. Mus Nat. Hist. 
(Ent.), 46(3): 256 (Sikkim).

Sastra purpurascens: Maulik, 1936, Fauna India, Galeruc. : 267 (Nepal, Burma).-Kimoto, 1970, Spec. Bull. Lep. Soc. Japan, 4 : 170 (Nepal).

Distribution : Nepal, Burma, Thailand, Laos.

Material examined. THAILAND : Chiengmai Prov., Doi Suthep, 1,000 m, 1 ex., 12. vi. 1965, Y. Miyatake (KU). LAOS : Vientiane Prov., Ban Van Eue, 2 exs., 15-31. iv. 1965, 1 ex., 15-31. v. 1966, 1 ex., 15. v. 1967, 1 ex., 30. v. 1967, Native collr (BISHOP) ; Umgeb. Vientiane, 4 exs., iii-vi. 1963 ; Umgeb. Paklay, 3 exs., 1963 (MUNCHEN).

\section{Sastroides tibialis Jacoby}

Sastroides tibialis Jac., 1900, Mém. Soc. Ent. Belg., 7 : 131 (India).

Sastra tibialis: Maulik, 1936, Fauna India, Galeruc. : 264 (Inida, Burma, Malaya).

Distribution : India, Burma, Laos, Vietnam, Malaya.

Material examined. LAOS : Vientiane Prov., Ban Van Eue, 1 ex., 15. v. 1965, 1 ex., 30. vi. 1966, 1 ex., 15. viii. 1966, 1 ex., 1-15. ix. 1967, Native collr (BISHOP). VIETNAM : Ban Me Thuot, 500 m, 1 ex., 16-18. v. 1960, S. Quate (BISHOP).

\section{Sastroides violaceus (Weise), new combination Fig. 1la}

Sastra violacea Weise, 1922, Tijdschr. Ent., 65 : 66 (Fokien ;Tonkin: Montes Mauson ; STOCKHOLM).

Mimastracella violacea: Gressitt \& Kimoto, 1963, Pac. Ins. Mon., 1B: 412, 414 (China).

Distribution : Laos, Vietnam, China.

Material examined, LAOS : Vientiane Prov., Ban Van Eue, 1 ex., 15. v. 1965, 1 ex., 29. iv. 1966, Native collr, 7 exs., 1-15. v. 1965, J.A. Rondon (BISHOP).

\section{Genus M eristoides Laboissière}

Meristoides Lab., 1929, Rev. Chil. Hist. Nat., 33 : 289 (type :Merista touzalini Laboissière; Yunnan).-Ogloblin, 1936, Fauna USSR, 26, 1 : 334, 375.-Gressitt \& Kimoto, 1963, Pac. Ins. Mon., 1B : 393, 554.

\section{M eristoides keani Laboissière Fig. 12c}

Meristoides keani Lab., 1929, Rev. Chil. Hist. Nat., 33 : 292 (Siam ; HAMBURG).

Distribution : Thailand.

Antenna filiform ; pronotum subquadrate, nearly impunctate medianly, and with a lateral and medio-basal depressions ; elytron wider than pronotum at base, and its surface confusedly and not closely impressed by fine punctures ; generally blackish violaceous, elytron ochraceous with basal and median bands violaceous ; abdomen ochraceous ; length $12.0 \mathrm{~mm}$.

Material examined. THAILAND : "Siam", 2 exs. (BANGKHEN).

\section{Genus Anadimonia Ogloblin}

Anadimonia Ogl., 1936, Fauna USSR, 26, 1: 128, 391 (type: Anadimonia potanini Ogloblin; China ; monobasic).-Wilcox \& Seeno, 1982, Entomography, 1 : 110. 
Trichocerophysa Gressitt \& Kimoto, 1963, Pac. Ins. Mon., 1B : 392, 471 (type : Trichocerophysa latifasciata Gressitt \& Kimoto ; SW. China).

\section{Anadimonic:latifasciata (Gressitt \& Kimoto), new combination Fig. 11c}

Trichocerophysa latifasciata Gressitt \& Kimoto, 1963, Pac. Ins. Mon., 1B: 472, fig. (SW. China :

Kweichow ; FREY).

Triochocerophysa hainana Gressitt \& Kimoto, 1963, ibid., 472, 473 (Hainan ; CAS).-Kimoto, 1969,

Esakia, Kyushu Univ., $7: 36$ (Taiwan). New synonym.

Distribution : Vietnam, S. China, Hainan, Taiwan.

Oblong ; elytron subparallel-sided and closely covered by fine hairs ; head, pro- and mesothorax yellowish brown ; ventral surfaces of metathorax and abdomen black ; elytron blackish with transverse band yellowish brown, in most dark colored specimen, elytron entirely blackish ; antenna pitchy black with anterior femur yellowish brown; length 4.3-4.8 mm.

Material examined. VIETNAM : Fyan, 900-1,000 m, 2 exs., 11. vii.-9. viii. 1961, N. R. Spencer (BISHOP).

\section{Genus Oides Weber}

Oides Weber, 1801, Obs. Ent. : 26 (type : Chrysomela bipunctata Fabricius= Oides andrewesi Jacoby ; Burma).-Maulik, 1936, Fauna India, Galeruc. : 105.-Ogloblin, 1936, Fauna USSR, 26, $1: 145,395$.-Gressitt \& Kimoto, 1963, Pac. Ins. Mon., 1B : 392, 474.

Adorium Fabricius, 1801, Syst. Ent., $1: 409$ (type :Chrysomela bipunctata Fabricius= Oides and rewesi Jacoby ; Burma).-Chapuis, 1875, Gen. Col., $11: 156$.

Ochralea Chevrolat, 1837, in Dejean, Cat. Col., ed. 3 : 399 (type : Adorium flavum Olivier ; India ; monobasic).

Rhombopalpa Chevrolat, 1837, ibid. : 399 (type :Adorium decempunctata Billberg; China ; monobasic).

Boisduvallia Montrouzier, 1855, Ann. Soc. Agr. Lyon, $7: 72$.

Botanoctona Fairmaire, 1877, Petites Nouvelles Ent., 2(185) : 185.

Allard (1891, Nouv. Arch. Mus. Paris, ser. 3 , 3 :230, from Luang Prabang, and 1904, Miss. Pavie Indo-chine, $3: 159$ from Cambodge) recorded Oides concolor (Fabricius) from Indo-China. According to Vachon (1980), this species distributes only in Africa. This species is not included in the text.

$$
\text { KEY to SPECIES of Oides }
$$

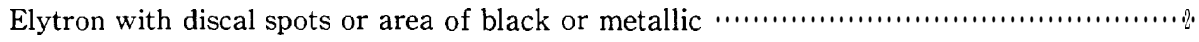

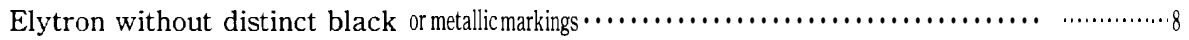

2(1) Elytral disc with a broad discal stripe or area extending most of elytral length ……........ 3

Elytral disc with several spots or a single subterminal dark area............................' 4

3(2) Elytral disc almost entirely metallic blue or green ; generally yellowish brown, antenna with apical four segments blackish ; length 13.0-15.0 $\mathrm{mm}$................... bowringii Elytral disc with a broad shiny black stripe less than $1 / 2$ as broad as elytron ; pronotum with an irregular transverse pitchy mark ; elytral epipleuron $1 / 3$ times as wide as elytron ; generally yellowish brown, with metasternum and most of abdominal segments pitchy black ; length $11.0-12.0 \mathrm{~mm} \ldots \ldots \ldots \ldots \ldots \ldots \ldots \ldots \ldots \ldots \ldots \ldots \ldots$ matata (part) 
Elytron with a single large subrounded postmedian black spot, occupying about $1 / 4$ area of disc; pronotum unspotted; generally yellowish brown with metasternum, middle of each abdominal segment, apical $2 / 3$ of antenna, tarsi and most of tibiae blackish ; length 12.0-14.5 mm

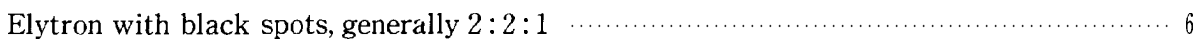

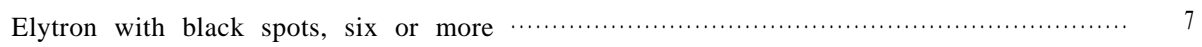

6(5) Elytral epipleuron at middle about $1 / 4$ as wide as disc ; pronotum unspotted ; elytron with five distinct subrounded spots; generally yellowish brown with metasternum and marking of each abdominal segment blackish ; length 10.0-12.0 mm …decempunctatus Elytral epipleuron at middle about $1 / 10$ as wide as disc ; pronotum with four black spots, each side with a large one near side and a small one near middle ; elytron with five large black spots, each more or less rhomboid in shape and the last appearing as if representing two merged spots; ventral surfaces entirely brownish ; length 12.0 $\mathrm{mm}$

7(5) Elytron with seven markings blackish; generally yellowish brown, pronotum with a

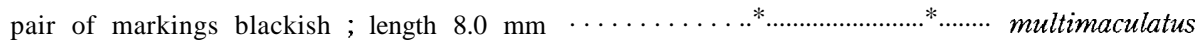
Elytron with six markings blackish; generally yellowish brown, pronotum with a pair of markings blackish ; length $11.5 \mathrm{~mm}$.............................. duodecimpunctatus

Elytral epipleuron about $1 / 3$ as wide as disc ; generally yellowish brown, with most of metasternum and a pair of markings of each abdominal segment blackish, antenna with apical four segments blackish ; length 11.0-12.0 $\mathrm{mm}$.................. maculatus (part)

9(8) Ventral surfaces partly black; body length $10.0-14.0 \mathrm{~mm}$ 10 Ventral surfaces entirely brownish ; basal margin of elytron straight ; generally yellowish brown ; length $8.5 \mathrm{~mm}$ flavus

10(9) Head pale; elytron impressed by distinct punctures; ventral surfaces of thorax pitchy black; abdomen yellowish brown with lateral marking blackish, in some

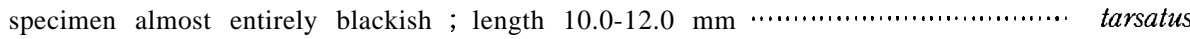
Head largely blackish ; elytral punctures of two sizes, the larger ones several times as large as the small ones ; antenna, legs and ventral surfaces of thorax largely black ; abdomen yellowish brown with lateral marking blackish ; length 11.0-12.0 $\mathrm{m} \mathrm{m}$

lividus

\section{Oides andrewesi Jacoby}

Chrysomela bipunctata Fabricius, 1781, Spec. Ins., 1: 127 (nec Linnaeus, 1758 ;Cryptocephalus) ("Cape of Good Hope") ; 1792, Ent. Syst., 1, 1 : 329 (Cap. Bon).-Hincks, 1949, Ann. Mag. Nat. Hist., ser. 12, $2: 618$ (nomenclature).

Adorium bipunctata : Fabricius, 1801, Syst. Eleuth., 1 : 409 (Cap. Bon).

Oides bipunctata : Weber, 1801, Obs. Ent., 53 (India orient)-Allard, 1889, Ann. Soc. Ent. France, ser. 6, $9: 307$ (Vietnam) ; 1891, Nouv. Arch. Mus Paris, ser. 3, $3: 230$ (Luang-Prabang) ; 1904, Miss. Pavie Indo-Chine, 3 : 159 (Cambodge).-Weise, 1922, Tijdschr. Ent., 65 : 56 (Darjeeling, Bangkok).-Kimoto, 1972, Tijdschr. Ent., 93(4): 145 (Siam).

Oides andrewesi Jacoby, 1900, Mém.Soc. Ent. Belg., 7 : 127 (Burma ;BM).-Hincks, 1949, Ann. Mag. Nat. Hist., ser. 12, $2: 618$ (independent species).-Gressitt \& Kimoto, 1963, Pac. Ins. Mon., 1B: 475 (Hainan).-Vachon, 1977, Ann. Soc. Ent. France, $82: 12$ (nomenclature).

Oides indosinensis Laboissière, 1927, Ann. Soc. Ent. France, 96 : 37 (Cochinchina, Annam, Laos, 
Tonkin, Assam ;HAMBURG).-Châjô, 1962, Phil. J. Sci., 91(1-2): 65 (Formosa).-Gressitt \& Kimoto, 1963, Pac. Ins. Mon., 1B: 476 (=andrewesi).

Oides bipunctata var. andrewesi : Maulik, 1936, Fauna India, Galeruc. : 109 (Burma).

Distribution : India, Burma, Thailand, Cambodia, Laos, Vietnam, Hainan, Taiwan.

Material examined. THAILAND : Mae Klang, Chieng Mai Prov., 340 m, 1 ex., 11. vi. 1965, P. D. Ashlock; Sattahib, 1 m, 1 ex., 24. vi. 1957, J. L. Gressitt ; Bangkhen, 1 ex., 24. iv. 1963, 1 ex., 24. vi. 1964, 1 ex., 26. vi. 1963, 1 ex., 10. viii. 1963, 1 ex., 20. viii. 1963, 1 ex., 7. iv. 1963, 1 ex., 10. ix. 1963, Native collr ; Kanchanaburi, 1 ex., 1. viii. 1963, 2 exs., 9. viii. 1967, 1 ex., 10. viii. 1963, 1 ex., 12. viii. 1963, Native collr ; Ratburi, 1 ex., 10. viii. 1964, 1 ex., 10. ix. 1964, Native collr ; Chantburi, 1 ex., 7. viii. 1964, 1 ex., 14. ix. 1963, Native collr; Kao Yai, 1 ex., 29. vii. 1963, 1 ex., 14. x. 1963, Native collr ; Prachuabkirikhan, 2 exs., 16. vii. 1963, Native collr; Phuket, 1 ex., 8. v. 1963, Native collr ; Pitsanuloke, 1 ex., 12. viii. 1964, Native collr ; Bangkok, 1 ex., 20. vi. 1963, Native collr ; Nakornrajsima, 1 ex., 13. x. 1963, Native collr ; Phuket, 1 ex., 8. v. 1963, Native collr ; Phukae, 1 ex., 9. v. 1963, Native coll- ; Kampaengpet, 1 ex., 10. ix. 1963, Native collr (BISHOP) ; Mae Klang Water Fall, nr. Chom Thong, 1 ex., 11. vi. 1965, K. Morimoto ; Chieng Mai, 1 ex., 18. vii. 1970, S. Nakao (KU) ; Tak, 1 ex., 6. vi. 1959, 1 ex., 20. vi. 1959, 1 ex., 21. vi. 1959, 1 ex., 27. vi. 1959, 1 ex., 29. vi. 1959, 6 exs., 28. vi. 1959 ; Kanchanaburi, 19 exs., 21. v. 1962 ; Prachuab Khiri Khan, 2 exs., 20. v. 1956 ; Phathomathani, 1 ex., 17. viii. 1956, 1 ex., 17. iv. 1956, 1 ex., 11. vi. 1958, 1 ex., 20. viii. 1956 ; Nakhon Ratchasima, 2 exs., 22. vi. 1958, 1 ex., 21. vi. 1958, 1 ex., 7. x. 1958 ; Saraburi, 4 exs., 21-22. vi. 1956 ; Bangkok, 2 exs., 12. viii. 1955 ; Nakhon Ratchasima, 1 ex., 6. ix. 1959, 1 ex., 22. vii. 1956 ; Prachuab Khiari Khan, 1 ex., v. 1956 (BANGKHEN). CAMBODIA : Ph. Chisau, 40 km, S. of P. Penh, 20 m, 1 ex., 29. iv. 1961, N. R. Spencer (BISHOP). LAOS : Vientiane Prov., Ban Van Eue, 5 exs., 30. vi. 1965, 21 exs., 31 vii. 1965, 4 exs., 15. vii, 1965, 1 ex., 30. ix. 1965, 2 exs., x. 1965, 5 exs., 30. vi. 1965, 1 ex., 2. ii. 1966, 8 exs., 31. v. 1966,1 ex., 15. vi. 1966, 3 exs., 30. vi. 1966, 1 ex., 15. vii. 1966, 2 exs., 30. vii. 1966, 5 exs., 30. iii. 1967, 5 exs., 1-15. ix. 1967, 4 exs., 31. xii. 1968, Native collr ; Sayaboury Prov., Sayaboury, 5 exs., 14. vii. 1965, 6 exs., 25. viii. 1966, 1 ex., 15. ix. 1966, 4 exs., 30. ix. 1966, Native collr ; Borikhane Prov., Pakkading, 1 ex., 17. v. 1965,1 ex., 13. vii. 1965, 1 ex., 14. vii. 1965, 1 ex., 31. vii. 1965, 1 ex., x. 1965, 3 exs., 28. vii. 1965, 1 ex., 8. ii. 1966, 1 ex., 29. iv. 1966, 1 ex., 15. vi. 1966, Native collr ; Attopu Prov., Houei Kong, 3 exs., 16. v. 1965, 2 exs., 31. v. 1965, 1 ex., 17. vii. 1965, 2 exs., 7. x. 1965, Native collr ; Sedone Prov., Pakse, 3 exs., 1. vi. 1965, 1 ex., 7. vi. 1965, 2 exs., 30. viii. 1965, 2 exs., Native collr ; Khammouane Prov., Phon Tiou, 3 exs., 17. v. 1965, 1 ex., 6. vii. 1965, Native collr ; Wapikhamthong Prov., Khong Sedone, 1 ex., 13. viii. 1965, Native collr ; Ban Theuong, $18 \mathrm{~km} \mathrm{NW}$ of Xieng Khouang, 1,035 m, 1 ex., 2-8. viii. 1960, R. E. Leech ; Xieng Kyouang Prov., Ban San Thang, 1 ex., 5. vi. 1965, Native collr ; Tonpheng, 1 ex., 30. xi. 1966, Native collr ; Dang Dok, 1 ex., 23. vii. 1965, Native collr (BISHOP) ; Umgeb. Vientiane, 16 exs., iii-vi. 1963 ; Umgeb. Paklay, 9 exs., 1963 ; Umgeb. Vanky, 3 exs., 1963-64 (MUNCHEN).

\section{Oides bowringii (Baly)}

Adorium bowringii Baly, 1863, Trans. Ent. Soc. London, ser. 3, 1: 623 (Hong Kong; BM). -Fairmaire, 1889, Ann. Soc. Ent. France, $58: 74$ (Korea, Moupin).

Oides bowringii: Laboissière, 1919, Bull. Soc. Ent. France, 1919 : 161 (“N. China”).-Ogloblin, 1936, Fauna USSR, 26, 1: 149 (Korea, Japan).-Gressitt \& Kimoto, 1963, Pac. Ins. Mon., 1B : 475, 476 (China).

Oides elegans Laboissière, 1919, Bull. Soc. Ent. France, 1919 : 161 (Tonkin ; HAMBURG) ; 1929, Ann. Soc. Ent. France, 98 : 252 (China).-Ogloblin, 1936, Fauna USSR, 26, 1 : 149, 396 (Corêe, China, Japon).-Gressitt \& Kimoto, 1963, Pac. Ins. Mon., 1B: 476 (= bowringii). 
O ides tonkinensis Laboissiere, 1929, Ann. Soc. Ent. France, 98 : 252 (Tonkin ; HAMBURG). New synonym.

Distribution : Vietnam, S. China, Japan, Korea.

No additional material was examined.

\section{Oides decempunctatus (Billberg)}

Adorium decempunctata Billb., 1808, in Schbnherr, Syn. Ins., I, $2: 230$, note (China).

Oides decemmaculata Laboissiere, 1927, Ann. Soc. Ent. France, 96 : 39 (Tonkin; HAMBURG). -Chajô, 1962, Phil. J. Sci., 91(1-2): 63 (Formosa). New synonym.

Solanophila gigantea Roubal, 1929, Bull. Soc. Ent. Ital., 61 : 96 (Korea ; described in Coccinellidae). -Ogloblin, 1936, Fauna USSR, 26, $1: 148$ (= decempunctata).

O ides decempunctata : Allard, 1891, Nouv. Arch. Mus. Paris, ser. 3, $3: 230$ (Luang-Prabang) ; 1904, Miss. Pavie Indo-Chine, 3 : 159 (Cambodge).-Ogloblin, 1936, Fauna USSR, 26, 1 : 148,396, fig. 61 (Korea, N. China).-Gressitt \& Kimoto, 1963, Pac. Ins. Mon., 1B: 475, 477 (China, Hainan, Vietnam).

Distribution : Cambodia, Laos, Vietnam, China, Hainan, Taiwan, Korea.

According to the study on the type of decemmaculata Laboissiere, this is only an infraspecific variation of decempunctata Billberg, in having the elytral markings larger.

Material examined. LAOS : Umgeb. Vientiane, 4 exs., iii-vi. 1963 ; 1 ex., Umgeb. Paklay, 4 exs., 1963 (MUNCHEN) ; Borikhane Prov., Pakkading, 1 ex., 22. iii. 1965, Native collr ; Sedone Prov., Pakse, 1 ex., 2. ix. 1965, Native collr ; Ile de Khong, 1 ex., 12. xi. 1965, Native collr ; Wapikhamthong Prov., Khong Sedone, 2 exs., 16. v. 1965, Native collr (BISHOP). VIETNAM : M'Drak, E. of Ban Me Thout, 4-600 m, 1 ex., 8-19. vii. 1960, C. M. Yoshimoto (BISHOP).

\section{Oides duodecimpunctatus (Clark)}

Rhombopalpa duodecimpunctata Clark, 1865, Ann. Mag. Nat. Hist., ser. 3, 15 : 143 (Siam).

O ides duodecimpunctata : Jacoby, 1883, Proc. 2001. Soc. London, 1883:400 (mentioned).

Distribution : Thailand.

I could not trace the type location of this species.

Oides duporti Laboissière Fig. lc

O ides duporti Lab., 1920, Bull. Soc. Ent. France, 1919 : 160 (Tonkin ; HAMBURG).

O ides leucomelaena Weise, 1922, Tijdschr. Ent., 65 : 58 (Fukien ; STOCKHOLM).-Gressitt \& Kimoto, 1963, Pac. Ins. Mon., 1B: 475, 478, fig. (S. China). New synonym

O ides leucomelaena varr. subsinuata, disjuncta Pic, 1928, Mel. Exot. Ent., 52 : 29 (Tonkin).

Distribution : Vietnam, S. China.

No additional material was examined.

\section{Oides duvaucelii (Guérin-Méneville)}

Adorium duvaucelii Guérin-Méneville, 1830, Voy. Coquille., Zool., 2 : 148 (Cochinchina).

Distribution : Vietnam.

I could not find the type series, Judging from the original description, this species is close to O ides bowringii (Baly) or same. This species is not included in the key. 


\section{Oides flavus (Olivier)}

Adotium flavum Oliv., 1807, Entomolog., 5 :611, pl. 1, fig. 5 (Bengale).-Vachon, 1977, Ann. Soc. Ent. France, $82: 12$ (nomenclature).

O ides inornata Baly, 1879, Cist. Ent., 2 : 444 (Assam ; BM).-Maulik, 1936, Fauna India, Galeruc. : 118 (=flava).

Oides albicans Duvivier, 1884, Notes Leyden Mus., $6: 237$ (E. Sumatra)-Allard, 1891, Nouv. Arch. Mus. Paris, ser. 3, 3 : 230 (Luang-Prabang) ; 1904, Miss. Pavie Indo-Chine, 3 : 159 (Cambodge).-Maulik, 1936, Fauna India, Galeruc. : 118 (=flava).

O ides coomani Laboissike, 1927, Ann. Soc. Ent. France, 96 : 42 (Tonkin; HAMBURG). New synonym.

O ides flava:Allard, 1891, Nouv. Arch. Mus. Paris, ser. 3, $3: 230$ (Luang-Prabang) ; 1904, Miss. Pavie Indo-Chine, 3 : 159 (Cambodge)-Maulik, 1936, Fauna India, Galeruc. : 118 (India, Malay Peninsula, Sumatra, Java, SE. Borneo, Philippines).-Gressitt \& Kimoto, 1963, Pac. Ins. Mon., 1B : 477 (Hainan, Vietnam).-Kimoto \& Takizawa, 1972, Kontyû, Tokyo, 40(4) :217( (Nepal).

Distribution : India, Nepal, Cambodia, Laos, Vietnam, Hainan, Malaya, Sumatra, Java, Borneo, Philippines.

Material examinaed. LAOS : Umgeb. Paklay, 1 ex., 1963 (MUNCHEN).

\section{Oides lividus (Fabricius)}

Adorium lividum Fabr., 1801, Syst. Eleuth., 1 : 410 (Sumatra).

O ides livida: Weber, 1801, Obs. Ent. : 53 (India orientali).-Vachon, 1980, Ann. Soc. Ent. France, $85: 15$ (nomenclature).

Adotium diardi Guérin-Méneville, 1830, Voy. Coquille, Zool., 2 : 148 (Cochinchina). New synonym.

Rhombopalpa pectoralis Clark, 1865, Ann. Mag. Nat. Hist., ser. 3, 15 : 144 (Siam ; BM).-Vachon, 1980, Bull. Soc. Ent. France, $85: 144$ (= livida).

O ides pectoralis: Jacoby, 1884, Notes Leyd. Mus., $6: 37$ (Sumatra).-Duvivier, 1891, C. R. Soc. Ent. Belg., 35 : 45 (Bengale).-Maulik, 1936, Fauna India, Galeruc. : 114 (India, Sikkim, Burma, Siam, Malaya, Sumatra).-Gressitt \& Kimoto, 1963, Pac. Ins. Mon., 1B: 475, 478 (China). -Kimoto \& Takizawa, 1972, Kontyû, Tokyo, 40 : 217 (Nepal).-Kimoto, 1977, Ent. Basiliensia, 2: 354 (Bhutan).

Oides nigripes Jacoby, 1891, Entomolog., 25 (Suppl) : 34 (Sikkim ; BM)--Maulik, 1936, Fauna India, Galeruc. : 114 (=pectoralis).

O ides pallidicornis Jacoby, 1899, Stett. Ent. Ztg., $60: 284$ (Sumatra).-Vachon, 1980, Bull. Soc. Ent. France, $85: 15$ (=livida).

Distribution : India, Nepal, Bhutan, Burma, Thailand, Laos, Vietnam, Malaya, Sumatra.

I could not check the type of Adorium diardi Guérin. Judging from the original description, this species is no doubt a synonym of livida Fabricius.

Material examined. THAILAND : Saraburi, 1 ex., 21. vii. 1956 ; Chanataburi, 2,000 ft, 1 ex., 2030. v. 1959 ; Kanchanaburi, 2 exs., 21. v. 1962 (BANGKHEN) ; Pattani, 1 ex., 31. viii. 1964, Native collr (BISHOP). LAOS : Umgeb. Vientiane, 2 exs., iii-vi. 1963 ; Umgeb. Paklay, 4 exs., 1963 ; Umgeb. Pakse, 1 ex., 1963 ; Umgeb. Vanky, 1 ex., 1964 (MUNCHEN) ; Vientiane Prov., Ban Van Eue, 1 ex., 15-31. iv. 1965, 1 ex., 1-15. v. 1965, 3 exs., 15-31. v. 1965, Native collr ; Vientiane Prov., Phou Kou Khouei, 1 ex., 30. vi. 1965, Native collr ; Khammouane Prov., Phon Tiou, 1 ex., 17. v. 1965, Native collr ; Borikhane Prov., Pakkading, 100 m, 1 ex., 23. iv. 1965, J. L. Gressitt ; Attopeu Prov., Houei 
Kong, 1 ex., 16. v. 1965, Native collr (BISHOP).

\section{Oides maculatus (Olivier)}

Adorium maculatum Oliv., 1807, Entomolg., 5: 611, pl. 1, fig. 4 (Java).

Adorium subhemisphaericum Guérin-Méneville, 1830, Voy. Coquille, Zool., 2 : 146 (Java).-Fairmaire, 1888, Ann. Soc. Ent. France, ser. 6, 8 : 374 (Tonkin).-Gahan, 1891, Ann. Mag. Nat. Hist., ser. 6, 7:460 (= maculata).

Oides indica Baly, 1879, Cist. Ent., 2 : 443 (Assam).- Jacoby, 1889, Ann. Mus. Civ. Genova, 26 : 206 (Burma).-Gahan, 1891, Ann. Mag. Nat. Hist., ser. 6, $7: 460$ (= maculata).

Adorium laticlavum Fairmaire, 1889, Ann. Soc. Ent. France, ser. 6, 9 : 74 (China : Kweichow, Maupin; PARIS). New synonym.

Oides subhemisphaerica :Allard, 1891, Nouv. Arch. Mus. Paris, ser. 3, 3 : 230 (Luang Prabang); 1904, Miss. Pavie Indo-Chine, 3 : 159 (Cambodge : Luang-Prabang à Theng).

Oides laticlava:Laboissière, 1929, Ann. Soc. Ent. France, 98 : '252, fig. 1 (China).-Ogloblin, 1936, Fauna USSR, 26, 1 : 148, 396 (China).-Gressitt \& Kimoto, 1963, Pac. Ins. Mon., 1B: 475, 478 (SW. China, Taiwan).

Oides epipleuralis Laboissière, 1929, Ann. Soc. Ent. France, 98 : 254, fig. (Formosa ; HAMBURG). —Chûjô, 1962, Phil. J. Sci., 91(1-2) : 68 (Formosa).-Gressitt \& Kimoto, 1963, Pac. Ins. Mon., 1B : 478 (= laticlava).

Oides maculata : Allard, 1891, Nouv. Arch, Mus. Paris, ser. 3, 3 : 230 (Luang-Prabang) ; 1904, Miss. Pavie Indo-China, 3 : 159 (Cambodge).-Maulik, 1936, Fauna India, Galeruc. : 106, 113 (India, Burma, Andaman Is., Penang, Java, Cambodia).-Chûjô, 1964, Nature and Life in SE Asia, Kyoto, 3 : 296 (Thailand).-Kimoto, 1982, Ent. Rev. Japan, 37(1) : 9 (Nepal).

Distribution : India, Nepal, Sikkim, Burma, Thailand, Cambodia, Laos, Vietnam, China, Taiwan, Malaya, Java.

This species is usually entirely pale above, but in most of the Chinese specimens it is characteristic in having the large blackish marking covering most of the surface of elytron. I could not check the type of Adorium maculatum Olivier. According to the study on the material identified by Laboissière, the specimens identified as laticlava or epipleuralis are the same as maculatus (Olivier), and its infraspecific variation.

Material examined. THAILAND: Cholburi, 19 exs., 16. x. 1956, Nakhon si thamarat, 7 exs., 7. ix. 1958 ; Saraburi, 2 exs., 21-22. vii. 1956 ; Phrae, 3 exs., 18. vi. 1938 ; Nakhon ratchasima, 2 exs., 23. vi. 1958, 1 ex., 15. vi. 1958 (BANGKHEN) ; Chiengmai Prov., Fang, 500 m, 1 ex., 13. vi. 1965, F. D. Ashlock; Chawang Co., 1 ex., 7. ix. 1958, J. L. Gressitt ; Banna, Chawang, nr. Nabon, 70 m, 1 ex., 5. ix. 1958, J. L. Gressitt ; Saraburi, 1 ex., 18. ix. 1964, Native collr; Kanchanaburi, 1 ex., 10. viii. 1967, Native collr ; Bang Khen, 1 ex., 7. ix. 1964, Native collr (BISHOP). LAOS : Umgeb. Vientiane, 10 exs., iii-vi. 1963 ; Umgeb. Pakse, 3 exs., 1963, Umgeb. Paklay, 3 exs., 1963-64 ; Umgeb. Vanky, 2 exs., 1963 (MUNCHEN) ; Sayaboury Prov., Sayaboury, 3 exs., 30. v. 1965, 5 exs., 14. vii. 1965, 5 exs., 25 . viii. 1966, 2 exs., 30. ix. 1966, Native collr ; Borikhane Prov., Pakkading, 3 exs., 14. vii. 1965, 1 ex., 3. viii. 1965, 1 ex., 13. v. 1966, Native collr ; Sedone Prov., Pakse, 3 exs., 5. vii. 1965, Native collr ; Vientiane Prov., Ban Van Eue, 1 ex., 29. iii. 1966, 1 ex., 15. iv. 1966, Native collr ; Khammouane Prov., Phon Tiou, 1 ex., 17. v. 1965, 1 ex., 10. vi. 1965, 1 ex., 6. vii. 1965, 1 ex., 4. viii. 1965, 1 ex., 28, ix. 1965, Native collr ; Vientiane Prov., 1 ex., 31. vii. 1965, Native collr (BISHOP). 


\section{Oides multimaculatus Pic}

Oides multimaculataPic, 19’28, Mêl. Exot. Ent., 52 : 29 (Tonkin ; PARIS).

Distribution : Vietnam.

No additional material was examined beside the type series.

\section{Oides tarsatus (Baly)}

Adorium tarsatum Baly, 1865, Trans. Ent. Soc. London, ser. 3, $2: 435$ (N. China ; BM).

Adorium sordidum Baly, 1865, ibid. : 435 (N. China ; BM)-Gressitt \& Kimoto, 1963, Pac. Ins. Mon., 1B : 479 (= tarsata).

Oides tibetana Jacoby, 1900, Mém.Soc. Ent. Belg., 7 : 128 (Tibet ; BM).-Gressitt \& Kimoto, 1963, Pac. Ins. Mon., 1B : 479 (Tibet; BM). New synonym.

Oides tibialis Laboissière, 1927, Ann. Soc. Ent. France, 96 : 40 (Tonkin; HAMBURG). New synonymy.

Oides sordida: Ogloblin, 1936, Fauna USSR, 26, $1: 147,396$ (China).

Oides tarsata: Ogloblin, 1936, ibid. : 147, 395 (Peking).-Gressitt \& Kimoto, 1963, Pac. Ins. Mon., 1B : 475, 479 (China).

Oides tibiella Wilcox, 1971, Col. Cat. Junk, Suppl., 7(1): 17 (new name for Oides tibialis Lab., nec Duvivier, 1884, from Philippines). New synonym.

Distribution : Vietnam, China.

No additional material was examined.

\section{Genus Hoplasoma Jacoby}

Hoplasoma Jacoby, 1884, Notes Leyden Mus., $6: 233$ (type : Hoplasoma apicalis Jacoby ; Celebes). -Gressitt \& Kimoto, 1963, Pac. Ins. Mon., 1B: 393, 497.

Hoplosoma Baly, 1889, Trans. Ent. Soc. London, $1889: 308$.

Haplosoma Jacoby, 1896, Ann. Soc. Ent. Belg., $40: 271,273$.

Paraulacophora Csiki, 1953, Ann. Hist.-Nat. Mus. Nat. Hung., 3 : 131.

\section{KEY TO SPECIES OF Hoplasoma}

Elytron with lateral costa starting from humeri; pronotum nearly $11 / 2$ times as wide as long; generally yellowish brown; ventral surfaces of metathorax and abdomen pitchy black ; antenna pitchy black with basal segments paler ; legs pitchy black with femora brownish; in some specimen elytron with an ill-difined longitudinal stripe blackish ; length $7.2-8.1 \mathrm{~mm}$ thailandicum

2(1) Pronotum less than twice as wide as long ; generally yellowish brown, ventral surfaces of abdomen pitchy black ; in male a pair of processes arised from middle of posterior margin of second abdominal segment ; length 7.0-8.5 mm unicolor Pronotum fully twice as wide as long; generally yellowish brown, ventral surfaces of metathorax and abdomen pitchy black, antenna with apical segments infuscate, posterior legs mostly pitchy brown; in male abdominal segment without any distinct processes ; length 9.0-10.5 $\mathrm{mm}$ 

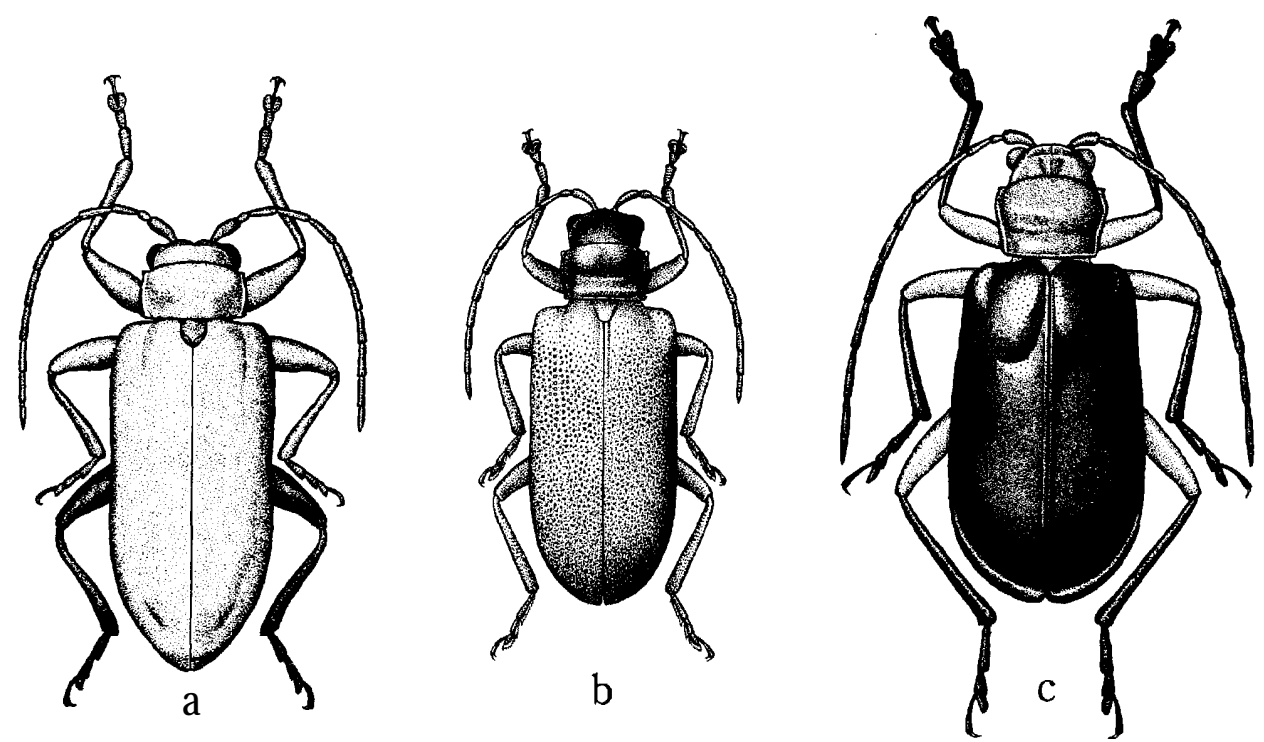

Fig. 13. a, Haplosoma majorinum Laboissiere ; b, H.unicolor (Illiger) ; c, Agetocera filicornis Laboissiere.

\section{Hoplasoma majorinum Laboissière Fig. 13a}

Hoplasoma majorina Lab., 1929, Ann. Soc. Ent. France, 98 : 258, fig. (Tonkin: Hanoi, Chapa ; Yunnan ;HAMBURG).-Chûjô, 1962, Phil. J. Sci., 91(1-2): 72 (Formosa).-Gressitt \& Kimoto, 1963, Pac. Ins. Mon., 1B : 497, fig. (China).-Kimoto, 1984, Ent. Rev. Japan, 39(1) : 49 (Taiwan). Distribution : Laos, Vietnam, S. China, Taiwan.

Material examined. LAOS :Xieng Kyouang Prov., Ban Sam Thang, 3 exs., 5. vi. 1965, Native collr (BISHOP).

\section{Hoplasoma thailandicum n. sp. Fig. 14a}

Generally yellowish brown, antenna pitchy black with two or three basal segments yellowish brown, ventral surfaces with metathorax and abdomen pitchy black, legs pitchy black with femora yellowish brown; in some specimen elytron with an ill-defined blackish stripe at middle.

Head with vertex smooth, shining, sparsely impressed by minute punctures, interocular space much wider than transverse diameter of single oculus, and interocular transverse impression distinct, frontal tubercle transverse, subquadrate, contiguous, distinctly raised, surface smooth, impunctate. Antenna slender, nearly $3 / 4$ as long as body length ; first segment long, robust, second shortest, nearly $1 / 3$ as long as first, third three times as long as second, fourth $11 / 3$ times as long as third, fourth to seventh subequal to each other in length and shape, eighth slightly shorter than seventh, ninth subequal to eighth in length and shape, tenth slightly shorter than ninth, eleventh subequal to ninth in length but its apex pointed. Pronotum transverse, $11 / 2$ times as broad as long, anterior margin nearly straight, lateral margin feebly rounded, widest almost at $1 / 3$ from anterior margin, slightly narrowed anteriorly and more strongly so posteriorly, basal margin slightly rounded posteriorly, dorsal surface smooth, shining, sparsely impressed by minute punctures, and with a distinct subbasal 
transverse depression. Scutellum subtriangular, finely granulate, impunctate. Elytron with lateral margin subparallel-sided, apex rounded, surface strongly and rather closely punctate and their interstices smooth, shining.

Length 7.2-8.1 mm.

Holotype (Type No. 2697, Kyushu Univ.) : THAILAND : Chiengmai Prov., Doi Suthep, 1,000 m, 12. v. 1965, K. Morimoto. Paratypes : THAILAND : 2 exs., same data as the holotype but 18. vi. 1965, K. Morimoto \& Y. Miyatake ; Chiengmai Prov., Doi Pui, 1,300 m, 1 ex., 8. vi. 1965, Y. Miyatake ; Chiengmai Prov., Chieng Dao, 1 ex., 15. vi. 1965, K. Morimoto (KU, KIMOTO) ; Chiengmai Prov., Doi Suthep, 1,300 m, 1 ex., 8. vi. 1965, P. D. Ashlock (BISHOP) ; "Siam”, 1 ex., Lot 319 (BANGKHEN).

This new species somewhat resembles Hoplasoma costatipennis Jacoby, from India, but differs in having mesothorax and the abdomen blackish, and elytron with the apex of the suture rounded.

\section{H oplasoma unicolor (Illiger) Fig. 13b}

Galleruca unicolor Ill., 1800, in Wiedemann, Arch. für 2001. u Zoot., 1, 2 : 135 (Bengal).

Haplosoma ventralis Baly, 1886, Trans. Ent. Soc. London, 1886 : 27 (Borneo : Sarawak ; BM). -Maulik, 1936, Fauna India, Galeruc. : 161 (= unicolor).

Haplosoma unicolor :Allard, 1888 (1889), Ann. Soc. Ent. France, ser. 6, $8: 327$ (Sumatra, Singapore, ile Bontang).--Jacoby, 1896, Ann. Soc. Ent. Belg., 40 : 271 (mentioned).

Haplosoma comiculata Allard, 1888 (1889), Ann. Soc. Ent. France, ser. 6, 8 : 328 (Sunda Is., Pnom Penh ; PARIS) ; 1891, Nouv. Arch. Mus. Paris, ser. 3, 3 : 231 (Luang Prabang).-Jacoby, 1896, Ann. Soc. Ent. Belg., $40: 271$ (= ventralis).-Allard, 1904, Miss. Pavie Indo-Chine, 3 : 160 (Laos, Luang Prabang).-Maulik, 1936, Fauna India, Galeruc. : 161 (= unicolor).

Hoplosoma javana: Allard, 1888 (1889), Ann. Soc. Ent. France, ser 6, $8: 327$ (Annam).

Phyllobrotica unicolor: Jacoby, 1889, Ann. Mus. Civ. Genova, $27: 230$ (Burma).

Haplasoma abdominalis Jacoby, 1896, Ann. Soc. Ent. Belg., 40 : 272 (Rangoon, Cashar, Tenasserim ; BM).-Maulik, 1936, Fauna India, Galeruc. : 161 (=unicolor).

Haplosoma simplicipennis Jacoby, 1896, Ann. Soc. Ent. Belg., 40 : 272 (Kanara, Belgaum, S. Bombay ; BM).-Maulik, 1936, Fauna India, Galelruc. : 161 (= unicolor).

Hoplasoma unicolor: Maulik, 1936, Fauna India, Galeruc.: 161 (India, Burma, Andaman Is., Siam, Malay Pen., Sumatra, Java, Billiton Is., Borneo, Philippines, China, Korea).-Laboissière, 1940, Bull. Mus. Hist. Nat. Belg., 16(3): 8 (Inde, Indochine, Indes neerlandaises).-Châjô, 1961, Nature and Life in SE Asia, Kyoto, $1: 350$ (Thailand).-Gressitt \& Kimoto, 1963, Pac. Ins. Mon., 1B : 497, 499, fig. (S. China, Hainan, Vietnam),-Chajô, 1964, Nature and Life in SE Asia, Kyoto, 3 : 292 (Thailand).-Kimoto, 1972, Tijdschr. Ent., 93(4) : 145 (Annam).-Kimoto \& Takizawa, 1972, Kontyâ, Tokyo, 40(4): 216 (Nepal).-Kimoto, 1977, Ent. Basiliensia, $2: 354$ (Bhutan) ; 1979, ibid., 4 : 469 (Bhutan, India).-Kimoto \& Takizawa, 1981, Ent. Rev. Japan, 35 (1-2) : 58 (Nepal).

Aulacophora (Paraulacophora) pannonica Csiki, 1953, Ann. Hist.-Nat. Mus. Nat. Hung., $3: 132$ (Hungary).-Kaszab, 1962, Fol. Ent. Hung., $15: 25$ (Hungary; suggested as imported one from the Oriental region).-Silfverberg, 1978, ibid., $31: 219$ (=unicolor).

Distribution : India, Burma, Nepal, Bhutan, Thailand, Laos, Vietnam, S. China, Hainan, Malaya, Philippines, Sumatra, Java, Billiton, Borneo.

Material examined. THAILAND : Krabi, 1 ex., 2. xii. 1963, 5 exs., 1. ix. 1964, Native collr; Trang Prov., Khaophapha Khaochong, 200-400 m, 1. ex., 30-31, xii, 1967, 2 exs., 10. i. 1964, G. A. Samuelson ; Banna, Nakhon, 100 m, 2 exs., 5-10. v. 1958, T. C. Maa (BISHOP) ; Chiengmai Prov., Doi Suthep, 1,000 m, 4 exs., 12. vi. 1965, K. Morimoto ; Chiengmai Prov., Mae Sa Watter Fall, 4 exs., 16. 
vi. 1965, K. Morimoto ; Khao Chong, nr. Trung, 1 ex., 25. vi. 1965, Y. Miyatake ; Sanpatong, 2 exs., 10. vii. 1970, K. Yano, 1 ex., 24. viii. 1973, S. Nakao (KU) ; Trang, 13 exs., 1-18. iv. 1960 ; Rannong, 3 exs., 15. x. 1959, 5 exs., 17. x. 1959; Tak, 2,800 ft., 8 exs., 20. viii. 1961 (BANGKHEN). LAOS : Vientiane Prov., Ban Van Eue, 11 exs., 15-31. v. 1965, J. A. Rondon, 2 exs., 15-31. v. 1965, 12 exs., 31. vii. 1965, 4 exs., 30. ix. 1965, 6 exs., 30. vi. 1965, 2 exs., 31. v. 1966, 1 ex., 30. vi. 1966, 4 exs., 15. xii. 1966, 1 ex., 30. xi. 1966, 1 ex., 15. ii. 1967, 6 exs., 30. iii. 1967, 1 ex., 30. vi. 1967, 6 exs., 30. viii. 1967, 2 exs., 1-15. ix. 1967, 6 exs., 31. xii. 1968, Native collr ; Vientiane Prov., Gi Sion Vill., de Tha Ngone, 1 ex., 10-24. x. 1965, Native collr ; Sayaboury Prov., Sayaboury, 2 exs., 15. iv. 1965, J. L. Gressitt, 2 exs., 17. v. 1965, 2 exs., 16. vii. 1965, Native collr ; Khammouane Prov., Phon Tiou, 1 ex., 30. iv. 1965, 2 exs., 17. v. 1965, 2 exs., 16. vii. 1965, 21 exs., 11. ix. 1965, Native collr ; Attopeu Prov., Howei Kong, 4 exs., 31. v. 1965, Native collr; Sedone Prov., Pakse, 1 ex., 31. v. 1967, Native collr; Tonpheng, 2 exs., 30. x. 1965, Native collr ; Borikhane Prov., Pakkading, 2 exs., 17. v. 1965, 1 ex., 13. vii. 1965, 2 exs., x. 1965, Native collr ; Ban Theuong, 18 km NW of Xieng Khouang, 1,035 m, 1 ex., 2-6. viii. 1960, 1 ex., 10-17. viii. 1960, R. E. Leech (BISHOP) ; Umgeb. Vientiane, 3 exs., 1963; Umgeb. Paklay, 16 exs., 1963 ; Umgeb. Pakse, 1 ex., 1964 ; Umgeb. Vankay, 2 exs., 1963 (MUNCHEN). VIETNAM : Dilinh (Djiring), 900 m, 4 exs., 22-28. iv. 1960, L. W. Quate ; $30 \mathrm{~km} \mathrm{~N}$. of Dilinh, 2 exs., 27. iv. 1960, S. Quate ; $50 \mathrm{~km} \mathrm{SW}$ of Pleiku, 250 m, 1 ex., 14. v. 1960, L. W. Quate ; Fyan, 1,200 m, 1 ex., 11. vii.-9. viii. 1961, N. R. Spencer (BISHOP).

\section{Genus Agetocera Hope}

Agetocera Hope, 1840, Col. Man., 3 : 170 (type :Aegeloceris mirabilis Hope ; India).-Chapuis, 1875, Gen. Col., 11 :177.-Maulik, 1936, Fauna India, Galeruc. :125.-Ogloblin, 1936, Fauna USSR, 26, 1: 158, 397.-Gressitt \& Kimoto, 1963, Pac. Ins. Mon., 1B : 393, 494.

$$
\text { Key to species of Agetocera }
$$

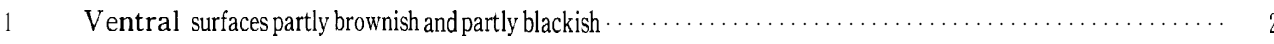

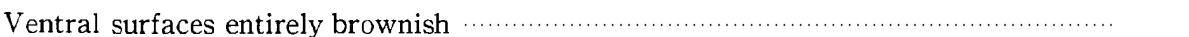

2(1) Ventral surfaces brownish with meso- and metathorax blackish ; antenna entirely brownish ; in male eighth antennal segment subequal to seventh in length and shape, ninth subcylindrical and tenth deeply emarginate at middle ; elytron black; generally reddish brown; legs pitchy black with anterior femur brownish; length 11.0 $\mathrm{mm}$

nigripennis

Ventral surfaces brownish with fifth abdominal segment blackish; elytron purplish blue ; generally yellowish brown, legs with tarsi and most of tibiae blackish ; length $12.0-13.0 \mathrm{~mm}$

duporti

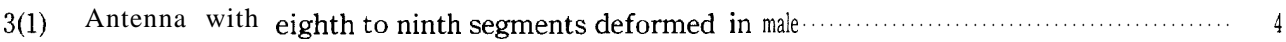
Antenna normal in male; pronotum strongly widened anteriorly ; elytron somewhat uneven, widened posteriorly, finely and irregularly punctured; generally reddish brown, elytron violaceous blue, antenna and legs mostly blackish; length 9.0-12.0

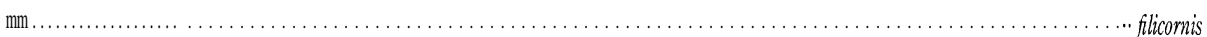

4(3) Fourth antennal segment of female not deformed 5

Fourth antennal segment of both sexes deformed, somewhat sinutate ; elytron purplish blue; generally yellowish brown, legs with tarsi and apical half of tibiae blackish, antenna with apical two segments blackish; length 13.5-16.0 mm ….............. mirabilis 
In male eighth antennal segment subequal to seventh in shape, ninth subcylindrical and tenth deeply emarginate at middle ; elytron black ; generally reddish brown, legs with tibiae and tarsi pitchy black, antenna entirely yellowish brown; length 10.0-12.0 $\mathrm{mm}$ m....................................................................... lobicornis

6(5) Ninth antennal segment of male excavated or angulate ; antenna yellowish brown

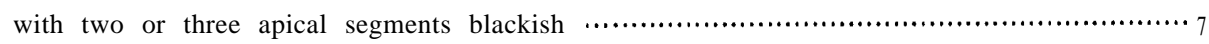
Ninth antennal segment of male subcylindrical, with depression bearing a small tubercle; generally yellowish to reddish brown, elytron dull violet purple ; antenna largely black; legs with tibiae and tarsi blackish; length $12.5-14.0 \mathrm{~mm}$.............. chapana

7(6) In male, ninth antennal segment with a very strong external protuberance at base and weaker one at apex, forming a deep crescent between ; elytron greenish to purplish black ; generally yellowish brown, legs with tarsi and apical half of tibiae blackish, and antenna with apical two segments blackish ; length 11.5-12.0 mm hopei In male, ninth antennal segment with angulately expanded; elytron cupreous violaceous; generally yellowish brown, legs with tibiae and tarsi largely blackish ; length $12.0 \mathrm{~mm}$ ....... orientalis

\section{Agetocera chapana Laboissière}

Agetocera chapana Lab., 1929, Ann. Soc. Ent. France, 98 : 260 (Tonkin; HAMBURG).-Maulik, 1936, Fauna India, Galeruc. : 132 (Burma, Chapa).-Gressitt \& Kimoto, 1963, Pac. Ins. Mon., 1B: 495 (N. Vietnam).

Distribution : Burma, Vietnam.

No additional material was examined beside the type.

\section{Agetocera duporti Laboissière}

Agetocera duporti Lab,, 1927, Ann. Soc. Ent. France, 96 : 44 (Tonkin; HAMBURG).

Distribution : Vietnam.

No additional material was examined beside the type.

\section{Agetocera filicornis Laboissière Fig. 13c}

Agetocera filicornis Lab., 1927, Ann. Soc. Ent. France, 96 : 49 (Tonkin ; HAMBURG).-Gressitt \& Kimoto, 1963, Pac. Ins. Mon., 1B : 496, fig. (China).

Distribution : Vietnam, China.

No additional material was examined beside the type.

\section{Agetocera hopei Baly}

Agetocera hopei Baly, 1865, Trans. Ent. Soc. London, ser. 3, 2(5): 438 (N. India ; BM).-Duvivier, 1891, C. R. Soc. Ent. Belg., 35 : 46 (Kurseong).-Maulik, 1936, Fauna India, Galeruc. : 130 (India, Assam, Burma).-Laboissière, 1927, Ann. Soc. Ent. France, 96 : 45, fig. 2 (Tonkin : Hanoi ;ChoGanh) ; 1929, ibid., 98 : 261 (Tonkin).-Gressitt \& Kimoto, 1963, Pac. Ins. Mon., 1B : 496 (China). -Kimoto, 1970, Spec. Bull. Lep. Soc. Japan, $4: 170$ (Nepal) ; 1977, Ent. Basiliensia, 2 : 354 (Bhutan) ; 1979, ibid., 4 : 469 (Nepal, India).-Kimoto \& Takizawa, 1983, Bull. Natn. Sci. Mus., Tokyo, ser. A, 9(2): 92 (Nepal). 
Agetocera pulchella Chapuis, 1875, Gen. Col., 11 : pl. 125, fig. 5.-Weise, 1902, Dtsche Ent. Z., 1902 : 367 ( = hopei).

Agetocera quadraticollis Laboissière, 1927, Ann. Soc. Ent. France, $96: 47$ (Tonkin; HAMBURG). New synonym.

Distribution : India, Burma, Nepal, Bhutan, Thailand, Laos, Vietnam, China.

It is almost impossible to separate this species from quadraticollis Laboissière, which is described by a single female specimen, except for the entirely brownish apical antennal segments in quadraticollis. In this paper, quadrataicollis is treated as a synonym of hopei.

Material examined. THAILAND : Musa nr. Fang, 1 ex., 22. vi. 1968, K. Hatta (EHIME). LAOS : Vientiane Prov., 1 ex., 30. ix. 1965, 1 ex., 30. vi. 1966, Native collr ; Vientiane Prov., Gi Sion Vill., de Tha Ngone, 2 exs., 10-24. x. 1965, 2 exs., 24-31. x. 1965, Native collr ; Vientiane Prov., Tha Ngone, 6 exs., 9. viii. 1965, Native collr ; Vientiane Prov., Phou Kou Khouei, 1 ex., 29. ix. 1965, Native collr ; Sedone Prov., Paksong, 6 exs., 4. ii. 1965, 1 ex., 1. vi. 1965, 5 exs., 5. vii. 1965,9 exs., 6. vii. 1965, Native collr ; Sayaboury Prov., Sayaboury, 1 ex., 15. ix. 1966, Native collr ; Xieng Khouang Prov., Muong Soui, 1 ex., 1. ix. 1965, Native collr ; Tonpheng, 4 exs., 30.ix. 1965, 1 ex., 15. viii. 1966, Native collr (BISHOP).

\section{Agetocera lobicornis Baly}

A getocera lobicornis Baly, 1865, Trans. Ent. Soc. London, ser. 3, 2(5): 437 (India ; BM).-Maulik, 1936, Fauna India, Galeruc. : 133 (India, Burma).

Distribution : India, Burma, Thailand, Laos.

Material examined. THAILAND : $50 \mathrm{~km}$ West of Tak, $900 \mathrm{~m}, 6$ exs., 7-8. iv. 1966, M. Sedlacek (BISHOP). LAOS : Vientiane Prov., Ban Van Eue, 1 ex., 15. vii. 1966, Native collr ; Borikhane Prov., Pakkading, 100 m, 1 ex., 23. iv. 1965, J. L. Gressitt, 1 ex., 15. iv. 1965, J. A. Rondon, 4 exs., 10. v. 1965, Native collr ; Borikhane Prov., Namkading, 100 m, nr. Pakkading, 2 exs., 22. iv. 1965, J. L. Gressitt ; Sedone Prov., Paksong, 1 ex., 16. v. 1965, Native collr ; Khammouane Prov., Phon Tiou, 2 exs., 30. iv. 1965, Native collr ; Muon Sing, NW of Luang Prabang, 650 m, 6 exs., 6-10. vi. 1960, S. Quate \& L. Quate (BISHOP) ; Umgeb. Paklay, 2 exs., 1963 (MUNCHEN).

\section{Agetocera mirabilis (Hope)}

Aegelocerus mirabilis Hope, 1831, in Gray, 2001. Miscell. : 29 (Nepal ; BM).

Aplosonyx heterocera Redtenbacher, 1868, Reise Novara, Zool., 2, Col. : 206 (Hong Kong ; WIEN). -Maulik, 1936, Fauna India, Galeruc. : 127 (= mirabilis).

Agetocera heterocea : Duvivier, 1891, C. R. Soc. Ent. Belg., $35: 46$ (India).

Agetocera orientalis: Weise, 1922 (nec 1902), Tijdschr. Ent., 65 : 63 (Darjeeling).-Laboissière, 1929, Ann. Soc. Ent. France, $98: 262$ (corrected as mirabilis).

Agetocera mirabilis:Laboissière, 1927, Ann. Soc. Ent. France, 96 : 43, fig. 4 (Nepaul ; Assam,

Tonkin, Laos).-Maulik, 1936, Fauna India, Galeruc. : 127, fig. 43 (India, Burma, Hainan, Laos).

-Gressitt \& Kimoto, 1963, Pac. Ins. Mon., 1B : 495, 496 (Hainan).-Kimoto \& Takizawa, 1972,

Kontyû, Tokyo, 40(4): 216 (Nepal).-Kimoto, 1979, Ent. Basiliensia, 4 : 469 (Nepal, India).

Distribution : India, Nepal, Bhutan, Burma, Laos, Vietnam, China, Hainan.

Material examined. LAOS : Vientiane Prov., Ban Van Eue, 3 exs., 30. vi. 1965, 3 exs., 31. vii. 1965, 1 ex., 15. viii. 1966, 1 ex., 30. iii. 1967, Native collr; Vientiane Prov., Phou Kou Khouei, 1 ex., 29. ix. 1965, Native collr ; Wapikhamthong, Khong Sedone, 1 ex., 27. ix. 1965, Native collr ; Attopeu Prov., Houei Kong, 1 ex., 17. vii. 1965, Native collr ; Sedone Prov., Paksong, 1 ex., 1. vi. 1965, Native collr ; Sedone Prov., Pakse, 1 ex., 30. viii. 1965, Native collr ; Thonpheng, 2 exs., 15. viii. 1966, Native collr 
(BISHOP) ; Umgeb. Vientiane, 6 exs., 1963 (MUNCHEN). VIETNAM : Tonkin, 1 ex., 10. iii. 1942, T. Esaki (KU).

\section{Agetocera nigripennis Laboissière}

A getocera nigripennis Lab., 1927, Ann. Soc. Ent. France, $96: 48$ (Tonkin; HAMBURG).

Distribution : Vietnam.

No additional material was examined beside the type specimen.

Agetocera orientalis Weise

A getocera orientalis Weise, 1902, Dtsche Ent. Z., 1902 : 367 (Tonkin).

Distribution : Vietnam.

No material was examined.

\section{Agetocera sokolovi Medvedev}

A getocera sokolovi Medvedev, 1981. Zool. Zh., 60(4): 618, fig. (Vietnam).

Distribution : Vietnam.

No material was examined. This species is not included in the key.

\section{Genus Pseudocophora Jacoby}

Pseudocophora Jac., 1884, Notes Leyden Mus., 6 : 69, 214 (type :Galleruca buquetii Guérin; Java). -Maulik, 1936, Fauna India, Galeruc. : 198.-Gressitt \& Kimoto, 1963, Pac. Ins. Mon., 1B:303, 490.

\section{Key to SPECIES of P seudocophora}

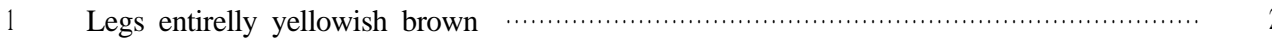

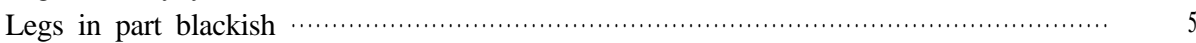

2(1) In male, postscutellar structure conspicuous, not nearly flat ; in female fifth abdominal

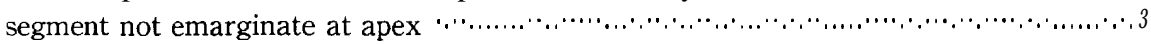

In male, postscutellar structure nearly flat, impunctate, with a fovea near suture ; in female fifth abdominal segment deeply and narrowly emarginate at apex ; generally yellowish brown with metathorax and part of basal four segments of abdomen black ;

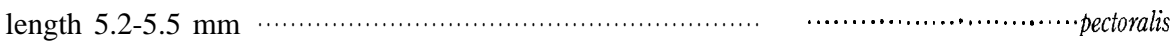

Elytron yellowish to reddish brown

Elytron black, in some specimen basal margin and part of epipleuron brownish ; generally yellowish to reddish brown ; length $4.8-5.0 \mathrm{~mm}$............................ nitens

4(3) Ventral surfaces yellowish brown with metathorax and basal part of each abdominal segment blackish in various degrees ; generally yellowish brown, in male postscutellar region entirely yellowish brown; length $5.5 \mathrm{~mm}$

Ventral surfaces yellowish brown, in some specimen metathorax blackish ; in male postscutellar region with small anterior and posterior markings black, but in some specimen entirely yellowish brown ; generally yellowish brown ; length 5.0-5.7 $\mathrm{mm}$ 
5(1) Legs black with anterior leg entirely reddish brown ; in male median lobe of fifth abdominal segment flat; generally reddish brown, ventral surfaces black ; in male postscutellar regions blackish in various degrees ; length $5.3-6.3 \mathrm{~mm}$.................. brunnea Legs reddish brown with middle and posterior femora largely blackish ; in male median lobe of fifth abdominal segment excavated ; generally yellowish brown, in male elytron with postscutellar marking blackish ; ventral surfaces entirely blackish ; length 5.3-5.7 mm

uniplagiata

\section{Pseudocophora brunnea Baly}

Pseudocophora brunnea Baly, 1886, J. Linn. Soc., Zool., 20 : 26 (Celebes) ; 1888, ibid., $20:$ 169, 170, 174 (Malacca, Celebes).- Jacoby, 1893, Entomolog., 225 (Suppl.) : 108 (mentioned).-Laboissière, 1940, Bull. Mus. Hist. Nat. Belg., 16(3): 14 (mentioned).

Distribution : Thailand, Cambodia, Laos, Malaya, Celebes.

Material examined. THAILAND : Chiengmai Prov., Chiengdao, 450 m, 2 exs., 5-11. iv. 1958, T. C. Maa ; Banna, Chawang, nr. Nabon, 700 m, 3 exs., 5-10. v. 1958, 2 exs., 5-6. ix. 1958, T. C. Maa (BISHOP) ; Kao Yai Nat. Park, 1 ex., 6. vi. 1965, Y. Miyatake (KU). CAMBODIA : Kiri Rom, 700 m, 1 ex., 31. iii-7. iv. 1961, N. R. Spencer (BISHOP). LAOS : Vientiane Prov., Ban Van Eue, 1 ex., 15. viii. 1965, 1 ex., 30. xi. 1965, 1 ex., 15. xi. 1966, Native collr ; Vientiane Prov., Gi Sion Vill., de Tha Ngone, 1 ex., 10-24. x. 1965 Native collr (BISHOP).

\section{Pseudocophora flaveola Baly Fig. $2 b$}

Pseudocophora flaveola Baly, 1888, J. Linn. Soc., Zool., 20 : 169 (Andaman Is. ; BM).-Maulik, 1936, Fauna India, Galeruc. : 206, figs. (Bengal, Burma, Tenasserim, Andaman Is.).-Gressitt \& Kimoto, 1963, Pac. Ins. Mon., 1B : 491, fig. (China).

Pseudocophora flava Allard, 1888 (1889), Ann. Soc. Ent. France, ser. 6, $8: 324$ (Annam; PARIS). -Baly, 1890, Ent. Monthly Mag., ser. 2, $1: 14$ (= flaveola ; as flavescens; error).

Distribution : India, Burma, Andaman Is., Thailand, Laos, Vietnam, Hainan.

As mentioned by Maulik (1936), this species is variable on the coloration of the ventral surfaces. On the type specimen taken from Andaman Is., the ventral surfaces are entirely pale. The several pale. However, the other five male specimens taken from Laos are characteristic in having the postscutellar region with small anterior and posterior markings and metathorax blackish. postscutellar region with small anterior and posterior markings and metathorax blackish.

Material examined. THAILAND : Pakchong, 100 m, NE of Bangkok, 1 ex., 3. xii. 1957, J. L. Gressitt (BISHOP) ; Khon Kaen, 2 exs., 24. vii. 1980, S. Azuma (RU). LAOS : Vientiane Prov., Ban Van Eue, 2 exs., 31. vii. 1965, 1 ex., 30. xi. 1965, 1 ex., 31. xii. 1965, 1 ex., 1-15. ix. 1967, Native collr; Borikhane Prov., Pakkading, 1 ex., 22. iii. 1965, Native collr ; Attopeu Prov., Houei Kong, 1 ex., 16. v. 1965, Native collr ; Dong Dok, 4 exs., 23. vii. 1965, Native collr ; Nongtevada, 1 ex., 17. iii. 1965, Native collr (BISHOP).

\section{Pseudocophora madoni Laboissière}

Pseudocophora madoni Lab., 1940, Bull. Mus. Hist. Nat. Belg., 16(3) : 16 (Tonkin ; BRUXELLES). Distribution : Vietnam.

The following specimen well agrees with the type of this species. However, this species might be a color variation of flaveola which is a very variable on the coloration of the ventral surfaces. 
Material examined. "Indo-China", 1 ex. (BASEL).

\section{Pseudocophora nitens Allard}

Pseudocophora nitens All., 1888 (1889), Ann. Soc. Ent. France, ser. 6, 8: 324 (Annam, Sumatra ; PARIS).

Distribution : Thailand, Laos, Vietnam, Sumatra.

Material examined. THAILAND : Chiengmai Prov., Chiengmai, 1 ex., 17. vi. 1965, P. D. Ashlock (BISHOP). LAOS : Vientiane Prov., Vientiane, 1 ex., 22. iii. 1966, Native collr ; Vientiane Prov., Ban Van Eue, 2 exs., 15. ix. 1967, 1 ex., 30. iii. 1967, Native collr (BISHOP). VIETNAM : Ban Me Thout, 500 m, 2 exs., 16-18. v. 1960, S. Quate (BISHOP).

\section{Pseudocophora pectoralis Baly}

Pseudocophora pectoralis Baly; 1888, J. Linn. Soc., Zool., 20 : 169,174 (Assam ;BM).- Jacoby, 1892, Ann. Mus. Civ. Genova, 32 : 958 (Burma).-Maulik, 1936, Fauna India, Galeruc. : 202, figs. (NE India).-Gressitt \& Kimoto, 1963, Pac. Ins. Mon., 1B: 491, 492 (S. China).

Distribution : India, Burma, Indo-China, S. China.

Material examined. "Indo-China", 6 exs. (BASEL).

\section{Pseudocophora uniplagiata Jacoby}

Pseudocophora uniplagiata Jac., 1884, Notes Leyd. Mus., 6 : 214 (Sumatra ; BM).-Baly, 1888, J. Linn. Soc.,Zool., 20 : 169, 173 (Sumatra, Siam).-Allard, 1888 (1889), Ann. Soc. Ent. France, ser. 6, 8: 324 (Sumatra).-Jacoby, 1893, Entomolog., 25 (Suppl.) : 108 (mentioned).-Laboissière, 1940, Bull. Mus. Hist. Nat. Belg., 16(3): 14 (note).-Châjô, 1964, Nature and Life in SE Asia, Kyoto, 3 : 298 (Thailand).

Pseudocophora femoralis Laboissière, 1940, Bull. Mus. Hist. Nat. Belg., 16(3) : 15 (Burma, Haut Tonkin : rivière Claire ; BRUXELLES). New synonym.

Distribution : Burma, Thailand, Sumatra.

Material examined. THAILAND : Chiengmai Prov., Chiengdao, 450 m, 2 exs., 5-11. iv. 1958, T. C. Maa (BISHOP); Kao Yai Nat, Park, 1 ex., 6. iv. 1965, Y. Miyatake (KU); Khon Kaen, 1 ex., 24. vii. 1980, 10 exs., 7. x. 1981, 3 exs., 8. x. 1981, S. Azuma (RU) ; Kanchanaburi, 1,300 ft., 4 exs., 21. v. 1962 (BANGKHEN). LAOS : Vientiane Prov., Ban Van Eue, 1 ex., 30. ix. 1965, 1 ex., 15. xi. 1966, 2 exs., 30. ix. 1967, Native collr; Vientiane Prov., Gi Sion Vill., de Tha Ngone, 1 ex., 24-31. x. 1966, Native collr (BISHOP) ; Umgeb. Vientiane, 2 exs., iii-vi. 1963 ; Umgeb. Pakse, 1 ex., 1963 (MUNCHEN).

\section{Genus Aulacophora Chevrolat}

Aulacophora Chevr., 1837, in Dejean, Cat. Col., ed. 3 : 402 (type :Galleruca quadraria Olivier ; Java).-Chapuis, 1875, Gen. Col., 11 :158.-Weise, 1886, Ins. Deutschl., 6(4):574.-Allard, 1888 (1889), Ann. Soc. Ent. France, ser. 6, 8 :307.-Baly, 1889, Trans. Ent. Soc. London, 1889 : 297. -Laboissière, 1929, Ann. Soc. Ent. France, 98(3):256.-Maulik, 1936, Fauna India, Galeruc. : 169.-Chen \& Kung, 1959, Acta Ent. Sinica, 9(4) :273.—Chajô, 1962, Phil. J. Sci., 91(1-2): 14, 74. -Gressitt \& Kimoto, 1963, Pac. Ins. Mon., 1B : 393, 482.-Kimoto, 1964, J. Fac. Agr. Kyushu Univ., 13(2) : 303.

Raphidopalapa Chevrolat, 1837, in Dejean, Cat. Col., ed. $3: 402$ (type :Crioceris abdominalis 


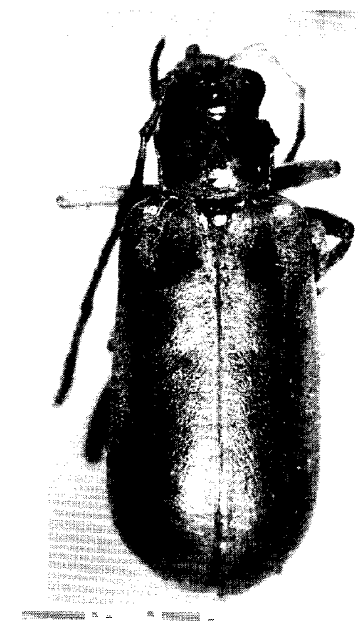

a

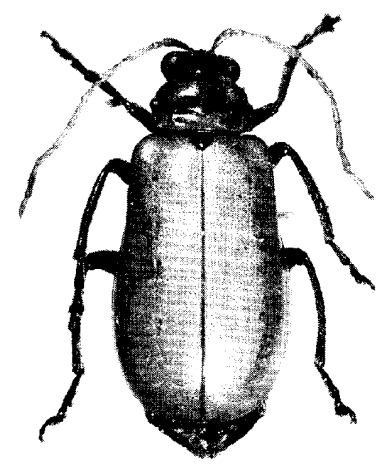

$\mathrm{b}$

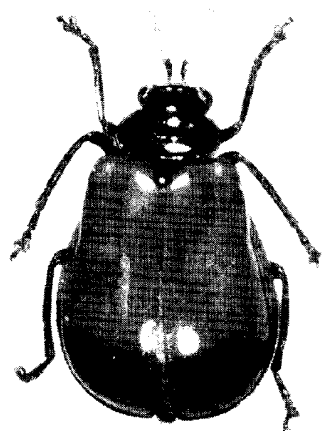

C

Fig. 14. a, Haplosomathailandica n. sp. ; b, Aulacophora coffeae (Hornstedt) ; c, A. luteicornis (Fabricius).

Fabricius ; Europe).

Rhaphidopalpa Rosenhauer, 1856, Tiere Andulsia. . 325 (type : Galleruca foveicollis Lucas ; Europe).-Allard, 1888 (1889), Ann. Soc. Ent. France, ser. 6, 8 :307.-Ogloblin, 1936, Fauna USSR, 26, $1: 19,150,370,396$.

Acuteipalpa Resenhauer, 1856, ibid. : 327.

Ceratia Chapuis, 1876, C. R. Soc. Ent. Belg., 19 : 100 (type : Aulacophora marginalis Chapuis ; Philippines).

Triaplatyps Fairmaire, 1877, Petit. Nouv. Ent., 2 : 186 (type :Triaplatyps quadripartita Fairmaire ; New Britain).

Pachypalpa Weise, 1892, Dtsche Ent. Z., 1892 : 392 (type : Galenucaluteicornis Fabricius ; Sumatra).-Laboissiere, 1929, Ann. Soc. Ent. France, 98(3): 256.

Orthaulaca Weise, 1892, ibid. : 396 (type :Galeruca similis Olivier ; SE Asia).

Cerania Weise, 1892, ibid. : 396 (type : Aulacophora cornuta Baly ; SE Asia)--Laboissière, 1929,

Ann. Soc. Ent. France, 98(3) : 256.

Spaerarthra Weise, 1892, ibid. : 396 (type : Aulacophora cyanoptera Boisduval ; New Guinea).

In this genus, it is almost impossible to correctly identify by the female specimens for some species.

Allard (1891, Nouv. Arch, Mus. Paris, ser. 3, 3 :231, from Luang Prabang, and 1908, Miss. Pavie Indo-Chine, $3: 160$, from Cambodge) recorded Aulacophora ioptera (Wiedemann) from Indo-China. However, this species is not included in the text, because Baly (1889, Trans. Ent. Soc. London, 1889 : 301) had some question on Allard's identification of ioptera.

\section{Key to SPeCies of A ulacophora}

1 In female apex of fifth abdominal segment trilobed 2 In female apex of fifth abdominal segment not trilobed 
2(1) Antenna filiform in both sexes; yellowish brown, ventral surfaces black, scutellum pitchy brown, legs entirely yellowish brown ; length $6.3 \mathrm{~mm}$...................... mouhoti

Antenna with apical segments incrassate in male ; coloration variable, 1) generally black, with lateral margin of pronotum and antenna yellowish brown ;2) pronotum entirely blackish, 3) elytron entirely brownish, 4) dorsal surfaces entirely blackish, 5) entirely reddish brown ; length $8.4 \mathrm{~mm}$

luteicornis

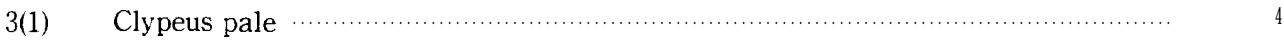

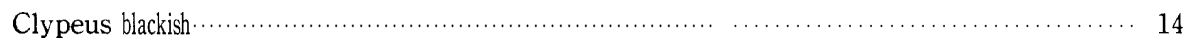

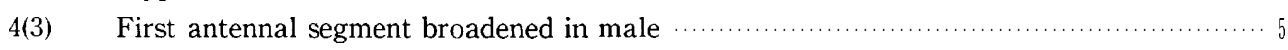

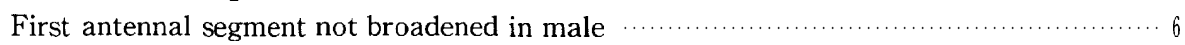

5(4) In male, antennal scape broadened, interantennal area specially modified, and humerus not covered with erect hairs; transverse sulcus of pronotum generally straight and sometimes interrupted in middle; elytron with suture and margin brown; generally yellowish brown, metathorax and abdomen blackish, legs pitchy black in various degrees, at least femora infuscate in most cases ; length 7.8-8.3

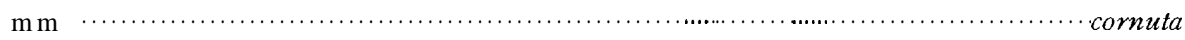

In male, antennal scape broadened but interantennal area not modified, and humerus covered with erect hairs; in female pygidum produced posteriorly and sharply pointed at apex ; transverse furrow of pronotum strongly curved at middle ; generally yellowish brown, metathorax and abdomen blackish, with apex of the latter brownish, in some specimen legs blackish in various degrees; length 6.4-7.5 $\mathrm{mm}$

6(4) In male, third to fifth antennal segments modified and vertical area of head with In male, third to fifth antennal segments not specially modified, but some times rather stout, and vertical area of head without structures $\ldots \ldots \ldots \ldots \ldots \ldots \ldots \ldots \ldots \ldots \ldots \ldots . . \ldots$

7(6) In male third antennal segment longer than broad, flat and subtriangular; legs entirely brownish

In male third antennal segment much broader than long, blade-like internally, and vertical area of head on each side with a longitudinal excavation ; legs nearly black; generally yellowish brown with elytron black, meso- and metathorax pitchy

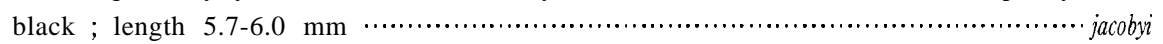

$8(7) \quad$ In male, fourth antennal segment broader than long, quite flat, produced endoapically, and vertical area of head on each side with a strongly thickened transverse ridge ; generally yellowish brown with elytron black; length $6.3-6.5 \mathrm{~mm} \ldots \ldots \ldots \ldots \ldots \ldots \ldots$ palliata In male, fourth antennal segment longer than broad, not very flat, incised apically and quite hairy around emargination, and vertical area of head on each side with a longitudinal excavation ; generally yellowish brown with elytron black ; length

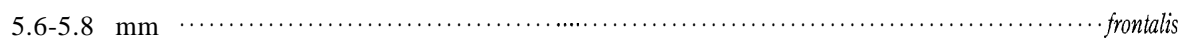

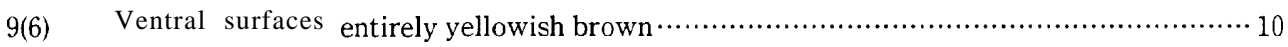

Ventral surfaces of metathorax and abdomen black; generally reddish to yellowish brown, elytron usually with three black markings $(2: 1)$, in some specimen these markings united, or with humeral markings only, legs brownish with femora infus-

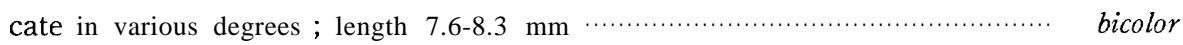

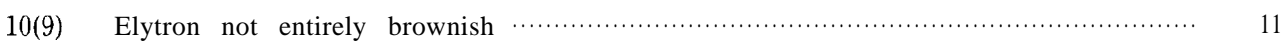
Elytron entirely yellowish brown ; generally yellowish brown, in some specimen scutellum partly infuscate ; length 6.3-6.8 mm 
11(10) Elytron with transverse furrow or depression subbasally, and its lateral margin rather widely explanate

Elytron without any distinct transverse furrow or depression subbasally, and its lateral margin rather narrowly explanate

12(11) In male apical segment of antenna incrassate, and its apex obliquely emarginate and armed with a short acute tooth ; generally reddish brown; legs pitchy black, elytron black ; length 7.7-9.8 $\mathrm{mm}$....

an tennata

In male apical segment of antenna not incrassate; generally yellowish brown; elytron black with apical half yellowish to reddish brown, in some specimen brownish with three pairs of black markings, or black with apex brownish; length 6.8$8.0 \mathrm{~mm}$

13(11) Elytron shining ; in male fifth abdominal segment longitudinally elevated and with a longitudinal deep sulcus in middle; generally yellowish brown, elytron black ; length 5.3-6.0 $\mathrm{mm}$

Elytron opaque ; in male fifth abdominal segment flat and with a shallow longitudinal short sulcus basally; generally yellowish brown, legs with tibiae and tarsi largely pitchy black, elytron black ; length 6.3-7.5 mm

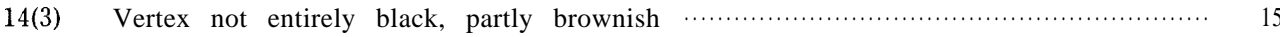
Vertex entirely black; yellowish brown with elytron, meso- and metathorax black ; length $5.1-5.7 \mathrm{~mm}$

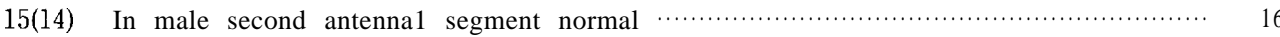
In male second antennal segment enlarged apically; generally black with head, pronotum and abdomen yellowish brown ; length 5.6-6.3 $\mathrm{mm}$

16(15) In male first segment of posterior leg normal, elongate, and median lobe of fifth abdominal segment flat ; femora of middle and posterior legs pitchy black ................ 17 In male first tarsal segment of anterior legs widened, round and median lobe of fifth abdominal segment longitudinally raised; generally yellowish brown with metathorax, abdomen, tibiae and tarsi largely pitchy black to brown; length 6.5-7.0 $\mathrm{mm}$

yunnanensis

17(16) In male interstices of eyes narrower, subequal to the length of first antennal segment, antenna thickly covered by long hairs; body length longer; scutellum yellowish brown ; posterior legs almost enirely pitchy black to black ; generally yellowish brown, in most specimens vertex partly blackish ; length $8.0-8.7 \mathrm{~mm}$.................. In male interstice of eyes wider, distinctly wider than the length of first antenna1 segment, antenna not thickly covered with long hairs ; body length shorter ;scutellum pitchy black; posterior legs yellowish brown with femora pitchy black in various degrees; generally yellowish brown, vertex always entirely yellowish brown, in some specimen elytron pitchy brown with lateral and apical margins broadly and suture narrowly yellowish brown ; length $6.5-7.0 \mathrm{~mm}$.................. semifusca

\section{Aulacophora antennata Baly}

Aulacophora antennata Baly, 1886, J. Linn. Soc., Zool., 1886: 301 (Java, Singapore, Sarawak) ; 1889, Trans. Ent. Soc. London, 1889 : 301 (notes).

Distribution : Thailand, Singapore, Borneo, Java.

Material examined. THAILAND : Trang Prov., Water fall to Khaochang, 150-300 m, 2 exs., 3. i. 1964, G. A. Samuelson (BISHOP) ; Khao Chong, nr. Trung, 1 ex., 26. vi. 1965, Y. Miyatake (KU). 


\section{Aulacophora bhamoensis Jacoby}

Aulacophora bhamoensis Jac., 1892, Ann. Mus. Civ. Genova, 32: 944 (Burma; GENOVA). -Maulik, 1936, Fauna India, Galeruc. : 172 (Burma).

Distribution : Burma, Thailand, Laos, Vietnam.

Material examined. THAILAND: Khao Yai Nat. Park, 1 ex., 6. vi. 1965, K. Morimoto ; Muak Lek, $40 \mathrm{~km}$ SE of Sara Buri, 1 ex., 5. vi. 1965, Y. Miyatake (KU). LAOS : Vientiane Prov., Ban Van Eue, 1 ex., 31. xii. 1968, Native collr ; Attopeu Prov., Houei Kong, 1 ex., 31. v. 1965, J. L. Gressitt ; Sayaboury Prov., Sayaboury, 1 ex., 16. iv. 1965, J. L. Gressitt (BISHOP). VIETNAM : Ban Me Thuot, 500 m, 1 ex., 16-18. v. 1960, S. Quate (BISHOP).

\section{Aulacophora bicolor (Weber)}

Galleruca bicolor Weber, 1801, Obs. Ent. : 56 (Sumatra).

Galleruca bicolor Fabricius, 1801 (nec Weber), Syst. Eleut., 1 : 482 (Sumatra)._Zimsen, 1964, Type Material Fabricius : 108 (= bicolor Weber).

Galleruca haemorrhoa Fabricius, 1803, in Illiger, Mag. Ins., 2 : 293 (new name for bicolor Fabricius, 1801).-Zimsen, 1964, Type Material Fabricius : 108.

Galeruca sexpunctata Olivier, 1808, Entomologie, $6: 627$, fig. (Timor).

Aulacophora hemichora Gistel, 1857, Achthundert und zwanzig neue order unbeschriebene wirbellose Tiere : 28 (Java).-Strand, 1916, Archiv Naturg., 82 (A5) : 91 (Java).-Monrós \& Bechyné, 1956, Ent., Arb. Mus. Frey, $7: 1132$ (= bicolor).

Aulacophora sexnotata Chapuis, 1876, C. R. Soc. Ent. Belg., 19 : 100 (Philippines).-Baly, 1886, J. Linn. Soc., Zool., $20: 19$ (as a var. of bicolor ; Ceylon, Celebes) ; 1889, Trans. Ent. Soc. London, 1889 : 300 (mentioned).-Weise, 1922, Tijdschr. Ent., $65: 62$ (= sexpunctata).

Aulacophora sexpunctata: Jacoby, 1884, Notes Leyden Mus., $6: 213$ (mentioned).-Allard, 1888 (1889), Ann. Soc. Ent. France, ser. 6, 8 : 314, 322 (Inde).-Schönfeldt, 1890, Ent. Nachr., 16(11) : 174 (Loo-Choo).-Duvivier, 1891, C. R. Soc. Ent. Belg., 35 : 146 (Java).

Aulacophora semiopaca Jacoby, 1886, Ann. Mus. Civ. Genova, 24 : 51 (Sumatra).-Baly, 1887, Ent. Monthly Mag., $23: 268$ (= bicolor).

Aulacophora bicolor : Baly, 1886, J. Linn. Soc.,Zool., 20 : 3, 4, 19 (Sumatra, Java, Celebes, Gilolo, Flores, Philippines, Lombok).-Allard, 1889, Ann. Soc. Ent. France, ser. 6, 9: 308 (Pnomh-Penh). -Baly, 1889, Trans. Ent. Soc. London, 1889 : 299 (mentioned).-Allard, 1891, Nouv. Arch. Mus. Paris, ser. 3, 3 : 230 (Luang-Prabang) ; 1904, Miss. Pavie Indo-Chine, 3: 159 (Cambodge). -Maulik, 1936, Fauna India, Galeruc. : 185, 187 (India, N. Andaman Is., Nicobars, Sumatra, Java, Formosa, Philippines).-Chen \& Kung, 1959, Acta Ent. Sinica, $9:$ 374, 393, fig. (China). -Chûjô, 1962, Phil. J. Sci., 91 : 77, 86 (Formosa).-Gressitt \& Kimoto, 1963, Pac. Ins. Mon., 1B: 483, 484 (Hainan, N. Vietnam).-Kimoto, 1964, J. Fac. Agr. Kyushu Univ., 13 : 304 (Ryukyu Is.). -Kimoto\& Gressitt, 1966, Pac. Ins., 8 : 477, 522 (Ryukyu Is.).-Kimoto, 1972, Tijdschr. Ent., 93(4) : 145 (Andaman).

Distribution : India, Ceylon, Andaman, Nicobar, Thailand, Cambodia, Laos, Vietnam, China, Hainan, Taiwan, Ryukyu Is., Philippines, Sumatra, Java, Celebes, Gilolo, Flores, Lombok, Timor.

Material examined. THAILAND : Chiengmai Prov., Chiengdao, 450 m, 14 exs., 5-11. iv. 1958, T. C. Maa (BISHOP). LAOS : Vientiane Prov., Ban Van Eue, 5 exs., 15-31. v. 1965, J. A. Rondon, 5 exs., 15-31. v. 1965, 2 exs., 31. vii. 1965, 2 exs., 30. ix. 1965, 1 ex., 30. xi. 1965, 1 ex., 15. xii. 1965, 1 ex., 18. i. 1966, 2 exs., 16. iii. 1966, 1 ex., 15. iv. 1966, 1 ex., 31. v. 1966, 1 ex., 15. vi. 1966, 1 ex., 15. xi. 1966, 1 ex., 15. ii. 1967, 1 ex., 30. ix. 1967, Native collr ; Vientiane Prov., Phou Kou Khouei, 1 ex., 31. v. 1966, 
2 exs., 16. vi. 1966, Native collr; Sayaboury Prov., Sayaboury, 3 exs., 29. iii. 1966, Native collr (BISHOP) ; Umgeb. Vientiane, 14 exs., iii-vi. 1963 ; Umgeb. Pakse, 2 exs., 1964 ; Umgeb. Vanky, 1 ex., 1963 ; Umgeb. Pakse, 1 ex., 1963 (MUNCHEN).

\section{Aulacophora coffeae (Hornstedt) Fig. 14b}

Chrysomela coffeae Hornstedt, 1788, Schrift. Ges. Naturf. Freunde Berlin, 8 : 5, fig. (Java : Bantamschen Koffee Plantagen).

Aulacophora coffeae : Baly, 1886, J. Linn. Soc., Zool., $20: 3$ 4, 18 (Java, Sumatra, Philippines, Tondano, Trigane, Cambodia, India).

Distribution : India, Thailand, Cambodia, Laos, Vietnam, Malaya, Sumatra, Java, Celebes, Philippines.

Material examined. THAILAND : Pakchong, $100 \mathrm{~m}$, NE of Bangkok, 1 ex., 2. xii. 1957, J. L. Gressitt ; Banna, 2 exs., 5-10. iv. 1958, T. C. Maa; Pakchong, 5 exs., 6. iii. 1963, 2 exs., 7. iii. 1963, 4 exs., 11. iii. 1964, Native collr ; Nakoarnsawan, 1 ex., 4. viii. 1963, 1 ex., 12. ix. 1963, Native collr ; Phu Kae, 1 ex., 8. iii. 1963, 1 ex., 30. vii. 1963, Native collr ; Muak Lek, 1 ex., 6. iii. 1963, Native collr ; Nakorn-nayok, 1 ex., 20. xi. 1963. Native collr ; Kampaengpet, 1 ex., 11. ix. 1963, Native collr ; Bang Khen, 1 ex., 11. ix. 1964, Native collr ; Prachuabkirikhan, 1 ex., 12. vii. 1963, Native collr ; Kanchanaburi, 1 ex., 10. viii. 1963, Native collr ; Saraburi, 1 ex., 10. ix. 1963, Native collr (BISHOP) ; Loe, 1 ex., 6. ix. 1962 ; Rayong, 6 exs., 27. vii. 1962 ; Bangkok, Honburi, 1 ex., 15. xii. 1957, 1 ex., i. 30.1958 ; Chanthaburi, 2 exs., 22. vii. 1962 ; Phetchabun, 1 ex., 17. iv. 1959 (BANGKHEN). LAOS : Vientiane Prov., Vientiane, 8 exs., 21. iv. 1965, J. A. Rondon, 1 ex., 25. iii. 1966, J. N. Sedlacek, 1 ex., 5. i. 1966, 20 exs., 21-22. ii. 1966, Native collr ; Vientiane Prov., Dong Dok, 1 ex., 23. vii. 1965, 7 exs., 3. vi. 1965, 1 ex., 26. xi. 1965, 1 ex., 4. ii. 1966, 8 exs., 15. ii. 1966, 1 ex., 26. ii. 1966, Native collr ; Vientiane Prov., Gi Sion Vill., de Tha Ngone, 2 exs., 10-24. x. 1965, 6 exs., 19-26. xii. 1965, 43 exs., 24-31. x. 1966, Native collr ; Vientiane Prov., Tha Ngone, 8 exs., 6. xi. 1965, J. L. Gressitt, 2 exs., 19. viii. 1965, 1 ex., 20 . xi. 1965, 3 exs., 25. xi. 1965, 2 exs., 21. xii. 1965, 1 ex., 2. ii. 1966, 1 ex., 23. ii. 1966, Native collr ; Vientiane Prov., Phou Kou Khouei, 1 ex., 4. xii. 1965, 1 ex., 29. iii. 1966, Native collr ; Vientiane Prov., Ban Van Eue, 1 ex., 11. iv. 1965, 3 exs., 15. xii. 1966, J. L. Gressitt, 15 exs., 1-15. v. 1965, J. A. Rondon, 1 ex., 15. vii. 1965, 3 exs., 31. vii. 1965, 1 ex., 31. viii. 1965, 2 exs., 30. ix. 1965, 2 exs., 30. xi. 1965, 1 ex., 15 . xii. 1965, 3 exs., 16. iii. 1966, 2 exs., 29. iv. 1966, 1 ex., 31. v. 1966. 9 exs., 15. vi. 1966, 1 ex.. 15. vii. 1966, 1 ex., 30. vii. 1966, 1 ex., 2. xi. 1966, 6 exs., 15. xi. 1966, 2 exs., 30. xi. 1966, 2 exs., 15. xii. 1966, 1 ex., 30. iii. 1967, 7 exs., 1-15. ix. 1967, 2 exs., 20. ix. 1967, 3 exs., 31. xii. 1967 ; Wapikhamthong Prov., Khong Sedone, Wapi, 2 exs., 15. vii. 1967, 44 exs., 15. ix. 1967, Native collr ; Wapikhamthong Prov., Khong Sedone, 9 exs., 13. x. 1965, Native collr ; Sayaboury Prov., Sayaboury, 1 ex., 15. i. 1966, J. L. Gressitt, 3 exs., 30. v. 1965, 5 exs., 3. viii. 1965, 17 exs., 6. xii. 1965, 2 exs., 15. i. 1966, 1 ex., 12. ii. 1966, 1 ex., 2. iii. 1966, 2 exs., 24. iii. 1966, 4 exs., 13. iv. 1966, 7 exs., 21. iv. 1966, 16 exs., 5. v. 1966, 8 exs., 20. v. 1966, Native collr ; Sedone Prov., Paksong, 1 ex., 6. ix. 1965, 1 ex., 20. ix. 1965. Native collr ; Sedone Prov., Pakse, 1 ex., iii. 1965, J. A. Rondon, 36 exs., 31. v. 1967, Native collr ; Khammouane Prov., Phon Tiou, 1 ex., 28. iv. 1965, 1 ex., 30. iv. 1965, 3 exs., 17. v. 1965, 1 ex., 6. vii. 1965, 1 ex., 16. viii. 1965, 1 ex., 11. ix. 1965, Native collr ; Borikhane Prov., Paksane, 1 ex., 31. viii. 1965, 2 exs., 1. xi. 1965, 4 exs., 5. xi. 1965, 2 exs., 8. ii. 1966, Native collr ; Borikhane Prov., Pakkading, 3 exs., 15. ix. 1965, J. L. Gressitt ; Borikhane Prov., Namkading nr. Pakkading, 1 ex., 21. iv. 1965, J. L. Gressitt ; Attopeu Prov., Houei Kong, 1 ex., 31. v. 1965, Native collr ; Savanakhet Prov., Savanakhet, 1 ex., 20. vii. 1965, 6 exs., 15. ix. 1965, Native collr ; Muong Sing, NW of Luang Prabang, 650 m, 1 ex., 6-10. vi. 1960, L. W. Quate ; Nongtevada, 1 ex., 26. vii. 1965, 4 exs., 27. vii. 1965, 3 exs., 6. viii. 1965, 8 exs., 4. vi. 1965, 1 ex., 13. xi. 1965, 7 exs., 18. xi. 1965, 6 exs., 23. xi. 1965, 1 ex., 2. xii. 1965, 1 ex., 22. xii. 1965, 6 exs., 7. ii. 1966,6 


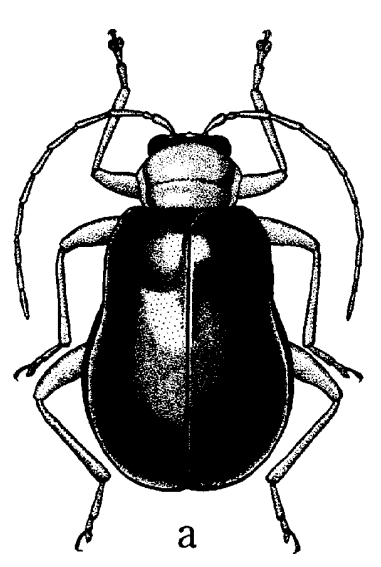

Fig. 15. a, Aulacophora coomani Laboissière;b, A. semifusca Jacoby ; c, A. nigripennis Motschulsky.

exs., 19. i. 1966, Native collr; Tonpheng, 1 ex., 14. ix. 1965, 4 exs., 27. x. 1965, Native collr ; Ile de Khong, 1 ex., 6. ii. 1965, 1 ex., 24. viii. 1965, Native collr (BISHOP) ; Umgeb. Pakse, 3 exs., 1964 ; Umgeb. Vanky, 1 ex., 1963 ; Umgeb. Vientiane, 1 ex., 1963 (MUNCHEN). VIETNAM : Ba Don, Tay Ninh, 15 m, 4 exs., 7. vii. 1960, R. E. Leech (BISHOP).

\section{Aulacophora coomani Laboissière Fig. 15a}

Aukzcophora coomani Lab., 1929, Ann. Soc. Ent. France, 98 : 257 (Tonkin; HAMBURG).-Chen \& Kung, 1959, Acta Ent. Sinica, 9(4) : 375, 380 (Vietnam).-Gressitt \& Kimoto, 1963, Pac. Ins. Mon., 1B: 484, 485, fig. (China, N. Vietnam).

Distribution : Laos, Vietnam, China.

Material examined. LAOS : Vientiane Prov., Ban Van Eue, 1 ex., 15. xii. 1966, Native collr (BISHOP). VIETNAM : Dilinh (Djiring), 1 ex., 27. ix.-14. x. 1960, C. M. Yoshimoto (BISHOP).

\section{Aulacophora cornuta Baly}

Aukzcophora cornuta Baly, 1879, Cist. Ent., $2: 445$ (Assam) ; 1886, J. Linn. Soc. London, $20: 3,5$, 15 (Assam, Siam, Celebes, Ceram, Timor, Sulu Is., Flores)-_Jacoby, 1884, Notes Leyden Mus., 6: 212 (Sumatra) ; 1905, Ann. Mus. Civ. Genova, 41 : 496 (New Guinea, Fergusson Is.).-Baly, 1886, J. Linn. Soc., Zool., $20:$ 3, 5, 15 (Assam, Celebes, Ceram, Timor, Sulu Is., Flores, Waigeau, New Guinea).-Maulik, 1936, Fauna India Galeruc.: 186 (Malacca, Celebes, Siam).-Chen \& Kung, 1959, Acta Ent. Sinica, 9(4): 375, 382, fig. (Yunnan).-Gressitt \& Kimoto, 1963, Pac. Ins. Mon., 1B: 483, 484, 486 (China).-Chûjô, 1964, Nature and Life in SE Asia, Kyoto, $3: 286$ (Thailand, Brunei).-Anand \& Cox, 1986, Ent. Abh. Staatl. Dresden, 50(1): 88 (India, Mallaca, Celebes, Siam, Nias, Andaman Is.).

Aulacophora bicornuta Allard, 1888 (1889), Ann. Soc. Ent. France, ser. 6, 8 : 309, 320 (Sumatra). -Maulik, 1936, Fauna India, Galeruc. : 192 (= comuta).

Aukzcophora (Cerania) cornuta: Laboissière, 1929, Ann. Soc. Ent. France, 98 : 258 (Tonkin). 
Distribution : India, Andaman, Thailand, Laos, Vietnam, China, Malaya, Borneo, Sumatra, Nias, Java, Batu Is., Celebes, Ceram, Timor, Sulu, Flores, Waigeau, New Guinea, Fergusson Is.

Material examined. THAILAND : Chiengmai Prov., Doi Suthep, 1 ex., 1-5. iv. 1958, T. C. Maa (BISHOP). LAOS : Vientiane Prov., Vientiane, 1 ex., 31. vii. 1965, Native collr ; Vientiane Prov., Ban Van Eue, 1 ex., 13. iv. 1965, J. L. Gressitt, 3 exs., 15-31. v. 1965, 3 exs., 15. vii. 1965, 1 ex., 30. ix. 1965, 9 exs., 30. xi. 1965, 1 ex., 30. vii. 1966, 3 exs., 15. xi. 1966, 3 exs., 1-15. ix. 1967, 1 ex., 15. x. 1967, Native collr ; Vientiane Prov., Phou Kou Khouei, 1 ex., 4. xii. 1965, Native collr ; Vientiane Prov., Gi Sion Vill., de Tha Ngone, 2 exs., 10-24. x. 1965, 1 ex., 6-13. iii. 1966, 1 ex., 24-31. x. 1966, Native collr; Borikhane Prov., Pakkading, 1 ex., 13. vii. 1965, J. L. Gressitt, 1 ex., 31. viii. 1965, 1 ex., 9. ix. 1965, 3 exs., 15. ix. 1965, 1 ex., 1. xi. 1965, Native collr ; Sayaboury Prov., Sayaboury, 2 exs., 30. v. 1965, 1 ex., 14. vii. 1965, 1 ex., 30. ix. 1965, 8 exs., 30. xii. 1965, Native collr ; Wapikhamthong Prov., Wapi, 1 ex., 15. ix. 1967, Native collr ; Sedone, 1 ex., 13. x. 1965, Native collr ; Tonphen, 1 ex., 14. ix. 1965,1 ex., 15. ix. 1965, 1 ex., 17. x. 1965, 1 ex., 30. viii. 1966, 1 ex., 15. x. 1966, Native collr ; Nongtavada, 1 ex., 6. viii. 1965, Native collr (BISHOP) ; Umgeb. Vientiane, 1 ex., iii-vi. 1963, 2 exs., iii-vi. 1964 ; Umgeb. Pakse, 5 exs., 1964 (MUNCHEN).

\section{Aulacophora cruenta (Fabricius)}

Chrysomela cruenta Fabr., 1781, Spec. Ins., 1 : 130 (India orientali) ; 1787, Mant. Ins., $1: 75$ (locality not cited).-Zimsen, 1964, Type Material Fabricius : 108.

Galleruca cmenta : Fabricius, 1792, Ent. Syst., 2 : 19 (E. Indies) ; 1801, Syst. Eleut., 1 : 483 (India orientali).

Galleruca rosea Fabricius, 1801, Syst. Eleut., 1 : 479 (Sumatra).-Zimsen, 1964, Type Material Fabricius : 107. New synonymy.

Galleruca variabilis Illiger, 1802, Mag. Ins. : 422 (locality not cited).-Maulik, 1936, Fauna India, Galeruc. : 196 (= rosea).

Aulacophora rosea: Chapuis, 1876, C. R. Soc. Ent. Belg., 19 : 99 (Philippines).-Baly, 1886, J. Linn. Soc., Zool., 20 : 2, 4, 9 (Sumatra, Java, Philippines, Malacca),-Allard, 1888 (1889), Ann. Soc. Ent. France, ser. 6, 8 : 310 (Java).-Jacoby, 1889, Ann. Mus. Civ. Genova, ser. 2, 7 : 206 (Burma). -Allard, 1891, Nouv. Arch. Mus. Paris, ser. 3, 3: 231 (Luang Prabang) ; 1904, Miss. Pavie IndoChine, 3 : 160 (Siam, Chantaboun à Battanbang).-Maulik, 1936, Fauna India, Galeruc. : 186, 196 (Assam, Tenasserim, Penang, Perak, Siam, Java, Sumatra, Philippines).

Aulacophora albicornis Chapuis, 1876, C. R. Soc. Ent. Belg., 19 : 99 (Philippines).-Maulik, 1936, Fauna India, Galeruc. : 196 (= rosea).

Aulacophora tibialis Chapuis, 1876, C. R. Soc. Ent. Belg., 19 : 99 (Luzon, Mindoro).-Allard, 1888 (1889), Ann. Soc. Ent. France, ser. 6, $8: 307$ (Philippines).-Maulik, 1936, Fauna India, Galeruc. : 196 (= rosea).

Aulacophora uniformis Chapuis, 1876, C. R. Soc. Ent. Belg., 19 : 99 (Luzon).-Allard, 1888 (1889), Ann. Soc. Ent. France, ser. 6, 8: 307 (Philippines).-Weise, 1913, Phil. J. Sci., ser. D, $8: 222$ (Philippines :Negros).-Maulik, 1936, Fauna India, Galeruc. : 196 (=rosea).

Aulacophoranigripeta Duvivier, 1885, Stett. Ent. Ztg., 46 : 389 (Ceylon).- Jacoby, 1887, Proc. 2001. Soc. London, 1887 : 103, fig. (Ceylon).-Weise, 1924, Col. Cat. Junk, $78: 13$ (= cmenta).

Aulacophoralata Baly, 1886, J. Linn. Soc., Zool., 20 : 2, 4, 8 (Ceram, Macassar, Celebes, Gilolo, Bachian).-Allard, 1888 (1889), Ann. Soc. Ent. France, ser. 6, 8: 311 (Celebes).-Weise, 1922, Tijdschr. Ent., 65 : 62 (Celebes).-Maulik, 1936, Fauna India, Galeruc. : 196 (=rosea).

Aulacophora biguttata Allard, 1888 (1889), Ann. Soc. Ent. France, ser. 6, 8 : 313, 322 (Ceylon). -Weise, 1924, Col. Cat. Junk, $78: 13$ (as ab. of cruenta). 
Aulacophora quadripunctata Allard, 1888 (1889), Ann. Soc. Ent. France, ser. 6, 8:314, 322 (Ceylon). -Weise, 1924, Col. Cat. Junk, $78: 13$ (= cmenta).

Aulacophora uniformis ab. bipunctata Weise, 1913, Phil. J. Sci., ser. D, 8(3) : 222 (Philippines: Negros).

Ceratia (Orthaulaca) mea : Weise, 1922, Tijdschr. Ent., $65: 62$ (Borneo ; ab. Zata).

Aulacophora stigmatica Weise, 1924, Col. Cat. Junk, $78: 16$ (new name for Aulacophora bipunctata Weise, 1913, nec Olivier, 1808).

Aulacophora tonkinensis Laboissière, 1929, Ann. Soc. Ent. France, 98 : 255 (Tonkin; HAMBURG). -Chen \& Kung, 1959, Acta Ent. Sinica, 9(4):375, 381 (=cruenta).-Gressitt\& Kimoto, 1963, Pac. Ins. Mon., 1B: 486 (=cruenta).-Chûjô, 1964, Nature and Life in SE Asia, Kyoto, $3: 288$ (Thailand).

Aulacophora cmenta : Maulik, 1936, Fauna India, Galeruc. : 187, 194 (S. India, Ceylon).-Chen \& Kung, 1959, Acta Ent. Sinica, 9(4): 375, 381, fig. (Hainan).-Gressitt \& Kimoto, 1963, Pac. Ins. Mon., 1B: 483, 486 (Hainan, N. Vietnam).

Distribution : India, Ceylon, Burma, Thailand, Laos, Vietnam, Hainan, Philippines, Malaya, Sumatra, Java, Celebes, Ceram, Macassar, Celebes, Gilolo, Bachian.

There is no reason to separate rosea from cmenta. This species is very variable on the markings of elytron. Those are, 1) ground color reddish brown with blackish markings, 2) basal half blackish and apical half reddish brown, 3) blackish with apical margin narrowly reddish brown. Except for two Laos specimens belonging to the type 2, the rest of them belongs to the type 1 .

Material examined. THAILAND : Trang Prew., Khaophapha, Khaochang, 200-400 m, 2 exs., lo11. i. 1965 G. A. Samuelson; Chanthaburi, Prov, 45 m, 1 ex., 24. iv.-1.v. 1958, T. C. Maa ; S. Banna, Nakhon, 108 m, 1 ex., 5-10. v. 1958, T. C. Maa; Kanchanaburi, 1 ex., 15. viii. 1964, Native collr ; Khao Yai, 1 ex., 12. viii. 1963, Native collr ; Saraburi, 1 ex., 16. ix. 1964, Native collr (BISHOP) ; Lahkon Si Thamarat, 1 ex., vii. 1961 ; Rayong, 1 ex., 17. vii. 1961 (BANGKHEN). LAOS : Khamouane Prov., Phon Tiou, 2 exs., 30. iv. 1965, 1 ex., 17. v. 1965, 1 ex., 6. vii. 1965, 1 ex., 4. viii. 1965, 1 ex., 1. ix. 1965 , Native collr ; Borikhane Prov., Pakkading, 1 ex., 30. vi. 1966 ; Borikhane Prov., Paksane, 1 ex., 28. ix. 1965 ; Tonpheng, 1 ex., 30. x. 1965 ; Ile de Khong, 1 ex., 20. vii. 1965, Native collr (BISHOP) ; Umgeb. Vientiane, 2 exs., iii-vi. 1963 ; Umgeb. Vanky, 1 ex., 1963 ; Umgeb. Paklay, 1 ex., 1963 (MUNCHEN).

\section{Aulacophora frontalis Baly}

Aulacophora frontalis Baly, 1888, J. Linn. Soc., Zool., 20 : 176, 181 (Borneo, Sarawak ; BM) -Jacoby, 1892, Ann. Mus. Civ. Genova, 32 : 942 (mentioned).-Bowditch, 1914, Psyche, $21: 134$ (mentioned).-Maulik, 1936, Fauna India, Galeruc.: 176, 183, fig. (India, Ceylon, Siam, Philippines).-Chen \& Kung, 1959, Acta Ent. Sinica, 9(4):375, 381, fig. (China).-Chajô, 1961, Nature and Life in SE Asia, Kyoto, 1: 350 (Cambodia) ; 1962, Phil. J. Sci., 91(1-2): 77, 96 (Formosa).-Gressitt \& Kimoto, 1963, Pac. Ins. Mon., 1B: 484,487 (China, N. Vietnam).-Châjô, 1964, Nature and Life in SE Asia, Kyoto, $3: 287$ (Thailand, Cambodia).

Aulacophora palliata:Allard, 1889, Ann. Soc. Ent. France, ser. 6, $9: 308$ (Hue, Saigon, Mutho, Pnomh-Penh, Tourane, Qui-Nhon) ; 1891, Nouv. Arch. Mus. Paris, ser. 3, $3: 230$ (Luang Prabang) ; 1904, Miss. Pavie Indo-Chine, $3: 160$ (Cambodge).-Laboissière, 1934, Ann. Ass. Nat. Levallois-Perret, $21: 140$ (corrected as frontalis).

Aulacophora (Ceratia) frontalis : Laboissiere, 1929, Ann. Soc. Ent. France, $98: 258$ (Tonkin, Cambodia, Saigon, Java).

Ceratia frontalis : Bryant, 1929, Bull. Raffles Mus., 2 : 90 (Mentawai : Siberut, Padong).

Distribution : India, Ceylon, Thailand, Cambodia, Laos, Vietnam, China, Taiwan, Mentawei, 
Borneo, Java, Philippines.

Material examined. THAILAND : Cholburi Prov., Sriracha Distr., 1 ex., '22. x. 1966, J. L. Gressitt ; Banna, Chanwang nr. Nabon, 70 m, 1 ex., 5. ix. 1958, J. L. Gressitt ; Patumthani, 2 exs., 10. vii. 1964, Native collr (BISHOP). CAMBODIA : Ph. Chisau, 40 km, S. of P. Penh, 20 m, 1 ex., 29. iv. 1961, N. R. Spencer (BISHOP). LAOS : Vientiane Prov., Vientiane, 2 exs., 4. viii. 1965, Native collr ; Vientiane Prov., Gi Sion Vill., de Tha Ngone, 2 exs., 6. ix. 1965, 1 ex., 10-24. x. 1965, Native collr ; Sedone Prov., Pakse, 1 ex., 5. vii. 1965, 1 ex., 31. v. 1967, Native collr; Borikhane Prov., Paksane, 1 ex., 28. viii. 1965, 1 ex., 9. ix, 1965, 1 ex., 1. xi. 1965, Native collr ; Khammouane Prov., Phon Tiou, 1 ex., 14. viii. 1965, 1 ex., 28. ix. 1965, Native collr ; Houei Kong, 1 ex., 7. x. 1965, Native collr ; Dong Dok, 2 exs., 23. viii. 1965, Native collr (BISHOP) ; Umgeb. Vientiane, 1 ex., iii-vi. 1963 ; Umgeb. Paklay, 1 ex., 1963 ; Umgeb. Vanky, 2 exs., 1963 (MUNCHEN). VIETNAM : Quang Tri, 2 km N., 1 ex., 8. v. 1970, A. R. Gillogly ; Ninh Hoa, N. of Nha Trang, 1 ex., 28. xi. 1960, C. M. Yoshimoto ; Blao (Balao), 600 m, 1 ex., 14-21. x. 1960, C. M. Yoshimoto (BISHOP).

\section{Aulacophora indica (Gmelin)}

Crioceris indica Gmelin, 1790, ed. Linnaeus, Syst. Nat., ed. 13, 1, $4: 170$ (India).

Crioceris testacea Fabricius, 1792, Ent. Syst., 1, $2: 4$ (India orientali)-Laboissiere, 1940, Bull. Mus. Hist. Nat. Belg., 16(3): 10 (= indica).

Galeruca similis Olivier, 1808, Entomologie, $6: 624$, fig. (Iles de L'Ocean Indien). New synonym. Raphidopalpa femoralis Motschulsky, 1857, Etud. Ent., 6 : 37 (Japan).-Baly, 1886, J. Linn. Soc., Zool., 20 : 16 (=similis).-Weise, 1892, Dtsche Ent. Z., 1892 : 395 (Philippines).-Okamoto, 1924, Bull. Agr. Expt. Stat. Chosen, 1(2) : 194 (Korea, Quelpart I.).-Laboissière, 1932, Result. Sci. Voy. Ind. Orient. Neerl., 4(4) : 151 (Japan, Coree, Chine)--Ogloblin, 1936, Fauna URSS, 26, 1 : 154,396, fig. (Korea, ?N. China).-Laboissike, 1940, Bull. Mus. Hist. Nat. Belg., 16(3) : 12 (Japan, Chine, Tonkin, Malacca, Andaman, Nicobar).-Anand \& Cox, 1986, Ent. Abh. Staatl. Mus. Dresden, 50(4): 85 (= similis).

Aulacophora testacea : Baly, 1878, Cist. Ent., 2 : 445 (Assam).-Anand \& Cox, 1986, Ent. Abh. Staatl. Mus. Dresden, 50(4) : 90 (India).

Aulacophora similis : Baly, 1886, J. Linn. Soc., Zool., 20 : 3, 5, 16 (Manchuria, Japan, N. India, China, Canton, Cochin China, Malay Archipelago).-Allard, 1888 (1889), Ann. Soc. Ent. France, ser. 6, 9 : 308 (Hue, Saigon, Myuthe, Pnomh-Penh, Lourane, Qui-Nhon).-Jacoby, 1889, Ann. Mus. Civ. Genova, ser. 2, 7 : 206 (Burma) ; 1896, ibid., 36 : 458 (Sumatra).-Weise, 1916, Ark. Zool., 10(20): 38, note (notes).-Maulik, 1929, Insects of Samoa, 4(3): 193 (Samoa ; Malay Archipelago to China and Japan, and to Australia).-Laboissiere, 1934, Ann. Ass. Nat. LevalloisPerret, 21 : 140 (Indochina).- Gressitt, 1957, Insects of Micronesia, 17(1):22, 28, fig. (Mi croneisa).-Chûjô, 1962, Phil. J. Sci., 9(1-2): 77, 82, fig. (Formosa).

Aulacophora flavipes Jacoby, 1883, Notes Leyden Mus., 5 : 202 (Saleyar).-Baly, 1886, J. Linn. Soc., Zool., $20: 17$ (=similis).

Rhaphidopalpa coffeae: Allard, 1888 (1889), Ann. Soc. Ent. France, ser. 6, 8: 306, 319 (Japan). -Weise, 1892, Dtsche Ent. Z., 1892 : 395 (=similis).

Rhaphidopalpa testacea : Allard, 1888 (1889), Ann. Soc. Ent. France, ser. 6, $8:$ 308, 320 (Celebes). Rhaphidopalpa abdominalis : Allard, 1889, Ann. Soc. Ent. France, ser. 6, $9: 308$ (Pnomh-Penh). -Laboissière, 1934, Ann. Ass. Nat. Levallois-Perret, 21 : 140 (= similis).

Rhaphidopalpabengalensis Weise, 1892, Dtsche Ent. Z., 1892 : 394 (Calcutta).-Laboissière, 1940, Bull. Mus. Hist. Nat. Belg., 16(3): 10 (= indica).

Orthaulaca similis : Weise, 1892, Dtsche Ent. Z., 1892 : 393 (Nias).-Weise, 1916, Arkiv Zool., 10 : 
38 (notes).

Raphidopalpa similis: Allard, 1891, Nouv. Arch, Mus. Paris, ser. 3, $3: 230$ (Luang-Prabang). -Duvivier, 1892, Ann. Soc. Ent. Belg., 36 : 430 (Chota-Nangpore)-Jacoby, 1903, ibid., 47 : 116 (India).-Allard, 1904, Miss. Pavie Indo-Chine, $3: 159$ (Cambodge).-Bryant, 1929, Bull. Raffles Mus., 2 : 90 (Mentawai : Siberut).

Rhuphidopalpa chinensis Weise, 1892, Dtsche Ent. Z., 1892 : 395 (Shanghai ;ZMB).-Laboissière, 1932, Result. Sci. Voy. Ind. Orient. Neerl., 4(4): 151 (Chine merid., Tonkin, Yunnan) ; 1934, Ann. Ass. Nat. Levallois-Perret, 21 : 140 (Indo-China).-Ogloblin, 1936, Fauna URSS, 26, 1 : 153, 396 (China, Mongolia, Annam).-Laboissière, 1940, Bull. Mus. Hist. Nat. Belg., 16(3) : 13 (China, Indochina, Siam, Mallaca).-Gressitt \& Kimoto, 1963, Pac. Ins. Mon., 1B: 486 (=femoralis).

Rhuphidopalpa indica : Laboissière, 1932, Result. Sci. Voy. Ind. Orient. Neerl., 4(4): 151 (India) ; 1940, Bull. Mus. Hist. Nat. Belg., 16(3): 10, 12, figs. (India, Iles Andaman).

Aulacophora foveicollis: Maulik, 1936, Fauna India, Galeruc. : 173, figs. (in part ; India, Ceylon, Burma, Andaman Is.).-Laboissière, 1940, Bull. Mus. Hist. Nat. Belg., 16(3) : 10 (India).—Chajô, 1959, Sci. Rep. Agr. Exped. Cambodia, Hyogo Univ. Agr., Japan, (2) : 60 (Cambodia).

Aulacophora femoralis: Châjô, 1941, Trans. Nat. Hist. Soc. Formosa, 31: 157 (Japan proper, Loochoo, Quelpart Is., Korea).-Chen \& Kung, 1959, Acta Ent. Sinica, 9 : 375, 385 (Taiwan, Japan, ? Korea).-Gressitt \& Kimoto, 1963, Pac. Ins. Mon., 1B: 483, 486, fig. (China, Hainan, N. Vietnam, Korea).-Kimoto, 1964, J. Fac. Agr. Kyushu Univ., 13 : 304, 305 (Japan, Ryukyu Is.). -Kimoto\& Gressitt, 1966, Pac. Ins., 8: 477, 523 (Ryukyu Is.).-Kimoto, 1972, Tijdschr. Ent., 93(4): 145 (Cochinchina).

Aulacophora femoralis chinensis : Chen \& Kung, 1959, Acta Ent. Sinica, 9 : 375, 384, fig. (China).

Aulacophora chinensis: Chûjô, 1961, Nature and Life in SE Asia, Kyoto, 1 : 350 (Cambodia, Thailand) ; 1964, ibid., $3: 285$ (Thailand).

Aulacophora indica : Kimoto, 1970, Khumbu Himal,3(3): 416 (Nepal).-Kimoto \& Takizawa, 1972, Kontyû, Tokyo, 40(4) : 216 (Nepal).-Kimoto, 1977, Ent. Basiliensia, 2 : 354 (Bhutan). -Kimoto \& Takizawa, 1981, Ent. Rev. Japan, 35(1-2) : 59 (Nepal).

Distribution : India, Ceylon, Burma, Nepal, Bhutan, Andaman, Nicobar, Thailand, Cambodia, Laos, Vietnam, Hainan, China, Taiwan, Philippines, Ryukyu Is., Japan, Korea, Siberia, Sunda Is., Micronesia, New Guinea, Samoa, Fiji.

Laboissière (1932) treated the material taken from India as indica, ones from S. China, Yunnan and Tonkin as chinensis, and ones from Japan, Korea and China as femoralis. According to the study on the materials taken from these areas, it came to conclusion that it is not necessary to separate these three species. Also, Chen \& Kung (1959) treated chinensis as a subspecies of femoralis. However, in central China, these two forms distribute in same areas. The color of legs is somewhat variable.

Material examined. THAILAND : Chiengami Prov., Fang, 600 m, 2 exs., 14. vi. 1965, P. D. Ashlock; Chiengami Prov., Mae Klang, 340 m, 1 ex., 11. vi. 1965, P. D. Ashlock; Chiengmai Prov., Chieng Dao, 1 ex., 15. vi. 1965, P. D. Ashlock; Saraburi Prov., Ban Muak Lek Nat. Park, 1 ex., 5. vi. 1965, P. D. Ashlock; Pantumthani, 7 exs., 10. ii. 1964, 3 exs., 10. vii. 1964, Native collr ; Kampaenget, 10 exs., 10-11. ix. 1963, Native collr; Pak Chong, 5 exs., 11. v. 1964, Native collr ; Nakoransawan, 4 exs., 12. ix. 1963, Native collr; Bang Khen, 1 ex., 10. viii. 1964, 1 ex., 9. ix. 1963, Native collr; Si Racha, 2 exs., 21. viii. 1963, Native collr; Pang-nga, 1 ex., 10. iv. 1963, Native collr;Prachuabkirikhan, 1 ex., 16. vii. 1963, Native collr; Khonkaen, 1 ex., 22. ix. 1964, Native collr; Trat, 1 ex., 10. vii. 1963, Native collr; Kanchanaburi, 1 ex., 6. vii. 1964, Native collr; Phliu, 1 ex., 23. iv. 1963, Native collr (BISHOP) ; Chiengmai Prov., Fang, 1 ex., 13. vi. 1965, K. Morimoto (KU) ; Bangkok, 27 exs., 7. ii. 1956, 3 exs., 14. iv. 1959, 2 exs., 10. ix. 1961 ; Chiengmai, 7 exs., 20. viii. 1961, Udon Thani, 4 exs., 
25. x. 1958 ; Rayong, 9 exs., 27. vii. 1962, Chanthaburi, 1 ex., 22. vii. 1962 ; Nakhon Ratchasima, 2 exs., 26. vii. 1959, Tak, 2,800 ft., 1 ex., 20. viii. 1961 (BANGKHEN). CAMBODIA : Damrey Phong, 2 exs., 14-16. iv. 1961, N. R. Spencer (BISHOP). LAOS : Umgeb. Vientiane, 5 exs., iii-vi. 1963 ; Umgeb. Pakse, 1 ex., 1963 ; Umgeb. Paklay, 5 exs., 1963 ; Umgeb. Vanky, 1 ex., 1963 (MUNCHEN) ;Borikhane Prov., Pakkading, 2 exs., 23. iv. 1965, 1 ex., vi. 1965, 3 exs., 13. vii. 1965, 176 exs., 15. x. 1965, 96 exs., x. 1965, J. L. Gressitt, 1 ex., 22. iii. 1965, 1 ex., 10. v. 1965, 3 exs., 14. vii. 1965, 5 exs., 3. viii. 1965, 91 exs., 15-31. viii. 1965, 43 exs., 9. ix. 1965, 1 ex., 26. iii. 1966, 5 exs., 5. v. 1966, 1 ex., 13. v. 1966, Native collr ; Namkading, nr. Pakkading, 100 m, 1 ex., 25. iv. 1965, J. L. Gressitt ; Borikhane Prov., Paksane, 3 exs., 7. viii. 1965, 3 exs., 17. viii. 1965, 1 ex., 31. viii. 1965, 2 exs., 9. ix. 1965, 1 ex., 28. ix. 1965, 1 ex., 19. xi. 1965, 1 ex., 3. xii. 1965, 1 ex., 8. xii. 1965, 2 exs., 28. xii. 1965, 1 ex., 15. viii. 1967, Native collr ; Sedon Prov., Paksong, 6 exs., 6. ix. 1965, Native collr ; Sedon Prov., Pakse, 1 ex., 12. v. 1965, 7 exs., v. 1965, P. D. Ashlock, 1 ex., 30. viii. 1965, 5 exs., 31. v. 1967, 3 exs., 15. viii. 1967, Native collr ; Khammouane Prov., Phon Tiou, 3 exs., 1-15. iv. 1965, J. L. Gressitt, 6 exs., 10-11. vi. 1965, N. Wilson, 13 exs., 28. iv. 1965, 14 exs., 30. iv. 1965, 1 ex., 17. v. 1965, 1 ex., 28. v. 1965, 52 exs., 6. vii. 1965, 34 exs., 4. viii. 1965, 31 exs., 14. viii. 1965, 14 exs., 16. viii. 1965, 214 exs., 18. viii. 1965, 97 exs., 28. ix. 1965, 27 exs., 11. xi. 1965, 6 exs., 8. iii. 1966, Native collr ; Vientiane Prov., Vientiane, 1 ex., 31. v.-3. vi. 1960, S. Quate \& L. Quate, 17 exs., 21. iv. 1965, J. A. Rondon, 2 exs., 28. vii. 1965, 5 exs., 31. vii. 1965, 1 ex., 2. viii. 1965, 3 exs., 3. viii. 1965, 1 ex., 15. iii. 1966, 3 exs., 21. iii. 1966, 1 ex., 22. iii. 1966, 1 ex., 25. iii, 1966, Native collr ; Vientiane Prov., Ban Van Eue, 2 exs., 13. iv. 1965, J. L. Gressitt, 10 exs., 15-31. v. 1965, J. A. Rondon, 43 exs., 15-31. v. 1965, 3 exs., 15. vii. 1965, 13 exs., 31. vii. 1965, 15 exs., 15. viii, 1965, 20 exs., 30. ix. 1965, 6 exs., 30. xi. 1965, 1 ex., 2. iii. 1966, 9 exs., 16. iii. 1966, 4 exs., 15. iv. 1966, 1 ex., 29. iv. 1966, 3 exs., 31. v. 1966, 3 exs., 15. vi. 1966, 7 exs., 15. xi. 1966, 4 exs., 15. xii. 1966, 1 ex., 31. i. 1967, 1 ex., 30. iii. 1967, 6 exs., 30. iii. 1967, 31 exs., 1-15. ix. 1967, 2 exs., 30. ix. 1967, 3 exs., 31. xii. 1968, Native collr ; Vientiane Prov., Dong Dok, 3 exs., 23. vii. 1965, 8 exs., 30. vii. 1965, 2 exs., 7. ix. 1965, 2 exs., 22. ix. 1965, 1 ex., 28. ix. 1965, 2 exs., 30. ix. 1965, 1 ex., 3. xi. 1965, 1 ex., 22. xi. 1965, 1 ex., 1. xii. 1965, 2 exs., 6. xii. 1965, 1 ex., 22. xii. 1965, 1 ex., 8. i. 1966, Native collr ; Vientiane Prov., Tha Ngone, 5 exs., 19. viii. 1965, 5 exs., 1. ix. 1965, 11 exs., 6. ix. 1965, 3 exs., 11. ix. 1965, 1 ex., 16. ix. 1965, 1 ex., 20. ix. 1965, 7 exs., 23. ix. 1965, 1 ex., 25. ix. 1965, 2 exs., 20. xi. 1965, 1 ex., 25. xi. 1965, 1 ex., 30. xi. 1965, 2 exs., 3. i. 1966, 1 ex., 2. ii. 1966, Native collr ; Vientiane Prov., Gi Sion Vill., de Tha Ngone, 30 exs., 10-24. x. 1965, 19 exs., 24-31. x. 1966, Native collr ; Vientiane Prov., Phou Ko Khouei, 1 ex., 30. i. 1966, Native collr ; Wapikhamthong Prov., Khong Sedone, 1 ex., 30. v. 1965, 11 exs., 17. vii. 1965, 2 exs., 13. viii, 1965, Native collr ; Wapikhamthong Prov., Wapi, 3 exs., 15. x. 1967, Native collr ; Sayaboury Prov., Sayaboury, 2 exs., 15. iv. 1965, J. L. Gressitt, 1 ex., 21. iv. 1966, J. A. Rondon, 1 ex., 23. iv. 1965, 25 exs., 5. v. 1965, 3 exs., 8. v. 1965, 2 exs., 17. v. 1965, 4 exs., 1 ex., 14. vii. 1965, 3 exs., 3. viii. 1965, 3 exs., 6. xii. 1965, 1 ex., 14. xii. 1965, 1 ex., 12. ii. 1966, 2 exs., 2. iii. 1966, 1 ex., 20. iii. 1966, 1 ex., 13. iv. 1966, 6 exs., 21. iv. 1966, 2 exs., 20. v. 1966, 2 exs., 30. ix. 1966, Native collr ; Attepeu Prov., Houei Kong, 1 ex., 7. ii. 1965, 1 ex., 16 v. 1965, 1 ex., 31. v. 1965, Native collr ; Houa Khong Prov., Houei Sai, 1 ex., 30. iii. 1965, Native collr ; Ban Theuong, 18 km NW of Xieng Khouang, 1,035 m, 1 ex., 2-6. viii. 1960, N. R. Spencer ; Savanakhet, 1 ex., 29. ii. 1967, Native collr ; Tonpheng, 1 ex., 30. iv. 1965, 4 exs., 14. ix. 1965, 3 exs., 30. ix. 1965, 30 exs., 30. x. 1965, 1 ex., 30. vi. 1965, 1 ex., 30. i. 1966, 2 exs., 15. ii. 1966, 1 ex., 30. iv. 1966, 1 ex., 15. vi. 1966, Native collr ; Ile de Khong, 2 exs., 12. xi. 1965, Native collr (BISHOP). VIETNAM : Ba Den Tay Ninh, 1 ex., 7. vii. 1960, R. E. Leech ; Dalat, 6 km S., 1,400-1,500 m, 1 ex., 9. vi.-7. vii. 1961, N. R. Spencer ; Lha Ho, 14 km N., Phan Rang, 2 exs., 15. xi. 1960, C. M. Yoshimoto ; Quant Tri, 2 km N., 1 ex., 14. i. 1970, 2 exs., 8. vi. 1970, A. R. Gillogly (BISHOP). 


\section{Aulacophora jacobyi (Weise)}

Aulacophora denticomis Jacoby, 1896 (Dec.), Ann. Mus. Civ. Genova, 37 : 137 (nec Blackburn, 1896) (Mentawei, Tenasserim).

Ceratia (Ceratia) jacobyi Weise, 1924, Col. Cat. Junk, 78: 10 (new name for denticomis Jacoby). Aulacophora (Ceratia) jacobyi :Laboissière, 1929, Ann. Soc. Ent. France, 98 : 258 (Tonkin).

Aulacophora jacobyi : Maulik, 1936, Fauna India, Galeruc. : 176, 184, fig. (India, Java, Penang, Mentawe Is.).-Chen \& Kung, 1959, Acta Ent. Sinica, 9(4): 375, 381, fig. (Hainan, Yunnan).

—Gressitt \& Kimoto, 1963, Pac. Ins. Mon., 1B : 484,488 (N. Vietnam).--Châjô, 1964, Nature and Life in SE Asia, Kyoto, $3: 287$ (Thailand).

Distribution : India, Burma, Thailand, Cambodia, Vietnam, China, Hainan, Malaya, Mentawei, Java.

Material examianed. THAILAND : Trang Prov., Khaophapha, Khaochong, 200-400 m, 3 exs., 2-3. i. 1964, 2 exs., 10-11. i. 1964, G. A. Samuelson (BISHOP). CAMBODIA : Kiri Rom, 700 m, 1 ex., 31. iii.-7. iv. 1961, N. R. Spencer (BISHOP).

\section{Aulacophora lewisii Baly}

Aulacophora lewisii Baly, 1886, J. Linn. Soc., Zool., 20 : 5, 24 (China ; BM) ; 1888, ibid., 22 : 179 (India, China, Malay Archipelago) ; 1889, Trans. Ent. Soc. London, 1889 : 301 (mentioned). -Duvivier, 1892, Ann. Soc. Ent. Belg., 36 : 430 (India : Mandar).-Chen, 1959, Acta Ent. Sinica, 9(4) : 375, 378 (Hong Kong, India).-Gressitt \& Kimoto, 1963, Pac. Ins. Mon., 1B : 484, 488 (China, N. Vietnam).-Kimoto, 1964, J. Fac. Agr. Kyushu Univ., 13 : 304, 305 (Japan, Ryukyu Is.).-Châjô, 1964, Nature and Life in SE Asia, Kyoto, $3: 288$ (Thailand).-Kimoto \& Gressitt, 1966, Pac. Ins., 8 : 477, 523 (Ryukyu Is.).-Kimoto, 1977, Ent. Basilienisa, 2 : 354 (Bhutan).

Orthaulaca (Ceratia) cattigarensis Weise, 1892, Dtsche Ent. Z., 2 : 942 (India).-Maulik, 1936, Fauna India, Galeruc. : 181 (India, Ceylon, Burma).-Chen \& Kung, 1959, Acta Ent. Sinica, 9(4):375(= cattigarensis).-Gressitt \& Kimoto, 1963, Pac. Ins. Mon., 1B:488 (= Zewisii).

Aulacophora intermedia Jacoby, 1892, Ann. Mus. Civ. Genova, 2(12) : 398 (China, Japan ; ZMB). -Kimoto, 1964, J. Fac. Agr. Kyushu Univ., 13:305 (=Zewisii).

Aulacophora (Ceratia) cattigarensis: Laboissière, 1929, Ann. Soc. Ent. France, 98(3) : 258, fig. (Tonkin, Cochinchine).

Aulacophora cattigarensis: Ogloblin, 1936, Fauna URSS, 26(1): 156, 397, fig. (Japan, China, Annam, Tonkin, Cochinchina).-Chen \& Kung, 1959, Acta Ent. Sinica, 9(4): 375, 379, fig. (China).-Chajô, 1962, Phil. J. Sci., 91(1-2): 78, 99, fig. (Formosa).

Distribution : India, Ceylon, Burma, Bhutan, Thailand, Cambodia, Laos, Vietnam, China, Taiwan, Ryukyu Is., Japan, Malay Archipelago.

Material examined. THAILAND : Chiengmai Prov., Fang, 500 m, 1 ex., 12. iv. 1958, T. C. Maa, 2 exs., 13. vi. 1965, P. D. Ashlock ; Chiengmai, 1 ex., 1966, J. Sedlacek ; Chiengmai Prov., Chiengdao, 5 exs., 5-11. iv. 1958, T. C. Maa ; S. Banna, Nakhon, 108 m, 3 exs., 5-10. v. 1958, T. C. Maa; Banna, Chawang, nr. Nabon, 4 exs., 4-5. ix. 1958, J. L. Gressitt ; Sattahib, 1 m, 1 ex., 24. xi. 1957, J. L. Gressitt ; Narathiwas Prov., 15 km W. of Waeng, 100 m, 1 ex., 7-11. ix. 1965, L. W. Quate ;Lum Poon Prov., 14 km W. of Lie, 1 ex., 4-6. ix. 1965, L. W. Quate ; Ratburi, 1 ex., 15. viii. 1966, T. C. Maa; Trang Prov., Khaophappha, Khaochang, 200-400 m, 1 ex., 13. i. 1964, G. A. Samuelson ; Muak Lek, 3 exs., 6. iii. 1963, Native collr ; Packchong, 2 exs., 7. iii. 1963, 1 ex., 11. iii. 1964, Native collr ; Saraburi, 2 exs., 4. iii. 1963, Native collr ; Patumthani, 2 exs., 10. vii. 1964, Native collr ; Khao Yai, 1 ex., 12. ii. 1964, Native collr ; Bangkhen, 1 ex., 7. ix. 1963, Native collr (BISHOP) ; Chiengmai Prov., Fang, 1 ex., 13. vi. 1965, 
K.Morimoto (KU) ; Rayong, 1 ex., 17. vii. 1962 ; Uthaithani, 1 ex., 13. iv. 1963 ; Lae, 2 exs., 6. ix. 1962 (BANGKHEN). CAMBODIA : Damrey Phong, 1 ex., 14-16. iv. 1968, N. R. Spencer; Kompong Chhnang, 7 m, 25. iv. 1961, N. R. Spencer (BISHOP). LAOS : Umgeb. Paklay, 21 exs., 1963 ; Umgeb. Pakse, 9 exs., 1963-64 ; Umgeb. Vientiane, 5 exs., iii-vi. 1963 (MUNCHEN); Khammouane Prov., Phon Tiou, 46 exs., 1-15. iv. 1965, J. L. Gressitt, 11 exs., 10-14. vi. 1965, N. Wilson, 1 ex., 28. iv. 1965, 5 exs., 30. iv. 1965, 1 ex., 13. vi. 1965, 2 exs., 14. vi. 1965, 9 exs., 6. vii. 1965, 4 exs., 4. viii. 1965, 1 ex., 14. viii. 1965, 23 exs., 11. ix. 1965, 1 ex., 28. ix. 1965, Native collr ; Borikhane Prov., Paksane, 8 exs., 7. viii. 1965, 2 exs., 9. ix. 1965, 13 exs., 1. xi. 1965, 2 exs., 10. xi. 1965, 1 ex., 29. xi. 1965, 1 ex., 14. ii. 1965, Native collr ; Borikhane Prov., Pakkading, 4 exs., 17. v. 1965, 1 ex., 14. vii. 1965, 1 ex., 9. ix. 1965, 2 exs., 15. ix. 1965, Native collr ; Namkading, nr. Pakkading, 100 m, 2 exs., 21. iv. 1965, J. L. Gressitt ; Pon-horn, N. of Pakkading, 1 ex., 20. iv. 1965, J. L. Gressitt ; Vientiane Prov., Vientiane, 21 exs., 21. iv. 1965, J. A. Rondon, 3 exs., 22. vii. 1965, 1 ex., 28. vii. 1965, 4 exs., 31. vii. 1965, 6 exs., 2-3. viii. 1965, 2 exs., 21-22. iii. 1966, Native collr ; Vientiane Prov., Tha Ngone, 1 ex., 27. ii.-6. iii. 1966, J. L. Gressitt, 1 ex., 19. viii. 1965, 1 ex., 30. ix. 1965, 1 ex., 16. xi. 1965, 4 exs., 25. xi. 1965, Native collr ; Vientiane Prov., Gi Sion Vill., de Tha Ngone, 10 exs., 24-31. x. 1965, Native collr ; Vientiane Prov., Ban Van Eue, 4 exs., 13. iv. 1965, J. L. Gressitt, 10 exs., 1-15. v. 1965, 2 exs., 31. vii. 1965, 1 ex., 30. xi. 1965, 1 ex., 30. i. 1966, 1 ex., 15. xii. 1966, 2 exs., 30. vii. 1967, Native collr ; Vientiane Prov., Dong Dok, 11 exs., 23. vii. 1965, 8 exs., 5. viii. 1965, 1 ex., 7. ix. 1965, 7 exs., 30. ix. 1965, 22 exs., 3. xi. 1965, 1 ex., 15 . ii. 1966, Native collr ; Sayaboury Prov., Sayaboury, 1 ex., 30. v. 1965, 1 ex., 14. vii. 1965, 1 ex., 9. viii. 1965, 1 ex., 14. xii. 1965, 1 ex., 5. v. 1966, 1 ex., 25. viii. 1966, Native collr; Attopeu Prov., Houei Kong, 2 exs., 16. v. 1965, 19 exs., 31. v. 1965, 1 ex., 20. vii. 1965, 2 exs., 7. x. 1965, Native collr ; Sedon Prov., Pakse, 4 exs., 21-23. v. 1965, P. D. Ashlock, 3 exs., 2. viii. 1965, 16 exs., 31. v. 1967, Native collr ; Sedon Prov., Pasksong, 1 ex., 20. ix. 1965, Native collr ; Wapikhamthong Prov., Wapi, 24 exs., 15. ix. 1967, Native collr ; Wapikhamthong Prov., Khong Sedone, 1 ex., 17. vii. 1965, 2 exs., 27. ix. 1965, Native collr ; Xieng Khouang Prov., Muong Soui, 1 ex., 1. ix. 1965, Native collr, Houa Khong Prov., Houei Sai, 2 exs., 3. ii. 1965, Native collr ; Tonpheng, 12 exs., 30. x. 1965, Native collr ; Nongtevda, 2 exs., 6. viii. 1965, 3 exs., 13. viii. 1965, Native collr ; Ile de Khong, 1 ex., 6. ii. 1965, 5 exs., 17. iv. 1965, Native collr (BISHOP), VIETNAM : Ban Den, Tay Ninh, 15 m, 2 exs., 7. vii. 1960, R. E. Leech ; Ninh Hoa, N. of Nha Trang, 2 exs., 28. xi. 1960, C. M. Yoshimoto ; 15-35 km NW of Phan Rang, 2 exs., 8-16. xi. 1960, C. M. Yoshimoto ; Quang Tri, 2 km N., 2 exs., 22. iv. 1970, A. R. Gillogly ; Danar, 200 m, 1 ex., 13-28. ii. 1961, N. R. Spencer ; Nha Ho, 14 km N., Phan Rang, 1 ex., xi. 1960, C. M. Yoshimoto ; 25 km SE of Pleiku, 400 m, 1 ex., 12. v. 1960, L. W. Quate (BISHOP).

\section{Aulacophora luteicornis (Fabricius) Fig. 14c}

Galleruca luteicornis Fabr., 1801, Syst. Eleu., 1 : 482 (Sumatra).

Aulacophora simplicipennis Clark, 1865, Ann. Mag. Nat. Hist., ser. 3, 15 : 145 (Pulo-Penang). - Jacoby, 1884, Notes Leyden Mus., $6: 40$ (Sumatra).-Baly, 1886, J. Linn. Soc., Zool., $20: 21$ (= luteicornis).

Aulacophora luteicornis: Jacoby, 1884, Notes Leyden Mus., 6:40 (Sumatra).-Baly, 1886, J. Linn. Soc., Zool., $20: 3,5,21$ (Malay Peninsula, Borneo, Sumatra).-Allard, 1888 (1889), Ann. Soc. Ent. France, ser. 6, $8: 310,321$ (Malacca, Borneo).

Aulacophora dilatata Jacoby, 1886, Ann. Mus. Civ. Genova, 24 : 49 (Sarawak).-Baly, 1887, Ent. Monthly Mag., 23 : 268 (as var. of luteicornis)-Allard, 1888 (1889), Ann. Soc. Ent. France, ser. 6, 8: 307, 319 (Borneo).-Baly, 1889, Trans. Ent. Soc. London, 1889 : 299 (as var. of luteicornis).

Aulacophora marginicollis Allard, 1888 (1889), Ann. Soc. Ent. France, ser. 6, 8 : 309, 320 (Singapour).-Baly, 1889, Trans. Ent. Soc. London, 1889 : 301 (as var. of luteicornis). 
Pachypalpa luteicornis: Weise, 1892, Dtche Ent. Z., 1892 : 392 (genus).

Pachypalpa Iuteicornis ab. marginicollis:Laboissière, 1934, Bull. Mus. Hist. Nat. Belg., 10(37):2

(Siam N. : Xieng-Sen).

Distribution : Thailand, Malaya, Borneo, Sumatra.

No additional material was examined.

\section{Aulacophora mouhoti Baly}

Aulacopkora mouhoti Baly, 1886, J. Linn. Soc.,Zool., 20 : 5, 25 (Cochinchina, Malacca ; BM) ; 1889, Trans. Ent. Soc. London, 1889 : 300 (mentioned).

Distribution : Vietnam, Malaya.

No additional material was examined beside the type series.

\section{Aulacophora nigripennis Motschulsky Fig. 15c}

Galeruca atripennis Hope, 1841, Proc. 2001. Soc. London, 1841 : 64 (nec Fabricius) (Canton).

Aulacopkora nigripennis Motsch., 1857, Etud. Ent., $6: 88$ (Japan).-Baly, 1866, J. Linn. Soc., Zool, $20:$ 2, 4, 5 (Manchuria, Japan, China).-Allard, 1888 (1889), Ann. Soc. Ent. France, ser. 6, 8 : 309 (key).-Ogloblin, 1936, Fauna USSR, 26, 1 : 156, figs. (Amur).-Chen \& Kung, 1959, Acta Ent. Sinica, 9(4): 375, 377, figs. (China, Vietnam).—Chajô, 1962, Phil. J. Sci., 91(1-2) : 79, 91 (Formosa).-Gressitt \& Kimoto, 1963, Pac. Ins. Mon., 1B : 484, 489 (China, Hainan).-Kimoto, 1964, J. Fac. Agr. Kyushu Univ., 13(2) : 306 (Japan, Ryukyu Is.).

Distribution : E. Siberia, Korea, Japan, Ryukyu Is., China, Taiwan, Hainan, Vietnam.

Material examined. "Indo-China", 5 exs. (BASEL).

\section{Aulacophora opacipennis Châjô}

Aulacophora opacipennis Châjô, 1962, Phil. J. Sci., 91(1-2):78, 98 (Formosa ;TARI) ; 1964, Nature and Life in SE Asia, Kyoto, $3: 283$ (Thailand).

Distribution : Thailand, Laos, Taiwan.

This species seems to be a closely related one to Aulacophora nigripalpis Chen \& Kung, 1959, described from Yunnan, China.

Material examined. THAILAND : Trang Prov., Khaophappha, Khaochang, 200-400 m, 4 exs., 1013. i. 1964, G. A. Samuelson ; Chiengmai Prov., Chiengami, 1,100-1,500 m., 1 ex., 1966, J. Sedlacek ; Khao Yai, 1 ex., 10. iv. 1963, 1 ex., 12. ii. 1964, Native collr (BISHOP). LAOS : Vientiane Prov., Vientiane, 1 ex., 21. iv. 1965, J. A. Rondon; Vientiane Prov., Ban Van Eue, 5 exs., 15-31. iv. 1965, 3 exs., 15-31. v. 1965, 2 exs., 15-31. vi. 1965, 3 exs., 15. vii. 1965, 1 ex., 31. vii. 1965, 1 ex., x. 1965, 34 exs., xi. 1965, 2 exs., 15. xii. 1965, 9 exs., 31. xii. 1965, 3 exs., 6. i. 1966, 1 ex., 15. i. 1966, 4 exs., 15. ii. 1966, 3 exs., 2. iii. 1966, 11 exs., 16. iii. 1966, 4 exs., 29. iv. 1966, 2 exs., 15. v. 1966, 1 ex., 31. v. 1966, 28 exs., 15. vi. 1966, 1 ex., 30. vii. 1966, 21 exs., 15. xii. 1966, 1 ex., 30. xii. 1966, 22 exs., 1-15. ix. 1967, 3 exs., 31. xii. 1968, Native collr; Vientiane Prov., Phou Kou Khouei, 1 ex., 16. vi. 1966, 1 ex., 15. vii. 1966, Native collr ; Vientiane Prov., Gi Sion Vill., de Tha Ngone, 1 ex., 19. viii. 1965, 15 exs., 10-24. x. 1965, Native collr; Sayaboury Prov., Sayaboury, 1 ex., 14. vii. 1965, 7 exs., 6. xii. 1965, 2 exs., 14. xii. 1965, 1 ex., 31. xii. 1965, 2 exs., 15. i. 1967, Native collr; Sayaboury Prov., Sayaboury, 3 exs., 14. xii. 1965, Native collr; Khammouane Prov., Phon Tiou, 2 exs., 1-15. iv. 1965, J. L. Gressitt, 1 ex., 6. vii. 1965, Native collr (BISHOP). 


\section{Aulacophora pubescens (Allard)}

Rhaphidopalpa pubescens Allard, 1888 (1889), Ann. Soc. Ent. France, ser. 6, $8: 306,319$ (Annam).

-Baly, 1889, Trans. Ent. Soc. London, 1889 : 298 (mentioned).

Distribution : Vietnam.

Judging from the original description, this species probably belongs to another genus, in having the dorsal surfaces covered with hairs. This species is not included in the key.

\section{Aulacophora semifusca Jacoby Fig. 15b}

Aulacophora semifusca Jac., 1892, Ann. Mus. Civ. Genova, 32 : 942 (Burma ;GENOVA).-Maulik, 1936, Fauna India, Galeruc. : 192 (Burma).

Aulacophora almora Maulik, 1936, Fauna India, Galeruc. : 170, fig. 51 (Himalayas, Assam ; BM). -Gressitt \& Kimoto, 1963, Pac. Ins. Mon., 1B : 483,484 (China, Hainan).-Kimoto \& Takizawa, 1972, Kontyû, Tokyo, 40(4) : 216 (Nepal).-Anand \& Cox, 1986, Ent. Abh. Staatl. Mus. Dresden, 50(4) : 83, 85 (India). New synonym.

Aulacophora similis: Chen \& Kung, 1959 (nec Olivier), Acta Ent. Sinica,9(4): 376, 382 (China). -Gressitt \& Kimoto, 1963, Pac. Ins. Mon., 1B: 484 (=almora).

Aulacophora simplex Châjô, 1962, Phil. J. Sci., 91(1-2): 77 (Formosa)-Anand \& Cox, 1986, Ent. Abh. Staatl. Mus. Dresden, 50(4): 83, 86 (= almora).

Distribution : India, Burma, Nepal, Thailand, Laos, Vietnam, S. China, Hainan, Taiwan,

This species is very variable on coloration of the dorsal surfaces and the legs, and almora is nothing but a infraspecific variation of semifusca. In female specimen, it is difficult to clearly separate this species from yunnanensis Chen \& Kung. The material here identified as female of semifusca Jacoby might includes female of yunnanensis. In the most pale colored specimen of semifuscus, the scutellum is entirely yellowish brown.

Material examined. Male specimens. THAILAND : Chiengmai Prov., Chiangdao, 450 m, 9 exs., 5-11. iv. 1958, T. C. Maa ; Chiengmai Prov., Fang (Agr. Exp. Station), 600 m, 2 exs., 14. vi. 1965, P. D. Ashlock (BISHOP) ; Chiengmai Prov., Fang, 1 ex., 19. vi. 1965, K. Morimoto (KU). LAOS : Vientiane Prov., Ban Van Eue, 2 exs., 11. iv. 1965, 6 exs., 13. iv. 1965, 1 ex., 14. iv. 1965, 1 ex., 15. iv. 1965, J. L. Gressitt, 14 exs., 1-15. v. 1965, J. A. Rondon, 1 ex., 15-31. v. 1965, 1 ex., 31. vii. 1965, 2 exs., 15. viii. 1965, 1 ex., 31. ix. 1965, 4 exs., 30. xi. 1965, 1 ex., 15. vi. 1966, 1 ex., 31. v. 1966, 1 ex., 30. viii. 1967, Native collr; Vientiane Prov., Gi Sion Vill., de Tha Ngone, 2 exs., 10-24. x. 1965, Native collr ; Vientiane Prov., Phou Kou Khouei, 1 ex., 15. vi. 1966, Native collr ; Khammouane Prov., Phou Tiou, 1 ex., 17. v. 1965, 1 ex., 28. ix. 1965, Native collr ; Sayaboury Prov., Sayaboury, 2 exs., 15-16. iv. 1965, J. L. Gressitt, 1 ex., 30. v. 1965, 1 ex., 5. v. 1966, 1 ex., 25. viii. 1966, Native collr ; Borikhane Prov. Pakkading, 1 ex., 13. vii. 1965, J. L. Gressitt ; Borikhane Prov., Uekinak, 100 m, nr. Pakkading, J. L. Gressitt (BISHOP) ; Umgeb. Vientiane, 2 exs., iii-vi. 1963 (MUNCHEN). VIETNAM : Ban Me Thuot, 500 m, 1 ex., 16-18. v. 1960, L. M. Quate ; Dilinh (Djiring), 900 m, 1 ex., 22-28. iv. 1960, L. W. Quate (BISHOP).

Female specimens. THAILAND : Chiengmai Prov., Chieng Dao, 450 m, 1 ex., 5-11. iv. 1958, T. C. Maa ; Chiengmai Prov., Dai Suthep, 1 ex., 28-31. viii. 1958, T. C. Maa; Chiegmai Prov., Fang, 500 m, 1 ex., 13. vi. 1965, P. D. Ashlock (BISHOP) ; Vientiane Prov., Fang, 6 exs., 13. vi. 1965, K. Morimoto (KU). LAOS : Vientiane Prov., Ban Van Eue, 1 ex., 11. iv. 11 exs., 13. iv. 1965, 1 ex., 14. iv. 1965, J. L. Gressitt, 37 exs., 1965, 8 exs., 15-31. v. 1965, J. A. Rondon, 2 exs., 15-31. iv. 1965, 11 exs., 15-31. v. 1965, 1 ex., 15-31. vi. 1965, 2 exs., 31. vii. 1965, 1 ex., 15. viii. 1965, 1 ex., 30. ix. 1965, 7 exs., 30. xi. 1965, 1 ex., 15. xii. 1965, 6 exs., 31. v. 1966, 1 ex., 15. vi. 1966, 2 exs., 15. viii. 1966, 4 exs., 30. xi. 1966, 1 ex., 


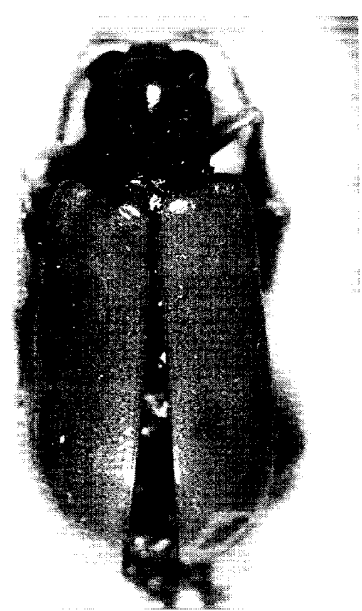

a

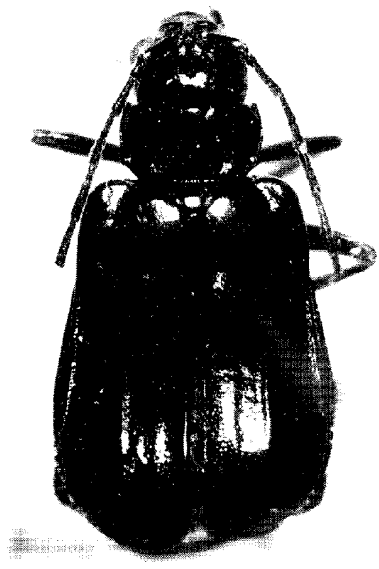

b

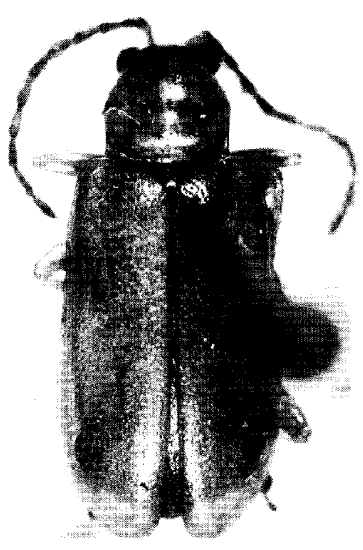

c

Fig. 16. a, Kanarella unicolor Jacoby ; b, Japonitata tricarinata (Laboissière) ; c, Cerophysella laosensis n. sp.

15. xii. 1966, 1 ex., 30. iii. 1967, 4 exs., 15-30. vi. 1967, 2 exs., 30. viii. 1967, 3 exs., 1-15. ix. 1967, 1 ex., 30. ix. 1967, 1 ex., 15. x. 1967, Native collr ; Vientiane Prov., Phou Kou Khouei, 1 ex., 31. vii. 1965, 2 exs., 16. vi. 1966, Native collr ; Vientiane Prov., Gision Vill., de Tha Ngone, 10 exs., 10-24. x. 1965, Native collr ; Sayaboury Prov., Sayaboury, 2 exs., 15. iv. 1965, J. L. Gressitt, 2 exs., 17. vii. 1965, 1 ex., 6. xii. 1965, 1 ex., 14. xii. 1965, 1 ex., 5. v. 1966, 4 exs., 25. viii. 1966, Native collr ; Borikhane Prov., Pakkading, 3 exs., 23. iv. 1965, 2 exs., 13. vii. 1965, J. L. Gressitt, 1 ex., 17. v. 1965, 1 ex., 29. iv. 1966, Native collr; Khammouane Prov., Phon Tiou, 1 ex., 28. iv. 1965, 1 ex., 17. v. 1965, 3 exs., 10. vi. 1965, 3 exs., 6. vii. 1965, 1 ex., 11. ix. 1965, 2 exs., 28. ix. 1965, Native collr ; Attopeu Prov., Hónei Kong, 1 ex., 16. v. 1965, 3 exs., 31. v. 1965, Native collr ; Wapikhamathong Prov., Khong Sedone, 1 ex., 16. v. 1965, 1 ex., 27. ix. 1965, Native collr ; Tonpheng, 1 ex., 14. ix. 1965, Native collr ; Dong Dok, 6 exs., 23. vii. 1965, Native collr (BISHOP). VIETNAM : Ban Me Thuot, 500 m, 2 exs., 16-18. v. 1960, L. W. Quate ; 76 km SW of Ban Me Thuot, 855 m, 1 ex., 20. v. 1960, R. E. Leech ; Blao (Balao), 500 m, 1 ex., 14-21. x. 1960, C. M. Yoshimoto (BISHOP).

\section{Aulacophora yunnanensis Chen \& Kung}

Aulacophora yunnanensis Chen \& Kung, 1959, Acta Ent. Sinica,9(4) : 383, 387, fig. (Yunnan). -Gressitt \& Kimoto, 1963, Pac. Ins. Mon., 1B : 483, 490 (China).

Distribution : Laos, China.

Material examined. LAOS : Vientiane Prov., Vientiane, 1 ex., 8. iv. 1965, J. L. Gressitt ; Vientiane Prov., Ban Van Eue, 2 exs., 11. iv. 1965, 5 exs., 13. iv. 1965, J. L. Gressitt, 4 exs., 15-31. iv. 1965, 1 ex., 15. viii. 1965, 1 ex., 15. iv. 1966, 1 ex., 15. vi. 1966, Native collr ; Vientiane Prov., Phou Kow Kuei, 1 ex., 16. iv. 1965, J. L. Gressitt ; Pakkading, Borikhane Prov., 4 exs., 23. iv. 1965, J. L. Gressitt ; Khammouane Prov., Phon Tiou, 1 ex., 28. iv. 1965, Native collr ; Sayaboury Prov., Sayaboury, 2 exs., 15-16. iv. 1965, J. L. Gressitt ; Dong Dok, 4 exs., Native collr (BISHOP) ; Umgeb. Vanky, 2 exs., 1963 ; Umgeb. Vientiane, 4 exs., iii-vi. 1963 ; Umgeb. Paklay, 3 exs., 1963 (MUNCHEN). 


\section{Genus Kanarella Jacoby}

Kanarella Jac., 1896, Ann. Soc. Ent. Belg., 40 : 279 (type : Kanarella unicolor Jacoby ; India). -Maulik, 1936, Fauna India, Galeruc. : 297.

Cneoranella Maulik, 1936, Fauna India, Galeruc. : 334 (type :Cneorane pallida Jacoby ; Ceylon).

\section{Kanarella unicolor Jacoby Fig. 16a}

Kanarella unicolor Jac., 1896, Ann. Soc. Ent. Belg., 40 : 280 (Kanara, Assam).-Maulik, 1936, Fauna India, Galeruc. : 299, fig. (India).-Kimoto, 1970, Khumbu Himal,3(3) : 421 (Nepal) ; 1979, Ent. Basiliensia, $4: 474$ (Nepal, Darjeeling, Maghalaya, Assam).

Distribution : India, Nepal, Laos, Vietnam.

Elongate ; pronotum nearly as long as wide, slightly widened anteriorly, and its surface smooth, shining, impunctate ; elytron subparallel-sided, confusedly impressed by distinct punctures ; entirely yellowish brown ; length 4.8-5.7 $\mathrm{mm}$.

Material examined. LAOS : Uekinak, nr. Pakkading, 1 ex., 22. iv. 1965, J. L. Gressitt (BISHOP). VIETNAM : Dalat, 1,300 m, 1 ex., 2. v. 1960, S. Quate (BISHOP).

\section{Genus Japonitata Strand}

Japonia Weise, 1922, Tijdschr. Ent. : 70 (nec Gould, 1859) (type : Phyllobroticanigrita Jacoby ; Japan).

Japonitata Strand, 1935, Folia Zool--Hydrobiol., $7: 294$ (new name for Japonia Weise, nec Gould). -Gressitt \& Kimoto, 1963, Pac. Ins. Mon., 1B: 501.

\section{KeY to species of Japonitata}

Elytron with a lateral costa; pronotum nearly $12 / 3$ times as wide as long; generally black, elytron violaceous blue, abdomen yellowish brown; length $5.0 \mathrm{~mm}$ (Laboissière, 1929 ; China)

unicostata

2(1) Pronotum nearly three times as wide as long ; in male elytron with two long and two short costae and in female with two long costae ; generally black, abdomen yellowish

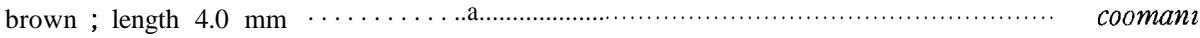
Pronotum nearly twice as wide as long; elytron with three costae of which median one interrupted at middle; generally black, elytron bluish black, abdomen yellowish brown ; length $5.0-6.5 \mathrm{~mm}$

tricarina ta

\section{Japonitata coomani (Laboissière)}

Japonia coomani Lab., 1932, Ann. Ass. Nat. Levallois-Perret, 20 : 138 (Tonkin; HAMBURG). Distribution : Vietnam.

No additional material was examined beside the type series.

\section{Japonitata tricarinata (Laboissière) Fig. 16b}

Japonia tricarinata Lab., 1929, Ann. Soc. Ent. France, 98 : 273 (Tonkin; HAMBURG). 
Japonitata tricarinata : Gressitt \& Kimoto, 1963, Pac. Ins. Mon., 1B: 501 (Tonkin :Hoa-Binh). Distribution : Vietnam.

No additional material was examined.

\section{Genus Euliroetis Ogloblin}

Euliroetis Ogl., 1936, Fauna USSR, 26, 1 : 197, 371, 403 (type : Aenidea ornata Baly ; Japan, China).-Gressitt \& Kimoto, 1963, Pac. Ins. Mon., 1B: 502.

\section{Euliroetis lameyi (Laboissière)}

Liroetis lameyi Lab., 1929, Ann. Soc. Ent. France, 98 : 278 (Tonkin; HAMBURG).

Euliroetis lameyi var. obscuripes Ogloblin, 1936, Fauna USSR, 26, 1 : 200, 405 (Amur).

Euliroetis lameyi: Ogloblin, 1936, Fauna USSR, 26, 1 : 199, 404 (Tonkin, Chine, Ussuri, Amur).

-Gressitt \& Kimoto, 1963, Pac. Ins. Mon., 1B: 503 (Tonkin).

Distribution : Vietnam, China, E. Siberia.

Oblong oval, pronotum with a pair of foveae ; elytron finely punctate, epipeluron extremely narrow. Yellowish brown, antenna, dorsal surfaces of femora, tibiae and tarsi black; elytron yellowish brown with basal, sutural and apical markings blackish; in some specimen legs almost entirely blackish ; in male fourth and fifth abdominal segments slightly depressed at middle ; length $6.3 \mathrm{~mm}$.

No additional material was examined.

\section{Genus Cerophysella Laboissière}

Cerophysella Lab., 1930, Ann. Soc. Ent. France, $99: 352$ (type : Cerophysella tonkinensis Laboissière ; Vietnam).-Ogloblin, 1936, Fauna USSR, 26, 1 :169.--Gressitt \& Kimoto, 1963, Pac. Ins. Mon., 1B:501.

Chonganiu Laboissière, 1930, Ann. Soc. Ent. France, 99 : 355 (type : Chongania duporti Laboissière; Tonkin). New synonym.

\section{Key to species of Cerophysella}

1 Posterior margin of pronotum without a pair of tubercles

Posterior margin of pronotum with a pair of tubercles ; in male elytron with a round depression behind scutellum; generally blackish, prothorax yellowish brown, legs reddish brown with middle and posterior legs partly infuscate ; length 4.5-5.0

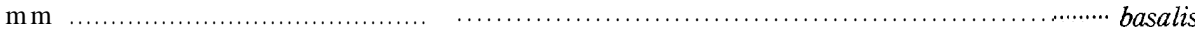

2(1) Elytral punctures finer, pronotum smooth 3 Elytral punctures stronger, pronotum granulate; in male specimens without any distinct secondary sexual character on dorsal surfaces; generally black, prothorax and legs yellowish to reddish brown, antenna pitchy black, in pale colored specimen elytron entirely brownish ; length 5.0-6.0 $\mathrm{mm}$

3(2) Antenna robuster, fifth and sixth segments nearly $11 / 2$ times as long as wide; in male pronotum with a pair of distinct tubercles slightly before transvere depression and elytron with a round depression behind scutellum ; generally black ; prothorax yellowish brown, coloration of elytron variable, 1) entirely black, 2) black with a transverse 
median band yellowish brown, 3) yellowish brown with a basi-sutural round marking blackish, 4) entirely yellowish brown ; in pale colored specimen abdomen and legs almost entirely brownish ; length $5.0-6.0 \mathrm{~mm}$ viridipennis

Antenna slenderer, fifth and sixth segments more than twice as long as wide ; in male third to eleventh antenna1 segments thickly covered by long hairs ; generally reddish brown, elytral coloration variable, 1) entirely blackish with basal margin narrowly reddish, 2) black with a transverse median band reddish, 3) entirely reddish brown ; in dark colored specimen ventral surfaces, antenna and legs almost entirely pitchy black ; length $5.0-5.5 \mathrm{~mm}$ ..plagiata

\section{Cerophysella basalis (Baly)}

Aenidea? basalis Baly, 1874, Trans. Ent. Soc. London, 1874 : 181 (Japan, China ; BM).

Phyllectrus ? ceylanicus Allard, 1889, C. R. Soc. Ent. Belg., 33 : 82 (Ceylon, Saigon ; PARIS). -Maulik, 1936, Fauna India, Galeruc. : 445 (Ceylon, Saigon). New synonym.

Cerophysella ceylanica: Laboissiere, 1930, Ann. Soc. Ent. France, $99: 355$ (mentioned) ; 1935, Ann. Ass. Nat. Levallois-Per-ret, $21: 141$ (mentioned).

Cerophysella basalis: Laboissiere, 1930, Ann. Soc. Ent. France, 99: 352 (genus).-Ogloblin, 1936, Fauna USSR, 26, 1 : 170, 399 (Japan, Chine).-Gressitt \& Kimoto, 1963, Pac. Ins. Mon., 1B : 501 (China).

Cerophysella tonkinensis Laboissiere, 1930, Ann. Soc. Ent. France, $99: 353$ (Tonkin; HAMBURG). New synonym.

Distribution : Thailand, Vietnam, Hainan, E. China, Japan.

Material examined. THAILAND: Nakhon Nayok Prov., Khoa Yai Nat. Park, 17 exs., 5-6. vi. 1965, P. D. Ashlock (BISHOP), 32 exs., 6. vi. 1965, S. Asahina, K. Morimoto \& Y. Miyatake (KU). VIETNAM : 10 km E. of Ban Me Thuot, 570 m, 9 exs., 10. v. 1960, R. E. Leech (BISHOP).

In the Chinese specimens, a pair of tubercles on the posterior margin of pronotum are distinct. However, in the Thailand and the Indochinese specimens these tubercles are more weaker.

\section{Cerophysella laosensis n. sp. Fig. 16c}

Generally black, prothorax yellowish to reddish brown, antenna pitchy black with one or two basal segments reddish brown, legs generally yellowish brown; in pale colored specimen elytron entirely yellowish to reddish brown.

Head with vertex finely granulate, impunctate, interocular space distinctly wider than transverse diameter of single oculus, and interocular transverse impression obsolate, frontal tubercle transverse, subtriangular, contiguous, distinctly raised, surface generally smooth, shining, not distinctly delimited posteriorly. Antenna robust, in preapical segments nearly twice as long as wide ; first robust, somewhat club-shaped, second shortest, nearly $2 / 5$ as long as first, third nearly twice as long as second, third to seventh subequal to each other in length and shape, eighth nearly $11 / 5$ times as long as seventh, ninth subequal to eighth in length and shape, eleventh $11 / 4$ times as long as tenth and its apex pointed. Pronotum transverse, 11/4 times as broad as long, anterior margin distinctly rounded anteriorly, lateral margin distinctly rounded, widest at $1 / 3$ from anterior margin, and slightly narrowed anteriorly and more strongly so posteriorly, basal margin distinctly rounded posteriorly, dorsal surface convex, finely granulate, sparsely impressed by minute punctures, with a pair of lateral transverse furrows which are united at middle. Scutellum subtriangular, surface finely granulate, impunctate. Elytron with lateral margins subparallel-sided and apex rounded, surface distinctly and 
closely punctate, and interstices of punctures finely granulate.

Length : 5.0-6.0 $\mathrm{mm}$.

Holotype (BISHOP) :LASO: Vientiane Prov., Gi Sion Vill., de Tha Ngone, 2. i. 1966, Native collr. Paratopotypes : 76 exs., same data as the holotype (BISHOP, KIMOTO). Paratypes : LAOS : Vientiane Prov., Gi Sion Vill., de Tha Ngone, 55 exs., 26. xii. 1965, Native collr ; Vientiane Prov., Ban Van Eue, 1 ex., 31. vii. 1965, 2 exs., 31. i. 1967, 1 ex., 30. ix. 1967, 1 ex., 31. xii. 1968, Native collr ; Borikhane Prov., Pakkading, 16 exs., 13. vii. 1965, Native collr ; Wapikhamthong Prov., Wapi, 1 ex., 31. v. 1967, 1 ex., 15. vii. 1967, Native collor (BISHOP).

This new species resembles Cerophysella viridipennis (Allard), but differs in having pronotum finely granulate and elytron with the punctures more strongly and closely impressed.

\section{Cerophysella plagiata Laboissière, resurrected from synonymy}

Cerophysella plagiata Laboissiere, 1930, Ann. Soc. Ent. France, 99 : 354 (Tonkin; HAMBURG). -Gressitt \& Kimoto, 1963, Pac. Ins, Mon. 1B: 501 (= basalis).

Distribution : Vietnam.

In Gressitt \& Kimoto (1963), Cerophysa plagiata Laboissiere was treated as a synonym of basalis Baly. According to the study on the type of Cerophysella plagiata Laboissiere, this specie is characteristic in having the posterior margin of pronotum without and distinct tubercles.

Material examined. THAILAND : Chiengmai Prov., Mae Sa, 1 ex., 16. vi. 1965, P. D. Ashlock; Chieng Dao, 2 exs., 5-11. iv. 1958, Native collr ; Khao Yai, 1 ex., 11. iv. 1963, Native collr (BISHOP) ; Khon Kaen, 6 exs., x-xii. 1972, M. Sato (EHIME). LAOS : Borikhane Prov., Pakkading, 200 m, 1 ex., 23. iv. 1965, J. L. Gressitt (BISHOP) ; Umgeb. Vientiane, 1 ex., iii-vi. 1963 ; Umgeb. Pakse, 1 ex., 1963 (MUNCHEN).

\section{Cerophysella viridipennis (Allard)}

Phyllectrus viridipennis Allard, 1889, C. R. Soc. Ent. Belg., 33 : 83 (Cochinchina ; PARIS).

Cerophysella viridipennis :Laboissière, 1930, Ann. Soc. Ent. France, $99: 355$ (mentioned).

Chongania duporti Laboissiere, 1930, Ann. Soc. Ent. France, 99 : 356, fig. (Tonkin; HAMBURG). New synonum.

Distribution : Ceylon, Thailand, Cambodia, Laos, Vietnam.

Material examined. THAILAND : Chiengmai Prov., Fang (Agr. Exp. Station), 600 m, 2 exs., 14. vi. 1965, P. D. Ashlock; Chiengmai Prov., Chieng Dao, 1 ex., 5-11. iv. 1958, Native collr; Nakhon Nayok Prov., Khao Yai Nat, Park, 1 ex., 6. vi. 1965, P. D. Ashlock; Kao Yai, 1 ex., 11. iv. 1963, Native collr (BISHOP). CAMBODIA : Kiri Rom, 700 m, 42 exs., 31. iii.-7. iv. 1961, N. R. Spencer (BISHOP). LAOS : Vientiane Prov., Vientiane, 1 ex., 30. iv. 1965, Native collr ; Vientiane Prov., Ban Van Eue, 2 exs., 11. iv. 1965, 10 exs., 12. iv. 1965, 8 exs., 13. iv. 1965, 2 exs., 14. iv. 1965, 3 exs., 15. iv. 1965, 1 ex., 16. iv. 1965, J. L. Gressitt, 6 exs., 1-15. v. 1965, J. A. Rondon, 6 exs., 15-31. iv. 1965, 1 ex., 15-31. v. 1965, Native collr ; Vientiane Prov., Phou-kow-kuei, 2 exs., 15. iv. 1965, 1 ex., 16. iv. 1965, 5 exs., 17. iv. 1965, J. L. Gressitt ; Sayaboury Prov., Sayaboury, 5 exs., 15-16. iv. 1965, J. L. Gressitt ; Muong Sing, NW of Luang Prabang, 650 m, 2 exs., 6-10. vi. 1960, L. W. Quate ; Phon Tiou, 1 ex., 1-15. iv. 1965, J. L. Gressitt (BISHOP) ; Umgeb. Vientiane, 14 exs., iii-vi. 1963 ; Umgeb. Vanky, 1 ex., 1963 ; Umgeb. Paklay, 2 exs., 1963 (MUNCHEN). VIETNAM : Dalat, 1,500 m, 4 exs., 29. iv.-4. v. 1960, S. Quate \& L. Quate ; NW of Dalat, 1,300 m, 5 exs., 4-5. v. 1960, S. Quate \& L. Quate ; $31 \mathrm{~km} \mathrm{S.} \mathrm{of} \mathrm{Dilinh} \mathrm{(Djiring),} 7$ exs., 29. iv. 1960, R. E. Leech ; $39 \mathrm{~km} \mathrm{~S}$. of Djiring, 1 ex., 29. iv. 1960, L. W. Quate ; $22 \mathrm{~km} \mathrm{S.} \mathrm{of} \mathrm{Nha} \mathrm{Trang,}$ 1 ex., 20-26. xi. 1960, C. M. Yoshimoto (BISHOP). 


\section{Genus Paridea Baly}

Paridea Baly, 1886, J. Linn. Soc. London, $20: 26$ (type : Paridea thoracica Baly $=$ Galleruca tetraspilota Hope ; India).-Maulik, 1936, Fauna India, Galeruc. :498.-Gressitt\& Kimoto, 1963, Pac. Ins. Mon., 1B :506.-Kimoto, 1965, J. Fac. Agr. Kyushu Univ., 13(3) : 375.

\section{KeY TO SPECIES OF Paridea}

Prosternum narrow but distinct, inserted between coxal cavities ; pygidum modified

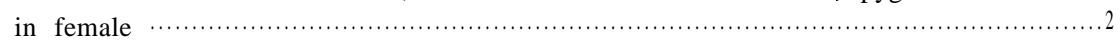

Prosternum not inserted between coxal cavities ; pygidium simple in both sexes

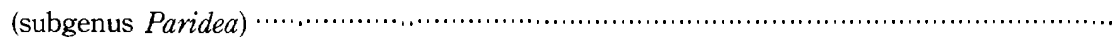

2(1) Pygidium of female entire, somewhat projecting beyond elytral apex and horn-like

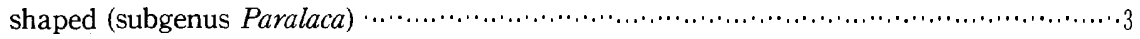

Pygidium of female deeply emarginate and projecting as a bilobed process beyond elytron (subgenus Semacia); yellowish brown with meso- and metathorax blackish ;

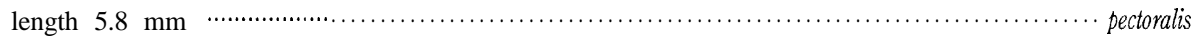

3(2) Pronotum pitchy brown with basal portion yellowish brown, elytron entirely yellowish to reddish brown; generally yellowish brown ; length $4.5-5.5 \mathrm{~mm} \cdots \cdots \cdots \cdot \ldots$ coomani Pronotum pitchy black; elytron pitchy black with a large median marking yellowish brown; head and ventral surfaces pitchy black with abdomen yellowish brown ; antenna and legs generally yellowish brown; length 5.7-6.0 mm …................. excavata

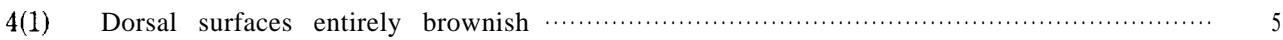

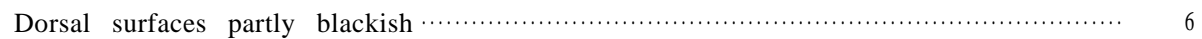

5(4) Elytron with a distinct lateral costa starting from humerus ; yellowish brown with

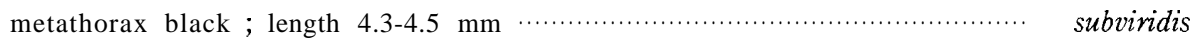
Elytron without any costa laterally; yellowish brown with metathorax black; in male elytron with a pair of foveae and several short irregular ridges basally, and tenth antennal segment depressed and infuscate ; length $5.4-6.5 \mathrm{~mm}$....................allardi

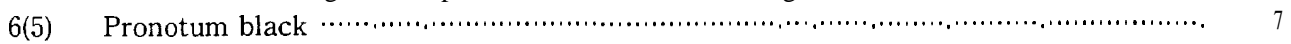

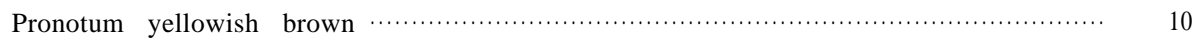

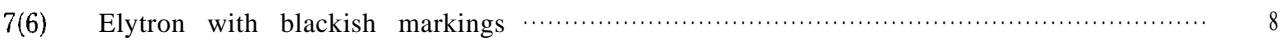
Elytron yellowish brown with margins blackish; generally blackish, vertex, apex of femora and base of tibiae reddish, and abdomen with lateral margin yellowish ; length $4.8 \mathrm{~mm}$

Elytral margins partly brownish

Elytral margins, together with basal and postmedian transverse band, entirely blackish ; generally blackish, abdomen with lateral margin yellowish ; length 3.9-5.3 $\mathrm{mm}$

9(8) Elytron yellowish brown with basal band and latero-apical marking blackish ; in female elytron with a distinct tubercle subapically ; generally black, vertex reddish brown, abdomen brownish ; legs blackish with apical $1 / 3$ of femora and base of tibiae brownish ; antenna blackish with basal segments brownish ; length $4.5 \mathrm{~mm}$ basalis Elytron yellowish brown with basal and apical band black; in male elytron without any distinct tubercle; generally blackish, abdomen with lateral margin brownish, antenna and legs blackish ; length $5 \mathrm{~mm}$ alternata

10(6) Legs entirely yellowish brown; head, pronotum and scutellum yellowish brown; 


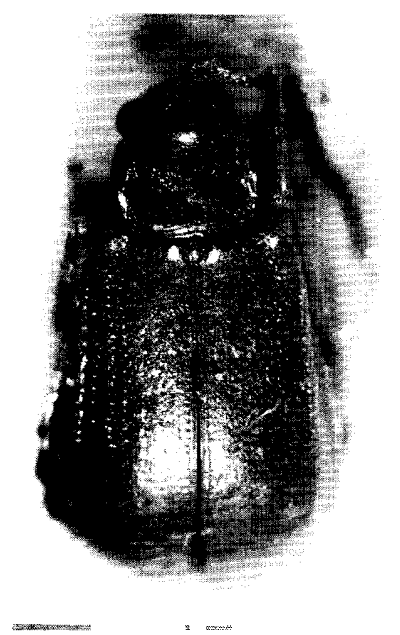

a

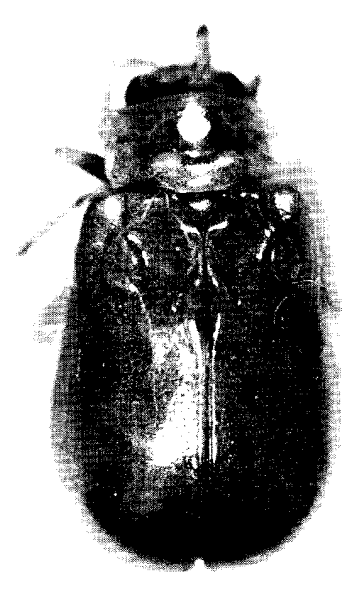

b

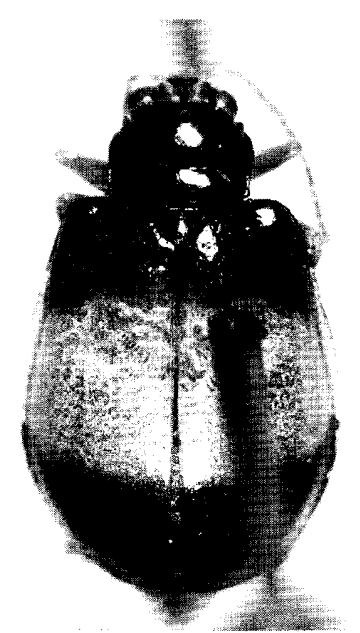

C

Fig. 17. a, Paridea (Paridea) subviridis Laboissiere ; b, P. (Parridea) allardin. sp.;c, P. (Paraulaca excavata n. sp.

elytron yellowish brown with basal and apical markings black, in some speicmen these two markings united; ventral surfaces yellowish brown with metathorax blackish, in some specimen abdomen blackish in various degrees ; length 5.7-6.0 $\mathrm{mm}$ tetraspilo ta

Legs yellowish brown with ventral surfaces of femora and tibiae black; dorsal surfaces yellowish brown, elytron with basal and apical markings blackish ; ventral surfaces yellowish brown with meso- and metathorax and four basal segments of abdomen blackish ; length 5.8-6.5 mm luteofascia ta

\section{Subgenus Paraulaca Baly}

Paraulaca Baly, 1888, J. Linn. Soc. London, 20 : 168 (type :Rhaphidopalpa angulicollis Motschulsky ; China).-Ogloblin, 1936, Fauna USSR, 26, $1: 165,397$ (type put in the subgenus Semacia !). Semacianella Laboissiere, 1930, Ann. Soc. Ent. France, 99 : 337 (type : Semacianella coomani Laboissiere; Tonkin). New synonym.

\section{Paridea (Paraulaca) coomani (Laboissière), new combination}

Semacianella coomani Lab., 1930, Ann. Soc. Ent. France, 99 : 338 (Tonkin; HAMBURG). Distribution : Vietnam.

Material examined. "Indo-China", 1 ex. (BASEL).

\section{Paridea (Paraulaca) excavata n. sp. Fig. 17c}

Generally pitchy black, elytron with a large median marking yellowish brown. Head with vertex smooth, shining, sparsely impressed by fine punctures, interocular space much 
wider than transverse diameter of single oculus, and interocular transverse impression distinct, frontal tubercle transverse, subquadrate, contiguous, distinctly raised, surface smooth, impunctate. Antenna slender, nearly $3 / 4$ as long as body length ; first segment long, robust, second shortest, nearly half as long as first, third twice as long as second, third to ninth subequal to each other in length and shape, tenth slightly shorter than ninth, eleventh slightly longer than tenth and its apex pointed. Pronotum transverse, $12 / 5$ times as broad as long, anterior margin nearly straight lateral margin feebly rounded, widest almost at $1 / 3$ from anterior margin, slightly narrowed anteriorly and posteriorly, basal margin distinctly rounded posteriorly, dorsal surface smooth, shining, sparsely impressed by minute punctures, and with a distinct transverse depression. Scutellum subtriangular, sparsely impressed by fine punctures. Elytron with lateral margin rounded, surface distinctly but not closely punctate and their interstices smooth, shining.

Male: Elytron with a distinct subbasal excavation; pygidium without horn- like projection apically. Female : Elytron without any distinct excavation subbasally ; pygidium with sharp hornlike shaped projection apically.

Length : 5.7-6.0 mm.

Holotype (BISHOP) : THAILAND : Chiengmai, 1,200 m, 11. iv. 1966, J. Sedlacek. Paratypes: THAILAND : Chiengmai Prov., Doi Suthep, 1,278 m, 1 ex., 29. iii.-4. v. 1958, T. C. Maa (BISHOP). LAOS : 100 m, Uekinak, ni-. Pakkading, 1 ex., 22. iv. 1965, J. L. Gressitt ; 100 m, Namakading, nr. Pakkading, 1 ex., 21. iv. 1965, J. L. Gressitt (BISHOP, KIMOTO).

This new species resembles Paridea coomani (Laboissière), but differs in having the general coloration pitchy black, and elytron with a median marking yellowish brown, and in the male elytron with a distinct subbasal excavation.

\section{Subgenus Paridea Baly \\ Paridea (Paridea) allardi n. sp. Fig. 17b}

Aulacophora chlorotica : Allard, 1888 (1889), Ann. Soc. Ent. France, ser. 6, $8: 308,319$ (Annam). Ceratia chlorotica: Weise, 1924, Col. Cat. Junk, 78 : 17 (Annam).

Generally yellowish brown, ventral surfaces with metathorax black.

Head with vertex smooth, shining, nearly impunctate, interocular space much wider than transverse diameter of single ocullus, and interocular transverse impression distinct, frontal tubercle transverse, subquadrate, contiguous, distinctly raised, surface smooth, impunctate. Antenna slender, nearly $3 / 4$ as long as body length ; first segment long, robust, second shortest, nearly $1 / 4$ as long as first, third $22 / 3$ times as long as second, fourth slightly shorter than third, fourth to ninth subequal to each other in length and shape, tenth slightly shorter than ninth, eleventh subequal to tenth in length but its apex pointed. Pronotum transverse, $13 / 4$ times as broad as long, anterior margin nearly straight, lateral margin feebly rounded, widest almost at middle, slightly narrowed anteriorly and posteriorly, basal margin slightly rounded posteriorly, dorsal surface smooth, shining, sparsely impressed by minute punctures, and with a distinct transverse depression. Scutellum subtriangular, convex, smooth, shining, impunctate. Elytron with lateral margin rounded, surface strongly and rather closely punctate and their interstices smooth, shining.

Male : Elytron with a pair of foveae and several short irregular ridges basally ; antenna with tenth segment depressed and infuscate. Female : Elytron normal ; antenna entirely brownish and normal.

Length : 5.4-6.5 mm.

Holotype (BISHOP) : LAOS : Vientiane Prov., Ban Van Eụe, 15. vi. 1966, Native collr.Para- 
topotypes : 3 exs., same data as the holotype (BISHOP). Paratypes : THAILAND : Khon Kaen, 1 ex., 24. vii. 1980, S. Azuma (RU). LAOS : 3 exs., same data as the holotype, but 30. xi. 1965, Native collr, 1 ex., 15-31. v. 1965, Native collr, 2 exs., 1-15. ix. 1967, Native collr, 3 exs., 15. vi. 1966, Native collr, 1 ex., 15. viii. 1965, Native collr, 1 ex., 15-31. iv. 1965, Native collr, 2 exs., 1-15. v. 1965, J. A. Rondon, 1 ex., 15. iv. 1965, J. L. Gressitt, 1 ex., 16. iv. 1965, J. L. Gressitt ; Phou-kow-kuei, N. of Vientiane, 1 ex., 17. iv. 1965, J. L. Gressitt ; Vientiane Prov., Gi Sion Vill., de Tha Ngone, 2 exs., 10-14. x. 1965, Native collr; Muong Sing, NW of Lang Praban, 650 m, 2 exs., 6-10. vi. 1960, S. Quate \& L. Quate ; Sayaboury Prov., Sayaboury, 1 ex., 15. iv. 1965, J. L. Gressitt, 1 ex., 16. iv. 1965, J. L. Gressitt, 1 ex., 14. xii. 1965, Native collr (BISHOP).

This new species somewhat resembles Paridea subviridis Laboissière, but differs in being the body length larger, and having elytron without any costa laterally and in the male with a pair of foveae and several short irregular ridges basally.

The material identified as "Aulacophorachlorotica Fabricius" by Allard is now preserved in Paris Museum. However, I could not trace any name referred by Allard.

\section{Paridea ( $\mathrm{P}$ aridea) alternata Laboissière}

Paridea alternata Lab., 1930, Ann. Soc. Ent. France, 99 : 345 (Tonkin; HAMBURG).

Distribution : Vietnam.

No additional material was examined beside the type series.

\section{Paridea ( $\mathrm{P}$ aridea) basalis Laboissière}

Paridea basalis Lab., 1930, Ann. Soc. Ent. France, 99 : 344 (Tonkin ; HAMBURG).

Distribution : Vietnam.

No additional material was examined beside the type series.

\section{Paridea ( $P$ aridea) circumdata Laboissière}

Paridea circumdata Lab., 1930, Ann. Soc. Ent. France, 99 : 345 (Tonkin).

Distribution : Vietnam.

I could not trace the type location of this species.

\section{Paridea ( $\mathrm{P}$ aridea) luteofasciata Laboissière}

Paridea luteofasciata Lab., 1930, Ann. Soc. Ent. France, 99 : 343 (Tonkin; HAMBURG).

Distribution : Vietnam.

No additional material was examined beside the type series.

\section{Paridea ( $P$ aridea) oculata Laboissière}

Paridea oculata Lab., 1930, Ann. Soc. Ent. France, 99 : 346 (Sikkim ; PARIS).

Distribution : N. India, Thailand, Vietnam.

Material examined. THAILAND : Chiengmai Prov., Chiengmai, 1,200 m, 4 exs., 11. iv. 1966, J. Sedlacek ; Chiengmai Prov., Doi Suthep, 1 ex., 28-31. iii. 1958, 2 exs., 1-8. iv. 1958, T. C. Maa ; 50 km W. of Tak, 900 m, 2 exs., 7-8. iv. 1966, J. Sedlacek (BISHOP) ; Khon Kaen, 1 ex., 24. vii. 1980, S. Azuma (RU). VIETNAM : Fyan, 1,200 m, 2 exs., 11. vii.-9. viii. 1961, N. R. Spencer (BISHOP). 


\section{Paridea ( $P$ aridea) perplexa (Baly)}

Aulacophora perplexa Baly, 1879, Cist. Ent., 2 : 447 (Assam ; BM).

Paridea perplexa:Baly, 1889, Trans. Ent. Soc. London, 1889 : 304 (genus).-Maulik, 1936, Fauna India, Galeruc. : 505 (India, Burma).-Kimoto, 1970, Khumbu Himal,3(3) : 417 (Nepal). -Kimoto\& Takizawa, 1972, Kontyâ, Tokyo, 40(4): 217 (Nepal) ; 1984, Bull. Natn. Sci. Mus. Tokyo, ser. A, 9(2) : 92 (Nepal).

Paridea harmandi Laboissière, 1930, Ann. Soc. Ent. France, 99 : 340 (NE Yunnan, Sikkim ; HAMBURG). New synonym.

Distribution : India, Nepal, Burma, Vietnam, S. China.

Material examined. VIETNAM : Dilinh (Djiring), 1,200 m, 1 ex., 22-28. iv. 1960, L. W. Quate ;24 km S. of Dilinh (Djiring), 1,050 m, 1 ex., 29. iv. 1960, R. E. Leech ; 17 km S. of Dilinh, 1,300 m, 1 ex., 6 -13. x. 1960, C. M. Yoshimoto ; $10 \mathrm{~km}$ E. of Banme Thuot, 1 ex., 10. v. 1960, R. E. Leech ; $20 \mathrm{~km} \mathrm{~N}$. of Pleiku, 650 m, 1 ex., 9. v. 1960, L. W. Quate (BISHOP).

\section{Paridea ( $P$ aridea) subviridis Laboissière Fig. 17a}

Paridea subviridis Lab., 1930, Ann. Soc. Ent. France, 99 : 341 (Tonkin; HAMBURG).

Distribution : Vientnam.

No additional material was examined beside the type series.

\section{Paridea ( $P$ aridea) tetraspilota (Hope)}

Galleruca tetraspilota Hope, 1831, in Gray, Zool. Misc. : 29 (Nepal ; BM).

Monolepta tetraspilota: Weise, 1924, Col. Cat. Junk, 78 : 173 (Nepal).

Paridea tetraspilota: Maulik, 1936, Fauna India, Galeruc.: 501 (India, Nepal, Burma, Yunnan, Siam, Formosa).

Paridea (Paridea) tetraspilota : Gressitt \& Kimoto, 1963, Pac. Ins. Mon., 1B: 516 (China).

Paridea thoracica Baly, 1886, J. Linn. Soc., Zool., 20 : 29 (India).-Laboissiere, 1930, Ann. Soc. Ent. France, 99 : 340 (Cambodia, Cochinchine : Mont de Chaudoc).-Maulik, 1936, Fauna India, Galeruc. :51 (= tetraspilota).

Distribution : India, Nepal, Thailand, Cambodia, Laos, Vietnam, S. China, Taiwan.

Material examined. THAILAND : Chiengmai Prov., Chiengdao, 3 exs., 5-11. iv. 1958, T. C. Maa (BISHOP) ; Khon Kaen, 3 exs., 24. vii. 1980, S. Azuma (RU). LAOS : Vientiane Prov., Ban Van Eue, 1 ex., 12. iv. 1965, J. L. Gressitt, 2 exs., 15-31. v. 1965, 2 exs., 30. xi. 1965, 1 ex., 31. xii. 1965, Native collr ; Sedone Prov., Paksong, 1 ex., 6. viii. 1965, Native collr ; Sayaboury Prov., Sayaboury, 1 ex., 15. iv. 1965, J. L. Gressitt (BISHOP). VIETNAM : $31 \mathrm{~km} \mathrm{S.} \mathrm{of} \mathrm{Dilinh} \mathrm{(Djiring),} \mathrm{1,050} \mathrm{m,} 2$ exs., 29. iv. 1960, R. E. Leech ;Banme Thuot, 500 m, 2 exs., 16-18. v. 1960, L. W. Quate (BISHOP).

\section{Subgenus Semacia Fairmaire}

Semacia Fairmaire, 1889, Ann. Soc. Ent. France, 58 : 82 (type : Semacia biplagiata Fairmaire ; China).-Ogloblin, 1936, Fauna USSR, 26, 1: 167, 398 (subgenus of Paraulaca, but included the type of the latter). 


\section{Par-idea (Semacia) pectoralis (Laboissière), new combination}

Semacia pectoralis Lab., 1930, Ann. Soc. Ent. France, 99 : 333 (Tonkin; PARIS).

Distribution : Vietnam.

No additional material was examined beside the type.

\section{Genus Trichomimastra Weise}

Trichomimastra Weise, 1922, Tijdschr. Ent., 65 : 75 (type :Mimastra seminigra Weise ; Banguay). -Ogloblin, 1936, Fauna USSR, 26, 1: 181.-Gressitt \& Kimoto, 1963, Pac. Ins. Mon., 1B: 543.

\section{Trichomimas tra hirsu ta (Jacoby), new combination Fig. 2a}

Mimastra hirsuta Jacoby, 1892, Ann. Mus. Civ. Genova, 32 : 945 (Burma ; GENOVA).

Mimastra (Trichomimastra) membranacea Weise, 1922, Tijdschr. Ent., 65 : 77 (Tonkin; STOCKHOLM). New synonym.

Mimastra (Trichomimastra) piceipennis Laboissière, 1929, Ann. Soc. Ent. France, 98 : 287 (Siam ; PARIS). New synonym.

Mimastra (Trichomimastra) pectoralis Laboisdire, 1929, Ann. Soc. Ent. France, 98 : 286 (Tonkin; HAMBURG). New synonym.

Trichomimastra pectoralis: Gressitt \& Kimoto, 1963, Pac. Ins. Mon., 1B: 546 (China).

Distribution : Burma, Thailand, Laos, Vietnam, Hainan.

The coloration of the dorsal surfaces is variable : 1) entirely yellowish to reddish brown, 2) pronotum yellowish to reddish brown, elytron pitchy black, 3) the dorsal surfaces generally yellowish to reddish brown, elytron with the sutural, the lateral and the apical margins blackish, 4) the dorsal surfaces entirely pitchy black.

Material examined. THAILAND : Chieng Mai Prov., Doi Suthep, 2 exs., 28-31. iii. 1958, T. C. Maa, 1 ex., 8. vi, 1965, P. D. Ashlock; Banna, Chawang, nr. Nabon, 1 ex., 5. ix. 1958, J. L. Gressitt, 1 ex., 5-10. iv. 1958, T. C. Maa (BISHOP) ; Chiengmai Prov., Doi Suthep, 1,000 m, 1 ex., 16. vi. 1965, K. Morimoto ; Khao Chong, nr. Trung, 1 ex., 25. vi. 1965 (KU). LAOS : Vientiane, Prov., Ban Van Eue, 800 m, 27 exs., 11-15. iv. 1965, J. L. Gressitt, 2 exs., 1-15. v. 1965, Malaise trap, J. A. Rondon, 3 exs., 15. xii. 1965, 1 ex., 29. iii. 1966, 1 ex., 30. xi. 1966, 1 ex., 31. xii. 1968, Native collr ; Sayaboury, 3 exs., 15. iv. 1965, J. L. Gressitt ; Borikhane Prov., Pakkading, 100-200 m, 1 ex., 23. iv. 1965, J. L. Gressitt (BISHOP) ; Umgeb. Vientiane, 1 ex., iii-vi. 1965 (MUNCHEN). VIETNAM : Mt. Lang Bian, 1,5002,000 m, 4 exs., 19. v.-8. vi. 1961, N. R. Spencer ; Fyan, 900-1,000 m, 4 exs., 11. vii.-9. viii. 1961, N. R. Spencer; Dilinh (Djiring), 1,200 m, 1 ex., 22-28. iv. 1960, L. W. Quate ; Dalat, 1,500 m, 1 ex., 29. iv.-4. v. 1960, L. W. Quate ; Dalat, 6 km S., 1,400-1,500 m, 2 exs., 9. vi.-7. vii. 1961, N. R. Spencer (BISHOP).

\section{Genus Siemssenius Weise}

Siemssenius Weise, 1922, Tijdschr. Ent., $65: 73$ (type: Siemssenius modesta Weise; China). -Gressitt \& Kimoto, 1963, Pac. Ins. Mon., 1B:555.-Seeno \& Wilcox, 1982, Entomography, 1 : 111.

Pseudoliroetis Laboissiere, 1929, Ann. Soc. Ent. France, $98: 280$ (type : Liroetis fulvipennis Jacoby ; China).-Ogloblin, 1936, Fauna USSR, 26, 1 :204.-Gressitt \& Kimoto, 1963, Pac. Ins. Mon., 1B : 529. 


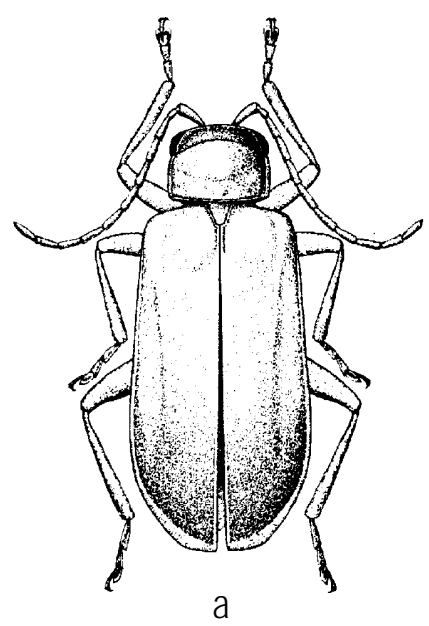

a

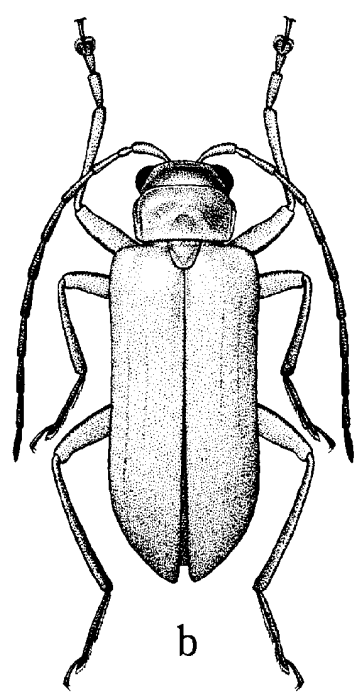

Fig. 18. a, Haplosomoides flavus Laboissike ; b, H. annamitus (Allard); c, H. costatus Baly.

Siemssenius fulvipennis(Jacoby), new combination

Fig. 22a

Liroetis fulvipennis Jac., 1890, Entomolog., $23: 215$, fig. (China ; BM).

Pseudoliroetis fulvipennis : Laboissiere, 1929, Ann. Soc. Ent. France, 98 : 281 (China).-Ogloblin, 1936, Fauna USSR, 26, $1:$ 206, 405 (China).-Gressitt \& Kimoto, 1963, Pac. Ins. Mon., 1B: 530, fig. (China).

Pseudoliroetis jeanvoinei Laboissière, 1929, Ann. Soc. Ent. France, 98 : 281 (Tonkin; HAM BURG). New synonym.

Distribution : Vietnam, China.

Oblong oval ; generally reddish to yellowish brown, antenna and legs entirely blackish ; length 11.0-14.0 mm.

No additional material was examined beside the type series.

\section{Genus Haplosomoides Duvivier}

Haplosomoides Duv., 1890, C. R. Soc. Ent. Belg., 34 :35 (type : Rhaphidopalpa serena Boheman ;E. Indies).-Maulik, 1936, Fauna India, Galeruc. : 162.-Gressitt\& Kimoto, 1963, Pac. Ins. Mon., 1B: 517 .

\section{KeY TO SPECIES OF Haplosomoides}

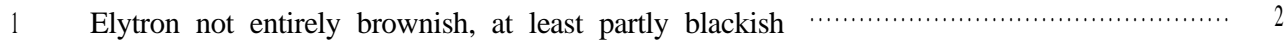
Elytron entirely brownish

2(1) Elytron black with posterior half yellowish brown; generally black, antenna yellowish

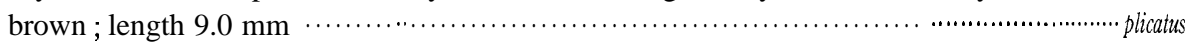

Elytron entirely black ; head, pronotum and legs yellowish brown ; length 6.7-7.0 $\mathrm{mm}$ costatus 
3(1) Abdomen entirely brownish

Abdomen entirely blackish ; generally yellowish brown; length $5.0-6.3 \mathrm{~mm} \cdots \cdots . .$. annamitus

4(3) Antenna blackish with basal segments brownish; pronotum $13 / 4$ times as wide as long ; generally yellowish brown ; in male, ventral surface of first abdominal segment with a distinct, long projection apically; length $5.7-6.3 \mathrm{~mm} \ldots \ldots \ldots \ldots \ldots \ldots \ldots$. Antenna entirely yellowish brown; pronotum $1 \mathrm{l} / \mathrm{Z}$ times as wide as long ; generally yellowish brown; in male without any long projection apically ; length 5.7-6.3 $\mathrm{mm}$

flavus

\section{Haplosomoides annamitus (Allard), new combination Fig. 18b}

Hoplasoma annamita Allard, 1888 (1889), Ann, Soc. Ent. France, ser. 6, 8: 328, 330 (Annam; PARIS).

Haplosomoides egena Weise, 1922, Tijdschr. Ent., 65 : 74 (China, Tonkin)._Laboissière, 1930, Ann. Soc. Ent. France, 99 : 326 (Tonkin).-Gressitt \& Kimoto, 1963, Pac. Ins. Mon., 1B: 518 (China, Vietnam).-Kimoto, 1984, Ent. Rev. Japan, 39(1) : 49 (Taiwan). New synonym.

Distribution : Nepal, Bhutan, Thailand, Laos, Vietnam, China, Taiwan.

I could not trace the type location of Haplosomoides egena Weise. My identification of this species is based on the material identified by Laboissike, preserved in Stockholm.

Material examined. THAILAND : Chiengmai, 3,600 ft., 5 exs., 9-10. iv. 1953, Tak, 2,800 ft., 4 exs., 21. iii. 1958 (BANGKHEN) ; Chiengmai Prov, Doi Suthep, 4 exs., 1-5. iv. 1958, T. C. Maa (BISHOP) ; Chiengmai Prov., Doi Suthep, 1,000 m, 2 exs., 18-19. vi. 1965, K. Morimoto \& Y. Miyatake ; Chieng Dao, 1 ex., 23. iv. 1973, Y. Yoshiyasu (KU). LAOS : Vientiane Prov., Ban Van Eue, 1 ex., 14-15. iv. 1965, J. L. Gressitt, 2 exs., 1-15. v. 1965, J. A. Rondon, 1 ex., 15-31. iv. 1965, Native collr ; Khamouane Prov., Phon Tiou, 1 ex., 17. v. 1965, Native collr (BISHOP) ; Umgeb. Vanky, 3 exs., 1963 ; Umgeb. Paklay, 2 exs., 1963 (MUNCHEN). VIETNAM : Dalat, 6 km S., 1,400-1,500 m, 2 exs., 9. vi.-7. vii. 1961, N. R. Spencer ; 7 km SE of Dilinh, 990 m, 1 ex., 2. v. 1960, R. E. Leech (BISHOP).

\section{H aplosomoides appendiculatus Laboissière}

Haplosomoides appendiculata Lab., 1930, Ann. Soc. Ent. France, 99 : 328 (Tonkin; BRUXELLES). -Gressitt \& Kimoto, 1963, Pac. Ins. Mon., 1B: 518 (Hainan).

Haplosomedia sarrata: Châjô, 1964, Nature and Life in SE Asia, Kyoto, 3 : 292 (Thailand).

Distribution : Thailand, Laos, Vietnam, Hainan.

Material examined. THAILAND: Banna, Nakhon, 108 m, 1 ex., 5-10. v. 1958, T. C. Maa (BISHOP). LAOS : Vientian Prov., Ban Van Eue, 7 exs., 13-15. iv. 1965, J. L. Gressitt, 4 exs., 1-15. v. 1965, J. A. Rondon, 1 ex., 15-31. iv. 1965, 15 exs., 15-31. v. 1965, 1 ex., 15. vii. 1965, 1 ex., 31. vii. 1965, 2 exs., 15. viii. 1965, 1 ex., 30. xi. 1965, 11 exs., 15. v. 1966, 1 ex., 30. vi. 1966, 9 exs., 31. v. 1966, 1 ex., 15. ii, 1967, 6 exs., 15. v. 1967, 5 exs., 30. vi. 1967, 7 exs., 30. viii. 1967, 5 exs., 15. ix. 1967, 8 exs., 30. ix. 1967, Native collr; Khamouane Prov., Phon Tiou, 11 ex., 11. vi. 1965, N. Wilson, 2 exs., 30. iv. 1965, Native collr ; Sedone Prov., Paksong, 1 ex., 26, vii. 1965, Native collr ; Borikhane Prov., Pakkading, 1 ex., 13. vii. 1965, J. L. Gressitt ; Sayaboury, 3 exs., 15. iv. 1965, J. L. Gressitt (BISHOP). VIETNAM : Ban Me Thuot, 500 m, 1 ex., 16-18. v. 1960, L. W. Quate ; Dak Sog, 76 km SW of Ban Me Thuot, 870 m, 1 ex., 19-21. 1960, L. W. Quate (BISHOP). 


\section{Haplosomoides costatus (Baly) Fig. 18c}

Mimastra costata Baly, 1878, Ann. Mag. Nat. Hist., ser. 5, 2 : 415 (China ; BM).-Weise, 1922, Tijdschr. Ent., 65 : 75 (Fokien, W. Formosa).

Haplosomoides costata :Laboissière, 1930, Ann. Soc. Ent. France, 99 : 325 (Tonkin, Kweichow). -Gressitt \& Kimoto, 1963, Pac. Ins. Mon., 1B: 518 (China).

Distribution : S. China, Hainan, Taiwan, Vietnam, Ryukyu Is.

No additional material was examined.

\section{Haplosomoides flavus Laboissière Fig. 18a}

Haplosomoides flava Lab., 1930, Ann. Soc. Ent. France, 99 : 326 (Tonkin ;BRUXELLES).-Gressitt \& Kimoto, 1963, Pac. Ins. Mon., 1B: 520 (Vietnam).

Haplosoma ceylonensis: Allard, 1889, Ann. Soc. Ent. France, 1889 : 308 (Hue).-Laboissiere, 1935, Ass. Nat. Levallois-Perret, $21: 140$ (corrected as Haplosomoides flava).

Distribution : Thailand, Laos, Vietnam.

Material examined. THAILAND : Trang, 3 exs., 1-18. iv. 1960 (BANGKHEN) ; Chieng Mai Prov., Doi Suthep, 5 exs., 1-5. iv. 1958, 1 ex., water margin, 29. iii.-4. v. 1958, T. C. Maa ; Khao Yai, 1 ex., 10. iv. 1963, Native collr ; Phliu, 1 ex., 22. iv. 1963, Native collr (BISHOP) ; Chiengmai Prov., Doi Suthep, 1,000 m, 1 ex., 12. vi. 1965, K. Morimoto ; Chiengmai Prov., Tankeo, Doi Suthep, 800 m, 1 ex., 10. vi. 1965, Y. Miyatake ; Kao Chong, nr. Trung, 9 exs., 25-26. vi. 1965, K. Morimoto \& Y. Miyatake (KU). LAOS : Umgeb. Vientiane, 1 ex., iii-vi. 1963 (MUNCHEN) ; Vientiane Prov., Ban Van Eue, 1 ex., 1-15. v. 1965, J. A. Rondon; Borikhane Prov., Pakkading, 1 ex., 13. vii. 1965, J. L. Gressitt ; Sayaboury, 1 ex., 13. vii. 1965, J. L. Gressitt (BISHOP). VIETNAM : Ban Me Thuot, 500 m, 9 exs., 1618. v. 1960, S. Quate \& L. W. Quate; Dilinh (Djiring), 1 ex., 27. ix.-14. x. 1960, C. M. Yoshimoto ;7 km SE of Dilinh, 1 ex., 990 m, 2. v. 1960, R. E. Leech ; 17 km S. of Dilinh, 1,300 m, 2 exs., 6-13. x. 1960, C. M. Yoshimoto ; $50 \mathrm{~km} \mathrm{SW}$ of Pleiku, $250 \mathrm{~m}, 2$ exs., 14. v. 1960, L. W. Quate ; $25 \mathrm{~km}$ SW of Pleiku, 400 m, 1 ex., 12. v. 1960, L. W. Quate (BISHOP).

\section{Haplosomoides plicatus (Allard) Fig. 19a}

Pseudocophora plicata All., 1887, Ann. Soc. Ent. France, ser. 6, 7: 201 (Malacca) ; 1888 (1889), ibid., ser. 6, $8: 325$ (Malacca).

Haplosomoides plicata: Duvivier, 1890, C. R. Soc. Ent. Belg., 34 : 35, anm 1 (Malacca).

Distribution : Thailand, Malaya.

Material examined. THAILAND: Cholburi, 1 ex., 4. vii. 1963, Native collr (BISHOP).

\section{Genus Fleutiauxia Laboissière}

Fleutiauxia Lab., 1933, Ann. Soc. Ent. France, 102 : 53 (type : Fleutiauxia cyanipennis Laboissière; Tonkin).-Ogloblin, 1936, Fauna USSR, 26, $1: 183$ (type : Aenida armata Baly ; Japan).

\section{KeY To SPECIES of Fleutiauxia}

Meso- and metathorax, and abdomen black ; generally yellowish brown, elytron bluish black, antenna somewhat infuscate ; in male head with a deep transverse excavation and a short tubercle at middle ; length 5.8-6.5 mm cyanipennis 


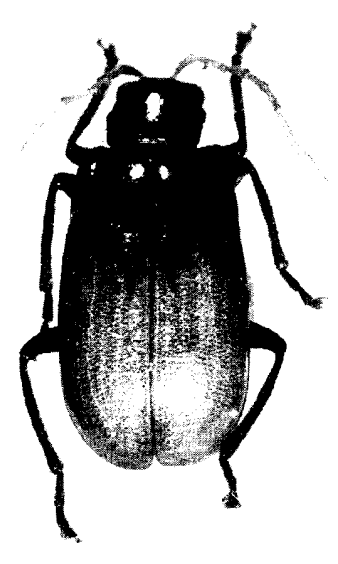

$\mathrm{a}$

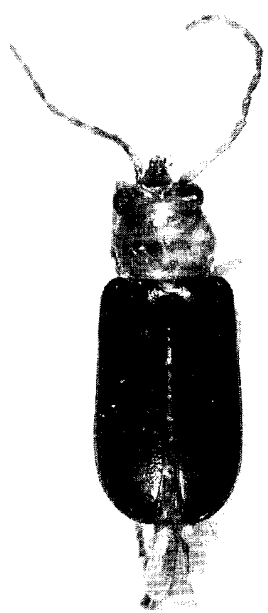

b

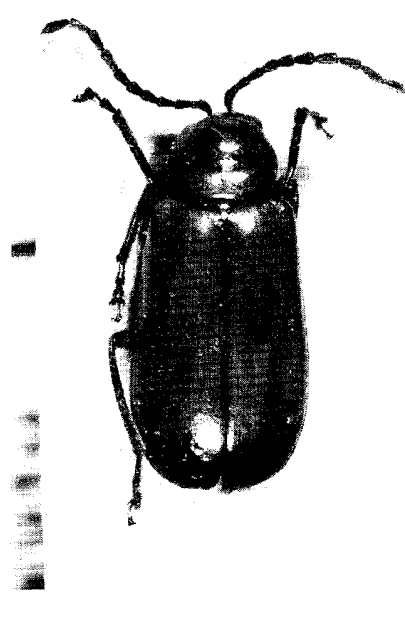

$\mathrm{C}$

Fig. 19. a, Haplosomoides plicatus (Allard) ; b, Fleutiauxia violaceipennis n. sp. ; c, Taumacera pulchella (Laboissière).

Ventral surfaces entirely yellowish to reddish brown; generally yellowish to reddish brown, elytron violaceous blue, antenna somewhat infuscate ; in male head with a long, subparallel-sided projection ; length $4.8-5.1 \mathrm{~mm}$ violaceipennis

\section{Fleutiauxia cyanipennis Laboissière}

Fleutiauxia cyanipennis Lab., 1933, Ann. Soc. Ent. France, 102 : 54 (Tonkin; HAMBURG). Distribution : Vietnam.

No additional material was examined beside the type series.

\section{Fleu tiauxia violaceipennis n. sp. Fig. 19b}

Generally yellowish to reddish brown, elytron violaceous blue, antenna dark reddish brown, with two or three basal segments paler, legs with tibiae and tarsi more or less infuscate.

Head with vertex finely granulate, sparsely impressed by minute punctures, interocular space much wider than transverse diameter of single oculus, and interocular transverse impression distinct, frontal tubercles, subtriangular distinctly separated to each other, slightly raised, surface finely granulate. Antenna slender, nearly $3 / 4$ as long as body length ; first segment robust, second nearly half as long as first, third $21 / 5$ times as long as second, fourth subequal to third in length and shape, fifth slightly shorter than fourth, fifth to ninth subequal to each other in length and shape, tenth slightly shorter than ninth, eleventh subequal to ninth in length but its apex pointed. Pronotum transverse, $12 / 5$ times as broad as long, anterior margin distinctly rounded anteriorly, lateral margin distinctly rounded, widest slightly before middle, slightly narrowed anteriorly and more strongly so posteriorly, basal margin slightly rounded posteriorly, almost straight at middle, dorsal surface convex, with a pair of shallow depressions laterally, finely granulate, nearly impunctate. Scutellum subtriangular, finely granulate, impunctate. Elytron with lateral margin subparallel-sided, apex 
rounded, surface finely granulate and impressed by distinct punctures and their interstices clearly wider than average diameter of punctures.

Male : Head with a long, subparallel-sided projection on frons. Female : Unknown.

Length : 4.8-5.1 mm.

Holotype (BISHOP) : LAOS : Sedone Prov, Pakse, 11. v. 1965, P. D. Ashlock. Paratype : LAOS : Sedone Prov., 5 km E. of Pakse, 1 ex., 13. v. 1965, P. D. Ashlock (KIMOTO).

This new species resembles Fleutiauxia cyanipennis Laboissière, but differs in having the ventral surfaces entirely yellowish to reddish brown, and in the male head with a long, subparallel-sided projection on frons.

\section{Genus Taumacera Thunberg}

Taumacera Thunb., 1814, Vet. Acad. Handl. : 48 (type : Taumacera deusta Thunberg ; India). -Maulik, 1936, Fauna India, Galeruc.: 479.-Gressitt \& Kimoto, 1963, Pac. Ins. Mon., 1B : 520.

Cerophysa Chevrolat, 1837, in Dejean, Cat. Col., ed. $3: 403$ (type : Galleruca nodicornis Wiedemann ; Java ; monobasic).-Ogloblin, 1936, Fauna USSR, 26, 1: 171, 372.

Ozomena Chevrolat, 1837, in Dejean, Cat. Col., ed. $3: 403$; 1845, in d'Orbingny, Dict. Univ. Hist. Nat., $6: 5$ (type :Galleruca nodicornis Wiedemann ; Java).

Cerophyta Blanchard, 1845, Hist. Insect., 2 :190.-Strand, 1935, Folia Zool.-Hydrobiol., 7 : 285.

Neocharis Jacoby, 1881, Proc. Zool. Soc. London, 1881 : 448 (type : Neocharis fulvicollis Jacoby ; Java ; monobasic).

Metallus Jacoby, 1886, Ann. Mus. Civ. Genova, 24 : 63 (new name for Neocharis Jacoby, nec Sharp, 1877, Eucnemidae).

Nacrea Baly, 1886, Trans. Ent. Soc. London, 1886 : 29 (type : Nacrea maculata Baly= Neocharis fulvicollis Jacoby ; Java).

\section{KEY TO SPECIES OF Taumacera}

1 Dorsal surfaces not entirely brownish

Dorsal surfaces entirely yellowish brown; generally yellowish brown; length 5.7-6.3

$\mathrm{mm}$

doisu thepica

2(1) Ventral surfaces partly brownish

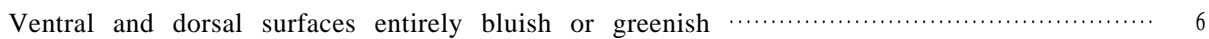

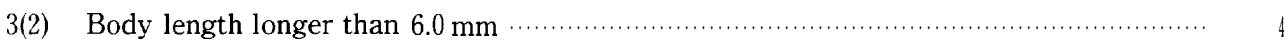

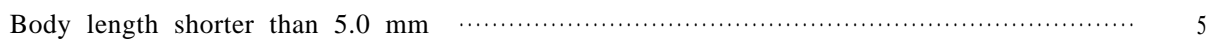

4(3) Dorsal surfaces entirely greenish ; generally bluish green, abdomen reddish brown ; length $7.0-7.5 \mathrm{~mm}$............................................................ me tallica

Dorsal surfaces brownish, elytron with apex bluish, in some specimen its basal margin also ; generally yellowish brown, antenna, tibiae and tarsi infuscate ; in male eighth antennal segments enlarged; length 6.0-7.0 mm pulchella

5(3) Dorsal surfaces brownish, sutural and lateral margins narrowly piceous; generally reddish brown with antenna and tarsi black; length $4.0 \mathrm{~mm} \ldots \ldots \ldots \ldots \ldots \ldots \ldots \ldots \ldots$. Elytron yellowish brown with basal and apical areas bluish ; generally pitchy black; in some specimen pronotum reddish brown; in male eighth antennal segment enlar-

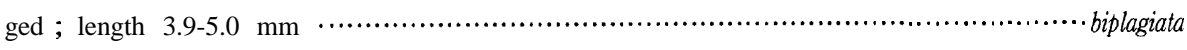

6(2) Pronotum $11 / 3$ times as wide as long, surface strongly and rather closely punctate, and interstices of punctues finely shagreened; in male eighth antennal segment enlar- 
ged; length 4.7-5.7 mm

Pronotum $11 / 4$ times as wide as long, surface rather finely and not closely punctate, and interstices of punctures smooth, shining ; in male third to fifth antennal segments deformed ; length 5.3-5.7 mm

laosensis

\section{Taumacera biplagiata (Duvivier)}

Cerophysa biplagiata Duviv., 1885, Stett. Ent. Ztg., 46: 393 (Hong Kong).-Weise, 1922, Tijdschr. Ent., 65 : 69 (China, Tonkin).

Cerophysa biplagiata var. muficollis Weise, 1922, Tijdschr. Ent., 65 : 69 (locality not cited).

Cerophysa biplagiata collaris Chen, 1942, Notes d'Ent. Chinoise, 9(3): 32 (China).

Taumacera (Cerophysa) biplagiata : Gressitt \& Kimoto, 1963, Pac. Ins. Mon., 1B: 523 (China).

Distribution : Thailand, Laos, Vietnam, S. China.

Material examined. THAILAND; Khao Yai Nat. Park, 1 ex., 6. v. 1965, Y. Miyatake (KU). LAOS : Borikhane Prov., Pakkading, 100-200 m, 1 ex., 23. iv. 1965, J. L. Gressitt (BISHOP) ; Umgeb. Vientiane, 1 ex., iii-iv. 1963 ; Umgeb. Pakse, 1 ex., 1963 ; Umgeb. Vanky, 1 ex., 1963 (MUNCHEN).

\section{Taumacera coomani (Laboissière), new combination Fig. 20b}

Cerophysa coomani Lab., 1930, Ann. Soc. Ent. France, 99 : 351 (Tonkin; HAMBURG).

Distribution : Laos, Vietnam.

Material examined, LAOS : Vientiane Prov., Ban Van Eue, 2 exs., 12. iv. 1965, 1 ex., 14. iv. 1965, J. L. Gressitt, 1 ex., 1-15. v. 1965, J. A. Rondon, 1 ex., 29. iv. 1966, Native collr ; Vientiane Prov., Phoukow-kuei, 1 ex., 15. iv. 1965, J. L. Gressitt ; Sayaboury Prov., Sayaboury, 1 ex., 15. iv. 1965, J. L. Gressitt (BISHOP).

\section{Taumacera doisuthepica n. sp. Fig. 20c}

Generally yellowish brown.

Head with vertex smooth, shining, nearly impunctate, interocular space much wider than transverse diameter of single oculus, and interocular transverse impression distinct, frontal tubercle subtriangular, contiguous, distinctly raised, surface somewhat wrinkled. Antenna slender, nearly $2 / 3$ as long as body length ; first segment long, robust, second shortest, nearly $1 / 3$ as long as first, third $21 / 5$ times as long as second, fourth $11 / 4$ times as long as third, fifth slightly shorter than fourth, fifth to seventh subequal to each other in length and shape, eighth slightly shorter than seventh, ninth subequal to eighth in length and shape, tenth slightly shorter than ninth, eleventh $11 / 2$ times as long as tenth and its apex pointed. Pronotum $13 / 4$ times as broad as long, anterior margin distinctly rounded posteriorly, lateral margin distinctly rounded, widest almost at $1 / 3$ from anterior margin, slightly narrowed anteriorly and more strongly so posteriorly, basal margin slightly rounded posteriorly, dorsal surface convex, smooth, shining, sparsely impressed by minute punctures, and with a deep subbasal transverse depression. Scutellum subtriangular, somewhat wrinkled, impunctate. Elytron with lateral margin subparallel-sided, apex rounded, surface finely impressed by distinct punctures and their interstices smooth and shining.

Length : 5.7-6.3 mm.

Holotype (Type No. 2698, Kyushu Univ.) : THAILAND : Chiengmai Prov., Doi Suthep, 1,000 m, 12. vi. 1965 , Y. Miyatake. Paratypes : 3 exs., same data as the holotype but K. Morimoto (KU, KIMOTO). 


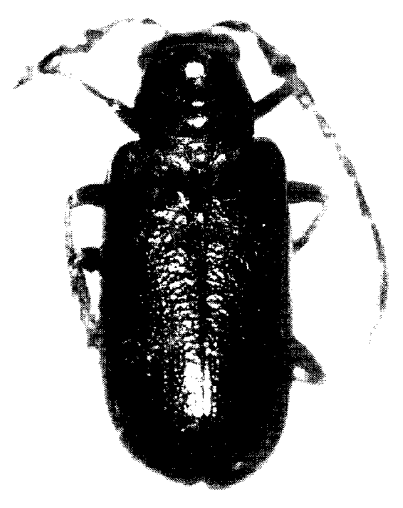

a

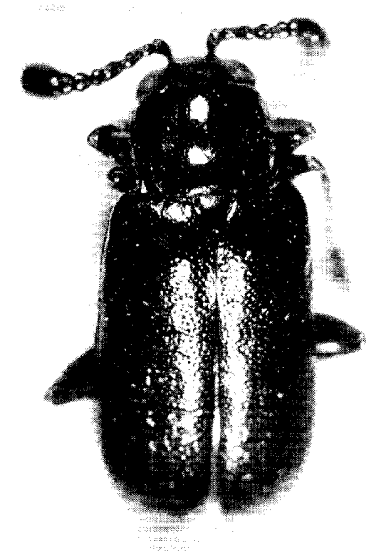

b

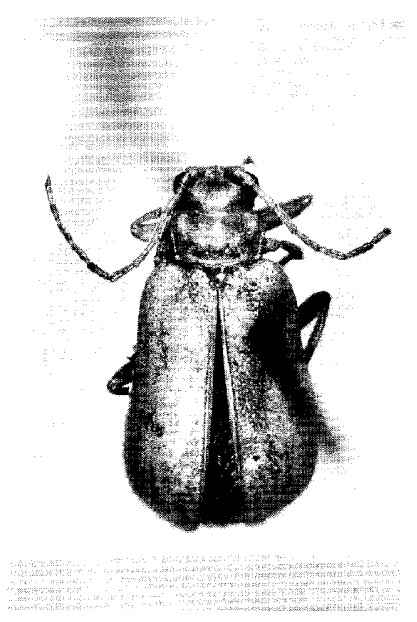

c

Fig. 20. a, Taumacera laosensis n. sp.;b, T. coomani (Laboissière); c, T. doisuthepica n.sp.

This new species somewhat resembles Taumacera pulchella (Laboissière), but differs in having elytron entirely yellowish brown, and antenna and the legs entirely yellowish brown.

Taumacera laosensis n. sp. Fig. 20a

Generally violaceous to greenish blue, antenna pitchy black with eleventh segment dark brown, legs generally pitchy black with bluish luster.

Head with vertex smooth, shining, sparsely impessed by fine punctures, interocular space distinctly wider than transverse diameter of single oculus, and interocular transverse impression feeble, frontal tubercle subtriangular, contiguous, slightly raised, surface smooth, shining, not distinctly delimited posteriorly. Antenna slender, nearly $3 / 4$ as long as body length ; in male third to eleventh segments thickly coverved by long hairs, first segment long, robust, second shortest, subspherical, nearly $1 / 4$ as long as first, third six times as long as second, and deeply sulcated apically, fourth short, nearly $1 / 3$ as long as third and sulcated as a whole, fifth elongate, nearly twice as long as fourth, sixth subequal to fifth in length and shape, seventh $21 / 4$ times as long as sixth, eighth subequal to seventh in length and shape, ninth $11 / 5$ times as long as eighth, tenth subequal to ninth in length and shape, eleventh $11 / 4$ times as long as tenth and its apex pointed. Pronotum transverse, $11 / 4$ times as broad as long, anterior margin distinctly rounded anteriorly, lateral margin slightly rounded, widest slightly before middle and slightly narrowed anteriorly and posteriorly, basal margin feebly rounded posteriorly, dorsal surface convex, smooth, shining, sparsely impressed by minute punctures and with a pair of deep depressions laterally. Scutellum subtriangular, smooth, shining, sparsely impressed by minute punctures. Elytron with lateral margin subparallel-sided, apex rounded, surface stronlgy and closely punctate, and interstices of punctures somewhat rugosed and clearly narrower than average diameter of punctures.

Male : Antenna with third to fifth segment deformed. Female : Unknown.

Length : 5.3-5.7 mm.

Holotype (BISHOP) : LAOS : Vientiane Prov., Vientiane, 31. v.-3. vi. 1960, S. Quate \& L. Quate. Paratypes: 2 exs., same data as the holotype (BISHOP, KIMOTO). 
This new species resembles Taumacera coomani (Laboissière), but differs in having pronotum more quadrate and with the surface smooth, shining, and in the male the third to the fifth antennal segments deformed.

\section{Taumacera metallica (Laboissière), new combination}

Cerophysa metallica Lab., 1930, Ann. Soc. Ent. France, 99 : 350 (Tonkin; HAMBURG).

Distribution : Vietnam.

No additional material was examined beside the type series.

\section{Taumacera pulchella (Laboissière), new combination Fig. 19c}

Cerophysa pulchella Lab., 1930, Ann. Soc. Ent. France, 99 : 348 (Tonkin; HAMBURG).

Taumacera (Cerophysa)pulchella: Gressitt \& Kimoto, 1963, Pac. Ins. Mon., 1B: 525 (China).

Distribution : Vietnam, China.

Material examined. "Indo-China", 1 ex. (BASEL).

\section{Taumacera siamensis (Jacoby), new combination}

Cerophysa siamensis Jac., 1905, Fascic. Malay. Zool., App., 2 : 5 (Siam : Biserat, Jalor; BM).

Distribution : Thailand.

No additional material was examined beside the type series. The description of this species was made by the female specimens only and the male has not been known.

\section{Genus Cneoranidea Chen}

Cneoranidea Chen, 1942, Notes d'Ent. Chinoise, 9 : 31 (type : Cneoranidea signatipes Chen; China). -Gressitt \& Kimoto, 1963, Pac. Ins. Mon., 1B: 531.

Cneoranidea maculata n. sp. Fig. 21a

Suboval; head, prothorax and scutellum reddish brown, elytron black with subbasal and postmedian markings yellowish brown; antenna black with one or two basal segments reddish brown, meso- and metathorax black, abdomen dark reddish brown, legs entirely black.

Head with vertex smooth, shining, sparsely impressed by minute punctures, interocular space distinctly wider than transverse diameter of single oculus, and interocular transverse impression distinct, deep, frontal tubercle oblique, subquadrate, contiguous, distinctly raised, surface smooth, shining. Antenna slender, nearly $2 / 3$ as long as body length ; first segment robust, club-shaped, second shortest, nearly $1 / 3$ as long as body length, third nearly six times as long as second, fourth nearly $3 / 4$ as long as third, fourth to ninth subequal to each other in length and shape, tenth slightly shorter than ninth, eleventh $11 / 3$ times as long as tenth and its apex pointed. Pronotum transverse, $21 / 5$ times as broad as long, anterior margin distinctly rounded posteriorly, lateral margin distinctly rounded, widest almost at middle, slightly narrowed anteriorly and posteriorly, basal margin strongly rounded posteriorly, anterior corner thickened, posterior corner pointed, dorsal surface convex ' side to side, nearly impunctate. Scutellum subtriangular, smooth, shining, impunctate. Elytron with lateral margin rounded, widened posteriorly, surface distinctly and closely punctate, and interstices of punctures smooth, shining. 
Length : 5.3-6.8 mm.

Holotype (BISHOP) : LAOS : Vientiane Prov., Ban Van Eue, 13. iv. 1965, J. L. Gressitt. Paratopotypes: 3 exs., same data as the holotype (BISHOP, KIMOTO). Paratypes : THAILAND: Chiengmai Prov., Doi Suthep, 1 ex., 28-31. iii. 1958, T. C. Maa (BISHOP). LAOS : Vientiane Prov., Ban Van Eue, 1 ex., 1-15. J. A. Rondon, 2 exs., 15-31. iv. 1965, 1 ex., 1-15. v. 1965, Native collr (BISHOP).

This new species resembles Cneoranidea signatipes Chen, but differs in having elytron blackish with the subbasal and the postmedian markings yellowish.

\section{Genus Liroetis Weise}

Liroetis Weise, 1889, Horae Soc. Ent. Ross., $23: 607$ (type : Liroetis aeneipennis Weise ; NW China).-Maulik, 1936, Fauna India, Galeruc. : 311.-Ogloblin, 1936, Fauna USSR, 26, $1: 207$, 372.-Gressitt \& Kimoto, 1963, Pac. Ins. Mon., 1B: 532.

\section{KEY TO SPECIES OF Liroetis}

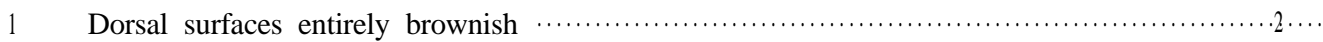

Elytron bluish green ; generally yellowish brown ; antenna with apical three or four

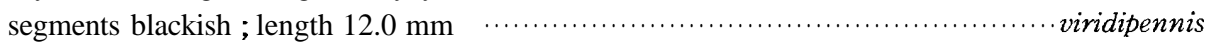

2(1) Entirely yellowish to reddish brown ; in male eleventh antenna1 segment with a small

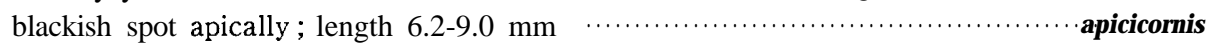
Generally yellowish to reddish brown ; antenna, tibiae and tarsi pitchy brown to black ; length $14.0 \mathrm{~mm}$ clermon ti

\section{Liroetis apicicornis (Jacoby)}

Liroetes apicicomis Jac., 1896, Ann. Soc. Ent. Belg., 40 : 282 (S. Bombay : Kanara ; BM).

Liroetis apicicomis : Maulik, 1936, Fauna India, Galeruc. : 313, fig. (India).

Distribution : India, Thailand, Laos, Vietnam.

Material examined. THAILAND : Chiengmai, 1,100 ft., 4 exs., 19. vi. 1936 ; Phrae, 3 exs., 2. v. 1938, 2 exs., 18. vi. 1938, 1 ex., 5. ix. 1938 (BANGKHEN). LAOS : Vientiane Prov., Ban Van Eue, 1 ex., 15-31. iv. 1965, 3 exs., 15-31. v. 1965, Native collr; Wapikhamthong Prov., Khong Sedone, 1 ex., 17. ix. 1965, Native collr (BISHOP) ; Umgeb. Vientiane, 1 ex., iii-vi. 1963 (MUNCHEN). VIETNAM : "Annam", 1 ex., 1927 (BASEL).

\section{Liroetis clermonti(Laboissière), new combination}

Pseudoliroetis clermonti Lab., 1929, Ann. Soc. Ent. France, 98 : 282 (Tonkin: Chapa ; HAMBURG).

Distribution : Vietnam.

This species closely resembles Liroetis nepalensis Chajô, from Nepal, but differs in being the body length longer $(14.0 \mathrm{~mm})$. In nepalensis, the body length is $8.0-10.0 \mathrm{~mm}$.

No additional material was examined beside the type series. 


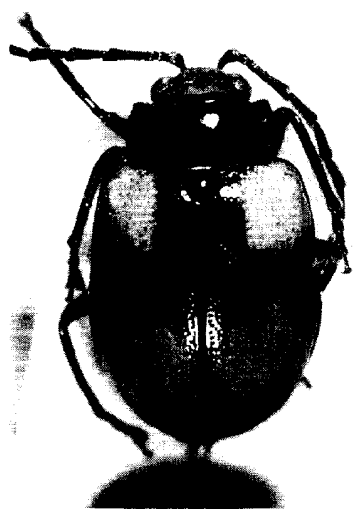

a

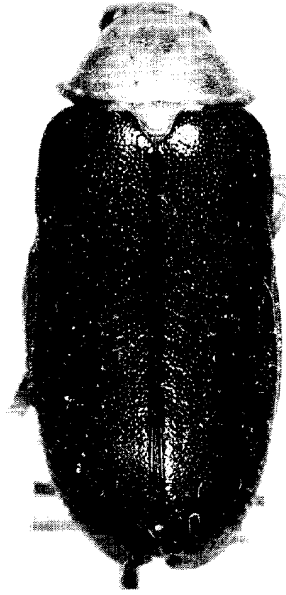

b

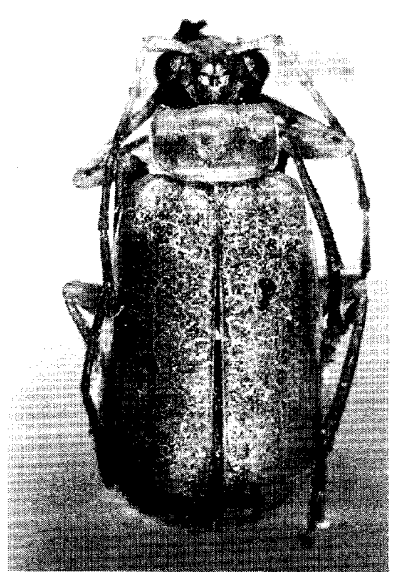

$\mathrm{C}$

Fig. 21. a, Cneorunidea maculatan. sp. ; b, Liroetis viridipennis n. sp.; c, Mimastra badia n. sp.

\section{Liroetis viridipennis n. sp. Fig. $21 \mathrm{~b}$}

Generally yellowish brown, antenna with three or four apical segments pitchy black.

Head with vertex closely impressed by large punctures and their interstices finely granulate, interocular space distinctly wider than transverse diameter of single oculus, and interocular transverse impression distinct only on middle, frontal tubercle subtriangular, contiguous, distinctly raised, surface impressed by fine punctures. Antenna slender, nearly $4 / 5$ as long as body length ; first segment robust, club-shaped, second shortest, nearly $1 / 3$ as long as first, third $22 / 3$ times,as long as second, fourth $12 / 3$ times as long as third, fifth to seventh subequal to each other in length and shape, eighth slightly shorter than seventh, ninth slightly shorter than eighth, tenth slightly shorter than ninth, eleventh subequal to ninth in length but its apex pointed. Pronotum transverse, $12 / 3$ times as broad as long, anterior margin slightly rounded posteriorly, lateral margin distinctly rounded, widest almost at middle, strongly narrowed anteriorly and less strongly so posteriorly, basal margin distinctly rounded posteriorly, and slightly concaved before scutellum, dorsal surface convex, sparsely impressed by large punctures and their interstices impressed by minute punctures, and with a pair of feeble depressions laterally. Scutellum subtriangular, impressed by distinct punctures and their interstices finely granulate. Elytron with lateral margin subparallel-sided, and apex rounded, surface distinctly and closely punctate, and interstices of punctures finely granulate.

Length : $12.0 \mathrm{~mm}$.

Holotype (BISHOP) : LAOS : Vientiane Prov., Ban Van Eue, 1 ex., 16. iii. 1966, Native collr. Paratype : LAOS : Umgeb. Vanky, 1 ex., 1964 (MUNCHEN).

This new species resembles Liroetis clermonti (Laboissière), but differs in having elytron entirely bluish green and the legs entirely yellowish brown.

\section{Genus Mimastra Baly}

Mimastra Baly, 1865, Ann. Mag. Nat. Hist., ser. 3, 16: 253 (type :Mimastra arcuata Baly ; Andaman Is.).-Chapuis, 1875, Gen. Col., 11: 178, 179.-Maulik, 1936, Fauna India, Galeruc. : 
524.-Ogloblin, 1936, Fauna USSR, 26, $1: 176$ - - Gressitt \& Kimoto, 1963, Pac. Ins. Mon., IB : 535.

Anthraxantha Fairmaire, 1878, Ann. Soc. Ent. France, ser. 5, 8: 137 (type : Anthraxanthadavidis Fairmaire ; China).

Brachita Allard, 1889, C. R. Soc. Ent. Belg., 33 : 103 (type : Brachitaterminata Allard).

\section{Key to species of Mimastra}

Ventral surfaces entirely pale

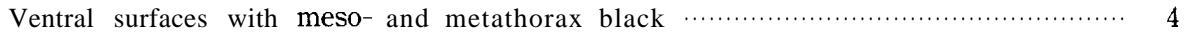

Legs not largely black

Legs almost entirely black; generally yellowish to reddish brown, antenna pitchy black ; length $6.8-8.0 \mathrm{~mm}$.............................................................. scutellata Elytron without costa latero-apically, elytral punctures stronger ; generally yellowish to reddish brown, with antenna, tibiae and tarsi blackish, in some specimen legs

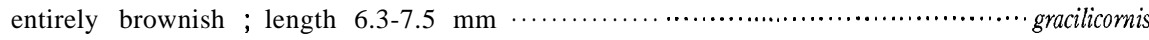
Elytron with a costa latero-apically, elytral punctures finer ; generally yellowish to reddish brown, with antenna, tibiae and tarsi blackish ; length $6.5-7.5 \mathrm{~mm} \cdots \cdots \cdots \cdots$ apicalis Abdomen black

Abdomen brownish ; generally yellowish brown, with metathorax, tibiae and tarsi blackish; in most dark colored specimen apex of femora and apical margin of elytron blackish ; length 6.8-7.5 $\mathrm{mm}$

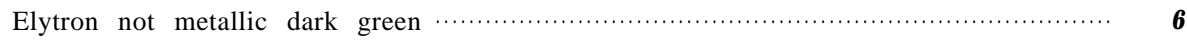
Elytron entirely metallic dark green; head, antenna and pronotum reddish brown, ventral surfaces of meso- and metathorax and abdomen blackish ; legs black with femora reddish brown ; length $8.0 \mathrm{~mm}$

annandalei Dorsal surfaces with blackish marking Dorsal surfaces entirely brownish, except pronotum with ill-defined dark markings

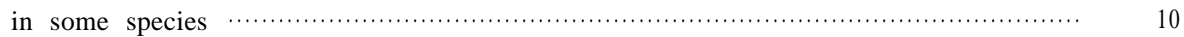
Elytron yellowish brown with blackish marking on basal $1 / 2 \ldots \ldots \ldots \ldots \ldots \ldots . . \ldots \ldots \ldots . . \ldots \ldots . .8$ Elytron yellowish brown with apical $1 / 3$ greenish or bluish, and some time a fine line extending forward; generally yellowish brown with metathorax and abdomen bluish black, legs yellowish brown, dorsal surfaces of femora and tibiae and entire tarsi blackish

Elytron yellowish brown, with a longitudinal wide stripe pitchy black ; generally yellowish brown, antenna, metathorax and abdomen pitchy black; legs with ventral surfaces of femora, and tibiae and tarsi blackish ; length 6.3-7.5 mm longicornis Elytron yellowish brown with large anterior and posterior markings blackish; generally yellowish brown with metathorax and abdomen largely blackish ; length $6.5 \mathrm{~mm}$ pygidia $l i s$ In male, first tarsal segment of anterior leg elongate with interior margin deeply emarginate ; length 7.5-9.0 $\mathrm{mm}$ In male, first tarsal segment of anterior leg enlarged, rounded ; length 9.0-10.0 mm (Hope, 1831 ; Kashmir, India, Nepal, S. China) Third antennal segment nearly $11 / 2$ times as long as second, and fourth more than twice as long as third; pronotum with lateral margin rounded, and with shallow a pair of lateral, and an anterior and a posterior median depressions 
Third antennal segment more than twice as long as second, and fourth $11 / 2$ times as long as third ; pronotum subquadrate, and with a deep transverse furrow which is interrupted at middle; generally yellowish brown, metathorax and abdomen pitchy black, antenna somewhat infuscate ; length $6.0-7.5 \mathrm{~mm}$ persimilis

11(10) Pronotum $13 / 4$ times as wide as long, smaller than $6.0 \mathrm{~mm}$; first segment of anterior tarsus normal in male; generally yellowish brown, with metathorax and abdomen pitchy black, antenna, tibiae and tarsi infuscate ; length 5.3-6.8 mm badia

Pronotum $11 / 2$ times as wide as long, larger than $7.0 \mathrm{~mm}$; in male first segment of anterior tarsus enlarged, rounded; generally yellowish brown, with metathorax and abdomen blackish, antenna pitchy brown with basal segments brownish, legs yellowish brown with most of tibiae and tarsi blackish ; length 7.8-8.4 mm

soreli

\section{Mimastra annandalei Jacoby}

Mimastra annandalei Jac., 1905, Fascic. Malay. Zool. Appl., 2 : 7 (Siamese Malaya : Bukit Besar, Nawngchick and Bansai Kau ; BM).

Distribution : Thailand.

No additional material was examined beside the type series.

\section{Mimastra apicalis n. sp. Fig. 24a}

Generally yellowish to reddish brown, antenna pitchy black with four or five basal segments brownish, legs with middle and posterior tibiae and tarsi and anterior tarsi pitchy brown to black.

Head with vertex smooth, shining, nearly impunctate, interocular space much wider than transverse diameter of single oculus, and interocular transverse impression distinct, frontal tubercle
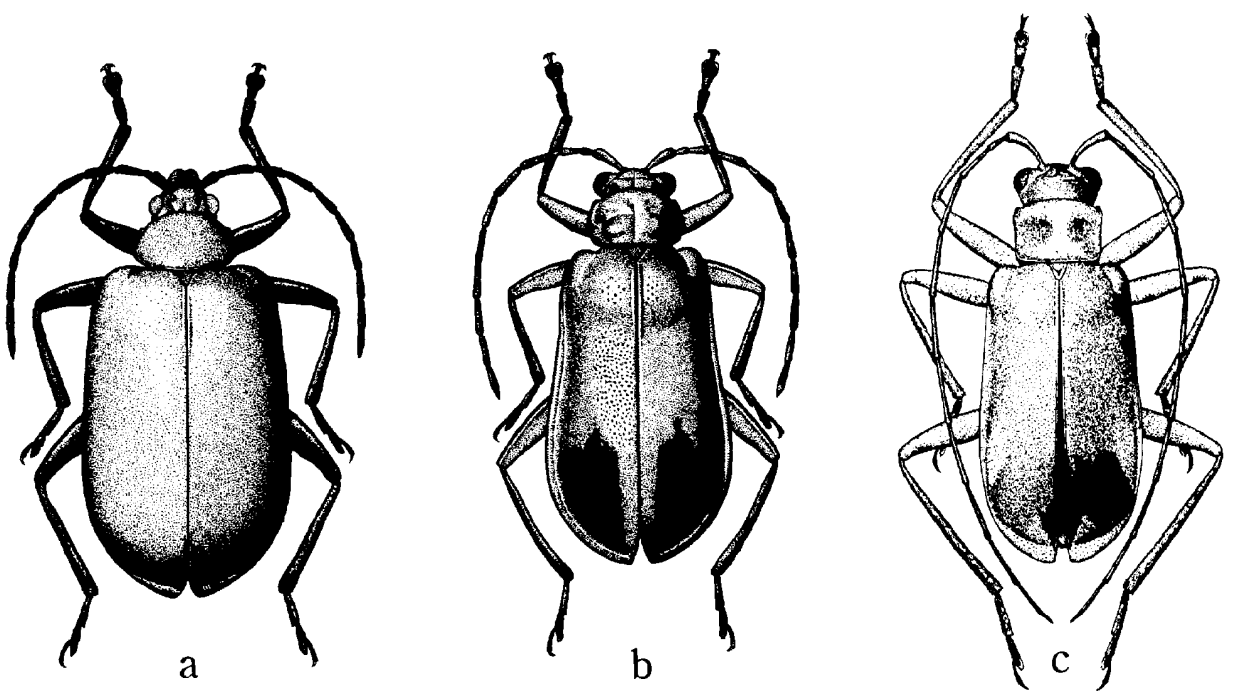

Fig. 22. a, Siemssenius fulvipennis (Jacoby) ; b, M imastra unicitarsis Laboissière ; c, M.longicornis Jacoby. 
strongly raised, subtriangular, contiguous, surface smooth, shining, impunctate. Antenna slender, nearly as long as body length ; first segment long, robust, second shortest, nearly $2 / 5$ as long as first, third $22 / 3$ times as long as second, fourth $11 / 4$ times as long as third, fifth slightly longer than fourth, fifth to ninth subequal to each other in length and shape, tenth slightly shorter than ninth, eleventh subequal to tenth in length and its apex pointed. Pronotum transverse, $12 / 5$ times as broad as long, anterior margin nearly straight, lateral margin distinctly rounded, widest almost at $1 / 3$ from anterior margin, slightly narrowed anteriorly and more strongly so posteriorly, basal margin slightly rounded posteriorly and slightly concaved before scutellum, dorsal surface smooth, shining, sparsely impressed by minute punctures, and with a distinct transverse depression. Scutellum subtriangular, smooth, shining, impunctate. Elytron with lateral margin subparallel-sided, apex rounded, and with a distinct costa latero-apically, running parallel to lateral margin, surface strongly and rather closely punctate and their interstices smooth, shining.

Length : 6.5-7.5 mm.

Holotype (BISHOP) : VIETNAM : $25 \mathrm{~km} \mathrm{SW}$ of Pleiku, $400 \mathrm{~m}, 12$. v. 1960, L. W. Quate. Paratopotypes: 3 exs., same data as the holotype (BISHOP, KIMOTO). Paratypes: LAOS: Sedone Prov., Pakse, 31. v. 1965, Native collr (BISHOP). VIETNAM : Pleiku, 700 m, 1 ex., 8-14. v. 1960, L. W. Quate (BISHOP).

This new species resembles Mimastra gracilicomis Jacoby, but differs in having elytron with a distinct costa latero-apically and elytral punctures much finer.

\section{Mimastra badia n. sp. Fig. 21c}

Generally yellowish brown, antenna pitchy black with four or five basal segments yellowish brown, ventral surfaces with metathorax and abdomen pitchy black, legs with tibiae and tarsi generally pitchy black.

Head with vertex somewhat wrinkled, sparsely impressed by minute punctures, interocular space much wider than transverse diameter of single oculus, and interocular transverse impression distinct, frontal tubercle subtriangular, contiguous, distinctly raised, surface smooth, impunctate. Antenna slender, nearly $3 / 4$ as long as body length ; first segment long, robust, second shortest, nearly $1 / 3$ as long as first, third $11 / 4$ times as long as second, fourth $24 / 5$ times as long as third, fifth slightly shorter than fourth, and fifth to eighth subequal to each other in length and shape, ninth slightly shorter than eighth, tenth slightly shorter than ninth, eleventh subequal to eighth in length and its apex pointed. Pronotum transverse, $13 / 4$ times as broad as long, anterior margin distinctly rounded posteriorly, lateral margin slightly rounded, widest almost at middle, slightly narrowed anteriorly and posteriorly, basal margin distinctly rounded posteriorly and slightly concaved before scutellum, dorsal surface smooth, shining, sparsely impressed by minute punctures, and with a distinct transverse depression. Scutellum subtriangular, finely granulate, impunctate. Elytron with lateral margin subparallel-sided, apex rounded, surface strongly and rather closely punctate and their interstices somewhat wrinkled.

Length : 5.3-6.8 mm.

Holotype (BISHOP) : LAOS : Vientiane Prov., Ban Van Eue, 31. vii. 1965, Native collr. Paratopotypes : 23 exs., same data as the holotype (BISHOP, KIMOTO). Paratypes : THAILAND : Chiengmai Prov., Doi Suthep, 1 ex., 1-8. iv. 1958, T. C. Maa; Khao Yai, 7 exs., 10. iv. 1963, Native collr (BISHOP). LAOS : Vientiane Prov., Ban Van Eue, 1 ex., 15. ii. 1967, 95 exs., 30. viii. 1967, Native collr ; Vientiane Prov., Gi Sion Vill., de Tha Ngone, 21 exs., 2. i. 1966, Native collr ; Borikhane Prov., Pakkading, 1 ex., 13. vii. 1966, Native collr ; Sedone Prov., Paksong, 1 ex., 16. v. 1965, Native collr ; Sayaboury Prov., Sayaboury, 1 ex., 25. viii. 1966, Native collr (BISHOP). VIETNAM : Dilinh 
(Djiring), 920-1,200 m, 2 exs., 22-28. iv. 1960, S. Quate \& L. Quate (BISHOP).

This new species closely resembles Mimastra soreli Baly, but differs in having pronotum much wider and in the male the first segment of the anterior tarsus not enlarged.

\section{M imastra gracilicornis Jacoby Fig. 23b}

Mimastra gracilicornis Jac., 1889, Ann. Mus. Civ. Genova, $27: 210$ (Burma ;GENOVA).-Maulik, 1936, Fauna India, Galeruc. : 536 (Burma).-Kimoto, 1972, Tijdschr. Ent., 93(4): 145 (Vietnam : Dalat).

Distribution : Burma, Thailand, Laos, Vietnam.

Material examined. THAILAND : Chieng Mai Prov., Doi Suthep, 1,000 m, 1 ex., 8. vi. 1965, S. Asahina, 1 ex., 12. vi. 1965, K. Morimoto ; Khao Yai Nat. Park, 1 ex., 5. vi. 1965, S. Asahina (KU) ; Chieng Mai Prov., Chieng Mai (Argoreturn), 300 m, 2 exs., 12. vi. 1965, P. D. Ashlock (BISHOP). LAOS : Khammouane Prov., Phon Tiou, 1 ex., 11. vi. 1965, N. Wilson, 1 ex., 11-12. vi. 1965, J. A. Rondon, 2 exs., 30. iv. 1965, 1 ex., 17. v. 1965, Native collr ; Savanakhet Prov., Savanakhet, 2 exs., 20. vii. 1965, Native collr ; Vientiane Prov., Ban Van Eue, 1 ex., 15-31. v. 1965, Native collr ; Pon-horn, N. of Pakkading, 1 ex., 20. iv. 1965, J. A. Samuelson (BISHOP) ; Umgeb. Vientiane, 1 ex., 1963, Umgeb. Paklay, 3 exs., 1963 (MUNCHEN). VIETNAM : Dalat, 1,500 m, 4 exs., 29. iv.-5. v. 1960, S. Quate \& L. W. Quate, 1 ex., 26-27. ix. 1960, J. L. Gressitt ; Dalat, 6 km S., 1,400-1,500 m, 9 exs., 9. vi.7. vii. 1961, N. R. Spencer ; Dilinh (Djiring), 1,200 m, 5 exs., 22-28. iv. 1960, L. W. Quate ; 7 km SE of Dilinh (Djiring), 990 m, 2 exs., 2. v. 1960, R. E. Leech ; 30 km N. of Dilinh, 1 ex., 27. iv. 1960, S. Quate (BIHSOP).

\section{M imastra longicornis Jacoby Fig. 22c}

Mimastra longicornis Jac., 1892, Ann. Mus. Civ. Genova, 32 : 944 (Burma ;GENOVA).-Maulik, 1936, Fauna India, Galeruc. : 535 (Burma).

Distribution : Burma, Thailand.

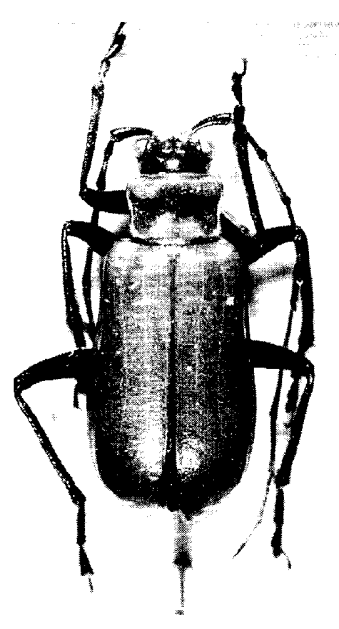

a

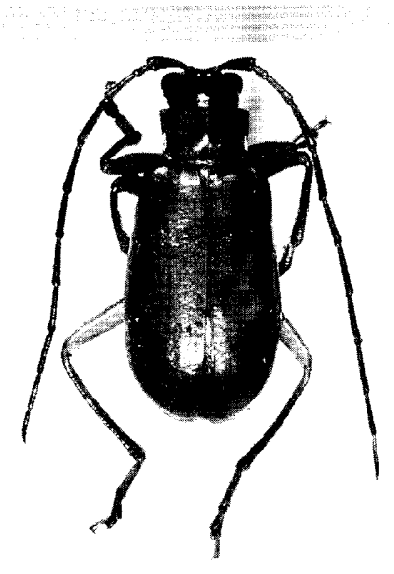

b

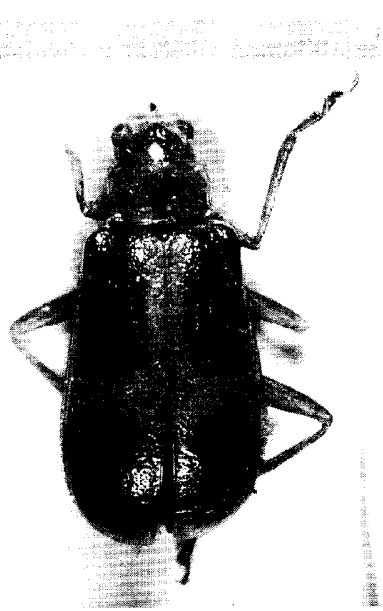

c

Fig. 23. a, Mimastra scutellata Jacoby ; b, M.gracilicornis Jacoby ; c, И. pygidialis Laboissière. 
Material examined. THAILAND : Chieng Mai Prov., Doi Pui, 1,300 m, 7 exs., 17. vi. 1965, K. Morimoto ; Chieng Mai Prov., Doi Suthep, 1,000 m, 1 ex., 18. vi. 1965, K. Morimoto (KU)

Mimastra pectoralis n. sp. Fig. 24c

Generally yellowish brown, ventral surfaces with metathorax black, legs with tibiae and tarsi pitchy black ; in most dark colored specimen apex of femora and apical margin of elytron blackish.

Head with vertex smooth, shining, sparsely impressed by minute punctures, interocular space much wider than transverse diameter of single oculus, and interocular transverse impression distinct, frontal tubercle subtriangular, contiguous, distinctly raised, surface smooth, impunctate. Antenna slender, nearly as long as body length ; first segment long, robust, second shortest, nearly $1 / 3$ as long as first, third $11 / 4$ times as long as second, fourth $31 / 5$ times as long as third, fifth slightly shorter than fourth, fifth to eighth subequal to each other in length and shape, ninth slightly shorter than eighth, tenth subequal to ninth in length and shape, eleventh subequal to tenth in length and its apex pointed. Pronotum transverse, nearly twice as broad as long, anterior margin rounded posteriorly, lateral margin feebly rounded, widest almost at middle, slightly narrowed anteriorly and posteriorly, basal margin distinctly rounded posteriorly, dorsal surface smooth, shining, sparsely impressed by minute punctures, and with a distinct transverse depression. Scutellum subtriangular, finely granulate, impunctate. Elytron with lateral margin subparallel-sided, apex rounded, surface strongly and rather closely punctate and their interstices smooth, shining.

Length : 6.8-7.5 mm.

Holotype (BISHOP) : LAOS : Sedone Prov., Paksong, 1 vi. 1965, Native collr. Paratypes : THAILAND : Nakhon Nayok Prov., Khao Yai Nat. Park, 1 ex., 6. vi. 1965, P. D. Asholock (BISHOP). LAOS : Vientiane Prov., Ban Van Eue, 2 exs., 15. x. 1967, 1 ex., 15. viii. 1968, Native collr ; Vientiane Prov., Gi Sion Vill., de Tha Ngone, 1 ex., 2. i. 1966, Native collr ; Borikhane Prov, Pakkading, 2 exs., 13. vii. 1965, Native collr ; Sayaboury Prov., Sayaboury, 4 exs., 25. viii. 1966, Native collr (BISHOP, KIMOTO). CAMBODIA : Kiri Rom, 700 m, 1 ex., 31. iii.-7. iv. 1961, N. R. Spencer (BISHOP). VIETNAM : Fyan, 900-1,200 m, 3 exs., 11. vii.-9. viii. 1961, N. R. Spencer ;M'Drak, E. of Ban Me Thuot, 4-600 m, 1 ex., 8-19. xii. 1960, C. M. Yoshimoto (BISHOP).

This new species resembles Mimastra soreli Baly, but differs in having pronotum much wider and the abdomen entirely brownish.

\section{Mimastra persimilis n. sp. Fig. 24b}

Generally yellowish brown, antenna pitchy brown with basal segments somewhat paler, ventral surfaces with metathorax and abdomen pitchy black, legs with tibiae and tarsi somewhat infuscate.

Head with vertex somewhat wrinkled, sparsely impressed by minute punctures, interocular space much wider than transverse diameter of single oculus, and interocular transverse impression distinct, frontal tubercle subtriangular, contiguous, distinctly raised, surface smooth, shining, impunctate. Antenna slender, nearly as long as body length ; first segment long, robust, second shortest, nearly $1 / 3$ as long as first, third $21 / 2$ times as long as second, fourth $11 / 2$ times as long as third, fifth slightly shorter than fourth, fifth to ninth subequal to each other in length and shape, tenth slightly shorter than ninth, eleventh slightly longer than tenth and its apex pointed. Pronotum transverse, 1 $2 / 3$ times as broad as long, anterior margin nearly straight, lateral margin rounded, widest almost at $1 / 3$ from anterior margin, slightly narrowed anteriorly and more strongly so posteriorly, basal margin distinctly rounded posteriorly and slightly concaved before scutellum, dorsal surface smooth, shining, sparsely impressed by minute punctures, and with a distinct transverse depression. Scutellum 
subtriangular, finely granulate, impunctate. Elytron with lateral margin subparallel-sided, apex rounded, surface strongly and rather closely punctate and their interstices smooth, shining.

Length : 6.0-7.5 mm.

Holotype (BISHOP) : VIETNAM : Dalat, 1,500 m, 29. iv.-4. v. 1960, S. Quate. Paratopotypes : 3 exs., same data as the holotype (BISHOP, KIMOTO). Paratypes : LAOS : Houei Kong, 1 ex., 7. x. 1965, Native collr (BISHOP). VIETNAM : Dalat, 6 km S., 1,400-1,500 m, 5 exs., 9. vi.-7. vii. 1961, N. R. Spencer; Mt. Lang Bian, 1,500-2,000 m, 2 exs., 19. v.-8. vi. 1961, N. R. Spencer (BISHOP).

This new species closely resembles Mimastra soreli Baly, but differs in having the third antennal segment longer and more than twice as long as the second, and the fourth $11 / 2$ times as long as the third.

\section{Mimastra pygidialis Laboissière Fig. 23c}

Mimastra pygidialis Lab., 1929, Ann. Soc. Ent. France, 98 : 285, fig. 14 (Tonkin:Hoa-binh, Chapa ; HAMBURG).-Gressitt \& Kimoto, 1963, Pac. Ins. Mon., 1B: 540 (Vietnam).

Distribution : Vietnam.

No additional material was examined beside the type series.

\section{Mimastra scutellata Jacoby Fig. 23a}

Mimastra scutellata Jac., 1904, Ann. Soc. Ent. Belg., 48 : 395 (India ; BM).-Maulik, 1936, Fauna India, Galeruc. : 537 (India, Burma).-Kimoto, 1982, Ent. Rev. Japan, 37 : 12, fig. (India, Burma). Distribution : India, Burma, Thailand, Laos.

Material examined. THAILAND : Chieng Mai Prov., Maesa, 1 ex., 16. vi. 1965, P. D. Ashlock (BISHOP). LAOS : Xieng Kyouang Prov., Ban Sam Thang, 16 exs., 5. vi. 1965, Native collr ; Sedone Prov., Pakse, 1 ex., 14. v. 1965, P. D. Ashlock (BISHOP).

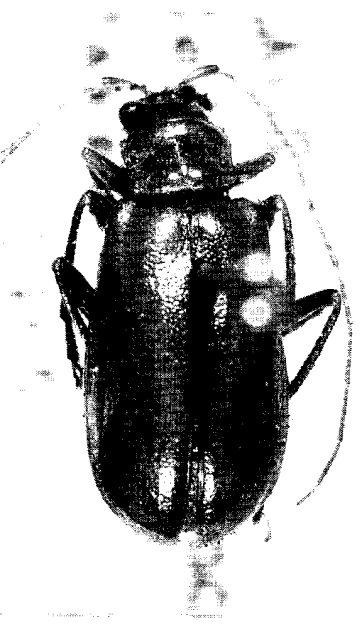

a

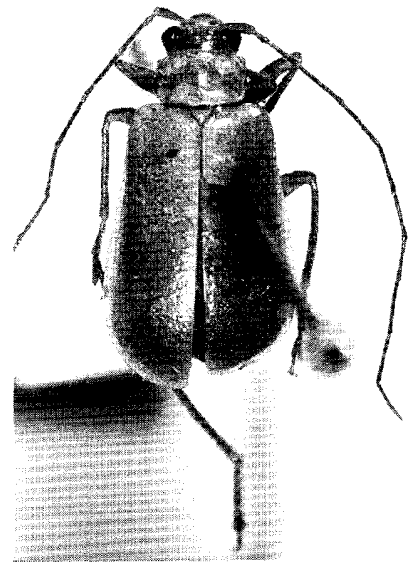

$\mathrm{b}$

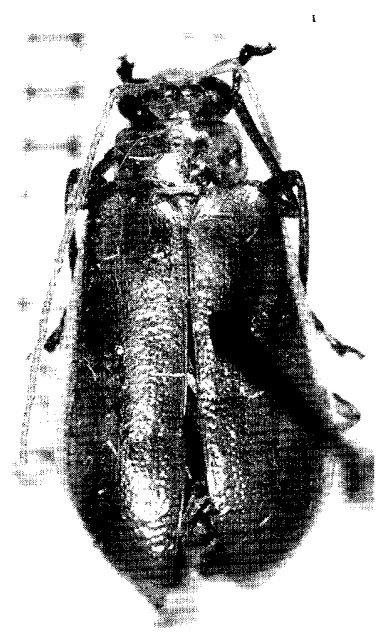

Fig. 24. a, Mimastra apicalis n. sp. ; b, M. persimilis n. sp. ; c, M. pectoralis n. sp. 


\section{Mimastra soreli Baly}

Mimastra soreli Baly, 1878, Ann. Mag. Nat. Hist., ser. 5, 2 : 414 (Upper Yangtze Kiang ; BM). -Ogloblin, 1936, Fauna USSR, 26, 1 : 178, 400 (Chine).-Gressitt \& Kimoto, 1963, Pac. Ins. Mon., 1B: 541 (China, Vietnam).

M imastra latimanus Allard, 1889, Ann. Soc. Ent. France, ser. 6, $9: 308$ (Annam; PARIS). New synonym.

Distribution : Thailand, Laos, Vietnam, S. China, Hainan.

Material examined. THAILAND : Chiengmai Prov., Chiengdao, $450 \mathrm{~m}, 1$ ex., 5-11. iv. 1958, T. C. Maa; Khao Yai, 1 ex., 10. iv. 1963, Native collr (BISHOP) ; Chiengdao, 1 ex., 23. iv. 1973, Y. Yoshiyasu (KU). LAOS : Vientiane Prov., Phou Kou Khouei, 1 ex., 10. iv. 1965, J. A. Rondon, 1 ex., 29. iii. 1966, 1 ex., 31. v. 1966, Native collr ; Vientiane Prov., Ban Van Eue, 1 ex., 29. iv. 1966, 1 ex., 30. viii. 1967, Native collr; Khammouane Prov., Phon Tiou, 1 ex., 10. iv. 1965, 9 exs., 28. iv. 1965, 44 exs., 30. iv. 1965, Native collr; Borikhane Prov., Pakkading, 3 exs., 22. iii. 1965, Native collr; Tonpheng, 3 exs., 30. iv. 1966, 2 exs., 15. v. 1966, Native collr (BISHOP) ; Umgeb. Vientiane, 237 exs., iii-vi. 1963 ; Umgeb. Vanky, 2 exs., 1963 ; Umgeb. Paklay, 1 ex., 1963 (MUNCHEN). VIETNAM : Dalat, 1,500 m, 36 exs., 29. iv.-4. v. 1960, S. Quate \& L. Quate; Dalat, 6 km S., 1,400-1,500 m, 5 exs., 9. vi.-7. vii. 1961, N. R. Spencer ; $18 \mathrm{~km} \mathrm{NW}$ of Dalat, 1,300 m, 1 ex., 4-5. v. 1960, L. W. Quate ; Dilinh (Djiring), 920-1,200 m, 60 exs., 22-28. iv. 1960, S. Quate \& L. Quate ; 17 exs., 9 km S. of Dilinh (Djiring), 1 ex., 24. iv. 1960, R. E. Leech ; 30 km NE of Dilinh, 1 ex., 26. iv. 1960, S. Quate ; Pleiku, 700 m, 1 ex., 8-14. v. 1960, L. W. Quate ; Mt. Lang Bian, 1,500-2,000 m, 2 exs., 19. v.-8. vi. 1961, N. R. Spencer ;Ini, N. Quang Tri, 1 ex., 14. i. 1970, A. R. Gillogly (BISHOP).

\section{Mimastra unicitarsis Laboissière Fig. 22b}

Mimastraunicitarsis Lab., 1940, Bull. Mus. Hist. Nat. Belg., 16(37) : 4, figs. (Burma).-Gressitt \& Kimoto, 1963, Pac. Ins. Mon., 1B: 542, figs. (China).-Kimoto, 1970, Khumbu Himal,3(3): 415 (Nepal).-Kimoto \& Takizawa, 1972, Kontyâ, Tokyo, 40(4): 218 (Nepal).-Kimoto, 1979, Ent. Basiliensia, $4: 472$ (Nepal, U. P.).

Distribaution : India, Nepal, Bhutan, Burma, Thailand, Laos, S. China.

This species closely resembles Mimastra cyanura (Hope), and is clearly separable only on the male specimen. Among the vast number of specimens taken from Thailand and Laos, I could not find any male specimen of cyanura. I could not trace the type location of this species. However, this species is able to clearly identify from Laboissière's illustration.

Material examined. THAILAND : Loe, 4,200 ft., 8 exs., 16. ii. 1941, 1 ex., 25. iv. 1959 ; Bangkok, 2 exs., 17. v. 1952 (BANGKHEN). LAOS : Vientiane Prov., Ban Van Eue, 56 exs., 11-15. iv. 1965, J. L. Gressitt, 1 ex., 1-15. v. 1965, J. A. Rondon, 18 exs., 15-31, iv. 1965, 8 exs., 15-31. v. 1965, 12 exs., 16. iii. 1966, 11 exs., 29. iv. 1966, 8 exs., 15. vi. 1966, Native collr ; Vientiane Prov., Phou Kou Khouei, 32 exs., 10. iv. 1965, 40 exs., 12-13. iv. 1965, J. L. Gressitt ; Xieng Kyouan Prov., Ban Sam Thang, 15 exs., 5. vi. 1965, Native collr ; Sayaboury, 2 exs., 15-16. iv. 1965, J. L. Gressitt ; Uekiang, nr. Pakkading, 100 m, 1 ex., 22. iv. 1965, J. L. Gressitt (BISHOP) ; Umgeb. Vanky, 3 exs., 1963 ; Umgeb. Vientiane, 6 exs., 1963, iii-vi, 1963 ; Umgeb. Pakse, 7 exs., 1963 (MUNCHEN).

\section{Genus Cneorane Baly}

Cneorane Baly, 1865, Ent. Monthly Mag., 2 : 97 (type :Cneorane fulvicollis Baly=Galleruca rubricollis Hope ; India).-Chapuis, 1875, Gen. Col., 11 : 178, 179.-Maulik, 1936, Fauna India, 
Galeruc. :335.-Ogloblin, 1936, Fauna USSR, 16, $1: 212,406 .-$ Gressitt \& Kimoto, 1963, Pac. Ins. Mon., 1B : 546.

\section{Key to SPECIES of C neorane}

1 Abdomen bluish or blackish $\cdot 2$

Abdomen entirely yellowish brown; generally reddish brown with elytron greenish to blackish blue; antenna pitchy black with basal two or three and apical segments brownish ; in male preapical segments of antenna widened ; length 7.3-7.8 $\mathrm{mm}$......

2(1) Rather large in size; larger than $5.5 \mathrm{~mm}$. subcoerulescens

Rather small in size; head and thorax reddish brown; elytron blackish blue; abdomen and legs infuscated, except femora of anterior and middle legs reddish ; length $4.5-5.0 \mathrm{~mm}$

rufocaerulea

3(2) Elytron with distinct transverse furrow subbasally ; interstices of elytral punctures narrower than diameter of each puncture

Elytron without distinct transverse furrow subbasally ; interstices of elytral punctures wider than diameter of each puncture

4(3) Metathorax blackish ; generally bluish to violaceous black; head, prothorax and

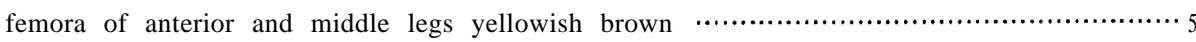
Metathorax and legs entirely yellowish brown ; generally yellowish brown, elytron greenish blue; abdomen pitchy black ; length $8.6 \mathrm{~mm}$

siamensis

5(4) Antenna entirely brownish ; elytron with punctures finer ; length $8.0 \mathrm{~mm}$........... fulvicornis Antenna blackish; elytron with punctures stronger ; length $6.8-8.0 \mathrm{~mm} \cdots \cdots . . . \cdots$ crassicornis

6(3) Head, pro- and mesothorax reddish brown ; elytron blackish or bluish ; legs, metathorax and abdomen bluish or blackish blue, except anterior and middle femora

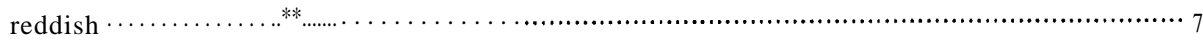
Head and thorax reddish brown with middle of mesothorax bluish black ; abdomen bluish black; legs yellowish brown, tarsi and apical portion of tibiae infuscate ; in male preapical segments widened ; length $8.0-8.6 \mathrm{~mm} \ldots \ldots \ldots \ldots \ldots \ldots \ldots \ldots \ldots \ldots$ rubricollis

7(6) Interstices of elytral punctures without irregular convexities ; length 8.5-9.0

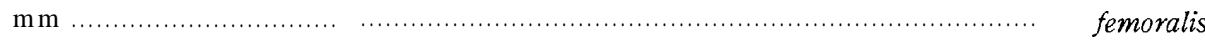
Interstices of elytral punctures convex and irregularly rugose ; length $11.0 \mathrm{~mm}$

cariosipennis

\section{Cneorane cariosipennis Fairmaire Fig. 25a}

Cneorane cariosipennis Fairm., 1888, Ann. Soc. Ent. Belg., 82 : 45 (Yunnan ; PARIS).-Ogloblin, 1936, Fauna USSR, 26, 1: 217 (China).-Gressitt \& Kimoto, 1963, Pac. Ins. Mon., 1B : 549 (China).

Distribution : Thailand, China.

As suggested by Gressitt \& Kimoto (1963, Pac. Ins. Mon., 1B:551), this species closely resembles Cneorane nigripennis Laboissière (1922, Bull. Soc. Ent. France, 1922 : 102 ; Yunnan). According to the study on the type of nigripennis preserved in Humburg Museum, the latter slightly differs in having the body length slightly shorter (about $9.0 \mathrm{~mm}$ ).

Material examined. THAILAND: Chiengmai Prov., Chiengmai, 1,10O-1,500 m, 4 exs., 11. iv. 1966, J. Sedlacek (BISHOP) ; Chiengmai Prov., Doi Pui, 1,300 m, 1 ex., 17. vi. 1965, K. Morimoto (KU). 


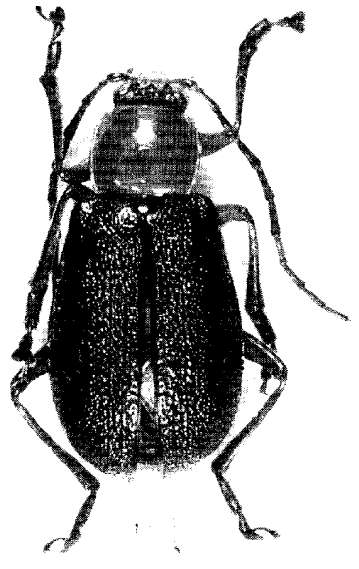

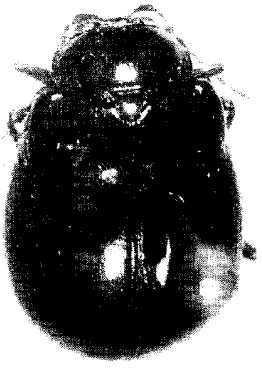

$\mathrm{b}$

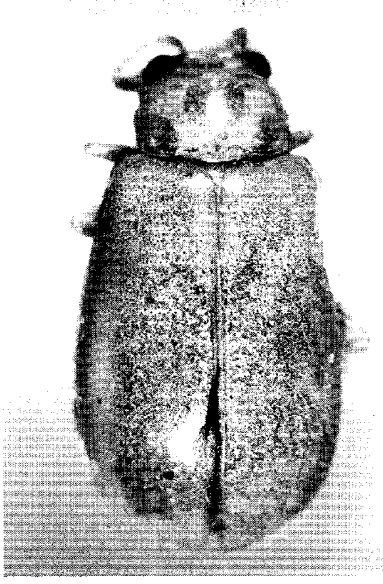

Fig. 25. a, Cneorane cariosipennis Fairmaire ; b, Cneorella laosensis n. sp. ; c, Bangprella fulva n. sp.

\section{Cneorane crassicornis Fairmaire}

Cneorane crassicomis Fairm., 1889, Ann. Soc. Ent. France, ser. 6, $9: 81$ (China : Koui-Tcheou ; PARIS).-Baly, 1890, Ent. Monthly Mag., ser. 2, 1 : 14 (=fulvicollis).-Gressitt \& Kimoto, 1963, Pac. Ins. Mon., 1B : 549 (Yunnan).

Distribution : Thailand, Vietnam, China.

Material examined. THAILAND : Chiengmai Prov., Doi Pui, 1,300 m, 1 ex., 17. vi. 1965, K. Morimoto (KU). VIETNAM : 18 km NW of Dalat, 1,300 m, 1 ex., 4-5. v. 1960, L. W. Quate ; Mt. Lang Bian, 1,500 m-2000 m, 1 ex., 19. v.-8. vi. 1961, N. R. Spencer (BISHOP).

\section{Cneorane femoralis Jacoby Fig. 2c}

Cneorane femoralis Jac., 1888, Proc.Zool.Soc. London, 1888 : 350 (Foochan ; BM).-Gressitt \& Kimoto, 1963, Pac. Ins. Mon., 1B : 549 (China, Hainan).-Kimoto, 1965, Ann. Naturhist. Mus. Wien, $68: 488$ (Taiwan).

Cneorane delatouchei Fairmaire, 1888, Ann. Soc. Ent. France, 32 : 45 (Fokien ; PARIS).-Jacoby, 1890, Entomolog., $23: 193$ (=femoralis).

Cneorane formosana Weise, 1922, Tijdschr. Ent. 65 :72 (Formosa).—Chajô, 1962, Phil. J. Sci., 91(12) : 204 (Formosa).-Kimoto, 1965. Ann. Naturhist. Mus. Wien, $68: 488$ (=femoralis).

Distribution : Laos, China, Hainan, Taiwan.

Material examined. LAOS : Vientiane Prov., Ban Van Eue, 1 ex., 15. xii. 1966, Native collr; Vientiane Prov., Gi Sion Vill., de Tha Ngone, 1 ex., 10-24. x. 1965, Native collr ; Borikhane Prov., Pakkading, 6 exs., 15. ix. 1965, light trap, J. L. Gressitt, 8 exs., 9. ix. 1965, 10 exs., 15. ix. 1965, Native collr ; Wapikhamthong Prov., Khong Sedone, Wapi, 1 ex., 15. vii. 1967, Native collr (BISHOP).

\section{Cneorane fulvicornis Jacoby}

Cneorane fulvicornis Jac., 1889, Ann. Mus. Civ. Genova, 27 : 209 (Burma ;GENOVA).-Maulik, 
1936, Fauna India, Galeruc. : 344 (Burma).

Distribution : Burma, Vietnam.

Material examined. VIETNAM : $17 \mathrm{~km} \mathrm{~S}$. of Dilinh, 1,300 m, 1 ex., 6-12. x. 1960, C. M. Yoshimoto ; Fyan, 900 m, 1 ex., 11. vii.-9. viii. 1961, N. R. Spencer (BISHOP).

\section{Cneorane rubricollis (Hope)}

Gallenca rubricollis Hope, 1831, in Gray, Zool.Miscell. : 29 (Nepal ; BM).

Cneorane fulvicollis Baly, 1865, Ent. Monthly Mag., 2 : 97 (India ; BM).- Jacoby, 1888, Proc.Zool.

Soc. London, 1888 : 350 (notes).

Cneorane rubicollis: Maulik, 1936, Fauna India, Galeruc. : 338 (India, Nepal).

Distribution : India, Nepal, Thailand, Laos.

Material examined. THAILAND: Chiengmai Prov., Doi Pui, 1,300 m, 1 ex., 17. vi. 1965, K. Morimoto (KU). LAOS : Attopeu Prov., Houei Kong, 1 ex., 26. vi. 1965, Native collr (BISHOP).

\section{Cneorane rufocoerulea Fairmaire}

Cneorane rufocoerulea Fairm., 1888, Ann. Soc. Ent. France, 57 : 374 (Tonkin).-Jacoby, 1890, Entomolog., 23 : 167 (mentioned).-Gressitt \& Kimoto, 1963, Pac. Ins. Mon., 1B: 551 (Vietnam). Distribution : Vietnam.

I could not trace the type location of this species. No material was examined.

\section{Cneorane siamensis Laboissière}

Cneorane siamensis Lab., 1929, Rev. Chil. Hist. Nat., 33 : 293 (Siam ; BM).

Distribution : Thailand.

Material examined. THAILAND : Chiengmai Prov., Doi Suthep, 1,000 m, 1 ex., 18. vi. 1965, K. Morimoto (KU).

\section{Cneorane subcoerulescens Fairmaire}

Cneorane subcoerulescens Fairm., 1888, Ann. Soc. Ent. Belg., 32 : 44 (Yunnan).-Gressitt \& Kimoto, 1963, Pac. Ins. Mon., 1B: 552 (China).

Distribution : Laos, Vietnam, China.

I could not trace the type location of this species.

Material examined. LAOS : Sedone Prov., Pakse, 1 ex., 31. v. 1965, Native collr ; Sedone Prov., Paksong, 1 ex., 17. v. 1965, P. D. Ashlock (BISHOP) ; Umgeb. Vientiane, 4 exs., iii-vi. 1963 (MUNCHEN). VIETNAM : Mt. Lang Bian, 1,500-2,000 m, 1 ex., 19. v.-8. vi. 1961, N. R. Spencer (BISHOP).

\section{Genus Cneorella M edvedev \& Dang Dap}

Cneorella Medvedev \& Dang Dap, 1981, Rev. d'Ent.l'URSS,60(3) : 629 (type : Cneorella chapaensis Medvedev \& Dang Dap ; Vietnam).

\section{KeY TO SPECIES OF Cneorella}




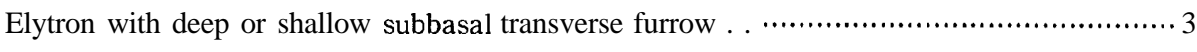

2(1) Interstices of elytral punctures shagreened ; generally greenish blue, abdomen yellowish brown ; length 4.8-5.2 $\mathrm{mm}$ suisapana Interstices of elytral punctures smooth ; generally blue to purplish, abdomen yellowish brown ; length 4.3-5.4 mm (Gressitt \& Kimoto, 1963 ; China, Taiwan ;Calomicrus ; new combination)

3(I) Elytral punctures stronger

Elytral punctures finer, subbasal transverse furrow very deep ; generally bluish black,

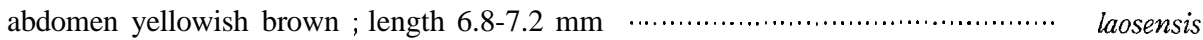

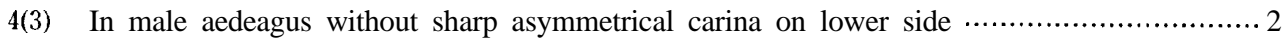
In male aedeagus with sharp asymmetrical carina on lower side ; generally blue, ventral surfaces of thorax and legs blackish, abdomen yellowish ; length $7.2 \mathrm{~mm}$.... vietnamica

4(3) Elytron dark green with violaceous blue basally and laterally ; generally violaceous blue, ventral surfaces and legs nearly black; elytron with apical angle square or slightly pointed ; in male median lobe of fifth abdominal segment with large, deep depression ; length 6.2-7.0 mm chapaensis Dorsal surfaces blue ; ventral surfaces, antenna and legs black ; elytron with apical angle broadly rounded ; in male median lobe of fifth abdominal segment with shallow, ill-defined depression ; length $5.4-7.0 \mathrm{~mm}$

cyanea

\section{Cneorella chapaensis Medvedev \& Dang Dap}

Cneorella chupaensis Medvedev \& Dang Dap, 1981, Rev. d'Ent.l'URSS, 60(3) : 630 (Vietnam), Distribution : Vietnam.

Material examined. VIETNAM : Fyan, 900-1,200 m, 7 exs., 11. vii.-9. viii. 1961, N. R. Spencer ; Dalat, 1,500 m, 1 ex., 29. iv.-4. v. 1960, S. Quate, 1 ex., 26-27. ix. 1960, C. M. Yoshimoto ; Dalat, 6 km S., 1,400-1,500 m, 1 ex., 9. vi.-7. vii. 1961, N. R. Spencer; Blao (Balao), 1 ex., 14-21. x. 1960, C. M. Yoshimoto (BISHOP).

\section{Cneorella cyanea Medvedev \& Dang Dap}

Cneorella cyanea Medvedev \& Dang Dap, 1981, Rev. d'Ent.l'URSS, 60(3) : 631 (Vietnam).

Distribution : Laos, Vietnam.

Material examined. LAOS : Vientiane Prov., Ban Van Eue, 6 exs., 13-14. iv. 1965, J. L. Gressitt, 2 exs., 15-31. v. 1965, 1 ex., 31. vii. 1965, 2 exs., 30. xi. 1965, 1 ex., 31. xii. 1968, Native collr ; Vientiane Prov., Gi Sion Vill., de Tha Ngone, 3 exs., 10-24. x. 1965, Native collr ; Sayaboury Prov., Sayaboury, 1 ex., 16. iv. 1965, J. L. Gressitt, 1 ex., 29. iii. 1966, Native collr (BISHOP) ; Umgeb. Vientiane, 1 ex., iii-vi. 1963 (MUNCHEN).

\section{Cneorella laosensis n. sp. Fig. 25b}

Generally bluish black, antenna with four of five apical segments reddish brown, abdomen entirely yellowish brown.

Head with vertex smooth, shining, sparsely impressed by fine punctures, interocular space distinctly wider than transverse diameter of single oculus, and interocular transverse impression distinct, frontal tubercle subtriangular, contiguous, distinctly raised, surface smooth, shining. Antenna slender, nearly $3 / 4$ as long as body length; first segment robust, club-shaped, second 
shortest, nearly half as long as first, third $12 / 3$ times as long as second, fourth to ninth subequal to each other in length and shape, tenth slightly shorter than ninth, eleventh $11 / 3$ times as long as tenth and its apex pointed. Pronotum transverse, nearly twice as broad as long, anterior margin distinctly rounded posteriorly, lateral margin distinctly rounded, widest almost at middle, slightly narrowed anteriorly and posteriorly, basal margin distinctly rounded posteriorly, dorsal surface convex side to side, sparsely impressed by fine punctures, and interstices of punctures smooth, shining. Scutellum subtriangular, smooth, shining, impunctate. Elytron convex, with lateral margin rounded, surface closely impressed by fine punctures, interstices of punctures smooth, shining, and with a subbasal transverse furrow very deep.

Length : 6.8-7.2 $\mathrm{mm}$.

Holotype (BISHOP) : LAOS : Ban Van Eue, 20 km E. of Phou-kow-kuei, 15-31. iv. 1965, Native collr. Paratopotypes: 14 exs., same data as the holotype (BISHOP, KIMOTO).

This new species resembles Cneorella chapaensis (Gressitt \& Kimoto), but differs in having elytron with a subbasal transverse furrow deeper and elytron with the punctures much strongly and closely impressed.

\section{Cneorella suisapana (Gressitt \& Kimoto), new combination Fig. 4b}

Calomicrus suisapanus Gressitt \& Kimoto, 1963, Pac. Ins. Mon., 1B: 577 (W. China : W. Hupeh, CAS).

Distribution : Thailand, China.

Material examined. THAIALAND : Chiengmai Prov., Doi Suthep, 1,000 m, 1 ex., 18. vi. 1965, Y. Miyatake (KU).

\section{Cneorella vietnamica Medvedev \& Dang Dap}

Cneorella vietnamica Medvedev \& Dang Dap, 1981, Rev. d'Ent. l'URSS, 60(3) : 632 (Vietnam).

Distribution : Vietnam.

No material was examined.

\section{Genus Bangprella n. gen.}

Antenna1 insertions widely separated by frons; labrum bilobed apically ; gena very narrow ; antenna with third segment distinctly longer than second ; prothorax convex side to side, without any distinct depression dorsally, and with lateral margin distinclty marginate, and basal margin not marginate except near side ; elytron with punctures irregularly impressed, without distinct subbasal furrow, epipleuron wide at base and gradually narrowed to apex ; prosternal process not elevated between anterior coxae; anterior coxal cavity open posteriorly ; middle and posterior tibiae each with a distinct spine apically ; tarsal claws appendiculate and first tarsal segment of posterior leg subequal to length of second and third segments combined.

Type species : Bangprella fulva Kimoto.

This genus somewhat resembles Kanarella Jacoby, but differs in having the middle and the posterior tibiae spined apically and pronotum much wider than long. 


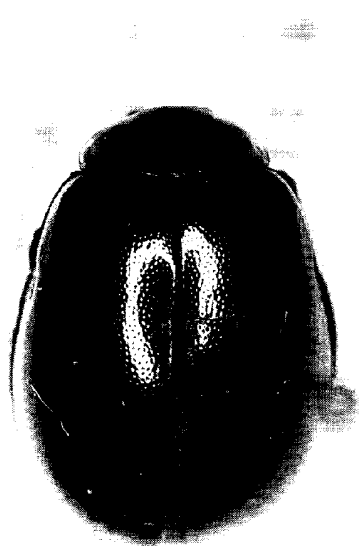

a

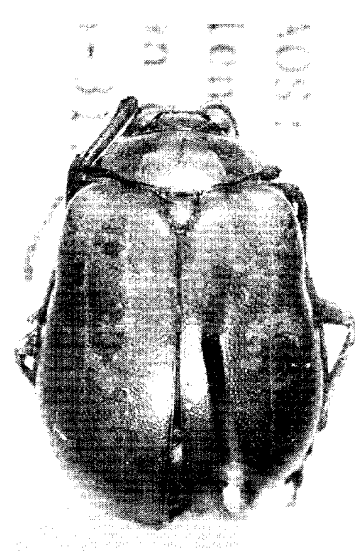

b

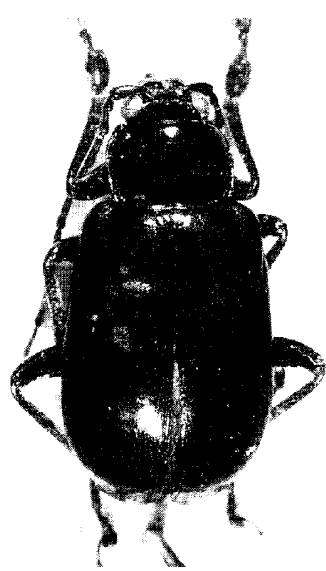

C

Fig. 26. a, Morphosphaera collaris Laboissière; b, Miltina dilatata Chapuis ; c, Parexosoma flaviventre (Baly).

\section{Bangprella fulva n. sp. Fig. 25c}

Generally yellowish brown, first to fourth abdominal segments reddish brown.

Head with vertex somewhat wrinkled, sparsely impressed by minute punctures, interocular space distinctly wider than transverse diameter of single oculus, and interocular transverse impression distinct, frontal tubercle subtriangular, contiguous, distinctly raised, surface smooth, shining. Antenna slender, nearly $2 / 3$ as long as body length ; first segment long, robust, somewhat club-shaped, second shortest, nearly $2 / 5$ as long as first, third twice as long as second, fourth $2 / 3$ as long as third, fifth subequal to fourth but wider, sixth subequal to fifth in length but much wider, and as long as wide apically, seventh nearly twice as long as sixth but widened, slightly longer than wide and with a small projection externally, eighth slightly shorter than seventh, widest at middle, slightly longer than wide, ninth elongate, twice as long as wide, subequal to eighth in length, tenth subequal to ninth in length

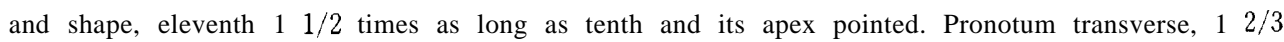
times as broad as long, anterior margin nearly straight, lateral margin distinctly rounded, widest almost at middle, slightly narrowed anteriorly and posteriorly, basal margin distinctly rounded posteriorly and slightly produced before scutellum, anterior corner thickened, slightly produced laterally, and posterior corner angulate, each with a setigerous pore, dorsal surface convex side to side, sparsely impressed by fine punctures, and interstices of punctures somewhat wrinkled. Scutellum subtriangular, smooth, shining, impunctate. Elytron with lateral margin rounded, surface distinctly and rather closely punctate, and interstices of punctures smooth, shining.

Male : Antenna deformed. Female : Unknown.

Length: 4.8-5.7 $\mathrm{mm}$.

Holotype (BISHOP) : THAILAND : Bangpra, 25 m, 23. xi. 1957, J. L. Gressitt. Paratopotypes : 3 exs., same data as the holotype (BISHOP, KIMOTO). Paratype : THAILAND : Pakchong, $100 \mathrm{~m}$, NE of Bangkok, 1 ex., 2. xii. 1957, J. L. Gressitt (BISHOP).

This new species somewhat resembles Kanarella unicolor Jacoby, but differs in having the middle and the posterior tibiae spined and pronotum much wider. 


\section{Genus M orphosphaera Baly}

Morphosphaera Baly, 1861, J. Ent., 1(4) : 298 (type :Chrysomela japonica Hornstedt ; Japan). -Chapuis, 1875, Gen. Col., 11:161, 170.-Maulik, 1936, Fauna India, Galeruc. :316.--Ogloblin, 1936, Fauna USSR, 26, $1:$ 230, 370, 408.—Gressitt\& Kimoto, 1963, Pac. Ins. Mon., 1B: 555.

\section{Key to species of Morphosphaera}

1 Pronotum with brownish with more than two pairs of blackish markings

Pronotum yellowish brown with a wide longitudinal median stripe and a pair of round markings purple ; elytron deep violaceous blue ; generally blackish ; abdomen entire-

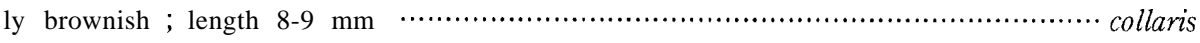

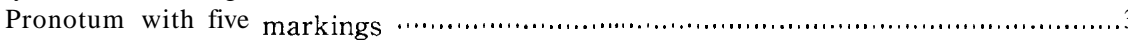

Pronotum with seven markings ; dorsal surfaces generally dark brown; head, ventral

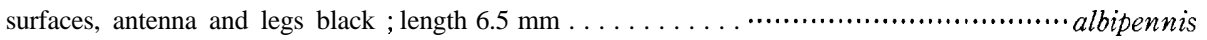

3(2) Head with vertex entirely reddish brown with bluish luster . . . . . . ............................ 4 Head generally black; generally brownish ; elytron brownish with violaceous luster ; antenna black; legs blackish with femora largely brownish; length 6.5-7.0 mm ...viridipennis

4(3) First to fourth abdominal segments with a pair of blackish markings ; ground color of pronotum ochraceous ; elytron brownish with violaceous luster ; ground color of ventral surfaces brownish; length $8 \mathrm{~mm}$.....

margaritacea Abdominal segments without any blackish markings; generally brownish ; antenna generally blackish ; legs blackish with most of femora brownish ; length $7.5 \mathrm{~mm} \cdots \cdots$ coomani

\section{M orphosphaera albipennis Allard}

Morphosphaera albipennis All., 1889, Ann. Soc. Ent. France, 58 : 309 (Cambodia ; HAMBURG) ; 1889, C. R. Soc. Ent. Belg., 33 : 67 (Cambodia).

Galerucida simplex Weise, 1922, Tijdschr. Ent., 65 :91 (Tonkin; STOCKHOLM). New synonym. Distribution : Thailand, Cambodia, Laos, Vietnam.

Material examined. THAILAND : "Siam", 5 exs., (BANGKEN). LAOS : Vientiane Prov., Ban Van Eue, 1 ex., 15-31. v. 1965, Native collr (BISHOP) ; Umgeb. Vientiane, 1 ex., iii-vi. 1963 ; Umgeb. Vanky, 1 ex., 1963 ; Umgeb. Pakse, 1 ex., 1964 ; Umgeb. Paklay, 1 ex., 1964 (MUNCHEN).

\section{M orphosphaera collaris Laboissière Fig. 26a}

Morphosphaera collaris Lab., 1930, Ann. Soc. Ent. France, 99: 362 (Tonkin; HAMBURG). -Gressitt \& Kimoto, 1963, Pac. Ins. Mon., 1B : 557 (China, Vietnam).

Distribution : Vietnam, China.

No additional material was examined beside the type series.

\section{M orphosphaera coomani Laboissière}

Morphosphaera coomani Lab., 1930, Ann. Soc. Ent. France, $99: 363$ (Tonkin; HAMBURG).

Distribution : Vietnam.

No additional material was examined besidethe type series. 


\section{Morphosphaera margaritacea Laboissière}

Morphosphaera margaritacea Lab., 1930, Ann. Soc. Ent. France, 99 :365 (Tonkin ; HAMBURG). Distribution : Vietnam.

Material examined. "Indo-China", 2 exs. (BASEL).

\section{Morphosphaera viridipennis Laboissière}

Morphosphaera viridipennis Lab., 1930, Ann. Soc. Ent. France, 99 : 366 (Tonkin; HAMBURG). Distribution : Thailand, Vietnam.

Material examined. THAILAND : Chiengmai Prov., Chiengdao, $450 \mathrm{~m}, 1$ ex., 5-11. iv. 1958, J. L. Gressitt (BISHOP).

\section{Genus Miltina Chapuis}

Miltina Chapuis, 1875, Gen. Col., 11 : 172 (type : Miltina dilatata Chapuis, SE Asia ; monobasic). -Maulik, 1936, Fauna India, Galeruc. : 313.-Gressitt \& Kimoto, 1963, Pac. Ins. Mon., 1B: 560.

\section{Miltina dilatata Chapuis Fig. 26b}

Miltina dilatata Chapuis, 1875, Gen. Col., 11 : 137, pl. 125, fig. 2 (no locality cited).-Jacoby, 1884, Notes Leyd. Mus., 6 : 42 (Sumatra).-Weise, 1922, Tijdschr. Ent., 65 : 99 (Tonkin).-Maulik, 1936, Fauna India, Galeruc. : 316, figs. $92-94$ (India, Tonkin, Malacca, Sumatra).-Gressitt \& Kimoto, 1963, Pac. Ins. Mon., 1B: 560 (S. China, Hainan, Vietnam).

Bonesia balyi Jacoby, 1889, Ann. Mus. Civ. Genova; 27 : 206 (Burma ;GENOVA).-Maulik, 1936, Fauna India, Galeruc. : 316 (= dilatata).

Distribution : India, Burma, Thailand, Laos, Vietnam, China, Malaya, Sumatra.

Subspherical, convex ; antenna flattened, in male fourth to tenth each with a long projection apically; yellowish brown; antenna and legs blackish, in some specimens brownish in various degrees ; length 7.5-11.5 mm.

Material examined. THAILAND : Chieng Mai Prov., Doi Suthep, 1,000 m, 1 ex., 12. vi. 1965, P. D. Ashlock (BISHOP) ; Chiengmai, Doi Suthep, 1 ex., 12. vi. 1965, Y. Miyatake (KU). LAOS : Vientiane Prov., Ban Van Eue, 1 ex., 1-15. v. 1965, J. A. Rondon, 6 exs., 15-31. v. 1967, Native collr ; Vientiane Prov., Phou Kou Khouei, 1 ex., 16. v. 1966, Native collr ; Sedone Prov., Pakse, 2 exs., 15. vi. 1967, Native collr (BISHOP) ; Umgeb. Vientiane, 1 ex., iii-vi. 1963 (MUNCHEN). VIETNAM : 7 km SE of Dilinh (Djiring), 990 m, 1 ex, 2. v. 1960, R. E. Leech (BISHOP).

\section{Genus Parexosoma Laboissière}

Parexosoma Lab., 1933, Bull. Mus. Hist. Nat. Paris, ser. 2, 4(8) : 96’7 (type: Makzcosoma flaviventre Baly ; N. India ; monobasic).-Ogloblin, 1936, Fauna USSR, 26, $1: 224,373$. -Gressitt \& Kimoto, 1963, Pac. Ins. Mon., 1B : 560.

Bijukta Maulik, 1936, Fauna India, Galeruc.: 321 (type : Malacosoma flaviventre Baly).

\section{Parexosoma flaviventre (Baly) Fig. 26c}

Malacosoma flaviventre Baly, 1878, Cist. Ent., 2 : 379 (Murree ; BM). 


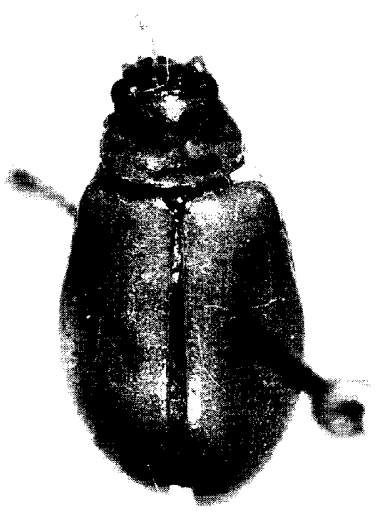

a

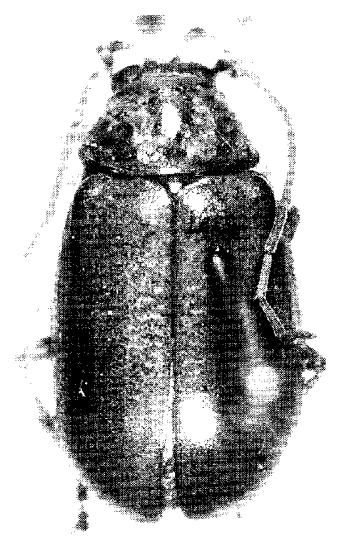

$\mathrm{b}$

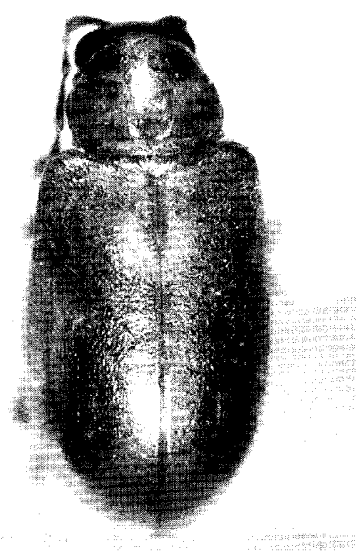

c

Fig. 27. a, Luperus thailandicus n. sp. ; b, Calomicrus persimilis n. sp. ; c, C. bicolor n. sp.

Parexosoma faviventre: Laboissihe, 1932, Bull. Mus. Hist. Nat. Paris, ser. 2, 4(8): 967 (type of genus).-Ogloblin, 1936, Fauna USSR, 26, 1 :225, fig. 98 (China).-Laboissière, 1940, Bull. Mus. Hist. Nat. Belg., 16(37) : 4 (Burma).-Gressitt \& Kimoto, 1963, Pac. Ins. Mon., 1B: 560 (China). -Kimoto \& Takizawa, 1972, Kontyâ, Tokyo, 40(4): 219 (Nepal).

Bijukta flaviventre: Maulik, 1936, Fauna India, Galeruc. : 323, fig. 96 (Punjub : Muree).

Parexosoma flaviventre var. tonkinensis Laboissière, 1932, Bull. Mus. Hist. Nat. Paris, ser. 2, 4(8): 967 (Tonkin).

Distribution : N. India, Nepal, Burma, Vietnam, W. China.

Oblong, antenna filiform ; pronotum transverse, convex from side to side ; elytron with a transverse furrow subbasally, its surface rather closely and confusedly impressed by fine punctures ; in male first segment of anterior tarsus widened ; entirely bluish black ; length $7.0 \mathrm{~mm}$.

No additinal material was examined.

\section{Genus H esperopenna Medvedev \& Dang Dap}

Hesperopenna Medvedev \& Dang Dap, 1981, Rev. d'Ent.l'USSR,60(3): 634 (type : Hesperopenna flava Medvedev \& Dang Dap ; Vietnam).

\section{Hesperopenna flava Medvedev \& Dang Dap}

Hesperopenna flava Medvedev \& Dang Dap, 1981, Rev. d'Ent.l'USSR,60(3): 634 (Vietnam). Distribution : Vietnam.

Body elongate-oval, more tapered forward. Body unicolorous yellow, apical half of elytron, except marginally, usually brighter and paler. Length 7.5-8.5 mm (after Ent. Rev. : 113).

No material was examined. 


\section{Genus Luperus Müller}

Luperus Müller, 1764, Fauna Insect. Fridrichsdalina :13.-Chapuis, 1875, Gen. Col., 9 : 186. -Ogloblin, 1936, Fauna USSR, 26, 1 : 243, 278, 415.-Gressitt \& Kimoto, 1963, Pac. Ins. Mon., 1B : 395, 561.-Kimoto, 1965, J. Fac. Agr. Kyushu Univ., 13(3) :378.-Silfverberg, 1979, Bull. Zool. Nomencl., 36(3): 161 (requested to have Chrysomela flavipes Linnaeus, 1767, designated as type).

\section{Luperus thailandicus n.sp. Fig. 27a}

Generally yellowish brown, head pitchy black with frontal tubercle reddish brown, scutellum pitchy black, meso- and metathorax pitchy brown.

Head with vertex somewhat granulate, sparsely impressed by minute punctures, interocular space distinctly wider than transverse diameter of single oculus, and interocular transverse impression distinct, frontal tubercle transverse, subtriangular, contiguous, and distinctly raised, surface smooth, shining. Antenna slender, nearly $3 / 4$ as long as body length ; first segment long, club-shaped, second shortest, nearly $1 / 5$ as long as first, third $21 / 2$ times as long as second, fourth $12 / 3$ times as long as third, fourth to ninth subequal to each other in length and shape, tenth slightly shorter than ninth, eleventh subequal to tenth in length but its apex pointed. Pronotum transverse, $12 / 3$ times as broad as long, anterior margin slightly rounded anteriorly, lateral margin distinctly rounded, widest almost at middle, distinctly narrowed anteriorly and posteriorly, basal margin distinctly rounded posteriorly, dorsal surface convex, with a pair of lateral transverse furrow starting from slightly behind anterior corner, sparsely impressed by distinct punctures, and interstices of punctures smooth, shining. Scutellum subtriangular, convex, smooth, shining, impunctate. Elytron with lateral margin rounded, surface more distinctly and closely punctate than pronotum and interstices of punctures smooth, shining.

Length: $7.5 \mathrm{~mm}$.

Holotype (BISHOP) : THAILAND : Chiengmai Prov., Doi Suthep, 1-5. iv. 1958, T. C. Maa. Paratopotype : 1 ex., same data as the holotype (KIMOTO). Paratype : THAILAND : Chiengmai, 1,200 m, 1 ex., 11. iv. 1966, J. Sedlacek (BISHOP).

This new species somewhat resembles Luperus iniquus (Weise), from China, but differs in having pronotum much wider and the general coloration yellowish brown.

\section{Genus Calomicrus Stephens}

Calomicrus Step., 1834, Brit. Ent. Mandb., 4 : 293 (type :Crioceris circumfusus Marsh; monobasic ; Europe).-Ogloblin, 1936, Fauna USSR, 26, 1 :252.-Gressitt \& Kimoto, 1963, Pac. Ins. Mon., 1B: 568.

\section{Key to species of Calomicrus}

Generally bluish black with abdomen yellowish brown; length $4.3-4.9 \mathrm{~mm}$........... coomani

2(1) Antenna entirely yellowish brown, dorsal surfaces entirey yellowish to reddish brown

Antenna generally pitchy brown to black; pronotum reddish brown and elytron yellowish brown ; pronotum nearly $11 / 3$ times as wide as long ; generally yellowish 
brown, head pitchy brown, legs with tibiae and tarsi somewhat infuscate; length 5.7 $-6.3 \mathrm{~mm}$ bicolor

3(2) Pronotum less transverse, nearly $11 / 2$ times as wide as long and widest slightly before middle ; generally yellowish to reddish brown ; length $6 \mathrm{X}-6.9 \mathrm{~mm}$................... persimilis Pronotum transverse, nearly $12 / 3$ times as wide as long and widest slightly behind middle ; generally yellowish brown, in some specimen tibiae and tarsi dark brown to pitchy black; length $4.8-6.0 \mathrm{~mm}$

flavus

\section{Calomicrus bicolor n. sp. $\quad$ Fig. 27c}

Generally yellowish brown, head pitchy brown, pronotum reddish brown, antenna pitchy brown to black with basal two or three segments reddish brown, legs yellowish brown with tibiae and tarsi somewhat infuscate.

Head with vertex finely wrinkled, sparsely impressed by minute punctures, interocular space distinctly wider than transverse diameter of single oculus, and interocular transverse impression distinct, and with several large punctures along this impression ; frontal tubercle subtriangular, contiguous, distinctly raised, surface smooth, shining. Antenna slender, nearly as long as body length ; first segment long, second shortest, nearly $1 / 3$ as long as first, third $11 / 2$ times as long as second, fourth twice as long as third, fourth to seventh subequal to each other in length and shape, eighth slightly shorter than seventh, ninth subequal to eighth in length and shape, tenth slightly shorter than ninth, eleventh subequal to ninth in length but its apex pointed. Pronotum transverse, $11 / 3$ times as broad as long, anterior margin slightly rounded anteriorly, lateral margin distinctly rounded, widest almost at middle, slightly narrowed anteriorly and posteriorly, basal margin distinctly rounded posteriorly, dorsal surface convex, with a pair of lateral transverse furrows starting from anterior corner, rather closely impressed by distinct punctures, and interstices of punctures smooth, shining. Scutellum subtriangular, smooth, shining, impunctate. Elytron with lateral margin rounded, surface more distinctly and closely punctate, and intersties of punctures smooth, shining.

Length : 5.7-6.3 mm.

Holotype (BISHOP) : LAOS : Sayaboury Prov., Sayaboury, 15. iv. 1965, J. L. Gressitt. Paratopotypes : 12 exs., same data as the holotype (BISHOP, KIMOTO). Paratype : LAOS : Vientiane Prov., Phou-kow-kuei, 1 ex., 14. iv. 1965, J. L. Gressitt (BISHOP).

This new species resembles Calomicrus flavus(Jacoby), but differs in having antenna generally pitchy brown, pronotum reddish brown and elytron yellowish brown.

\section{Calomicrus coomani Gressitt \& Kimoto Fig. 28a}

Calomicrus coomani Gressitt \& Kimoto, 1963, Pac. Ins. Mon., 1B : 569, fig. (Tonkin:Hoa-Binh; FREY).-Kimoto, 1966, Esakia, Kyushu Univ., 5 : 31 (Taiwan).

Distribution : Thailand, Laos, Vietnam, Taiwan.

Material examined. THAILAND : Trang Prov., Khaophappha, Khaochang, '200 m, 1 ex., 1-3. i. 1964, light trap, G. A. Samuelson; Phu Kae, 1 ex., 15. v. 1963, Native collr (BISHOP) ; Khao Chong, nr. Trung, Y. Miyatake leg. (KU). LAOS : Ban Van Eue, Vientiane Prov., 1 ex., 15-31. v. 1965, Native collr ; Sedone Prov., Pakse, 1 ex., 31. v. 1967, Native collr ; Uekinak, nr. Pakkading, 100 m, 4 exs., 22. iv. 1965, J. L. Gressitt (BISHOP) ; Umgeb. Vientiane, 2 exs., iii-vi. 1963 (MUNCHEN). 

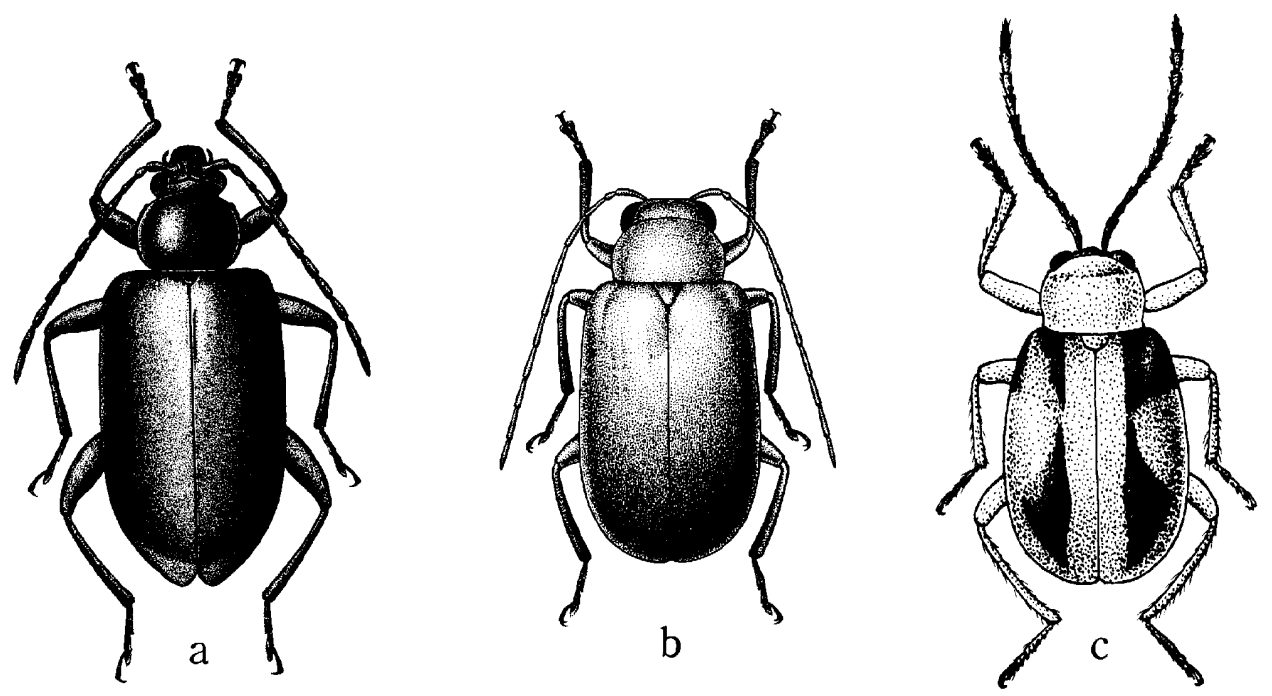

Fig. 28. a, Calomicrus coomani Gressitt \& Kimoto;b, Sinoluperus subcostatus Gressitt \& Kimoto ; c, Medythia suturalis (Motschulsky).

\section{Calomicrus flavus (Jacoby), new combination}

Agelastica ? flava Jac., 1892, Ann. Mus. Civ. Genova, 32 : 967 (Burma ;GENOVA).-Weise, 1924, Col. Cat. Junk, $78: 131$ (Burma).

Distribution : Burma, Thailand, Laos, Vietnam.

This species is not listed in Maulik, 1936, Fauna India, Galerucinae.

Material examined. THAILAND : Chiengmai Prov., Mae Sa, 2 exs., 16. vi. 1965, P. D. Ashlock (BISHOP) ; Utkaithani, 3 exs., 13. iv. 1963 (BANGKHEN) ; Chiengmai Prov., Mae Sa Water Fall, 2 exs., 16. vi. 1965, Y. Miyatake (KU). LAOS : Borikhane Prov., Pakkading, 100 m, 2 exs., 22-23. iv. 1965, J. L. Gressitt, 1 ex., 31. viii. 1965, Native collr; Pon-horn, N. of Pakkading, 2 exs., 20. iv. 1965, J. L. Gressitt ; Vientiane Prov., Ban Van Eue, 1 ex., 11. iv. 1965, 1 ex., 15-31. v. 1965, Native collr ; Sedone Prov., 5 km E. of Pakse, 3 exs., 13. v. 1965, P. D. Ashlock (BISHOP) ; Umgeb. Vientiane, 1 ex., iii-vi. 1963 (MUNCHEN). VIETNAM : 25 km SW of Pleiku, 400 m, 1 ex., 12. v. 1960, L. W. Quate (BISHOP).

\section{Calomicrus persimilis n. sp. Fig. $27 \mathrm{~b}$}

\section{Entirely yellowish brown.}

Head with vertex slightly wrinkled, sparsely impressed by minute punctures, interocular space distinctly wider than transverse diameter of single oculus, and interocular transverse impression distinct, frontal tubercle subtriangular and distinctly raised, surface smooth, shining. Antenna slender, slightly shorter than body length ; first segment long, second shortest, nearly $1 / 4$ as long as first, third $11 / 2$ times as long as second, fourth $22 / 3$ times as long as third, fourth to eighth subequal to each other in length and shape, ninth slightly shorter than eighth, tenth subequal to ninth in length and shape, eleventh slightly longer than ninth and subequal to eighth in length but its apex pointed. Pronotum transverse, $11 / 2$ times as broad as long, anterior margin slightly rounded anteriorly, lateral 
margin distinctly rounded, widest slightly before middle, slightly narrowed anteriorly and posteriorly, basal margin distinctly rounded posteriorly, dorsal surface convex, with a pair of lateral transverse furrow starting from anterior corner, closely impressed by fine punctures, and interstices of punctures smooth, shining. Scutellum subtriangular, smooth, shining, impunctate. Elytron with lateral margin rounded, surface distinctly and closely punctate, and interstices smooth, shining.

Length : 6.6-6.9 mm.

Holotype (BISHOP) : LAOS : Sedone Prov., Pakse, 31. v. 1965, Native collr. Paratopotypes : 3 exs., same data as the holotype (BISHOP, KIMOTO). Paratypes : LAOS : Ile de Khong, 7 exs., 17. iv. 1965, J. L. Gressitt (BISHOP). VIETNAM : $20 \mathrm{~km} \mathrm{~N}$. of Pleiku, $650 \mathrm{~m}, 1$ ex., 9. v. 1960, S. Quate \& L. Quate ; Ban Me Thuot, 500 m, 1 ex., 16-18. v. 1960, S. Quate (BISHOP).

This new species closely resembles Calomicrus flavus(Jacoby), but differs in having pronotum less transverse, and the legs entirely yellowish brown.

\section{Genus Trichosepharia Laboissière}

Trichosepharia Lab., 1936, Ann. Soc. Ent. France, 105 : 254 (type : Ttichosepharia pubescens Laboissiere ; Vietnam).

\section{Trichosepharia pubescens Laboissière Fig. 29a}

Ttichosepharia pubescens Lab., 1936, Ann. Soc. Ent. France, 105 : 254 (Tonkin; HAMBURG).

Ttichosepharia pubescens basalis Laboissiere, 1936, Ann. Soc. Ent. France, 105 : 255 (Tonkin; HAMBURG).

Distribution : Vietnam.

Suboval; dorsal surfaces thickly covered by fine hairs ; generally reddish brown ; elytron reddish basally and yellowish apically and with a large blackish transverse median marking, which is also extending along sutural margin towards apex ; antenna, tibiae and tarsi infuscate; length $8.0 \mathrm{~mm}$.

No additional material was examined beside the type series.

\section{Genus Sinoluperus Gressitt \& Kimoto}

Sinoluperus Gressitt \& Kimoto, 1963, Pac. Ins. Mon., 1B: 583 (type: Sinoluperus subcostatus Gressitt \& Kimoto ; S. China).

\section{Sinoluperus subcostatus Gressitt \& Kimoto Fig. 28b}

Sinoluperus subcostata Gressitt \& Kimoto, 1963, Pac. Ins. Mon., 1B: 584, fig. (S. China, Hainan ; CAS).

Distribution : Laos, S. China, Hainan.

Suboval; interantennal space wide; antenna filiform ; pronotum transverse, convex from side to side ; elytron subparallel-sided, its surface closely and confusedly impressed by distinct punctures ; yellowish brown, in some specimen elytron almost entirely blackish ; length 5.2-5.5 mm.

Material examined. LAOS : Sedone Prov., $5 \mathrm{~km}$ of Pakse, 1 ex., 13. v. 1965, P. D. Ashlock (BISHOP). 


\section{Genus Medythia Jacoby}

Medythia Jacoby, 1887, Notes Leyden Mus., $9: 242$ (type: Medythia quadrimaculata Jacoby; Sumatra ; monobasic).-Kimoto, 1981, Ent. Rev. Japan, $35: 7$.

Paraluperodes Ogloblin, 1936, Fauna USSR, 26, 1: 310, 375, 431 (type : Cnecodes suturalis Motschulsky ; Burma ; monobasic).-Gressitt \& Kimoto, 1963, Pac. Ins. Mon., 1B:585.-Kimoto, 1965, J. Fac. Agr. Kyushu Univ., 13(3) : 386.

\section{KeY TO SPECIES OF Medythia}

Pronotum as broad as long, widest almost at $1 / 3$ from anterior margin, and strongly narrowed posteriorly ; antenna pitchy black with first and eighth to tenth segments pale brown, in some specimen tenth segment partly or entirely blackish ; generally yellowish brown with apex and basal half of tibiae blackish; elytron with blackish stripe ; length

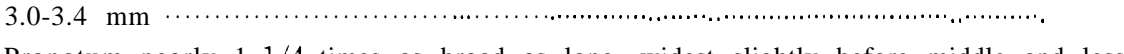

Pronotum nearly $11 / 4$ times as broad as long, widest slightly before middle and less strongly narrowed posteriorly ; antenna pitchy brown with basal segments paler ; generally yellowish brown, elytron with broad stripe blackish ; head pitchy brown ; length $2.3-$ $2.6 \mathrm{~mm}$ siamensis

\section{Medythia siamensis n. sp. Fig. 29b}

Generally yellowish brown, head pitchy brown, elytron yellowish brown with broad longitudinal stripe black, antenna pitchy brown with basal segments generally paler.

Head with vertex somewhat wrinkled, sparsely impressed by minute punctures, interocular space slightly wider than transverse diameter of single oculus, frontal tubercle subtriangular, contiguous, distinctly raised, surface finely granulate, and delimited posteriorly by several large punctures arranged in a transverse row, clypeus impressed by large punctures, surface finely granulate. Antenna robust, in preapical segments, nearly three times as long as wide, nearly $2 / 3$ as long as body length ; first segment long, second shortest, nearly half as long as first, third subequal to second in length but more slenderer, fourth $11 / 2$ times as long as third, fourth to ninth subequal to each other in length and shape, tenth slightly shorter than ninth, eleventh subequal to ninth in length but its apex pointed. Pronotum transverse, nearly $11 / 4$ times as broad as long, anterior margin slightly rounded posteriorly, lateral margin distinctly rounded, widest slightly before middle, slightly narrowed anteriorly and posteriorly, dorsal surface convex side to side, rather closely impressed by distinct punctures, and interstices of punctures smooth, shining. Scutellum semicircular, smooth, shining, impunctate. Elytron with lateral margin rounded, surface more distinctly and closely punctate than pronotum, and interstices of punctures smooth, shining.

Length : 2.3-2.6 $\mathrm{mm}$.

Holotype (BANGKHEN) : THAILAND : Bangkok, 1960, lot no. 2728. Paratopotypes : 4 exs., same data as the holotype (BANGKHEN, KIMOTO).

This new species resembles Medythia suturalis (Motschulsky), but differs in having the characteristic coloration of antenna, and pronotum slightly wider.

\section{Medythia suturalis (Motschulsky) Fig. 28c}

Cnecodes suturalis Motsch., 1858, Etud. Ent., 7 : 100 (Burma).-Weise, 1910, Phil. J. Sci., ser. D, 5 : 


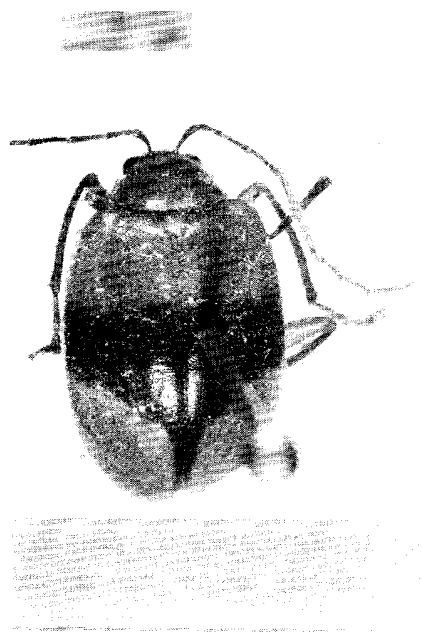

a

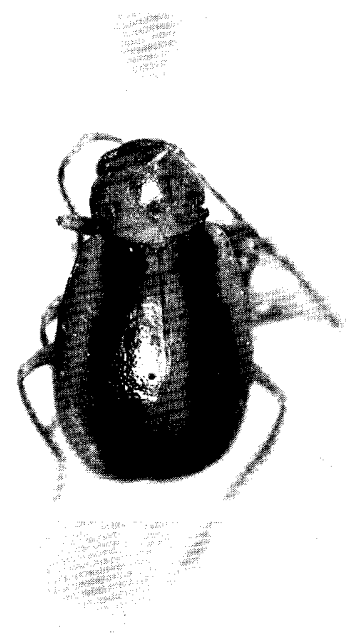

$\mathrm{b}$

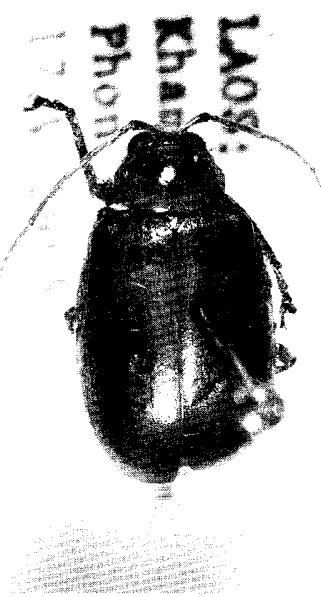

$\mathrm{C}$

Fig. 29. a, Trichosepharia pubescens Laboissière; b, Medythia siamensis $n$. $\mathrm{sp}$; c, Liroetiella tibialis $\mathrm{n}$. $\mathrm{sp}$.

141 (Philippines).

Luperodes suturalis :Allard, 1889, Ann. Soc. Ent. France, ser. 6, $9: 310$ (Pnom-Penh).-Weise, 1922, Tijdschr. Ent., $65: 80$ (Canton).

Monolepta suturalis : Duvivier, 1892, Ann. Soc. Ent. Belg., 36 : 444 (India).

Luperodes suturalis abb. abbreviatus, vittatus Weise, 1922, Tijdschr. Ent., $65: 81$ (locality not cited). Paraluperodes suturalis : Ogloblin, 1936, Fauna USSR, 26, 1 : 312, 433, fig. 312 (India, S. China, Sumatra, Java, Philippines).-Chûjô, 1961, Nature and Life in SE Asia, Kyoto, 1 : 352, pl. 12, fig. 8 (Thailand) ; 1962, Phil. J. Sci., 91(1-2) : 233 (Formosa) ; 1964, Nature and Life in SE Asia, Kyoto, 3 : 298 (Thailand).-Gressitt \& Kimoto, 1963, Pac. Ins. Mon., 1B: 585 (S. China, Hainan, Vietnam).-Kimoto, 1965, J. Fac. Agr. Kyushu Univ., 13(3) : 387 (Ryukyu Is.).

Medythia suturalis : Kimoto, 1981, Ent. Rev. Japan, $35: 8$ (Ryukyu Is., China, Taiwan, Hainan, Thailand, Vietnam, Malaya, Java, Celebes).

Distribution : India, Burma, Thailand, Cambodia, Laos, Vietnam, Malaya, Philippines, Hainan, S. China, Taiwan, Ryukyu Is., Sumatra, Java, Celebes.

Material examined. THAILAND : Bangkok, 50 exs., 1960 (BANGKHEN) ; Chiengmai Prov., Chiengmai, 7 exs., 11. vi. 1965, 10 exs., 17. vi. 1965, P. D. Ashlock, 3 exs., 14. xi. 1957, J. L. Gressitt ; Chiangmai Prov., Doi Aneka, 1 ex., 9. iv. 1953, T. C. Maa; Pangmakampon (Pankampawng), nr. Fang, 450 m, 2 exs., 15-16. xi. 1957, T. C. Maa ; Pak Chong, 100 m, NE of Bangkok, 20 exs., 1-3. xii. 1957, J. L. Gressitt, 1 ex., 3. xii. 1957, T. C. Maa; Scattahib Area, 5 exs., 24. xi. 1957, J. L. Gressitt ; Nakhon Nayok Prov., Khao Yai Nat. Park, 1 ex., 6. xi. 1965, P. D. Ashlock ; Si Racha, 1 ex., 21. xii. 1963, Native collr (BISHOP) ; Chiengmai, 8 exs., 11. vi. 1965, at light, K. Morimoto \& Y. Miyatake, 1 ex., 12. viii. 1973, 4 exs., 17. viii. 1973, at light, K. Yano ; Sanpatong, 1 ex., 7. vii. 1970, soybean, K. Yano ; Khao Chong, nr. Trung, 1 ex., 24. vi. 1965, at light, Y. Miyatake (KU) ; Khon Kaen, 1 ex., 20. xii. 1979, S. Azuma ; Lom Sak, 1 ex., 2. i. 1980, S. Azuma (RU) ; Sanpatong, R. E. S., 2 exs., 25. xi. 1968, Jeraphanatha (EHIME) ; Bangsaen, 5 exs., 16-17. xi. 1968, 2 exs., 25. xii. 1968, M. Sato ; Sanpatong, 1 ex., 25. xii. 1968, M. Sato ; Nakorn Nayok, 4 exs., 24. i. 1968, K. Baba ; Khao Yai, 1 ex., 29-31. i. 1968, 
K. Baba ; Saraburi, 2 exs., 28. i. 1968, K. Baba (KIMOTO). CAMBODIA : Damrey Phong, 1 ex., 14-16. iv. 1961, N. R. Spencer (BISHOP) ; Chaon, 1 ex., T. Shibata (OMNH). LAOS : Vientiane, 1 ex., 31. v.3. vi. 1960, S. Quate \& L. Quate, 4 exs., 8-9. v. 1965, P. D. Ashlock; Vientiane Prov., Ban Van Eue, 2 exs., 30. ii. 1965, J. L. Gressitt ; Vientiane Prov., Gi Sion Vill., de Tha Ngone, 3 exs., 28. ii. 1965, 1 ex., 27. ii.-6. iii. 1966, J. L. Gressitt (BISHOP). VIETNAM : Nha Trang, 5 exs., 17-26. xi. 1960, C. M. Yoshimoto ; $22 \mathrm{~km} \mathrm{~S}$. of Nha Trang, 4 exs., 20-26. xi. 1960, C. M. Yoshimoto ; $22 \mathrm{~km} \mathrm{S.} \mathrm{of} \mathrm{Nha} \mathrm{Trang,}$ 4 exs., 20-26. xi. 1960, C. M. Yoshimoto ; 15-35 km, NW of Phan Rang, 12 exs., 8-16. xi. 1960, C. M. Yoshimoto ; Nha Ho, 14 km N., Phan Rang, 1 ex., 15. xi. 1960, C. M. Yoshimoto ; Quang Tri, 2 km N., 4 exs., 9. iii. 1970, 1 ex., 1. iv. 1970, 14 exs., 30. iv. 1970, 6 exs., 5. v. 1970, 1 ex., 27. v. 1970, 2 exs., 11. vi. 1970, A. R. Gillogly (BISHOP).

\section{Genus Liroetiella $\mathbf{n}$. gen.}

Antenna1 insertions widely separated by frons ; labrum rounded, truncate apically ; gena very narrow ; frontal tubercles contiguous ; antenna with third segment distinctly longer than second ; prothorax with lateral and posterior margins distinctly marginate, elytron with punctures irregularly impressed, without distinct subbasal furrow, epipleuron wide at base and gradually narrowed to apex ; prostemal process not elevated between anterior coxae; anterior coxal cavity open posteriorly; middle and posterior tibiae distinctly spined apically ; tarsal claws appendiculate and first tarsal segment of posterior legs distinctly longer than second and third segments combined.

Type species : Liroetiella tibialis Kimoto.

This new genus somewhat resembles Siemssenius Weise (= Pseudoliroetis Laboissière), but differs in having the middle and the posterier tibiae spined apically. Also from Liroetis Weise, differs in having elytral epipleuron wide in base.

\section{Key to SPECIES of Liroetiella}

Thorax entirely black; generally yellowish brown; head black with middle of vertex pitchy brown; length $3.8-4.5 \mathrm{~mm}$

2(1) Antenna with third segment nearly twice as long as second $\ldots \ldots \ldots \ldots \ldots \ldots \ldots \ldots \ldots \ldots \ldots \ldots$ Antenna with third segment nearly four times as long as second ; generally yellowish brown ; length 4.5-5.7 $\mathrm{mm}$ antennalis

3(2) Smaller ; body length $3.8-5.4 \mathrm{~mm}$ Larger ; generally yellowish brown, with tibiae and tarsi pitchy black ; length 8.4$10.0 \mathrm{~mm}$

4(3) Pronotum with surface smooth, not granulate Pronotum with surface finely granulate and distinctly punctate; generally yellowish brown ; antenna pitchy brown with basal segments paler ; length 4.8-5.4 mm $\cdots$ granulicollis

5(4) Legs yellowish brown with tibiae and tarsi pitchy black; generally reddish brown with elytron and abdomen yellowish brown, antenna pitchy black; length 3.9-4.5 $\mathrm{mm}$

Legs entirely brownish ; generally yellowish brown, in some specimens antenna infuscate; length $3.8-4.5 \mathrm{~mm}$ 
Liroetiella antennalis n. sp. Fig. 30a

Entirely yellowish brown, in some specimens legs with middle and posterior tibiae and tarsi pitchy black.

Head with vertex somewhat wrinkled, sparsely impressed by minute punctures, interocular space subequal to transverse diameter of single oculus, and interocular transverse impression distinct, frontal tubercle subtriangular, contiguous, distinctly raised, surface finely granulate. Antenna slender, nearly as long as body length ; first segment long, second shortest, nearly $1 / 4$ as long as first, nearly four times as long as second, fourth $11 / 2$ times as long as third, fourth to seventh subequal to each other in length and shape, eighth slightly shorter than sixth, eighth to tenth subequal to each other in length and shape, eleventh subequal to tenth in length but its apex pointed. Pronotum transverse, $11 / 4$ times as broad as long, anterior margin nearly straight, lateral margin feebly rounded, widest almost at middle, slightly narrowed anteriorly and posteriorly, basal margin distinctly rounded posteriorly, dorsal surface convex, with a pair of lateral transverse furrows starting from anterior corners, sparsely impressed by fine punctures, and interstices of punctures smooth, shining. Scutellum subtriangular, smooth, shining, impunctate. Elytron with lateral margin rounded, surface more distinctly and closely punctate than pronotum, and interstices of punctures smooth, shining.

Length : 4.5-5.7 mm.

Holotype (Type No. 2699, Kyushu Univ.) : THAILAND : Khao Chong, nr. Trung, 26. vi. 1965, K. Morimoto \& Y. Miyatake. Paratopotypes: 8 exs., same data as the holotype (KU). Paratypes: THAILAND: Kao Chong Park, 1 ex., 1. iii. 1965, K. Yasumatsu (KU) ; Trang, Khaophappha, Kaochong, 1 ex., 11. i. 1964, 2 exs., 13. i. 1964, G. A. Samuelson ; Chiengmai, 1,1OO-1,500 m, no date, 1966, J. Sedlacek (BISHOP) ; Slika Fall, 1 ex., 24. i. 1968, K. Baba (KIMOTO). LAOS : Ban Van Eue, 800 m, SE of Phou-kow-kuei, 3 exs., 13. iv. 1965, J. L. Gressitt, 1 ex., 15. iii. 1966, Native collr; Sayaboury Prov., Sayaboury, 1 ex., 15. iv. 1965, J. L. Gressitt (BISHOP). VIETNAM : Fyan, 900-1,000 m, 1 ex., 11. vii.-9. viii. 1961, N. R. Spencer ; Ban Me Thuot, 500 m, 1 ex., 16-18. v. 1960, S. Quate ; 20 km N. of Pleiku, 650 m, 1 ex., 9. v. 1960, L. W. Quate ; Kontum, N. of Pleiku, 550 m, 1 ex., 13. v. 1960, L. W. Quate (BISHOP).

This new species resembles Liroetiella minor Kimoto, but differs in having the third antenna 1 segment nearly four times as long as the second.

\section{Liroetiella bicolor $\mathbf{n} . \mathbf{s p} . \quad$ Fig. 31a}

Generally reddish brown, elytron and abdomen yellowish brown, antenna pitchy brown with first segment reddish brown, legs yellowish brown with tibiae and tarsi pitchy black.

Head with vertex finely granulate, sparsely impressed by minute punctures, interocular space subequal to transverse diameter of single oculus, and interocular transverse impression distinct, frontal tubercle subtriangular, contiguous, distinctly raised, surface finely granulate. Antenna slender, nearly as long as body length ; first segment long, second shortest, nearly $1 / 4$ as long as first, third twice as long as second, fourth three times as long as third, fifth subequal to fourth in length and shape, sixth slightly shorter than fifth, sixth to eight subequal to each other in length and shape, ninth slightly shorter than eighth, tenth subequal to ninth in length and shape, eleventh subequal to tenth in length but its apex pointed. Pronotum transverse, $11 / 4$ times as broad as long, anterior margin slightly rounded anteriorly, lateral margin distinctly rounded, widest almost at middle, slightly narrowed anteriorly and posteriorly, basal margin distinctly rounded posteriorly, dorsal surface convex, with a pair of lateral transverse furrows starting from anterior corner, closely impressed by strong punctures, and interstices of punctures smooth, shining. Scutellum subtriangular, smooth, 

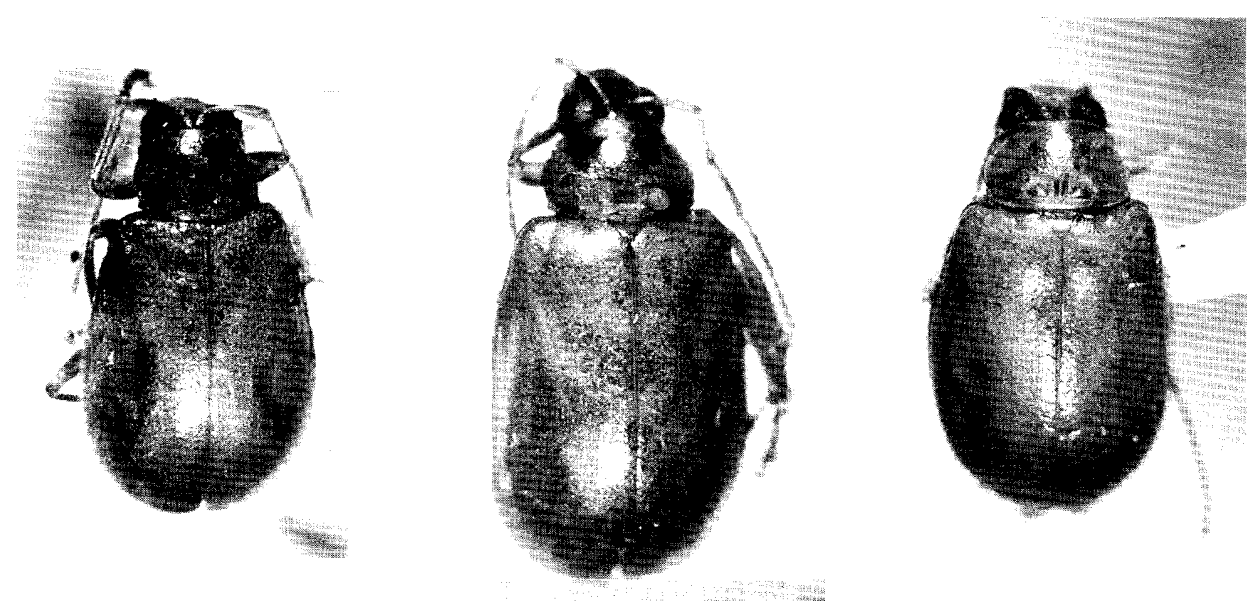

a

Fig. 30. a, Liroetiella antennalis n. sp. ; b, L. minor n. sp. ; c, L. granulicollis n. sp.

shining, impunctate. Elytron with lateral margin rounded, surface distinctly and rather closely punctate, and interstices of punctures smooth, shining.

Length : $3.9-4.5 \mathrm{~mm}$.

Holotype (BISHOP) : THAILAND : Chiengmai Prov., Chieng Dao, 5-11. iv. 1958, Native collr. Paratopotype: 1 ex., same data as the holotype (BISHOP). Paratypes: THAILAND: Chiengrnai Prov., Chieng Dao, 1 ex., 15. vi. 1965, Y. Miyatake (KU) ; Chieng Dao, 1 ex., 5-11. iv. 1958, T. C. Maa (BISHOP) ; Khao Yai, 1 ex., 29-31. i. 1968, K. Baba ; Saraburi, 1 ex., 28. i. 1968, K. Baba (KIMOTO). LAOS : Ban Van Eue, 20 km E. of Phou Kow Kuei, 1 ex., 15-31. v. 1965, Native collr ; Sayaboury Prov., Sayaboury, 1 ex., 17. ii. 1965, Native collr (BISHOP).

This new species resembles Liroetiella minor Kimoto, but differs in having the legs yellowish brown with tibiae and tarsi pitchy black, and pronotum reddish and elytron yellowish brown.

\section{Liroetiella granulicollis n.sp. Fig. 30c}

Generally yellowish brown, antenna pitchy brown with basal three segments yellowish brown.

Head with vertex finely granulate, sparsely impressed by minute punctures, interocular space distinctly wider than transverse diameter of single oculus, and interocular transverse impression deep, distinct, frontal tubercle subtriangular, contiguous, distinctly raised, surface finely granulate. Antenna slender, nearly as long as body length ; first segment long, second shortest, nearly $1 / 5$ as long as first, third $21 / 2$ times as long as second, fourth three times as long as third, fifth subequal to fourth in length and shape, sixth slightly shorter than fifth, and sixth to tenth subequal to each other in length and shape, eleventh slightly shorter than tenth and its apex pointed. Pronotum transverse, $13 / 4$ times as broad as long, anterior margin slightly rounded posteriorly, lateral margin distinctly rounded, widest almost at middle, distinctly narrowed anteriorly and posteriorly, basal margin distinctly rounded posteriorly, dorsal surface convex, with a pair of lateral transverse furrow starting from anterior corners, sparsely impressed by distinct punctures, and interstices of punctures finely granulate. Scutellum subtriangular, smooth, shining, impunctate. Elytron with lateral margin rounded, surface more distinctly and closely punctate than pronotum, and interstices of punctures granulate. 
Length : 4.8-5.4 mm.

Holotype (BISHOP) : LAOS : Sayaboury Prov., Sayaboury, 16. iv. 1965, J. L. Gressitt. Paratopotype : 1 ex., same data as the holotype (KIMOTO). Paratypes : 2 exs. "Indo-China" (BASEL).

This new species resembles Liroetiellaantennalis Kimoto, but differs in having pronotum finely granulate, and the third antennal segment nearly twice as long as the second.

\section{Liroetiella minor n. sp. $\quad$ Fig. $30 \mathrm{~b}$}

Entirely yellowish brown, in some specimen head somewhat infuscate.

Head with vertex smooth, shining, sparsely impressed by minute punctures, interocular space subequal to transverse diameter of single oculus, and interocular transverse impression distinct, frontal tubercule subtriangular, congtiguous, distinctly raised, surface smooth, shining. Antenna slender, nearly as long as body length ; first segment long, second shortest, nearly $1 / 3$ as long as first, third $11 / 2$ times as long as second, fourth twice'as long as third, fourth to seventh subequal to each other in length and shape, eighth slightly shorter than seventh, seventh to tenth subequal to each other in length and shape, eleventh subequal to tenth in length but its apex pointed. Pronotum transverse, $11 / 4$ times as broad as long, anterior margin slightly rounded anteriorly, lateral margin distinctly rounded, widest almost at middle, slightly narrowed anteriorly and posteriorly, basal margin distinctly rounded posteriorly, dorsal surface convex, with a pair of lateral transverse furrows starting from anterior corners, rather closely impressed by distinct punctures, and interstices of punctures smooth, shining. Scutellum subtriangular, smooth, shining, impunctate. Elytron with lateral margin rounded, surface more distinctly and closely punctate than pronotum, and interstices of punctures smooth, shining.

Length : $3.8-4.5 \mathrm{~mm}$.

This new species resembles Liroetiella tibialis Kimoto, but differs in being the body length shorter, and having the legs entirely brownish.

Holotype (BISHOP) : LAOS : Vientiane Prov., Ban Van Eue, 1-15. ix. 1967, Native collr. Paratopotypes : 47 exs., same data as the holotype (BISHOP). Paratypes : THAILAND : Fang, 5 exs., 13-14. vi. 1965, K. Morimoto \& Y. Miyatake (KU). LAOS : Vientiane Prov., Van Ban Eue, 14 exs., no date, 1965, Native collr. VIETNAM : Fyan, 900-1,200 m, 4 exs., 11. vii.-9. viii. 1961, N. R. Spencer ; Ban Me Thuot, 1 ex., 16-18. v. 1960, L. W. Quate (BISHOP).

\section{Liroetiella nigricollis $\mathbf{n}$. sp. $\quad$ Fig. 31b}

Generally yellowish brown, head black with middle of vertex pitchy brown, thorax entirely black.

Head with vertex finely wrinkled, sparsely impressed by minute punctures, interocular space subequal to transverse diameter of single oculus, and interocular transverse impression distinct, frontal tubercle subtriangular, contiguous, distinctly raised, surface finely wrinkled. Antenna slender, nearly as long as body length ; first segment long, second shortest, nearly $1 / 4$ as long as first, third 1 $2 / 3$ times as long as second, fourth $21 / 2$ times as long as third, fifth slightly shorter than fourth, sixth slightly shorter than fifth and subequal to seventh in length and shape, eighth slightly shorter than seventh, eighth to tenth subequal to each other in length and shape, eleventh subequal to tenth in length but its apex pointed. Pronotum transverse, $11 / 4$ times as broad as long, anterior margin nearly straight, lateral margin distinctly rounded, widest almost at middle, slightly narrowed anteriorly and posteriorly, basal margin distinctly rounded posteriorly, dorsal surface convex, with a pair of lateral transverse furrows starting from anterior corners, rugosely impressed by strong punctures, and interstices of punctures smooth, shining. Scutellum subtriangular, smooth, shining, impunctate. 
Elytron with lateral margin rounded, surface strongly and closely punctate, and interstices of punctures smooth, shining.

Length : $3.8-4.5 \mathrm{~mm}$.

Holotype (BISHOP) : THAILAND : Chiengmai Prov., Doi Suthep, 1-15. iv. 1958, T. C. Maa. Paratopotypes : 2 exs., same data as the holotype (BISHOP, KIMOTO).

This new species somewhat resembles Liroetiella minor Kimoto, but differs in having thorax entirely blackish.

\section{Liroetiella tibialis n. sp. Fig. $29 \mathrm{c}$}

Generally yellowish brown, legs with tibiae and tarsi pitchy black.

Head with vertex smooth, shining, sparsely impressed by minute punctures, interocular space distinctly wider than transverse diameter of single oculus, and interocular space distinctly wider than transverse diameter of single oculus, and interocular transverse impression distinct, frontal tubercule subtriangular, contigous, distinctly raised, surface smooth, shining. Antenna slender, nearly as long as body length ; first segment long, second shortest, nearly $1 / 4$ as long as first, third twice as long as second, fourth twice as long as third, fifth slightly shorter than fourth, fifth to ninth subequal to each other in length and shape, tenth slightly shorter than ninth, eleventh subequal to tenth in length but its apex pointed. Pronotum transverse, $12 / 3$ times as broad as long, anterior margin nearly straight, lateral margin distinctly rounded, widest almost at middle, slightly narrowed anteriorly and posteriorly, basal margin distinctly rounded posteriorly, dorsal surface convex, with a pair of lateral transverse furrows starting from anterior corners, sparsely impressed by fine punctures, and interstices of punctures smooth, shining. Scutellum subtriangular, smooth, shining, impunctate. Elytron with lateral margin rounded, surface more distinctly and closely punctate than pronotum, and interstices of punctures smooth, shining.

Length : 8.4-10.0 mm.

Holotype (BISHOP) : LAOS : Khammouane Prov., Phon Tiou, 17. v. 1965, Native collr. Paratopotypes : 4 exs., same data as the holotype (BISHOP). Paratypes : THAILAND : Chiengmai, 1,100-

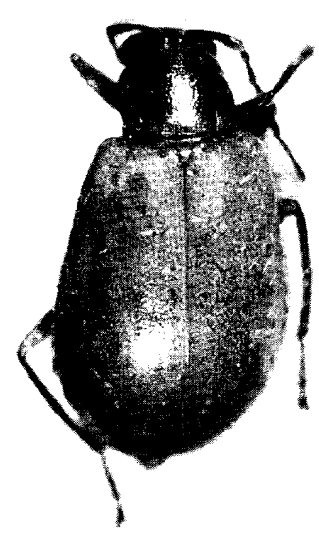

a

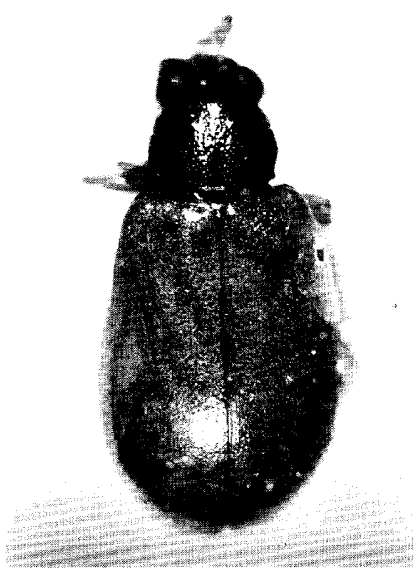

b

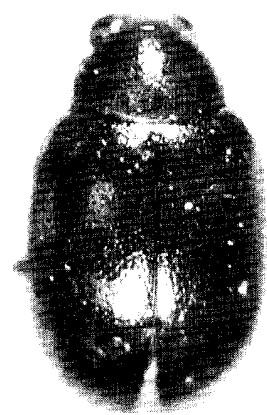

c

Fig. 31. a, Liroetiella bicolor n. sp. ; b, L. nigricollis n. sp. ; c, Desbordesius piceus n. sp. 
1,500 m, no data, 1966, J. H. Sedlacek (BISHOP). LAOS : Khammouane Prov., Phon Tiou, 1 ex., 30. iv. 1965, 4 exs., 28. v. 1965, 3 exs., 8. vi. 1965, at light, 1 ex., 9. vi. 1965, at light, 1 ex., 28. vi. 1965, Native collr ; Borikhane Prov., Pakkading, 1 ex., 10. v. 1965, Native collr ; Vientiane Prov., Vientiane, 1 ex., 15. iii. 1966, J. H. Sedlacek ; Vientiane Prov., Ban Van Eue, 1 ex., 16. iii. 1966, 1 ex., 15. v. 1966, Native collr (BISHOP, KIMOTO) ; Umgeb. Vientiane, 1 ex., iii.-vi. 1963 (MUNCHEN).

This new species resembles Liroetiella minor Kimoto, but differs by the longer body and having tibiae and tarsi pitchy black.

\section{Genus Desbordesius Laboissière}

Desbordesius Lab., 1933, Bull. Mus. Hist. Nat. Paris, ser. 2, 5 : 203 (type : Desbordesius laevigatus Laboissière ; Vietnam).

\section{Key to species of Desbordesius}

1 Dorsal surfaces with pronotum sparsely covered by fine punctures and elytron more strongly punctate ; generally pitchy black, basal segments of antenna reddish brown, legs with most of anterior legs and part of middle leg brownish ; length $1.8-3.0 \mathrm{~mm} \cdots \cdots \cdot$ laevigatus Dorsal surfaces with pronotum rather closely impressed by distinct punctures and elytron distictly and rather closely punctate ; generally pitchy black, antenna yellowish brown with three or four apical segments pitchy black, legs yellowish brown with femora generally pitchy black ; length $2.1-2.3 \mathrm{~mm}$

piceus

\section{Desbordesius laevigatus Laboissière}

Desbordesius leavigatus Lab., 1933, Bull, Mus. Hist. Nat., Paris, 5 : 204, fig. (Tonkin: Hoa-Binh ; HAMBURG) ; 1934, Ann. Ass. Nat. Levallois-Perret, 21 : 137 (Tonkin: Hoa-Binh).

Distribution : Thailand, Vietnam.

Material examined. THAILAND : Trang Prov, Khaophappha, Khaochong, 200-400 m, 1 ex., 10. i. 1964, G. A. Samuelson ; Banna, Chawang, nr. Nabon, 70 m, 1 ex., 6. ix. 1958, J. L. Gressitt (BISHOP) ; Chiengmai Prov., Doi Suthep, 12 exs., 18. vi. 1965, K. Morimoto \& Y. Miyatake ; Chiengmai Prov., Doi Pui, 1 ex., 17. vi. 1965, K. Morimoto ; Khao Chong, nr. Trung, 2 exs., 25. vi. 1965, K. Morimoto (KU). VIETNAM : Dilinh (Djiring), 1,200 m, 2 exs., 22-28. iv. 1960, L. W. Quate ; Blao (Balao), 500 m, 2 exs., 14-21. x. 1960, C. M. Yoshimoto ; Fyan, 900-1,200 m, 18 exs., 11. vii.-9. viii. 1961, N. R. Spencer ; Dalat, 6 km S., 1,400-1,500 m, 2 exs., 12. ix. 1960, J. L. Gressitt, 3 exs., 9. vi.-7. vii. 1961, N. R. Spencer ; Dalat, 1,500 m, 3 exs., 26-27. ix. 1960, C. M. Yoshimoto ; 20 km S., Dalat, 1,300-1,500 m, 2 exs., 11-12. ix. 1960, J. L. Gressitt ; 20 km N. of Pleiku, 1 ex., 9. v. 1960, L. W. Quate ; Kontum, N. of Pleiku, 550 m, 1 ex., 13. v. 1960, L. W. Quate (BISHOP).

\section{Desbordesius piceus n. sp. Fig. 31c}

Generally pitchy black, antenna yellowish brown with apical three or four segments pitchy black, legs yellowish brown with femora generally pitchy black.

Head with vertex smooth, shining, sparsely impressed by minute punctures, interocular space distinctly narrower than transverse diameter of single oculus, and interocular transverse impression distinct, frontal tubercle subtriangular, contiguous, distinctly raised, surface smooth, shining, impunctate. Antenna robuster, in preapical segments $1 / 3$ as wide as long, and nearly $2 / 3$ as long as body 
length ; first segment long, robust, club-shaped, second nearly half as long as first, third slightly shorter and slenderer than second, fourth nearly twice as long as third, fifth subequal to fourth in length and shape, sixth slightly shorter than fifth, sixth to tenth subequal to each other in length and shape, eleventh $12 / 3$ times as long as tenth and its apex pointed. Pronotum transverse, $12 / 3$ times as broad as long, anterior margin slightly rounded posteriorly, lateral margin distinctly rounded, widest almost at middle, and distinctly narrowed anteriorly and posteriorly, basal margin distinctly rounded posteriorly, dorsal surface convex side to side, smooth, shining, rather closely impressed by distinct punctures. Scutellum subtrianglar, smooth, shining, impunctate. Elytron strongly convex, with lateral margin distinctly rounded, surface distinctly and rather closely punctate and their interstices smooth, shining.

Length: 2.1-2.3 $\mathrm{mm}$.

Holotype (Type No. 2700, Kyushu Univ.) : THAILAND : Pupan National Park (Nakhom Phanom), 26. ii. 1965, K. Yasumatsu. Paratopotypes: 4 exs., same data as the holotype (KU).

This new species resembles Desbordesius Zaevigatus Laboissière, but differs in having the dorsal surfaces more closely and strongly punctate.

\section{Genus Atrachya Dejean}

Atrachya Dejean, 1837, Cat. Col., ed. 3 : 401 (type : Gallerucamenetriesii Faldermann ; N. China ; monobasic).-Gressitt \& Kimoto, 1963, Pac. Ins. Mon., 1B: 587.

Luperodes Motschulsky, 1858, Etud. Ent., $7: 102$ (type : Luperodes alboplagiatus Motschulsky ; Ceylon).-Ogloblin, 1936, Fauna USSR, 26, $1: 306,374$.

Iphidea Baly, 1865, Ent. Monthly Mag., 2 : 127 (type :Iphidea discrepans Baly ; Japan).

\section{KEY TO SPECIES of Atrachya}

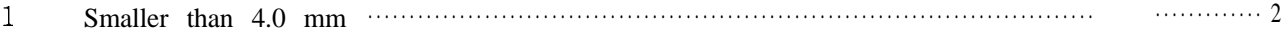

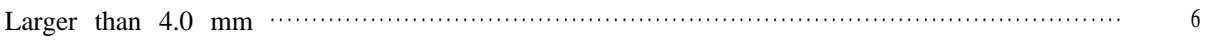

2(1) Pronotum with a pair of distinct depressions, and covered by distinct punctures …......... 3 Pronotum convex side to side and covered by minute punctures ; generally yellowish brown, elytron black with basal and postmedian markings yellowish brown, in some specimen these markings united ; vertex, meso- and metathorax black; antenna large-

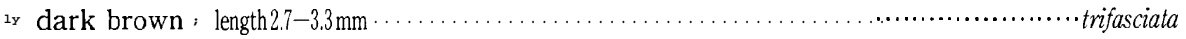

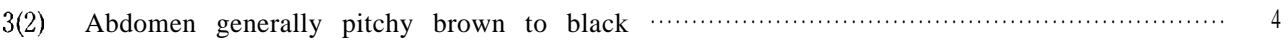

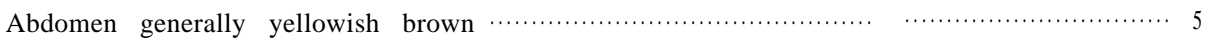

4(3) Head black; generally black; elytron black with a brownish large median marking from sutural to lateral margins ; prothorax yellowish brown, legs yellowish brown with apical part of tibiae and entire tarsi pitchy brown ; antenna generally dark

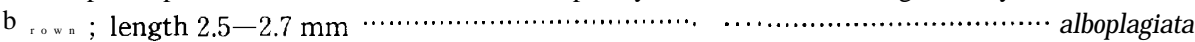

Head and prothorax yellowish brown; elytron pitchy black with an elongate marking on disc yellowish brown; legs yellowish brown with tibiae and tarsi more or less infuscate; antenna pitchy black with basal segments brownish; length 3.3-3.8 $\mathrm{mm}$

5(3) Pronotum $13 / 4$ times as wide as long ; elytron yellowish brown with basal margin and base of sutural and lateral margins, together with basal, postmedian and apical markings, blackish ; in most dark colored specimen elytral margins entirely blackish ; generally yellowish brown with vertex and meso- and metathorax pitchy black; in 
some specimen fifth abdominal segment partly infuscate; length $2.3-3.2 \mathrm{~mm} \cdots \cdots$ chiengmaica Pronotum $11 / 2$ times as wide as long; elytron yellowish brown with basal margin together with base of lateral and sutural margins blackish ; generally yellowish brown, with vertex and meso- and metathorax pitchy black; fifth abdominal segment black ; length 2.7-3.2 $\mathrm{mm}$

6(1) Dorsal surfaces sparsely impressed by fine punctures; in male elytron with a short elongate fovea behind scutellum

Dorsal surfaces closely impressed by strong punctures ; in male elytron without any distinct fovea ; generally bluish black with abdomen brownish ; length 4.8-5.3 $\mathrm{mm}$

7(6) Legs largely brownish ; generally yellowish brown, elytron with humeral and post median markings, meso- and metathorax, tibiae and tarsi blackish ; length 4.0-5.0

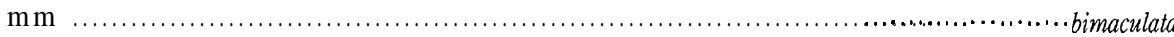

Legs largely blackish ; generally yellowish brown with meso- and metathorax and abdomen black; antenna blackish with basal segments brownish ; elytron with an illdefined blackish marking behind scutellum in most specimens, and in some cases surface of elytron infuscate in various degrees ; length $5.0 \mathrm{~mm}$................ impressipennis

\section{Atrachya alboplagiata (Jacoby), new combination}

Charaea ? alboplagiata Jac., 1892, Ann. Mus. Civ. Genova, 32 : 975 (Burma ; GENOVA).

Distribution : Burma, Thailand.

This species is listed neither in Weise, 1924, Col. Cat Junk, part 78, Galerucinae, nor in Maulik, 1936, Fauna India, Galerucinae.

Material examined. THAILAND : Chiengmai Prov., Doi Pui, 1685 m, 1 ex., 17. vi. 1965, Y. Miyatake ; Chiengmai Prov., Doi Suthep, 1 ex., 18. viii. 1973, S. Nakao (KU).

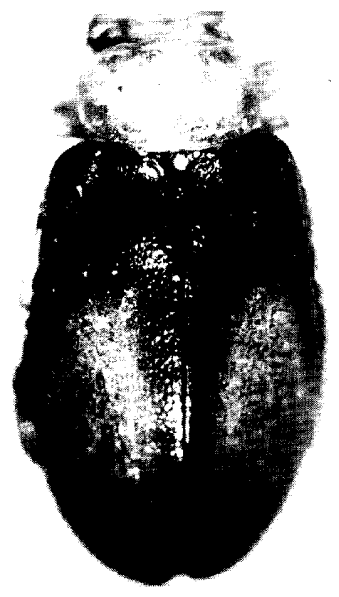

a

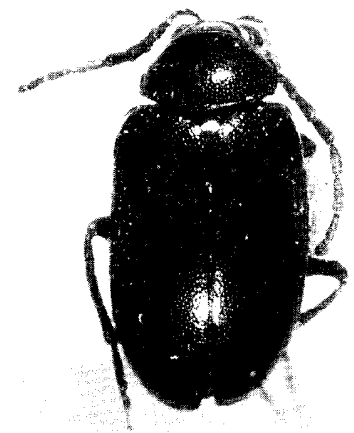

b

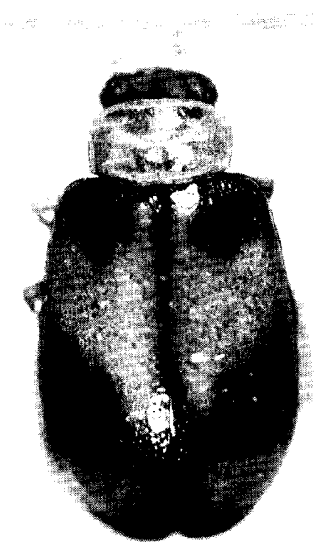

C

Fig. 32. a, Atrachya maeklangica n. sp. ; b, A. atridorsata n. sp. ; c, A. chiengmaica n. sp. 


\section{A trachya a tridorsa ta n. sp. Fig. $32 \mathrm{~b}$}

Generally bluish black, abdomen yellowish brown.

Head with vertex smooth, shining, sparsely impressed by fine punctures; interocular space distinctly wider than transverse diameter of single oculus, and interocular transverse impression distinct, frontal tubercle subtriangular and distinctly raised. Antenna slender, nearly $2 / 3$ as long as length of body ; first segment long, robust, second shortest, nearly $2 / 3$ as long as first, third $11 / 4$ times as long as second, fourth $21 / 2$ times as long as third, fifth subequal to fourth in length and shape, sixth slightly shorter than fifth, sixth to tenth subequal to each other in length and shape, eleventh slightly longer than tenth and its apex pointed. Pronotum transverse, $14 / 5$ times as broad as long, anterior margin slightly rounded anteriorly, lateral margin distinctly rounded, widest almost basal margin, and narrowed anteriorly, basal margin strongly rounded posteriorly, dorsal surface convex side to side, without any trace of depression laterally, rather closely impressed by strong punctures, and interstices of punctures smooth, shining. Scutellum subtriangular, smooth, shining, impunctate. Elytron broader than prothorax, more strongly and closely punctate, and interstices of punctures smooth, shining.

Male : Fifth abdominal segment trilobed, median lobe generally flat. Female : Fifth abdominal segment entire.

Length : 4.8-5.3 mm.

Holotype (BISHOP) : LAOS : Muong Sing, NW of Luang Prabang, 650 m, 6-10. vi. 1960, L. W. Quate. Paratopotype : 1 ex., same data as the holotype (KIMOTO). Paratypes : LAOS : Ban Van Eue, 20 km W. of Phou-kow-kuei, 1 ex., 15-31. v. 1965, Native collr (BISHOP). VIETNAM : Fyan, 900-1000 m, 1 ex., 11. vii.-9. viii. 1961, N. R. Spencer (BISHOP).

This new species resembles Atrachyaerythrocephala (Baly), from Punjab, but differs in having the abdomen yellowish brown.

\section{Atrachya bimaculata (Hornstedt)}

Chrysomela bimaculata Hornst., 1788, Schriften Ges. Naturf. Freunde, $8: 4$, fig. (Sumatra, Java).

Cnecodes bisignatus Motschulsky, 1858, Etud. Ent., 7 : 99 (Burma).-Weise, 1982, Dtsche Ent. Z., $1892: 400$ (= bimaculata).

Luperodes scutellatus Jacoby, 1884, Notes Leyden Mus., $6: 46$ (Sumatra); 1889, Ann. Mus. Civ. Genova, 27 : 284 (Sumatra, Isl. of Nias) ; 1896, ibid., $36: 466$ (as var. of bimaculatus).-Maulik, 1936, Fauna India, Galeruc. : 401 (as var. of bimculatus).

Monolepta bimucuclata : Jacoby, 1889, Ann. Mus. Civ. Genova., $27: 284$ (Isl. of Nias).-Maulik, 1936, Fauna India, Galeruc. : 401 (Burma, Ceylon, Sumatra, Java).

Luperodes bisignatus : Allard, 1889, Ann. Soc. Ent. France, 1889:310 (Mytho).

Candezea sculpta Blackbum, 1890, Proc. Linn. Soc. N. S. W., ser. 2, $5: 363$ (Queensland) ; 1896, Trans. Roy. Soc. S. Aust., $20: 89$ (= bimaculata).

Candezea bimaculata: Blackburn, 1896, Trans. Roy. Soc.S. Aust., 20 : 89 (New Guinea).

Cnecodes bimaculatus: Weise, 1892, Dtsche Ent. Z., 1892: 400 (notes).

Luperodes (Cnecodes) bisignatus: Jacoby, 1896, Ann. Mus. Civ. Genova, $36: 466$ (Sumatra).

Distribution : Ceylon, Burma, Thailand, Sumatra, Java, New Guinea, Australia.

Material examined. THAILAND: Samut Prakan, 2 exs., 18. x. 1935; Bangkok, 2 exs., 20. ix. 1949 (BANGKHEN). 


\section{Atrachya chiengmaica n. sp. Fig. 32c}

Generally yellowish brown, head with vertex pitchy black, elytron yellowish brown, with basal margin and base of sutural and lateral margins, together with basal, postmedian and apical markings blackish ; in most dark colored specimen elytral margins entirely blackish ; antenna pitchy black with three or four basal segments yellowish brown, ventral surfaces with meso- and metathorax pitchy black.

Head with vertex smooth, shining, sparsely impressed by minute punctures, interocular space distinctly wider than transverse diameter of single oculus, and interocular transverse impression distinct, frontal tubercle subtriangular and distinctly raised. Antenna slender, nearly $2 / 3$ as long as length of body ; first segment long, second shortest, nearly $1 / 3$ as long as first, third subequal to second in length but more slender, fourth $12 / 3$ times as long as third, fifith subequal to fourth in length and shape, sixth slightly shorter than fifth, and sixth to tenth subequal to each other in length and shape, eleventh slightly longer than tenth and its apex pointed. Pronotum transverse, $12 / 3$ times as broad as long, anterior margin slightly rounded anteriorly, lateral margin slightly rounded, widest at $1 / 3$ from anterior margin, and slightly narrowed anteriorly and more strongly so posteriorly, basal margin strongly rounded posteriorly, dorsal surface convex, with a pair of deep depressions laterally, sparsely impressed by distinct punctures, and interstices of punctures smooth, shining. Scutellum subtriangular, smooth shining, impunctate. Elytron broader than prothorax, more strongly and closely punctate, and interstices of punctures smooth, shining.

Male : Fifth abdominal segment trilobed, median lobe generally flat. Female : Fifth abdominal segment entire.

Length: $2.3-3.2 \mathrm{~mm}$.

Holotype (Type No. 2701, Kyushu Univ.) : THAILAND : Chiengmai Prov., Doi Pui, 1,300 m, 17. vi. 1965, K. Morimoto. Paratypes : THAILAND: Chiengmai Prov., Doi Pui, 1,300 m, 1 ex., 17. vi. 1965, Y. Miyatake ; Chiengmai Prov., Doi Suthep, 2 exs., 18. vi. 1965, Y. Miyatake (KU) ;Doi Suthep, 1 ex., 1-15. iv. 1958, T. C. Maa (BISHOP).

This new species resembles Atrachya trifasciata (Jacoby), but differs in having pronotum with a pair of distinct depressions and more strongly punctate.

\section{Atrachya impressipennis (Jacoby)}

Luperodes impressipennis Jac., 1890, Ann. Mus. Civ. Genova, 32 : 950 (Burma ; GENOVA).

Monolepta impressipennis : Maulik, 1936, Fauna India, Galeruc. : 394 (Burma).

Distribution : Burma, Thailand, Laos.

Material examined. THAILAND: Mae Taeng, 1 ex., 16. viii. 1973, sweeping of paddy field, K. Yano (KU). LAOS : Sedon Prov., Paksong, 8 exs., 18. v. 1965, P. D. Ashlock (BISHOP).

\section{Atrachya indica (Jacoby), new combination}

Monolepta indica Jac., 1896, Ann. Soc. Ent. Belg., 40 : 296 (Kanara, Belgaum, S. Bombay ; BM). -Maulik, 1936, Fauna India, Galeruc. : 439 (India).

Distribution : India, Thailand.

Material examined. THAILAND : Chiengmai Prov., Chieng Dao, 18 exs., 15-16. vi. 1965, K. Morimoto \& Y. Miyatake (KU). 


\section{Atrachya maeklangica n. sp. Fig. $32 \mathrm{a}$}

Head and prothorax yellowish brown ; elytron pitchy black with an elongate marking on disc yellowish brown ; scutellum pitchy black ; antenna pitchy black with two or three basal segments yellowish brown; meso- and metathorax and abdomen pitchy brown to black ; legs yellowish brown with tibiae and tarsi more or less infuscate.

Head with vertex smooth, shining, sparsely impressed by minute punctures, interocular space distinctly wider than transverse diameter of single oculus, and interocular transverse furrow feebly impressed, frontal tubercle subtrianglar, contiguous, distinctly raised, surface smooth, shining. Antenna slender, nearly $3 / 4$ as long as body length ; first segment robust, club-shaped, second short, nearly $2 / 5$ as long as first, third $11 / 3$ times as long as second, fourth nearly twice as long as third, fifth slightly shorter than fourth, and fifth to ninth subequal to each other in length and shape, tenth slightly shorter than ninth, eleventh $11 / 3$ times as long as tenth and its apex pointed. Pronotum transverse, $12 / 3$ times as broad as long, anterior margin slightly narrowed anteriorly, lateral margin distinctly rounded, widest slightly before middle, slightly narrowed anteriorly and more strongly so posteriorly, basal margin distinctly rounded posteriorly, dorsal surface convex, with a pair of feeble depressions laterally, sparsely impressed by distinct punctures, and interstices of punctures smooth, shining. Scutellum subtriangular, smooth, shining, impunctate. Elytron with lateral margin rounded, surface more distinctly and closely punctate than pronotum, and interstices of punctures smooth, shining.

Male : Third antenna1 segment shortest, and nearly $2 / 3$ as long as second, fourth nearly twice as long as length of second and third combined ; fifth abdominal segment trilobed and its median lobe generally flat. Female : Fifth abdominal segment entire.

Length : 3.3-3.8 mm.

Holotype (Type No. 2702, Kyushu Univ.) : THAILAND: Mae Klang Water Fall, nr. Chom Thong, 1 ex., 11. vii. 1965, Y. Miyatake. Paratypes : THAILAND : Ban Tap Dua, Chieng Dao, 1 ex., 15. viii. 1973, K. Yano (KU) ; 1 ex., same data as the holotype but K. Morimoto (KIMOTO).

This new species somewhat resembles Atrachya alboplagiata (Jacoby), but differs in being the body length longer, and having the head entirely brownish.

\section{A trachya trifasciata (Jacoby), new combination}

Monolepta trifasciata Jac., 1896, Ann. Soc. Ent. Belg., 40 : 295 (Belgaum, S. Bombay ; BM). -Maulik, 1936, Fauna India, Galeruc. : 409 (India).

Distribution : India, Thailand.

Material examined. THAILAND : Chiengmai Prof., Fang (Agr. Exp. Station), 600 m, 4 exs., 14. vi. 1965, P. D. Ashlock ; Chiengmai Prov., Chieng Dao, 2 exs., 15. vi. 1965, P. D. Ashlock (BISHOP); Chieng Dao, 14 exs., 23. iv. 1965, Y. Yoshiyasu ; Chieng Dao, Ban Tap Dua, 3 exs., 15. viii. 1973, K. Yano ; Mae Talai, nr. Chieng Dao, 1 ex., 3. vii. 1970, upland rice, K. Yano ; Fang, 4 exs., 21-22. viii. 1973, 2 exs., 22. viii. 1973, S. Nakao (KU).

\section{Genus M onolepta Chevrolat}

Monolepta Chevrolat, 1837, in Dejean, Cat. Col., ed. 3.. 408 (type : Crioceris bioculata Fabricius ; South Africa).-Maulik, 1936, Fauna India, Galeruc.: 373.-Ogloblin, 1936, Fauna USSR, 26,1 : 25, 313, 376.-Chajô, 1962, Phil. J. Sci., 91 (1-2) : 14, 109.-Gressitt\& Kimoto, 1963, Pac. Ins. Mon., 1B :602.-Kimoto, 1965, J. Fac. Agr. Kyushu Univ., 13 (3) : 389. 


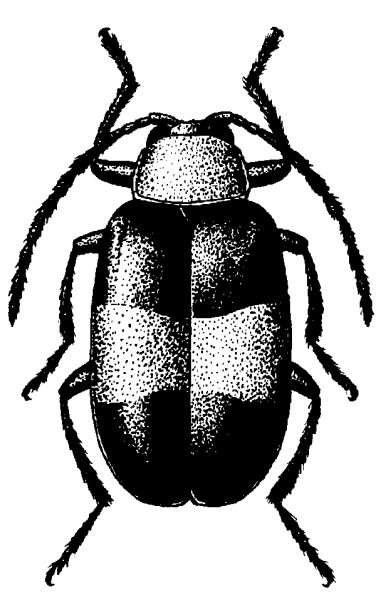

a
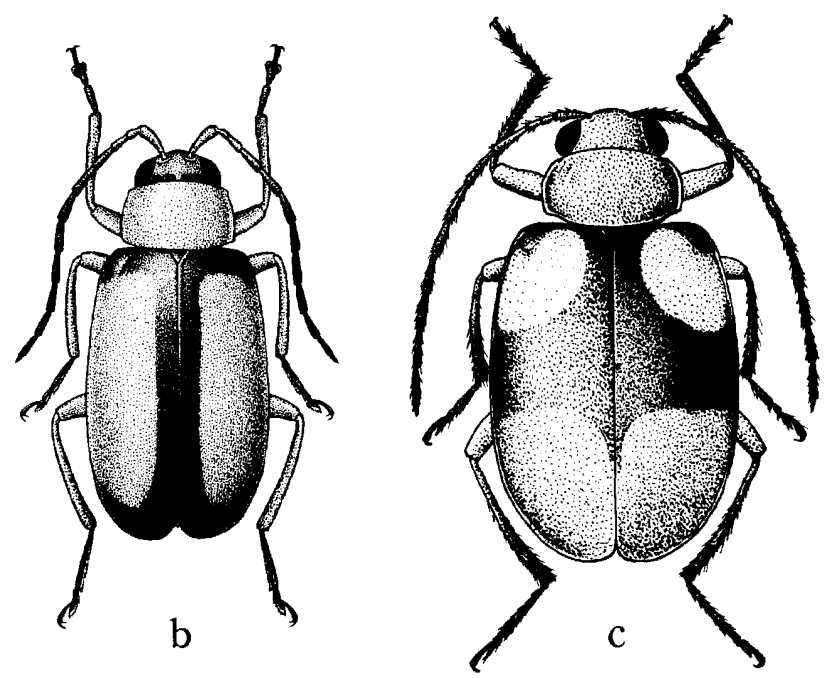

Fig. 33. a, Monolepta leechi Jacoby ; b, M. flavovittata Chen ; c, M. signafa (Olivier).

Cnecodes Motschulsky, 1858, Etud. Ent., 7 : 100.

Ochralea Clark, 1865, Ann. Mag. Nat. Hist., ser. 3, 15 : 144 (type :Ochralea nigricornis Clark).

-Chapuis, 1875, Gen. Col., $11: 235,336$.

Candezea Chapuis, 1879, Ann. Mus. Civ. Genova, $15: 24$.

Alaotra Duvivier, 1891, C. R. Soc. Ent. Belg., ser. 4, $21: 318$.

Damais Jacoby, 1908, Ann. Soc. Ent. Belg., 48: 118.

\section{KEY TO SPECIES OF Monolepta}

In male, elytron with a cavity on basal part at or near suture, or near middle of disc

In male, elytron without any distinct cavity

Elytron brownish with subapical and/or basal markings pitchy or blackish

Elytron brownish with three transverse bands blackish ; generally yellowish brown, with head and meso- and metathorax blackish ; in male elytron with a longitudinal cavity on outer part of disc ; length $5.0-5.7 \mathrm{~mm}$......................................... cavipennis

4(3) Pronotum slightly depressed laterally and sparsely impressed by minute punctures ; generally yellowish brown; head and pronotum reddish brown ; antenna generally pitchy brown, meso- and metathorax and legs blackish ; in male elytron with an elongate deep fovea contiguous to suture and with a distinct tubercle on outer margin of fovea, and apical $1 / 3$, humeral marking and basal $1 / 3$ of sutural and lateral margins and entire basal margin blackish ; in female elytron with basisutural marking blackish, and in some specimen this marking enlarged and connected with humeral marking ; in some specimen elytron entirely yellowish brown ; 
Pronotum with a pair of deep oblique depressions laterally and closely impressed by distinct punctures; generally yellowish to reddish brown; abdomen yellowish brown, elytron yellowish brown with basal and apical $1 / 3$ blackish ; in male elytron with a longitudinal shallow depression along suture and with a tuft of hairs behind scutellum ; length 5.3-6.0 mm

5(3) Pronotum subquadrate, $11 / 4$ times as wide as long, distinctly but not closely punctate ; generally reddish brown, elytron with a large round yellowish marking, which is almost completely surrounded by pitchy black, and connected with sutural margin ; abdomen yellowish brown ; in male elytron with an elongate fovea, somewhat oblique, contiguous to suture ; length $4.1 \mathrm{~mm}$. ..*......

Pronotum transverse, $11 / 2$ times as wide as long, widest almost at anterior margin, and its surface strongly and rather closely punctate; generally reddish brown, elytron with basal $2 / 3$ yellowish brown, and humerus, basal $1 / 4$ of sutural and basal half of lateral margins and entire basal margin pitchy brown, and apical half blackish brown and gradually become paler towards apex ; in male elytron with an elongate fovea contiguous to suture ; length $4.1-4.5 \mathrm{~mm}$.......................... vietnamica

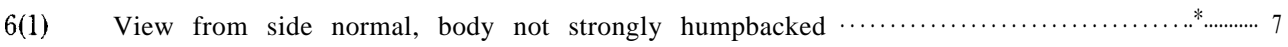
View from side, body strongly humpbacked, with great depth ; coloration variable : brown, pitchy brown, or pitchy black in various degrees, in most pale colored specimen entirely brown and most dark colored specimen, almost entirely pitchy

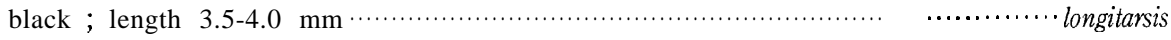

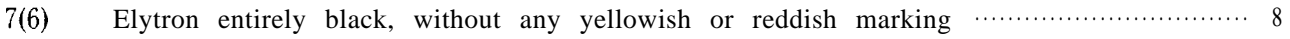

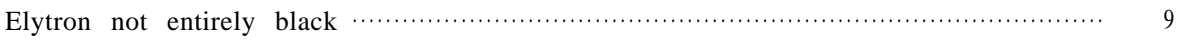

8(7) Legs entirely yellowish brown ; generally yellowish brown, ventral surfaces pitchy black; pronotum distinctly punctate, and with a pair of shallow depressions ; antenna relatively robust, and with second and third segments subequal to each

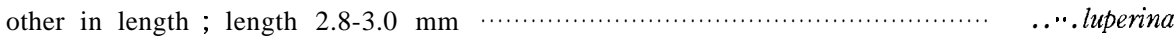
Legs entirely black; generally pitchy black, head and prothorax reddish brown ; length $2.7-3.5 \mathrm{~mm}$ ........... semiluperina

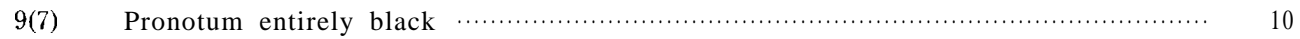

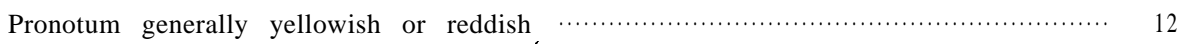

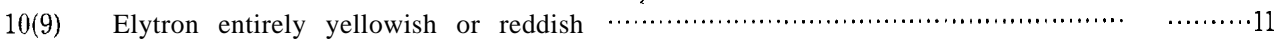

Elytron black with a large median marking yellowish brown; generally pitchy black, meso- and metathorax black, abdomen reddish brown; length 5.3-5.4 $\mathrm{mm}$

11(10) Legs entirely yellowish brown ; generally yellowish brown; head black, in some specimen vertex pitchy black medianly; length $4.2-4.8 \mathrm{~mm}$........................ langbianica Legs entirely black; generally reddish brown, head, prothorax, and antenna black ; length $3.8-3.9 \mathrm{~mm}$ subflavipennis

12(9) Elytron generally yellowish to reddish brown; generally dorsal surfaces brown-

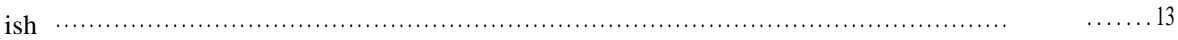

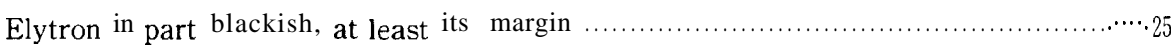

13(12) Interstices of eyes much narrower than transverse diameter of single eye $\cdots \cdots \cdots \cdots \cdots \cdots \cdots \cdot \cdots \cdot \cdots \cdots \cdot 14$ Interstices of eyes subequal to or much wider than width of transverse diameter of

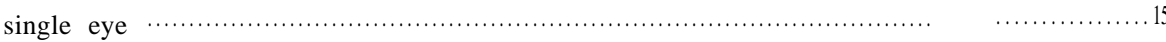

14(13) Prdnotum $12 / 3$ times as wide as long and its surface impressed by a pair of depressions laterally ; elytron impressed by strong punctures ; generally reddish 
brown ; in some specimen vertex blackish to pitchy brown in various degrees, and in some female specimen fifth abdominal segment blackish ; length 4.2-5.7

$\mathrm{mm}$ nigrifrons

Pronotum $11 / 3$ times as wide as long, and convex side to side ; elytron impressed by finer punctures; elytral margin widely brownish basally ; generally yellowish brown, elytron with reddish brown basally ; length $5.7-7.5 \mathrm{~mm} \ldots \ldots \ldots \ldots \ldots \ldots \ldots$ rubrobasali $\mathrm{s}$

15(13) Legs brownish with tibiae and tarsi blackish …................................................ 16

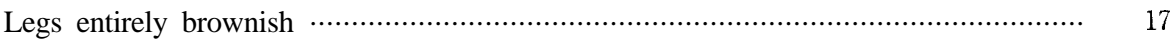

16(15) Smaller ; punctures of dorsal surfaces stronger ; generally yellowish brown ; length

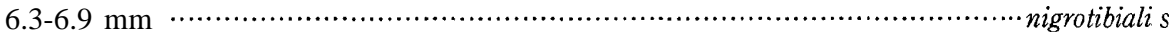
Larger ; punctures of dorsal surfaces finer ; generally ochraceous ; length 9.3-12.0

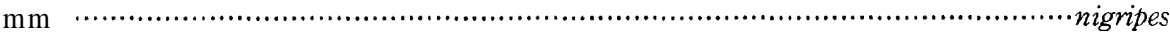

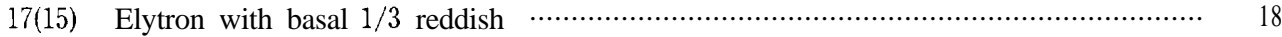

Elytron generally brownish, in some cases basal margin narrowly reddish ….................19

18(17) Elytron brownish with basal $1 / 3$ reddish ; generally reddish brown, antenna generally blackish ; length $4.8 \mathrm{~mm}$ ………....................................................... hagen $i$

Elytron with basal $1 / 3$ and apical $1 / 3$ reddish brown and median $1 / 3$ yellowish brown ; generally yellowish brown, head black, metathorax and posterior leg red-

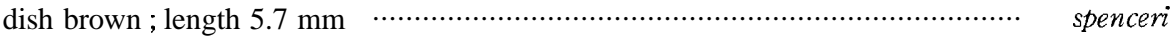

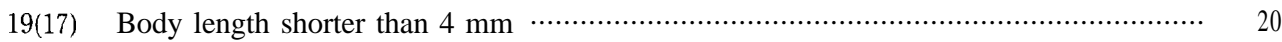

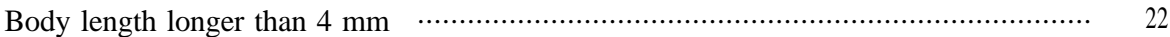

20(19) Pronotum convex side to side, without any trace of depression …….......................... 21 Pronotum with a pair of depressions laterally ; generally yellowish brown, antenna dark brown, in some specimen elytron reddish brown ; length $3.0-3.3 \mathrm{~mm} \cdots \cdots \cdots \cdots$ severin $i$

21(20) Body subparallel-sided ; pronotum sparsely impressed by minute punctures, and elytron more distinctly punctate; antenna yellowish brown with apical segments slightly infuscate ; length $3.0-4.0 \mathrm{~mm}$

Body oval ; dorsal surfaces of prothorax and elytron rather closely and strongly punctate; generally yellowish brown; antenna pitchy black with basal segments

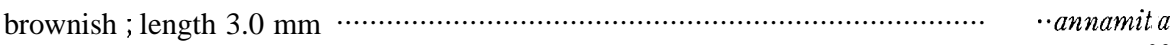

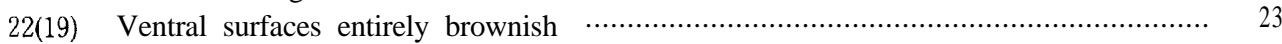
Ventral surfaces brownish with metathorax black ; generally yellowish brown ;

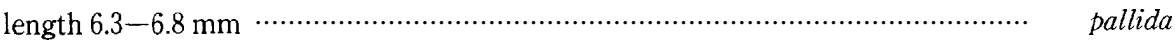

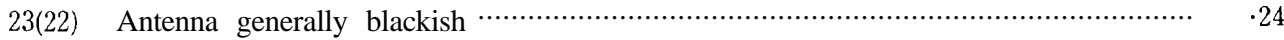

Antenna generally brownish ; generally yellowish brown, in some specimen elytron with $b_{\mathrm{asa}} 1$ margin reddish ; length $4.2-5.0 \mathrm{~mm}$............................................pallidula

24(23) Antenna blackish with apical four or five segments brownish ; generally yellowish brown ; length 4.2-5.0 $\mathrm{mm}$ ……...........................................................pallidulella Antenna blackish with basal four segments brownish ; generally yellowish brown ;

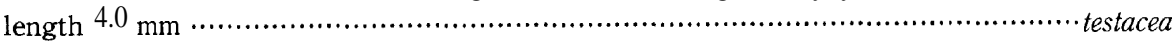

25(12) Elytron brownish with basal and apical margins narrowly blackish, and sutural and lateral margins generally brownish, without any discal stripe or marking; generally

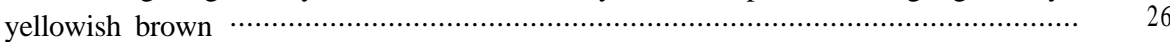
Not as above combination of characters ……….............................................28

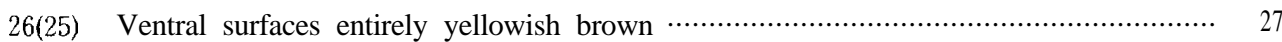
Ventral surfaces with metathorax blackish; generally yellowish brown, antenna generally pitchy black, tarsi partly infuscate ; length $3.3-4.1 \mathrm{~mm}$........................fyanic $a$ 
27(26) Larger ; pronotum more transverse, nearly $12 / 3$ times as wide as long; generally yellowish brown with tibiae and tarsi pitchy brown to black; length $7.2-9.3$

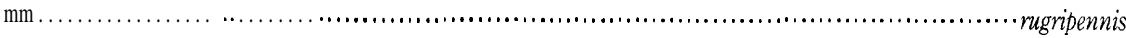

Smaller ; pronotum more elongate, nearly $11 / 2$ times as wide as long ; generally yellowish brown with antenna, tibiae and tarsi pitchy brown to black; length 5.4$6.3 \mathrm{~mm}$

banmethuotica

28(25) Elytron brownish with basal, lateral, sutural and apical margins narrowly blackish,

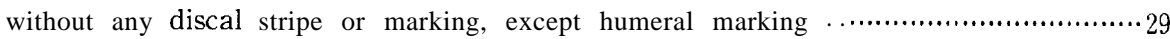

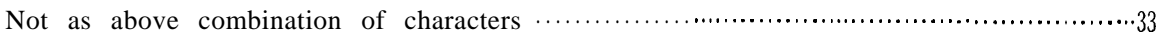

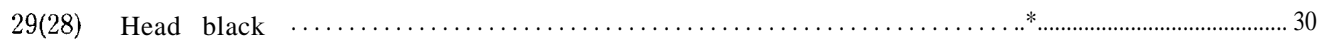

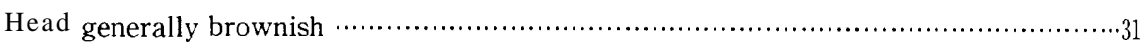

30(29) Smaller; abdomen pitchy black with fifth abdominal segments brownish ; elytron yellowish brown with margins, together with humerus black to pitchy brown, and basal part of sutural margin somewhat widely blackish; prothorax yellowish brown, meso- and metathorax pitchy black ; antenna generally pitchy black; legs yellowish brown with tibiae and tarsi somewhat infuscate; length $2.7 \mathrm{~mm} \cdots$ semihumeralis Larger; abdomen yellowish brown with apex of fifth segment and middle of pygidium blackish ; elytron yellowish brown with margins somewhat widely blackish ; prothorax yellowish brown, meso- and metathorax and antenna black ; legs yellowish brown with tibiae and tarsi black, and dorsal surface of anterior femur

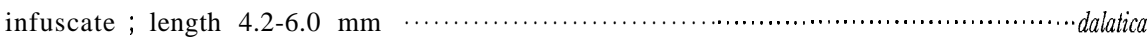

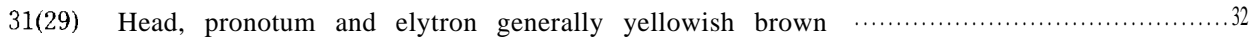
Head and pronotum reddish brown; generally yellowish brown, elytron with margins, including humerus, entirely blackish, ventral surface with meso- and metathorax slightly infuscate, antenna generally black, legs with tibiae and tarsi black-

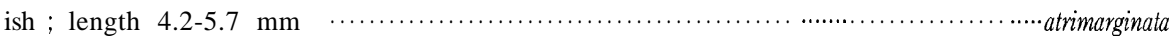

32(31) Ventral surfaces entirely brownish; generally yellowish brown with vertex,

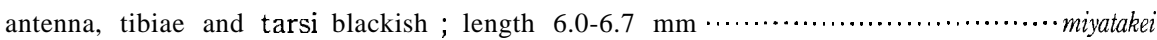
Ventral surfaces with metathorax and fifth abdominal segment blackish ; generally yellowish brown, elytron with humerus blackish, tibiae and tarsi pitchy black ;

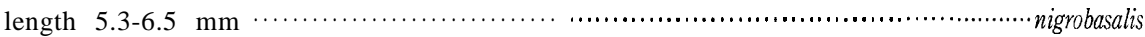

33(28) Elytron of two colors ; ground color black with single band or marking of yellowish

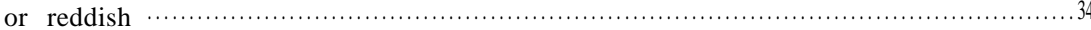

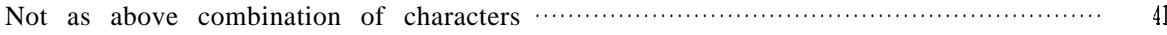

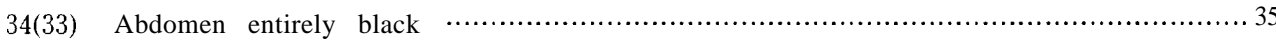

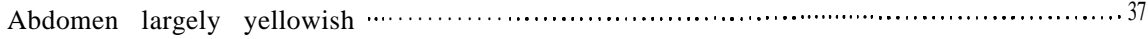

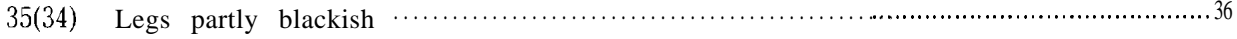
Legs entirely yellowish brown; generally yellowish brown with meso-and meta-

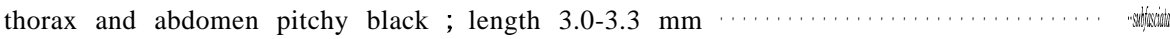

36(35) Legs blackish with anterior leg brownish; head, prothorax and antenna yellowish brown, meso- and metathorax and abdomen blackish ; length $3.8-5.1 \mathrm{~mm}$............. leechii Legs yellowish brown with middle and posterior tibiae and tarsi blackish; head, prothorax and antenna yellowish brown, meso- and metathorax and abdomen blackish ; length $3.5-4.2 \mathrm{~mm}$................................................. birmanensis

37(34) Elytron black with an elongate yellowish marking covering about $3 / 4$ of length and $4 / 5$ of width of disc and free from lateral and sutural margins $\ldots \ldots \ldots \ldots \ldots \ldots \ldots \ldots \ldots \ldots, \ldots \ldots$ Elytron with a transverse band covering from lateral margin to sutural mar- 


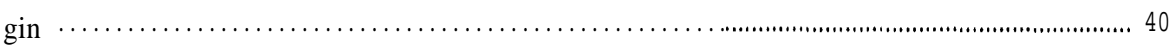

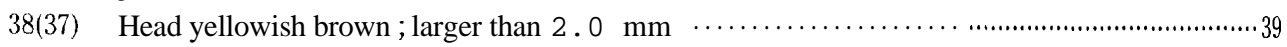

Head black ; generally yellowish brown ; meso- and metathorax and basal part of first abdominal segment, tibiae and tarsi blackish; antenna pitchy black with basal

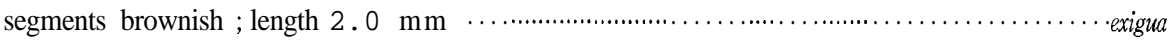

39(38) Pronotum closely impressed by strong punctures ; generally yellowish to reddish brown ; meso- and metathorax black ; antenna pitchy black with basal segments

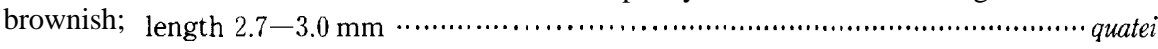

Pronotum sparsely impressed by minute punctures; head, prothorax and abdomen yellowish brown with fifth abdominal segment blackish ; meso- and metathorax

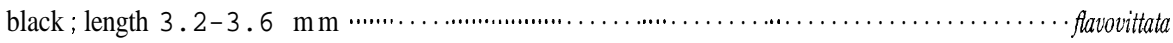

40(37) Head yellowish brown ; generally yellowish brown, with meso- and metathorax and

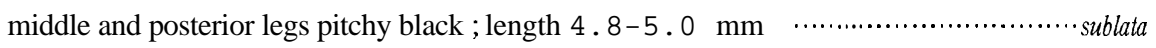
Head black ; prothorax yellowish brown, meso- and metathorax pitchy brown, abdomen reddish brown, antenna and legs pitchy brown to black; length 5.4 mm

41(33) Elytron with four or six bands of black, red and yellow; never with discal spots,

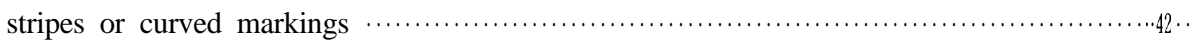

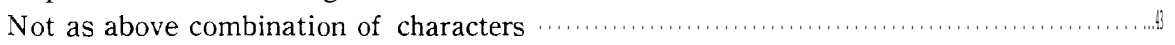

42(41) Elytron with four bands of black, red and yellow ; band one black, rather narrow ; band two red, occupying almost basal half ; band three yellowish ; band four black, covering apex; head, thorax and abdomen almost entirely reddish or brownish ; antenna and legs largely pitchy brown to black ; length 5.4-6.0 $\mathrm{mm}$ …................ zonalis Elytron with six bands of black, red and yellow ; band one black, rather narrow ; band two red, twice as wide as band one ; band three black, curved interiorly ; band four yellowish, widest ; band five black ; band six reddish brown, covering apex ; head, prothorax and antenna yellowish brown, meso- and metathorax, and middle and posterior legs pitchy black, abdomen reddish brown; length 4.5-5.0

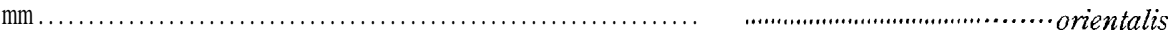

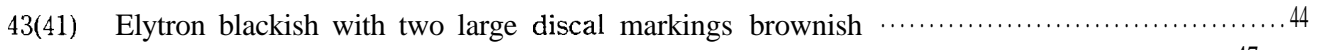

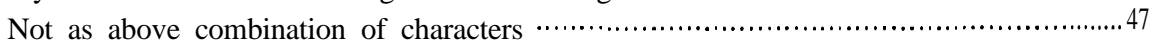

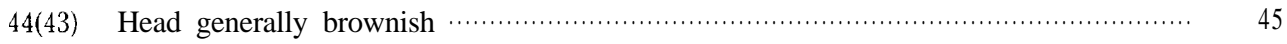

Head with vertex pitchy black ; generally reddish brown, meso- and metathorax blackish ; antenna and most of tibiae and tarsi pitchy brown ; length 3.9-4.2 $\mathrm{mm}$ quadrinotata

45(44) Elytron with basal marking closely situated to basal margin ; generally yellowish to reddish brown, with meso- and metathorax blackish $\ldots \ldots \ldots \ldots \ldots \ldots \ldots \ldots \ldots \ldots \ldots \ldots \ldots \ldots \ldots . \ldots \ldots$ Elytron with basal marking widely separated from basal margin ; generally reddish brown, and antenna and legs mostly pitchy black ; length $5.3-6.0 \mathrm{~mm}$.............. laosensis

46(45) Larger ; elytron with apex brownish ; length $4.5 \mathrm{~mm}$.............................pseudosignata Smaller ; elytron with apex blackish ; length $3.5-3.9 \mathrm{~mm}$............................. signata

47(43) Elytron with lateral, apical and sutural margins narrowly blackish $\ldots \ldots \ldots \ldots \ldots \ldots \ldots \ldots \ldots 48$ Elytron with lateral, apical and sutural margins in part brownish $\ldots \ldots \ldots \ldots \ldots \ldots \ldots \ldots \ldots . \ldots \ldots$

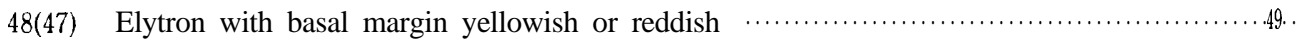
Elytron with basal, lateral, sutural and apical markings narrowly blackish, and with six discal markings $(2: 2: 2)$ blackish ; generally yellowish brown, head, metathorax and pygidium largely pitchy black, antenna, tibiae and tarsi pitchy brown ; length 
$4.2 \mathrm{~mm}$ wilsoni

49(48) Smaller ; elytron with a longitudinal blackish stripe on disc, in some specimen entirely brownish dorsally ; generally yellowish to reddish brown, with antenna,

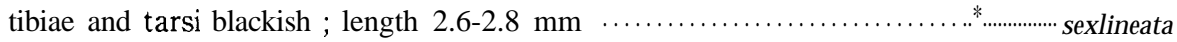
Larger ; elytron reddish basally ; head black; generally yellowish brown with fifth abdominal segment, tibiae and tarsi blackish; length $5.7-7.2 \mathrm{~mm}$ ..rondoni

50(47) Elytron generally brown with margins in part narrowly blackish, and without any

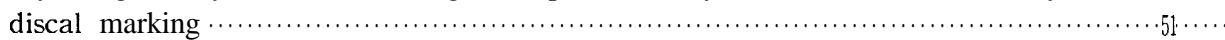

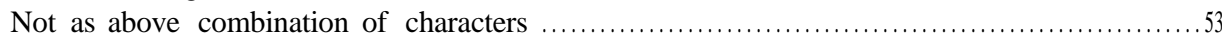

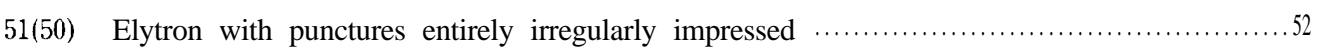
Elytron with punctures arranged in semiregular longitudinal rows, and their interstices longitudinally raised ; generally yellowish brown with metathorax blackish ; antenna generally pitchy black with basal segments brownish; length 3.0-4.1

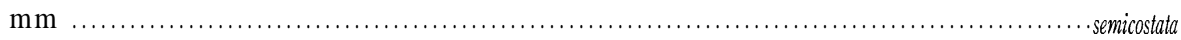

52(51) Elytron with basal, basal $2 / 3$ of lateral and most of sutural margins blackish; generally yellowish brown, meso- and metathorax and basal part of first to fourth abdominal segments blackish ; length 5.7-6.3 mm trangica Elytron with basal margin blackish; generally yellowish brown with metathorax blackish ; length 3.6-4.5 mm azumai

53(50) Elytron generally back with apical half to $1 / 3$ reddish brown and its boundary illdefined, and without any discal markings ……....................................................5 54 Elytron with discal markings 54

54(53) Antenna and legs entirely yellowish brown ; head, meso- and metathorax generally pitchy brown to black, and prothorax and middle of metathorax and abdomen yellowish to reddish brown; length $4.8 \mathrm{~mm}$ semiapicalis Antenna and legs largely pitchy black to black ; generally pitchy black with head and prothorax reddish brown ; length 3.2-3.6 $\mathrm{mm}$ -apicipennis

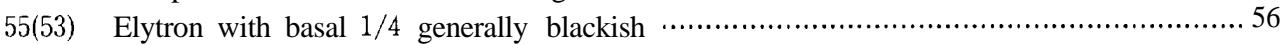

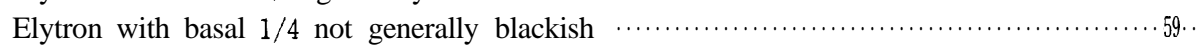

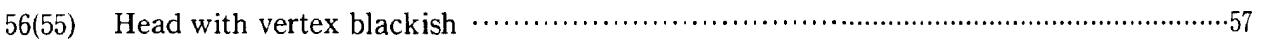
Head generally brownish …................................................................. 58

57(56) Legs entirely yellowish brown; head entirely black; generally yellowish brown with metathorax largely pitchy black, elytron with basal $1 / 3$ and large subapical

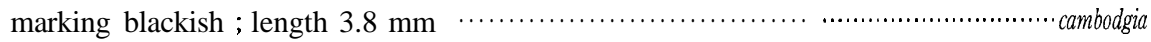
Legs yellowish brown with posterior femur black; head yellowish brown with vertex black; generally yellowish brown, elytron yellowish brown with basal $1 / 4$

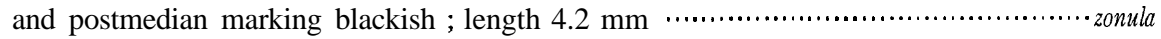

58(56) Antenna slenderer, entirely yellowish brown ; generally yellowish brown, with metathorax, in some specimen middle and posterior femora also, pitchy brown to black ; elytron with basal $1 / 4$ and a large subapical marking pitchy brown to black ; length $3.5-4.0 \mathrm{~mm}$ bifasciata Antenna robuster, generally pitchy brown with first and apical three segments brownish ; generally yellowish brown, elytron with basal $1 / 4$ and a large subapical marking blackish ; length $3.3 \mathrm{~mm}$ thailandica

59(55) Elytron with dark spots or postmedian band, or apical $1 / 3$ blackish... a......................... 60 Elytron with basal margin and basal $1 / 2$ of lateral and sutural margins, in some specimen entire lateral and apical margins also, rather broadly blackish, and 
another transverse or oblique black markings situated before middle, connected with sutural and lateral margins ; generally yellowish brown, metathorax always black,

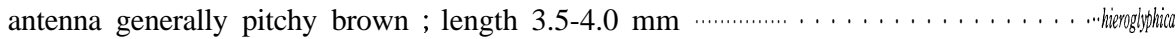

60(59) Elytral markings defined, with seven paris of markings, viz. scutellar, humeral, basal, lateral, postmedian, latero-apical and apical markings, together with basal part of sutural margin, blackish ; generally yellowish brown, pronotum with blackish lateral marking which is attached to lateral margin ; metathorax black, antenna generally pitchy black, legs in part infuscate ; length $5.7-6.8 \mathrm{~mm}$........ duodecimmaculata Elytral markings ill-defined, basal, apical and basal half of lateral and sutural margins, together with basal, postmedian and latero-subapical markings blackish ; generally yellowish brown, metathorax black, pronotum with lateral margin widely blackish in most specimens; head with vertex in part infuscate ; length $3.0-4.2$ $\mathrm{mm}$

\section{Monolepta annamita Laboissière}

Luperodes ferruginea Allard, 1889, Ann. Soc. Ent. France, 1889 : 311 (Hue; PARIS).

Monolepta annamita Laboissière, 1935, Ann. Ass. Nat. Levallois-Perret, 21 : 143 (new name for

Luperodes ferruginea Allard, nec Karsch).

Distribution : Thailand, Laos, Vietnam.

Material examined. THAILAND : Chiengmai Prov., Chiengdao, 1 ex., 15. vi. 1965, K. Morimoto (KU). LAOS : Vientiane Prov., Vientiane, 1 ex., 26. v. 1965, P. D. Ashlock (BISHOP). VIETNAM : Ban Me Thuot, 3 exs., 16-18. v. 1960, L. W. Quate ; 55 km NW of Phan Rang, 1 ex., 8-16. xi. 1960, C. M. Yoshimoto ; Quang Tri, 2 km N., 1 ex., 30. iv. 1970, A. R. Gillogly (BISHOP).

\section{Monolepta apicipennis n. sp. Fig. 36c}

Head and prothorax reddish brown ; elytron pitchy black with apical 1/3 reddish brown, antenna

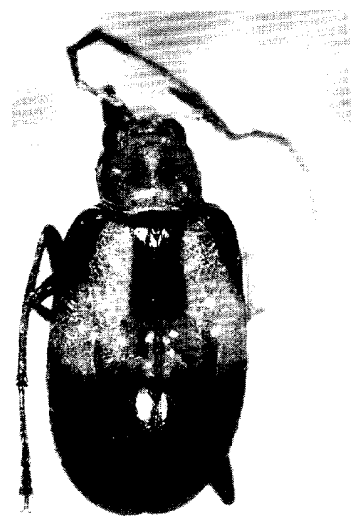

a

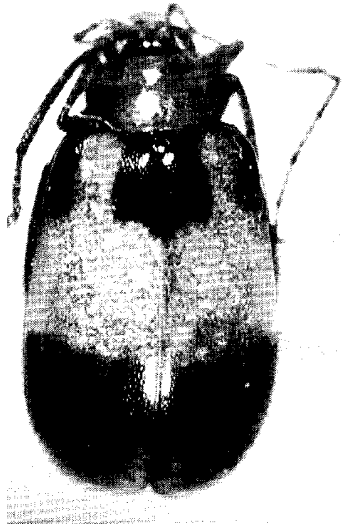

$\mathrm{b}$

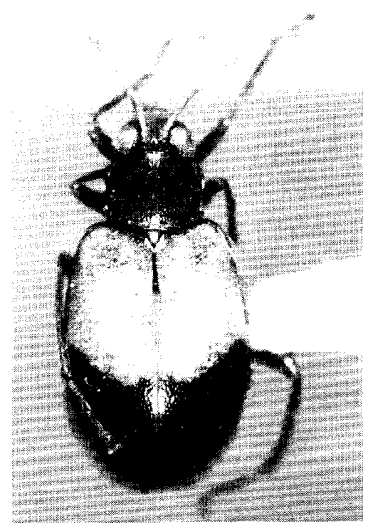

C

Fig. 34. a, Monolepta scutellaris n. sp.; b, M.marginipennis (Jacoby) ; c, M. vietnamica n. sp. 
pitchy black, meso- and metathorax, abdomen and legs black.

Head with vertex slightly wrinkled, sparsely impressed by minute punctures, interocular space distinctly wider than transverse diameter of single oculus, and interocular transverse impression distinct, frontal tubercle subtriangular and feebly raised. Antenna slender, nearly $2 / 3$ as long as length of body ; first segment long, second shortest, nearly $1 / 3$ as long as first, third subequal to second in length but slenderer, fourth nearly four times as long as third, fifth slightly longer than fourth, fifth to eighth subequal to each other in length and shape, ninth slightly shorter than eighth, ninth subequal to tenth in length and shape, eleventh subequal to tenth in length but its apex pointed. Pronotum transverse, $12 / 3$ times as broad as long, anterior margin nearly straight, lateral margin slightly rounded, widest at $1 / 3$ from anterior margin and slightly narrowed anteriorly and more strongly so posteriorly, dorsal surface convex side to side, without any trace of depression laterally, sparsely impressed by distinct punctures, and interstices of punctures smooth and shining. Scutellum subtriangular, smooth, shining, sparsely impressed by minute punctures. Elytron broader than prothorax, more strongly and closely punctate, and interstices of punctures smooth, shining.

Male: Fifth abdominal segment trilobed, median lobe generally flat. Female : Fifth abdominal segment entire.

Length : 3.2-3.6 mm.

Holotype (BISHOP) : VIETNAM : Mt. Lang Bian, 1,500-2,000 m, 19. v.-8. vi. 1961, N. R. Spencer. Paratopotype : 1 ex., same data as the holotype (KIMOTO). Paratypes : VIETNAM : Dilinh (Djiring), 1 ex., 1,200 m, 22-28. iv. 1960, L. W. Quate ; Dalat, 1,500 m, 1 ex., 29. iv.-4. v. 1960, S. Quate \& L. Quate ; Dalat, 6 km S., 1,400-1,500 m, 1 ex., 9. vi.-7. vii. 1961, N. R. Spencer (BISHOP).

This new species somewhat resembles Monoleptasemiapicalis Kimoto, but differs by the shorter body, and having antenna and the legs largely pitchy black.

Monolep ta a trimargina ta n. sp. $\quad$ Fig. 44b

Generally yellowish brown, head and pronotum reddish brown, elytron with margins, including humerus, entirely blackish, ventral surfaces with meso- and metathorax slightly infuscate, antenna generally black, with basal three segments more or less brownish, legs with tibiae and tarsi blackish.

Head with vertex smooth, shining, sparsely impressed by minute punctures, interocular space distinctly wider than transverse diameter of single oculus, and interocular transverse impression distinct, frontal tubercle subtriangular and distinctly raised. Antenna slender, nearly $2 / 3$ as long as body length ; first segment long, second shortest, nearly $1 / 3$ as long as first, third as long as second in length and slenderer, fourth nearly four times as long as third, fifth slightly longer than fourth, fifth to eighth subequal to each other in length and shape, ninth slightly shorter than eighth, ninth subequal to tenth in length and shape, eleventh slightly longer than tenth and subequal to eighth in length but its apex pointed. Pronotum transverse, $11 / 2$ times as broad as long, anterior margin nearly straight, lateral margin slightly rounded, widest almost at middle, slightly narrowed anteriorly and posteriorly, basal margin strongly rounded posteriorly, dorsal surface convex side to side, without any trace of depression laterally, sparsely impressed by distinct punctures, and interstices of punctures smooth and shining. Scutellum subtriangular, smooth, shining, impunctate. Elytron broader than prothorax, more strongly and closely punctate, and interstices of punctures impressed by minute punctures.

M ale : Fifth abdominal segment trilobed, median lobe generally flat. Female : Fifth abdominal segment entire.

Length : 4.2-5.7 $\mathrm{mm}$.

Holotype (BISHOP) : LAOS : Vientiane Prov., Ban Van Eue, 16. iii. 1966, Native collr.Paratypes : LAOS : Vientiane Prov., Ban Van Eue, 1 ex., 14-16. iv. 1965, J. L. Gressitt, 1 ex., 13. vii. 1965, 
1 ex., 31. vii. 1965, 1 ex., 15. xii. 1966, 1 ex., 1-15. ix. 1967, 2 exs., 31. xii. 1968, Native collr (BISHOP, KIMOTO).

This new species resembles Monolepta semihumeralis Kimoto, but differs in having the head yellowish brown, and the ventral surfaces yellowish brown with meso- and metathorax blackish.

Monolepta arumai n. sp. Fig. 39c

Generally yellowish brown, elytron with basal margins and basal part of lateral and sutural margins black ; antenna black with basal three or four segments brownish ; ventral surfaces with meso- and metathorax and fifth abdominal segment black.

Head with vertex smooth, shining, sparsely impressed by minute punctures, interocular space distinctly narrower than transverse diameter of single oculus, and interocular transverse impression distinct, frontal tubercle subtriangular and distinctly raised. Antenna slender, nearly $2 / 3$ as long as body length ; first segment long, second shortest, nearly half as long as first, third nearly as long as second in length, fourth nearly twice as long as third, fifth slightly longer than fourth, sixth slightly shorter than fifth, and sixth to ninth subequal to each other in length and shape, tenth slightly shorter than ninth, eleventh slightly longer than tenth and subequal to ninth in length but its apex pointed. Pronotum transverse, $11 / 2$ times as broad as long, anterior margin nearly straight, lateral margin slightly rounded, widest almost at middle, slightly narrowed anteriorly and posteriorly, basal margin strongly rounded posteriorly, dorsal surface convex, with a pair of deep oblique depressions laterally, distinctly impressed by large punctures, and interstices of punctures smooth, shining. Scutellum subtriangular, finely granulate, impunctate. Elytron broader than prothorax, more strongly and closely punctate, and interstices of punctures smooth, shining.

Male : Fifth abdominal segment trilobed, median lobe distinctly depressed as a whole. Female : Fifth abdominal segment entire.

length : 3.6-4.5 mm.

Holotype (RU) : THAILAND : Khon Kaen, 24. viii. 1980, S. Azuma. Paratopotypes : 6 exs., same data as the holotype (RU, KIMOTO). Paratypes : THAILAND : Khon Kaen, 1 ex., 20. xii. 1978, 1 ex., 22. xii. 1979, 1 ex., 7. x. 1981, 1 ex., 8. x. 1981, S. Azuma (RU) ; Chieng Dao, Ban Tap Dua, 3 exs., 15. viii. 1973, K. Yano ; Samptong, 1 ex., 17. viii. 1973, S. Nakao (KU) ; Chiengmai Prov., 2 exs., 28-31. iii. 1958, 3 exs., 29. iii.-vi. 4. 1958, 4 exs., 1-5. iv. 1958, T. C. Maa; Chiengmai, 1,200 m, 1 ex., 11. iv. 1966, J. Sedlacek (BISHOP).

This new species resembles Monolepta semicostata Kimoto, but differs in having elytron with punctures confusedly impressed and without any longitudinal costa dorsally.

\section{Monolepta banmethuotica n. sp. Fig. 40c}

Generally yellowish brown, elytron with basal, apical and basal part of lateral margins black ; head reddish brown; antenna pitchy brown with basal three segments yellowish brown, legs with tibiae and tarsi pitchy black.

Head with vertex smooth, shining, sparsely impressed by fine punctures, interocular space distinctly wider than transverse diameter of single oculus, and interocular transverse impression distinct, frontal tubercle subtriangular and distinctly raised. Antenna slender, nearly $2 / 3$ as long as body length; first segment long, second shortest, nearly $1 / 3$ as long as first, third $11 / 2$ times as long as second, fourth $21 / 2$ times as long as third, fifth slightly longer than fourth, fifth to eighth subequal to each other in length and shape, ninth slightly shorter than eighth, tenth subequal to ninth in length and shape, eleventh subequal to ninth in length but its apex pointed. Pronotum transverse, $11 / 2$ times 
as broad as long, anterior margin nearly straight, lateral margin slightly rounded, widest almost at middle, slightly narrowed anteriorly and posteriorly, basal margin strongly rounded posteriorly, dorsal surface convex, with a pair of deep oblique depressions laterally, closely impressed by distinct punctures, and interstices of punctures smooth and shining. Scutellum subtriangular, smooth, shining, impunctate. Elytron broader than prothorax, more strongly and closely punctate, and interstices of punctures smooth, shining.

Male : Fifth abdominal segment trilobed, median lobe generally flat. Female : Fifth abdominal segment entire.

Length : 5.4-6.3 mm.

Holotype (BISHOP) : VIETNAM : Ban Me Thuot, 500 m, 20-24. xii. 1960, L. W. Quate. Paratopotype : 1 ex., same data as the holotype (BISHOP). Paratypes : VIETNAM : Ban Me Thuot, 4 exs., 20-24. xii. 1960, L. W. Quate \& C. M. Yoshimoto ; Phon Tiou, 1 ex., 1-15. iv. 1965, J. L. Gressitt (BISHOP ; KIMOTO).

This new species resembles Monolepta fyanica Kimoto, but differs by the longer body and having the ventral surfaces entirely yellowish brown.

\section{M onolepta bifasciata (Hornstedt) Fig. 45c}

Chrysomela bifasciata Hornst., 1788, Schrif. Naturf. Freunde, Berlin, $8: 3$, fig. 6 (Java).

Cryptocephalus multicolor Gmelin, 1790, ed. Linnaeus, Systema Nat., 1(4) : 1712 (Java ; new name for Chrysomela bifasciata Hornstedt).

Crioceris quadrinotata Fabricius, 1801, Syst. Eleu., 1 : 460 (Sumatra).-Weise, 1910, Phil. J. Sci., ser. D, 5(4): 255 (= bifasciata)

Luperodes latefascia Motschulsky, 1858, Etud. Ent., 7 : 104 (Indes orientales).-Jacoby, 1884, Notes Leyden Mus., $6: 54$ (= bifasciata).

Monolepta rubrosignata Boheman, 1859, Eugenies Resa, 2(1): 182 (Manila).-Weise, 1910, Phil. J. Sci., ser. D, 5(4): 225 (= bifasciata).

Monolepta latefascia: Chapuis, 1875, Gen. Col., 11 : 236 (genus).

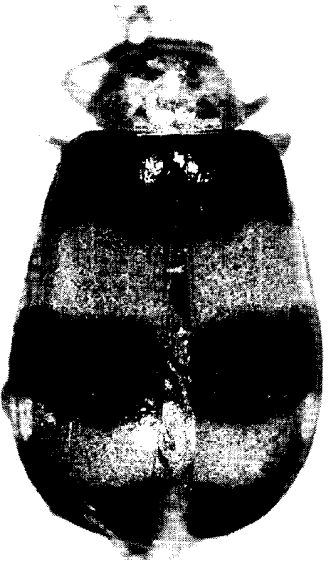

a

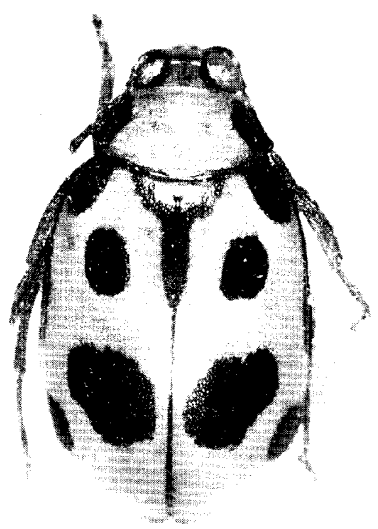

$\mathrm{b}$

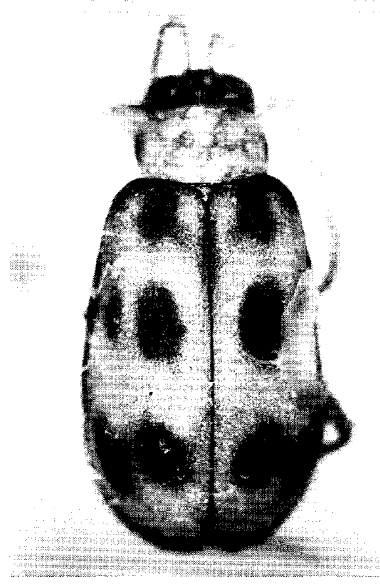

-

Fig. 35. a, Monolepta cavipennis Baly ; b, M. duodecimmaculata(Jacoby); c, M. wilsoni n. sp. 
Monoleptabifasciata: Jacoby, 1884, Notes Leyd. Mus., $6: 53$ (Sumatra, India).-Weise, 1910, Phil.

J. Sci., ser. D, 5(4) : 225 (Philippines).-Maulik, 1936, Fauna India, Galeruc. : 408 (India, Ceylon,

Penang, Sumatra, Singapore).

Monolepta quadrinotata: Jacoby, 1889, Ann. Mus. Civ. Genova, 27: 284 (Sumatra, Nias).

-Kimoto, Izmay \& Samuelson, 1984, Esakia, Kyushu Univ., 21 : 50, Fig. (Papua New Guinea).

Distribution: India, Ceylon, Thailand, Cambodia, Laos, Malaya, Singapore, Sumatra, Philippines, New Guinea.

Material examined. THAILAND : Khao Chong, nr. Trung, 1 ex., 24, vi. 1965, Y. Miyatake (KU) ; Kao Yai, 1 ex., 29-31. i. 1968, K. Baba (KIMOTO). CAMBODIA : Ph. Chisau, 40 km, S. of P. Penh, 20 m, 1 ex., 29. iv. 1961, N. R. Spencer (BISHOP). LAOS : Khammouane Prov., Phon Tiou, 1 ex., 10. vi. 1965, Native collr (BISHOP).

\section{M onolepta birmanensis Jacoby}

Monolepta birmanensis Jac., 1892, Ann. Mus. Civ. Genova, 32 : 982 (Burma ; GENOVA). -Duvivier, 1892, Ann. Soc. Ent. Belg., 36: 444 (Kurseong ; as birmaensis).-Maulik, 1936, Fauna India, Galeruc. : 405 (Burma, Darjeeling).

Distribution : India, Burma, Thailand, Vietnam.

Material examined. THAILAND : Chiengmai Prov., Doi Pui, 1,300 m, 5 exs., 17. vi. 1965, K. Morimoto \& Y. Miyatake (KU). VIETNAM : Mt. Lang Bian, 1,500-2,000 m, 19. v.-8. vi. 1961, N. R. Spencer (BISHOP).

\section{M onolepta cambodgia Laboissière}

Monolepta cambodgia Lab., 1935, Ann. Ass. Nat. Levallois-Perret, $21: 142$ (Cambodia).

Luperodes latefasciata: Allard, 1889, Ann. Soc. Ent. France, 1889: 310 (Pnomh-Penh).

Distribution : Cambodia, Laos.

Material examined. LAOS : Vientiane Prov., Vientiane, 1 ex., 31. v.-3. vi. 1960, light trap, S. Quate \& L. Quate (BISHOP).

\section{M onolepta cavipennis Baly Fig. 35a}

Monolepta cavipennis Baly, 1878, Cist. Ent., 2 : 459 (Assam; BM).-Bryant, 1923, Ann. Mag. Nat. Hist., ser. 9, 12 : 146 (India).-Maulik, 1936, Fauna India, Galeruc. : 405 (Cochin-China, HongKong).-Gressitt \& Kimoto, 1963, Pac. Ins. Mon., 1B: 612 (China, Hainan, Vietnam).

Diacantha fasciata Allard, 1888, Ann, Soc. Ent. France, ser. 6, $8: 318,323$ (Cambodia ; PARIS). New synonym.

Luperodes albofasciata Allard, 1889, Ann. Soc. Ent. France, ser. 6, 9: 310 (Annam; PARIS, HAMBURG). New synonym.

Candezea trifasciata Jacoby, 1900, Mém. Soc. Ent. Belg., 7 : 139 (Siam, Tringanee, Cochin-China ; BM)--Maulik, 1936, Fauna India, Galeruc. : 405 (= cavipennis).

Monolepta siamensis Weise, 1915, Ergebn. 2. Dtsche Zentr.-Exp., Zool., 1: 177 (new name for Candezea trifasciata Jacoby, 1900, nec Jacoby 1896, from Belgaum).

Candezea albofasciata: Laboissière, 1935, Ann. Ass. Nat. Levallois-Perret, 21 : 142 (Indo-China).

Distribution : India, Thailand, Cambodia, Laos, Vietnam, China, Hainan.

Material examined. THAILAND : Chanthaburi, 5 exs., 22. vii. 1962 ; Rayong, 12 exs., 27. vii. 1962 ; Uthaithani, 1 ex., 13. iv. 1963 (BANGKHEN) ; Nakhon Nayok Prov., Khao Yai Nat. Park, 5 
exs., 5-6. vi. 1965, P. D. Ashlock; Saraburi Prov., Ban Muak Lek Nat. Park, 2 exs., 5. vi. 1965, P. D. Ashlock; Saraburi, 1 ex., 4. iv. 1963, Native collr ; Pakchong, 100 m, NE of Bangkok, 3 exs., 3. xii. 1957, J. L. Gressitt ; Sattahib, 1 m, 1 ex., 24. xi. 1957, J. L. Gressitt ; Kampaengpet, 1 ex., 10. ix. 1963 (BISHOP) ; Khao Yai. Nat. Park, 13 exs., 6. vi. 1965, S. Asahina, K. Morimoto \& Y. Miyatake ; Sikiew, 1 ex., 5. xi. 1966, sweeping of paddy field, K. Yasumatsu \& K. Yano (KU) ; Khon Kaen, 1 ex., X-xii. 1972, M. Sato (EHIME) ; Khao Yai, 2 exs., 29-31. i. 1968, K. Baba (KIMOTO). LAOS : Umgeb. Vientiane, 7 exs., iii-vi. 1963, Umgeb. Pakse, 2 exs., 1963-64 (MUNCHEN) ; Vientiane Prov., Vientiane, 1 ex., 28. vii. 1965, 1 ex., 31. vii. 1965, 1 ex., 5. i. 1966, 2 exs., 6. vi. 1966, Native collr ; Vientiane Prov., Dong Dok, 2 exs., 23. vii. 1965, 6 exs., 7. ix. 1965, 1 ex., 3. xi. 1965, 2 exs., 22. xi. 1965, 4 exs., 26. xi. 1965, 2 exs., 1. xii. 1965, 1 ex., 6. xii. 1965, Native collr ; Vientiane Prov., Ban Van Eue, 6 exs., 15-31. 1965, 1 ex., 15. ii. 1966, 1 ex., 15. iii. 1966, 1 ex., 30. xi. 1966, 4 exs., 31. i. 1967, 1 ex., 15. ii. 1967, 1 ex., 15. viii. 1968, 4 exs., 31. xii. 1968, Native collr ; Vientiane Prov., Gi Sion Vill., de Tha Ngone, 1 ex., 19-16. i. 1966, 20 exs., 14-31. x. 1966, Native collr ; Khammouane Prov., Phon Tiou, 1 ex., 6. vii. 1965, 1 ex., 4. viii. 1965, 22 exs., 11. ix. 1965, 32 exs., 28. ix. 1965, Native collr ; Borikhane Prov., Paksane, 1 ex., 7. viii. 1965, 9 exs., 31. viii. 1965, 4 exs., 9. ix. 1965, 2 exs., 15. xi. 1965, 3 exs., 24. xi. 1965, 3 exs., 29. xi. 1965, 2 exs., 28. xii. 1965, Native collr; Borikhane Prov., Pakkading, 1 ex., 14. vii. 1965, 2 exs., 3. viii. 1965, 4 exs., 31. viii. 1965, 1 ex., 29. iv. 1966, Native collr ; Wapikhamthong Prov., Wapi, 28 exs., 15. ix. 1967, Native collr ; Sedone Prov., Pakse, 14 exs., 31. v. 1967, Native collr; Sedone Prov., Paksong, 12 exs., 6. ix. 1965, Native collr ; Sayaboury Prov., Sayaboury, 1 ex., 12. xii. 1965, Native collr ; Tongphen, 1 ex., 30. x. 1966, Native collr ; Nongtevda, 2 exs., 27. vii. 1965, 5 exs., 6. viii. 1965, 11 exs., 30. viii. 1965, 4 exs., 18. xi. 1965, 2 exs., 23. xi. 1965, 3 exs., 2. xii. 1965, 2 exs., 7. xii. 1965, Native collr (BISHOP). VIETNAM : Ban Me Thuot, 500 m, 1 ex., 20-24. xii. 1960, L. W. Quate ;M'Drak, E. of Ban Me Thuot, 4-600 m, 3 exs., 8-19. xii. 1960, C. M. Yoshimoto ; Fyan, 9001,200 m, 2 exs., 11. vii.-9. viii. 1961, N. R. Spencer ; Karyu Danar, 200 m, 1 ex., 13-28. ii. 1961, N. R. Spencer ; Nha Trang, 1 ex., 17-26. xi. 1960, C. M. Yoshimoto ; $25 \mathrm{~km} \mathrm{SW}$ of Peiku, $400 \mathrm{~m}, 1$ ex., 12. v. 1960, L. W. Quate (BISHOP).

\section{Monolepta dalatica n. sp. Fig. 44c}

Head black, prothorax yellowish brown, elytron yellowish brown with margins somewhat widely blackish ; antenna pitchy black ; meso- and metathorax black, abdomen yellowish brown, with fifth segment partly or entirely blackish; legs yellowish brown with tibiae and tarsi black, and dorsal surface of anterior femur infuscate.

Head with vertex smooth, shining, sparsely impressed by minute punctures; interocular space distinctly wider than transverse diameter of single oculus, and interocular transverse impression distinct, frontal tubercle subtriangular and distinctly raised. Antenna slender, nearly $2 / 3$ as long as body length ; first segment long, second shortest, nearly $2 / 5$ as long as first, third slightly longer than second, fourth twice as long as third, fourth to eighth subequal to each other in length and shape, ninth slightly shorter than eighth, tenth subequal to ninth, eleventh slightly longer than tenth and subequal to eighth but its apex pointed, Pronotum transverse, $12 / 3$ times as broad as long, anterior margin nearly straight, lateral margin slightly rounded, widest almost at middle, slightly narrowed anteriorly and posteriorly, basal margin strongly rounded posteriorly, dorsal surface convex, with a pair of shallow depressions laterally, sparsely impressed by fine punctures, and interstices of punctures smooth, shining. Scutellum subtriangular, finely granulate, impunctate. Elytron broader than prothorax, more strongly and closely punctate, and interstices of punctures smooth, shining.

Male : Unknown. Female : Fifth abdominal segment entire.

Length : 4.2-6.0 $\mathrm{mm}$. 
Holotype (BISHOP) : VIETNAM: Dalat, 6 km S., 1,400-1,500 m, 9. vi.-7. vii. 1961, N. R. Spencer. Paratypes : THAILAND : Khon Kaen, 2 exs., x-xii. 1972, M. Sato (EHIME). VIETNAM : Fyan, 9001,000 m, 2 exs., 11. vii.-9. viii. 1961, N. R. Spencer ; Dalat, 1,500 m, 1 ex., 29. iv.-4. v. 1960, S. Quate (BISHOP, KIMOTO).

This new species somewhat resembles Monolepta nigrobasalis Jacoby, but differs in being the body length shorter, and having the head entirely black. The specimens from Thailand slightly differ from the Vietnam specimens, in having the dorsal surface of the anterior femur entirely brownish.

\section{Monolepta discoidalis (Jacoby)}

Candezea discoidalis Jac., 1895, Stett. Ent. Ztg., $56: 76$ (Perak; BM).

Monolepta (Candezea) discoidalis : Weise, 1924, Col. Cat. Junk, 78 : 175 (Perak).

Lomirana discoidalis : Wilcox, 1973, Col. Cat. Junk, Suppl., 78: 597 (Malaya).

Distribution : Thailand, Malaya.

Material examined. THAILAND: Banna, Chawang, nr. Nabon, 70 m, 5. ix. 1958, J. L. Gressitt (BISHOP).

\section{Monolepta duodecimmaculata (Jacoby) Fig. 35b}

Luperodes duodecimmaculuta Jac., 1889, Ann. Mus. Civ. Genova, 27 : 212 (Burma).

Monolepta duodecimmaculata : Maulik, 1936, Fauna India, Galeruc. : 403 (Burma).

Distribution : Burma, Laos.

Material examined. LAOS : Vientiane Prov., Ban Van Eue, 1 ex., 15-31. v. 1965, Native collr ; Savanakhet Prov., Savanakhet, 1 ex., 15. iv. 1967, Native collr; K. Sedon, 1 ex., 18. iv. 1965, J. A. Rondon (BISHOP) ; Umgeb. Vientiane, 2 exs., iii-vi. 1963 ; Umgeb. Paklay, 1 ex., 1963 (MUNCHEN).

\section{Monolepta exigua n. sp. Fig. $45 \mathrm{~b}$}

Generally yellowish brown, head black, elytron black with an elongate yellowish marking covering about $3 / 4$ of length and $4 / 5$ of width of disc and free from lateral and sutural margins, antenna pitchy black with basal three segments yellowish brown, ventral surfaces with meso- and metathorax black, abdomen with basal part of first segment and apical part of fifth segment black ; legs with tibiae and tarsi generally pitchy black.

Head with vertex smooth, shining, sparsely impressed by minute punctures ; interocular space distinctly wider than transverse diameter of single oculus, and interocular transverse impression deep, frontal tubercle subtriangular and distinctly raised. Antenna slender, nearly $2 / 3$ as long as body length ; first segment long, second short, nearly $2 / 5$ as long as first, third subequal to second in length but more slender, fourth $21 / 2$ times as long as third, fifth slightly longer than fourth, fifth to seventh subequal to each other in length and shape, eighth slightly shorter than seventh, and eighth to tenth subequal to each other in length and shape, eleventh longer than tenth and subequal to seventh in length but its apex pointed. Pronotum transverse, $11 / 2$ times as broad as long, anterior margin nearly straight, lateral margin slightly rounded, widest at $1 / 2$ from anterior margin, and slightly narrowed anteriorly and more strongly so posteriorly, basal margin distinctly rounded posteriorly, dorsal surface convex side to side, without any trace of depression laterally, sparsely impressed by fine punctures, and interstices of punctures smooth, shining. Scutellum subtriangular, smooth, shining, impunctate. Elytron broader than prothorax, more strongly and closely punctate, and interstices of punctures smooth, shining. 
Male : Fifth abdominal segment trilobed, median lobe longitudinally sulcate. Female : Fifth abdominal segment entire.

Length : $2.0 \mathrm{~mm}$.

Holotype (BISHOP) : VIETNAM : Dalat, 1,500 m, 26 - 27. ix. 1960, C. M. Yoshimoto. Paratopotypes : 4 exs., same data as the holotype (BISHOP, KIMOTO). Paratypes : VIETNAM : Dalat, 1,550 m, 1 ex., 11. ix. 1960, J. L. Gressitt ; 6 km S. of Dalat, 2 exs., 12. iv. 1960, J. L. Gressitt ; 15 km NE of Dalat, 1,850 m, 1 ex., 5. v. 1960, L. W. Quate ; 25 km SW of Pleiku, 400 m, 1 ex., 12. v. 1960, L. W. Quate (BISHOP).

This new species somewhat resembles Monolepta quatei Kimoto, but differs in being the body length shorter, and having the head entirely black.

\section{Monolepta flavovittata Chen Fig. 33b}

Monolepta flavovittata Chen, 1942, Notes d'Ent. Chinoise, 9 : 61 (China : Hupeh).-Gressitt \& Kimoto, 1963, Pac. Ins. Mon., 1B: 614 (China).

Distribution : Vietnam, China.

Material examined. VIETNAM : Nha Trang, 2 exs., 17-26. xi. 1960, C. M. Yoshimoto ; Dailanh, N. of Nha Trang, 3 exs., 29. xi.-5. xii. 1960, C. M. Yoshimoto ; Ninh Hoa, N. of Nha Trang, 1 ex., 28. xi. 1960, C. M. Yoshimoto ; 15-35 km, NW of Phan Rang, 1 ex., 8-16. xi. 1960, C. M. Yoshimoto (BISHOP).

\section{Monolepta fyanica n. sp. Fig. $40 \mathrm{~b}$}

Generally yellowish brown; elytron with basal and apical margins black ; head with narrow blackish area behind eye ; antenna pitchy black with basal three segments brownish ; ventral surfaces with metathorax black.

Head with vertex smooth, shining, sparsely impressed by minute punctures, interocular space

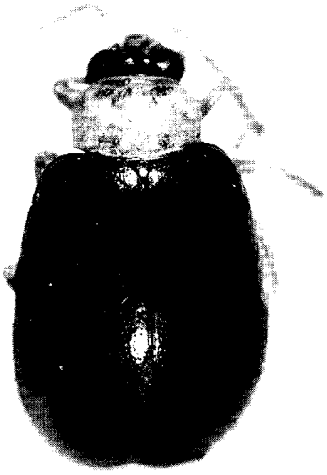

a

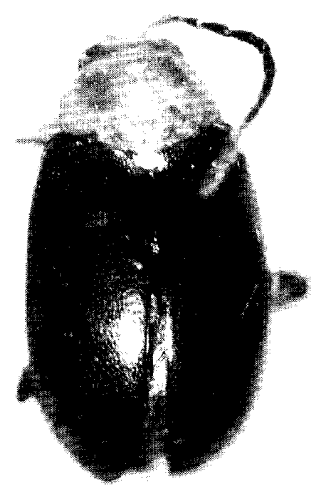

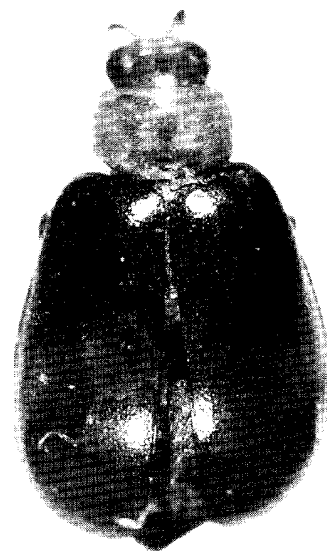

$\mathrm{C}$

Fig. 36. a, Monolepta semiapicalis n. sp. ; b, M. semiluperina n. sp. ; c, M. apicipennis n. sp. 
distinctly wider than transverse diameter of single oculus, and interocular transverse impression distinct, frontal tubercle subtriangular and distinctly raised. Antenna slender, nearly $2 / 3$ as long as body length ; first segment long, second shortest, nearly $1 / 3$ as long as first, third as long as second but slenderer, fourth nearly three times as long as third, fifth slightly longer than fourth, sixth to ninth subequal to each other in length and shape, tenth slightly shorter than ninth in length, eleventh slightly longer than tenth and subequal to ninth in length but its apex pointed. Pronotum transverse, $11 / 2$ times as broad as long, anterior margin nearly straight, lateral margin slightly rounded, widest at $1 / 3$ from anterior margin and slightly narrowed anteriorly and more strongly so posteriorly, basal margin rounded posteriorly, dorsal surface convex, with a pair of deep transverse depressions which are united at middle, sparsely impressed by fine punctures, and interstices of punctures smooth and shining. Scutellum subtriangular, finely granulate, impunctate. Elytron broader than prothorax, more strongly and closely punctate, and interstices of punctures smooth and shining.

Male : Unknown. Female : Fifth abdominal segment entire.

Length: 3.3-4.1 mm.

Holotype (BISHOP) : VIETNAM : Fyan, 900-1,200 m, 11. vii.-9. viii. 1961, N. R. Spencer. Paratopotypes : 3 exs., same data as the holotype (BISHOP, KIMOTO).

This new species resmbles Monolepta banmethuotica Kimoto, but differs by the shorter body and having the ventral surfaces with metathorax blackish.

\section{Monolepta gisionica n. sp. $\quad$ Fig. $37 \mathrm{c}$}

Head black, prothorax yellowish brown, elytron black with a transverse postmedian band together with its apex yellowish brown, antenna pitchy brown with second and third segments ochraceous, ventral surfaces reddish brown with meso- and metathorax pitchy black, legs pitchy brown to black.

Head with vertex somewhat wrinkled, sparsely impressed by fine punctures, interocular space distinctly wider than transverse diameter of single oculus, and interocular transverse impression shallow, frontal tubercle subtriangular and slightly raised. Antenna somewhat robust, nearly $2 / 3$ as long as body length ; first segment long, second shortest, nearly $1 / 3$ times as long as first, third slightly longer than second, fourth three times as long as third, fifth slightly shorter than fourth, sixth slightly shorter than fifth and sixth to ninth subequal to each other in length and shape, tenth slightly shorter than ninth, eleventh slightly longer than tenth and subequal to fourth in length but its apex pointed. Pronotum transverse, $13 / 4$ times as broad as long, anterior margin nearly straight, lateral margin slightly rounded, widest at slightly before basal margin and narrowed anteriorly, basal margin strongly rounded posteriorly, dorsal surface convex side to side, without any trace of depression laterally, rather closely impressed by fine punctures, and interstices of punctures smooth, shinging. Scutellum subtriangular, finely granulate, impunctate. Elytron broader than prothorax, more strongly and closely punctate, and interstices of punctures finely granulate.

M ale : Unknown. Female : Fifth abdominal segment entire.

Length : $5.4 \mathrm{~mm}$.

Hoiotype (BISHOP) : LAOS : Vientiane Prov., Gision Vill., de Tha Ngone, 2. i. 1966, Native collr. Paratopotypes : 2 exs., same data as the holotype (BISHOP, KIMOTO). Paratypes: LAOS : Vientiane Prov., Ban Van Eue, 1 ex., I-15. v. 1965, J. A. Rondon, 1 ex., 15-31. v. 1965, Native collr (BISHOP).

This new species somewhat resembles Monolepta sublata Gressitt \& Kimoto, but differs in having antenna robuster, pronotum widest at slightly before the basal margin, and the head generally blackish. 


\section{Monolepta hageni Weise}

Monoleptabasalis Jacoby, 1884, Notes Leyden Mus., $6: 55$ (nec Harold, 1880) (Sumatra).

Monolepta hageni Weise, 1916, Dtsche Ent. Z., 1916 : 40 (new name for Monolepta basalis Jacoby, nec Harold) (Sumatra).-Chûjô, 1964, Nature and Life in SE Asia, Kyoto, 3 : 293 (Thailand : Naborn).

Distribution : Thailand, Sumatra.

Material examined. THAILAND : Trang Prov., Khaophappha, Khaochang, ZOO-400 m, 1 ex., 10. i. 1964, G. A. Samuelson ; Banna, Chawang, nr. Nabon, 70 m, 1 ex., 5. ix. 1958, T. C. Maa ; Banna, Nakhon, 108 m, 3 exs., 5-10. v. 1958, T. C. Maa (BISHOP).

\section{Monolepta hieroglyphica (Motschulsky)}

Luperodes hieroglyphicus Motsch., 1858, Etud. Ent., 7: 104 (Indes or.).

Monolepta elegantula Boheman, 1859, Eugenies Resa, 2 : 183 (Malacca)._Jacoby, 1889, Ann. Mus. Civ. Genova, 27 : 226 (Malacca).

Monolepta quadrigutta: Fairmaire, 1887, Rev. d'Ent., 6: 333 (China).-Yuasa, 1936, Rep. First Sci. Exp. Manchoukuo, 5, 1, 10 (51) : 19, pl. 2, Fig. 6 (Johol).

Monolepta biarcuata Weise, 1889, HoraeSoc. Ent. Ross., 23 : 632 (C. Mongolia).

Monolepta hieroglyphica ab. simplex Weise, 1913, Phil. J. Sci., ser. D, 8 : 229 (Philippines) ; 1922, Tijdschr. Ent., 65 : 104 (Sumatra).-Ogloblin, 1936, Fauna URSS, 26(1): 318, 319 (Philippines).

Monolepta hieroglyphica: Weise, 1922, Tijdschr. Ent., $65: 104$ (Sumatra).-Maulik, 1936, Fauna India, Galeruc. : 380, 411, fig. 100 (Burma, Singapore, Sumatra, Philippines, China).-Ogloblin, 1936, Fauna URSS, 26, $1:$ 315, 318, 434 (Tonkin, Burma, Sumatra, Philippines).--Chûjô, 1962, Phil. J. Sci., 91 (1-2) : 113, 129 (Formosa).

Monolepta hieroglyphica ab. biarcuata : Weise, 1922, Tijdschr. Ent., 65: 104 (China).

Monolepta hieroglyphica biarcuata : Ogloblin, 1936, Fauna URSS, 26(1): 315, 318, 319, 434, fig. 131 (S. Ussuri Distr., Manchuria, Korea, Japan, Formosa, China, Ordos, Alachan).

Monolepta hieroglyphica:Chajô, 1964, Nature and Life in SE Asia, Kyoto, 3 : 293 (Thailand). -Kimoto, 1972, Tijdschr. Ent., 93 (4) : 145 (Annam).

Distribution : Burma, Thailand, Cambodia, Laos, Vietnam, Malaya, China, Taiwan, Mongolia, E. Siberia,, Korea, Philippines, Sumatra.

Material examined. THAILAND : Trang Prov., Khaophappha, Khaochang, 200-400 m, 1 ex., 3031. xii. 1963, G. A. Samuelson ; Nakorn-nayok, 1 ex., 14. v. 1963, Native collr ; Phu Kae, 1 ex., 17. v. 1963, Native collr ; Chiengmai Prov., Chieng Dao, 450 m, 1 ex., 5-11.1958, T. C. Maa (BISHOP) ; Kao Yai, 1 ex., 29-31. i. 1968, K. Baba (KIMOTO). LAOS : Vientiane Prov, Ban Van Eue, 1 ex., 15. i. 1967, Native collr ; Luang Prabang, 300 m, 1 ex., 4-5. vi. 1960, L. W. Quate ; Sedone Prov, Pakse, 1 ex., 21. v. 1965, P. D. Ashlock (BISHOP) ; Umgeb. Paklay,1 ex., 1963 (MUNCHEN). VIETNAM : Mt. Lang Bian, 14 exs., 19. v.-8. vi. 1961, N. R. Spencer ; 17 km NE of Dalat, 2,000 m, 1 ex., 3. v. 1960, L. W. Quate ; Karyu Danar, 200 m, 2 exs., 13-28. ii. 1961, N. R. Spencer ; Fyan, 1,200 m, 1 ex., 11. vii.-9. viii. 1961, N. R. Spencer ;M’Drak, E. of Ban Me Thuot, 4-600 m, 1 ex., 8-19. ii. 1960, C. M. Yoshimoto (BISHOP).

\section{Monolepta langbianica n. sp. Fig. 37b}

Generally yellowish brown, head and prothorax generally black, in some specimen middle of vertex pitchy black. 
Head with vertex smooth, shining, sparsely impressed by minute punctures, interocular space distinctly wider than transverse diameter of single oculus, and interocular transverse impression deeper, frontal tubercle subtriangular and distinctly raised. Antenna slender, nearly $2 / 3$ as long as body length ; first segment long, second shortest, nearly $1 / 3$ as long as first, third $11 / 2$ times as long as second, fourth $11 / 2$ times as long as third, fifth slightly longer than fourth, sixth to eighth subequal to each other in length and shape, ninth slightly shorter than eighth, tenth subequal to ninth in length and shape, eleventh slightly longer than tenth and subequal to eighth in length but its apex pointed. Pronotum transverse, $11 / 2$ times as broad as long, anterior margin nearly straight, lateral margin slightly rounded, widest at $1 / 2$ from anterior margin, slightly narrowed anteriorly and more strongly so posteriorly, basal margin strongly rounded posteriorly, dorsal surface convex, with a pair of deep oblique depressions laterally, sparsely impressed by distinct punctures, and interstices of punctures smooth and shining. Scutellum subtriangular, smooth, shining, impunctate. Elytron broader than prothorax, more strongly and closely punctate, and interstices of punctures smooth, shining.

Male : Fifth abdominal segment trilobed, median lobe generally fiat. Female : Fifth abdominal segment entire.

Length : $4.2-4.8 \mathrm{~mm}$.

Holotype (BISHOP) : VIETNAM : Mt. Lang Bian, 1,500-2,000 m, 19. v.-8. vi. 1961, N. R. Spencer. Paratopotypes : 6 exs., same data as the holotype (BISHOP, KIMOTO).

This new species resembes Monoleptabicavipennis Chen, from China, but differs in having the antenna and the legs entirely yellowish brown, and in the male elytron without any cavity.

\section{Monolepta laosensis n. sp. Fig. 42c}

Generally reddish brown, elytron black with basal and subapical markings yellowish brown, and basal marking widely separated from basal margin, antenna entirely pitchy black, legs generally pitchy black with ventral surface of anterior femur reddish brown.

Head with vertex somewhat wrinkled, sparsely impressed by minute punctures; interocular

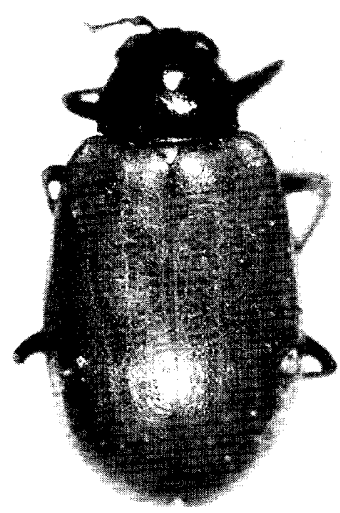

a

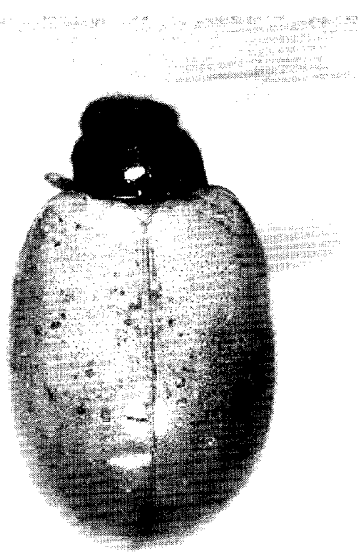

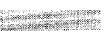

$\mathrm{b}$

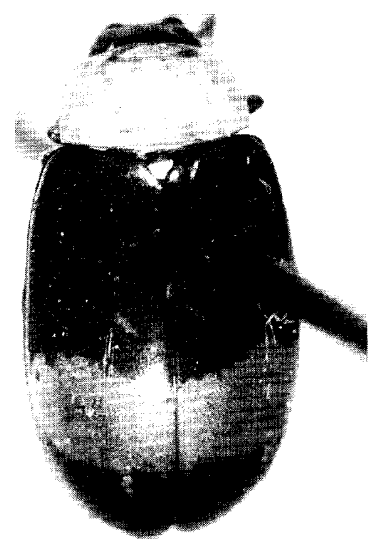

Fig. 37. a, Monolepta subflavipennis n. sp. ; b, M. langbianica n. sp. ; c, $M$. gisionica n. sp. 
space distinctly wider than transverse diameter of single oculus, and interocular transverse impression distinct, frontal tubercle subtriangular and distinctly raised. Antenna slender, nearly $2 / 3$ as long as length of body ; first segment long, second shortest, nearly $1 / 3$ as long as first, third twice as long as second, fourth $11 / 2$ times as long as third, fifth slightly longer than fourth, fifth to ninth subequal to each other in length and shape, tenth slightly shorter than ninth, eleventh slightly longer than tenth and subequal to ninth in length but its apex pointed. Pronotum transverse $12 / 3$ times as broad as long, anterior margin nearly straight, lateral margin slightly rounded, widest at $1 / 3$ from anterior margin, and slightly narrowed anteriorly and more strongly so posteriorly, basal margin strongly rounded posteriorly, dorsal surface convex, with a pair of shallow oblique depressions laterally, sparsely impressed by fine punctures, and interstices of punctures smooth, shining. Scutellum subtriangular, smooth, shining, impunctate. Elytron broader than prothorax, more strongly and closely punctate, and interstices of punctures smooth, shining.

Male : Fifth abdominal segment trilobed, median lobe generally flat. Female : Fifth abdominal segment entire.

Length : $5.3-6.0 \mathrm{~mm}$.

Holotype (BISHOP) : LAOS : Sayaboury Prov., Sayaboury, 1 ex., 15. iv. 1965, J. L. Gressitt. Paratopotype : 1 ex., same data as the holotype (KIMOTO). Paratypes : Vientiane Prov., Ban Van Eue, 1 ex., 1-15. v. 1965, J. A. Rondon, 1 ex., 15-31. iv. 1965, 1 ex., 15. viii. 1965, 1 ex., 31. xii. 1965, 2 exs., 16. iii. 1966, 1 ex., 15. xi. 1966, 1 ex., 30. viii. 1967, Native collr ; Sayaboury Prov., Sayaboury, 1 ex., 16. iv. 1965, J. L. Gressitt ; Sedon Prov., Paksong, 1 ex., 18. v. 1965, P. D. Ashlock (BISHOP).

This new species somewhat resembles Monolepta quadrinotata Kimoto, but differs in being the body length longer, and having the head entirely reddish brown.

\section{M onolepta lauta Gressitt \& Kimoto}

M onolepta lauta Gressitt \& Kimoto, 1963, Pac. Ins. Mon., 1B: 604, 617, fig. (Hainan ; CAS).

Distribution : Laos, Vietnam, Hainan.

Material examined. LAOS : Vientiane Prov., Gi Sion Vill., de Tha Ngone, 2 exs., 26. xii. 1965, Native collr ; Ban Van Eue, 20 km E. of Phou-kow-kuei, 1 ex., 15-31. v. 1965, J. A. Rondon (BISHOP). VIETNAM : Pleiku, 700 m, 1 ex., 8-14. v. 1960, L. W. Quate ; 20 km N. of Pleiku, 650 m, 3 exs., 9. v. 1960, S. Quate \& L. Quate ; 25 km SW of Pleiku, 400 m, 1 ex., 12. v. 1960, L. W. Quate ; Blao (Balao), 500 m, 1 ex., 14-21. x. 1960, C. M. Yoshimoto (BISHOP).

\section{M onolepta leechi Jacoby Fig. 33a}

M onolepta leechi Jac., 1890, Entomologist, 23 :21.6 (China; MCZ).-Gressitt \& Kimoto, 1963. Pac. Ins. Mon., 1B: 618 (S. China).-Kimoto, 1976, Ent. Rev. Japan, 29 : 5 (Taiwan).

Distribution : Thailand, Laos, Vietnam, S. China, Taiwan.

Material examined. THAILAND : Chiengmai Prov., Doi Suthep, 1,000 m, 1 ex., 18. vi. 1965, K. Morimoto ; Fang, 2 exs., 13-14. vi. 1965, K. Morimoto (KU). LASO: Vientiane Prov., Ban Van Eue, 1 ex., 31 . xii. 1968, Native collr ; Vientiane Prov., Gi Sion Vill., de Tha Ngone, 1 ex., 2. i. 1966, Native collr ; Khammouane Prov., Phon Tiou, 1 ex., 8. vi. 1965, Native collr (BISHOP). VIETNAM : Dalat, 1,500 m, 26-27. ix. 1960, C. M. Yoshimoto (BISHOP).

\section{M onolepta longitarsis Jacoby}

M onolepta longitarsis Jac., 1896, Ann. Soc. Ent. Belg., 40 : 297 (Bombay: Belgaum ; BM). 
-Maulik, 1936, Fauna India, Galeruc. : 377, 386, figs (India).-Châjô, 1964, Nature and Life in SE Asia, Kyoto, 3 : 294 (Thailand : Chieng Mai, Doi Suthep).

Distribution : India, Thailand, Vietnam.

Material examined. THAILAND : Chieng Mai Prov., Mak Klang, 340 m, 1 ex., 11. vi. 1965, P. D. Ashlock (BISHOP) ; Chieng Mai Prov., Doi Suthep, 1,000 m, 18. vi. 1965, Y. Miyatake ; Chieng Mai Prov., Chieng Dao, 15. vi. 1965, 13 exs., K. Morimoto \& Y. Miyatake (KU). VIETNAM : Fyan, 9001,200 m, 11. vii.-9. vii. 1961, 10 exs., N. R. Spencer; Dalat, 6 km S., 1,400-1,500 m, 4 exs., 9. vi.-7. vii. 1961, N. R. Spencer; Ban Me Thuot, 500 m, 1 ex., 20-24. xii. 1960, N. R. Spencer (BISHOP).

\section{Monolepta luperina $\mathbf{W}$ eise}

Monolepta luperina Weise, 1922, Tijdschr. Ent., 65 : 103 (Tonkin; STOCKHOLM).

Distribution : Vietnam.

Materail examined. VIETNAM : $15 \mathrm{~km}$ from Dalat, 1,500 m, 1 ex., 29. iv.-5. v. 1960, S. Quate (BISHOP).

\section{Monolepta marginipennis (Jacoby) Fig. 34b}

Candezea marginipennis Jac., 1892, Ann. Mus. Civ. Genova, 32 : 979 (Bumra ; BM).

Monolepta marginipennis: Maulik, 1936, Fauna India, Galeruc. : 435 (Bumra).

Distribution : Burma, Thailand, Laos, Vietnam.

Material examined. THAILAND : Chiengmai Prov., Doi Suthep, 1 ex., 8. vi. 1965, P. D. Ashlock (BISHOP) ; Chiengmai Prov., Doi Suthep, 1,000 m, 10 exs., 18. vi. 1965, Y. Miyatake (KU). LAOS : Sedon Prov., Paksong, 9 exs., 17-18. v. 1965, light trap, P. D. Ashlock (BISHOP). VIETNAM : Fyan, 900-1,200 m, 82 exs., 11. vii.-9. viii. 1961, N. R. Spencer ; Dalat, 1,500 m, 1 ex., 26-27. ix. 1960, C. M. Yoshimoto ; Dalat, 6 km S., 1,400-1,500 m, 5 exs., 9. vi.-7. vii. 1961, N. R. Spencer ; Dilinh (Djiring), 3 exs., 27. ix.-14. x. 1960, C. M. Yoshimoto (BISHOP).

\section{Monolepta miyatakei n. sp. Fig. 40a}

Generally yellowish brown; elytron with basal, lateral, sutural and apical margins narrowly black; head with vertex generally black; antenna pitchy black with basal three segments brownish ; legs with tibiae and tarsi blackish; abdomen with apex of pygidium blackish.

Head with vertex distinctly but not closely punctate, interocular space subequal to transverse diameter of single oculus and interocular transverse impression distinct, frontal tubercle subtriangular and distinctly raised. Antenna slender, slighly longer than body length ; first segment long, second shortest nearly $1 / 3$ as long as first, third subequal to second in length, fourth nearly three times as long as third, fifth slightly longer than fourth, fifth to seventh subequal to each other in length and shape, eighth slightly shorter than seventh, eighth to tenth subequal to each other in length and shape, but eleventh subequal to tenth in length but its apex pointed. Pronotum transverse, $11 / 2$ times as broad as long, anterior margin nearly straight, lateral margin distinctly rounded, widest almost at middle and distinctly narrowed anteriorly and posteriorly, basal margin strongly rounded posteriroly, dorsal surface convex, with a pair of shallow depressions laterally, and rather closely impressed by distinct punctures. Scutellum subtriangular, impunctate, finely granulate at middle. Elytron broader than prothorax, more strongly and closely punctate.

Male : Fifth abdominal segment trilobed, median lobe feebly depressed at middle. Female : Fifth abdominal segment entire. 
Length : $6.0-6.7 \mathrm{~mm}$.

Holotype (Type No, 2703, Kyushu Univ.) : THAILAND : Khao Yai Nat. Park, 6. vi. 1963, Y. Miyatake. Paratopotype : 1 ex., same data as the holotype (KIMOTO). Paratypes : THAILAND : Songkhla, 1 ex., 28. vi. 1965, Y. Miyatake (KU) ; Khon Kaen, 1 ex., 24. vii. 1980, S. Azuma (RU) ; Uthaithani, 8 exs., 13. iv. 1965 (BANGKHEN).

This new species somewhat resembles Monolepta nigrobasalis Jacoby, but differs in having the middle of vertex blackish and the ventral surfaces entirely brownish.

\section{Monolepta nigrifrons (Allard), new combination Fig. 38c}

Luperodes nigrifrons All., 1889, Ann. Soc. Ent. France, ser. 6, $9: 310$ (Annam; PARIS).

Nadrana facialis Laboissière, 1936, Ann. Soc. Ent. France, 105 : 262 (Tonkin; HAMBURG). New synonym.

Distribution : Thailand, Laos, Vietnam.

The coloration of head is somewhat variable. In some specimens vertex and the interstices of antennae are entirely brownish instead of blackish.

Material examined. THAILAND : Chiengmai Prov., Doi Suthep, 1 ex., 14. xi. 1957, J. L. Gressitt, 1 ex., 28-31. iii. 1958, T. C. Maa; Chiengmai Prov., Chieng Dao, 1 ex., 5-11. iv. 1958, T. C. Maa (BISHOP) ; Chiengmai Prov., Mae Sa Water Fall, 1 ex., 16. vi. 1965, Y. Miyatake (KU) ; Chanthaburi, 1 ex., 22. vii. 1962 (BANGKHEN). LAOS : Wapikhamthong Prov., Wapi, 1 ex., 15. ix. 1967, Native collr; Vientiane Prov., Ban Van Eue, 1 ex., 31. xi. 1965, Native collr (BISHOP). VIETNAM : Fyan, 1,200 m, 8 exs., 11. vii.-9. viii. 1961, N. R. Spencer ; Ban Me Thuot, 500 m, 2 exs., 20-24. xii. 1960, C. M. Yoshimoto ; Dak Song, 76 km SW of Ban Me Thuot, 870 m, 1 ex., 19-21. v. 1960, S. Quate \& L. Quate ; Blao (Balao), 500 m, 1 ex., 14-21. x. 1960, C. M. Yoshimoto ; Dilinh (Djiring), 1,200 m, 1 ex., 22-28. iv. 1960, C. M. Yoshimoto; 17 km S. of Dilinh, 1,300 m, 2 exs., 6-12. x. 1960, C. M. Yoshimoto ; Karyu Danar, 200 m, 2 exs., 13-28. ii. 1961, N. R. Spencer (BISHOP).

\section{M onolepta nigripes (Olivier)}

Galeruca nigripes Oliv., 1808, Entomologie, $6: 648$, pl. 4, fig. 58 (Bengal).

Monolepta concolor Boheman, 1859, Eugenies Resa, 2 : 182 (Manila).-Weise, 1922, Tijdschr. Ent., 65 : 105 (Java, Borneo).-Maulik, 1936, Fauna India, Galeruc. : 414 (= nigripes).

Ochralea nigricornis Clark, 1865, Ann. Mag. Nat. Hist., ser. 3, 15 : 144 (Penang ; BM).-Weise, 1924, Col. Cat. Junk, $78: 167$ (= nigripes).

Ochralea nigripes: Harold, 1880, Stett. Ent. Ztg., $41: 147$ (India, Java, Manila, Sumatra). - Jacoby, 1889, Ann. Mus. Civ. Genova, $27: 230$ (Tenasserim).

Ochralea fulva Baly, 1886, Trans. Ent. Soc. London, 1886 : 39 (Himalayas ; Burma).-Maulik, 1936, Fauna India, Galeruc. : 414 (= nigripes).

Monolepta concolor ab. nigripes : Weise, 1922, Tijdschr. Ent., 65 : 105 (Philippines, Sunda Is., Hinterindien, Tonkin).

Ochralea concolor: Laboissière, 1932, Mém. Mus. Hist. Nat. Belg., hors ser., 4(4) : 179 (Java).

Monolepta nigripes: Maulik, 1936, Fauna India, Galeruc. : 414 (Himalayas, India, Burma, Ceylon, Siam, Malaya, Celebes, Borneo, Philipines).-Châjô, 1964, Nature and Life in SE Asia, Kyoto, 3 : 294 (Cambodia).-Kimoto, 1972, Tijdschr. Ent., 93(4): 145 (Siam, Burma).

Distribution : India, Ceylon, Burma, Thailand, Cambodia, Laos, Vietnam, Malaya, Celebes, Borneo, Java, Sumatra, Philippines.

Material examined. THAILAND : Tak, 26 exs., 25. v. 1959, 5 exs., 29. v. 1959, 4 exs., 30. v. 1959, 
5 exs., 31. v. 1959, 1 ex., 9. vi. 1959, 1 ex., 11. vi. 1959, 7 exs., 12. vi. 1959 ; Trang, 7 exs., 1-18. iv. 1960 ; Uthaithani, 5 exs., 13. iv. 1963 ; Nakhon Rathchiasima, 3 exs., 22. vi. 1958 ; Chanthaburi, 2 exs., 31. v.4. iv. 1959 ; Chiengmai, 2,000 ft., 1 ex., 16. iv. 1958 (BANGKHEN) ; Chiengmai Prov., Doi Suthep, 900 m, 1 ex., 14. xi. 1957, J. L. Gressitt ; Banna Chawang, nr. Nabon, 70 m, 6. ix. 1958, J. L. Gressitt (BISHOP) ; Khao Chong, nr. Trung, 4 exs., 25. vi. 1965, Y. Miyatake ; Kor Hong, 1 ex., 23. vi. 1965, K. Morimoto ; Song Khla, 1 ex., 22. vi. 1965, Y. Miyatake (KU) ; Khon Kaen, 1 ex., 24. vii. 1980, S. Azuma (RU). CAMBODIA : Phnom-Antiang, nr. Sisophon, 1 ex., 20. xi. 1962, T. Shibata (OMNH). LAOS : Umgeb. Vientiane, 38 exs., iii-vi. 1963 (MUNCHEN) ; Khammouane Prov., Phon Tiou, 5 exs., iii. 1965, 1 ex., 1-15. iv. 1965, J. L. Gressitt, 13 exs., 30. iv. 1965, 1 ex., 17. v. 1965, 2 exs., 6. vii. 1965, 1 ex., 4. viii. 1965, Native collr ; Borikhane Prov., Pakkading, 2 exs., 21. iv. 1965, 1 ex., x. 1965, J. L. Gressitt, 1 ex., 5. iv. 1965, 6 exs., 22. iv. 1965, 12 exs., 23. iv. 1965, 3 exs., 17. v. 1965, 2 exs., 15. vi. 1965, Native collr ; Ponhom, N. of Pakkading, 2 exs., 20. iv. 1965, J. L. Gressitt ; Namkading, nr Pakkading, 1 ex., 21. iv. 1965, J. L. Gressitt ; Sedon Prov., Pakse, 3 exs., iii. 1965, J. A. Rondon, 1 ex., 31. iv. 1965, 1 ex., 16. v. 1965, 16 exs., 31. v. 1965, 1 ex., 5. vii. 1965, Native collr ; Sedon Prov., Paksong, 1 ex., 26. vii. 1965, Native collr ; Vientiane Prov., Vientiane, 3 exs., 21. iv. 1965, J. A. Rondon, 2 exs., iii. 1966, J. Sedlacek, 7 exs., 22. vii. 1965, 2 exs., 30. iv. 1966, 1 ex., 15. v. 1967, Native collr ; Vientiane Prov., Ban Van Eue, 4 exs., 15-31. iv. 1965, 2 exs., 31. vii. 1965, Native collr ; Vientiane Prov., Phou Kou Khouei, 1 ex., 1-15. iv. 1965, 1 ex., 17. iv. 1965, J. L. Gressitt, 1 ex., 15. vi. 1966, Native collr ; Wapikhamthong Prov., Khong Sedone, 2 exs., 30. iv. 1965, 2 exs., 16. v. 1965, 2 exs., 30. v. 1965, Native collr ; Sayaboury Prov., Sayaboury, 1 ex., 5. v. 1966, 1 ex., 20. v. 1966, Native collr ; Savanakhet Prov., Savanakhet, 1 ex., 5. vii. 1965, Native collr ; Ile de Khong, 15 exs., 17. iv. 1965, J. L. Gressitt, 2 exs., 6. ii. 1965, 1 ex., 20. vii. 1965, 1 ex., 7. ix. 1965, Native collr (BISHOP). VIETNAM : $25 \mathrm{~km}$ SE of Pleiku, 400 m, 1 ex., 12. v. 1960, L. W. Quate (BISHOP).

\section{Monolepta nigrobasalis Jacoby}

Monolepta nigrobasalis Jacoby, 1903, Ann, Soc. Ent. Belg., 47 : 127 (Nilgiri Hills).-Maulik, 1936,

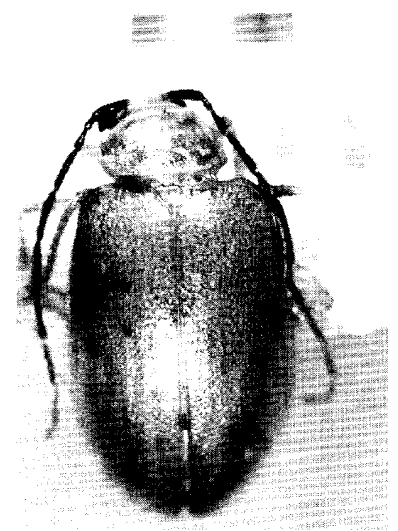

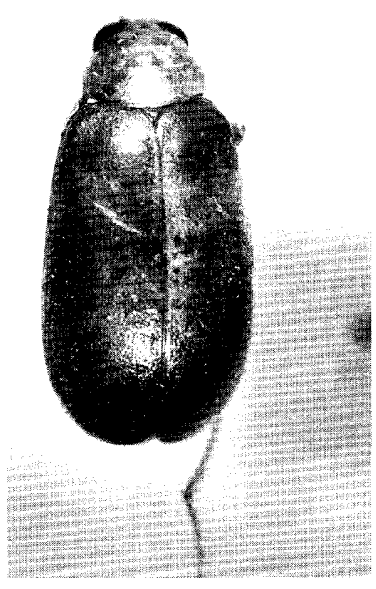

b

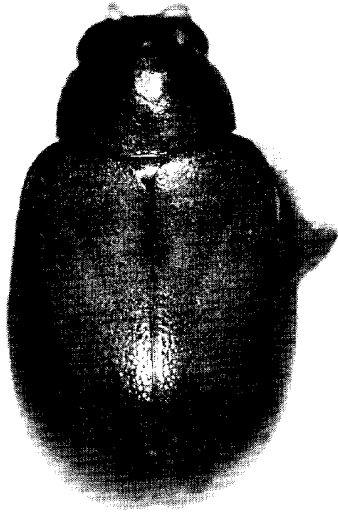

C

Fig. 38. a, Monolepta pallidulella n. sp. ; b, M. rubrobasalis n. sp. ; c, M. nigrifrons (Allard). 
Fauna India, Galeruc. : 425, 442, fig. (India).-Chajô, 1961, Nature and Life in SE Asia, Kyoto, $1: 351$ (Thailand : Chieng Mai); 1964, ibid., $3: 295$ (Chieng Mai).

Damais humeralis Jacoby, 1903, Ann. Soc. Ent. Belg., 47 : 119 (Nilgiri Hills; BM).-Maulik, 1936,

Fauna India, Galeruc. : 442 (= nigrobasalis).

Distribution : India, Thailand.

Material examined. THAILAND : Chiengmai Prov., Chiengmai, 3 exs., 11. iv. 1966, J. Sedlacek ; Chiengmai Prov., Doi Suthep, 10 exs., 28. iii.-5. iv. 1958, T. C. Maa; Chiengmai Prov., Doi Pui, 1 ex., 2. iv. 1958, T. C. Maa ; 50 km West of Tak, 900 m, 2 exs., 7-8. iv. 1966, J. Sedlacek (BISHOP) ; Khon Kaen, 1 ex., 20. xii. 1979, S. Azuma (RU).

\section{Monolepta nigrotibialis (Allard), new combination}

Luperodes nigrotibialis All., 1889, Ann. Soc. Ent. France, ser. 6, $9: 311$ (Cambodia ; PARIS).

Neolepta nigro tibialis : Laboissière, 1935, Ann. Ass. Nat. Levallois-Perret, $21: 143$ (genus).

Distribution : Laos, Vietnam.

Material examined. LAOS : Vientiane Prov., Ban Van Eue, 2 exs., 31. xii. 1968, Native collr ; Wapikhamthong Prov., Khong Sedon, 1 ex., 30. iv. 1965, Native collr (BISHOP).

\section{M onolepta orientalis Jacoby}

M onolepta orientalis Jac., 1889, Ann. Mus. Civ. Genova, 27 : 227 (Burma ;GENOVA).-Duvivier, 1892, Ann. Soc. Ent. Belg., 36: 444 (Mandar).-Maulik, 1936, Fauna India, Galeruc. : 407 (India, Burma).-Kimoto, 1970, Spec. Bull. Lep. Soc. Japan, $4: 171$ (Nepal).-Kimoto \& Takizawa, 1972, Kontyû, Tokyo, 40 (4) : 220 (Nepal) ; 1983, Bull. Natn. Sci. Mus. Tokyo, ser. A, 9 (2) : 86 (Nepal).

Monolepta konbirensis Duvivier, 1891, C. R. Soc. Ent. Belg., 35 : 47 (Konbir-Nowatoli).-Maulik, 1936, Fauna India, Galeruc. : 408 (= orientalis).

M onolepta orientalis var. konbirensis : Jacoby, 1896, Ann. Soc. Ent. Belg., 40 : 294 (India).

Distribution : India, Nepal, Burma, Thailand.

Material examined. THAILAND: Nakorn-nayok, 3 exs., 14. v. 1963, Native collr (BISHOP).

\section{M onolepta pallida (Jacoby)}

Ochralea pallida Jac., 1892, Ann. Mus. Civ. Genova, 32 : 978 (Burma ; BM).

Monolepta pallida: Maulik, 1936, Fauna India, Galeruc. : 398 (Burma).

Distribution : Burma, Thailand, Laos, Vietnam.

Material examined. THAILAND : Chiengmai Prov., Chieng Dao, 1 ex., 5-11. iv. 1958, J. L. Gressitt ; Khao Yai, 1 ex., 12. ii. 1964, Native collr (BISHOP) ; Chiengmai Prov., Chieng Dao, 1 ex., 23. iv. 1973, Y. Yoshiyasu ; Khao Yai Nat. Park, 1 ex., 6. vi. 1965, Y. Miyatake (KU). LAOS : Attopeu Prov., Houei Kong, 1 ex., 15. iv. 1965, J. A. Rondon, 1 ex., 16. v. 1965, 4 exs., 31. v. 1965, 3 exs., 20. vii. 1965, 1 ex., 4. ix. 1965, Native collr ; Sedon Prov, Paksong, 1 ex., 18. v. 1965, P. D. Ashlock, 1 ex., 7. vi. 1965, Native collr ; Sedon Prov., Pakse, 1 ex., 15. v. 1965, P. D. Ashlock, 1 ex., 31. v. 1965, Native collr ; Borikhane Prov., Pakkading, 1 ex., 21. iv. 1965, J. L. Gressitt ; Khammouane Prov., Phon Tiou, 1 ex., 28. iv. 1965, Native collr (BISHOP) ; Umgeb. Vanky, 1 ex., 1963 (MUNCHEN). VIETNAM : Ban Me Thuot, 500 m, 2 exs., 16-18. v. 1968, S. Quate \& L. Quate ; Dalat, 6 km S., 1,400-1,500 m, 2 exs., 9. vi.-7. vii. 1961, N. R. Spencer ; Kontum, N. of Pleiku, 550 m, 2 exs., 13. v. 1960, S. Quate \& L. Quate ; $25 \mathrm{~km} \mathrm{SW}$ of Pleiku, 400 m, 3 exs., 12. v. 1960, S. Quate \& L. Quate ; Fyan, 900-1,000 m, 1 ex., 11. vii. 
-9. viii, 1961, N. R. Spencer (BISHOP).

\section{Monolepta pallidula (Baly)}

Luperodes pallidulus Baly, 1874, Trans. Ent. Soc. London, 1874 ; 187 (Japan : Nagasaki ; BM). -Ogloblin, 1936, Fauna USSR, 26, 1: 307, 310, 431 (Japan).

Monolepta gracilipes Chûjô, 1938, Arb. Morph. Taxon. Ent. Berlin, 5 (2) : 145 (Formosa ;TARI); 1962, Phil. J. Sci., 91 (1-Z) : 123 (Formosa).-Kimoto, 1969, Esakia, Kyushu Univ., 7 : 47 (= pallidula).

Monolepta pallidula:Chajô, 1962, Phil. J. Sci., 91 (1-2) : 123 (Formosa).-Gressitt \& Kimoto, 1963, Pac. Ins. Mon., 1B: 604, 626, fig. (China).

Distribution : Thailand, Laos, Vietnam, S. China, Taiwan, Ryukyu Is., Japan.

Material examined. THAILAND : Pangmakampo (Pankampawng), nr. Fang, $450 \mathrm{~m}, 2$ exs., 15 -16. xi. 1957, J. L. Gressitt ; Nakorn-nayok, 1 ex., 14. v. 1963, Native collr (BISHOP) ; Chiengmai Prov., Chiengdao, 1 ex., 15. vi. 1965, Y. Miyatake (KU) ; Khon Kaen, 3 exs., 22. xii. 1979, 3 exs., 24. vii. 1980, 8 exs., 7. x. 1981, 2 exs., 8. x. 1981, S. Azuma (RU) ; Musa, nr. Fang, 2 exs., 22. xi. 1968, K. Hatta (EHIME). LAOS : Vientiane Prov., Ban Van Eue, 20 exs., 13. iv. 1965, 1 ex., 15. iv. 1965, J. L. Gressitt, 6 exs., 1-15. v. 1965, 1 ex., 15-31. v. 1955, J. A. Rondon, 1 ex., 15. iv. 1965, 1 ex., 15. v. 1967, 2 exs., 30. ix. 1967, 2 exs., 2. ii. 1967, 1 ex., 31. xii. 1968, Native collr; Vientiane Prov., Phou-kow-kuei, 14 exs., 12-17. iv. 1965, J. L. Gressitt ; Vientiane Prov., Tha Ngone, 1 ex., 16. xi. 1965, Native collr ; Borikhane Prov., Paksane, 1 ex., 29. xi. 1965, Native collr ; Sayaboury Prov., Sayaboury, 13 exs., 15-16. iv. 1965, J. L. Gressitt, 1 ex., 12. ii. 1966, Native collr (BISHOP). VIETNAM : Fyan, 7 exs., 11. vii.-9. viii. 1961, N. R. Spencer (BISHOP).

Monolepta pallidulella n. sp. Fig. 38a

Generally yellowish brown, antenna black with apical four or five segments brownish.

Head with vertex smooth, shining, sparsely impressed by minute punctures, interocular space distinctly wider than transverse diameter of single oculus, and interocular transverse impression distinct, frontal tubercle subtriangular and distinctly raised. Antenna slender, nearly $2 / 3$ as long as length of body ; first segment long, second shortest, nearly $1 / 3$ as long as first, third $11 / 2$ times as long as second, fourth nearly twice as long as third, fifth slightly longer than fourth, fifth to eighth subequal to each other in length and shape, ninth slightly shorter than eighth, tenth slightly shorter than ninth, eleventh slightly longer than tenth and subequal to ninth in length but its apex pointed. Pronotum transverse, $13 / 4$ times as broad as long, anterior margin nearly straight, lateral margin slightly rounded, widest at $1 / 3$ from anterior margin, and slightly narrowed anteriorly and more strongly so posteriorly, basal margin strongly rounded posteriorly, dorsal surface convex, with a pair of deep transverse depressions which are united at middle, closely impressed by distinct punctures and interstices of punctures smooth, shining. Scutellum subtriangular, smooth, shining, impunctate. Elytron broader than prothorax, more strongly and closely punctate, and interstices of punctures smooth, shining.

Male : Fifth abdominal segment trilobed, median lobe generally flat. Female : Fifth abdominal segment entire.

Length : 4.2-5.0 mm.

Holotype (BISHOP) : VIETNAM : Fyan, 900-1,000 m, 11. vii.-9. viii. 1961, N. R. Spencer. Paratopotypes : 39 exs., same data as the holotype (BISHOP, KIMOTO). Paratypes : LAOS : Vientiane Prov., Vientiane, 1 ex., 31. v.-3. vi. 1960, S. Quate \& L. Quate (BISHOP). VIETNAM : Mt. Lang Bian, 
1,500-2,000 m, 13 exs., 19. v.-8. vi. 1961, N. R. Spencer ; Dalat, 1,500 m, 5 exs., 29. iv.-4. v. 1960, S. Quate \& L. Quate ; Dalat, 6 km S., 1,400-1,500 m, 8 exs., 9. vi.-7. vii. 1961, N. R. Spencer ; 15 km NW of Dalat, 1,850 m, 1 ex., 5. v. 1960, L. W. Quate ; 6 km SW, Dalat, 1,550 m, 1 ex., 11. ix. 1960, J. L. Gressitt ; Blao (Balao), 500 m, 2 exs., 14-21. x. 1960, C. M. Yoshimoto ; Dak Song, 76 km SWof Ban Me Thuot, 3 exs., 19-21. v. 1960, S. Quate \& L. Quate (BISHOP).

This new species closely resembles Monolepta pallidula (Baly), but differs in having antenna black with the apical four or five segments brownish, and pronotum with a transverse furrow much deeper.

Monolepta pseudornata n. sp. Fig. 43b

Generally pitchy black; elytron black with a large median marking yellowish brown ; meso- and metathorax black; abdomen reddish brown.

Head with vertex smooth, shining, sparsely impressed by minute punctures; interocular space subequal to transverse diameter of single oculus, and interocular transverse impression distinct, frontal tubercle subtriangular and distinctly raised. Antenna slender, nearly $2 / 3$ as long as body length ; first segment long, second shortest, nearly $1 / 4$ as long as first, third $21 / 4$ times as long as second, fourth $11 / 2$ times as long as third, fifth slightly longer than fourth, fifth to eighth subequal to each other in length and shape, ninth slightly shorter than eighth, tenth subequal to ninth in length and shape, eleventh longer than tenth and subequal to eighth in length but its apex pointed. Pronotum transverse, $11 / 2$ times as broad as long, anterior margin nearly straight, lateral margin slightly rounded, widest slightly before middle, and slightly narrowed anteriorly and posteriorly, basal margin strongly rounded posteriorly, dorsal surface convex, with a pair of deep transverse depressions laterally, rather closely impressed by fine punctures, and interstices of punctures smooth, shining. Scutellum subtriangular, smooth shining, impunctate. Elytron broader than prothorax, more strongly and closely punctate, and interstices of punctures smooth, shining, and with a feebly impressed transverse depression subbasally.

Male : Unknown. Female : Fifth abdominal segment entire.

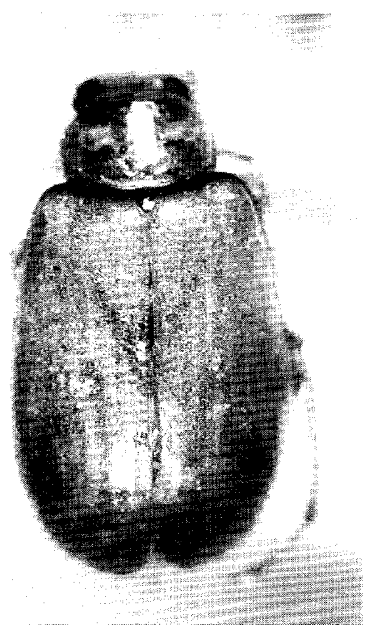

a

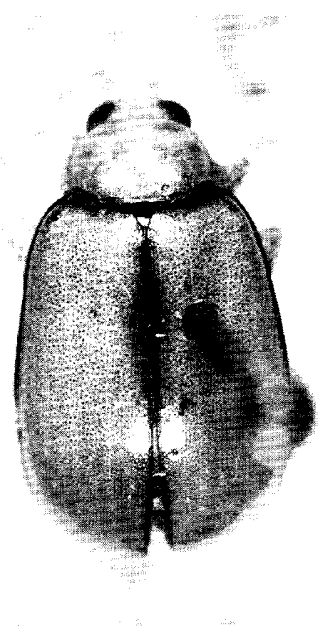

b

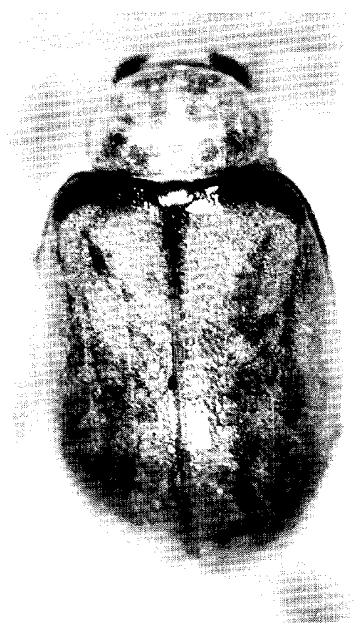

C

Fig. 39. a, Monolepta semicostata n. sp. ; b, M. trangica n. sp. ; c, M. azumai n. sp. 
Length : 5.3-5.4 mm.

Holotype (BISHOP) : VIETNAM : Fyan, 1,200 m, 11. vii.-9. viii. 1961, N. R. Spencer. Paratype : 1 ex same as holotype but $900-1,000 \mathrm{~m}$ (KIMOTO).

This new species resembles Monolepta ornata(Jacoby), from Burma, but differs in having the abdominal segments generally reddish brown, and elytral marking much elongate.

\section{Monolepta pseudosignata n.sp. Fig. 42b}

Generally yellowish to reddish brown; elytron black with two large discal markings, together with apex, yellowish brown, and basal marking closely situated to basal margin, antenna pitchy brown with basal two or three segments brownish, ventral surfaces with meso- and metathorax black, legs with tibiae and tarsi pitchy brown.

Head with vertex somewhat wrinkled, sparsely impressed by minute punctures, interocular space distinctly wider than transverse diameter of single oculus, and interocular transverse impression feebly impressed only on middle, frontal tubercle subtriangular and slightly raised. Antenna slender, nearly $2 / 3$ as long as length of body ; first segment long, second shortest, nearly $1 / 3$ as long as first, third $11 / 3$ times as long as second, fourth $21 / 3$ times as long as third, fourth to eighth subequal to each other in length and shape, ninth slightly shorter than eighth, tenth subequal to ninth in length and shape, eleventh longer than tenth and subequal to eighth in length but its apex pointed. Pronotum transverse, $11 / 2$ times as broad as long, anterior margin nearly straight, lateral margin slightly rounded, widest slightly before middle and slightly narrowed anteriorly and posteriorly, basal margin strongly rounded posteriorly, dorsal surface convex side to side, without any trace of depressions laterally, rather closely impressed by fine punctures, and interstices of punctures smooth, shining. Scutellum subtriangular, smooth, shining, impunctate. Elytron broader than prothorax, strongly and rather closely punctate, and interstices of punctures smooth, shining.

Male : Fifth abdominal segment trilobed, median lobe generally flat. Female : Fifth abdominal segment entire.

Length : $4.5 \mathrm{~mm}$.

Holotype (BISHOP) : LAOS : Sayaboury Prov., Sayaboury, 31. xii. 1965, Native collr. Paratypes : LAOS : Sayaboury Prov., Sayaboury, 2 exs., 6. xii. 1965, 1 ex., 12. xii. 1965, Native collr (BISHOP, KIMOTO). VIETNAM: Ban Me Thuot, 500 m, 1 ex., 16-18. v. 1960, L. W. Quate (BISHOP).

This new species closely resembles Monolepta signata (Olivier), but differs in being the body length longer, and having elytron with the apex yellowish brown.

\section{Monolepta quadrinotata n. sp. Fig. 42a}

Generally reddish brown; head dark reddish brown with vertex pitchy black, elytron black with anterior and posterior markings yellowish brown; antenna pitchy brown with basal one or two segments reddish brown; ventral surfaces with meso- and metathorax black; legs with most of tibiae and tarsi pitchy brown.

Head with vertex smooth, shining, sparsely impressed by minute punctures ; interocular space distinctly wider than transverse diameter of single oculus, and interocular transverse depression distinct, frontal tubercle subtriangular and distinctly raised. Antenna slender, nearly $2 / 3$ as long as body length ; first segment long, second shortest, nearly $L / 5$ as long as first, third slightly longer than second, fourth nearly twice as long as third, fourth to seventh subequal to each other in length and shape, eighth slightly shorter than seventh, eighth to tenth subequal to each other in length and shape, 
eleventh slightly longer than tenth and subequal to seventh in length but its apex pointed. Pronotum transverse, $11 / 2$ times as broad as long, anterior margin slightly rounded anteriorly, lateral margin distinctly rounded, widest almost at middle, slightly narrowed anteriorly and posteriorly, basal margin strongly rounded posteriorly, dorsal surface convex side to side, without any trace of depression laterally, sparsely impressed by fine punctures, and interstices of punctures smooth, shining. Scutellum subtriangular, smooth, shining, sparsely impressed by minute punctures. Elytron broader than prothorax, more strongly and closely punctate, and interstices of punctures smooth, shining.

Male : Fifth abdominal segment trilobed, median lobe generally flat. Female : Fifth abdominal segment entire.

Length: $3.9-4.2 \mathrm{~mm}$.

Holotype (BISHOP) : LAOS : Ban Van Eue, SE of Phou-kow-kuei, 12. iv. 1965, J. L. Gressitt. Paratopotypes : 2 exs., same data as the holotype (BISHOP, KIMOTO). Paratypes: THAILAND : Chiengmai Prov., Mak Klang, 340 m, 1 ex., 11. vi. 1965, P. D. Ashlock (BISHOP) ; Chieng Dao, 2 exs., 15. vi. 1965, K. Morimoto \& Y. Miyatate (KU). LAOS : Ban Van Eue, SE of Phou-kqw-kuei, 6 exs., 13. iv. 1965, 1 ex., 11. iv. 1965, J. L. Gressitt, 1 ex., 15-31. v. 1965, Native collr ; Phou-kow-kuei, 800 m, N. of Vientiane, 2 exs., 10. iv. 1965, J. L. Gressitt ;Vientiane Prov., Gi Sion Vill., de Tha Ngone, 7 exs., 2. i. 1966, Native collr ; Borikhane Prov., Pakkading, 2 exs., 13. vii. 1965, Native collr ; Sedon Prov., Pakse, 1 ex., 31. v. 1965, Native collr ; Wapikhamthong Prov., Khong Sedon, 1 ex., 30. v. 1965, Native collr ; Sayaboury Prov., Sayaboury, 1 ex., 15. iv. 1965, J. L. Gressitt (BISHOP).

This new species somewhat resembles Monoleptalaosensis Kimoto, but differs in being the body length shorter and having the head generally dark reddish brown to black.

Monolepta quatei n. sp. Fig. 44a

Generally yellowish brown to reddish brown; elytron black with yellowish marking covering nearly $4 / 5$ of width of disc and free from lateral and sutural margins; antenna pitchy black with basal two or three segments yellowish brown ; ventral surfaces with meso- and metathorax pitchy black.

Head with vertex smooth, shining, sparsely impressed by distinct punctures, interocular space distinctly wider than transverse diameter of single oculus, and interocular transverse impression distinct, frontal tubercle subtriangular and slightly raised. Antenna slender, nearly $2 / 3$ as long as length of body ; first segment long, second shortest, nearly $1 / 4$ as long as first, third as long as second but slenderer, fourth three times as long as third, fifth subequal to fourth in length and shape, sixth slightly shorter than fifth and sixth to ninth subequal to each other in length and shape, tenth slightly shorter than ninth, eleventh slightly longer than tenth and subequal to ninth in length but its apex pointed. Pronotum transverse, $13 / 4$ times as broad as long, anterior margin slightly rounded posteriorly, lateral margin distinctly rounded, widest almost at middle, distinctly narrowed anteriorly and posteriorly, basal margin strongly rounded posteriorly, dorsal surface convex side to side, without any trace of depression laterally, rather closely impressed by strong punctures, and interstices of punctures smooth and shining. Scutellum subtriangular, smooth, shining, impunctate. Elytron broader than prothorax, more strongly and closely punctate, and interstices of punctures smooth, shining.

Male : Unknown. Female : Fifth abdominal segment entire.

Length : 2.7-3.0 mm.

Holotype (BISHOP) : VIETNAM : Ban Me Thout, 16-18. v. 1960, L. W. Quate. Paratopotype : 1 ex., same data as the holotype (KIMOTO). Paratypes : THAILAND : Chiengmai Prov., Chieng Dao, 


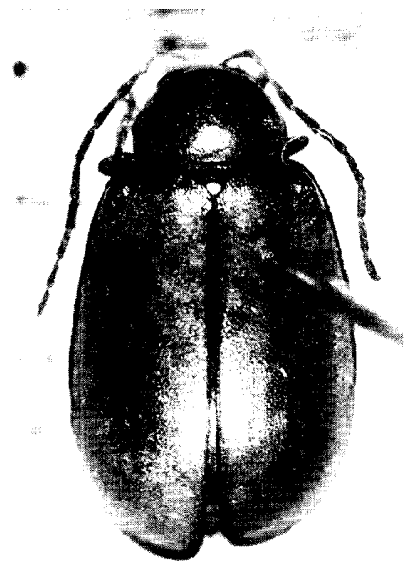

a

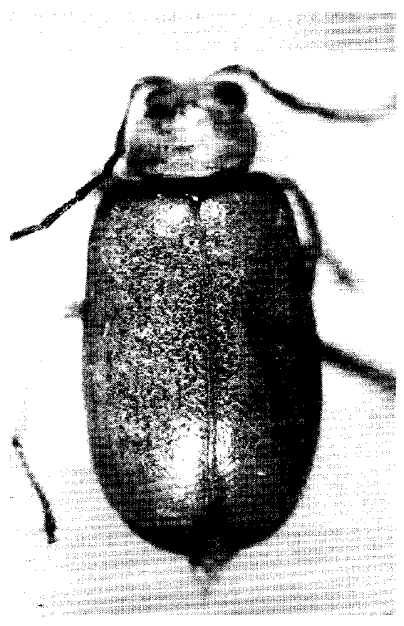

b

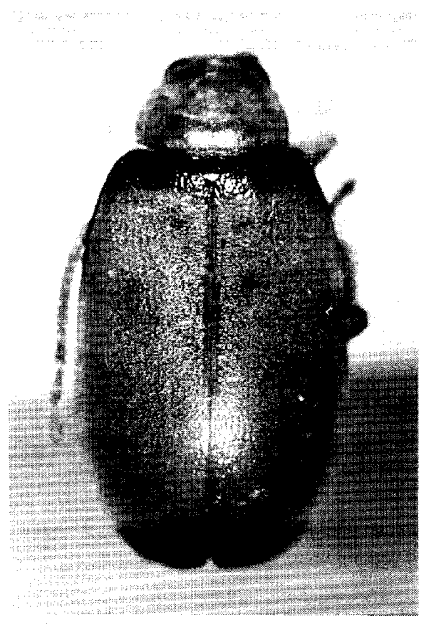

C

Fig. 40. a, Monolepta miyatakei n. sp. ; b, M. fyanica n. sp. ; c, M. banmethuotica n. sp.

1 ex., 15. vi. 1965, Y. Miyatake (KU). LAOS : Sedone Prov., Pakse, 1 ex., 31. v. 1967, Native collr (BISHOP).

This new species resembles Monolepta flavovittata Chen, but differs in having pronotum more strongly and closely punctate, and the head and the fifth abdominal segment generally brownish.

\section{Monolepta rondoni $\mathrm{n}$. sp. Fig. $41 \mathrm{a}$}

Generally yellowish brown; elytron with basal margin reddish brown and lateral, sutural and apical margins blackish ; head black; metathorax reddish brown, legs with tibiae and tarsi black; antenna reddish brown.

Head with vertex very finely and sparsely punctate, interocular space wider than transverse diameter of single' oculus, and interocular transverse impression distinct, frontal tubercle subtriangular and distinctly raised. Antenna slender, nearly $2 / 3$ as long as length of body ; first segment long, second shortest, nearly $1 / 3$ as long as first, third subequal to second in length, fourth $21 / 3$ times as long as third, fourth to sixth subequal to each other in length and shape, seventh slightly shorter than sixth, seventh to ninth subequal to each other in length and shape, eleventh subequal to ninth in length but its apex pointed. Pronotum transverse, $11 / 2$ times as broad as long, anterior margin nearly straight, lateral margin slightly rounded, widest at slightly before middle, narrowed anteriorly and posteriorly, dorsal surface convex, with a pair of shallow depressions laterally, closely impressed by fine punctures. Scutellum subtriangular, smooth, shining, impunctate. Elytron broader than prothorax, more strongly and closely punctate, and interstices of punctures smooth, shining.

Male : Fifth abdominal segment trilobed, median lobe generally flat. Female : Fifth abdominal segment entire.

Length : 5.7-7.2 $\mathrm{mm}$.

Holotype (BISHOP) : LAOS : Vientiane Prov., Ban Van Ėue, 1-15. v. 1965, J. A. Rondon. Paratopotypes : 13 exs., same data as the holotype (BISHOP, KIMOTO). Paratypes : Same locality as the holotype, 1 ex., 16. iv. 1965, J. L. Gressitt, 1 ex., 15-31. v. 1965, 1 ex., 15. xii. 1966, Native collr, 
Sayaboury Prov., Sayaboury, 2 exs., 15. iv. 1965, J. L. Gressitt ; Wapikhamthong Prov., Wapi, 2 exs., 15. x. 1967, Native collr (BISHOP) ; Umgeb. Vientiane, 6 exs., iii-vi. 1963 (MUNCHEN).

This new species resembles Monoleptamiyatakei Kimoto, but differs in having the basal margin of elytron reddish brown, and in the male the median lobe of the fifth abdominal segment generally flat.

\section{Monolepta rubrobasalis n.sp. $\quad$ Fig. $38 \mathrm{~b}$}

Generally yellowish brown, elytron with basal margin reddish brown.

Head with vertex very finely granulate, impunctate, interocular space distinctly narrower than transverse diameter of single oculus, and interocular transverse impression distinct, frontal tubercle subtriangular and distinctly raised. Antenna slender, nearly $2 / 3$ as long as length of body, first segment long, second shortest, nearly $1 / 4$ as long as first, third $11 / 2$ times as long as second, fourth $21 / 2$ times as long as third, fifth slightly longer than fourth, fifth to eighth subequal to each other in length and shape, ninth slightly shorter than eighth, tenth subequal to ninth in length and shape, eleventh subequal to ninth in length but its apex pointed. Pronotum transverse, $11 / 3$ times as broad as long, anterior margin nearly straight, lateral margin slightly rounded, widest almost at middle, slightly narrowed anteriorly and posteriorly, basal margin strongly rounded posteriorly, dorsal surface convex, with a pair of shallow depressions laterally, sparsely impressed by fine punctures, and interstices of punctures finely granulate. Scutellum subtriangular, finely granulate, impunctate. Elytron broader than prothorax, more strongly and closely punctuate, and interstices of punctures finely granulate.

Male : Fifth abdominal segment trilobed, median lobe longitudinally sulcate. Female : Fifth abdominal segment entire.

Length : 5.7-7.5 mm.

Holotype (BISHOP) : THAILAND : Chiengmai Prov., Fang, 500 m, 15. iv. 1958, T. C. Maa. Paratypes: THAILAND: Chiengmai Prov., Chiengmai, 5 exs., 11. iv. 1966, J. Sedlacek ; Chiengmai Prov., Fang, 500 m, 12-19. iv. 1958, T. C. Maa ; Chiengmai Prov., Chiengdao, 450 m, 12 exs., 5-11. iv. 1958, T. C. Maa ; Chiengmai Prov., Doi Suthep, 6 exs., 29. iii.-4. v. 1958, 1 ex., 28-31. iii. 1958, 4 exs., 1-8. iv. 1958, T. C. Maa ; $50 \mathrm{~km} \mathrm{W.} \mathrm{of} \mathrm{Tak,} 900$ m, 2 exs., 7-8. iv. 1966, J. \& J. H. Sedlacek (BISHOP) ; Chiengmai Prov., Doi Pui, 1.300 m, 2 exs., 8. vi. 1965, Y. Miyatake (KU).

This new species resembles Monolepta nigrifrons (Allard), but differs in having pronotum more narrower, and elytron yellowish brown with the basal margin reddish brown.

\section{Monolepta rugipennis (Laboissière), new combination}

Nadrana rugipennis Lab., 1936, Ann. Soc. Ent. France, 105 : 261 (Tonkin; HAMBURG).

Distribution : Thailand, Laos, Vietnam.

Material examined. THAILAND : Chiengmai Prov., Doi Suthep, 2 exs., 1-5. iv. 1958, T. C. Maa (BISHOP) ; Khao Yai Nat. Park, 2 exs., 6. vi. 1965, Y. Miyatake (KU) ; Khon Kaen, 1 ex., 24. vii. 1980, 2 exs., 8. x. 1981, S. Azuma ; Nam Proam, 2 exs., 19. i. 1980, S. Azumạ (RU). LAOS : Vientiane Prov., Ban Van Eue, 1 ex., 16. iii. 1966, 1 ex., 15. xii. 1966, Native collr ; Sayaboury Prov., Sayaboury, 2 exs., 21. iv. 1966, Native collr ; Phon Tiou, 4 exs., iii. 1965, 7 exs., 1-15. iv. 1965, J. L. Gressitt, 3 exs., 28 . iv. 1965, 40 exs., 30. iv 1965, 2 exs., 17. v. 1965, 2 exs., 13. vi. 1965, 8 exs., 6. vii. 1965, 1 ex., 4. viii. 1965, 6 exs., 11. ix. 1965, Native collr (BISHOP) ; Umgeb. Vientiane, 20 exs., 1963 ; Umgeb. Paklay, 16 exs., 1963 ; Umgeb. Vanky, 11 exs., 1963, 2 exs., 1964 ; Umgeb. Pakse, 1 ex., 1963, 8 exs., 1964 (MUNCHEN). 


\section{Monolepta scripta (Motschulsky)}

Luperodes scriptus Motsch., 1866, Bull. Soc. Nat. Moscow, 39 (1) : 416 (Ceylon).

Luperodes multimaculatus Jacoby, 1887, Proc. 2001. Soc. London, 1887 : 111 (Ceylon ; BM). -Maulik, 1936, Fauna India, Galeruc. : 403 (=scripta).

Monolepta multimaculata: Jacoby, 1889, Ann. Mus. Civ. Genova, 27 : 226 (Burma ; GENOVA). -Maulik, 1936, Fauna India, Galeruc. : 403 (=scripta).

Monolepta piceomaculata Jacoby, 1896, Ann. Soc. Ent. Belg., 40 : 295 (India ; BM).-Maulik, 1936, Fauna India, Galeruc. : 403 (=scripta).

Monolepta scripta: Maulik, 1936, Fauna India, Galeruc. : 403 (India, Ceylon, Burma).

Distribution : India, Ceylon, Burma, Laos, Vietnam.

Material examined. LAOS : Ban Van Eue, 800 m, SE of Phou-kow-kuei, 1 ex., 11. iv. 1965, J. L. Gressitt (BISHOP). VIETNAM : Fyan, 900-1,000 m, 2 exs., 11. vii.-9. viii. 1961, N. R. Spencer ; Dalat, 6 km S., 1,400-1,500 m, 1 ex., 9. vi.-7. vii. 1961, N. R. Spencer (BISHOP).

\section{Monolep ta scutellaris n. sp. Fig. 34a}

Generally yellowish to reddish brown, elytron with scutellar and humeral marking and apical $1 / 3$ blackish in male and with basal and apical $1 / 3$ blackish in female, legs generally pitchy black.

Head with vertex somewhat wrinkled, sparsely impressed by fine punctures, interocular space subequal to transverse diameter of single oculus, and interocular transverse impression distinct, frontal tubercle subtriangular and distinctly raised. Antenna slender, nearly as long as body length ; first segment long, second shortest, nearly $1 / 3$ as long as first, third $11 / 2$ times as long as second, fourth twice as long as third, fifth to tenth subequal to each other in length and shape, eleventh slightly shorter than tenth and its apex pointed. Pronotum transverse, $11 / 4$ times as broad as long in male and $11 / 2$ times as broad as long in female, anterior margin nearly straight, lateral margin feebly rounded, widest slightly before middle and slightly narrowed anteriorly and more strongly so posteriorly, basal margin distinctly rounded posteriorly, and almost straight at middle, dorsal surface convex, and with a pair of deep oblique depressions and closely impressed by distinct punctures, and their interstices smooth, shining. Scutellum subtriangular, smooth, shining, impunctate. Elytron broader than prothorax, finely and closely punctate, and interstices of punctures smooth, shining.

Male : Elytron with a longitudinal shallow depression along suture and with a tuft of hairs behind scutellum ; fifth abdominal segment trilobed, median lobe slightly depressed as a whole. Female : Elytron normal ; fifth abdominal segment entire.

Length : 5.3-6.0 mm.

Holotype (BISHOP) : LAOS : Sedone Prov., Paksong, 17-18. v. 1965, light trap, P. D. Ashlock. Paratopotypes : 3 exs., same data as the holotype (BISHOP, KIMOTO). Paratypes : LAOS : Attopeu Prov., Houei Kong, 1 ex., 31. v. 1965, 6 exs., 20. vii. 1965, Native collr ; Vientiane Prov., Ban Van Eue, 1 ex., 15. ii. 1966, 1 ex., 30. iii. 1967, 1 ex., 15-30. viii. 1968, Native collr (BISHOP).

This new species resembles Monolepta marginipennis(Jacoby), but differs in having pronotum with a pair of deep oblique depressions laterally, and in male elytron with a longitudinal shallow depression and a tuft of hairs behind scutellum.

Monolepta semiapicalis n. sp. Fig. 36a

Head black, prothorax yellowish brown, elytron pitchy black with apical $1 / 3$ reddish brown and its boundary ill-defined ; ventral surfaces yellowish to reddish brown with most of meso- and 


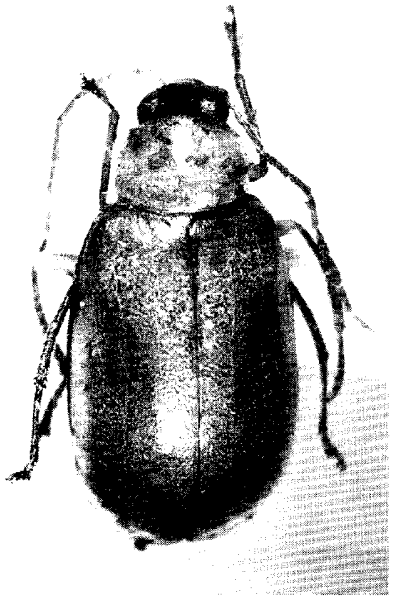

a

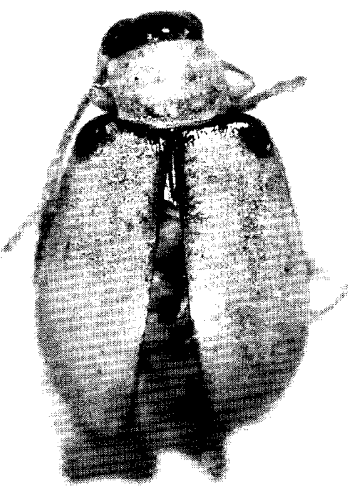

b

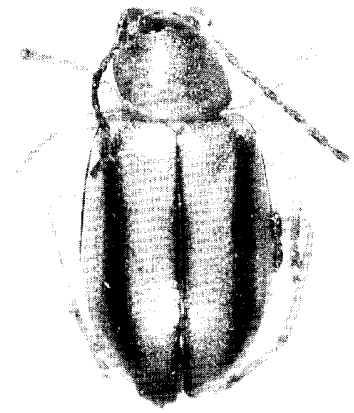

c

Fig. 41. a, Mondepta rondoni n. sp. ; b, M. semihumeralis n. sp. ; c, M. sexlineata Chajô.

metathorax pitchy black ; antenna and legs entirely yellowish brown.

Head with vertex sparsely impressed by fine punctures and their interstices smooth and shining; interocular space distinctly wider than transverse diameter of single oculus, and interocular transverse impression distinct, frontal tubercle subtriangular and distinctly raised. Antenna slender, nearly $2 / 3$ as long as body length; first segment long, second shortest, nearly $1 / 3$ as long as first, third subequal to second in length, fourth $31 / 4$ times as long as third, fifth slightly longer than fourth, fifth to eighth subequal to each other in length and shape, ninth slightly shorter than eighth, tenth subequal to ninth in length and shape, eleventh subequal to tenth in length but its apex pointed. Pronotum transverse, $11 / 2$ times as broad as long, anterior margin nearly straight, lateral margin slightly rounded, widest slightly before middle and slightly narrowed anteriorly and posteriorly, basal margin strongly rounded posteriorly, dorsal surface convex, with a pair of shallow oblique depressions laterally, sparsely impressed by distinct punctures, and interstices of punctures smooth and shining. Scutellum subtriangular, smooth, shining, impunctate. Elytron broader than prothorax, more strongly and closely punctate, and interstices of punctures smooth and shining.

Male : Unknown. Female : Fifth abdominal segment entire.

Length : $4.8 \mathrm{~mm}$.

Holotype (BISHOP) : VIETNAM : Mt. Lang Bian, 1,500-2,000 m, N. R. Spencer. Paratopotypes : 2 exs., same data as the holotype (BISHOP, KIMOTO).

This new species somewhat resembles Monolepta luperina Weise, but differs in having elytron with the apical $1 / 3$ reddish brown, and the head generally blackish.

\section{Monolepta semicostata n. sp. Fig. 39a}

Generally yellowish brown, elytron with basal margin blackish ; scutellum pitchy black ; antenna pitchy black with basal two or three segments paler; head with narrow blackish area behind eye; ventral surfaces with metathorax largely blackish.

Head with vertex sparsely impressed by minute punctures, surface smooth, shining interocular 
space distinctly wider than transverse diameter of single oculus, and interocular transverse impression distinct, frontal tubercle subtriangular and distinctly raised. Antenna slender, nearly $2 / 3$ as long as length of body ; first segment long, second shortest, nearly $2 / 5$ as long as first, third as long as second but slenderer, fourth $21 / 2$ times as long as third, fourth to eighth subequal to each other in length and shape, ninth slightly shorter than eighth, tenth subequal to ninth in length and shape, eleventh slightly longer than tenth and subequal to eighth in length but its apex pointed. Pronotum transverse, nearly $12 / 3$ times as broad as long, anterior margin nearly straight, lateral margin distinctly rounded, widest at $1 / 3$ from anterior margin, slightly narrowed anteriorly and more strongly so posteriorly, basal margin distinctly rounded posteriorly, dorsal surface convex, with a pair of shallow transverse depressions laterally, sparsely impressed by fine punctures, and interstices of punctures smooth, shining. Scutellum subtriangular, smooth, shining, impunctate. Elytron broader than prothorax, more strongly and closely punctate, and punctures arranged in semiregular longitudinal rows, and their interstices smooth, longitudinally raised.

M ale : Fifth abdominal segment trilobed, median lobe generally flat. Female : Fifth abdominal segment entire.

Length : 3.0-4.1 mm.

Holotype (BISHOP) : LAOS: Khuammouane Prov., Phon Tiou, 11-12. vi. 1965, J. A. Rondon. Paratypes : LAOS : Khuammouane Prov., Phon Tiou, 1 ex., 3. iv. 1965, 1 ex., 9. vi. 1965, N. Wilson ; Vientiane Prov., Ban Van Eue, 1 ex., 14-15. iv. 1965, J. L. Gressitt, 1 ex., 28. ii. 1966, Native collr ; Borikhane Prov., Pakkading, 100-200 m, 1 ex., 23. iv. 1965, J. L. Gressitt (BISHOP, KIMOTO).

This new species somewhat resembles Monoleptaazumai Kimoto, but differs in having elytron impressed by the punctures semiregularly arranged in rows and their interstices distinctly raised longitudinally.

\section{Monolepta semihumeralis $\mathbf{n}$. $\mathbf{s p}$. Fig. $41 \mathrm{~b}$}

Head black, prothorax yellowish brown, elytron yellowish brown with margins, together with humerus, black to pitchy brown, and basal part of sutural margin somewhat widely blackish ; antenna pitchy black, with two or three basal segments brownish ; meso- and metathorax pitchy black; abdomen pitchy black with fifth segment brownish; legs yellowish brown with tibiae and tarsi pitchy brown.

Head with vertex smooth, shining, sparsely impressed by minute punctures; interocular space distinctly wider than transverse diameter of single oculus, and interocular transverse impression distinct, frontal tubercle subtriangular and distinctly raised. Antenna slender, nearly $2 / 3$ as long as length of body ; first segment long, second shortest, nearly $1 / 3$ as long as first, third subequal to second in length but slenderer, fourth twice as long as third, fifth slightly longer than fourth, sixth slightly shorter than fifth, sixth to eighth subequal to each other in length and shape, ninth slightly shorter than eighth and robuster, tenth subequal to ninth in length and shape, eleventh subequal to eighth in length but its apex pointed. Pronotum transverse, $11 / 2$ times as broad as long, anterior margin nearly straight, lateral margin slightly rounded, widest almost at anterior margin, and slightly narrowed posteriorly, basal margin strongly rounded posteriorly, dorsal surface convex, with a pair of deep depressions laterally, rather closely impressed by distinct punctures, and interstices of punctures smooth, shining. Scutellum subtriangular, smooth, shining, impunctate. Elytron broader than prothorax, more strongly and closely punctate, and interstices of punctures smooth, shining.

Male : Unknown. Female : Fifth abdominal segment entire.

Length : $2.7 \mathrm{~mm}$.

Holotype (BISHOP) : VIETNAM : Mt. Lang Bian, 1,500-2,000 m, 19. v.-8. vi. 1961, N. R. Spencer. 
Paratopotypes : 3 exs., same data as the holotype (BISHOP, KIMOTO). Paratype : VIETNAM : Dalat, 1,500 m, 1 ex., 29. iv.-4. v. 1960, S. Quate \& L. Quate (BISHOP).

This new species somewhat resembles Monolepta nigrobasalis Jacoby, but differs in being the body length shorter, and having the head entirely blackish.

\section{Monolep ta semiluperina n. sp. Fig. 36b}

Generally pitchy black, head, prothorax and scutellum reddish brown, antenna pitchy black with first segment generally reddish brown ; legs pitchy black with anterior leg generally reddish brown.

Head with vertex smooth, shining, sparsely impressed by minute punctures, interocular space subequal to transverse diameter of single oculus, frontal tubercle subtriangular and slightly raised and not delimited posteriorly by interocular transverse impression. Antenna slender, nearly as long as body length ; first segment long, second shortest, nearly $1 / 3$ as long as first, third $11 / 2$ times as long as second, fourth nearly $12 / 3$ times as long as third, fifth slightly longer than fourth, fifth to seventh subequal to each other in length and shape, eighth slightly shorter than seventh, eighth to tenth subequal to each other in length and shape, eleventh longer than tenth and subequal to fifth in length but its apex pointed. Pronotum transverse, $12 / 3$ times as broad as long, anterior margin nearly straight, lateral margin slightly rounded, widest almost at $1 / 3$ from basal margin, and narrowed anteriorly and posteriorly, basal margin strongly rounded posteriorly, dorsal surface convex side to side, without any trace of depression laterally, sparsely impressed by fine punctures, and interstices of punctures smooth, shining. Scutellum subtriangular, smooth, shining, impunctate. Elytron broader than prothorax, more strongly and closely punctate, and interstices of punctures smooth, shining.

Male : Fifth abdominal segment trilobed, median lobe generally flat. Female : Fifth abdominal segment entire.

Length : 2.7-3.5 mm.

Holotype (BISHOP) : VIETNAM : $6 \mathrm{~km} \mathrm{S.,} \mathrm{Dalat,} \mathrm{12.} \mathrm{ix.} \mathrm{1960,} \mathrm{J.} \mathrm{L.} \mathrm{Gressitt.} \mathrm{Paratopotype} \mathrm{:} 1$ ex., same data as the holotype (KIMOTO). Paratypes : VIETNAM :6 km S. of Dalat, 1,550 m, 1 ex., 11. ix. 1960, J. L. Gressitt ; Fyan, 1,200 m, 1 ex., 11. vii.- 9. viii. 1961, N. R. Spencer ; Blao (Balao), 500

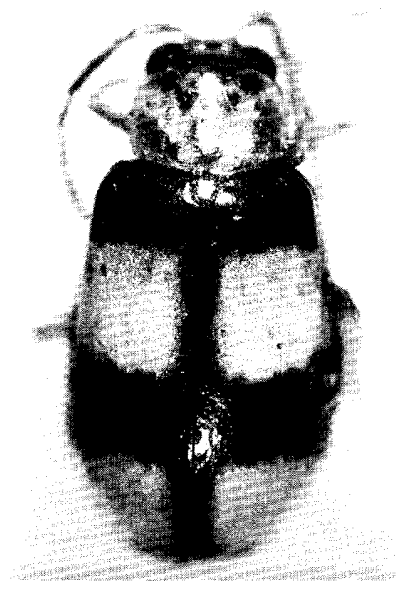

a

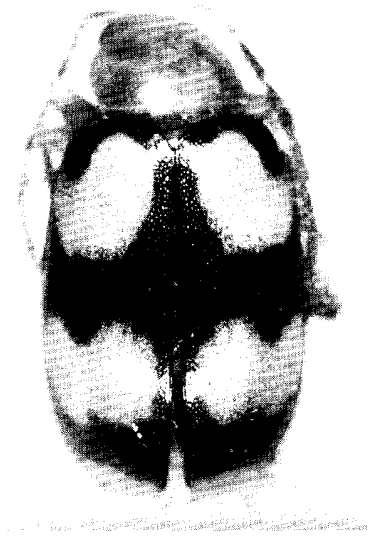

$\mathrm{b}$

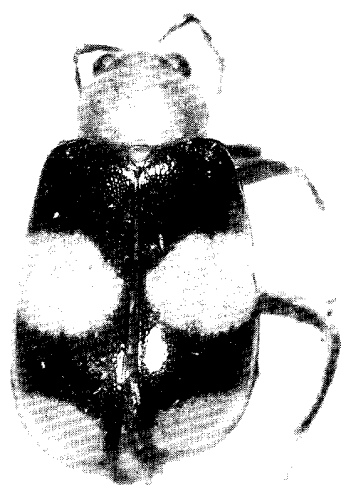

ᄂ

Fig. 42. a, Monolepta quadrinotata n. sp. ; b, M. pseudosignata n. sp. ; c, M. laosensis n. sp. 
m, 1 ex., 14-21. x. 1960, C. M. Yoshimoto (BISHOP).

This new species resembles Monoleptaluperina Weise, but differs in having the legs blackish with the anterior leg brownish, and meso- and metathorax and the abdomen entirely blackish.

\section{Monolepta severini (Jacoby)}

Luperus severini Jac., 1896, Ann. Soc. Ent. Belg., 40 : 276 (Belgaum ; BM).

Monolepta severini: Manlik, 1936, Fauna India, Galeruc. : 424, 431 (India, Burma).-Châjô, 1961, Nature and Life in SE Asia, Kyoto, 1 : 351 (Thailand : Chieng Mai); 1964, ibid., 3 : 295

(Thailand : Chieng Mai, Li).

Distribution : India, Burma, Thailand, Laos, Vietnam.

Material examined. THAILAND: Chiengmai Prov., Chiengdao, 2 exs., 15. vi. 1965, P. D. Ashlock, 5 exs., 5-11. iv. 1958, T. C. Maa, 1 ex., 5-11. iv. 1958, Native collr ; Chiengmai Prov., Doi Suthep, 7 exs., 28-31. iii. 1958, 1 ex., 1-15. iv. 1958, T. C. Maa, 1 ex., 14. xi. 1958, J. L. Gressitt ; Chiengmai Prov., Fang, 3 exs., 12-19. iv. 1958, T. C. Maa; Chiengmai Prov., Doi Pui, 1 ex., 2. iv. 1958, T. C. Maa ; Chiengmai Prov., Ban-tin-doi, 310 m, 1 ex., 14. xi. 1957, J. L. Gressitt ; Pangmakampon (Pakampawng), nr. Fang, 2 exs., 16. xi. 1957, J. L. Gressitt ;Doi Angka nr. Chiengmai Phon, 2,436 m, 1 ex., 9. iv. 1958, T. C. Maa ; Pakchong, 100 m, NE of Bangkok, 1 ex., 3. xii. 1957, J. L. Gressitt, 1 ex., 1-3. xii. 1957, J. L. Gressitt ; Chantaburi, Prew, 45 m, 1 ex., 25-30. iv. 1958, T. C. Maa ; Pangkan Pawng, 1 ex., 15. xi. 1957. J. L. Gressitt (BISHOP) ; Chiengmai Prov., Doi Suthep, 5 exs., 18-19. vi. 1965, Y. Miyatake ; Chiengmai Prov., Doi Pui, 1,200-1,300 m, 5 exs., 17. vi. 1965, K. Morimoto \& Y. Miyatake ; Chieng Dao, 7 exs., 15. vi. 1965, K. Morimoto \& Y. Miyatake, 2 exs., 10. xi. 1966, sweeping of paddy field, K. Yasumatsu \& K. Yano, 4 exs., 23. iv. 1973, Y. Yoshiyasu ; Chiengmai Prov., Fang, 1 ex., 14. vi. 1965, K. Morimoto, 1 ex., 22. viii. 1965, S. Nakao ; Khao Yai Nat. Park, 1 ex., 6. vi. 1965, Y. Miyatake ; Samptong, 1 ex., 17. viii. 1973, S. Nakao ; Ratapoom, 3 exs., 27. vi. 1965, Y. Miyatake ; Kor Hong, 2 exs., 23. vi. 1965, K. Morimoto ; Songkhla, 7 exs., 23. vi. 1965, Y. Miyatake ; Mae Trang, 3 exs., 10. xi. 1966, sweeping of paddy field, K. Yasumatsu \& K. Yano ;Pasang, 1 ex., 9. xi. 1966, sweeping of paddy field, K. Yasumatsu \& K. Yano ; Parabattakpa, 2 exs., 9. xi. 1966, sweeping of paddy field, K. Yasumatsu \& K. Yano ; Lampoon, 1 ex., 17. vii. 1970, K. Yano ; Mae Rim, nr. Chiengmai, 1 ex., 10. xi. 1966, sweeping of paddy field, K. Yasumatsu \& K. Yano, 1 ex., 3. vii. 1970, sweeping of paddy field, K. Yano (KU) ; Fang Spa, 2 exs., 21. xi. 1968, K. Hatta (EHIME) ; Khon Kaen, 2 exs., 7-8. x. 1981, S. Azuma (RU) ; Saraburi, 1 ex., 28. i. 1968, K. Baba (KIMOTO). LAOS : Vientiane Prov., Vientiane, 1 ex., 31. v.-3. vi. 1960, S. Quate \& L. Quate ; Vientiane Prov., Ban Van Eue, 1 ex., 1-15. v. 1965, J. A. Rondon; Vientiane Prov., Phou-kow-kuei, 1 ex., 17. iv. 1965, J. L. Gressitt ; Vientiane Prov., Tha Ngone, 1 ex., 6. ix. 1965, Native collr ; Luang Prabang, 300 m, 4 exs., 4-5. vi. 1960, S. Quate \& L. Quate ; Muong Sing, NW of Luang Prabang, 650 m, 1 ex., 6-10. vi. 1960, S. Quate \& L. Quate ; Borikhane Prov., Pakkading, 100 m, 1 ex., 21. iv. 1965, J. L. Gressitt ; Sayaboury Prov., Sayaboury, 2 exs., 15. i. 1966, 1 ex., 12. ii. 1966, Native collr (BISHOP). VIETNAM : Fyan, 9001,200 m, 49 exs., 11. vii.-9. viii. 1961, N. R. Spencer ; Mt. Lang Bian, 1,500-2,000 m, 23 exs., 19. v.-8. vi. 1961, N. R. Spencer ; Dalat, 1,500 m, 6 exs., 29. iv.-4. v. 1960, S. Quate \& L. Quate, 8 exs., 26-27. ix. 1960, C. M. Yoshimoto, 1 ex., 11. ix. 1960, J. L. Gressitt ; 5 km S. of Dalat, 1,300 m, 1 ex., 2. v. 1960, S. Quate \& L. Quate ; $6 \mathrm{~km} \mathrm{SW}$ of Dalat, 1,550 m, 1 ex., 11. ix. 1960, J. L. Gressitt ; $6 \mathrm{~km} \mathrm{S.} \mathrm{of} \mathrm{Dalat,} \mathrm{1,550} \mathrm{m,}$ 3 exs., 12. ix. 1960, J. L. Gressitt, 19 exs., 9. vi.-7. vii. 1961, N. R. Spencer ; 15 km, from Dalat, 1,500 m, 1 ex., 29. iv.-5. v. 1960, S. Quate ; 17 km NE of Dalat, 2,000 m, 1 ex., 3. v. 1960, L. W. Quate ; $37 \mathrm{~km} \mathrm{SE}$ of Dalat, 780 m, 1 ex., 25. iv. 1960, L. W. Quate ; 20 km S., Dalat, 1,300 m, 5 exs., 12. ix. 1960, J. L. Gressitt ; Quang Tri, 2 km N., 1 ex., 9. iii. 1970, 1 ex., 30. iv. 1970, 4 exs., 9-11. vi. 1970, A. R. Gillogly ; 34 km N., Phan Rang, 1 ex., 9. xi. 1960, C. M. Yoshimoto ; Blao (Balao), 600 m, 1 ex., 14-21. x. 1960, 
C. M. Yoshimoto ; Dak Song, $76 \mathrm{~km} \mathrm{SW}$ of Ban Me Thuot, 870 m, 2 exs., 19-21. v. 1960, S. Quate \& L. Quate ; Ap Hung-Lam, 21 km NW of Dilinh, 1,100 m, 1 ex., 29. ix.-5. x. 1960, C. M. Yoshimoto ; 50 km SW of Pleiku, 250 m, 4 exs., 14. v. 1960, S. Quate \& L. Quate ; 25 km SW of Pleiku, 400 m, 4 exs., 12. v. 1960, L. W. Quate ; 20 km N. of Pleiku, 650 m, 5 exs., 9. v. 1960, L. W. Quate ;M'Drak, E. of Ban Me Thuot, 4-600 m, 1 ex., 8-19. xii. 1960, C. M. Yoshimoto ; Dilin (Djiring), 1,200 m, 9 exs., 22-28. iv. 1960, L. W. Quate (BISHOP).

\section{M onolepta sexlineata Chajô $\quad$ Fig. 41c}

Monolepta duvivieri Jacoby, 1904, Ann. Soc. Ent. Belg., 48 : 404 (nec Jacoby, 1897) (India : Nilgiri Hills ; BM).

Monolepta lineata Weise, 1915, Ergebnisse 2. Dtsche Zentr. Afr. Exp., Zool., 1: 177 (new name for Monolepta duvivieri Jacoby, 1904 ; nec Monolepta lineata Karsch, 1882)._Maulik, 1936, Fauna India, Galeruc. : 398 (India).-Chûjô, 1961, Nature and Life in SE Asia, Kyoto, 1 : 351, Fig. (Thailand).

Monolepta sexlineata Chûjô, 1938, Arb. Morph. Taxon. Ent. Berlin-Dahlem, 5 (2) : 150, fig. (Formosa ; DEI) ; 1962, Phil. J. Sci., 91 (1-2) : 127, fig. (Formosa).-Gressitt \& Kimoto, 1963, Pac. Ins. Mon., 1B: 632 (China, Hainan).

Monolepta tarsalis:Chûjô, 1961, Nature and Life in SE Asia, Kyoto, 1 : 352 (Thailand : Chiengmai).

Monolepta madrasensis Wilcox, 1973, Col. Cat. Junk, Suppl., 78 (3) : 562 (new name for Monolepta duvivieri Jacoby, 1904, and Monolepta Zineata Weise, 1911).-Kimoto \& Takizawa, 1983, Bull. Natn. Sci. Mus. Tokyo, ser. A, 9 (2) : 89 (Nepal). New synonym.

Distribution : India, Nepal, Thailand, Cambodia, Laos. Vietnam, China, Hainan, Taiwan.

Material examined. THAILAND : Chiengmai, 1,100-1,500 m, 1 ex., 11-12. iv. 1966, J. Sedlacek ; 20 km E. of Chiengmai, 330 m, 2 exs., 13-15. iv. 1966, J. Sedlacek (BISHOP) ; Chiengmai, 1 ex., 9. vi. 1965, K. Morimoto ; Takhane Distr., 1 ex., 24. ii. 1965, K. Yasumatsu ; Chieng Dao, 19 exs., 16. viii. 1973, sweeping of paddy field, K. Yano ; Tung Saew, 2 exs., 8. xi. 1966, sweeping of paddy field, K. Yasumatsu \& K. Yano ; Mae Sai, 1 ex., 13. vii. 1970, sweeping of paddy field, K. Yano ; Saraburi, 1 ex., 31. vii. 1970, sweeping of paddy field, K. Yano ; Amphoe Phan, Chieng Rai, 1 ex., 25. iv. 1973, Y. Yoshiyasu (KU) ; Fang Spa, 1 ex., 21. xi. 1968, K. Hatta (EHIME). CAMBODIA : Ph. Chisau, 40 km S. of P. Penh, 20 m, 1 ex., 29. iv. 1961, N. R. Spencer (BISHOP). LAOS : Sedon Prov., Paksong, 8 exs., 18. v. 1965, P. D. Ashlock; Muong Sing, NW of Luang Prabang, 650 m, 3 exs., 6-10. vi. 1960, L. W. Quate (BISHOP). VIETNAM : Mt. Lang Bian, 1,500-2,000 m, 2 exs., 19. v.-8. vi. 1961, N. R. Spencer (BISHOP).

\section{M onolepta signata (Olivier) Fig. 33c}

Galeruca signata Oliv., 1808, Entomologie, $6: 665$, pl. 5, fig. 89 (Indies or. : Bengal).

Crioceris neglecta Sahlberg, 1829, in Thomson, Ent. Arch., 2 (1) : 29, pl. 2, fig. 36 (Ostindien: Bengal).

Luperodes quadripustulatus Motschulsky, 1858, Etud. Ent., 7 : 105 (India or., Ceylon).-Jacoby, 1887, Proc. 2001. Soc. London, 109 (Ceylon).-Allard, 1891, Nouv. Arch. Mus. Paris, ser. 3, 3: 231 (Luang-Prabang); 1904, Miss. Pavie Indo-Chine, $3: 160$ (Cambodge).

Monolepta signata : Jacoby, 1889, Ann. Mus. Civ. Genova, 27 : 229 (Burma, Tenasserim).-Allard, 1889, Ann. Soc. Ent. France, ser. 6, 9 : 312 (Saigon, Pnom-Penh, Mythgo, Zui-Nhon) ; 1891, Nouv. Arch. Mus. Paris, ser. 3, 3 : 231 (Luang-Prabang).-Duvivier, 1891, C. R. Soc. Ent. Belg., 


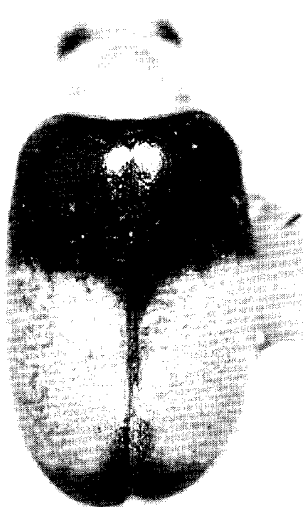

a

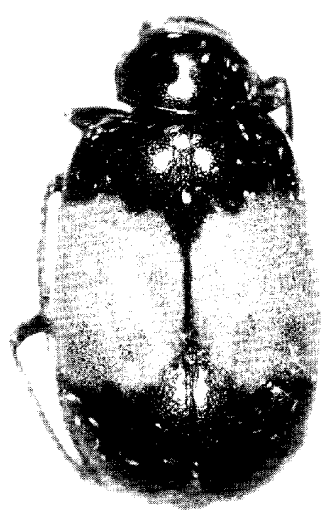

b

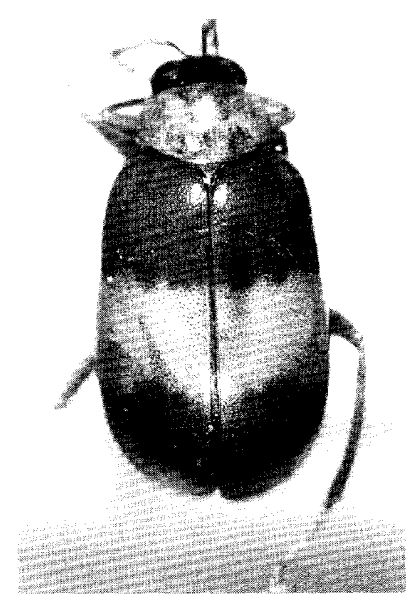

Fig. 43. a, Monolepta subfasciata n. sp. ; b, M. pseudornata n. sp. ; c, M. spenceri n. sp.

35 : 153 (Java) ; 1892, Ann. Soc. Ent. Belg., 36 : 444 (India)-Allard, 1904, Miss. Pavie IndoChine, 3 : 160 (Cambodge).-Maulik, 1936, Fauna India, Galeruc. : 410 (India, Ceylon, Siam, China).-Bryant, 1952, Ann. Mag. Nat. Hist., ser. 12, 5 : 602 (Nepal).-Chûjô, 1961, Nature and Life in SE Asia, Kyoto, 1 : 351 (Cambodia, Thailand) ; 1964, ibid., 3 : 296 (Thailand).-Gressitt \& Kimoto, 1963, Pac. Ins. Mon., 1B: 606, 633, fig. (China, Vietnam).-Kimoto, 1970, Khumbu Himal, 3 (3) : 421 (Nepal).-Kimoto \& Takizawa, 1972, Kontyâ, Tokyo, 40 (4) : 220 (Nepal) ; 1983, Bull. Natn. Sci. Mus., Tokyo, ser. A, 9 (2) : 90 (Nepal).

Monolepta neglecta: Weise, 1922, Tijdschr. Ent., 65 : 102 (Bengalen).

Monolepta signata abb. lutea, coalita, bipunctata, pici, yunnanensis, sexmaculata, belmonti Piton, 1943, Bull. Soc. Linn. Lyon, 12 ; 138 (Yunnan).

Distribution : India, Ceylon, Burma, Nepal, Thailand, Cambodia, Vietnam, S. China, Hainan, Java.

Material examined. THAILAND : Chiang Dao, 1 ex., 10. xi. 1966, sweeping of paddy field, K. Yano, 10 exs., 16. viii. 1973, sweeping of paddy field, K. Yano ; Mae Taeng, 3 exs., 16. viii. 1973, sweeping of paddy field, K. Yano ; Fang, 9 exs., 21. viii. 1973, K. Yano ; Chieng Mai, 12 exs., 17. viii. 1973, sweeping of paddy field, K. Yano ; Sampatong, 5 exs., 24. viii. 1973, sweeping of paddy field, K. Yano, 1 ex., 7. viii. 1970, soybean, K. Yano, 1 ex., 29. xi. 1963, K. Yasumatsu, 1 ex., 8. xi. 1966, sweeping of paddy field, K. Yasumatsu \& K. Yano ; Mae Tang, 12 exs., 10. xi. 1966, sweeping of paddy field, K. Yasumatsu \& K. Yano ; Tung Saew, 6 exs., 8. xi. 1966, sweeping of paddy field, K. Yasumatsu \& K. Yano ; Sikiew, 3 exs., 5. xi. 1966, sweeping of paddy field, K. Yasumatsu \& K. Yano ; Ban-Prom, 2 exs., 4. xi. 1966, sweeping of paddy field, K. Yasumatsu \& K. Yano ; Rajburi, 3 exs., 1. xi. 1966, sweeping of paddy field, K. Yasumatsu \& K. Yano ; Rangsit, nr. Bangkok, 1 ex., 7. xi. 1966, sweeping of paddy field, K. Yasumatsu \& K. Yano ; Chomtong, $40 \mathrm{~km} \mathrm{SW}$ of Chieng Mai, 2 exs., 2. vii. 1970, sweeping of paddy field, K. Yano ; Songquare, nr. Sampatong, 3 exs., 4. vii. 1970, sweeping of paddy field, K. Yano ; Mae Sai, 3 exs., 13. vii. 1970, sweeping of paddy field, K. Yano ;Sarapi, nr. Chieng Mai, 1 ex., 18. vii. 1970, sweeping of paddy field, K. Yano ; Mae Talai, nr. Chieng Dao, 1 ex., 3. vii. 1970, upland rice, K. Yano ; Ban-Tai Gud Hin, 1 ex., 4. xi. 1966, sweeping of paddy field, K. Yasumatsu \& K. Yano ; Sakon Nakhon, 4 exs., 25. ii. 1965, sweeping of paddy field, K. Yasumatsu, Kao Chong 
Park, Phattalung, 3 exs., 1. iii. 1965, sweeping of paddy field, K. Yasumatsu ; Takhae Distr., 5 exs., 24. ii. 1965, K. Yasumatsu; Ban Tap Dua, Chiang Dao, 1 ex, 15. viii. 1973, sweeping of paddy field, K. Yano ; Botanical Garden, Chieng Mai, 1 ex, 13. viii. 1973, K. Yano ; Prew, 1 ex., 29. viii. 1973, sweeping of paddy field, K. Yano ; Khon Kaen, 1 ex., 3. xi. 1966, sweeping of paddy field, K. Yasumatsu \& K. Yano ; Pasang, 1 ex., 9. xi. 1966, sweeping of paddy field, K. Yasumatsu \& K. Yano ; Prabattakpa, 1 ex., 9. xi. 1966, sweeping of paddy field, K. Yasumatsu \& K. Yano ; Chiengmai Prov., Doi Pui, 5 exs., 17. vi. 1965, K. Morimoto \& Y. Miyatake ; Chiengmai Prov., Doi Suthep, 1 ex., 10. vi. 1965, K. Morimoto ; Mae Sa Water Fall, 2 exs., 16. vi. 1965, K. Morimoto ; Chiengmai, 7 exs., 9. vi. 1965, 3 exs., 12. vi. 1965, K. Morimoto ; Chieng Dao, 1 ex., 15. vi. 1965, K. Morimoto ; Fang, 13 exs., 14. vi. 1965, K. Morimoto ; Khao Yai Nat. Park, 6 exs., 6. vi. 1965, K. Morimoto \& Y. Miyatake ; Khao Chong, nr. Trung, 1 ex., 27. vi. 1965, K. Morimoto (KU) ; Khon Kaen, 3 exs., 20. xii. 1979, 1 ex., 17. i. 1980, S. Azuma ; Nam Proam, 2 exs., 6. i. 1980, S. Azuma (RU) ; Fang Spa, 5 exs., 21. xi. 1968, K. Hatta ; Khon Kaen, 1 ex., 2. x. 1972, M. Sato (EHIME) ; Chieng Dao, 2 exs., 23. iv. 1973, Y. Yoshiyasu ; Amphoe Phan, Chieng Rai, 1 ex., 25. iv. 1978, Y. Yoshiyasu ; Khao Yai, 2 exs., 29-31. i. 1968, K. Baba (KIMOTO) ; Rayong, 14 exs., 27. vii. 1962 ; Cholburi, 7 exs., 26. iii. 1962 ; Udon thani, 6 exs., 15. vii. 1958 ; Chiengmai, 2 exs., 17. iv. 1958 ; Tak, 2,800 ft., 1 ex., 20. viii. 1961 (BANGKHEN) ; Chiengmai Prov., Chiengmai, 4 exs., 9. vi. 1965, P. D. Ashlock, 1 ex., 1. xi. 1963, Native collr ; Chiengami Prov., Fang, 5-600 m, 5 exs., 14. vi. 1965, 5 exs., 13. vi. 1965, 2 exs., 19. iv. 1958, T. C. Maa ; Chiengmai Prov., Chieng Dao, 450 m, 2 exs., 5-11. iv. 1958, T. C. Maa; Chiengmai Prov., Chieng Dao, 450 m, 2 exs., 5-11. iv. 1958, T. C. Maa; Chiengmai Prov., Mae Sa, 1 ex., 16. vi. 1965, P. D. Ashlock; Chiengmai Prov., Mae Klang, 8 exs., 11. vi. 1965, P. D. Ashlock; Trang Prov., Khaophappha, Kaochang, 200-400 m, 3 exs., 30-31. xii. 1963, 1 ex., 1. i. 1964, 1 ex., 4. i. 1964,1 ex., 10. i. 1964, G. A. Samuelson ; Pakchong, 100 m, NE of Bangkok, 1 ex., 1. xii. 1957, J. L. Gressitt, 6 exs., 11. iii. 1964, Native collr ; Nakhon Nayok Prov., Khao Yai Nat. Park, 8 exs., 5. vi. 1965, P. D. Ashlock; Saraburi Prov., Ban Muak Lek Nat. Park, 3 exs., 6. vi. 1965, P. D. Ashlock; Saraburi, 2 exs., 4. iii. 1963, Native collr ; Muak Lek, 1 ex., 19. xi. 1963, Native collr ; Chanthaburi, Prew, 45 m, 3 exs., 24. iv.-1. v. 1958, T. C. Maa (BISHOP). CAMBODIA : Kompong Chhnang, 7 m, 1 ex., 25. iv. 1961, N. R. Spencer (BISHOP). LAOS : Umgeb. Vientiane, 3 exs., iii-vi. 1963 ; Umgeb. Paklay, 2 exs., 1963 (MUNCHEN) ; Borikhane Prov., Pakkading, 1 ex., 13. vii. 1965, J. L. Gressitt \& K. A. J. Wise, 11 exs., 31. viii. 1965, 34 exs., 15-31. viii. 1965, 12 exs., 9. ix., 79 exs., 15. ix, 1965, 2 exs., x. 1965, Native collr ; Borikhane Prov., Paksane, 1 ex., 17. viii. 1965, 1 ex., 31. viii. 1965, 1 ex., 19. xi. 1965, 2 exs., 13. xii. 1965,3 exs., 8. xii. 1965, 1 ex., 7. i. 1966, 13 exs., 1. ii. 1966, 3 exs., 8. ii. 1966, Native collr ; Khammouane Prov., Phon Tiou, 1 ex., 10. v. 1965, N. Wilson ; Wapikhamthong Prov., Khong Sedone, 1 ex., 21. ix. 1965, Native collr ; Attopeu Prov., Houei Kong, 2 exs., 31. v. 1965, Native collr ; Sedon Prov., Pakse, 2 exs., 11. v. 1965, 1 ex., 22. v. 1965, 2 exs., 13. v. 1965,1 ex., 15. v. 1965, 4 exs., 21. v. 1965, 1 ex., 23. v. 1965, P. D. Ashlock; K. Sedon, 7 exs., 13. x. 1965, Native collr ; Savanakhet, 1 ex., 15. x. 1965, Native collr ; Muong Sing, NW of Luang Prabang, 650 m, 17 exs., 6-10. vi. 1960, S. Quate \& L. W. Quate ; Dong Dok, 1 ex., 5. viii. 1965, 1 ex., 7. ix. 1965, 1 ex., 1. xii. 1965, 1 ex., 6. xii. 1965, 2 exs., 8.. i. 1966, Native collr ; Nongtevda, 1 ex., 27. vii. 1965, 1 ex., 6. viii. 1965, 1 ex., 30. viii. 1965, 2 exs., 18. xi. 1965, 1 ex., 7. ii. 1966, Native collr (BISHOP). VIETNAM : Vientiane Prov., Vientiane, 1 ex., 8. v. 1965, 2 exs., 26. v. 1965, 2 exs., 27. v. 1965, 1 ex., 28. v. 1965, P. D. Ashlock, 3 exs., 22. vii. 1965, 2 exs., 2. viii. 1965, 4 exs., 3. viii. 1965, 5 exs., 3. ii. 1966, Native collr ; Vientiane Prov., Namgueng River, Phou Kou Khouei, 100 m, 1 ex., 10. iv. 1965, Native collr ; Vientiane Prov., Ban Van Eue, 3 exs., 15-31. v. 1965, 2 exs., 31. xii. 1965, 1 ex., 15. ii. 1965, 1965, 1 ex., 31. xii. 1968, Native collr ; Vientiane, Tha Ngone, 1 ex., 1. ix. 1965, 1 ex., 11. ix. 1963, 1 ex., 20. ix. 1965, 1 ex., 16. xi. 1965, 1 ex., 20. xi. 1965, 1 ex., 25. xi. 1965, 1 ex., 4. xii. 1965, 1 ex., 9. ii. 1966, Native collr ; Ban Me Thuot, 500 m, 2 exs., 16-18. v. 1960, S. Quate ;M'Drak, SE of Ban Me Thuot, 4-600 m, 23 exs., 8-19. xii. 1960, C. M. Yoshimoto ; Dak Song, 76 km SE of Ban Me Thuot, 870 
m, 1 ex., 19-21. v. 1960, L. W. Quate ; Dilin (Djiring), 1,200 m, 12 exs., 2'2-28. iv. 1960, S. Quate \& L. W. Quate, 1 ex., 27. ix.-14. x. 1960, C. M. Yoshimoto ; 9 km S. of Dilin (Djiring), 3 exs., 24. iv. 1960, R. E. Leech ; Dalat, 6 km S., 1,400-1,500 m, 16 exs., 9. vi.-7. vii. 1961, N. R. Spencer; 18 km NW of Dalat, 1,300 m, 2 exs., 4-5. v. 1960, L. W. Quate ; Karyu Danar, 200 m, 3 exs., 13-28. ii. 1961, N. R. Spencer ; Fyan, 1,200 m, 1 ex., 11. vii.-9. viii. 1961, N. R. Spencer ; Pleiku, 700 m, 1 ex., 8-14. v. 1960, L. W. Quate ; Mt. Lang Bian, 1,500-2,000 m, 1 ex., 19. v.-8. vi. 1961, N. R. Spencer ; Quang Tri, 2 km N, 1 ex., 9. vi. 1970, A. R. Gillogly (BISHOP).

\section{Monolepta spenceri n. sp. Fig. 43c}

Generally yellowish brown, elytron with basal $1 / 3$ and apical $1 / 3$ reddish brown ; head black ; ventral surfaces with metathorax reddish brown ; posterior legs more reddish.

Head with vertex sparsely impressed by fine punctures and their interstices finely wrinkled ; interocular space distinctly narrower than transverse diameter of single oculus, and interocular transverse impression distinct, frontal tubercle subtriangular and distinctly raised. Antenna slender, nearly $2 / 3$ as long as length of body ; first segment long, second shortest, nearly $1 / 3$ as long as first, third as long as second, fourth nearly three times as long as third, fourth to sixth subequal to each other in length and shape, seventh slightly shorter than fifth, and seventh to tenth subequal to each other in length, eleventh subequal to tenth in length but its apex pointed. Pronotum transverse, $12 / 3$ times as broad as long, anterior margin nearly straight, lateral margin slightly rounded, widest slightly before middle and slightly narrowed anteriorly and posteriorly, basal margin strongly rounded posteriorly, dorsal surface convex side to side, without any trace of depression laterally, rather closely impressed by fine punctures, and interstices of punctures smooth and shining. Scutellum subtriangular, finely granulate, impunctate. Elytron broader than prothorax, more strongly and closely punctate, and interstices of punctures smooth and shining.

M ale : Unknown. Female : Fifth abdominal segment entire.

Length : $5.7 \mathrm{~mm}$

Holotype (BISHOP) : VIETNAM : Mt. Lang Bian, 1,500-2,000 m, 19. v.-8. vi. 1961, N. R. Spencer. Paratopotype ; 1 ex., same data as the holotype (KIMOTO). Paratype : VIETNAM : Fyan, 900-1,000 m, 1 ex., 11. vii.-9. viii. 1961, N. R. Spencer (BISHOP).

This new species somewhat resembles Monolepta hageni Weise, but differs in having elytron with the apical $1 / 3$ reddish, the head entirely black and antenna and the legs yellowish to reddish brown.

\section{Monolepta subfasciata n. sp. Fig. 43a}

Generally yellowish brown, elytron black with a large transverse band covering from sutural to lateral margins yellowish brown; ventral surfaces entirely black.

Head with vertex slightly wrinkled, sparsely impressed by minute punctures, interocular space distinctly wider than transverse diameter of single oculus, and interocular transverse impression distinct only on median portion, frontal tubercle subtriangular and feebly raised. Antenna slender, nearly $2 / 3$ as long as length of body ; first segment long, second shortest, nearly $2 / 5$ as long as first, third subequal to second in length but slightly slenderer, fourth nearly three times as long as third, and fourth to eighth subequal to each other in length and shape, ninth slightly shorter than eighth, tenth subequal to ninth in length and shape, eleventh slightly longer than tenth and its apex pointed. Pronotum transverse, $12 / 3$ times as broad as long, anterior margin slightly rounded posteriorly, lateral margin almost straight, gradually narrowed posteriorly, basal margin distinctly rounded posteriorly, dorsal surface convex side to side, without any trace of depression laterally, sparsely 


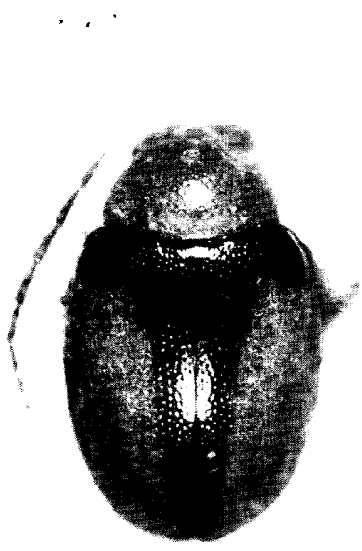

a

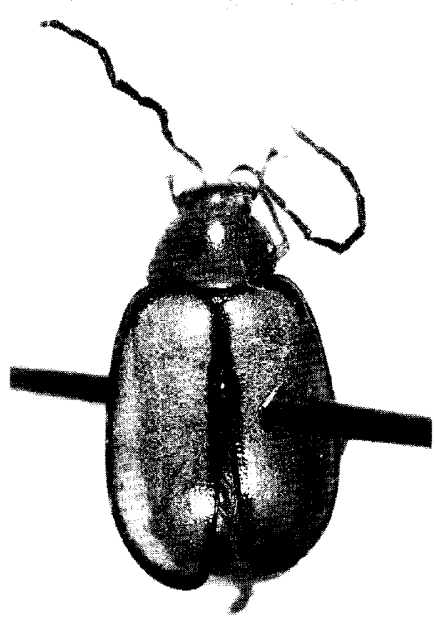

$\mathrm{b}$

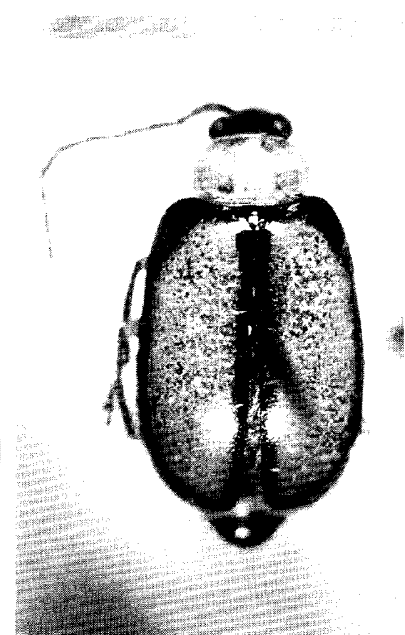

c

Fig. 44. a, Monolepta quatei n. sp. ; b, M.atrimarginata n. sp. ; c, M. dalatica n. sp.

impressed by distinct punctures, and interstices of punctures smooth, shining. Scutellum subtriangular, smooth, shining, impunctate. Elytron broader than prothorax, more strongly and closely punctate, and interstices of punctures smooth, shining.

M ale : Fifth abdominal segment trilobed, median lobe generally flat. Female : Fifth abdominal segment entrire.

Length : $3.0-3.3 \mathrm{~mm}$.

Holotype (BISHOP) : VIETNAM : Mt. Lang Bian, 1,500-2,000 m, 19. v.-8. vi. 1961, N. R. Spencer. Paratopotypes : 7 exs., same data as the holotype (BISHOP, KIMOTO). Paratypes : LAOS : Namkading, nr. Pakkading, $100 \mathrm{~m}, 1$ ex., 21. iv. 1965, J. L. Gressitt (BISHOP). VIETNAM : $17 \mathrm{~km} \mathrm{NE}$ of Dalat, 2,000 m, 1 ex., 3. v. 1960, L. W. Quate (BISHOP).

This new species somewhat resembles Monolepta birmanensis Jacoby, but differs by the shorter body and having the legs entirely yellowish brown.

\section{Monolepta subflavipennis n. sp. Fig. 37a}

Generally reddish brown, prothorax black, head, antenna and legs generally pitchy black.

Head with vertex smooth, shining, sparsely impressed by minute punctures, interocular space subequal to transverse diameter of single oculus, and interocular transverse impression shallower, frontal tubercle subtriangular and distinctly raised. Antenna slender, nearly $2 / 3$ as long as length of body ; first segment long, second shortest, nearly $1 / 3$ as long as first, third $12 / 3$ times as long as second, fourth $11 / 2$ times as long as third, fifth subequal to fourth, sixth to ninth subequal to each other in length and shape, tenth slightly shorter than ninth, eleventh slightly longer than tenth and subequal to ninth in length but its apex pointed. Pronotum transverse, $11 / 4$ times as broad as long, anterior margin nearly straight, lateral margin slightly rounded, widest almost at middle, slightly narrowed anteriorly and posteriorly, basal margin strongly rounded posteriorly, dorsal surface convex, with a pair of distinct depressions laterally, sparsely impressed by fine punctures, and interstices of punctures smooth and shining. Scutellum subtriangular, finely granulate, impunctate. 
Elytron broader than prothorax, more strongly and closely punctate, and interstices of punctures smooth and shining, and with a shallow transverse furrow subbasally.

Male : Fifth abdominal segment trilobed, median lobe slightly depressed at middle. Female : Fifth abdominal segment entire.

Length : 3.8-3.9 mm.

Holotype (BISHOP) : VIETNAM : Fyan, 900-1,000 m, 11. vii.-9. viii. 1961, N. R. Spencer. Paratopotypes: 3 exs., same data as the holotype (BISHOP, KIMOTO).

This new species resembles Monolepta langbianica Kimoto, but differs in being the body length slightly shorter, and having elytron and the ventral surfaces reddish and antenna entirely blackish.

\section{Monolepta sublata Gressitt \& Kimoto}

Monolepta sublata Gressitt Az Kimoto, 1963, Pac. Ins. Mon. 1B: 605, 636 (S. China ; BISHOP). -Kimoto, 1976, Ent. Rev. Japan, 29 : 6 (Taiwan).

Distribution : Thailand, S. China, Taiwan.

Material examined. THAILAND : Khao Yai Nat. Park, 1 ex., 6. vi. 1965, Y. Miyatake (KU) ; Khao Yai, 1 ex., 10. iv. 1968, Native collr (BISHOP).

\section{Monolepta testacea (Jacoby)}

Luperodes testacea Jacoby, 1903, Ann. Soc. Ent. Belg., $47: 119$ (India ; BM).

Monolepta testacea : Maulik, 1936, Fauna India, Galeruc.: 428 (India).-Chajô, 1961, Nature and Life in SE Asia, Kyoto, $1: 352$ (Thailand, Chieng Mai).

Distribution : India, Thailand.

No additional material was examined.

\section{Monolepta thailandica n. sp. Fig. 45a}

Generally yellowish brown, elytron with basal $1 / 4$ and a large subapical marking pitchy black to black, antenna pitchy brown with first and apical three segments reddish brown, ventral surfaces with meso- and metathorax pitchy black; legs with tibiae and tarsi generally pitchy black.

Head with vertex somewhat wrinkled, sparsely impressed by minute punctures ; interocular space distinctly wider than transverse diameter of single oculus, and frontal tubercle slightly raised but ill-defined, and not delimited posteriorly by transverse depression. Antenna slender, in preapical segments three times as long as wide, nearly $2 / 3$ as long as body length ; first segment long, second shortest, nearly $1 / 3$ as long as first, third slightly longer than second but more slenderer, fourth $21 / 4$ times as long as third, fifth subequal to fourth in length and shape, sixth slightly shorter than fifth, sixth to ninth subequal to each other in length and shape, tenth slightly shorter than ninth, eleventh subequal to ninth in length but its apex pointed. Pronotum transverse, $12 / 5$ times as broad as long, anterior margin nearly straight, lateral margin slightly rounded, widest slightly before middle, and slightly narrowed anteriorly and more strongly so posteriorly, basal margin strongly rounded posteriorly, dorsal surface convex side to side, without any trace of depression laterally, sparsely impressed by fine punctures, and interstices of punctures smooth, shining. Scutellum subtriangular, smooth, shining, impunctate. Elytron broader than prothorax, more strongly and closely punctate, and interstices of punctures smooth, shining.

Male : Fifth abdominal segment trilobed, median lobe generally flat. Female : Fifth abdominal segment entire. 
Length : $3.3 \mathrm{~mm}$.

Holotype (BISHOP) : THAILAND : Trang Prov., Khaophappha, Khaochang, 200-400 m, 30-31. xii. 1963, G. A. Samuelson. Paratypes : THAILAND : Trang Prov., Khaophappha, Khaochong, 200400 m, 2 exs., 1-3. i. 1964, 3 exs., 10-12. i. 1964, G. A. Samuelson ; Nakhon Nayok Prov., Khao Yai Nat. Park, 1 ex., 6. vi. 1964, P. D. Ashlock (BISHOP) ; Chiengmai Prov., Chieng Dao, 1 ex., 23. iv. 1973, Y. Yoshiyasu (KU) ; Khao Yai, 1 ex., 29-31. i. 1968, K. Baba (KIMOTO).

This new species resembles Monolepta cambodgia Laboissière, but differs in having the head generally brownish, and antenna pitchy brown with the first and the apical three segments brownish.

\section{Monolepta trangica n. sp. $\quad$ Fig. $39 \mathrm{~b}$}

Generally yellowish brown ; elytron with basal, basal $2 / 3$ of lateral and most of sutural margins blackish ; scutellum pitchy black ; ventral surfaces with meso- and metathorax and basal part of first to fourth abdominal segments blackish.

Head with vertex sparsely impressed by minute punctures, surface slightly wrinkled, interocular space subequal to transverse diameter of single oculus, and interocular transverse impression feebly impressed, frontal tubercle subtriangular and distinctly raised. Antenna slender, nearly $2 / 3$ as long as length of body ; first segment long, second shortest, nearly $1 / 3$ as long as first, third slightly longer than second, fourth $21 / 2$ times as long as third, fourth to eighth subequal to each other in length and shape, ninth slightly shorter than eighth, tenth subequal to ninth in length and shape, eleventh subequal to tenth in length but its apex pointed. Pronotum transverse, $12 / 3$ times as broad as long, anterior margin nearly straight, lateral margin slightly rounded, widest almost at middle, slightly narrowed anteriorly and posteriorly, basal margin strongly rounded posteriorly, dorsal surface convex, with a pair of feeble depressions laterally, sparsely impressed by fine punctures, and interstices of punctures smooth and shining. Scutellum subtriangular, finely granulate, impunctate. Elytron broader than prothorax, more strongly and closely punctate, and interstices of punctures smooth, shining.

Male : Fifth abdominal segment trilobed, median lobe generally flat. Female : Fifth abdominal segment entire.

Length : 5.7-6.3 mm.

Holotype (BISHOP) : THAILAND : Trang Prov., Khaophappha, Kaochang, 200-400 m, 13. i. 1964, G. A. Samuelson. Paratopotypes: 3 exs., same data as the holotype (BISHOP, KIMOTO). Paratypes : Same locality and collector as the holotype, 1 ex., 4. i. 1964, 1 ex., 10. i. 1964, 1 ex., 11-12. i. 1964 (BISHOP).

This new species somewhat resembles Monolepta pallida(Jacoby), but differs in having elytron with the basal, the basal $2 / 3$ of the lateral and most of the sutural margins blackish.

\section{Monolepta vietnamica n. sp. Fig. 34c}

Generally reddish brown ; elytron with basal $2 / 3$ yellowish brown, humerus, basal $1 / 4$ of sutural and basal half of lateral margins and entire basal margin pitchy brown, and apical half blackish brown and gradually becomes paler towards apex ; antenna entirely yellowish brown, legs pitchy black, abdomen entirely yellowish brown.

Head with vertex finely granulate, sparsely impressed by fine punctures, interocular space subequal to transverse diameter of single oculus, and interocular transverse impression distinct, frontal tubercle subtriangular and distinctly raised. Antenna slender, nearly as long as body length ; first segment long, second shortest, nearly $1 / 3$ as long as first, third $11 / 3$ times as long as second, 


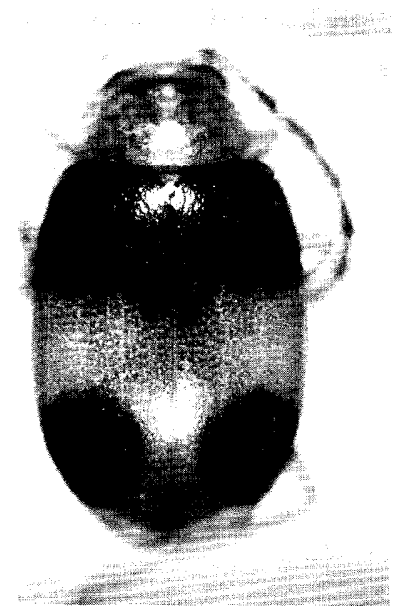

a

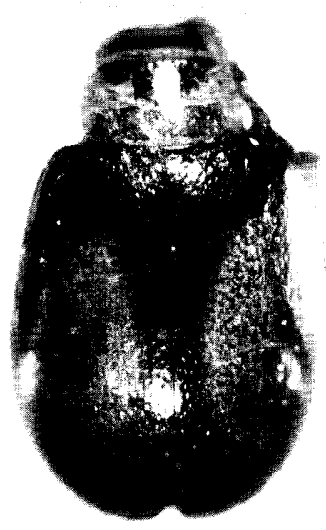

$\mathrm{b}$

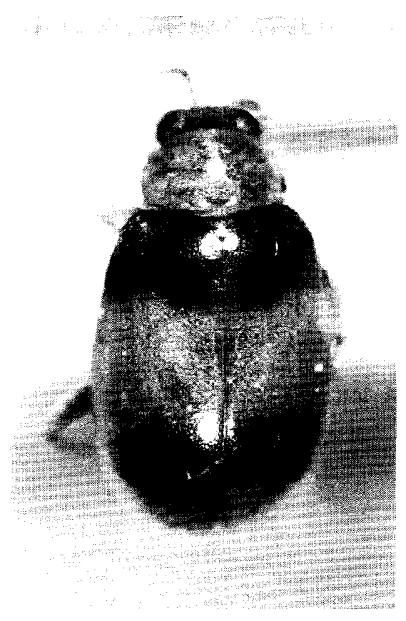

C

Fig. 45. a, Monolepta thailandican. sp. ; b, M.exigua n. sp. ; c, M. bifasciata (Hornsted).

fourth nearly twice as long as third, fifth slightly longer than fourth, fifth to eighth subequal to each other in length and shape, ninth slightly shorter than eighth, tenth subequal to ninth in length and shape, eleventh subequal to eighth in length but its apex pointed. Pronotum transverse, $11 / 2$ times as broad as long, anterior margin nearly straight, lateral margin nearly straight, widest slightly behind anterior comer and slightly narrowed posteriorly, basal margin rounded posteriorly and almost straight at middle, dorsal surface convex, with a pair of shallow depressions laterally and a slight convexity at middle of anterior margin, closely impressed by strong punctures, and interstices of punctures smooth, slightly convex. Scutellum subtriangular, smooth, shining, impunctate. Elytron broader than prothorax, closely impressed by distinct punctures, and their interstices smooth, shining.

M ale : Elytron with an elongate fovea contiguous to suture ; fifth abdominal segment trilobed, median lobe slightly depressed as a whole. Female : Elytron normal ; fifth abdominal segment entire.

Length: $4.1-4.5 \mathrm{~mm}$.

Holotype (BISHOP) : VIETNAM : Dalat, 6 km S., 1,400-1,500 m, 9. vi.-7. vii. 1961, N. R. Spencer. Paratopotypes : 3 exs., same data as the holotype (BISHOP). Paratypes : VIETNAM : Fyan, 9001,200 m, 3 exs., 11. vii.-9. viii. 1961, N. R. Spencer (BISHOP, KIMOTO).

This new species resembles Monolepta discoidalis (Jacoby), but differs in having pronotum more transverse and the surface more strongly and closely punctate.

\section{Monolepta wilsoni n. sp. Fig. $35 \mathrm{c}$}

Generally yellowish brown, head pitchy brown, elytron yellowish to reddish brown, with basal, lateral, sutural and apical margins narrowly black, and with six discal markings $(2: 2: 2)$ black, ventral surfaces with meso- and metathorax and fifth abdominal segment pitchy brown, antenna pitchy brown, legs with tibiae and tarsi pitchy brown.

Head with vertex very finely granulate, sparsely impressed by distinct punctures, interocular space distinctly narrower than transverse diameter of single oculus, and interocular transverse impression distinct, frontal tubercle subtriangular and distinctly raised. Antenna slender, nearly $2 / 3$ as long as body length ; first segment long, second shortest, nearly $1 / 4$ as long as first, third $11 / 2$ times 
as long as second, fourth nearly $21 / 2$ times as long as third, fifth slightly longer than fourth, fifth to seventh subequal to each other in length and shape, eighth slightly shorter than seventh, ninth subequal to eighth in length and shape, tenth slightly shorter than ninth, and eleventh longer than tenth, and subequal to ninth in length but its apex pointed. Pronotum transverse, $12 / 3$ times as broad as long, anterior margin nearly straight, lateral margin slightly rounded, widest at $1 / 3$ from anterior margin, and slightly narrowed anteriorly and more strongly so posteriorly, basal margin strongly rounded posteriorly, dorsal surface convex, with a pair of deep oblique depressions laterally, sparsely impressed by distinct punctures, and interstices of punctures finely granulate. Scutellum subtriangular, finely granulate, impunctate. Elytron broader than prothorax, sparsely impressed by distinct punctures, and interstices of punctures finely granulate.

Length : $4.2 \mathrm{~mm}$.

Holotype (BISHOP) : LAOS : Khammouane Prov., Phon Tiou, 13. iv. 1965, N. Wilson. Paratypes: THAILAND: Chiengmai Prov., Doi Suthep, 1,000 m, 1 ex., 18. vi. 1965, Y. Miyatake (KU). LAOS : Khammouane Prov., Phon Tiou, 1 ex., 10. vi. 1965, N. Wilson (KIMOTO).

This new species is characteristic in having elytron yellowish brown with the margins and the discal six markings blackish.

\section{Monolepta zonalis Gressitt \& Kimoto}

Monolepta zonalis Gressitt \& Kimoto, 1963, Pac. Ins. Mon., 1B:638 (SW China, Vietnam; BISHOP).

Distribution : Laos, Vietnam, SW China.

Material examined. LAOS : Umgeb. Vientiane, 3 exs., iii-vi. 1963 ; Umgeb. Paklay, 1 ex., 1963 (MUNCHEN).

\section{Monolepta zonula Weise}

Monolepta albofasciata Jacoby, 1889, Ann. Mus. Civ. Genova, $27: 228$ (nec Monolepta albofasciata

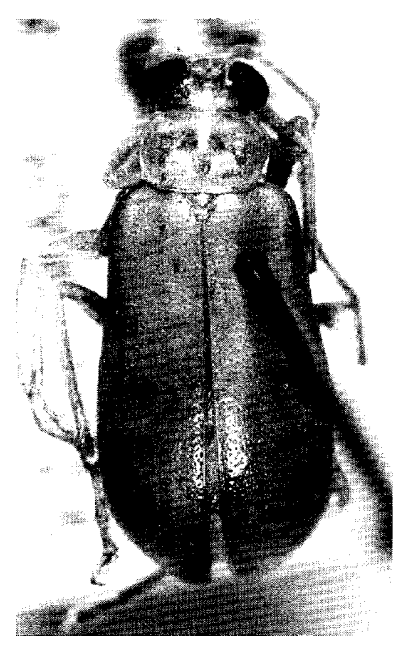

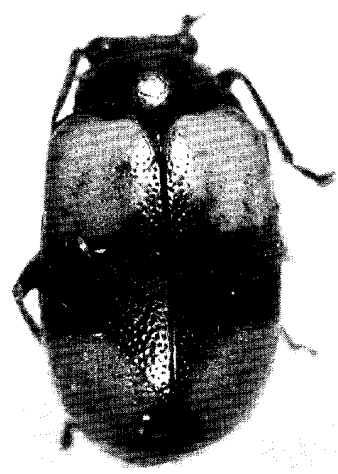

b

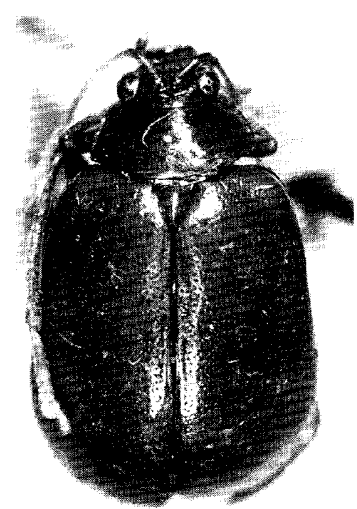

C

Fig. 46. a, Liroetoides fulvus n. sp. ; b, Sermyloides maculatipennis n. sp.; c, s. majorn. sp. 
Jacoby, 1884, from Java) (Burma ;GENOVA).-Allard, 1891, Nouv. Arch. Mus. Paris., ser. 3, 3 : 231 (Luang-Prabang à Theng) ; 1904, Miss. Pavie Indo-Chine, 3 : 160 (Cambodge ; Siam, Chantaboun à Battambang).

Monolepta fasciatipennis Jacoby, 1892, Ann. Mus. Civ. Genova, 32 : 983 (Burma ; GENOVA). -Maulik, 1936, Fauna India, Galeruc. : 413 (=albofasciata).

Monolepta zonula Weise, 1916, Dtshe Ent. Z., 1916 : 40 (new name for Monolepta albofascifata Jacoby, 1889, nec 1884).-Maulik, 1936, Fauna India, Galeruc. : 413 (Burma).

Distribution : Burma, Cambodia, Laos.

No material was examined.

\section{Genus Liroetoides n. gen.}

Antennal insertions distinctly separated by frons ; labrum rounded, truncate apically, frontal tubercles contiguous ; gena $1 / 4$ as deep as oculus ; third antennal segment much longer than second ; prothorax with anterior margin not marginate and lateral and basal margins entirely marginate, dorsal surface convex with a pair of feeble depressions laterally ; elytron with punctures irregularly impressed, without distinct subbasal furrow subbasally, epipleuron wide at base and gradually narrowed posteriorly ; prosternal process not elevated between anterior coxae; anterior coxal cavity closed posteriorly ; middle and posterior tibiae with many short spines apically ; tarsal claws appendiculate and first tarsal segment of posterior leg distinctly longer than length of second and third segments combined.

Type species : Liroetoides fulvus Kimoto.

This genus somewhat resembles Liroetis Weise, but differs in having the anterior coxal cavity closed posteriorly and the anterior margin not marginate. From Dercetina Gressitt \& Kimoto, differs in having the first tarsal segments of posterior leg longer than the following segments combined.

\section{Liroetoides fulvus n. sp. Fig. 46a}

Entirely yellowish brown.

Head with vertex smooth, shining, sparsely impressed by minute punctures, interocular space much narrower than transverse diameter of single oculus, and interocular transverse impression distinct, frontal tubercle elongate, subtriangular, contiguous, distinctly raised, surface smooth, shining, impunctate. Antenna slender, nearly as long as body length ; first segment long, robust, clubshaped, second nearly $2 / 5$ as long as first, third $32 / 3$ times as long as second, fourth slightly longer than third, fourth to seventh subequal to each other in length and shape, eighth slightly shorter than seventh, ninth subequal to eighth in length and shape, eleventh subequal to ninth in length but its apex pointed. Pronotum transverse, $12 / 3$ times as broad as long, anterior margin slightly rounded posteriorly, lateral margin distinctly rounded, widest slightly before middle, and distinctly narrowed anteriorly and posteriorly, basal margin slightly rounded posteriorly, anterior corner thickened, produced laterally, and posterior corner angulate; dorsal surface convex, with a pair of feeble depressions laterally, smooth, shining, sparsely impressed by distinct punctures and rather closely by minute ones. Scutellum subtriangular, finely granulate, impunctate. Elytron with lateral margin subparallel-sided, apex rounded, surface strongly and rather closely punctate and their interstices smooth, shining.

Length : 8.8-9.8 $\mathrm{mm}$.

Holotype (BISHOP) : VIETNAM : Fyan, 900-1,000 m, 11. vii.-9. viii. 1961, N. R. Spencer. Paratopotypes : 12 exs., same data as the holotype (BISHOP, KIMOTO). Paratypes : VIETNAM : Dalat, 
6 km S., 1,400-1,500 m, 5 exs., 9. vi.-7. vii. 1961, N. R. Spencer (BISHOP).

This new species somewhat resembles Mimastra gracilis Baly, from Kashgar, but differs in having the anterior coxal cavity closed posteriorly and the ventral surfaces entirely yellowish brown.

\section{Genus Sermyloides Jacoby}

Sermyloides Jac., 1884, Notes Lyden Mus., 6: 24 (type : Sermyloides basalis Jacoby ; Sumatra). -Gressitt \& Kimoto, 1963, Pac. Ins. Mon., 1B : 639,

Praeochralea Duvivier, 1885, Stett. Ent. Ztg., 46 : 245 (type : Praeochralea antennalis Duvivier ; Philippines).

\section{KeY to SPECIES OF Sermyloides}

1 Ventral surfaces partly blackish

Ventral surfaces entirely brownish

2(1) Metathorax black; generally reddish brown, antenna black with basal segments brownish, legs with posterior femur and tibia black; elytron black with apical part reddish brown, in some specimen blackish only on basal $1 / 4$; length $5.0-5.7 \mathrm{~mm} \cdots \cdots$ variabilis Metathorax and abdomen black; generally reddish brown, antenna yellowish brown,

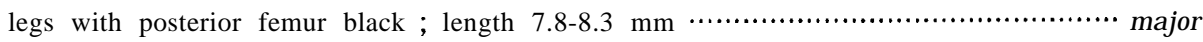

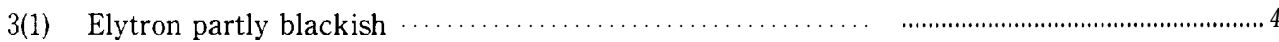
Elytron entirely yellowish to reddish brown; generally yellowish to reddish brown ; antenna black with basal one or two segments in part brownish ; length 5.7-6.3 $\mathrm{m} \mathrm{m}$

4(3) Elytron yellowish brown with margins and a transverse band at middle blackish ; generally reddish brown, scutellum blackish, antenna generally pitchy black with basal segments brownish ; legs pitchy black with femora dark reddish brown ; length 4.8$5.3 \mathrm{~mm}$ maculatipennis

Elytron reddish brown with apical portion blackish; generally yellowish brown; length $4.5-5.0 \mathrm{~mm}$ - apicalis

\section{Sermyloides apicalis Laboissière}

Sermyloides apicalis Lab., 1936, Ann. Soc. Ent. France, 105 : 258 (Tonkin; HAMBURG).

Distribution : Vietnam.

No additional material was examined beside the type series.

\section{Sermyloides coomani Laboissière}

Sermyloides coomani Lab,, 1936, Ann. Soc. Ent. France, 105 : 256 (Tonkin;HAMBURG).-Gressitt \& Kimoto, 1963, Pac. Ins. Mon., 1B: 641 (Vietnam).

Distribution : Thailand, Laos, Vietnam.

Material examined. THAILAND: Khon Kaen, 1 ex., 24. vii. 1980, S. Azuma (RU). LAOS : Vientiane Prov., Ban Van Eue, 1 ex., 15-31. v. 1965, J. A. Rondon, 1 ex., 15-31. vii. 1965, Native collr (BISHOP). 


\section{Sermyloides maculatipennis $\quad$ n. sp. Fig. $46 \mathrm{~b}$}

Generally reddish brown, elytron yellowish brown with basal, lateral, sutural and apical margins and a transverse band at middle black; antenna pitchy black with one or two basal segments reddish brown ; legs pitchy black with femora dark reddish brown.

Head with vertex finely granulate, sparsely impressed by minute punctures, interocular space distinctly wider than transverse diameter of single oculus, and interocular transverse impression distinct, frontal tubercle subtriangular, transverse, contiguous, distinctly raised, surface finely granulate. Antenna slender, nearly $2 / 3$ as long as length of body ; first segment long, second shortest, nearly $1 / 5$ as long as first, third four times as long as second, fourth $3 / 4$ as long as third, fifth slightly shorter than fourth, fifth to eighth subequal to each other in length and shape, ninth slightly shorter than eighth, tenth subequal to ninth in length, eleventh subequal to eighth in length but its apex pointed. Pronotum transverse, $22 / 5$ times as broad as long, anterior margin slightly rounded posteriorly, lateral margin slightly rounded, widest almost at base, slightly narrowed anteriorly, basal margin distinctly rounded posteriorly and nearly straight at middle, dorsal surface convex side to side, sparsely impressed by distinct punctures, and interstices of punctures finely granulate. Scutellum subtriangular, finely granulate, impunctate. Elytron gradually widened posteriorly, apex rounded, more strongly and closely punctate, and interstices of punctures finely granulate.

Male : Head with clypeus widely depressed at middle ; fifth abdominal segment with a pair of short notches apically. Female : Head with clypeus convex ; fifth abdominal segment entire.

Length : 4.8-5.3 mm.

Holotype (BISHOP) : LAOS : Vientiane Prov., Ban Van Eue, 29. iv. 1966, Native collr. Paratypes : 1 ex., same data as the holotype, but 1-15. v. 1965, J. A. Rondon; Sayaboury Prov., Sayaboury, 1 ex., 15. iv. 1965 Native collr (BISHOP, KIMOTO).

This new species resembles Sermyloidesapicalis Laboissière but differs in having elytron yellowish brown with the margins and a transverse band at middle blackish.

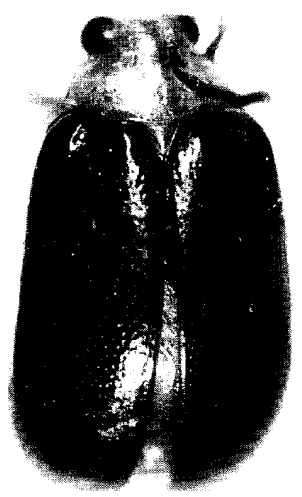

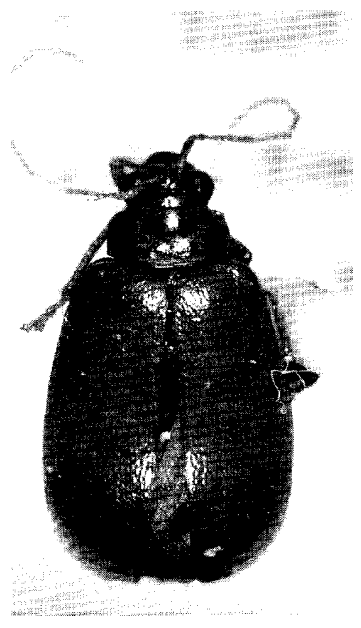

b

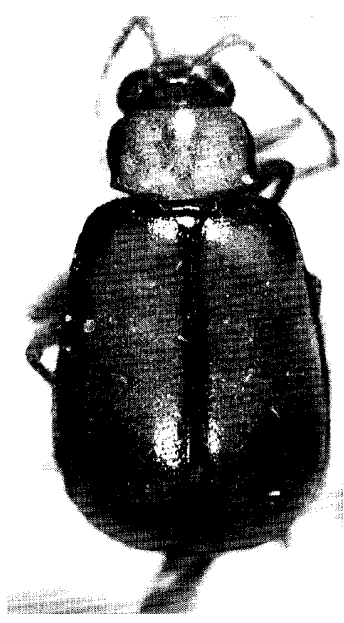

$\mathrm{C}$

Fig. 47. a, Sermyloides variabilis n. sp. ; b, Paleosepharia unicolor n. sp. ; c, $P$. tenasserimensis (Maulik). 


\section{Sermyloides major n. sp. Fig. 46c}

Generally reddish brown, ventral surfaces with metathorax and abdomen black ; legs with posterior femur black.

Head with vertex finely granulate, sparsely impressed by fine punctures, interocular space distinctly wider than transverse diameter of single oculus, and interocular transverse impression distinct, frontal tubercle subtriangular, contiguous, distinctly raised. Antenna slender, nearly $2 / 3$ as long as body length ; first segment long, second shortest, nearly $1 / 5$ as long as first, third five to six times as long as second, fourth slightly shorter than third, fifth slightly shorter than fourth, fifth to seventh subequal to each other in length and shape. Pronotum transverse, $21 / 3$ times as broad as long, anterior margin distinctly narrowed posteriorly, lateral margin slightly rounded, widest almost at base, distinctly narrowed anteriorly, basal margin strongly rounded posteriorly, dorsal surface convex side to side, without any trace of depression, sparsely impressed by fine punctures, and interstices of punctures finely granulate. Scutellum subtriangular, finely granulate, sparsely covered by fine setae. Elytron gradually widened posteriorly and its apex rounded, strongly and rather closely punctate, and interstices of punctures somewhat wrinkled.

Male : Head with frons depressed as a whole ; third antennal segment widened subbasally ; fifth abdominal segment with a pair of short notches apically. Female : Head with frons convex ; antenna normal ; fifth abdominal segment entire.

Length : 7.8-8.3 mm.

Holotype (BISHOP) : CAMBODIA: Kiri Rom, 700 m, 31. iii.-7. iv. 1961, N. R. Spencer. Paratopotype : 1 ex., same data as the holotype (KIMOTO).

This new species resembles Semyloides coomani Laboissière, but differs in being the body length longer, and having mesothorax, the abdomen and the posterior femur blackish.

\section{Sermyloides varia bilis n. sp. $\quad$ Fig. 47a}

Generally reddish brown; elytron black with apical part reddish brown, in some specimen blackish only on basal 1/4; antenna black with one or two basal segments reddish brown ; ventral surfaces with metathorax black, legs with posterior femur and tibia black.

Head with vertex finely granulate, sparsely impressed by fine punctures, interocular space distinctly wider than transverse diameter of single oculus, interocular tranverse impression obsolete, frontal tubercle subtriangular, contiguous, feebly raised, surface finely granulate. Antenna slender ; first segment long, second shortest, nearly $1 / 6$ as long as first, third seven times as long as second, fourth slightly shorter than third, fifth slightly shorter than fourth, sixth subequal to fifth in length and shape. Pronotum transverse, $21 / 4$ times as broad as long, anterior margin slightly rounded posteriorly, lateral margin nearly straight, widest almost at base, distinctly narrowed anteriorly, basal margin strongly rounded posteriorly, dorsal surface convex side to side, sparsely impressed by distinct punctures, and interstices of punctures finely granulate. Scutellum subtriangular, finely granulate, covered by fine setae. Elytron gradually widened posteriorly and rounded at apex, more strongly and closely punctate, and interstices of punctures somewhat wrinkled.

Male : Antenna with third segment curved at middle ; fifth abdominal segment with a pair of short notches apically. Female : Antenna normal ; fifth abdominal segment entire.

Length : 5.0-5.7 mm.

Holotype (BISHOP) : VIETNAM : Fyan, 900-1,000 m, 11. vii.-9. viii. 1961, N. R. Spencer. Paratopotype : 1 ex., same data as the holotype (KIMOTO).

This new species somewhat resembles Sermyloides maculatipennis Kimoto, but differs in having 
metathorax blackish and in the male antenna with the third segment curved.

\section{Genus Paleosepharia Laboissière}

Paleosepharia Lab., 1936, Ann. Soc. Ent. France, 105 : 251 (type : Paleosepharia truncata Laboissière ; Cochin China ; monobasic).-Gressitt \& Kimoto, 1963, Pac. Ins. Mon., 1B: 644.

\section{Key to SPECIES of Paleosepharia}

1 Legs generally yellowish brown

Legs yellowish brown with tibiae and tarsi blackish; elytron yellowish brown with humeral and postmedian transverse markings, together with basal, sutural and lateral margins narrowly, blackish ; in some specimen postmedian elytral marking somewhat ill-defined; generally yellowish brown with metathorax, tibiae, tarsi, and lateral margin of pronotum blackish; antenna pitchy black with basal segments brownish ; $4.8 \mathrm{~mm}$

tenasserimensis

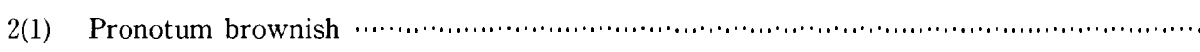

Pronotum pitchy black ; generally yellowish brown ; head, thorax and fifth abdominal

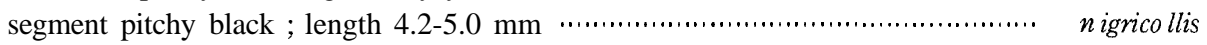

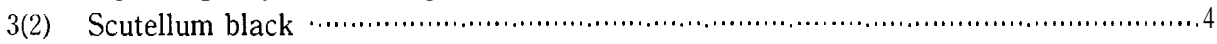

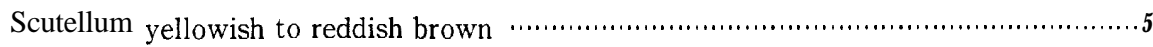

4(3) Elytron dark brown with humeral and postbasal depression areas somewhat paler; generally yellowish brown, with metathorax blackish ; in male elytron with deep postbasal depression distinct and curving obliquely outward posteriorly ; length 4.5 $\mathrm{mm}$

Elytron yellowish brown with basal margin and interior margin of epipleuron, in some cases lateral margin also, blackish basally; generally yellowish brown ; meso- and metathorax black, in some specimen reddish brown; in male elytron with postbasal depression indistinct and only slightly depressed along suture subbasally ; length 4.2-

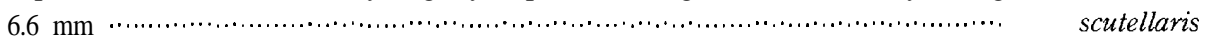

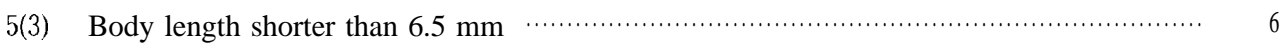

Body length longer than $6.5 \mathrm{~mm}$; in male elytron with postbasal depression curving obliquely outward posteriorly and median lobe of fifth abdominal segment with a deep longitudinal sulcus at middle; generally yellowish brown with metathorax reddish brown to black ; length $6.8-8.1 \mathrm{~mm}$...................................................... truncata

6(5) Ventral surfaces entirely yellowish brown …............................................... 7

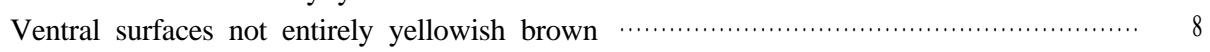

$7(6)$ Pronotum nearly $11 / 2$ times as wide as long, and anterior margin nearly straight ; generally yellowish brown; length $4.8 \mathrm{~mm}$

fulva Pronotum $21 / 2$ times as wide as long, and anterior margin distinctly rounded posteri-

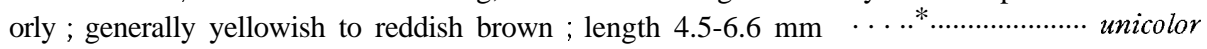

$8(6)$ In male elytron with postbasal depression curving obliquely outward posteriorly and median lobe of fifth abdominal segment with a pair of short longitudinal costae basally and their interstices distinctly depressed ; generally yellowish brown, elytron yellowish brown with basal margin and interior margin of epipleuron infuscate, metathorax reddish brown ; length $5.0-6.3 \mathrm{~mm}$ persimilis In male elytron with postbasal depression subparallel to suture and closely situated, 
and median lobe of fifth abdominal segment feebly depressed apically; generally yellowish brown, metathorax and basal part of interior margin of elytral epipleuron blackish ; length 4.7-5.7 $\mathrm{mm}$

fulvicornis

\section{Paleosepharia fulvan. sp. $\quad$ Fig. 49c}

Entirely yellowish brown.

Head with vertex finely granulate, sparsely impressed by fine punctures; interocular space subequal to transverse diameter of single oculus, and interocular transverse impression distinct, frontal tubercles subtriangular, contiguous, distinctly raised, surface granulate. Antenna slender, nearly as long as length of body ; first segment long, second shortest, nearly $1 / 3$ as long as first, third $11 / 3$ times as long as second, fourth $21 / 2$ times as long as third, fifth slightly longer than fourth, fifth to eighth subequal to each other in length and shape, ninth slightly shorter than eighth, tenth subequal to ninth in length and shape, eleventh subequal to eighth in length but its apex pointed. Pronotum transverse, $12 / 5$ times as broad as long, anterior margin nearly straight, lateral margin slightly rounded, widest slightly before middle, and slightly narrowed anteriorly and posteriorly, basal margin feebly rounded posteriorly, dorsal surface convex with a feeble depression at middle, rather closely impressed by fine punctures, and interstices of punctures finely granulate. Scutellum subtriangular, finely granulate, impunctate. Elytron with lateral margin rounded, strongly and closely punctate, and interstices of punctures finely granulate.

Male : Fifth abdominal segment trilobed, median lobe generally flat. Female : Fifth abdominal segment entire.

Length : $4.8 \mathrm{~mm}$.

Holotype (BISHOP) : VIETNAM : Blao (Balao), 500 m, 14-21. x. 1960, C. M. Yoshimoto. Paratopotype : 1 ex., same data as the holotype (KIMOTO). Paratypes : VIETNAM : Fyan, 1,200m, 2 exs., 11. vii.-9. viii, 1961, N. R. Spencer (BISHOP).

This new species resembles Paleosepharia fulvicornis Chen, but differs in having meso- and metathorax entirely yellowish brown and pronotum with a feeble depression at middle.

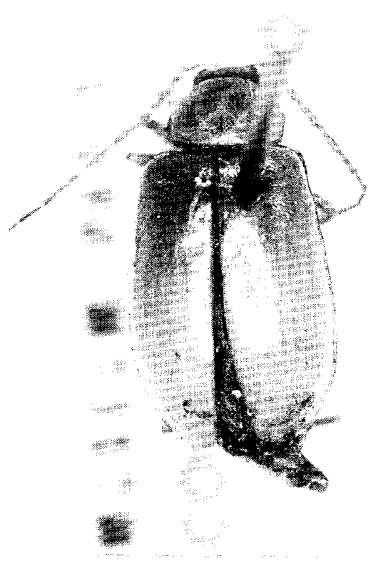

a

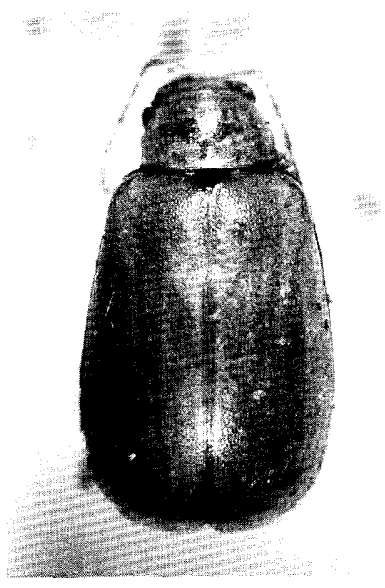

$\mathrm{b}$

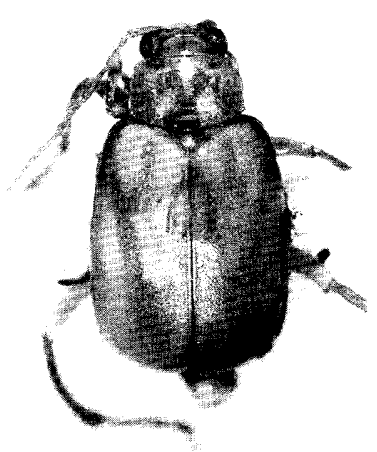

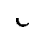

Fig. 48. a, Paleosepharia truncata Laboissière; b, P. scutellaris n. sp. ; c, P. persimilis n. sp. 


\section{Paleosepharia fulvicornis Chen}

Paleosepharia fulvicornis Chen, 1942, Notes d'Ent. Chinoise, 9: 51 (China: Chekiang).-Gressitt \& Kimoto, 1963, Pac. Ins. Mon., 1B: 644, 647 (China).

Distribution : Vietnam, China.

Material examined. VIETNAM : Dalat, 1,500 m, 1 ex., 29. iv.-4. v. 1960, L. W. Quate ; Fyan, 1,200 m, 1 ex., 11. vii.-9. viii. 1961, N. R. Spencer (BISHOP).

\section{Paleosepharia nigricollis n. sp. Fig. 49b}

Generally yellowish brown, head, thorax, and fifth abdominal segment pitchy black.

Head with vertex finely granulate, sparsely impressed by distinct punctures, interocular space distinctly wider than transverse diameter of single oculus, and interocular transverse impression distinct, frontal tubercle subtriangular, contiguous, distinctly raised. Antenna slender, nearly as long as length of body ; first segment long, second shortest, nearly $1 / 4$ as long as first, third $12 / 3$ times as long as second, fourth twice as long as third, fourth to eighth subequal to each other in length and shape, ninth slightly shorter than eighth, tenth subequal to ninth in length and shape, eleventh subequal to eighth in length but its apex pointed. Pronotum transverse, twice as broad as long, anterior margin slightly rounded posteriorly, lateral margin slightly rounded, widest almost at middle, slightly narrowed anteriorly and more strongly so posteriorly, basal margin distinctly rounded posteriorly, dorsal surface convex with a shallow transverse depression at middle, sparsely impressed by distinct punctures, and interstices of punctures finely granulate. Scutellum subtriangular, finely granulate, impunctate. Elytron with lateral margin subparallel-sided, more strongly and closely punctate, and interstices of punctures finely granulate.

Length : 4.2-5.0 $\mathrm{mm}$.

Holotype (Type No. 2704, Kyushu Univ.) : THAILAND : Chiengmai Prov., Fang, 14. vi. 1965, Y. Miyatake. Paratypes: THAILAND : Chiengmai Prov., Doi Suthep, 1,000 m, 1 ex., 18. vi. 1965, Y. Miyatake (KU) ; Khon Kaen, 1 ex., 24. vii. 1980, 1 ex., 7. x. 1981, S. Azuma (RU, KIMOTO).

This new species somewhat resembles Paleosepharia scutellaris Kimoto, but differs in having pronotum pitchy black, more wider, and with a transverse depression at the middle.

\section{Paleosepharia persimilis n. sp. Fig. $48 \mathrm{c}$}

Generally yellowish brown, elytron with basal margin and interior margin of epipleuron infuscate, metathorax reddish brown.

Head with vertex smooth, shining, sparsely impressed by fine punctures, interocular space slightly wider than transverse diameter of single oculus, and interocular transverse impression distinct, frontal tubercle subtriangular, contiguous, slightly raised. Antenna slender, nearly as long as body length ; first segment long, second shortest, nearly $1 / 3$ as long as first, third $11 / 2$ times as long as second, fourth nearly twice as long as third, fourth to eighth subequal to each other in length and shape, ninth slightly shorter than eighth, tenth subequal to ninth in length and shape, eleventh subequal to eighth in length but its apex pointed. Pronotum transverse, $12 / 3$ times as broad as long, anterior margin nearly straight, lateral margin slightly rounded, widest slightly before middle, and slightly narrowed anteriorly and posteriorly, basal margin slightly rounded posteriorly, and almost straight at middle, dorsal surface convex with a shallow depression at middle, sparsely impressed by distinct punctures, and interstices of punctures finely granulate. Scutellum subtriangular, finely granulate, impunctate. Elytron with lateral margin rounded, more strongly and closely punctate, and 
interstices of punctures smooth, shining.

Male : Elytron with postbasal depression curving obliquely outward posteriorly ; fifth abdominal segment trilobed and median lobe of fifth abdominal segment with a pair of short longitudinal costae basally and their interstices distinctly depressed. Female : Elytron normal ; fifth abdominal segment entire.

Length : $5.0-6.3 \mathrm{~mm}$.

Holotype (BISHOP) : LAOS : Sayaboury Prov., Sayaboury, 16. iv. 1965, J. L. Gressitt. Para types : THAILAND: Chiengmai, Ban-Tin-Doi, $350 \mathrm{~m}, 1$ ex., 13. vii. 1957, J. L. Gressitt (BISHOP) ; Chiengmai Prov., Doi Pui, 1 ex., 8. vi. 1965, Y. Miyatake (KU). LAOS : 2 exs., same data as the holotype but 15. iv. 1965 ; Vientiane Prov., Vientiane, 8 exs., 22. vii. 1965, Native collr ; Phou-kowkuei, N. of Vientiane, 2 exs., 16-17. iv. 1965, J. L. Gressitt ; Vientiane Prov., Ban Van Eue, 2 exs., 31. viii. 1965, Native collr ; Borikhane Prov., Pakkading, 1 ex., 23. iv. 1965, 2 exs., 13. vii. 1965, J. L. Gressitt (BISHOP). VIETNAM : Fyan, 900-1,200 m, 3 exs., 11. vii.-9. viii. 1961, N. R. Spencer (BISHOP).

This new species resembles Paleosepharia scutellaris Kimoto, but differs in having metathorax reddish brown, and in male elytron with a postbasal depression deeper and curving obliquely outward posteriorly.

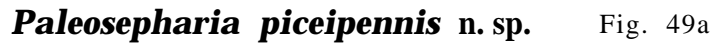

Generally yellowish brown, elytron dark brown with humeral and postbasal areas somewhat paler, scutellum pitchy black; ventral surfaces with metathorax pitchy brown to black.

Head with vertex somewhat wrinkled, sparsely impressed by fine punctures ; interocular space distinctly wider than transverse diameter of single oculus, and interocular transverse impression feeble, frontal tubercle subtriangular, congtiguous, slightly raised, surface finely granulate. Antenna slender, nearly as long as length of body ; first segment long, second shortest, nearly $1 / 3$ as long as first, third $12 / 5$ times as long as second, fourth $21 / 2$ times as long as third, fifth to ninth subequal to each other in length and shape. Pronotum transverse, $12 / 3$ times as broad as long, anterior margin nearly straight, lateral margin slightly rounded, widest almost at middle, slightly narrowed anteriorly and posteriorly, basal margin distinctly rounded posteriorly, dorsal surface convex with a pair of shallow depressions laterally, sparsely impressed by fine punctures, and interstices of punctures finely granulate. Scutellum subtriangular, finely granulate, impunctate. Elytron with lateral margin slightly rounded, strongly and closely punctate, and interstices of punctures smooth, shining.

Male : Elytron with deep postbasal depression distinct and curving obliquely outward posteriorly; fifth abdominal segment trilobed, median lobe generally flat. Female : Elytron normal ; fifth abdominal segment entire.

Length : $4.5 \mathrm{~mm}$.

Holotype (BISHOP) : LAOS : Sayaboury Prov., Sayaboury, 25. iii. 1966, Native collr. Paratopotype: 1 ex., same data as the holotype (KIMOTO). Paratype : LAOS : Sayaboury Prov., Sayaboury, 1 ex., 13. iv. 1966, Native collr (BISHOP).

This new species somewhat resembles Paleosepharia persimilis Kimoto, but differs in having elytron generally dark brown with the humeral and the postbasal areas paler.

\section{Paleosepharia scutellaris n. sp. Fig. $48 \mathrm{~b}$}

Monolepta piceicollis: Chajô, 1964, Nature and Life in SE Asia, Kyoto, 3 : 295 (Thailand :Chiegmai). 


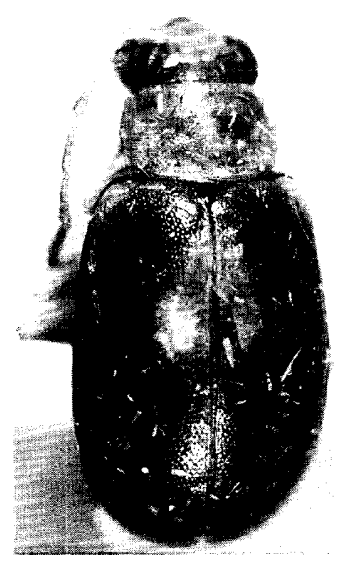

a

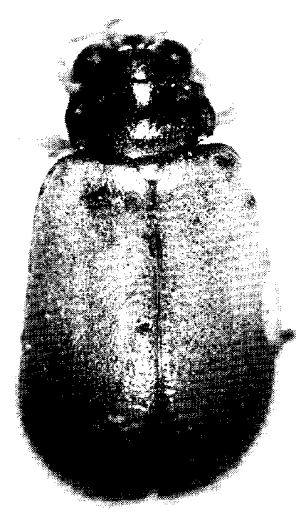

$\mathrm{b}$

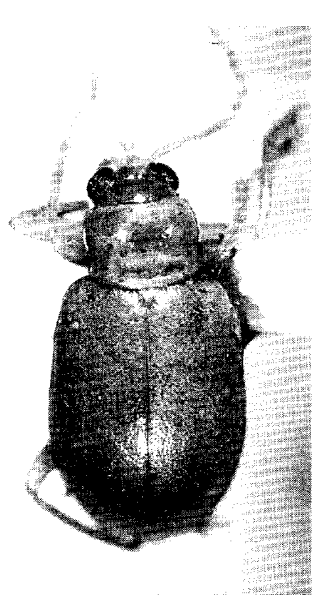

C

Fig. 49. a, Paleosepharia piceipennis n. sp. ; b, P. nigricollis n. sp. ; c, P. fulva n. sp.

Generally yellowish brown, scutellum black, elytron with basal margin blackish, and interior margin of epipleuron, in some cases lateral margin also, blackish basally, antenna entirely yellowish brown, in some specimen apex of eleventh segment infuscate; ventral surfaces with meso- and metathorax black, in some specimen reddish brown.

Head with vertex finely granulate, sparsely impressed by fine punctures, interocular space distinctly wider than transverse diameter of single oculus, and interocular transverse impression distinct, frontal tubercle subtriangular, contiguous, distinctly raised, surface finely granulate. Antenna slender, nearly $2 / 3$ as long as length of body ; first segment long, second shortest, nearly $1 / 3$ as long as first, third $21 / 2$ times as long as second, fourth $11 / 4$ times as long as third, fourth to seventh subequal to each other in length and shape, eighth slightly shorter than seventh, eighth to tenth subequal to each other in length and shape, eleventh subequal to tenth in length but its apex pointed. Pronotum transverse, $11 / 2$ times as broad as long, anterior margin nearly straight, lateral margin slightly rounded, widest almost at middle, slightly narrowed anteriorly and posteriorly, basal margin distinctly rounded posteriorly, dorsal surface convex, with a pair of shallow depressions laterally, closely impressed by fine punctures, and interstices of punctures finely granulate. Scutellum subtriangular, finely granulate, impunctate. Elytron with lateral margin rounded, strongly and rather closely punctate, and interstices of punctures smooth, shining.

Male : Elytron with postbasal depression indistinct and only slightly depressed along suture subbasally; fifth abdominal segment trilobed, median lobe generally flat. Female : Elytron normal ; fifth abdominal segment entire.

Length : 4.2-6.6 mm.

Holotype (BISHOP) : VIETNAM : Dalat, 1,500 m, 29. iv.-4. v. 1960, L. W. Quate. Paratopotypes : 9 exs., same data as the holotype (BISHOP, KIMOTO). Paratypes : THAILAND : Chiengmai Prov., Doi Suthep, 1,000 m, 1 ex., 8. vi. 1965, S. Asahina (KU) ; Chiengmai Prov., Ban-tin-doi, 350 m, 1 ex., 13. xi. 1957, J. L. Gressitt (BISHOP). CAMBODIA : Kiri Rom, 700 m, 1 ex., 31. iii.-7. iv. 1961, N. R. Spencer (BISHOP) ; Pailin, 1 ex., 26. xi. 1962, Maejima (OMNH). LAOS : Sayaboury Prov., Sayaboury, 5 exs., 15-16. iv. 1965, J. L. Gressitt, 2 exs., 2. iii. 1966, 1 ex., 25. viii. 1966, Native collr ; Vientiane Prov., Ban Van Eue, 3 exs., 12-13. iv. 1965, J. L. Gressitt, 1 ex., 15-31. iv. 1965, 3 exs., 15. xi. 
1966, Native collr (BISHOP) ; Umgeb. Vientiane, 3 exs., iii-vi. 1963 (MUNCHEN). VIETNAM : Dalat, 1,500 m, 1 ex., 29. iv.-4. v. 1960, S. Quate ; 15 km NW of Dalat, 1,850 m, 2 exs., 5. v. 1960, L. W. Quate ; 20 km S., Dalat, 1,300 m, 1 ex., 12. ix. 1960, J. L. Gressitt ; 20 km N. of Pleiku, 650 m, 1 ex., 9. v. 1960, L. W. Quate ; 24 km E. of Dilinh (Djiring), 900 m, 1 ex., 25. iv. 1960, R. E. Leech ; Ap HungLam, 21 km NW of Dilinh, 1,100 m, 1 ex., 29. ix.-9. x. 1960, C. M. Yoshimoto (BISHOP).

This new species resembles Paleosepharia fulva Chen, but differs in having scutellum and mesoand metathorax blackish.

\section{Paleosepharia tenasserimensis (Maulik), new combination $\quad$ Fig. 47c}

Monolepta tenasserimensis Maulik, 1936, Fauna India, Galeruc. : 393 (Burma : Tenasserim ; BM). Distribution : Burma, Thailand.

Material examined. THAILAND : Trang Prov., Khaophappha, Khaochong, 200-400 m, 5 exs., 11. i. 1964, G. A. Samuelson ; Chiengmai, 1,000-1,500 m, 1 ex., no date, 1966, J. Sedlacek (BISHOP) ; Khao Chong, nr. Trang, 2 exs., 25. vi. 1965, Y. Miyatake (KU). LAOS : Khammouane Prov., Phon Tiou, 1 ex., 11. vi. 1965, N. Wilson (BISHOP) ; Umgeb. Vanky, 1 ex., 1963 (MUNCHEN).

\section{Paleosepharia truncata Laboissière Fig. 48a}

Paleosepharia truncata Lab., 1936, Ann. Soc. Ent. France, 105 : 252, fig. (Cochin China ; HAMBURG).

Distribution : Thailand, Laos, Vietnam.

Material examined. THAILAND : Trang Prov., Kaochong, 2 exs., 1-18. iv. 1960 (BANGKHEN). LAOS : Sedone Prov., Paksong, 1 ex., 17. v. 1965, P. D. Aslock; Wapikhamthong Prov., Khong Sedone, 1 ex., 30. v. 1965, Native collr (BISHOP).

\section{Paleosepharia unicolor n. sp. Fig. 47b}

Entirely yellowish to reddish brown.

Head with vertex finely granulate, sparsely impressed by fine punctures, interocular space distinctly wider than transverse diameter of single oculus, and interocular transverse impression feeble, frontal tubercle subtriangular, contiguous, distinctly raised, surface finely granulate. Antenna slender, nearly as long as length of body ; first segment long, second shortest, nearly $2 / 5$ as long as first, third twice as long as second, fourth twice as long as third, fifth slightly longer than fourth, fifth to seventh subequal to each other in length and shape, eighth slightly shorter than seventh, ninth subequal to eighth in length and shape, eleventh subequal to tenth in length but its apex pointed. Pronotum transverse, $21 / 2$ times as broad as long, anterior margin distinctly rounded posteriorly, lateral margin slightly rounded, widest almost at middle, slightly narrowed anteriorly and more distinctly so posteriorly, basal margin distinctly rounded posteriorly, almost straight at middle, dorsal surface convex, with a pair of distinct transverse depressions which are united at middle, sparsely impressed by distinct punctures, and interstices of punctures finely granulate. Scutellum subtriangular, finely granulate, impunctate. Elytron gradually widened posteriorly and truncate at apex, surface distinctly and rather closely punctate, and interstices of punctures granulate.

Male : Fifth abdominal segment trilobed, median lobe longitudinally sulcate. Female : Fifth abdominal segment entire.

Length : 4.5-6.6 mm.

Holotype (BISHOP) : VIETNAM : Fyan, 900-1,000 m, 11. vii.-9. viii. 1961, N. R. Spencer. Para- 
topotypes: 4 em., same data as the holotype (BISHOP, KIMOTO). Paratypes : LAOS : Vientiane Prov., Ban Van Eue, 1 ex., 30. xi. 1965, 1 ex., 15. xii. 1966, Native collr (BISHOP). VIETNAM: 18 km NW of Dalat, 1,300 m, 1 ex., 4-5. v. 1960, L. W. Quate (BISHOP).

This new specks resembles Paleosepharia fulva Kimoto, but differs in having pronotum much wider, and with a pair of transverse furrows which are united at middle.

\section{Genus A plosonyx Chevrolat}

Aplosonyx Chevr., 1837, in Dejean, Cat. Col., ed. 3 : 399 (type :Galeruca albicornis Wiedemann; Java).-Maulik, 1936, Fauna India, Galeruc.. 612.-Gressitt\& Kimoto, 1963, Pac. Ins. Mon., $1 \mathrm{~B}: 653$.

Haplosonyx Gistel, 1848, Nat. Thierr. : 14 (emendation for Aplosonyx Chevrolat).

Caritheca Baly, 1977, Ent. Monthly Mag., 123 :226 (type : Caritheca quadripustulata Baly; Sumatra ; monobasic). New synonym.

\section{$\mathrm{K}$ EY to species of Aplosonyx}

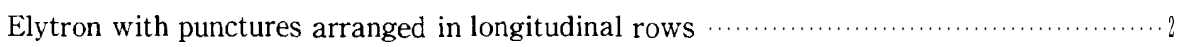

Elytron with punctures confusedly impressed …........................................... 3

2(1) Elytron reddish brown; head and pronotum black; ventral surfaces black with abdomen and middle of metasternum brown; elytron with punctures partly disappearing and postbasal transverse furrow distinct ; length $10.5 \mathrm{~mm} \cdots \cdots \cdots \cdots$ mufipennis Entirely yellowish brown, in some specimen metathorax and part of abdomen infuscate ; elytron with punctures distinctly impressed on entire surface and without any transverse furrow ; length $8.3-8.6 \mathrm{~mm}$............................................ inornatus

3(1) Elytral punctures stronger, interstices of punctures narrower than diameter of single puncture in lateral portion ……....................................................................... Elytral punctures finer, interstices of punctures wider than diameter of single puncture in lateral portion

4(3) Pronotum pale with basal black area or a pair of black spots ; elytron pale with a broad purplish band anterior to middle, which extends forward along suture and expands again on base ; in some specimens dorsal surface entirely reddish brown ; reddish brown with lateral part of meso- and metathorax infuscate ; length $10.0-$ $13.0 \mathrm{~mm}$

Elytron violaceous blue with basal and postmedian markings yellowish brown; yellowish brown with a longitudinal stripe on pronotum, vertex, meso- and metathorax pitchy black ; antenna pitchy black with most of first and eleventh segments brownish, and legs pitchy black with femora mostly brownish ; length 8.3-9.1 $\mathrm{mm}$ quadripustulatus

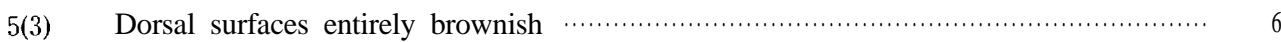
Dorsal surfaces partly or largely bluish, violaceous or blackish $\ldots \ldots \ldots \ldots \ldots \ldots \ldots \ldots \ldots \ldots \ldots . . . \ldots$

6(5) Legs with tibiae and tarsi blackish …........................................................ 7 Legs entirely ochraceous ; generally ochraceous ; length X2.0-14.0 mm ….......... spenceri

7(6) Leg with tibiae and tarsi black; yellowish brown with meso- and metathorax largely pitchy black, in pale colored specimen ventral surfaces entirely yellowish brown ; antenna pitchy black with first segment brownish ; length 11.0-12.0 $\mathrm{mm}$ 


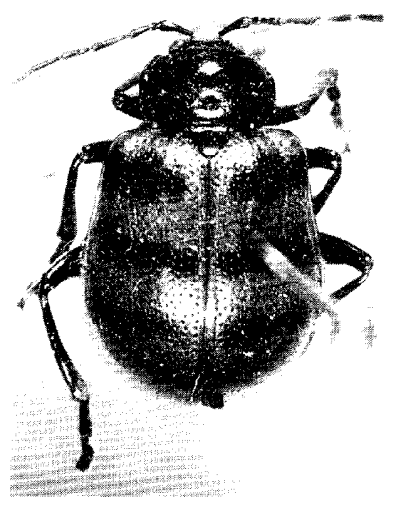

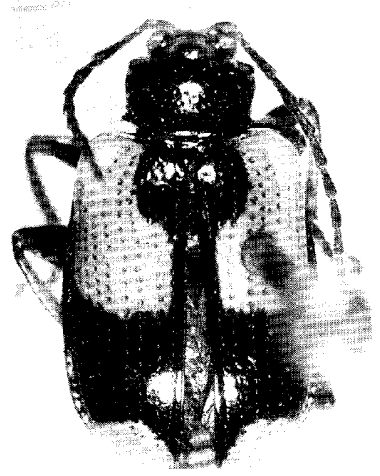

b

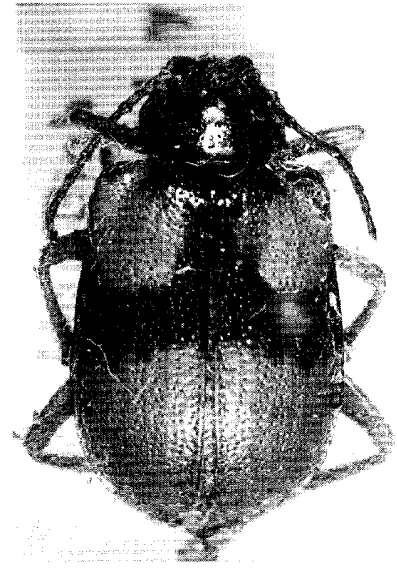

C

Fig. 50. a, Leptarthra pici Laboissière ; b, Aplosonyx ornatus (Jacoby); c, A. ancora Laboissiere.

Legs entirely blackish ; yellowish brown with ventral surfaces of meso- and metathorax and abdomen pitchy black; antenna yellowish brown with apical two segments blackish ; length $\mathbf{1 5 . 0} \mathrm{m} \mathrm{m}$ robinsoni

Pronotum mostly or entirely black

Pronotum not entirely black

9(8) Elytron yellowish brown with a broad blackish band at middle, which extends along suture and expands again on base ; pronotum mostly black ; ochraceous with vertex, ventral surfaces of thorax pitchy black; antenna pitchy black with two or three basal segments brownish; legs pitchy black with most parts of femora brownish; length $5.8-6.3 \mathrm{~mm}$

Elytron entirely yellowish brown; pronotum entirely black; generally black, labrum, antenna and tibiae and tarsi generally yellowish brown ; length 8.5-10.0 $\mathrm{mm}$ nigricollis

10(8) Elytron entirely bluish or violaceous

Elytron yellowish brown with basal half violaceous blue; ochraceous, antenna pitchy black with two or three basal and two or three apical segments brownish ; length $9.6 \mathrm{~mm}$

mouhoti

11(10) Pronotum with four raised areas in front of transverse furrow; elytron blue or purplish blue; reddish brown, antenna with fifth to eighth segments pitchy black, and legs with tibiae and tarsi largely blackish ; length 13.0-15.0 mm chalybaeus Pronotum without any distinctly raised areas in front of transverse furrow ; elytron violaceous; reddish brown, legs with tibiae and tarsi pitchy black; length 10.0 $\mathrm{mm}$

sublaevicollis

\section{A plosonyx ancora Laboissière Fig. 50c}

Aplosonyx ancora Lab., 1935, Ann. Ass. Nat. Levallois-Perret, '21 : 110 (Tonkin; HAMBURG). -Gressitt \& Kimoto, 1963, Pac. Ins. Mon., 1B: 653 (China, Vietnam). 
Distribution : Vietnam, China.

Material examined. "Indo-China”, 2 exs. (BASEL).

\section{Aplosonyx chalybaeus (Hope)}

Galleruca chalybaeus Hope, 1831, in Gray, 2001. Misc., 28 (Nepal ; BM).

Aplosonyx elongata Baly, 1863, Trans. Ent. Soc. London, ser. 3, $1: 642$ (N. India ; BM) ; 1879, Cist. Ent., 2 : 452 (= chalybaeus).

Haplosonyx chalybaeus: Baly, 1879, Cist. Ent., 2 : 452 (Assam) ; Duvivier, 1892, Ann. Soc. Ent. Belg., 36 : 440 (India).

Aplosonyx chalybeus var. jeanvoinei Laboissike, 1935, Ann. Ass. Nat. Levallois-Perret, 21 : 109 (Tonkin).

Aplosonyx chalybaeus: Maulik, 1936, Fauna India, Galeruc.: 614 (Himalaya from Nepal to Burma).-Gressitt \& Kimoto, 1963, Pac. Ins. Mon., 1B: 653 (Tibet, Vietnam).

Distribution: Nepal, Sikkim, N. India, Burma, Vietnam, China (Tibet).

No additional material was examined.

\section{Aplosonyx inornatus (Jacoby)}

Haplosonyx inornatus Jac., 1892, Ann. Mus. Civ. Genova, 32 : 964 (Burma ; GENOVA).

Aplosonyx inornatus : Maulik, 1936, Fauna India, Galeruc. : 617 (Burma).

Distribution : Burma, Thailand, Laos.

Material examined. THAILAND : Chieng Mai Prov., Doi Suthep, 1,000 m, 1 ex., 12. vi. 1965, Y. Miyatake (KU). LAOS : Vientiane Prov., Gi Sion Vill., de Tha Ngone, 1 ex., 10-24. x. 1965, Native collr (BISHOP).

\section{Aplosonyx mouhoti (Baly) Fig. 51c}

Haplosonyx mouhoti Baly, 1879, Ann. Mag. Nat. Hist., ser. 5, 4 : 114 (Siam, Cambodja ; BM).

Distribution : Thailand, Cambodia.

Material examined. THAILAND : Chieng Mai Prov., Doi Suthep, 1,000 m, 1 ex., 8. vi. 1965, S. Asahina (KU).

\section{Aplosonyx nigricollis (Duvivier) Fig. 52c}

Haplosonyx nigricollis Duviv., 1885, Stett. Ent. Z., $46: 244$ (Nias, Malacca).-Jacoby, 1889, Ann. Mus. Civ. Genova, 27 : 284, fig. (Nias) ; 1897, ibid., 38 : 410 (Batu Is.).

Aplosonyx nigricollis : Laboissière, 1940, Bull. Mus. Hist. Nat. Belg., 16(3): 22 (Nias). Distribution : Thailand, Malaya, Nias, Mentawei, Batu.

I could not examined the type of this species. My identification is based on Laboissière's material. Material examined. THAILAND : Musa, nr. Fang, 1 ex., 22. xi. 1968, Hatta (EHIME).

\section{Aplosonyx orientalis (Jacoby)}

Haplosonyx orientalis Jac., 1892, Ann. Mus. Civ. Genova, 32 : 962 (Burma ; GENOVA).

Haplosonyx varipes Jac., 1892, Ann. Mus. Civ. Genova, 32 : 964 (Burma ; GENOVA). New synonym. 
Galerucida indica: Allard, 1889, Ann. Soc. Ent. France, ser. 6, $9: 310$ (Saigon, Pnomh-Penh). -Laboissière, 1922, Bull. Soc. Ent. France, 1922 : 102 (=tonkinensis).

Sphenoraia tonkinensis Laboissière, 1922, Bull. Soc. Ent. France, 1922 : 102 (Saigon, Pnomh-Penh, Lao-Lay, Ha Lang; HAMBURG). New synonym.

Aplosonyx varipes : Laboissière, 1934, Ann. Ass. Nat. Levallois-Perret, 21 : 109 (Tonkin). -Maulik, 1936, Fauna India, Galeruc. : 619 (India, Burma, Tonkin).-Gressitt \& Kimoto. 1963, Pac. Ins. Mon., 1B : 654 (India, Burma, Tonkin).-Chajô, 1964, Nature and Life in SE Asia, Kyoto, $3: 284$ (Thailand).

Aplosonyx orientalis: Maulik, 1936, Fauna India, Galeruc.: 619 (Burma).-Gressitt \& Kimoto, 1963, Pac. Ins. Mon., 1B: 654 (Vietnam).

Distribution : India, Burma, Thailand, Laos, Vietnam.

Material examined. THAILAND : Trang Prov., Khaophappha, Khaochang, 200-400 m, 1 ex., 1. i. 1964, 4 exs., 4. i. 1964, 1 ex., 10. i. 1964, 1 ex., 11. i. 1964, 2 exs., 13. i. 1964, G. A. Samuelson ; Chieng Mai Prov., Maesa, 2 exs., 16. vi. 1965, P. D. Ashlock;Doi Suthep, 1,278 m, 1 ex., 29. iii.-4. v. 1958, T. C. Maa; Loey Prov., 17 km NW of City, 1 ex., 18. v. 1967, J. S. Burton ; Khao Yai, 1 ex., 7. ix. 1963, Pakchong, 1 ex., 7. iii. 193 Native collr (BISHOP) ; Fang, 1 ex., 14. vi. 1965, K. Morimoto ; Khao Yai Nat. Park, 1 ex., 6. vi. 1965, S. Asahina ; Khao Chong, nr. Trung, 1 ex., 27. vi. 1965, K. Morimoto ; Chiengmai, 3 exs., 20. vii. 1970, K. Yano (KU) ; Trang, 8 exs., 1-18. iv. 1960, 1 ex., 4. iv. 1960, 1 ex., 9. iv. 1960, Phrae; 1 ex., 7. viii. 1938 ; Chiengmai, 2,800 ft., 1 ex., 24-27. iv. 1953 ; Chiengmai, 3,300 ft, 2 exs., 21. vi. 1936, Nakhon Ratchasima, 1 ex., 19. v. 1961 (BANGKHEN). LAOS : Vientiane Prov., Ban Van Eue, 1 ex., 12. iv. 1965, J. L. Gressitt, 17 exs., 15-31. v. 1965, 13 exs., 31. vii. 1965, 2 exs., 30. xi. 1965, 2 exs., 15. iv. 1966, 1 ex., 15. vi. 1966, Native collr ; Vientiane Prov., Phou Kou Khouei, 1 ex., 31. v. 1966, Native collr; Tha Ngone, 1 ex., 10-24. x. 1965, Native collr ; Sedone Prov., Pakse, 3 exs., 31. v. 1967, 3 exs., 15. vi. 1967, 1 ex., 30. vi. 1967, Native collr ; Wapikhamthong Prov., Khong Sedone, Wapi, 1 ex., 30. v. 1965, 1 ex., 17. vii. 1965, 2 exs., 17. ix. 1965, 1 ex., 31. v. 1967, 1 ex., 31. viii. 1967, 1 ex., 15. x. 1967, Native collr ; Sayaboury Prov., Sayaboury, 1 ex., 21. ix. 1965, 1 ex., 29. iii. 1966, 1 ex., 25. viii. 1966, Native collr ; Muong Sing, NW of Luang Prabang, 650 m, 6 exs., 6-10. vi. 1960, S. Quate \& L. Quate ; Tonpheng, 1 ex., 30. vi. 1966, Native collr ; Ile de Khong, 1 ex., 6. ii. 1965, 1 ex., 20. vii. 1965, 1 ex., 24.

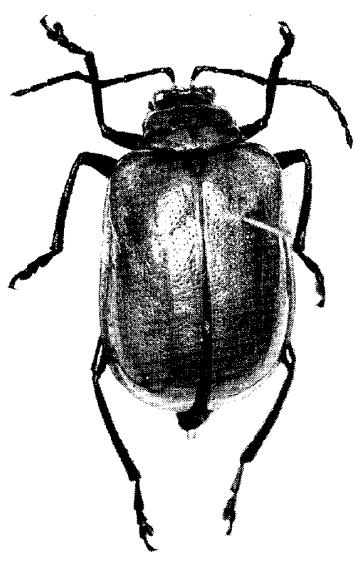

a

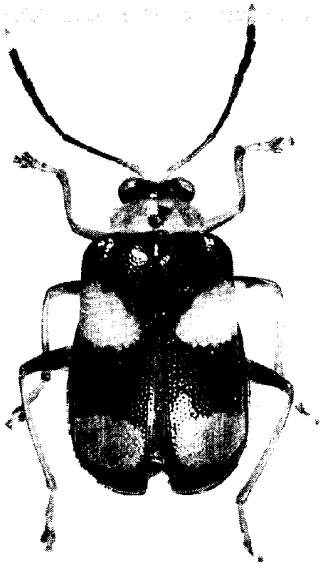

b

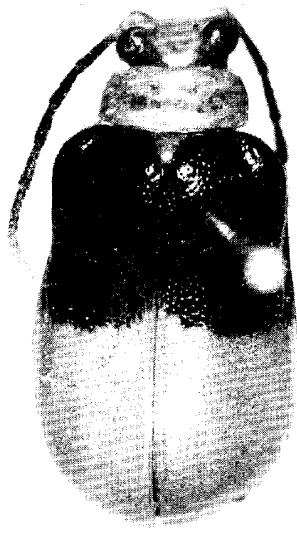

C

Fig. 51. a, Aplosonyx robinsoni (Jacoby); b, A. quadripustulatus(Baly); c, A. mouhoti (Baly). 
viii. 1965, 1 ex., 7. ix. 1965, Native collr (BISHOP) ; Umgeb. Vientiane, 5 exs., iii-vi. 1963 ; Umgeb. Paklay, 1 ex., 1963 ; Umgeb. Vanky, 1 ex., 1963 (MUNCHEN). VIETNAM : Kontum, N. of Pleiku, 500 m, 1 ex., 13. v. 1960, S. Quate (BISHOP).

\section{Aplosonyx ornatus (Jacoby) Fig. 50b}

Haplosonyx ornata Jac., 1892, Ann. Mus. Civ. Genova, 32 : 963 (Burma ; GENOVA).

A plosonp ornata : Laboissière, 1940, Bull. Mus. Hist. Nat. Belg., 16(3): 21 (Burma).

Distribution : Burma, Laos.

Material examined. LAOS : Vientiane Prov., Ban Van Hue, 1 ex., 31. vii. 1965, Native collr (BISHOP).

\section{Aplosonyx quadripus tula tus (Baly), new combination Fig. 51b}

Caritheca quadripustulata Baly, 1877, Ent. Monthly Mag., 13 : 226 (Sumatra ;BM).-Laboissière, 1932, M\&m. Mus. Hist. Nat. Belg., hors ser., 4(4): 172 (Sumatra) (as Carithea).

Distribution : Thailand, Sumatra.

Material examined. THAILAND: Narathiwas Prov., $15 \mathrm{~km} \mathrm{W.} \mathrm{of} \mathrm{Waeng,} 100 \mathrm{~m}, 3$ exs., 7-11. ix. 1965, L. W. Quate (BISHOP) ; Khao Chong, nr. Trung, 3 exs., 26-27. vi. 1965, K. Morimoto \& Y. Miyatake (KU).

\section{Aplosonyx robinsoni (Jacoby) Fig. 51a}

Haplosonyx robinsoni Jac., 1905, Fasciculi Malayenses, 2 (Appl.) : 6 (Siamese Malay : Bukit Besar, Nawangchik and Tor; BM).

Aplosonp robinsoni : Maulik, 1936, Fauna India, Galeruc. : 618 (Burma, Thailand, Perak, Sumatra).

Distribution : Burma, Thailand, Malaya, Sumatra.

Material examined. THAILAND : Chieng Mai Prov., Doi Suthep, 1,000 m, 1 ex., 12. vi. 1965, P. D. Ashlock (BISHOP).

\section{A plosonyx rufipennis (Duvivier) Fig. 52a}

Haplosonyx rufipennis Duviv., 1892, Ann. Soc. Ent. Belg., 36 : 439 (Pedong).

A plosonyx rufipennis:Laboissière, 1934, Ann. Ass. Nat. Levallois-Perret, 21 : 110 (Tonkin, Yunnan) ; 1940, Bull. Mus. Hist. Nat. Belg., 16(3): 21 (Tonkin).-Gressitt \& Kimoto, 1963, Pac. Ins. Mon., 1B: 654 (China).

Aplosonyx rubra Maulik, 1936, Fauna India, Galeruc. : 620 (Burma ;BM).-Laboissière, 1940, Bull. Mus. Hist. Nat. Belg., 16(3): 21 (= rufipennis).

Distribution : India, Vietnam, W. China.

No additional material was examined.

\section{Aplosonyx spenceri n. sp. Fig. 52b}

Entirely ochraceous.

Head with vertex smooth, shining, nearly impunctate, and with a short longitudinal furrow in front, interocular space distinctly wider than transverse diameter of single oculus, and interocular 
transverse impression distinct, frontal tubercle transverse, subquadrate, distinctly raised, surface smooth, shining. Antenna slender, nearly $2 / 3$ as long as body length; first segment robust, clubshaped, second shortest, nearly $1 / 4$ as long as first, third nearly half as long as second, fourth nearly three times as long as third, fifth slightly shorter than fourth, sixth slightly shorter than fifth, seventh subequal to seventh in length and shape, eighth slightly shorter than seventh, and eighth to tenth subequal to each other in length and shape, eleventh subequal to seventh in length but its apex pointed. Pronotum transverse, twice as broad as long, anterior margin distinctly rounded posteriorly, lateral margin distinctly constricted at middle, basal margin distinctly rounded posteriorly and concaved before scutellum, dorsal surface convex, smooth, shining, sparsely impressed by distinct punctures, and with a pair of oblique lateral furrows very deep. Scutellum subtriangular, smooth, shining, impunctate. Elytron with lateral margin slightly rounded, surface sparsely and confusedly impressed by distinct punctures and interstices of punctures smooth, shining, and with a shallow postbasal transverse furrow.

Length : 12.0-14.0 mm.

Holotype (BISHOP) : CAMBODIA : Kiri Room, 700 m, 31. iii.-7. iv. 1961, N. R. Spencer. Paratopotype : 1 ex., same data as the holotype (KIMOTO).

This new species somewhat resembles Aplosonyx orientalis (Jacoby), but differs in being the body length longer and having the legs and the ventral surfaces entirely ochraceous.

\section{Aplosonyx sublaevicollis (Jacoby)}

Haplosonyx sublaevicollis Jac., 1889, Ann. Mus. Civ. Genova, 27 : 218 (Tenasserim ; GENOVA).

Aplosonyx sublaevicollis: Maulik, 1936, Fauna India, Galeruc.: 615 (Burma).-Laboissière, 1940, Bull. Mus. Hist. Nat. Belg., 16 (3) : 21 (Tenasserim).-Châjô, 1961, Nature and Life in SE Asia, Kyoto, $1: 349$, fig. (Thailand).

Distribution : Burma, Thailand, Laos.

Material examined. THAILAND : Chiengmai, 3,200 ft, 4 exs., 25. iv. 1939, Chiengmai, 5,500 ft, 1 ex., 7. iv. 1939 (BANGKHEN). LAOS : Muong Sing, NW of Luang Prabang, 650 m, 1 ex., 6-10. vi. 1960, S. Quate (BISHOP).

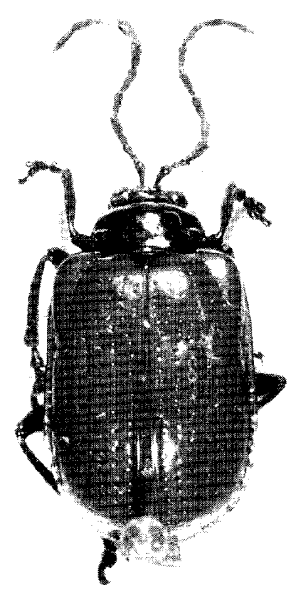

a

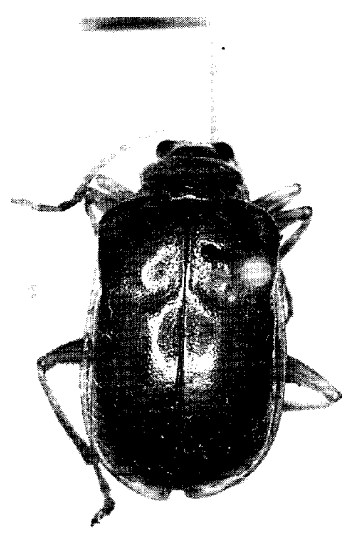

b

Fig. 52. a, Aplosonyx rufipennis (Duvivier) ; b, A. spenceri n. sp. ; c, A. nigricollis (Duvivier). 


\section{Genus Leptarthra Baly}

Leptarthra Baly, 1861, J. Ent., 1: 202 (type : Leptarthra abdominalis Baly ; E. Himalayas). -Chapuis, 1875, Gen. Col., 11 : 224, 227-Maulik, 1936, Fauna India, Galeruc. : 609.-Gressitt \& Kimoto, 1963, Pac. Ins. Mon., 1B: 654.

\section{Leptarthra pici Laboissière Fig. 50a}

Leptarthra pici Lab., 1934, Ann. Ass. Nat. Levallois-Perret, 21 : 112, fig. (Tonkin; HAMBURG). Distribution : Vietnam.

Subovate; head, pronotum, scutellum, ventral surfaces of thorax, antenna and legs largely violaceous blue; elytron ochraceous with three transverse bands violaceous blue; abdomen reddish brown with basal segments in part bluish. Length $10.2 \mathrm{~mm}$.

No additional material was examined.

\section{Genus Sphenoraia Clark}

Sphenoraia Clark, 1865, Ann. Mag. Nat. Hist., ser. 3, 16 :262.--Gressitt \& Kimoto, 1963, Pac. Ins. Mon., 1B : 655 (type :Galleruca bicolor Hope ; Nepal).

\section{KeY TO SPECIES of Sphenoraia}

Short oval, antenna robust, strongly compressed (Sphenoraioides) $\ldots \ldots \ldots \ldots \ldots \ldots \ldots \ldots \ldots \ldots \ldots \ldots 2$

Elongate, subparallel-sided ; antenna filiform (Sphenoraia) ; reddish brown, pronotum with a pair of and elytron with seven pairs of blackish markings, ventral surfaces and legs in part blackish ; length $8.3 \mathrm{~mm}$ (Hope, 1831 ; type of subgenus ; India, Burma,

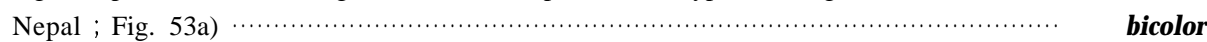

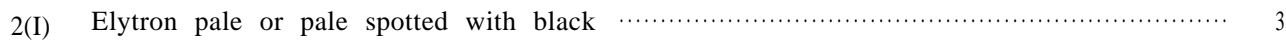

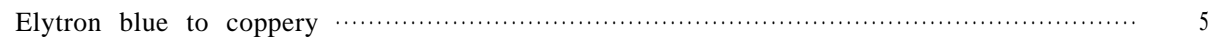

3(2) Punctures of elytron not arranged in a pair of rows or semiregularly arranged $\cdots \cdots \cdots \cdots \cdots \cdots \cdot \cdots$ Punctures of elytron regularly arranged in a pair of rows ; yellowish brown, tibiae and tarsi black, antenna black with basal two or three segments brownish; length 9.3

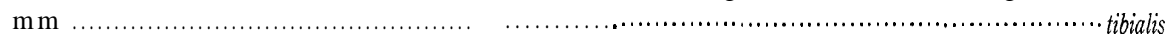

4(3) Elytron impressed by regular fairly strong punctures alternating with impunctate stripes; elytron and abdomen reddish brown, head, thorax, antenna and legs entirely violaceous blue ; antenna slenderer ; length 7.5-8.0 $\mathrm{mm}$.................................duvivieri Elytron entirely irregularly punctured ; elytron reddish to yellowish brown with seven black spots, and pronotum pale with two black spots ; in most pale colored specimen dorsal surfaces entirely reddish brown, and in most dark colored specimen elytron almost entirely blackish ; antenna robuster ; length $5.5-7.0 \mathrm{~mm}$...................... nebulosa

5(2) Interstices of elytral punctures wider than diameter of single puncture, sometimes punctures semiregularly arranged in rows; dorsal surfaces varying color, purplish

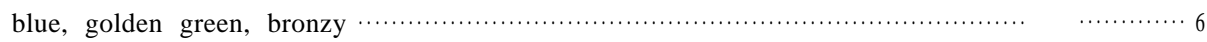
Interstices of elytral punctures narrower than diameter of single puncture and confusedly punctate throughout ; entirely bluish black ; pronotum with a pair of deep transverse furrows ; length 7.2-8.2 $\mathrm{mm}$ 
6(5) Pronotum with a pair of deep furrows laterally, sides strongly rounded and widest almost at middle ; length 8.5-9.0 mm (Hope, 1831; Kashmir, N. India, Nepal, Bhutan ; W. China ?) …................................................................................ Pronotum without any distinct furrow laterally, sides slightly rounded and widest at $1 / 3$ from anterior corner ; length 7.5-8.5 mm

\section{Subgenus Sphenoraioides Laboissière}

Sphenoraioides Laboissiere, 1934, Ann. Ass. Nat. Levallois-Perret, 21 : 131 (type :Galleruca fulgida Redtenbacher ; Himalaya : Maussuri).

\section{Sphenoraia (Sphenoraioides) duvivieri (Laboissière)}

Sphenoraia indica Duvivier, 1887, C. R. Soc. Ent. Belg., $1887: 48$ (nec Harold, 1880) (Inde borealae ; BRUXELLES).

Galerucidaduvivieri Lab., 1929, Encycl. Ent., Col., 1 (2) : 53 (new name for Sphenoraia indica Duvivier).

Sphenoraia (Sphenoraioides) duvivieri: Laboissiere, 1934, Ann. Ass. Nat. Levallois-Perret, $21: 134$ (Tonkin, Yunnan).-Gressitt \& Kimoto, 1963, Pac. Ins. Mon., 1B: 656 (China).

Gallerucida amala Maulik, 1936, Fauna India, Galeruc. : 549 (Burma)-Laboissike, 1940, Bull. Mus. Hist. Nat. Belg., 16 (3) : 30 (=duvivieri).

Distribution : India, Burma, Thailand, Laos, Vietnam, China.

Material examined. THAILAND : Khon Kaen, 1 ex., 24. vii. 1980, S. Azuma (RU). LAOS : Vientiane Prov., Vientiane, 1 ex., iv. 1967, Native collr; Vientiane Prov., Ban Van Eue, 1 ex., 29. iv. 1966, 1 ex., 31. v. 1966, 1 ex., 15. vi. 1966, Native collr ; Vientiane Prov., Gi Sion Vill., de Tha Ngone, 1 ex., 24-31. x. 1966, Native collr ; Xing Kyouong Phov., Ban Sam Thung, 2 exs., 5. vi. 1965, Native

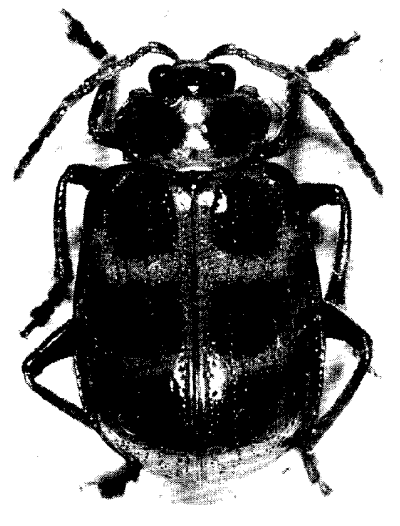

a

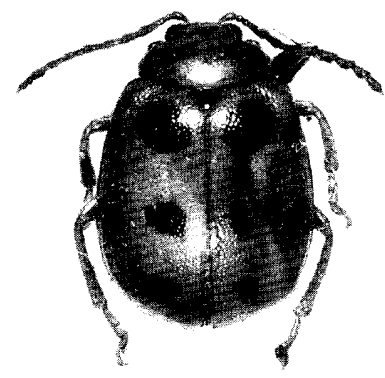

b

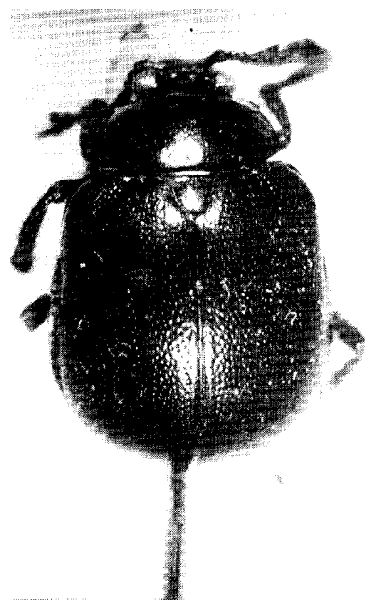

C

Fig. 53. a, Sphenoraia (Sphenoraia)bicolor (Hope) ; b, S. (Sphenoraioides) nebulosa (Gyllenhal) ; c, $S$. (Sphenoraioides) paviei Laboissiere. 
collr ; Sayaboury Prov., Sayaboury, 1 ex., 16. iv. 1965, J. L. Gressitt, 1 ex., 21. iv. 1966, Native collr (BISHOP). VIETNAM : Tonkin, 1 ex., 10. viii. 1942, T. Esaki (KU).

\section{Sphenoraia (Sphenoraioides) micans (Fairmaire) Fig. 4a}

Eustetha micans Fairmaire, 1888, Ann. Soc. Ent. Belg., 32 : 42 (Moupin).

Galemcida fulgida: Weise, 1922, Tijdschr. Ent., 65 : 90 (Fukien).

Galerucida fulgida var. coerulescens Weise, 1922, ibid. : 91 (Fukien).

Sphenoraia (Sphenoraioides) micans : Laboissière, 1934, Ann. Ass. Nat. Levallois-Perret, 21 : 31 (China).-Gressitt \& Kimoto, 1963, Pac. Ins. Mon., 1B: 657, fig. (China).

Sphenoraia (Sphenoraioides) micans var. cyanella Laboissiere, 1934, ibid. : 132 (Tibet).

Galemcida fulgida: Ogloblin, 1936, Fauna USSR, 26, $1: 363$ (China).

Distribution : China, Indo-China.

Material examined. "Indo-China": Pa Kha, 1 ex., 1944 (BASEL).

\section{Sphenoraia (Sphenoraioides) nebulosa (Gyllenhal) Fig. 53b}

Galleruca nebulosa Gyll., 1808, in Schönherr, Syn. Ind., 1 (2) : 292, pl. 4, fig. 10 (E. India).

Galemcida nebulosa : Maulik, 1936, Fauna India, Galeruc. : 552, fig. 135 (East Indes).

Sphenoraia (Sphenoraioides) nebulosa :Laboissière, 1934, Ann. Ass. Nat. Levallois-Per-ret, 21 : 132, fig. 7 (Tonkin, India, Burma, Siam).-Gressitt \& Kimoto, 1963, Pac. Ins. Mon., 1B: 256 (China, Vietnam).

Doryida balyi: Allard, 1889, Ann. Soc. Ent. France, ser. 6,9: 310 (Hue, Pnomh-Penh) ; 1891, Nouv. Arch. Mus. Paris, ser. 3, $3: 231$; 1904, Miss. Pavie Indo-Chine, $3: 160$ (Cambodge).-Laboissière, 1934, Ann. Ass. Nat. Levallois-Perret, 21 : 132, fig. 7 (corrected as nebulosa ;Tonkin, India, Burma, Siam).

Distribution : India, Burma, Thailand, Cambodia, Laos, Vietnam, S. China, Hainan.

Material examined. THAILAND : Chieng Mai Prov., Fang, 500 m, 2 exs., 12-19. iv. 1958, T. C. Maa, 1 ex., 13. vi, 1965, P. D. Ashlock; Chieng Mai Prov., Mae Klang, 340 m, 1 ex., 11. vi. 1965, P. D. Ashlock; Saraburi Prov., Ban Muak Lek Nat. Park, 1 ex., 6. vi. 1965, P. D. Ashlock; Pangmakampon (Pankmpawng), nr. Fang, 450 m, 2 exs., 16. xi. 1957, J. L. Gressitt ;Nakorn-nayok, 1 ex., 6. ix. 1963, Native collr (BISHOP) ; Saraburi, 1 ex., 21. vii. 1956, 1 ex., 23. v. 1958 ; Cholburi, 1 ex., 1. ix. 1956 ; Chiengmai, 1,000 ft., 1 ex., 16-21. iv. 1953 (BANGKHEN). LAOS : Khammouane Prov., Phon Tiou, 2 exs., 18. viii. 1965, J. A. Rondon, 1 ex., 28. iv. 1965, 3 exs., 30. iv. 1965, 2 exs., 17. v. 1965, 1 ex., 28. v. 1965, 1 ex., 14. vii. 1965, 1 ex., 11. ix. 1965, 3 exs., 28. ix. 1965, 1 ex., 8. iii. 1966, Native collr ; Vientiane Prov., Vientiane, 1 ex., 21. iv. 1965, J. A. Rondon, 1 ex., 21. ii. 1966, 1 ex., 22. vii. 1966, Native collr ; Vientiane Prov., Gi Sion Vill., de Tha Ngone, 1 ex., 24-31. x. 1966, Native collr ; Sayaboury Prov., Sayaboury, 1 ex., 14. vii. 1965, Native collr ; Borikhane Prov., Paksane, 1 ex., 7. viii. 1965, Native collr ; Borikhane Prov., Pakkading, 1 ex., 5. iv. 1966, Native collr ; Sedone Prov., Pakse, 1 ex., 15. vii. 1967, Native collr ; Savanakhet, 1 ex., 18. iv. 1965, J. A. Rondon; Dong Dok, 1 ex., 23. vii. 1965, 1 ex., 11. ix. 1965, 1 ex., 26. ii. 1966, Native collr; Nongteva, 1 ex., 27. vii. 1965, Native collr (BISHOP) ; Umgeb. Vientiane, 10 exs., iii-vi. 1963 ; Umgeb. Vanky, 2 exs., 1963 ; Umgeb. Paklay, 2 exs., 1963 (MUNCHEN).

\section{Sphenoraia (Sphenoraioides) paviei Laboissière Fig. 53c}

Sphenoraia paviei Lab., 1934, Ann. Ass. Nat. Levallois-Perret, 21 : 134 (Tonkin;PARIS).-Gres- 
sitt \& Kimoto, 1963, Pac. Ins. Mon., 1B: 657 (Laos).

Galenucida magica:Allard, 1891, Nouv. Arch. Mus. Paris, ser. 3, 3 : 231 ; 1904, Miss. Pavie Indo-

Chine, 3 : 160 (Laos : Luang-Prabang à Theng).

Distribution : Thailand, Laos, Vietnam.

Material examined. "Thailand", 2 exs. (BANGKHEN). LAOS : Sayaboury Prov., Sayaboury, 1 ex., 21. iv. 1965, J. A. Rondon, 1 ex., 21. iv. 1966, Native collr (BISHOP) ; Umgeb. Vientiane, 1 ex., iiivi. 1963 (MUNCHEN).

\section{Sphenoraia (Sphenoraioides) tibialis (Baly), new combination}

Berecynthatibialis Baly, 1865, Ent. Monthly Mag., 2 : 98 (Cambodia ; BM).

Sphenoraia convexa Laboissière, 1940, Bull. Mus. His. Nat. Belg., 16 : 29 (Siam ; BRUXELLES). New synonym.

Distribution : Thailand, Cambodia, Laos.

Material examined. THAILAND : Pak Chong, 1 ex., 16. v. 1963, Native collr (BISHOP). LAOS : Tonphen, 29, v. 1966, Native collr (BISHOP).

\section{Genus Cassena Weise}

Cassena Weise, 1892, Dtsche Ent. Z., 1892 :388.-Bryant, 1962, Ann. Mag. Nat. Hist., ser. 13, 5 : 369 (type: Cassena vorax Weise = Cassena celebensis Weise, nec Jacoby, 1886 ; Celebes). -Gressitt \& Kimoto, 1963, Pac. Ins., 5(4) : 925.

Euphyma Baly, 1879, Cist. Ent., 2 : 457 (nec Mulsant, 1875, Baly, 1877) (type : Euphyma collaris Baly ; Assam ; monobasic).

Solenia Jacoby, 1886, Ann. Mus. Civ. Genova, 34 : 87 (new name for Euphyma Baly, 1879).

Solephyma Maulik, 1936, Fauna India, Galeruc., 329 (new name for Euphyma Baly, 1879, nec Baly, 1877, Cryptocephalinae and Solenia Jacoby, 1886, nec Mulsant, 1875, Staphylinidae).-Bryant, 1962, Ann. Mag. Nat. Hist., ser. 13, 5 :369.-Gressitt \& Kimoto, 1963, Pac. Ins. Mon., 1B: 657.

\section{Key to species of Cassena}

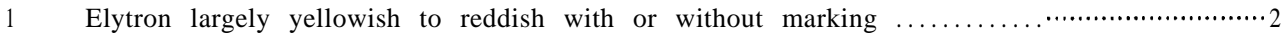

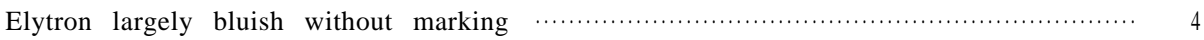

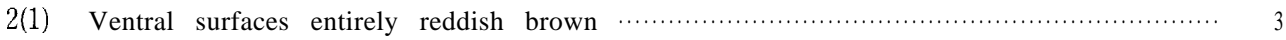

Ventral surfaces reddish brown with abdomen pitchy brown ; elytron yellowish brown, sutural margin widely blackish ; head and pronotum reddish brown, scutellum pitchy

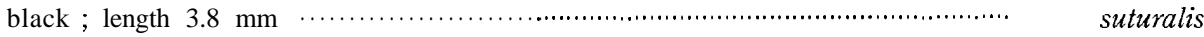

3(2) Elytron reddish brown with yellowish apical marking, which is margined by black in most specimens ; general color reddish brown, legs reddish brown with tibiae and tarsi

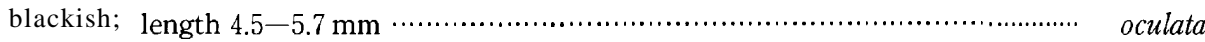

Elytron yellowish brown with margins narrowly infuscate, and in some specimen dark brown with a large discal marking yellowish ; generally reddish brown, legs almost

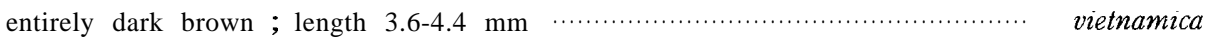

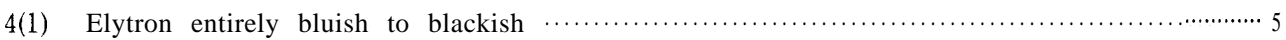
Elytron bluish black with apex yellowish brown; generally bluish black with abdomen yellowish brown; length 3.1-4.7 $\mathrm{mm}$

terminalis

5(4) Ventral surfaces not entirely brownish 

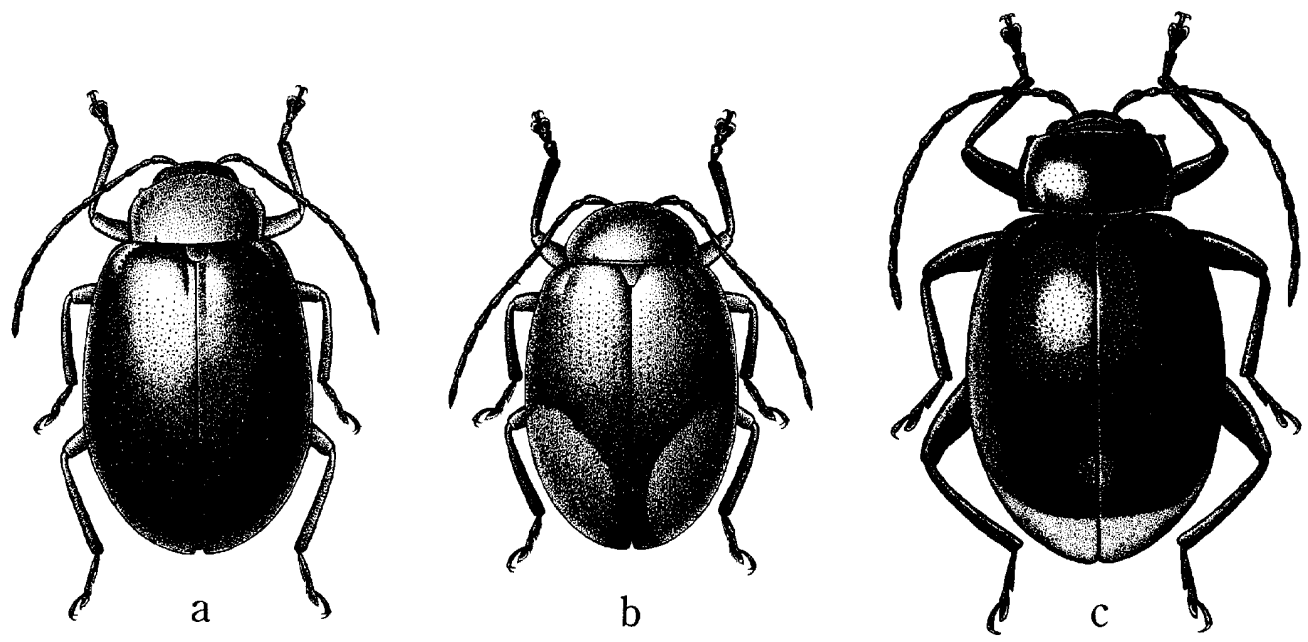

Fig 54. a, Cassena collaris (Baly) ; b, C. oculata Laboissière; c, C. terminalis (Gressitt \& Kimoto).

Ventral surfaces, head, pronotum, scutellum and legs entirely brownish ; elytron bluish black, antenna pitchy black with basal segments brownish ; length $4.8-5.6 \mathrm{~mm} \cdots \cdots \cdots$....indica

6(5) Legs at least partly brownish

Legs almost entirely pitchy black to bluish black; generally pitchy black, elytron bluish black, head and prothorax reddish brown, antenna largely pitchy black ; length 7.1-7.8 mm rubyana

7(6) Body length longer; legs pitchy black with anterior and middle legs brownish; generally reddish brown with most of metathorax and abdomen pitchy brown; elytron bluish black ; length $4.8-5.7 \mathrm{~mm}$

collaris

Body length shorter ; legs entirely brownish ; generally reddish to yellowish brown with metathorax pitchy brown, elytron pitchy black ; length 3.3-4.2 $\mathrm{mm}$............ integricollis

\section{Cassena collaris (Baly) Fig. 54a}

Euphyma collaris Baly, 1879, Cist. Ent., 2 : 457 (Assam ; BM).

Solenia collaris: Jacoby, 1889, Ann. Mus. Civ. Genova, ser. 2, $7: 236$ (Burma).

Solenia robusta Jacoby, 1896, Ann. Mus. Civ. Genova, 36: 500 (Padang, Perak ; BM).-Maulik, 1936, Fauna India, Galeruc. : 331 (=collaris).

Cassena tonkinensis Weise, 1922, Tijdschr. Ent., 65 : 128 (Tonkin; STOCKHOLM). New synonym.

Cassena collaris :Laboissière, 1932, Mém. Mus. Hist. Nat. Belg., hors ser., 4(4) : 182 (Indes, Tonkin).

Cassena robusta:Laboissière, 1932, Mém. Mus. Hist. Nat. Belg., hors ser., 4(4) : 182 (Java, Perak). Solephyma collaris: Maulik, 1936, Fauna India, Galeruc.: 331 (India, Burma, Siam, Perak, Sumatra).

Solephyma tikhami Gressitt \& Kimoto, 1963, Pac. Ins. Mon., 1B:663, fig. (Tonkin: Lao-kay, SinoVietnam border; China ; BISHOP). New synonym.

Cassena tinkhami : Gressitt \& Kimoto, 1963, Pac. Ins., 5(4) : 925 (N. Vietnam, China). 
Distribution : India, Burma, Thailand, Vietnam, China, Malaya, Sumatra, Java.

Material examined. THAILAND : Chieng Mai Prov., Fang, 1 ex., 12-19. iv. 1958, T. C. Maa; Chieng Mai Prov., Fang (Agr. Exp. Station), 600 m, 1 ex., 14. vi. 1965, P. D. Ashlock; Pakchong, NE of Bangkok (light trap), 1 ex.. 1-3. xii. 1957, T. C. Maa; Banna, 1 ex., 5-10. iv. 1958, T. C. Maa (BISHOP) ; Chieng Mai Prov., Doi Suthep, 5 exs., 10. vi. 1965, K. Morimoto ; Chieng Mai Prov., Tan Keo, Doi Suthep, 800 m, 1 ex., vi. 1965, Y. Miyatake ; Chieng Mai Prov., Mae Sa Water Fall, 1 ex., 16. vi. 1965, K. Morimoto ; Khao Yai Nat. Park, 3 exs., 6. vi. 1965, K. Morimoto ; Pew, Chanta Buri, 2 exs., 29. viii. 1973, S. Nakao ; Fang, 1 ex., 22. viii. 1973, K. Yano (KU) ; Khon Kaen, 1 ex., 20. xii. 1979, S. Azuma (RU) ; Kanchanaburi, 2,500 ft, 2 exs., 31. v. 1962, Chiengmai, 1 ex., 6. vi. 1963 ; Kanchanaburi, 1,300 ft, 1 ex., 21. v. 1962 ; Nakhon Ratchasima, 2 exs., 30. vi. 1962 ; Uthaithani, 4 exs., 13. iv. 1963 (BANGKHEN). LAOS: Vientiane Prov. Vientiane, 2 exs., 22. vii. 1965, Native collr ; Vientiane Prov., Ban Van Eue, 1 ex., 15. v. 1966, Native collr ; Vientiane Prov., Gi Sion Vill., de Tha Ngone, 1 ex, 21-28. ii. 1966, 1 ex, 24-31. x. 1966, Native collr ; Khammouane Prov, Bon Nong Sun, 1 ex., 15. vii. 1965, N. Wilson ; Sayaboury Prov, Sayaboury, 1 ex., 25. viii. 1966, Native collr ; Namkading, 100 m, nr. Pakkading, 1 ex., 15. iv. 1965, J. L. Gressitt, Ile de Khong, 1 ex., 24. viii. 1965, J. A. Rondon (BISHOP) ; Umgeb. Vientiane, 1 ex., iii-vi. 1963 (MUNCHEN). VIETNAM : $24 \mathrm{~km} \mathrm{S.} \mathrm{of} \mathrm{Dilinh}$ (Djiring), 1,050 m, 29. iv. 1960, R. E. Leech (BISHOP).

\section{Cassena indica (Jacoby), new combination}

Solenia (Euphyma) indica Jac., 1889, Ann. Mus. Civ. Genova, 27 : 235 (Burma ; GENOVA).

Solephyma indica: Maulik, 1936, Fauna India, Galeruc. : 332 (Burma).

Distribution : Burma, Thailand.

Material examined. THAILAND : Chiengmai Prov., Doi Pui, 1,360 m, 16 exs., 2. v. 1958, T. C. Maa (BISHOP). LAOS : Vientiane Prov., Ban Van Eue, 2 exs., 15-31. v. 1965, Native collr (BISHOP). VIETNAM : $50 \mathrm{~km} \mathrm{SW}$ of Pleiku, $250 \mathrm{~m}, 1$ ex., 14. v. 1960, L. W. Quate (BISHOP).

\section{Cassena integricollis (Jacoby) Fig. 56a}

Solenia integricollis Jac., 1892, Ann. Mus Civ. Genova, 32 : 987 (Burma ; GENOVA).

Solephyma integricollis: Maulik, 1936, Fauna India, Galeruc. : 333 (Burma).

Cassena integricollis :Laboissière, 1932, Mém. Mus. Hist. Nat. Belg., hors ser., 4(4): 182 (Burma).

Distribution : Burma, Thailand, Vietnam.

Material examined. THAILAND : Chiengmai Prov., Fang, 500 m, 2 exs., 13. vi. 1965, P. D. Ashlock (BISHOP) ; Chieng Mai Prov., Fang, 4 exs., 13. vi. 1965, K. Morimoto, 2 exs., 14. vi. 1965, Y. Miyatake (KU). VIETNAM : Fyan, 1,200 m, 1 ex., 11. vii.-9. viii. 1961, N. R. Spencer ;M'Drak, E. of Ban Me Thuot, 4-600 m, 1 ex., 8-19. xii. 1960, C. M. Yoshimoto (BISHOP).

\section{Cassena oculata Laboissière Fig. 54b}

Cassena oculata Lab., 1934, Ann. Ass. Nat. Levallois-Perret, 21 : 137 (Tonkin; HAMBURG).

Solephyma ocellata Gressitt \& Kimoto, 1963, Pac. Ins. Mon., 1B: 661 (Tonkin:Hoa-bin ; FREY). New synonym.

Cassena ocellata: Gressitt \& Kimoto, 1963, Pac. Ins., 5(4) : 925 (N. Vietnam).

Distribution : Thailand, Laos, Vietnam.

Material examined. THAILAND : Khao Yai Nat. Park, 1 ex., 6. vi. 1965, K. Morimoto (KU). LAOS : Attopeu Prov., Houei Kong, 1 ex., 17. vii. 1965, 1 ex., 7. x. 1965, Native collr (BISHOP). 
VIETNAM : Dilinh (Djiring), 1 ex., 27. ix.-14. x. 1960, C. M. Yoshimoto ; $17 \mathrm{~km}$ S. of Dilinh, 1,300 m, 1 ex., 6-13. x. 1960, C. M. Yoshimoto (BISHOP).

\section{Cassena rubyana (Maulik), new combination Fig. 55a}

Cneoranerubyana Maulik, 1936, Fauna India, Galeruc. : 343 (Burma ; BM).

Distribution : Burma, Thailand, Laos, Vietnam.

Material examined. THAILAND: Chiengmai Prov., Doi Pui, 1,300 m, 1 ex., 17. vi. 1965, K. Morimoto (KU). LAOS : Vientiane Prov., Vientiane, 1 ex., 30. iv. 1965, Native collr ; Vientiane Prov., Ban Van Eue, 2 exs., 1-15. v. 1965, 1 ex., 15-31. v. 1965, J. A. Rondon; Sedon Prov, Pakscng, 13 exs., 16. v. 1965, Native collr ; Sayaboury Prov., Sayaboury, 1 ex., 30. v. 1965, Native collr ; Khammouane Prov., Phon Tiou, 1 ex., 6. vii. 1965, Native collr (BISHOP) ; Umgeb. Ventiane, 4 exs., iii.-vi. 1963 ; Umgeb. Paklay, 1 ex., 1963 (MUNCHEN). VIETNAM : Ban Me Thuot, 500 m, 21 exs., 16-18. v. 1960, L. W. Quate ; $20 \mathrm{~km} \mathrm{~N}$. of Pleiku, 1 ex., 650 m, 9. v. 1960, L. W. Quate (BISHOP).

Cassena suturalis n. sp. Fig. $56 \mathrm{~b}$

Head and pronotum reddish brown, scutellum pitchy black; elytron yellowish brown with sutural margin widely and basal, lateral and apical margins narrowly black; antenna pitchy black with two or three basal segments brownish; ventral surfaces reddish brown with abdomen pitchy brown ; legs pitchy brown to black with anterior and middle femora reddish brown.

Head with vertex smooth, shining, sparsely impressed by minute punctures, interocular space distinctly wider than transverse diameter of single oculus, and interocular transverse impression distinct, frontal tubercle subtriangular, oblique, contiguous, distinctly raised, surface smooth, shining. Antenna slender, nearly $3 / 4$ as long as length of body ; first segment long, robust, second shortest, nearly half as long as first, third $11 / 2$ times as long as second, fourth slightly longer than second, fifth subequal to fourth in length and shape, sixth slightly shorter than fifth, sixth to eighth subequal to

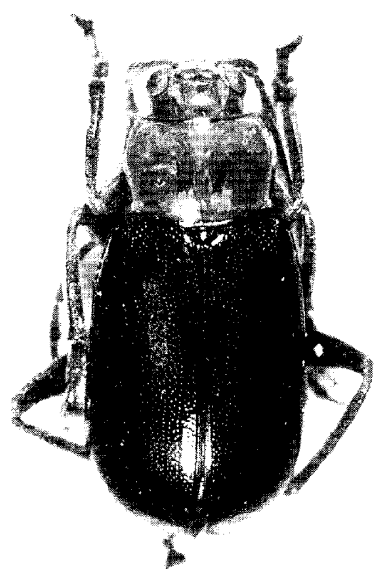

a

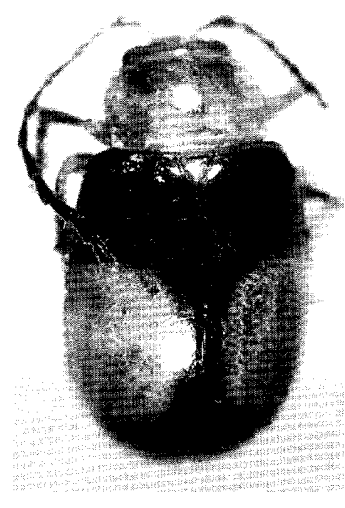

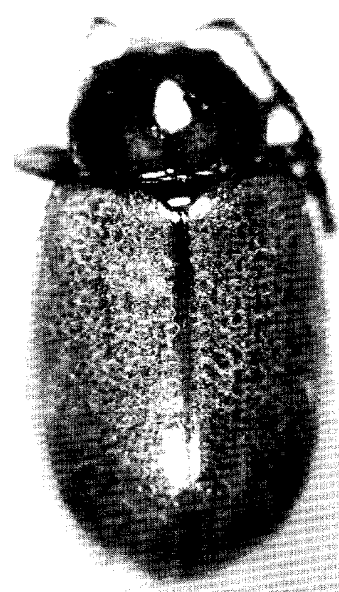

C

Fig. 55. a, Cassena mbyana (Maulik) ; b-c, C. vietnamica n. sp. 
each other in length and shape, ninth slightly shorter than eighth, tenth subequal to ninth in length and shape, eleventh subequal to eighth in length but its apex pointed. Pronotum transverse, $12 / 3$ times as broad as long, anterior margin nearly straight, lateral margin distinctly rounded, widest almost at middle, distinctly narrowed anteriorly and posteriorly, basal margin distinctly rounded posteriorly, dorsal surface convex side to side, with a pair of short longitudinal furrows basally, sparsely impressed by fine punctures, and interstices of punctures smooth, shining. Scutellum subtriangular, smooth, shining, sparsely impressed by minute punctures. Elytron with lateral margin distinctly rounded, surface distinctly and closely punctate, and interstices of punctures smooth, shining.

Length : $3.8 \mathrm{~mm}$.

Holotype (Type No. 2705, Kyushu Univ.) : THAILAND : Chieng Mai Prov., Doi Suthep, 1,000 m, 18. vi. 1965, K. Morimoto (KU). Paratypes : 7 exs., same data as the holotype ; 1 ex., collected by Y. Miyatake (KU, KIMOTO).

This new species somewhat resembles Cassena oculata Laboissière, but differs in having elytron yellowish brown with the sutural margin widely and the basal, the lateral and the apical margins narrowly blackish, and the ventral surfaces reddish brown with the abdomen pitchy brown.

\section{Cassena terminalis (Gressitt \& Kimoto) Fig. 54c}

Solephyma terminalis Gressitt \& Kimoto, 1963, Pac. Ins. Mon., 1B: 662, fig. (S. China ; BISHOP). Cassena terminalis: Gressitt \& Kimoto, 1963, Pac. Ins., 5(4) : 925 (S. China).

Distribution : Thailand, China.

Material examined. THAILAND: Chiengmai, 1,200 m, 1 ex., 11. iv. 1966, J. Sedlacek (BISHOP).

Cassena vietnamica n. sp. Fig. 55b, c

Generally reddish brown; elytron yellowish brown with margins narrowly infuscate, in some specimen dark brown with a large discal markings yellowish brown; antenna pitchy black with three basal segments reddish brown; legs dark brown with femora reddish brown.

Head with vertex smooth, shining, sparsely impressed by minute punctures, interocular space distinctly wider than transverse diameter of single oculus, and interocular transverse impression distinct, frontal tubercle transverse, subquadrate, contiguous, distinctly raised, surface smooth, shining. Antenna slender, nearly $3 / 4$ as long as body length ; first segment long, robust, second shortest, nearly half as long as first, third $12 / 5$ times as long as second, fourth slightly longer than third, fourth to eighth subequal to each other in length and shape, ninth slightly shorter than eighth, tenth subequal to ninth in length and shape, eleventh $11 / 5$ times as long as tenth and its apex pointed. Pronotum transverse, $11 / 2$ times as broad as long, anterior margin distinctly rounded anteriorly, lateral margin distinctly rounded, widest almost at middle, distinctly narrowed anteriorly and posteriorly, basal margin slightly rounded posteriorly, dorsal surface convex side to side, with a pair of short longitudinal furrows basally, sparsely impressed by fine punctures, and interstices of punctures smooth, shining. Scutellum subtriangular, smooth, shining, impunctate. Elytron with lateral margin rounded, surface distinctly and rather closely punctate, and interstices of punctures smooth, shining.

Length : $3.6-4.4 \mathrm{~mm}$.

Holotype (вISнор) : VIETNAM : $31 \mathrm{~km} \mathrm{~S}$. of Dilinh (Djiring), 1,050 m, 29. iv. 1960, R. E. Leech. Paratopotypes : 10 exs., same data as the holotype (BISHOP, KIMOTO). Paratype :20 km N. of Pleiku, 650 m, 1 ex., 9. v. 1960, S. Quate (BISHOP). 


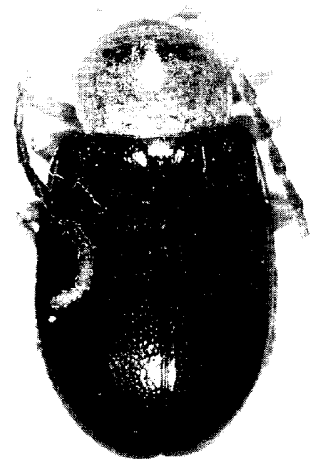

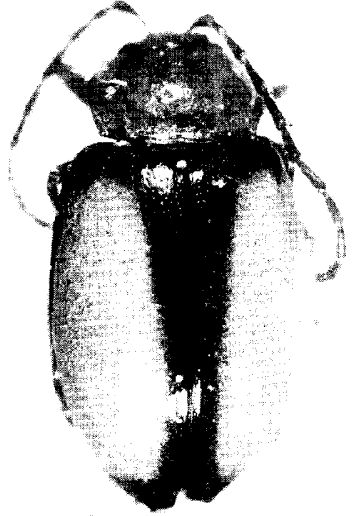

b

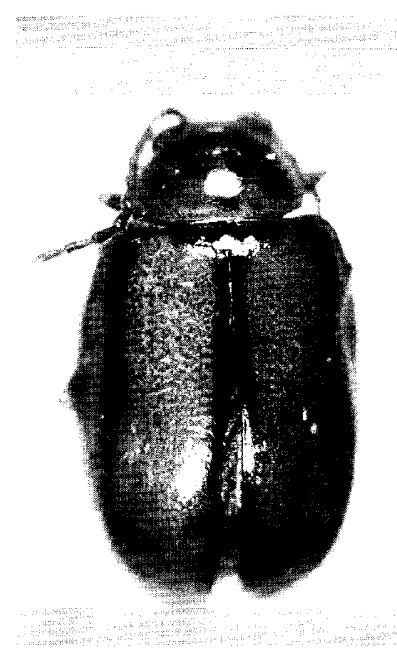

C

Fig. 56. a, Cassena integricollis (Jacoby); b, C. suturalis n. sp.; c, Pseudoides flavovittis (Motschulsky).

This new species resembles Cassena oculata Laboissière, but differs in being the body length shorter, and having elytron yellowish brown with the margins narrowly infuscate and in the pale colored specimen dark drown with a large discal marking yellowish brown.

\section{Genus Cyclanthipha Laboissière}

Cyclanthipha Lab, 1932, Ann. Ass. Nat. Levallois-Perret, 20 : 136 (type : Cyclanthipha ornata Laboissière ; Vietnam).

\section{Cyclanthipha ornata (Laboissière)}

Cyclanthipha ornata Lab., 1932, Ann. Ass. Nat. Levallois-Perret, 20 : 137, fig. (Tonkin ;BRUXELLES).

Distribution : Thailand, Laos, Vietnam.

Short oval ; generally reddish brown, elytron yellowish with a large median marking blackish and basal $2 / 3$ of sutural margin narrowly reddish ; antenna, tibiae and tarsi blackish ; length 5.0-5.5 $\mathrm{mm}$.

No additional material was examined beside the type series.

\section{Genus Vietoluperus Medvedev \& Dang Dap}

Vietoluperus Medvedev \& Dang Dap, 1981, Rev. d'Ent.l'USSR, 60 (3) : 632 (type :Vietoluperus alleculoides Medvedev \& Dang Dap ; Vietnam). 


\section{Vietoluperus alleculoides Medvedev \& Dang Dap}

Vietoluperus alleculoides Medvedev \& Dang Dap. 1981, Rev. d'Ent.l'USSR, 60 (3) : 632 (Vietnam). Distribution : Vietnam.

Body oblong, rather broad; generally reddish brown, fourth to eleventh antennal segments, elytron, tibiae and tarsi blackish brown ; usually with base of tibiae and elytral humerus somewhat reddish brown and pronotum darkened. In general specimens, the color is uniformly reddish. Length 6.0-7.5 mm (after translation by Entomological Abs. : 111).

No material was examined.

\section{Genus Pseudoides Jacoby}

Pseudoides Jac., 1892, Ann. Mus. Civ. Genova, 32 : 966 (type : Pseudoides bivittata Jacoby ; monobasic).-Maulik, 1936, Fauna India, Galeruc. : 302.

Erganoides Jacoby, 1903, Ann. Soc. Ent. Belg., $47: 125$ (type : Erganoides flavicollis Jacoby ; S . India).-Ogloblin, 1936, Fauna USSR, 26, 1 : 300, 431.-Gressitt \& Kimoto, 1963, Pac. Ins. Mon., 1B : 592. New synonym.

Key to species of Pseudoides

Generally yellowish brown with vertex, scutellum and elytral margins pitchy black ; antenna pitchy brown with basal segments brownish; legs yellowish brown with tibiae and tarsi blackish ; pronotum widest almost at middle and strongly narrowed anteriorly ;

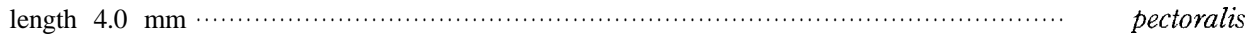

Generally yellowish brown, with vertex, scutellum, metathorax and abdomen pitchy brown to black; elytron with a discal stripe, and sutural margins widely blackish ; antenna pitchy black with basal segments brownish; pronotum widest almost at $1 / 3$ from anterior margin and more strongly narrowed posteriorly ; length $3.3-3.8 \mathrm{~mm}$........... flavovittis

\section{Pseudoides flavovittis (Motschulsky) Fig. 56c}

Calomicrus flavovittis Motsch., 1858, Etud. Ent., 7 : 101 (Burma).

Luperus jlavovittis : Allard, 1889, Ann. Soc. Ent. France, 58 : 309 (Pnomh-Penh).

Pseudoides bivittata Jacoby, 1892, Ann. Mus. Civ. Genova, 32 : 966 (Burma ;GENOVA).-Maulik, 1936, Fauna India, Galeruc. : 303, fig. (Burma).-Wilcox, 1971, Col. Cat. Junk, Suppl., $78: 153$ (= flavovittis).

Erganoides flavovittis: Laboissike, 1935, Ann. Ass. Nat. Levallois-Per-ret, 21 : 141 (Cambodge, Birmanie, Tonkin); 1940, Bull. Mus. Hist. Nat. Belg., 16 (37) :2(Tonkin, Burma, Bhamo, Cambodia).

Pseudoides flavovittis: Wilcox, 1971, Col. Cat. Junk, Suppl., 78 : 153 (Burma, Cambodia, Vietnam). -Kimoto \& Takizawa, 1973, Kontyâ, Tokyo, 41 (2) : 177 (Nepal).-Kimoto, 1979, Ent. Basiliensia, $4: 474$, fig. (Bhutan).

Distribution : Burma, Nepal, Bhutan, Thailand, Cambodia, Laos, Vietnam.

Material examined. THAILAND : Trang Prov., Khaophappha, 2 exs., 30-31. vii. 1963, 4 exs., 1. i. 1964, 1 ex., 4. i. 1964, G. A. Samuelson ; Sattahib, 1 m, 1 ex., 24. xi. 1957, J. L. Gressitt ; 20 km NW of Ratburi, 1 ex., 15. viii. 1966, T. C. Maa (BISHOP) ;Sarapi, nr. Chiengmai, 7 exs., 18. vii. 1970, K . Yano (KU). LAOS : Vientiane Prov., Ban Van Eue, 1 ex., 1-15. v. 1965, J. A. Rondon, 14 exs., 15-31. 
v. 1965, 35 exs., 31. v. 1966, 1 ex., 15. x. 1967, 1 ex., 30. ix. 1967, Native collr ; Vientiane Prov., Gi Sion Vill., de Tha Ngone, 1 ex., 7-21. ii. 1965, 50 exs., '2. i. 1966, Native collr ; Vientiane Prov., Dong Dok, 6 exs., 23. vii. 1965, Native collr ; Sedone Prov., Paksong, 1 ex., 26. vii. 1965, Native collr ;Nongtevada, 1 ex., 13. viii. 1965, Native collr (BISHOP). VIETNAM : Fyan, 1,200 m, 2 exs., 11. vii.-9. viii. 1961, N. R. Spencer ; Ninh Hoa, N. of Nha Trang, 12 ex., 28. xi. 1960, C. M. Yoshimoto ; Nha Trang, 1 ex., 17-26. xi. 1960, C. M. Yoshimoto ; 22 km S. of Nha Trang, 1 ex., 20-26. xi. 1960, C. M. Yoshimoto (BISHOP).

\section{Pseudoides pectoralis (Laboissière), new combination Fig. 3b}

Erganoides pectoralis Lab., 1933, Bull. Mus. Hist. Nat. Paris, ser. 2, $5: 205$ (Tonkin:Hoa-Binh; HAMBURG).

Distribution : Vietnam.

No additional material was examined.

\section{Genus Eumelepta Jacoby}

Eumelepta Jac., 1892, Ann. Mus. Civ. Genova, 32: 988 (type: Eumelepta biplagiata Jacoby Burma).-Maulik, 1936, Fauna India, Galeruc. : 304.

\section{Eumelepta biplagiata Jacoby Fig. 57a}

Eumelepta biplagiata Jac., 1892, Ann. Mus. Civ. Genova, 32 : 988 (Burma ; GENOVA) ; 1900, Mém. Soc. Ent. Belg., 7 : 136 (mentioned).-Maulik, 1936, Fauna India, Galeruc. : 306 (Burma, Sumatra, Malay Peninsula :Kina Balu).

Distribution : Burma, Thailand, Laos, Vietnam, Borneo, Sumatra.

Suboval. Antenna robust ; pronotum smooth, shining, nearly impunctate ; elytron distinctly and closely punctate; general color pitchy black, pronotum yellowish brown, elytron black with a large elongate marking yellowish brown, antenna pitchy brown, legs yellowish brown; length $2.7 \mathrm{~mm}$.

Material examined. THAILAND : Trang Prov., Khaophappha, Khaochang, 1 ex., 3-5. i. 1964, G. A. Samuelson, Bangkok, 1 m, 1 ex., 4. xii. 1957, T. C. Maa (BISHOP) ; Chiengmai Prov., Doi Suthep, 1,000 m, 2 exs., 18. vi. 1965, K. Morimoto \& Y. Miyatake ; Chiengmai Prov., Chieng Dao, 1 ex., 15. vi. 1965, K. Morimoto (KU). LAOS: Borikhane Prov., Pakkading, 1 ex., 15. ix. 1965, J. L. Gressitt ; Nongtevada, 1 ex., 17. vii. 1965, Native collr (BISHOP). VIETNAM : Fyan, 1,200 m, 12 exs., 11. vii.9. viii. 1961, N. R. Spencer ; Dalat, 1,550 m, 1 ex., 11. ix. 1960, J. L. Gressitt ; Dalat, 6 km S., 1,300-1,500 m, 17 exs., 9. vi.-7. vii. 1961, N. R. Spencer (BISHOP).

\section{Genus Hoplosaenidea Laboissière}

Cynorta Baly, 1865, Ann. Mag. Nat. Hist., ser. 3, 16 : '249 (nec Koch, 1839) (type :Cynorta porrecta Baly ; Java).-Laboissière, 1940, Bull. Mus. Hist. Nat. Belg., 16 (37) : 19 (as a synonym of Diaphaenidea Laboissike).

Hoplosaenidea Laboissière, 1933, Ann. Soc. Ent. France, 102 : 59 (type : Hoplosaenidea touzalini Laboissière; Yunnan).-Gressitt \& Kimoto, 1963, Pac. Ins. Mon., 1B: 666 (as Haplosaenidea: error).

Diaphaenidea Laboissière, 1933, ibid: 61 (type : Diaphaenidea aerosa Laboissière; China). Micraenidea Laboissière, 1933, ibid. : 64 (type :Micraenidea coomani Laboissière;Tonkin). 
Cynorita Laboissière, 1940, Bull. Mus. Hist. Nat. Belg., 16 (37) : 28 (type : Cynorta citrina Jacoby ; Philippines). New synonym.

Cynortana Strand, 1942, Folia Zool.-Hydrobiol., 11 : 391 (new name for Cynorta Baly, nec Koch).

\section{KEY TO SPECIES OF Hoplosaenidea}

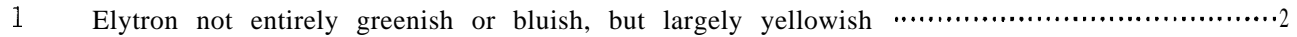

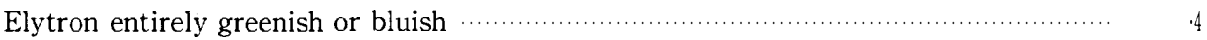

2(1) Ventral surfaces entirely yellowish brown ; dorsal surfaces, antenna and legs yellow-

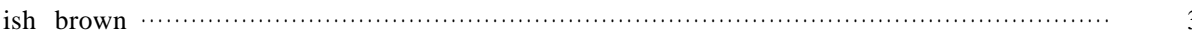

Ventral surfaces black with pro- and mesothorax yellowish brown; generally yellowish brown, elytron with sutural and lateral margins blackish, in some specimen elytron almost entirely yellowish brown; length $6.5-6.8 \mathrm{~mm} \ldots \ldots \ldots \ldots \ldots \ldots \ldots \ldots \ldots \ldots \ldots \ldots \ldots \ldots$ nigrolimbata

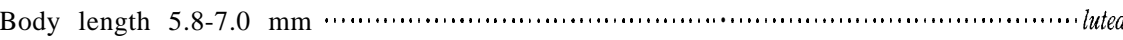

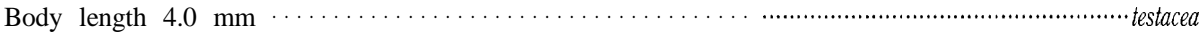

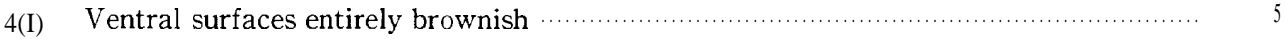
Ventral surfaces at least in part blackish

5(4) Elytron impressed by strong punctures and their interstices subequal to or narrower than average diameter of punctures ; in male head without large cavity on middle

Elytron impessed by fine punctures and their interstices clearly wider than average diameter of punctures ; in male head with a deep and large cavity at middle ; generally yellowish brown; elytron bluish black ; length $3.6 \mathrm{~mm} \ldots \ldots \ldots \ldots \ldots \ldots \ldots \ldots \ldots \ldots \ldots \ldots \ldots$ elegans

6(5) Antenna with third segment nearly twice as long as second and fourth subequal to length of second and third combined; yellowish to reddish brown; elytron golden green; antenna pitchy brown with basal segments much paler; length 3.3-3.5

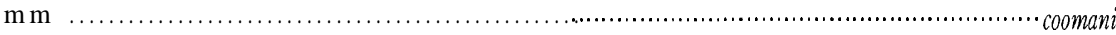

Antenna with second segment subequal to third in length, and fourth longer than length of second and third combined ; generally reddish brown, elytron golden green ;

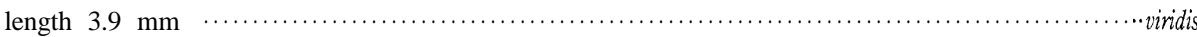

7(4) Body length shorter than $4.0 \mathrm{~mm}$; elytron with punctures closely and rugosely impressed, and their interstices distinctly narrower than average diameter of punctures, and distinctly raised

Body length over $5.0 \mathrm{~mm}$; elytron with punctures finely impressed, and their interstices much wider than average diameter of punctures and finely granulate; generally yellowish brown with elytron, metathorax and abdomen largely bluish black; in male head with a large and deep cavity on middle; length 5.0-6.5 mm

8(7) Head entirely yellowish brown ; legs with tibiae and tarsi largely or partly blackish; generally yellowish brown, elytron greenish blue, antenna, meso- and metathorax and abdomen pitchy black ; in male head with a large and deep cavity on middle ; length

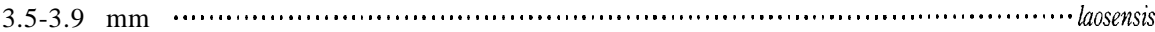

Head yellowish brown with vertex more or less greenish; legs entirely yellowish brown; generally yellowish brown, elytron golden green, antenna pitchy brown, meso- and metathorax and abdomen pitchy black; in male head without distinct cavity ; length $3.3-3.8 \mathrm{~mm}$ 


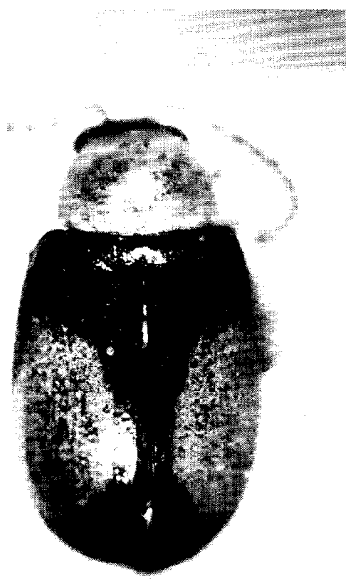

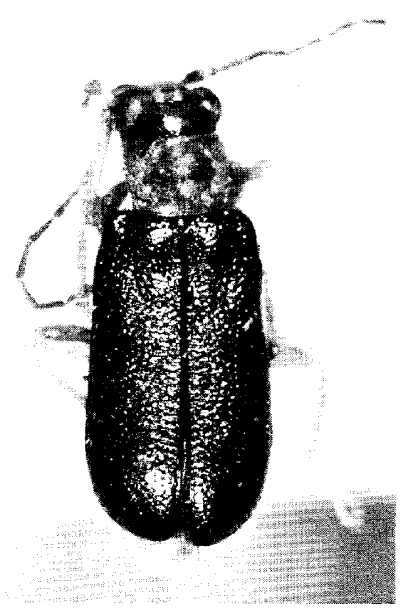

b

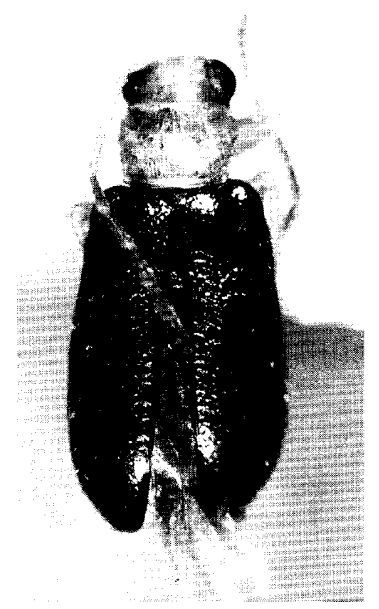

Fig. 57. a, Eumelepta biplagiata Jacoby ; b, Hoplosaenidea rugosa n. sp. ;c, H. coomani (Laboissière).

\section{Hoplosaenidea coomani (Laboissière) Fig. 57c}

Micraenidea coomani Lab., 1933, Ann. Soc. Ent. France, 102 : 64 (Tonkin; HAMBURG).

Hoplosaenidea coomani : Gressitt \& Kimoto, 1963, Pac. Ins. Mon., 1B : 669 (Tonkin:Hoa-binh). Distribution : Laos, Vietnam.

Material examined. LAOS: Sedone Prov., Paksong, 18. v. 1965, P. D. Ashlock (BISHOP). VIETNAM : Dalat, 1,500 m, 5 exs., 29. iv.-4. v. 1960, L. W. Quate ; Dalat, 6 km S, 1,400-1,500 m, 2 exs., 9. vi.-7. vii. 1961, N. R. Spencer; 18 km NW of Dalat, 1300 m, 7 exs., 4-5. v. 1960, L. W. Quate ; Blao (Balao), 500 m, 4 exs., 14-21. x. 1960, C. M. Yoshimoto ; Mt. Lang Bian, 1,500 m, 6 exs., 29. iv.-4. v. 1960, L. W. Quate ; Fyan, 900-1,200 m, 4 exs., 11. vii.-9. viii. 1961, N. R. Spencer ; Ap Hung-Lam, 21 km NW of Dilinh, 1,100 m, 1 ex., 29. ix.- 5. x. 1960, C. M. Yoshimoto (BISHOP).

\section{Hoplosaenidea cornuta (Laboissière)}

Diaphaenidea comuta Lab., 1933, Ann. Soc. Ent. France, $102: 63$ (Tonkin; HAMBURG).

Hoplosaenidea cornuta: Gressitt \& Kimoto, 1963, Pac. Ins. Mon., 1B : 669 (Tonkin :Hoa-Binh). Distribution : Vietnam.

No additional material was examined.

\section{Hoplosaenidea elegans n. sp. Fig. 58c}

Generally yellowish brown, elytron bluish black; antenna dark yellowish brown, with three or four basal segments yellowish brown.

Head with vertex finely granulate, sparsely impressed by minute punctures, interocular space much wider than transverse diameter of single oculus, and interocular transverse impression distinct, frontal tubercle elongate, subtriangular, contiguous, distinctly raised, surface finely granulate. Antenna slender, nearly $2 / 3$ as long as body length ; first segment long, robust, second shortest, nearly 
$1 / 5$ as long as first, third four times as long as second, fourth twice as long as third, fourth to eighth subequal to each other in length and shape, ninth slightly shorter than eighth, tenth subequal to ninth in length and shape, eleventh subequal to eighth in length but its apex pointed. Pronotum transverse, $12 / 5$ times as broad as long, anterior margin nearly straight, lateral margin slightly rounded, widest almost at $1 / 3$ from anterior margin, slightly narrowed anteriorly and more strongly so posteriorly, basal margin slightly rounded posteriorly, dorsal surface convex, with a pair of large depressions laterally, finely granulate, nearly impunctate. Scutellum subtriangular, finely granulate, impunctate. Elytron with lateral margin subparallel-sided, apex rounded, surface impressed by an oblique subbasal and an elongate lateral furrows, and finely granulate and impressed by fine punctures and their interstices clearly wider than average diameter of punctures.

Male : Head with vertex impressed by a deep and large cavity at middle ; fifth abdominal segment trilobed, median lobe generally flat. Female : Head with vertex convex; fifth abdominal segment entire.

Length : $3.6 \mathrm{~mm}$.

Holotype (BISHOP) : LAOS : Vientiane Prov., Ban Van Eue, 15. viii. 1965, Native collr. Paratypes : THAILAND : Chiengmai, 1,500 m, 1 ex., 11-12. iv. 1966, J. Sedlacek, 1 ex., 1,100-1,500 m, no data, 1966, J. Sedlacek (BISHOP). LAOS : Vientiane Prov., Ban Van Eue, 1 ex., 12. iv. 1965, J. L. Gressitt (KIMOTO).

This new species resembles Hoplosaenidea coomani (Laboissière), but differs in having elytron covered by much finer punctures, and in the male the head with a deep and large cavity at the middle.

\section{Hoplosaenidea laosensis n. sp. Fig. 58b}

Generally yellowish brown, scutellum pitchy brown, elytron greenish blue, antenna pitchy black with one or two basal segments brownish, ventral surfaces with meso- and metathorax and abdomen pitchy black, legs with tibiae and tarsi pitchy black.

Head with vertex smooth, shining, sparsely impressed by minute puntures, interocular space slightly wider than transverse diameter of single oculus, and interocular transverse impression distinct, frontal tubercle subtriangular, contiguous, distinctly raised, surface smooth, shining. Antenna slender, nearly $2 / 3$ as long as body length ; first segment long, second shortest, nearly $1 / 3$ as long as first, third twice as long as second, fourth $11 / 2$ times as long as third, fifth slightly shorter than fourth, fifth to seventh subequal to each other in length and shape, eighth slightly shorter than seventh, eighth to tenth subequal to each other in length and shape, eleventh subequal to tenth in length but its apex pointed. Pronotum transverse, $12 / 5$ times as broad as long, anterior margin nearly straight, lateral margin distinctly rounded, widest almost at $1 / 3$ from anterior margin, slightly narrowed anteriorly and more strongly so posteriorly, basal margin feebly rounded posteriorly, dorsal surface convex, smooth, shining, with a pair of deep, oblique depressions which are united at middle, sparsely impressed by minute punctures. Scutellum subtriangular, smooth, shining, impunctate. Elytron with lateral margin subparallel-sided, apex rounded, surface strongly and closely punctate and their interstices clearly narrower than average diameter of punctures, and finely granulate.

M ale : Head with vertex impressed by a deep and large cavity at middle ; fifth abdominal segment trilobed apically. Female : Unknown.

Length : $3.5-3.9 \mathrm{~mm}$.

Holotype (BISHOP) : LAOS : Vientiane, 31. v.-3. vi. 1960, S. Quate \& L. Quate. Paratopotypes : 2 exs., same data as the holotype (BISHOP, KIMOTO).

This new species resembles Hoplosaenidea mugosa Kimoto, but differs in having the legs yellowish brown with tibiae and tarsi pitchy black and in the male the head with a large and deep cavity at the 
middle.

\section{Hoplosaenidea lutea (Laboissière), new combination}

Macrimalutea Laboissiere, 1936, Ann. Soc. Ent. France, 105 : 245 (Tonkin: Hoa-Binh ; HAMBURG).

Cynorita lutea : Laboissière, 1940, Bull. Mus. Hist. Nat. Belg., 16 (37) : 30 (Tonkin : Hoa-Binh).

Distribution : Thailand, Cambodia, Laos, Vietnam.

Material examined. THAILAND : Chiengmai Prov., Doi Suthep, 900 m, 1 ex., 14. xi. 1958, J. L. Gressitt, 1 ex., 29. iii.-4. iv. 1958, 2 exs., 1-5. iv. 1958, T. C. Maa ; Chiengmai Prov., Chieng Dao, 5-11. iv. 1958, T. C. Maa ; Pakchong, 100 m, NE of Bangkok, 3 exs., 3. xii. 1957, J. L. Gressitt ; 50 km West of Tak, 900 m, 1 ex., 7-8. iv. 1966, J. Sedlacek ; Banna, Nakhon, 108 m, 1 ex., 5-10. v. 1958, T. C. Maa; Nakhon Nayok Prov., Khao Yai Nat. Park, 1 ex., 6. vi. 1965, P. D. Ashlock; Muak Lek, 1 ex., 6. iii. 1963, Native collr (BISHOP) ; Chiengmai Prov., Doi Suthep, 1,000 m, 2 exs., 12. vi. 1965, K. Morimoto (KU) ; Nakhon Ratchasima, 2 exs., 30. vi. 1962 (BANGKHEN). CAMBODIA : Damarey Phong, 3 exs., 14-16. iv. 1961, N. R. Spencer (BISHOP). LAOS : Vientiane Prov., Vientiane, 1 ex., iii. 1966, J. Sedlacek ; Vientiane Prov., Ban Van Eue, 1 ex., 15. v. 1965, 2 exs., 31. vii. 1965, Native collr ; Sedone Prov., Paksong, 1 ex., 1. vi. 1965, Native collr ; Borikhane Prov., Pakkading, 1 ex., 23. iv. 1965, J. L. Gressitt, 1 ex., 13. vii. 1965, 1 ex., 15-31. viii. 1965, Native collr ; Namkading nr. Pakkading, 1 ex., 21. iv. 1965, J. L. Gressitt ; Pon-horn, N. of Pakkading, 1 ex., 20. iv. 1965, J. L. Gressitt (BISHOP) ; Umgeb. Vientiane, 1 ex., iii-vi. 1963 (MUNCHEN). VIETNAM : $20 \mathrm{~km} \mathrm{~N}$. of Pleiku, $650 \mathrm{~m}, 1$ ex., 9. v. 1960, L. W. Quate ; Dalat, 6 km S., 1,500 m, 1 ex., 9. v. 1960, L. W. Quate ; Chute de Bola, 7 km W. of Dilinh (Djiring), 840 m, 1 ex., 27. iv. 1960, R. E. Leech (BISHOP).

\section{Hoplosaenidea nigrolim ba ta (Jacoby), new combination $\quad$ Fig. 59a}

Macrima nigrolimbata Jac., 1889, Stett. Ent. Ztg., 60 : 308 (Sumatra).-Laboissiere, 1936, Ann. Soc.

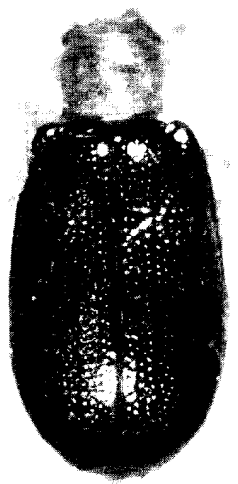

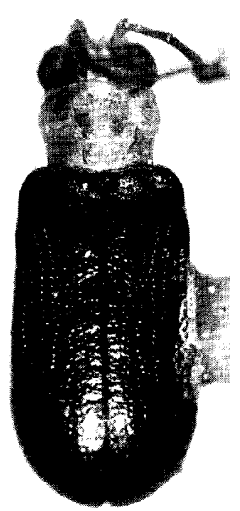

b

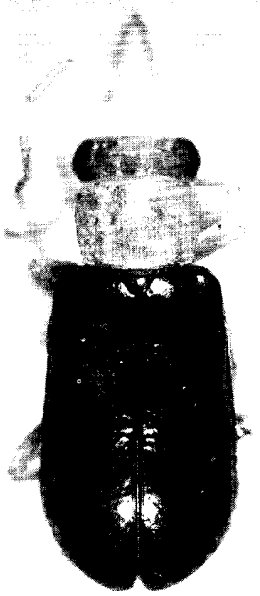

C

Fig. 58. a, Hoplosaenidea viridis n. sp. ; b, H. laosensis n. sp. ; c, H. elegans n. sp. 
Ent. France, 105: 245 (Tonkin:Hoa-Binh).

Cynorta nigrolimbata:Laboissière, 1940, Bull. Mus. Hist. Nat Belg., 16 (37) : 30 (Tonkin:HoaBinh).

Cynorta trilineata:Châjô, 1964, Nature and Life in SE Asia, Kyoto, 3 : 289, fig. (Thailand).

Distribution : Thailand, Laos, Vietnam, Sumatra.

Material examined. THAILAND : Trang Prov., Khaophappha, Kaochong, 200-400 m, 1 ex., 13. i. 1964, G. A. Samuelson (BISHOP) ; Khao Yai Nat. Park, 1 ex., 6. vi. 1965, Y. Miyatake ; Khao Chong, nr. Trung, 1 ex., 26. vi. 1965, Y. Miyatake ; Kao Chong Park, 1 ex., 1. iii. 1965, K. Yasumatsu (KU) ; Khon Kaen, 1 ex., 8. x. 1981, S. Azuma (RU). LAOS : Vientiane Prov., Ban Van Eue, 1 ex., 13. iv. 1965, J. L. Gressitt, 1 ex., 15. ii. 1965, 1 ex., 17. ii. 1965, 2 exs., 15-31. iv. 1965, 1 ex., 15-31. v. 1965, 7 exs., 30. vi. 1965, 1 ex., 31. vii. 1965, 1 ex., 15. viii. 1965, 1 ex., 30. xi. 1965, 1 ex., 15. v. 1967, 1 ex., 15. ix. 1967, 1 ex., 30. ix. 1967, Native collr ; Wapithamthong Prov., Khong Sedone, 1 ex., 17. ix. 1965, Native collr ; Sayaboury Prov., Sayaboury, 1 ex., 15. iv. 1965, J. L. Gressitt ; Namkading, nr. Pakkading, 1 ex., 21. iv. 1965, J. L. Gressitt (BISHOP) ; Umgeb. Vientiane, 2 exs., iii.-vi. 1963 ; Umgeb. Vanky, 2 exs., 1963 (MUNCHEN). VIETNAM : Karyu Danar, 200 m, 4 exs., 13-28. ii. 1961, N. R. Spencer ; Dilinh (Djiring), 1 ex., 27. ix.-14. x. 1960, C. M. Yoshimoto ; Banme Thuot, 500 m, 1 ex., 16-18. v. 1960, R. E. Leech (BISHOP).

\section{Hoplosaenidea rugosa n. sp. Fig. 57b}

Generally yellowish to reddish brown, scutellum pitchy black, elytron golden green, antenna pitchy brown with three or four basal segments reddish brown, ventral surfaces with meso- and metathorax and abdomen pitchy black; in some specimen head with vertex pitchy brown with slightly greenish luster.

Head with vertex finely granulate, sparsely impressed by minute punctures, interocular space much wider than transverse diameter of single oculus, and interocular transverse impression distinct, frontal tubercle subtriangular, contiguous, distinctly raised, surface finely granulate. Antenna slender, nearly as long as body length ; first segment long, robust, second shortest, nearly $1 / 4$ as long as first, third $21 / 2$ times as long as second, fourth $12 / 3$ times as long as third, fifth slightly shorter than fourth, fifth to eighth subequal to each other in length and shape, ninth slightly shorter than eighth, tenth subequal to ninth in length and shape, eleventh subequal to eighth in length but its apex pointed. Pronotum transverse, $11 / 3$ times as broad as long, anterior margin nearly straight, lateral margin slightly rounded, widest almost at $1 / 3$ from anterior margin, slightly narrowed anteriorly and more strongly so posteriorly, basal margin slightly rounded posteriorly, dorsal surface convex, with a pair of deep depressions laterally, finely granulate, sparsely impressed by fine punctures. Scutellum subtriangular, finely granulate, impunctate. Elytron with lateral margin subparallel-sided, apex rounded, surface impressed by an oblique subbasal depression, and closely and rugosely punctate, and their interstices finely granulate.

Male : Fifth abdominal segment trilobed, median lobe generally flat. Female : Fifth abdominal segment entire.

Length : 3.3-3.8 $\mathrm{mm}$.

Holotype (BISHOP) : VIETNAM : $20 \mathrm{~km} \mathrm{~N}$. of Pleiku, $650 \mathrm{~m}, 9$. v. 1960, S. Quate. Paratopotype : 1 ex., same data as the holotype. Paratypes : VIETNAM : Blao (Balao), 500 m, 2 exs., 14-21. x. 1960, C. M. Yoshimoto ; $28 \mathrm{~km} \mathrm{~N}$. of Dilinh (Djiring), 1 ex., 22-28. iv. 1960, S. Quate (BISHOP, KIMOTO).

This new species resembles Hoplosaenidea coomani (Laboissihe), but differs in having elytron closely and rugosely punctate and the ventral surfaces with meso- and metathorax and the abdomen pitchy black. 


\section{Hoplosaenidea testacea (Allard), new combimation}

Luperus testaceus All., 1889, Ann. Soc. Ent. France, ser. 6, 9 : 309 (Cambodia ; PARIS, HAMBURG).

Macrima testacea :Laboissière, 1935, Ann. Ass. Nat. Levallois-Perret, 21 : 141 (genus) ; 1936, Ann. Soc. Ent. France, 105 : 244 (Cambodge : Pnom Penh).

Cynorita testacea :Laboissière, 1940, Bull. Mus. Hist. Nat. Belg., 16(37) : 30 (genus).

Distribution : Cambodia.

No additional material was examined beside the type specimen. This species closely resembles Hoplosaenidea lutea (Laboissiere), but is much smaller.

\section{Hoplosaenidea viridis n. $\mathbf{s p .} \quad$ Fig. 58a}

Generally reddish brown, scutellum pitchy black, elytron golden green.

Head with vertex smooth, shining, sparsely impressed by minute punctures, interocular space much wider than transverse diameter of single oculus, and interocular transverse impression distinct, frontal tubercle transverse, contiguous, distinctly raised, surface smooth, shining. Antenna slender, nearly $2 / 3$ as long as body length ; first segment long, robust, second shortest, nearly $2 / 3$ as long as first, third subequal to seventh in length but more slenderer, fourth twice as long as third, fourth to seventh subequal to each other in length and shape, eighth slightly shorter than seventh, eighth to tenth subequal to each other in length and shape, eleventh subequal to seventh in length but its apex pointed. Pronotum transverse, $11 / 4$ times as broad as long, anterior margin slightly rounded anteriorly, lateral margin distinctly rounded, widest almost at $1 / 3$ from anterior margin, slightly narrowed anteriorly and more strongly so posteriorly, basal margin slightly rounded posteriorly, dorsal surface convex, smooth, shining, with a transverse depression subbasally, sparsely impressed by fine punctures. Scutellum subtriangular, smooth, shining, sparsely impressed by minute punctures. Elytron with lateral margin slightly rounded, surface closely and distinctly punctate, and their interstices subequal to or narrower than average diameter of punctures, and their interstices smooth, shining.

Male : Fifth abdominal segment with a pair of short notches apically. Female : Fifth abdominal segment entire.

Length : $3.9 \mathrm{~mm}$.

Holotype (BISHOP) : VIETNAM : Fyan, 900-1,200 m, 11. vii.-9. viii. 1961, N. R. Spencer. Paratopotypes : 6 exs., same data the holotype (BISHOP, KIMOTO). Paratypes : VIETNAM : Dalat, 6 km S., 1,400-1,500 m, 1 ex., 9. vi.-7. vii. 1961, N. R. Spencer ; 6 km SE, Dalat, 1,500 m, 1 ex., 11. ix. 1960, J. L. Gressitt (BISHOP).

This new species resembles Hoplosaenidea coomani (Laboissiere), but differs in being the body length longer, and having the second antennal segment subequal to the third segment in the length.

\section{Genus Sinoluperoides n. gen.}

Antenna1 insertions widely separated by frons ; labrum bilobed apically ; gena very narrow ; frontal tubercles widely separated by frons; antenna with third segment distinctly longer than second; prothorax with a transverse furrow subbasally, and with lateral and basal margins distinctly marginate ; elytron with punctures irregularly impressed, with distinct subbasal furrow, epipleuron wide at base and gradually narrowed to apex ; prosternal process not elevated between anterior coxae; anterior coxal cavity closed posteriorly ; middle and posterior tibiae distinctly spined apical- 


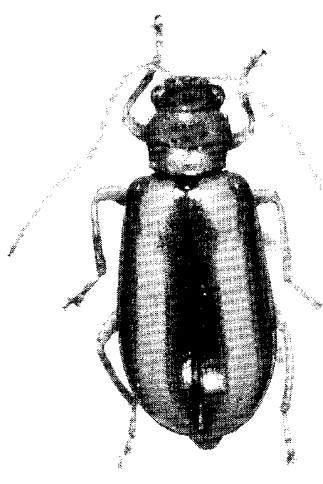

a

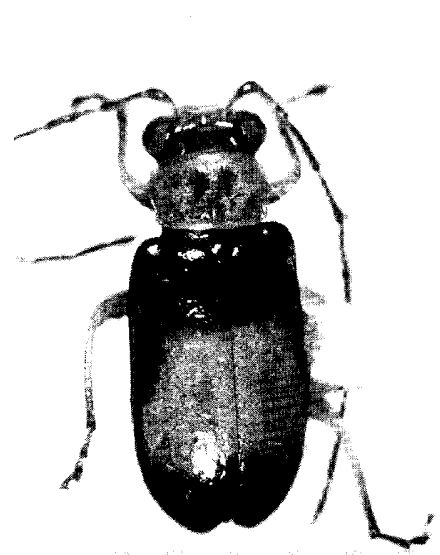

b

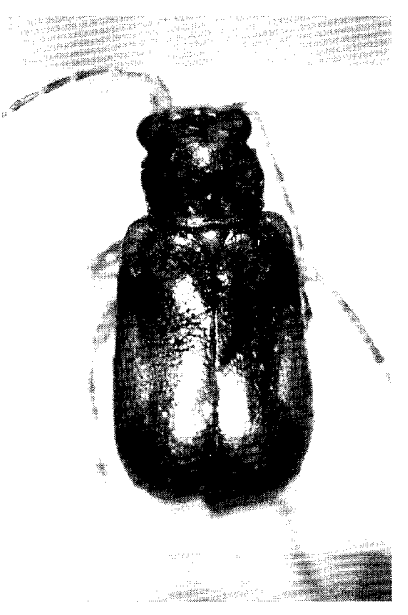

C

Fig. 59. a, Hoplosaenidea nigrolimbata(Jacoby); b, Sinoluperoides antennatus n. sp.; c, S. maculatus n. sp.

ly; tarsal claws appendiculate and first tarsal segment of posterior leg longer than length of second and third segments combined.

Type species : Sinoluperoides maculatus Kimoto.

This new genus closely resembles Hoplosaenidea Laboissière, but differs in having frontal tubercles widely separated by frons, and the anterior margin of clypeus emarginate. Also, from Sinoluperus Gressitt \& Kimoto, this new genus is separable in having the middle and the posterior tibiae with a spine at the apex.

\section{KeY tO SPECIES OF Sinoluperoides}

1 Elytron without distinct subbasal transverse furrow

Elytron with distinct subbasal transverse furrow ; pronotum with a transverse furrow deeper and the furrow distinctly punctate especially on lateral portion ; coloration variable, in most pale colored specimen generally yellowish brown, in dark colored specimen elytron with lateral and apical margins, in some cases post-humeral area also, blackish, pronotum partly blackish, especially on lateral part in various degrees, head with middle and lateral parts of vertex blackish, antenna pitchy black, ventral surfaces with metathorax dark brown, legs in part dark brown ; length 3.8-4.2 $\mathrm{mm}$

2(1) Pronotum with transverse furrow deeper ...3

Pronotum with transverse furrow shallower and sparsely impressed by fine punctures ; generally yellowish to reddish brown, elytron with margins widely pitchy black ; meso-and metathorax, antenna and legs generally pitchy brown to black ; in dark colored specimen pronotum and abdomen pitchy black; length $4.2 \mathrm{~mm}$ marginalis

3(2) Body length shorter ; pronotum with transverse furrow which is sparsely impressed by fine punctures ; generally yellowish brown; in male elytron black with median mark- 
ing yellowish brown, metathorax and abdomen pitchy brown, and antenna thickly covered by long hairs from second to eleventh segments ; in female elytron yellowish brown with basal margin and basal part of lateral and sutural margins blackish, ventral surfaces with metathorax blackish ; length $3.8 \mathrm{~mm} \ldots \ldots \ldots \ldots \ldots \ldots \ldots \ldots$ antennatus Body length longer ; pronotum with a transverse furrow which is impressed by strong punctures rather closely ; generally yellowish brown ; in male antenna not covered by long hairs; length $6.2 \mathrm{~mm}$

major

\section{Sinoluperoides antenna tus n.sp. Fig. 59b}

Generally yellowish brown ; in male elytron black with median marking yellowish brown, and metathorax and abdomen pitchy brown; in female elytron yellowish brown with basal margin and basal part of lateral and sutural margins blackish ; antenna generally pitchy black.

Head with vertex smooth, shining, sparsely impressed by minute punctures, interocular space much wider than transverse diameter of single oculus, and interocular transverse impression distinct, frontal tubercle oblique, subquadrate, separated to each other, distinctly raised, surface smooth, shining. Antenna slender, nearly as long as body length ; first segment long, second shortest, nearly $1 / 5$ as long as first, third five times as long as second, fourth $11 / 2$ times as long as third, fifth slightly shorter than fourth, fifth to eighth subequal to each other in length and shape, ninth slightly shorter than eighth, tenth subequal to ninth in length and shape, eleventh subequal to eighth in length and its apex pointed. Pronotum transverse, $12 / 3$ times as broad as long, anterior margin slightly rounded anteriorly, sides strongly rounded, widest at $1 / 3$ from anterior margin, and slightly narrowed anteriorly and more strongly so posteriorly, basal margin distinctly rounded posteriorly, dorsal surface convex, smooth, shining, with a transverse subbasal depression, and sparsely impressed by distinct punctures. Scutellum subtriangular, smooth, shining, sparsely impressed by minute punctures. Elytron with lateral margin subparallel-sided, surface closely impressed by distinct punctures and their interstices smooth, shining.

Male : Antenna with second to eleventh segments thickly covered by long hairs ; fifth abdominal segment trilobed, median lobe longitudinally sulcate. Female : Antenna not covered by long hairs ; fifth abdominal segment entire.

Length: $3.8 \mathrm{~mm}$.

Holotype (BISHOP): LAOS: 800 m, Ban-van-eue, SE of Phou-kow-kuei, 11. iv. 1965, J. L. Gressitt. Paratopotype : 1 ex ., same data as the holotype (BISHOP). Paratypes : LAOS : Ban Van Eue, 1 ex., 12. iv. 1965, 1 ex., 13. iv. 1965, J. L. Gressitt, 1 ex., 15-31. v. 1965, 1 ex., 15. ii. 1966, Native collr ; 800 m, Phou-kow-kuei, 1 ex., 16. iv. 1965, J. L. Gressitt ; Sayaboury Prov., Sayaboury, 1 ex., 15. iv. 1965, J. L. Gressitt (BISHOP, KIMOTO).

This new species resembles Sinoluperoidesmarginalis Kimoto, but differs in havihg prontoum with a transverse subbasal furrow and in the male antenna with the second to the eleventh segments covered by long hairs.

Sinoluperoides macula tus n.sp. Fig. 59c

Coloration variable, in most pale colored specimen generally yellowish brown, in dark colored specimen elytron with lateral and apical margins, in some cases post humeral area also, blackish, pronotum partly blackish, especially on lateral part in various degrees, head with middle and lateral parts of vertex blackish, antenna pitchy black, ventral surfaces with metathorax dark brown, legs in part dark brown. 
Head with vertex somewhat wrinkled, sparsely impressed by fine punctures, interocular space much wider than transverse diameter of single oculus, and interocular transverse impression distinct, frontal tubercle oblique, separated to each other, distinctly raised, surface smooth, shining. Antenna slender, nearly as long as body length ; first segment long, second shortest, nearly $1 / 3$ as long as first, third $21 / 3$ times as long as second, fourth slightly longer than third, fifth slightly shorter than fourth, sixth to seventh subequal to each other in length and shape, eighth slightly shorter than seventh, eighth to tenth subequal to each other in length and shape, eleventh subequal to seventh in length and its apex pointed. Pronotum transverse, $11 / 2$ times as broad as long, anterior margin nearly straight, lateral margin slightly rounded, widest slightly before middle, slightly narrowed anteriorly and more strongly so posteriorly, basal margin distinctly rounded posteriorly, dorsal surface convex, smooth, shining, with a pair of transverse depressions subbasally, punctures generally finer and sparser, but more stronger and closer on subbasal depression. Scutellum subtriangular, smooth, shining, sparsely impressed by minute punctures. Elytron with lateral margin subparallel-sided, surface impressed by a transverse furrow subbasally, and closely impressed by distinct punctures and their interstices smooth, shining.

Length: $3.8-4.2 \mathrm{~mm}$.

Holotype (BISHOP) : VIETNAM : Dalat, 1,500 m, 29. iv.-4. v. 1960, S. Quate \& L. Quate. Paratopotypes: 5 exs., same-data as the holotype (BISHOP, KIMOTO). Paratypes : VIETNAM : Dalat, 6 km S., 1,400-1,500 m, 6 exs., 9. vi.-7. vii. 1961, N. R. Spencer; 18 km NW of Dalat, 1 ex., 1,300 m, 4-5. v. 1960, L. W. Quate ; Fyan, 900-1,200 m, 9 exs., 11. vii.-9. viii. 1961, N. R. Spencer ; Mt. Lang Bian, 1,500-2,000 m, 4 exs., 19. v.-8. vi. 1961, N. R. Spencer ; 25 km SW of Pleiku, 400 m, 1 ex., 12. v. 1960, L. W. Quate ; 20 km N. of Pleiku, 650 m, 1 ex., 9. v. 1960, S. Quate ; Ban Me Thuot, 500 m, 1 ex., 16-18. v. 1960, L. Quate ; 76 km SW of Ban Me Thuot, 1 ex., 855 m, 1 ex., 20. v. 1960, R. E. Leech ; Dak Song, $76 \mathrm{~km} \mathrm{SW}$ of Ban Me Thuot, 870 m, 1 ex., 19-21. v. 1960, light trap, S. Quate \& L. Quate (BISHOP).

This new species somewhat resembles Sinoluperoides major Kimoto, but differs in being the body length shorter, and having elytron with a subbasal transverse furrow.
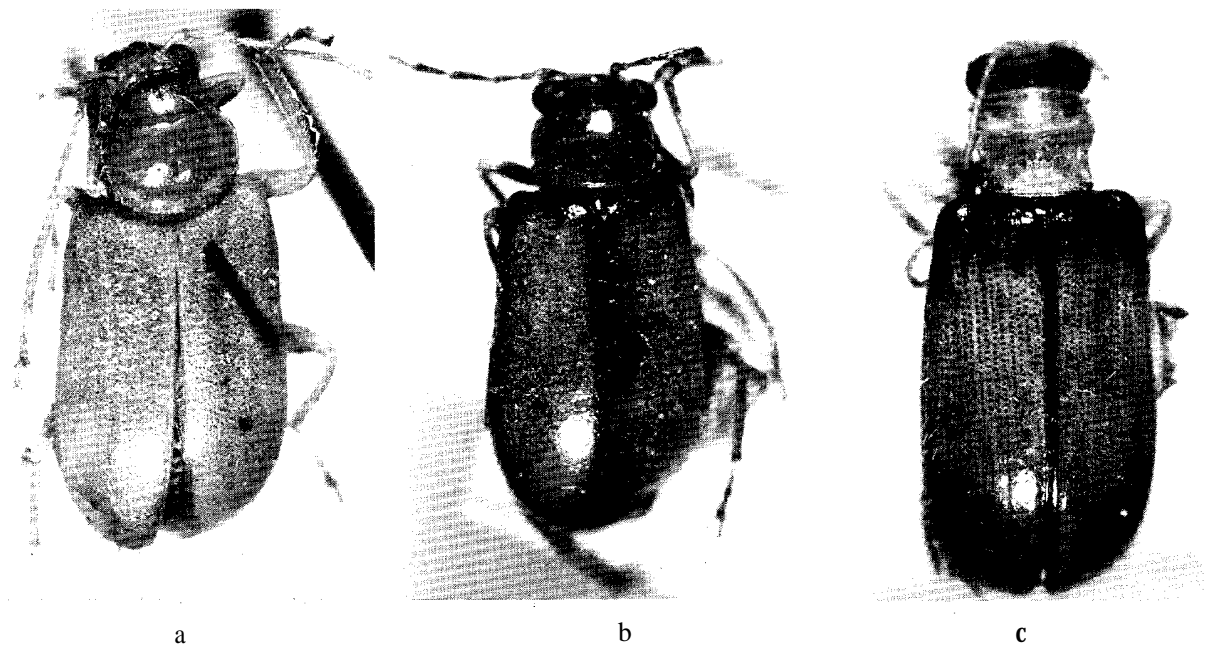

Fig. 60. a, Sinoluperoides major n. sp. ; b, S. marginalis n. sp. ; c, Doryscus testaceus Jacoby. 
Sinoluperoides major n. sp. Fig. 60a

Entirely yellowish brown.

Head with vertex somewhat wrinkled, sparsely impressed by minute punctures, interocular space much wider than transverse diameter of single oculus, and interocular transverse impression distinct, frontal tubercle oblique, subquadrate, separated to each other, distinctly raised, surface smooth, shining. Antenna slender, nearly as long as body length ; first segment long, second shortest, nearly $1 / 4$ as long as first, third four times as long as second, fourth slightly longer than third, fifth slightly shorter than fourth, sixth slightly shorter than fifth, seventh slightly shorter than sixth, seventh to ninth subequal to each other in length and shape, tenth slightly shorter than ninth, eleventh subequal to ninth in length and its apex pointed. Pronotum transverse, $11 / 2$ times as broad as long, anterior margin nearly straight, lateral margin strongly rounded, widest slightly before middle, strongly narrowed anteriorly and posteriorly, basal margin distinctly rounded posteriorly, dorsal surface convex, smooth, shining, with a transverse subbasal depression, punctures generally finer and sparser, but more stronger and closer on subbasal depression. Scutellum. subtriangular, smooth, shining, sparsely impressed by minute punctures. Elytron with lateral margin slightly rounded, surface closely impressed by distinct punctures and their interstices smooth, shining.

Length : $6.2 \mathrm{~mm}$.

Holotype (BISHOP) : VIETNAM : Dak Song, $76 \mathrm{~km}$ SE of Ban Me Thuot, 870 m, 19-21. v. 1960, L. W. Quate. Paratypes: VIETNAM : $10 \mathrm{~km} \mathrm{E.} \mathrm{of} \mathrm{Ban} \mathrm{Me} \mathrm{Thuot,} 570 \mathrm{~m}, 1$ ex., 10. v. 1960,. R. E. Leech ; 76 km SW of Ban Me Thuot, 855 m, 1 ex., 20. v. 1960, R. E. Leech (BISHOP).

This new species resembles Sinoluperoides antennatus Kimoto, but differs in being the body length longer, and having pronotum with a subbasal transverse furrow which is more closely and strongly punctate.

\section{Sinoluperoides marginalis $\mathrm{n}$. sp. Fig: $60 \mathrm{~b}$}

Generally yellowish to reddish brown, elytron yellowish brown, with basal, lateral, sutural and apical margins widely pitchy black, antenna pitchy black with one or two basal segments reddish brown; ventral surfaces with meso- and metathorax pitchy black; in dark colored specimen pronotum and abdomen generally pitchy black.

Head with vertex smooth, shining, sparsely impressed by minute punctures, interocular space much wider than transverse diameter of single oculus, and interocular transverse impression distinct, frontal tubercle oblique, subquadrate, separated to each other, distinctly raised, surface smooth, shining. Antenna slender, nearly as long as body length ; first segment long, second shortest, nearly $1 / 4$ as long as first, third three times as long as second, fourth slightly longer than third, fifth slightly shorter than fourth, fifth to tenth subequal to each other in length and shape, eleventh slightly longer than tenth and its apex pointed. Pronotum transverse, twice as broad as long, anterior margin nearly straight, lateral margin slightly rounded, widest slightly before middle, slightly narrowed anteriorly and more strongly so posteriorly, basal margin distinctly rounded posteriorly, dorsal surface convex, smooth, shining, with a pair of feeble depressions laterally, sparsely impressed by fine punctures. Scutellum subtriangular, smooth, shining, sparsely impressed by minute punctures. Elytron with lateral margin subparallel-sided, apex rounded, surface rather closely impressed by distinct punctures, and their interstices smooth, shining.

Length : $4.2 \mathrm{~mm}$.

Holotype (BISHOP) : VIETNAM : Fyan, 900-1,000 m, 11. vii.-9. viii. 1961, N. R. Spencer. Paratopotypes : 5 exs., same data as the holotype (BISHOP, KIMOTO). 
This new species resembles Sinoluperoides major Kimoto, but differs in having pronotum with a pair of feeble depressions laterally, and elytron with the margins entirely blackish.

\section{Genus Doryscus Jacoby}

Doryscus Jac., 1887, Proc. 2001. Soc. London, 1887 : 115 (type : Doryscus testaceus Jacoby; monobasic).-Maulik, 1936, Fauna India, Galeruc.:75.-Châjô, 1962, Phil. J. Sci., 91(1-2): 16.

\section{Doryscus testaceus Jacoby Fig. 60c}

Doryscus testaceus Jac., 1887, Proc. Zool. Soc. London, 1887 : 115 (Ceylon ;BM).—Jacoby, 1896, Ann. Mus. Civ. Genova, 36 : 498 (Sumatra).- Jacoby, 1896, Ann. Soc. Ent. Belg., $40: 300$ (India, Sumatra)-Maulik, 1936, Fauna India, Galeruc. : 77, fig. (Ceylon, India, Tonkin:Hoa-Binh). -Chajô, 1962, Phil. J. Sci., 91(1-2): 17, figs. (Taiwan).-Kimoto, 1969, Esakia, Kyushu Univ., (7) : 57 (Taiwan).-Kimoto \& Takizawa, 1972, Kontyâ, Tokyo, 40(4): 222 (Nepal).-Kimoto, 1979, Ent. Basiliensia, 4 : 474 (Nepal, Darjeeling).

Trichobalya varians Gressitt \& Kimoto, 1963, Pac. Ins. Mon., 1B:674 (SE China ; BISHOP) ; 1965, Pac. Ins., $7: 802$ (= testaceus).

Distribution : India, Ceylon, Thailand, Vietnam, China, Taiwan, Philippines, Sumatra.

Elongate, pronotum distinctly constricted behind middle and with some distinct erect hairs marginally ; elytron impressed by regularly arranged longitudinal rows of punctures, and covered with fine erect hairs; general color yellowish to reddish brown, in some specimen partly blackish in various degrees ; length $3.2-4.8 \mathrm{~mm}$.

Material examined. THAILAND : Chiengmai Prov., Doi Suthep, 1,300 m, 1 ex., 8. vi. 1965, P. D. Ashlock (BISHOP) ; Chiengmai Prov., Doi Pui, 1 ex., 8. vi. 1965. 1 ex., 17. vi. 1965, Y. Miyatake; Chiengmai Prov., Doi Suthep, 1,000 m, 2 ex., 18. vi. 1965, K. Morimoto \& Y. Miyatake (KU) ; Khon Kaen, 3 exs., 7-8. x. 1981, S. Azuma (RU). VIETNAM : Dalat, 1,500 m, 1 ex., 29. iv.-5. v. 1960, L. Quate ; Dalate, 6 km S., 1,400-1,500 m, 5 exs., 9. vi.-7. vii. 1961, N. R. Spencer ; $18 \mathrm{~km} \mathrm{NW}$ of Dalate, 1,300 m, 1 ex., 4-5. v. 1960, L. W. Quate ; Mt. Lang Bian, 1,500-2,000 m, 3 exs., 19. v.-8. vi. 1961, N. R. Spencer (BISHOP).

\section{Genus Strobiderus Jacoby}

Strobiderus Jac., 1884, Notes Leyden Mus., 6 : 61 (type :Strobiderus excavatus Jacoby ; Sumatra). -Maulik, 1936, Fauna India, Galeruc. : 283.-Gressitt \& Kimoto, 1963, Pac. Ins. Mon., 1B : 397.

\section{Key to sPecies of Strobiderus}

1 Elytron covered by depressed and erect hairs; yellowish to reddish brown with pygidium pitchy black, in some specimen head dark brown to pitchy black ; length 4.0-4.8

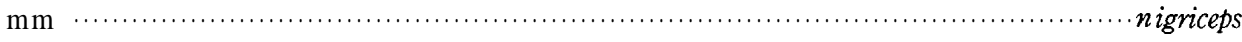

Elytron covered by erect hairs only, entirely yellowish brown ; length $4.1-5.8 \mathrm{~mm} \cdots \cdots \cdots$ fulvus

\section{Strobiderus fulvus Kimoto Fig. 61a}

Strobiderus fulvus Kimoto, 1977, Ent. Basiliensia, 2 : 379 (Bhutan ; BASEL).

Distribution : Bhutan, Laos. 
Material examined. LAOS : Vientiane Prov., Ban Van Eue, 20 km E. of Phou-kow-kuei, 2 exs., 1-15. v. 1965, J. A. Rondon ; Borikhane Prov., Pakkading, 1 ex., 23. iv. 1965, J. L. Gressitt ; Sayaboury Prov., Sayaboury, 2 exs., 15. iv. 1965, J. L. Gressitt (BISHOP).

\section{Strobiderus nigriceps Laboissière}

Strobidems nigriceps Lab., 1936, Ann. Soc. Ent. France, 105 : 258 (Tonkin; HAMBURG).

Strobidems tonkinensis Lab., 1936, Ann. Soc. Ent. France, 105 : 259 (Tonkin; HAMBURG). New synonym.

Distribution : Thailand, Laos, Vietnam.

According to the study on the types, Strobidems tonkinensis is only a infraspecific variation in having the head yellowish to reddish brown.

Material examined. THAILAND : Trang Prov., Waterfall to Kaochang, 150-300 m, 2 exs., 3. i. 1964, 1 ex., 9-11. i. 1964, G. A. Samuelson ; 50 km West of Tak, 900 m, 1 ex., 7-8. iv. 1966, J. Sedlacek (BISHOP) ; Chiengmai Prov., Doi Suthep, 1,000 m, 1 ex., 18. vi. 1965, Y. Miyatake (KU) ; Khon Kaen, 1 ex., 4. viii. 1980, 1 ex., 8. x. 1981, S. Azuma (RU). LAOS : Vientiane Prov., Vientiane, 2 exs., 31. v.3. vi. 1960, S. Quate \& L. Quate ; Vientiane Prov., Ban Van Eue, 1 ex., 15. ii. 1966, 1 ex., 15. ii. 1967, 1 ex., 30. ix. 1967, 1 ex., 15. x. 1967, Native collr; Wapikhamthong Prov., Wapi, 1 ex., 15. x. 1967, Native collr (BISHOP). VIETNAM : M'Drak, E. of Banme Thuot, 4-600 m, 1 ex., 8-19. xii. 1960, C. M. Yoshimoto; Ap Hung-Lam, $21 \mathrm{~km} \mathrm{NW}$ of Dilinh, 1,100 m, 3 exs., 29. ix.-5. x. 1960, C. M. Yoshimoto ; Dalat, 6 km S., 1,400-1,500 m, 2 exs., 9. vi.-7. vii. 1961, N. R. Spencer (BISHOP).

\section{Genus Trichobalya Weise}

Trichidea Baly, 1890, Ent. Monthly Mag., 26 : 18 (nec Haan, 1838) (type: Ttichidea bowringii Baly ; Hongkong ; monobasic).

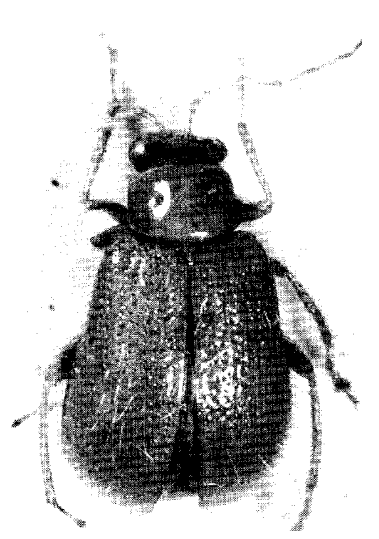

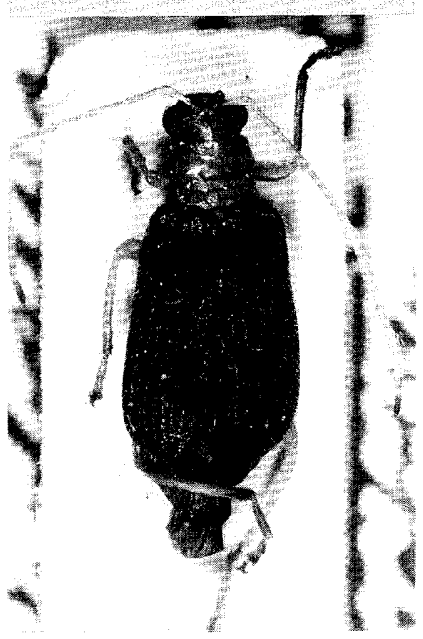

b

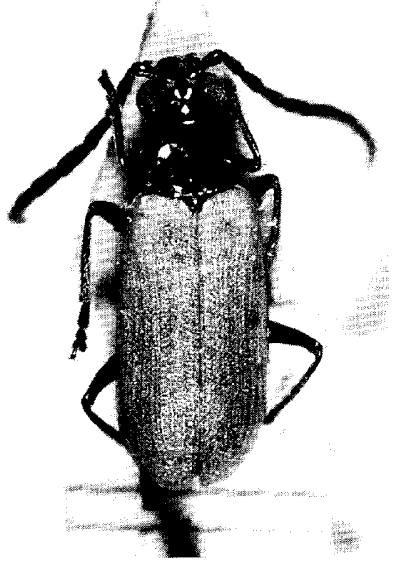

Fig. 61. a, Strobiderus fulvus Kimoto ; b, Trichobalya bowringii (Baly) ; c, Theopea bicolor $\mathrm{n}$. $\mathrm{sp}$. 
Ttichobalya Weise, 1924, Col. Cat,, 78:152 (new name for Trichidea Baly, 1890).-Gressitt \& Kimoto, 1963, Pac. Ins. Mon., 1B: 673.

\section{KeY TO SPECIES OF Trichobalya}

1 Head pitchy black; pronotum nearly $11 / 2$ times as wide as long ; generally yellowish to reddish brown; elytron bluish to violaceous black; length $6.5-7.3 \mathbf{m ~ m}$............. melanocephala Head reddish brown; pronotum nearly $11 / 4$ times as wide as long; generally yellowish to reddish brown; elytron violaeous to bluish black; length $5.0-8.0 \mathrm{~mm}$ bowringii

\section{Trichobalya bowringii (Baly) Fig. $61 \mathrm{~b}$}

Trichidea bowringii Baly, 1890, Ent. Monthly Mag., 26: 13 (Hong Kong; BM).

Trichobalya bowringii : Weise, 1924, Col. Cat. Junk, 78 : 152 (Hong Kong).-Gressitt \& Kimoto, 1963, Pac. Ins. Mon., 1B : 673 (China, Vietnam).

Ttichobalya tonkinensis Laboissière, 1936, Ann. Soc. Ent. France, 105 :260, fig. 58 (Tonkin: Choganh, Hoa-binh, Yen-bei ; Yunnan ; HAMBURG).-Gressitt \& Kimoto, 1963, Pac. Ins. Mon., $1 \mathrm{~B}: 673$ (= bowringii).

Distribution : Thailand, Laos, Vietnam, China.

Material examined. THAILAND : Khon Kaen, 1 ex., 20. xii. 1979, S. Azuma (RU). LAOS : Vientiane Prov., Ban Van Eue, 1 ex., 12. iv. 1965, J. L. Gressitt ; Vientiane Prov., Phoukou Khouei, 2 exs., 31. v. 1966, Native collr ; Sedone Prov., 5 km E. of Pakse, 1 ex., 13. v. 1965, P. D. Ashlock (BISHOP). VIETNAM : $20 \mathrm{~km} \mathrm{~N}$. of Pleiku, 550-650 m, 1 ex., 9. v. 1960, 1 ex., 13. v. 1960, L. W. Quate (BISHOP).

\section{Trichobalya melanocephala (Jacoby), new combination}

Cynorta melanocephala Jac., 1889, Ann. Mus. Civ. Genova, 27 : 220 (Burma ;GENOVA).-Maulik, 1936, Fauna India, Galeruc. : 514 (Burma).

Trichidea mouhoti Baly, 1890, Ent. Monthly Mag., ser. 2, 1: 14 (Siam, Laos ; BM). New synonym.

Trichobalya gularis Laboissière, 1936, Ann. Soc. Ent. France, 105 : 261 (Tonkin; HAMBURG).

-Gressitt \& Kimoto, 1963, Pac. Ins. Mon., 1B: 674 (Vietnam). New synonym.

Distribution : Burma, Thailand, Laos, Vietnam.

Material examined. THAILAND : Packhong, $100 \mathrm{~m}$, NE of Bangkok, 1 ex., 3. xii. 1957, J. L. Gressitt; 50 km West of Tak, 900 m, 2 exs., 7-8. iv. 1966, J. Sedlacek ; Kao Yai, 1 ex., 30. viii. 1963, Native collr (BISHOP) ; Fang, 1 ex., 13. vi. 1965, K. Morimoto ; Khao Y ai Nat. Park, 1 ex., 6. vi. 1965, Y. Miyatake (KU) ; Phet buri, 5 exs., 13. i. 1963 (BANGKHEN). LAOS : Vientiane Prov., Ban Van Eue, 1 ex., 30. xi. 1965, Native collr ; Sayaboury Prov., Sayaboury, 1 ex., 15. iv. 1965, J. L. Gressitt, 1 ex., 6. xii. 1965, 1 ex., 14. xii. 1965, Native collr (BISHOP).

\section{Genus Theopea Baly}

Theopea Baly, 1864, Trans. Ent. Soc. London, ser. 3, $2: 237$ (type : Crioceris impressa Fabricius ; Sumatra).-Chapuis, 1875, Gen. Col., $11: 242$.-Maulik, 1936, Fauna India, Galeruc. : 285. -Gressitt \& Kimoto, 1963, Pac. Ins. Mon., 1B: 675.

Ozomena Harold, 1876, Col. Hefte, 15: 132. 
Key to species of Theopea

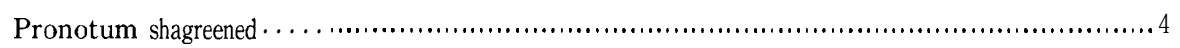

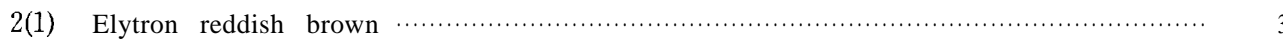

Elytron violaceous to bluish black; head, thorax, abdomen and legs blackish; antenna blackish with ninth and tenth segments yellowish white ; length $6 \mathrm{~mm} \cdots \cdot$ elegantula

3(2) Pronotum black; generally black; elytron reddish brown ; antenna and legs pitchy

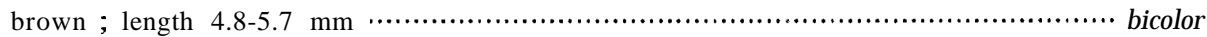

Pronotum reddish brown; ventral surfaces pitchy black; head and elytron reddish brown; antenna and legs pitchy brown; length $7.2 \mathrm{~mm} \ldots \ldots \ldots \ldots \ldots \ldots \ldots \ldots \ldots \ldots \ldots \ldots \ldots$ mouhoti

4(1) Elytral costa distinct at apex ; violaceous to greenish blue, antenna pitchy black, legs yellowish brown; length $5.8-6.2 \mathrm{~mm}$.................................................. sauteri Elytral costa indistinct at apex; bluish black, antenna pitchy black, legs almost entirely yellowish brown ; length 5.3-5.7 $\mathrm{mm}$

similis

\section{Theopea bicolor n. sp. Fig. 61c}

Generally black ; elytron reddish brown ; antenna and legs generally pitchy brown.

Head with vertex somewhat wrinkled, sparsely impressed by minute punctures, interocular space distinctly wider than transverse diameter of single oculus, and interocular transverse impression distinct, frontal tubercle subtriangular, contiguous, distinctly raised, surface smooth, shining. Antenna slender, slightly longer than body length ; first segment long, second shortest, nearly $1 / 4$ as long as first, third $31 / 3$ times as long as second, fourth slightly longer than third, fifth subequal to fourth in length and shape, sixth slightly shorter than fifth, sixth to tenth subequal to each other in length and shape, eleventh slightly longer than tenth and its apex pointed. Pronotum transverse, $11 / 4$ times as broad as long, anterior margin nearly straight, lateral margin slightly rounded, widest slightly before middle, slightly narrowed anteriorly and posteriorly, basal margin distinctly rounded posteriorly, dorsal surface convex, smooth, shining, with a pair of lateral depressions, sparsely impressed by minute punctures, Scutellum subtriangular, smooth, shining, sparsely impressed by minute punctures. Elytron with lateral margin subparallel-sided, surface closely impressed by strong punctures arranged in semiregular rows, and their interstices smooth, shining, longitudinally costate.

Length : 4.8-5.7 mm.

Holotype (BISHOP) : VIETNAM : $20 \mathrm{~km} \mathrm{N.} \mathrm{of} \mathrm{Pleiku,} 650 \mathrm{~m}$, 9. v. 1960, L. W. Quate. Paratopotypes: 2 exs., same data as the holotype (BISHOP, KIMOTO). Paratypes: LAOS: Umgeb. Vientiane, 5 exs., iii-vi. 1963 ; Umgeb. Vanky, 8 exs., 1963 ; Umgeb. Paklay, 5 exs., 1963 (MUN CHEN).

This new species resembles Theopea mouhoti Baly, but differs in having pronotum smooth and blackish.

\section{Theopea elegantula Baly}

Theopea elegantula Baly, 1864, Trans. Ent. Soc. London, ser. 3, 2 : 238 (Sarawak ; BM).

Theopea nigricollis Jacoby, 1892, Entomolog., 25, Suppl. : 87 (Borneo, Perak, Burma ; BM).

-Maulik, 1936, Fauna India, Galeruc.: 286 (Burma, Perak). New synonym.

Distribution : Burma, Laos, Borneo.

Material examined. LAOS : Vientiane Prov., Vientiane, 3 exs., 31. v.-3. vi. 1960, light trap, S. 
Quate \& L. Quate (BISHOP).

\section{Theopea mouhoti Baly Fig. 62b}

Theopea mouhoti Baly, 1864, Trans. Ent. Soc. London, ser. 3, 2 : 238 (Siam ; BM)

Distribution : Laos, Vietnam.

Material examined. LAOS : Vientiane Prov., Ban Van Eue, 1 ex., 15. v. 1966, Native collr (BISHOP) ; Umgeb. Vientiane, 2 exs., iii-vi. 1963 (MUNCHEN).

\section{Theopea sauteri Chajô}

Theopea sauteri Châjô, 1935, Arb. Morph. Tax. Ent. Berlin-Dahlem, 2 : 169 (with var. minuta Chûjô ; Formosa) ; 1962, Phil. J. Sci., 91(1-2): 158 (Formosa).

Theopea coerulea Gressitt \& Kimoto, 1963, Pac. Ins. Mon., 1B : 679 (China, Tonkin :Hoa-binh ; BISHOP). New synonym.

Distribution : Laos, Vietnam, China, Taiwan.

Material examined. LAOS : Vientiane Prov., Ban Van Eue, 1 ex., 12. iv. 1965, 3 exs., 13. iv. 1965, 1 ex., 15. iv. 1965, J. L. Gressitt, 2 exs., 1-15. v. 1965, J. A. Rondon, 2 exs., 15-31. v. 1965, 1 ex., 15. v. 1966, 1 ex., 31. v. 1966, Native collr ; Vientiane Prov., Phou-kow-kuei, 1 ex., 16. iv. 1 1965, J. L. Gressitt ; Sayaboury Prov., Sayaboury, 3 exs., 15-16. iv. 1965, J. L. Gressitt ; Sayaboury Prov., Sayaboury, 3 exs., 15-16 iv. 1965, J. L. Gressitt (BISHOP) ; Umgeb Vientiane, 3 exs., iii-vi. 1963 ; Umgeb. Vanky, 3 exs., 1963 ; Umgeb. Paklay, 3 exs. (MUNCHEN). VIETNAM : Dalat, 6 km S., 1,4001,500 m, 21 exs., 9. vi.-7. vii. 1961, N. R. Spencer; $18 \mathrm{~km} \mathrm{NW}$ of Dalat, 1,300 m, 1 ex., 4-5. v. 1960, L. W. Quate ; Fyan, 900-1,000 m, 5 exs., 11. vii.-9. viii. 1961, N. R. Spencer ; Ban Me Thuot, 500 m, 1 ex., 16-18. v. 1960, S. Quate ; 10 km E. of Ban Me Thuot, 570 m, 1 ex., 18. v. 1960, R. E. Leech ; 7 km SE of Dilinh (Djiring), 990 m, 1 ex., 2. v. 1960, R. E. Leech (BISHOP).

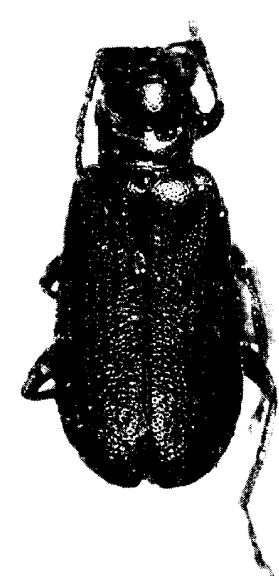

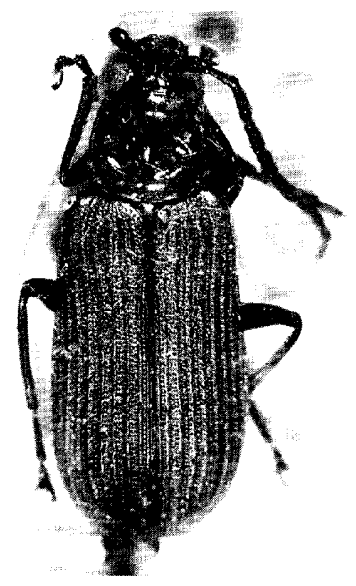

$\mathrm{b}$

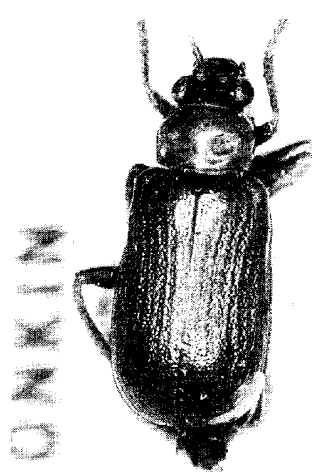

$\mathrm{c}$

Fig. 62. a, Theopea similis n. sp.; b, T. mouhoti (Baly) ; c, Platyxantha variceps (Laboissière). 
Theopea similis n. sp. Fig. 62a

Generally bluish black, antenna pitchy black, legs almost entirely yellowish brown.

Head with vertex finely granulate, sparsely impressed by distinct punctures, interocular space distinctly wider than transverse diameter of single oculus, and interocular transverse impression distinct, frontal tubercle subtriangular, contiguous, distinctly raised, surface smooth, shining. Antenna slender, slightly longer than body length ; first segment long, second shortest, nearly $1 / 5$ as long as first, third $52 / 3$ times as long as second, fourth $11 / 3$ times as long as third, fourth to ninth subequal to each other in length and shape, tenth slightly shorter than ninth, eleventh slightly longer than tenth and its apex pointed. Pronotum transverse, slightly wider than long, anterior margin slightly rounded anteriorly, lateral margin slightly rounded, widest slightly before middle, slightly narrowed anteriorly and more strongly so posteriorly, basal margin distinctly rounded posteriorly, dorsal surface convex, finely granulate, with a pair of deep lateral depressions, rather closely impressed by large punctures. Scutellum subtriangular, smooth, shining, sparsely covered by fine setae. Elytron with lateral margin subparallel-sided, surface closely impressed by strong punctures which are partly arranged in semiregular geminate rows, and their interstices finely granulate and longitudinally costate especially on lateral area.

Length : 5.3-5.7 mm.

Holotype (BISHOP) : LAOS : Vientiane Prov., Vientiane, 31. v.-3. vi. 1960, light trap, S. Quate \& L. Quate. Paratopotype: 1 ex., same data as the holotype (BISHOP). Paratypes: THAILAND: Trang Prov, Khaophappha, Kaochong, 200-400 m, 1 ex., 11. i. 1964, G.A. Samuelson (BISHOP). LAOS : Borikhane Prov., Pakkading, 2 exs., 10. v. 1965, Native collr (BISHOP, KIMOTO).

This new species very closely resembles Theopea sauteri Chajô, but differs in having elytron with the longitudinal costae weaker.

\section{Genus Paraplotes Laboissière}

Paraplotes Lab., 1933, Ann. Soc. Ent. France, 102 : 51 (type : Paraplotes mgosa Laboissière; Tonkin).-Gressitt \& Kimoto, 1963, Pac. Ins. Mon., 1B:681.

\section{KeY TO SPECIES OF Paraplotes}

1 Abdomen brownish ; generally yellowish brown; elytron blackish ; length $5.0 \mathrm{~mm} \cdots \cdots \cdot \cdots \cdot \cdot \mathrm{rugosa}$ Abdomen, metathorax, vertex and scutellum blackish; elytron violaceous brown with

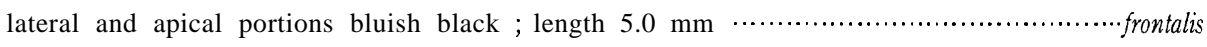

\section{Paraplotes frontalis Laboissière}

Paraplotes frontalis Lab., 1933, Ann. Soc. Ent. France, 102 : 53 (Tonkin; HAMBURG).

Distribution : Vietnam.

No additional material was examined beside the type series.

\section{Paraplotes rugosa Laboissière}

Paraplotes mgosa Lab., 1933, Ann. Soc. Ent. France, 102 : 52 (Tonkin).-Gressitt \& Kimoto, 1963, Pac. Ins. Mon., 1B : 684 (Vietnam).

Distribution : Vietnam. 
I could not trace the type location of this species.

\section{Genus Platyxan t ha Baly}

Platyxantha Baly, 1864, Trans. Ent. Soc. London, ser. 3, 2 : 233 (type :Platyxantha apicalis Baly ; Sumatra).-Chapuis, 1875, Gen. Col., 11 :244.-Gressitt \& Kimoto, 1963, Pac. Ins. Mon., 1B: 686.

Paraenidea Laboissière, 1933, Ann. Soc. Ent. France, 102 : 66 (type : Paraenidea azurea Labossière; Yunnan). New synonym.

Platyxanthoides Laboissière, 1933, ibid. : 71 (type :Platyxanthoides variceps Laboissière ; Tonkin). New synonym.

\section{KEY TO SPECIES OF Platyxantha}

1 Ventral surfaces in part blackish

Ventral surfaces entirely yellowish brown ; generally yellowish brown, head largely blackish ; length $3.8 \mathrm{~mm}$

2(1) In male, third antenna1 segment broadly triangular or globose . . . . . . . . ..............................3 In male, third antennal segment much longer than broad, fairly slender; generally reddish ochraceous ; vertex, metathorax and abdomen largely bluish black ; length $5.5-8.5 \mathrm{~mm}$

variceps

3(2) In male, third antenna1 segment broadly triangular or globose and second very small, eighth arched, fairly slender; generally reddish ochraceous, metathorax and abdomen largely bluish black; elytron purplish red to green or blue ; in some specimen elytron reddish ochraeous with basal margin bluish; length $6.0-9.0 \mathrm{~mm} \ldots \ldots \ldots \ldots \ldots \ldots \ldots \ldots \ldots$ indica In male, third antennal segment broadly triangular, oblique apically, hairy; eighth and ninth arched; tenth and eleventh greatly flattened and broadened; generally yellowish brown; elytron purplish to greenish blue; vertex, metathorax and abdomen bluish black ; length $6.8 \mathrm{~mm}$

........occipitalis

\section{Platyxantha indica (Jacoby) Fig. 3a}

Dorydea indica Jac., 1889, Ann. Mus. Civ. Genova, $27: 231$ (Burma ; GENOVA).

Platyxantha indica : Maulik, 1936, Fauna India, Galeruc. : 589 (Burma).

Paraenidea azurea Laboissière, 1933, Ann. Soc. Ent. France, 102 : 67 (Hoa-Binh, Luang-Prabang ; HAMBURG).-Gressitt \& Kimoto, 1963, Pac. Ins. Mon., 1B: 686 (Tonkin :Hoa-binh). New synonym.

Paraenidea azurea var. hoabinhia Laboissiere, 1933, Ann. Soc. Ent. France, 102 : 69 (Hoa-Binh, environs de Tuyen-Quan ; Tien-Yen ; Siam ; Lakhon ; PARIS).

Distribution : Thailand, Laos, Vietnam.

Material examined. THAILAND : Chiengmai Prov., Mae Sa, 1 ex., 16. vi. 1965, P. D. Ashlock; $50 \mathrm{~km}$ West of Tak, 900 m, 2 exs., 7-8. iv. 1966, M. Sedlacek ; Khao Yai, 2 exs., 10. iv. 1963, Native collr ; Bang Khen, 1 ex., 26. ix. 1963, Native collr (BISHOP) ; Fang, 1 ex., 14. vi. 1965, K. Morimoto ; Khao Yai Nat. Park, 1 ex., 6. vi. 1965, K. Morimoto (KU). LAOS : Vientiane Prov., Vientiane, 1 ex., 21. iv. 1965, J. A. Rondon; Vientiane Prov., Ban Van Eue, 2 exs., 13-14. iv. 1965, J. L. Gressitt, 2 exs., 1-15. v. 1965, J. A. Rondon, 5 exs., 15-31. vi. 1965, Native collr ; Sayaboury Prov., Sayaboury, 4 exs., 15. iv. 1965, J. L. Gressitt, 1 ex., 30. v. 1965, Native collr ; Muong Sing, NW of Luang Prabang, 650 m, 
4 exs., 6-10. vi. 1960, L. W. Quate ; Khammouane Prov., Phon Tiou, 1 ex., 10. vi. 1965, N. Wilson, 1 ex., 28. iv. 1965, 1 ex., 30. iv. 1965, 8 exs., 17. v. 1965, 10 exs., 28. v. 1965, 5 exs., 28. vi. 1965,1 ex., 6. vii. 1965, Native collr ; Borikhane Prov., Pakkading, 9 exs., 23. iv. 1965, J. L. Gressitt, 1 ex., 15. vi. 1966, J. A. Rondon, 1 ex., 22. iii. 1965, 1 ex., 10. v. 1965, 3 exs., 15. vi. 1965, Native collr ; Borikhane Prov., Namkading, nr. Pakkading, 100 m, 5 exs., 21. iv. 1965, 4 exs., 22. iv. 1965, J. L. Gressitt ; Borikhane Prov., Uekinak, nr. Pakkading, 100 m, 1 ex., 22. iv. 1965, J. L. Gressitt (BISHOP) ; Umgeb. Vientiane, 15 exs., iii-vi. 1963 ; Umgeb. Vanky, 19 exs., 1963 ; Umgeb. Paklay, 5 exs., 1963 (MUNCHEN). VIETNAM : Fyan, 1,200 m, 11. vii.-9. viii. 1961, N. R. Spencer (BISHOP).

\section{Platyxantha occipitalis (Laboissière), new combination}

Paraenidea occipitalis Lab., 1933, Ann. Soc. Ent. France, 102 : 70 (Tonkin:Hoa-Binh;BRUX-

ELLES).-Gressitt \& Kimoto, 1963, Pac. Ins. Mon., 1B: 686 (Tonkin:Hoa-binh).

Distribution : Vietnam.

No additional material was examined beside the type series.

\section{Platyxantha tonkinensis Laboissière}

Platyxantha tonkinensis Lab., 1936, Ann. Soc. Ent. France, 105 : 239 (Tonkin; HAMBURG).

Distribution : Vietnam.

No additional material was examined beside the type series.

\section{Pla tyxan tha variceps (Laboissière), new combination Fig. 62c}

Platyxanthoides varipes Lab., 1933, Ann. Soc. Ent. France, 102 : 71 (Tonkin:Hoa-binh ; BRUXELLES).

Paraenidea variceps : Gressitt \& Kimoto, 1963, Pac. Ins. Mon., 1B: 686 (Tonkin:Hoa-binh). Distribution : Vietnam.

No additional material was examined beside the type series.

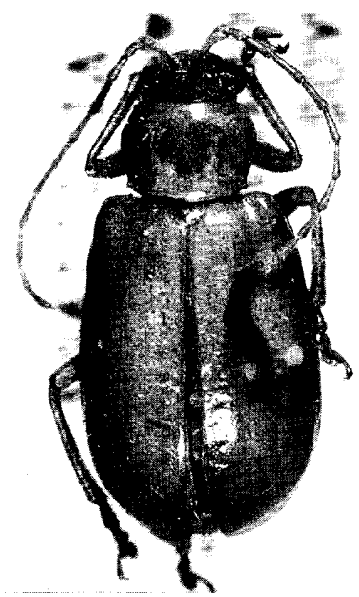

a

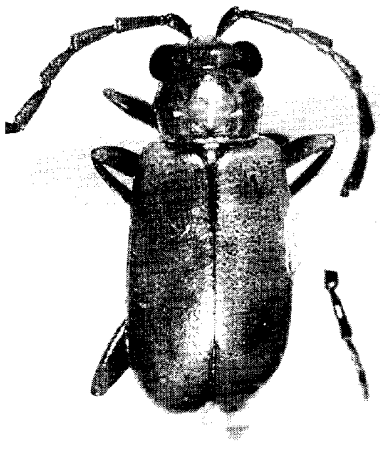

b

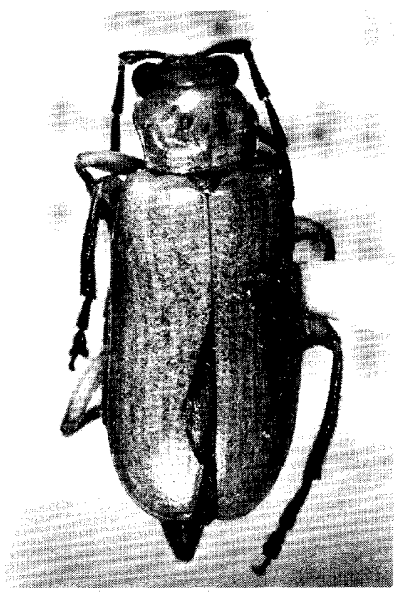

C

Fig. 63. a, Laosixantha fulva n. sp. ; b, Hyphaenia frontalis n. sp. ; c, H.nigricornis n. sp. 


\section{Genus Palpoxena Baly}

Palpoxena Baly, 1861, J. Ent., 1 : 203 (type : Palpoxena laeta Baly ; Borneo)-Chapuis, 1875, Gen. Col., 11 : 244, 246.-Maulik, 1936, Fauna India, Galeruc.: 568.-Gressitt \& Kimoto, 1963, Pac. Ins. Mon., 1B: 687.

Aenidea Baly, 1874, Trans. Ent. Soc. London, 1874 : 179 (by substitution of Aenidea for Palpoxena Baly, 1861).-Jacoby, 1885, Proc. 2001. Soc. London, 1885 : 749 (type : Palpoxena Zaeta Baly).

Neochrolea Jacoby, 1887, Proc. Zool.Soc. London, $1887: 117$, pl. 11, fig. 4 [type : Neoleptacavifrons Jacoby $=$ Palpoxena fascialis (Baly); monobasic ; Ceylon $]$.

\section{Key to SPECIES OF Palpoxena}

Generally yellowish brown

Generally bluish black ; antenna and legs largely yellowish brown ; length 12.0-13.0

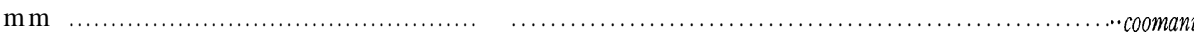

2(1) Elytron violaceous blue, with apical angle brownish ; in some specimen dorsal surfaces entirely violaceous ; generally yellowish brown ; in male head widely depressed on frons, and palpus widened and deformed ; length $9.5 \mathrm{~mm}$ (Baly, 1861 ; Borneo) ….......... laeta Elytron bluish black; generally yellowish brown; in male head widely excavated on frons and palpus widened but not deformed ; length $8.0-9.5 \mathrm{~mm} \cdots \ldots \ldots \ldots \ldots \ldots$. coeruleipennis

\section{Palpoxena coomani (Laboissière)}

Aenidea coomani Lab., 1933, Ann. Soc. Ent. France, 102 : 59 (Tonkin; BRUXELLES).

Palpoxena coomani : Wilcox, 1973, Col. Cat. Junk, Suppl., 78 (3) : 648 (Vietnam).

Distribution : Vietnam.

No additional material was examined beside the type series.

\section{Palpoxena coeruleipennis (Baly) Fig. 3c}

Aenidea coeruleipennis Baly, 1888, J. Linn. Soc.Zool., 20 : 159 (Siam ; BM).

A enidea crassipalpis Jacoby, 1892, Ann. Mus. Civ. Genova, 32 : 986 (Burma : Tenasserim ; GENOVA). New synonym.

A enidea ulyssis Laboissière, 1933, Ann. Soc. Ent. France, 102 : 58 (Tonkin ; HAMBURG). New synonym.

Palpoxena crassipalpis: Maulik, 1936, Fauna India, Galeruc. : 586 (Burma).

Palpoxena coemuleipennis :Chûjô, 1959, Sci. Rep. Agr. Exped. Cambodia, Hyogo Univ. Agr., (2) : 60 (Cambodia) ; 1964, Nature and Life in SE Asia, Kyoto, $3: 297$ (Thailand).

Palpoxena Zaeta : Gressitt \& Kimoto, 1963, Pac. Ins. Mon., 1B: 687 (Tonkin :Hoa-binh)

Distribution : Thailand, Cambodia, Laos, Vietnam.

Material examined. THAILAND : Chiengmai Prov., Chiengdao, 450 m, 3 exs., 5-11. iv. 1958, T. C. Maa (BISHOP). CAMBODIA : $20 \mathrm{~km}$ E. of Siem Reap, 2 exs., 11. iii. 1968, D. E. Hardy (BISHOP). LAOS: Vientiane Prov., Ban Van Eue, 1 ex., 15. iii. 1966, Native collr; Khammouane Prov., Phon Tiou, 3 exs., 8. iii. 1966, Native collr ; Sayaboury Prov., Sayaboury, 1 ex., 1-10. iv. 1965, J. A. Rondon, 2 exs., 2. iii. 1966, Native collr ; Pakse, 1 ex., iii. 1965, J. A. Rondon (BISHOP). VIETNAM : $20 \mathrm{~km}$ N. of Pleiku, 650 m, 1 ex., 9. v. 1960, L. W. Quate ; 25 km SW of Pleiku, 400 m, 2 exs., 12. v. 1960, L. W. Quate; Karyu Danar, 200 m, 1 ex., 13-28. ii. 1961, N. R. Spencer (BISHOP). 


\section{Genus Laosixantha n. gen.}

Antenna1 insertions widely separated by frons ; labrum rounded, truncate apically ; gena nearly half as deep as oculus; antenna with third segment distinctly longer than second ; prothorax convex side to side, without any trace of depression dorsally, and with anterior, lateral and basal margins entirely marginate ; elytron with punctures irregularly impressed, without distinct subbasal furrow, epipleuron wide at base and gradually narrowed to apex ; prosternal process not elevated between anterior coxae; anterior coxal cavity closed posteriorly ; middle and posterior tibiae with many short spines apically; tarsal claws appendiculate and first tarsal segment of posterior leg distinctly shorter than length of second and third combined.

Type species :Laosixantha fulva Kimoto.

This new genus resembles Palpoxena Baly, but differs in having the first segment of the posterior tarsus distinctly shorter than the following segments combined, pronotum convex side to side, without any trace of depression dorsally and elytron without any trace of transverse depression behind the subbasal area.

\section{Laosixantha fulva n. sp. Fig. 63 a}

Entirely ochraceous.

Head with vertex smooth, shining, sparsely impressed by minute punctures, interocular space much wider than transverse diameter of single oculus, and interocular transverse impression distinctly raised, surface smooth, shining. Antenna slender, nearly $3 / 4$ as long as body length ; first segment long, robust, somewhat club-shaped, second nearly half as long as first, third almost twice as long as second, fourth slightly longer than third, fourth to tenth subequal to each other in length and shape, eleventh slightly longer than tenth and its apex pointed. Pronotum transverse, $12 / 5$ times as broad as long, anterior margin slightly rounded posteriorly, lateral margin distinctly rounded, widest almost at $1 / 3$ from anterior margin, distinctly narrowed anteriorly and posteriorly, basal margin slightly rounded posteriorly, dorsal surface convex side to side, smooth, shining, very sparsely impressed by distinct punctures. Scutellum subtriangular, smooth, shining. Elytron with lateral margin rounded, surface rather closely impressed by distinct punctures.

Length : 7.5-9.5 mm.

Holotype (BISHOP) : LAOS : Borikhane Prov., Pakkading, 15-31. viii. 1965, Native collr. Paratopotypes: 9 exs., same data as the holotype (BISHOP). Paratypes : LAOS : Borikhane Prov., Pakkading, 5 exs., 13. vii. 1965, malaise trap, J. L. Gressitt \& K. A. J. Wise, 13 exs., 15. ix. 1965, light trap, J. L. Gressitt, 2 exs., 3. viii. 1965, 2 exs., 31. viii. 1965, 3 exs., 9. ix. 1965, Native collr ; Sayaboury Prov., Sayaboury, 1 ex., 15. iv. 1965, J. L. Gressitt ; Vientiane Prov., Vientiane, 9 exs., 15. v. 1967, Native collr ; Vientiane Prov., Gi Sion Vill., de Tha Ngone, 1 ex., 10-24. x. 1965, Native collr ; Khammouane Prov., Phon Tiou, 1 ex., 17. v. 1965, Native collr ; Wapikhamthong Prov., Khong Sedone, 1 ex., 30. v. 1965, Native collr (BISHOP, KIMOTO) ; Umgeb. Vientiane, 21 exs., iii-vi. 1963 (MUNCHEN).

This new species somewhat resembles Calomicrus persimilis Kimoto, but differs in having the anterior coxal cavity closed posteriorly.

\section{Genus Hyphaenia Baly}

Hyphaenia Baly, 1865, Ann. Mag. Nat. Hist., ser. 3, 16: 410 (type: Luperus pilicornis Motschulsky; Burma).-Chapuis, 1875, Gen. Col., 11 :242.-Maulik, 1936, Fauna India, Galeruc. : 486. 


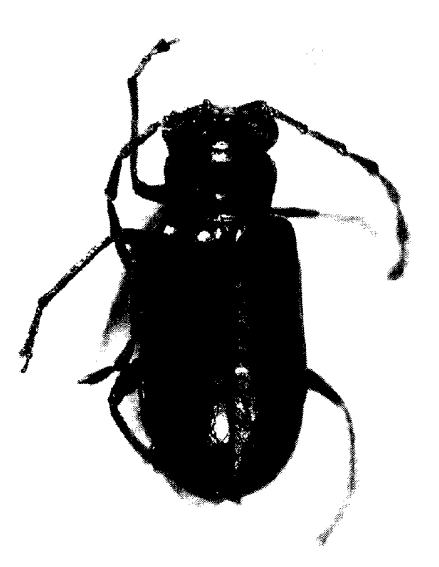

a

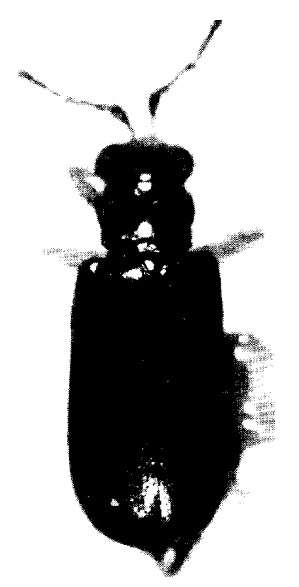

$\mathrm{b}$

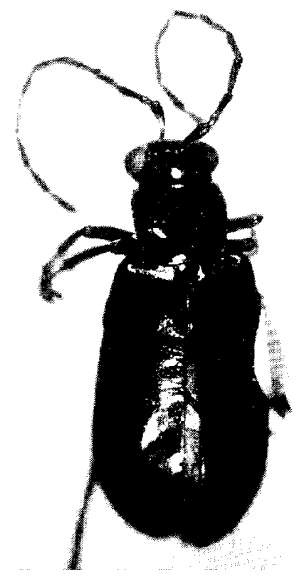

C

Fig. 64. a, Hyphaenia antennalis n. sp. ; b, H. minor n. sp. ; c, H. elongata n. sp.

-Gressitt \& Kimoto, 1963, Pac. Ins. Mon., 1B: 688.

Trichocerastes Motschulsky, 1866, Bull. Soc. Nat. Moscou, 39, 1 (2) : 413 (type :Luperus pilicornis Motschulsky ; Burma).

\section{K EY TO SPECIES of Hyphaenia}

1 Dorsal surfaces generally or at least partly yellowish to dark reddish brown $\cdots \cdots \cdots \cdots \cdots \cdots \cdots \cdot \ldots+\cdots$ Dorsal surfaces entirely greenish to bluish black

2(1) Pronotum transverse, nearly $11 / 2$ times as wide as long Pronotum subquadrate, slightly longer than wide ; elytron yellowish brown with basal, lateral and apical margins bluish black in various degrees, in some specimen elytron entirely yellowish brown ; generally yellowish brown, antenna pitchy brown to black ;

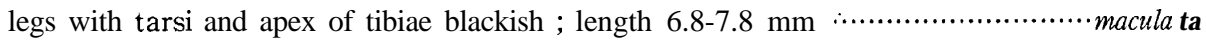

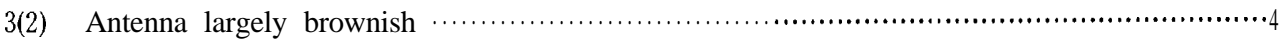
Antenna largely blackish ; generally yellowish brown, antenna, tibiae and tarsi pitchy black ; in some specimen head pitchy black with vertex brownish, and also in some specimen elytron largely pitchy brown ; in male head with a large excavation on middle of frons ; length $6.8-7.5 \mathrm{~mm}$ nigincorints

4(3) In male head without any distinct cavity on frons and antennal segments slightly depressed ; generally yellowish brown ; in some specimen pronotum with two pair of ill-defined markings dark brown, and in dark colored specimen tibiae and tarsi mostly pitchy black ; length $5.0-7.0 \mathrm{~mm}$............ *..*. fulva In male head with a transverse cavity on frons and with a distinct projection on posterior margin of clypeus medianly ; generally yellowish brown, antenna dark brown, legs in part infuscate ; in some specimen metathorax and abdomen pitchy black ; length 4.5-5.7 $\mathrm{mm}$

5(1) Abdomen yellowish brown ; in male third to eleventh antenna1 segments thickly covered by long hairs $*$.. 
Abdomen entirely bluish

6(5) Pronotum nearly $11 / 2$ times as wide as long ; interocular space distinctly wider than

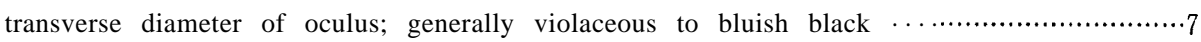
Pronotum slightly wider than long; interocular space distinctly narrower than transverse diameter of single oculus ; generally bluish black; length 4.2-4.8 mm . . *abdominalis

7(6) Dorsal surfaces entirely violaceous ; length $6.0 \mathrm{~mm}$ (Laboissière, 1936 ; Yunnan) ….....aenea Dorsal surfaces entirely bluish ; length $5.0-6.0 \mathrm{~mm} \ldots \ldots \ldots \ldots \ldots \ldots \ldots \ldots \ldots \ldots \ldots \ldots \ldots \ldots \ldots \ldots$.cyanscens

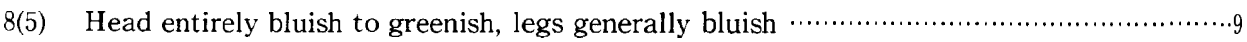
Head greenish blue with anterior half yellowish, legs generally brownish ; generally greenish to bluish black, antenna pitchy black, ventral surfaces of pro- and meso-

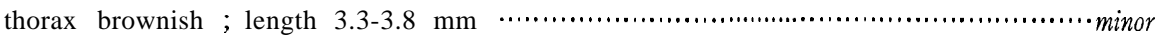

9(8) Pronotum $11 / 2$ times as wide as long; in male antenna with third to fifth segments thickly covered by long hairs and seventh segment distinctly curved ; length 3.6-3.9

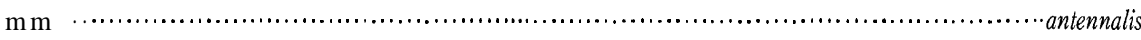

Pronotum $11 / 4$ times as wide as long; antenna slender, not modified in male ; generally bluish black, antenna pitchy black; length $3.9-4.5 \mathrm{~mm}$

."longata

\section{Hyphaenia abdominalis n.sp. Fig. 65b}

Generally bluish black, abdomen yellowish brown,

Head with vertex smooth, shining, sparsely impressed by minute punctures, interocular space distinctly narrower than transverse diameter of single oculus, and interocular transverse impression obsolete, frontal tubercle subquadrate, contiguous, slightly raised, surface smooth, shining. Antenna slender, nearly as long as body length ; first segment long, second shortest, nearly $1 / 4$ as long as first, third four times as long as second, third to eighth subequal to each other in length, ninth slightly shorter than eighth, tenth slightly shorter than ninth, eleventh subequal to ninth in length and its apex pointed. Pronotum transverse, slightly wider than long, anterior margin distinctly rounded anteriorly, lateral margin slightly rounded, widest slightly before middle, slightly narrowed anteriorly and posteriorly, basal margin slightly rounded posteriorly, dorsal surface convex, smooth, shining, with a deep transverse subbasal depression, punctures finely and sparsely punctate. Scutellum subtriangular, smooth, shining, sparsely impressed by minue punctures. Elytron with lateral margin subparallelsided, apex rounded, surface distinctly but not closely punctate and their interstices finely granulate.

Length : 4.2-4.8 $\mathrm{mm}$.

Holotype (BISHOP) : THAILAND : Trang Prov., Khaophappha, Khaochang, 200-400 m, 11. i. 1964, G. A. Samuelson. Paratopotype : 1 ex., same data as the holotype (KIMOTO).

This new species resembles Hyphaenia cyanescens Laboissière, but differs in having pronotum slightly wider than long, and the interocular space distinctly narrower than the transverse diameter of single oculus.

\section{Hyphaenia antennalis n. sp. Fig. 64a}

Entirely bluish black.

Head with vertex smooth, shining, sparsely impressed by minute punctures, interocular space as long as transverse diameter of single oculus, and interocular transverse impression distinct, frontal tubercle transverse, subquadrate, contiguous, distinctly raised, surface smooth, shining. Antenna slender in basal segments and robuster in preapical segments, nearly as long as body length ; first long, second shortest, nearly $1 / 4$ as long as first, third four times as long as second, fourth $11 / 4$ times 
as long as third, fifth $3 / 4$ as long as fourth, and fifth to eleventh segments much robuster than basal segments, sixth slightly shorter than fifth, seventh slightly shorter than sixth, eighth subequal to seventh in length and slightly curved, ninth slightly shorter than eighth, tenth subequal to ninth in length and shape, eleventh subequal to eighth in length and its apex pointed. Pronotum transverse, $11 / 2$ times as broad as long, anterior margin nearly straight, lateral margin slightly rounded, widest slightly before middle, slightly narrowed anteriorly and more strongly so posteriorly, basal margin distinctly rounded posteriorly, dorsal surface convex, smooth, shining, with a pair of deep transverse subbasal depressions which are united at middle, punctures finely and sparsely impressed. Scutellum subtriangular, smooth, shining, sparsely impressed by minute punctures. Elytron with lateral margin subparallel-sided, rounded at apex, surface closely impressed by distinct punctures and their interstices smooth, shining.

Male : Antenna with third to fifth segments thickly covered by long hairs. Female : Unknown. Length : $3.6-3.9 \mathrm{~mm}$.

Holotype (RU) : THAILAND : Khon Kaen, 24. vii. 1968, S. Azuma. Paratopotypes : '2 exs., same data as the holotype (RU, KIMOTO). Paratypes: THAILAND : Chiengmai Prov., Doi Suthep, 1 ex., 1-15. iv. 1958, T. C. Maa (BISHOP). LAOS : Vientiane Prov., Ban Van Eue, 1 ex., 15-31. v. 1965, 1 ex., 15. v. 1966, Native collr (BISHOP).

This new species resembles Hyphaenia cyanescens Laboissière, but differs in having the ventral surfaces entirely bluish black.

\section{H yphaenia cyanescens Laboissière}

Hyphaenia cyanescens L a b ., 1936, Ann. Soc. Ent. France, $105: 242$ (Tonkin; HAMBURG). -Gressitt \& Kimoto, 1963, Pac. Ins. Mon., 1B: 688 (Tonkin;Hoa-binh).

Distribution : Vietnam.

No additional material was examined.

\section{Hyphaenia elongata n. sp. Fig. $64 \mathrm{c}$}

Generally bluish black, antenna pitchy black.

Head with vertex finely granulate, impunctate, interocular space distinctly wider than transverse diameter of single oculus, and interocular transverse impression distinct, frontal tubercle subtriangular, contiguous, distinctly raised, surface finely granulate. Antenna slender, nearly as long as body length ; first segment long, second shortest, nearly $1 / 4$ as long as first, third $21 / 2$ times as long as second, fourth $11 / 3$ times as long as third, fourth to ninth subequal to each other in length and shape, tenth slightly shorter than ninth, eleventh subequal to ninth in length and its apex pointed. Pronotum transverse, $11 / 4$ times as broad as long, anterior margin slightly rounded anteriorly, lateral margin slightly rounded, widest at $1 / 3$ from anterior margin and slightly narrowed anteriorly and more strongly so posteriorly, basal margin distinctly rounded posteriorly, dorsal surface with a pair of deep transverse depressions which are united at middle, and punctures distinctly but not closely impressed, and interstices of punctures finely granulate. Elytron with lateral margin subparallel-sided and apex rounded, surface closely impressed by distinct punctures and their interstices finely granulate.

Length : 3.9-4.5 mm.

Holotype (BISHOP) : VIETNAM : Mt. Lang Bian, 1,500-2,000 m, 19. v.-8. vi. 1961, N. R. Spencer. Paratopotypes : 4 exs., same data as the holotype (BISHOP, KIMOTO). Paratypes : Fyan, 900-1,200 m, 17 exs., 11. vii.-9. viii. 1961, N. R. Spencer; Dalat, 6 km S., 1,400-1,500 m, 1 ex., 9. vi.-7. vii. 1961, N. R. Spencer ; 20 km SW, Dilinh (Djiring), 900 m, 1 ex., 22-28. iv. 1960, S. Quate (BISHOP). 
This new species resembles Hyphaenia antennalis Kimoto, but differs in having pronotum more elongate and antenna much slender.

\section{Hyphaenia frontalis n. sp. Fig. $63 \mathrm{~b}$}

Generally yellowish brown, antenna dark brown, legs with tibiae and tarsi pitchy brown, in some specimen metathorax and abdomen pitchy black.

Head with vertex finely granulate, sparsely impressed by distinct punctures, interocular space much wider than transverse diameter of single oculus, frontal tubercle transverse, subquadrate, contiguous, slightly raised, hardly delimited posteriorly by interocular transverse impression, surface finely granulate. Antenna slender, longer than body length ; first segment long, second shortest, nearly $1 / 5$ as long as first, third $52 / 3$ times as long as second, fourth slightly shorter than third, fourth to tenth subequal to each other in length and shape, eleventh $11 / 3$ times as long as tenth and its apex pointed. Pronotum transverse, $12 / 5$ times as broad as long, anterior margin nearly straight, lateral margin strongly rounded, widest slightly before middle, slightly narrowed anteriorly and more strongly so posteriorly, basal margin slightly rounded posteriorly and nearly straight at middle, dorsal surface convex, with a pair of deep lateral depressions, punctures generally finer and sparser, but more stronger and closer on lateral depression. Scutellum subtriangular, finely granulate, sparsely impressed by minute punctures. Elytron with lateral margin subparallel-sided, with apex rounded, surface closely impressed by distinct punctures and their interstices finely granulate.

Male : Head with a transverse cavity on frons and with a distinct projection on posterior margin of clypeus medianly; fifth abdominal segment trilobed. Female : Head without any distinct cavity ; fifth abdominal segment entire.

Length : 4.5-5.7 mm.

Holotype (BISHOP) : LAOS : Sedone Prov., Paksong, 18. v. 1965, P. D. Ashlock. Paratopotypes : LAOS : Sedone Prov., Paksong, 2 exs., 18. v. 1965, P. D. Ashlock (BISHOP, KIMOTO).

This new species resembles Hyphaenia fulva Kimoto, but differs in having the head with a

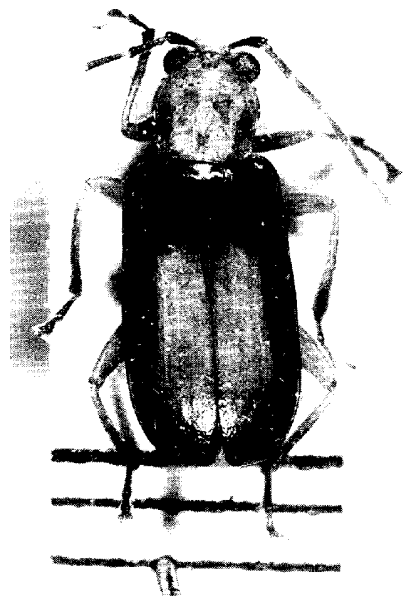

a

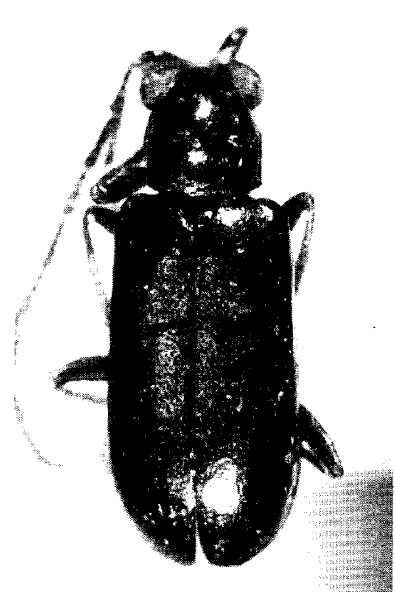

$\mathrm{b}$

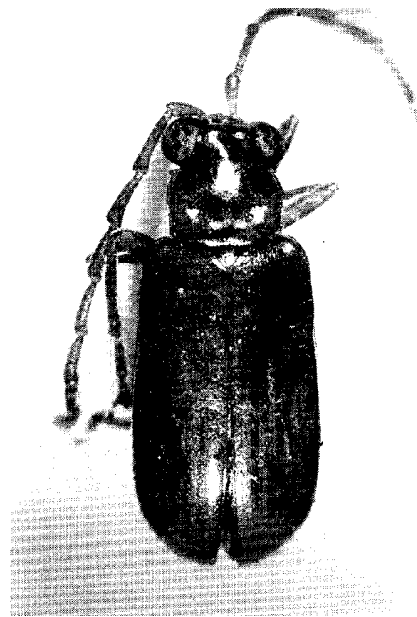

C

Fig. 65. a, Hyphaeniamaculata n. sp. ; b, H. abdominalis n. sp. ; c, H. fulva n. sp. 
transverse cavity on frons and with a distinct projection on the posterior margin of clypeus medianly and antenna much darker.

\section{Hyphaenia fulva n. sp. Fig. 65c}

Generally yellowish brown; in some specimen pronotum with a pair of ill-defined markings dark brown, and in dark colored specimen ventral surfaces with meso- and metathorax and abdomen pitchy black, and legs with tibiae and tarsi mostly pitchy brown.

Head with vertex finely granulate, sparsely impressed by distinct punctures, interocular space much wider than transverse diameter of single oculus, frontal tubercle transverse, subquadrate, contiguous, slightly raised, hardly delimited posteriorly by interocular transverse impression, surface finely granulate. Antenna slender, longer than body length ; first segment long, second shortest, nearly $1 / 5$ as long as first, third seven times as long as second, fourth slightly shorter than third, fourth to tenth subequal to each other in length and shape, eleventh $11 / 3$ times as long as tenth and its apex pointed. Pronotum transverse, $12 / 5$ times as broad as long, anterior margin nearly straight, lateral margin strongly rounded, widest slightly before middle, slightly narrowed anteriorly and more strongly so posteriorly, basal margin slightly rounded posteriorly and nearly straight at middle, dorsal surface convex, with a pair of deep lateral depressions, punctures generally finer and sparser, but stronger and closer on lateral depression. Scutellum subtriangular, finely granulate, sparsely impressed by minute punctures. Elytron with lateral margins subparallel-sided, with apex rounded, surface closely impressed by distinct punctures and their interstices finely granulate.

Male : Antenna with eighth and ninth segments slightly curved; fifth abdominal segment trilobed. Female : Fifth abdominal segment entire.

Length : $5.0-7.0 \mathrm{~mm}$.

Holotype (BISHOP) : VIETNAM : Dalat, 1,500 m, 28. iv.-4. v. 1960, L. W. Quate. Paratopotypes : 4 exs., same data as the holotype (BISHOP, KIMOTO). Paratypes : VIETNAM : $18 \mathrm{~km} \mathrm{NW}$ of Dalat, 1,300 m, 6 exs., 4-5. v. 1960 L. W. Quate (BISHOP).

This new species somewhat resembles Hyphaenianigricornis Kimoto, but differs in having antenna entirely brownish and in the male the head without any large excavation at the middle.

\section{Hyphaenia maculata n. sp. Fig. 65a}

Generally yellowish brown ; elytron yellowish brown with basal, lateral and apical margins bluish black in various degrees, in most pale colored specimen entirely yellowish brown; antenna pitchy brown to black; legs with tarsi and apex of tibiae blackish.

Head with vertex somewhat wrinkled, sparsely impressed by distinct punctures, interocular space much wider than transverse diameter of single oculus, frontal tubercle subtriangular, contiguous, slightly raised, surface finely wrinkled, delimited posteriorly by interocular transverse impression. Antenna slender, nearly as long as body length ; first segment long, second shortest, nearly $1 / 5$ as long as first, third $41 / 4$ times as long as second, fourth $11 / 3$ times as long as third, fifth subequal to fourth in length and shape, sixth slightly shorter than fifth, sixth to tenth subequal to each other in length and shape, eleventh subequal to tenth and its apex pointed. Pronotum subquadrate, slightly longer than wide; anterior margin slightly rounded posteriorly, lateral margin slightly rounded, widest almost at $1 / 3$ from anterior margin, slightly narrowed anteriorly and more strongly so posteriorly, basal margin slightly rounded posteriorly and nearly straight at middle, dorsal surface convex, with a pair of shallow depressions laterally, and punctures distinctly and rather closely impressed and their interstices smooth, shining. Scutellum subtriangular, finely granulate, nearly impunctate. Elytron 
with lateral margins subparallel-sided, with apex rounded, surface closely impressed by distinct punctures and their interstices finely granulate, and punctures arranged in semiregular rows at lateral area and their interstices slightly raised as several longitudinal costae.

Length : 6.8-7.8 $\mathrm{mm}$.

Holotype (MUNCHEN) : LAOS : Umgeb. Paklay, 1963. Paratypes : LAOS : Umgeb. Vientiane, 2 exs., iii-vi. 1963 ; Umgeb. Vanky, 3 exs., 1963 ; Umgeb. Paklay, 2 exs., 1963 (MUNCHEN, KIMOTO) ; Ile de Khong, 1 ex., 17. v. 1965, Native collr (BISHOP).

This new species resembles Hyphaenianigricomis Kimoto, but differs in having pronotum slightly longer than the wide and in the male the head without any distinct excavation.

\section{Hyphaenia minor n. sp. Fig. $64 \mathrm{~b}$}

Generally greenish to bluish black, head yellowish to reddish brown with vertex greenish blue, antenna generally pitchy black ; ventral surfaces with pro- and mesothorax brownish ; legs generally pitchy brown.

Head with vertex somewhat wrinkled, sparsely impressed by distinct punctures, interocular space much wider than transverse diameter of single oculus, frontal tubercle transverse, subquadrate, contiguous, distinctly raised, delimited posteriorly by deep interocular transverse impression, surface smooth, shining. Antenna slender, nearly as long as body length ; first segment long, second shortest, nearly $1 / 4$ as long as first, third three times as long as second, fourth $12 / 3$ times as long as third, fifth slightly shorter than fourth, fifth to seventh subequal to each other in length and shape, eighth slightly shorter than seventh, eighth to tenth subequal to each other in length and shape, eleventh subequal to seventh in length but its apex pointed. Pronotum transverse, $11 / 2$ times as broad as long, anterior margin nearly straight, lateral margin feebly rounded, widest at slightly behind anterior margin, distinctly narrowed posteriorly, basal margin slightly rounded posteriorly, dorsal surface convex, with a pair of deep lateral depressions, punctures finely and sparsely impressed and their interstices finely granulate. Scutellum subtriangular, finely granulate, nearly impunctate. Elytron with lateral margin subparallel-sided, with apex rounded, surface not closely impressed by distinct punctures and their interstices finely granulate.

Length : 3.3-3.8 mm.

Holotype (BISHOP) : VIETNAM : Dalat, 1,500 m, 1 ex., 29. iv.-4. v. 1960, L. W. Quate. Paratypes : VIETNAM : Fyan, 900-1,200 m, 6 exs., 11. vii.-9. viii. 1961, N. R. Spencer; Dalat, 6 km S., 1,400-1,500 m, 1 ex., 9. vi.-7. vii. 1961, N. R. Spencer ; $15 \mathrm{~km} \mathrm{NW}$ of Dalat, 1,850 m, 1 ex., 5. v. 1960, L. W. Quate (BISHOP, KIMOTO).

This new species resembles Hyphaenia cyanescens Laboissière, but differs in being the body length shorter and having the head greenish blue with the anterior half yellowish brown.

\section{Hyphaenia nigricornis $\quad$ n. sp. Fig. 63c}

Generally yellowish brown, antenna entirely pitchy black; legs yellowish brown with tibiae and tarsi pitchy black; in dark colored specimen elytron largely pitchy brown, and in some specimen head pitchy black with vertex yellowish brown.

Head with vertex somewhat wrinkled, sparsely impressed by distinct punctures, interocular space much wider than transverse diameter of single oculus, frontal tubercle transverse, subquadrate, contiguous, slightly raised, delimited posteriorly by a shallow transverse impression, surface finely granulate. Antenna slender, longer than body length ; first segment long, second shortest, nearly $1 / 5$ as long as first, third five times as long as second, fourth $11 / 4$ times as long as third, fifth slightly 


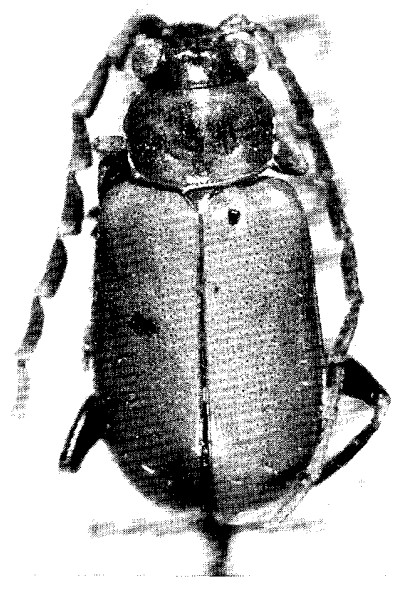

a

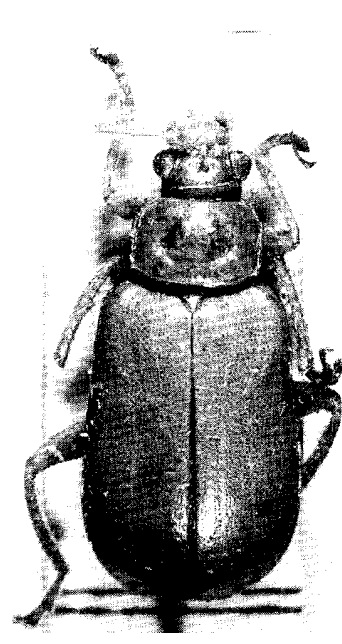

b

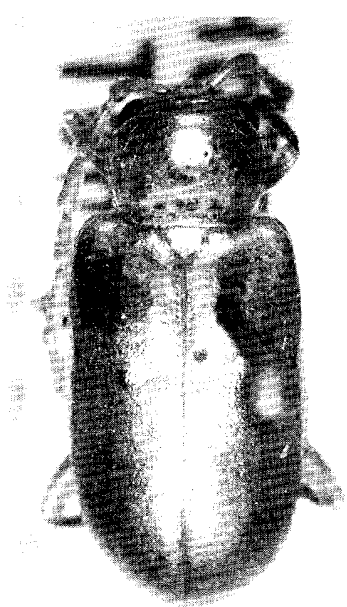

Fig. 66. a, Acroxena femoralis n. sp. ; b, A. fulva n. sp. ; c, A. nasuta Baly.

shorter than fourth, fifth to eighth subequal to each other in length and shape, ninth slightly shorter than eighth, tenth subequal to ninth in length and shape, eleventh subequal to tenth in length but its apex pointed. Pronotum transverse, $12 / 5$ times as broad as long, anterior margin nearly straight, lateral margin distinctly rounded, widest at $1 / 3$ from anterior margin, slightly narrowed anteriorly and more strongly so posteriorly, basal margin slightly rounded posteriorly and nearly straight at middle, dorsal surface convex, with a pair of deep lateral depressions which are united at middle, punctures sparsely and finely impressed, and interstices of punctures finely granulate. Scutellum subtriangular, finely granulate, sparsely impressed by minute punctures. Elytron with lateral margin subparallel-sided, with apex rounded, surface closely impressed by distinct punctures which are arranged in semiregular geminate rows and their intersitces finely granulate and slightly raised as several longitudinal costae.

Male : Head with a large excavation on middle of frons ; fifth abdominal segment with a pair of short longitudinal notches apically. Female : Head without large excavation ; fifth abdominal segment entire.

Length : 6.8-7.5 mm.

Holotype (BISHOP) : LAOS : 100 m, Namkading, nr. Pakkading, 21. iv. 1965, J. L. Gressitt. Paratypes: THAILAND: Chiengmai Prov., Fang, 1 ex., 14. vi. 1965, K. Morimoto (KU). LAOS: Savanakhet Prov., Savanakhet, 13 exs., 15. iv. 1967, Native collr ; Borikhane Prov, Pakkading, 1 ex., 22. iv. 1965, '2 exs., 23. iv. 1965, J. L. Gressitt, 1 ex., 15. iv. 1965, 1 ex., 17. v. 1965, Native collr ; 100 m, Namkading, nr. Pakkading, 1 ex., 21. iv. 1965, 1 ex., 22. iv. 1965, J. L. Gressitt ; Vientiane Prov., Vientiane, 3 exs., 15. v. 1967, Native collr ; Vientiane Prov., Ban Van Eue, 1 ex., 1-15. v. 1965, J. A. Rondon, 1 ex., 15-31. v. 1965, Native collr ; Muong Sing, NW of Luang Prabang, 550 m, 1 ex., 6-10. vi. 1960, at light, L. Quate ; Wapikhamthong Prov., Khong Sedone, 1 ex., 30. v. 1965, Native collr ; Ile de Khong, 2 exs., 3. v. 1965, 7. v. 1965, Native collr (BISHOP) ; Umgeb. Paklay, 5 exs., 1963 ; Umgeb. Vanky, 1 ex., 1963 ; Umgeb. Vientiane, 6 exs., iii-vi. 1963 (MUNCHEN). VIETNAM : 60 km SW, Blao (Balao), 2 exs., 22. iv. 1960, L. W. Quate ; Kontum, N. of Pleiku, 550 m, 2 exs., 13. v. 1960, L. W. Quate (BISHOP). 
This new species somewhat resembles Hyphaenia frontalis Kimoto, but differs in having antenna generally blackish, and elytron with punctures arranged in some semiregular geminate rows and their interstices slightly costate.

\section{Genus Cassenoides n. gen.}

Antennal insertions widely separated by frons ; labrum slightly bilobed apically ; gena $1 / 5$ as deep as oculus ; antenna with third segment distinctly longer than second; prothorax convex side to side without any distinct depression dorsally, and with lateral and basal margins distinctly marginate; prostemal process distinctly elevated between anterior coxae; anterior coxal cavity closed posteriorly ; middle and posterior tibiae with many short spines apically; tarsal claws appendiculate and first tarsal segment of posterior leg subequal to length of second and third segments combined.

Type species: Cassenoides flavomarginatus Kimoto.

This new genus somewhat resembles Cassena Weise, but differs in having pronotum with the anterior margin immarginate and without a pair of short longitudinal furrows basally.

\section{Cassenoides flavomarginatus n. sp. Fig. $67 \mathrm{a}$}

Generally ochraceous, elytron greenish blue with lateral and apical margins entirely ochraceous.

Head with vertex smooth, shining, sparsely impressed by minute punctures, interocular space much wider than transverse diameter of single oculus, and interocular transverse impression distinct, frontal tubercle transverse, subtriangular, contiguous, distinctly raised, surface smooth, impunctate. Antenna slender, nearly $2 / 3$ as long as body length ; first segment long, robust, somewhat club-shaped, second shortest, nearly $2 / 5$ as long as first, third twice as long as second, third to ninth subequal to each other in length and shape, tenth slightly shorter than ninth, eleventh $11 / 4$ times as long as tenth and its apex pointed. Pronotum transverse, nearly twice as broad as long, anterior margin slightly rounded posteriorly, lateral margin distinctly rounded, widest almost at middle, distinctly narrowed anteriorly and posteriorly, basal margin distinctly rounded posteriorly, dorsal surface convex side to side, smooth, shining, sparsely impressed by minute punctures. Scutellum subtriangular, smooth, shining, impunctate. Elytron with lateral margin rounded, surface strongly and rather closely punctate, and their interstices smooth, shining and narrower than average diameter of punctures.

Length : 5.7-6.3 $\mathrm{mm}$.

Holotype (BISHOP) : THAILAND : Chantaburi, 29. vi. 1963, Native collr. Paratypes : THAILAND : Chanthaburi, Prew, 45 m, 1 ex., 24. iv.-1.v. 1956, T. C. Maa; Saraburi, 1 ex., iii. 1963, Native collr ; Phou Kae, 1 ex., 17. v. 1963, Native collr (BISHOP) ; Nakhon Ratchasima, 1 ex., 19. v. 1961; Chanthaburi, 2 exs., 20-31. iv. 1959 (BANGKHEN ; KIMOTO).

This new species somewhat resembles Cassena collaris (Baly), but differs in having pronotum without short longitudinal furrow basally, and elytron with the lateral and the apical margins ochraceous.

\section{Genus Acroxena Baly}

Acroxena Baly, 1879, Cist. Ent., $2: 462$ (type : Acroxena nasuta Baly ; Assam).-Maulik, 1936, Fauna India, Galeruc. :564.-Gressitt \& Kimoto, 1963, Pac. Ins. Mon., 1B: 687. 
1 At least ventral surfaces or legs in part infuscate

Entirely yellowish brown; in male head with frons transversely excavated and with a tuft of hairs on middle of anterior and posterior margins of excavation, and antenna with ventral surfaces of third to fifth segments infuscate ; length $7.8-8.3 \mathrm{~mm}$.............. fulva

2(1) Legs pitchy black with basal $2 / 3$ of femora brownish; generally yellowish to reddish brown, metathorax and abdomen largely pitchy black; in male head with large cavity on middle of frons, and antenna with ventral surfaces of third to eighth segments infuscate ; length $6.5-7.7 \mathrm{~mm}$ femoralis Legs blackish brown with femora entirely brownish; generally yellowish brown; metathorax and abdomen somewhat infuscate; in male head with frons slightly excavated at middle and clypeus enlarged, and antenna with ventral surfaces of third to seventh infuscate ; length $9.3-11.0 \mathrm{~mm}$

nasuta

\section{Acroxena femoralis n. sp. Fig. 66a}

Generally yellowish to reddish brown, ventral surfaces with metathorax and abdomen largely pitchy black ; legs generally pitchy black with basal $2 / 3$ of femora and base of tibiae yellowish brown ; in male antenna with exterior margins of third to eighth segments infuscate.

Head with vertex finely granulate, nearly impunctate except several large punctures along interocular transverse impression which is feebly impressed, interocular space much wider than transverse diameter of single oculus, frontal tubercle transverse, subquadrate, contiguous, distinctly raised, surface finely granulate. Antenna slender, nearly as long as body length ; first segment robust, especially in male, second shortest, nearly $1 / 6$ as long as first, third eight times as long as second in male and six times as long as second in female, fourth slightly longer than third, fourth to tenth subequal to each other in length and shape, eleventh $1 \frac{1 / 4}{4}$ times as long as tenth and its apex pointed.

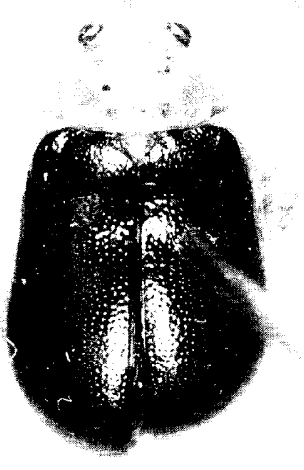

a

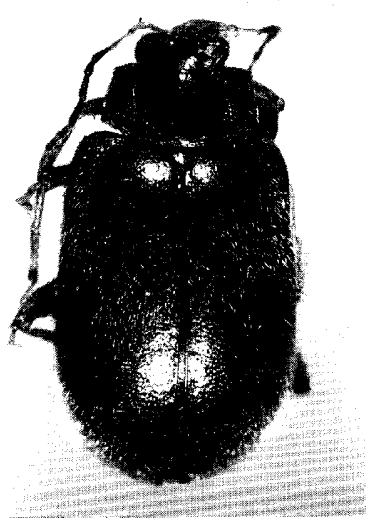

b

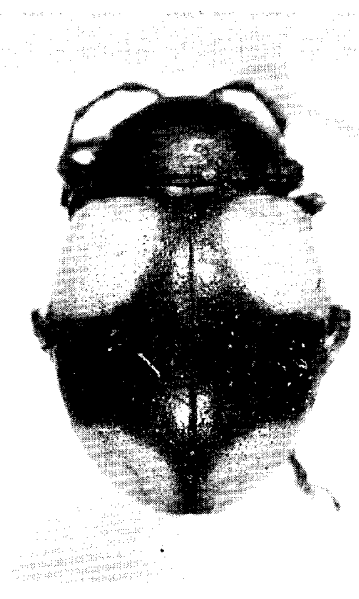

C

Fig. 67. a, Cassenoides flavomarginatus n. sp. ; b, Pseudeustetha hirsuta (Jacoby); c, Emathea aptera n. sp. 
Pronotum transverse $12 / 3$ times as broad as long, anterior margin slightly rounded posteriorly, lateral margin distinctly rounded, widest slightly before middle and distinctly narrowed anteriorly and more strongly so posteriorly, basal margin slightly rounded posteriorly, dorsal surface convex, finely granulate, sparsely impressed by fine punctures, and with a pair of deep oblique depressions laterally. Scutellum subtriangular, somewhat wrinkled, sparsely impressed by minute punctures. Elytron with lateral margin slightly rounded, surface distinctly and rather closely punctate and their interstices finely granulate.

M ale : Head with large cavity on middle of frons ; fifth abdominal segment trilobed apically.

Female : Head with frons convex; fifth abdominal segment entire.

Length : 6.5-7.7 mm.

Holotype (MUNCHEN) : LAOS : Umgeb. Vientiane, iii-vi. 1963. Paratypes : LAOS : 2 exs., same data as the holotype ; Umgeb. Paklay, 1 ex., 1963 (MUNCHEN) ; Ban Van Eue, $20 \mathrm{~km}$ E. of Phoukow-kuei, 1 ex., 15-31. v. 1965, J. A. Rondon (BISHOP).

This new species somewhat resembles Acroxena nasuta Baly, but differs in having the legs pitchy black with the basal $2 / 3$ of femora brownish and in the male the head with a large cavity on the middle of frons.

\section{Acroxena fulva n. sp. Fig. $66 \mathrm{~b}$}

Entirely yellowish brown; in male antenna with exterior margin of third to fifth segments infuscate.

Head with vertex finely granulate, sparsely impressed by minute punctures, interocular space much wider than transverse diameter of single oculus, interocular transverse impression feebly impressed, and with several large punctures along the impression, frontal tubercle transverse, subquadrate, contiguous, distinctly raised, surface finely granulate. Antenna slender, nearly as long as body length ; first segment robust, especially in male, second shortest, nearly $1 / 6$ as long as first in male, second shortest, nearly $1 / 6$ as long as first, third eight times as long as second in male and five times as long as second in female, fourth slightly longer than third, fifth slightly shorter than fourth, fifth to tenth subequal to each other in length and shape, eleventh $11 / 4$ times as long as tenth and its apex pointed. Pronotum transverse, $12 / 3$ times as broad as long, anterior margin slightly rounded posteriorly, lateral margin distinctly rounded, widest slightly before middle and distinctly narrowed anteriorly and posteriorly, basal margin slightly rounded posteriorly, dorsal surface convex, finely granulate, and with a pair of deep oblique depressions laterally, punctures generally finely and sparsely impressed but more strongly and closely impressed on the depression. Scutellum subtriangular, somewhat wrinkled, sparsely impressed by minute punctures. Elytron with lateral margin slightly rounded, surface distinctly and rather closely punctate and their interstices finely granulate.

M ale : Head with frons transversely excavated and with a tuft of hairs on middle of anterior and posterior margins of excavation; fifth abdominal segment trilobed apically; antenna robuster than female. Female : Head with frons convex; fifth abdominal segment entire.

Length : 7.8-8.3 mm.

Holotype (Type No. 2706, Kyushu Univ.) : THAILAND : Chiengmai Prov., Chieng Dao, 23 iv. 1973, Y. Yoshiyasu. Paratypes : LAOS : Umgeb. Vanky, 1 ex., 1963 (MUNCHEN). VIETNAM : Chute de Bourg, $37 \mathrm{~km}$ SE of Dalat, 780 m, 1 ex., 25. iv. 1960, R. E. Leech (BISHOP).

This new species closely resembles Acroxena indica Jacoby, from India, but differs in having pronotum widest almost at $1 / 3$ from the anterior corner and slightly narrowed anteriorly and posteriorly, and in the male the head with frons transversely sulcated and with a tuft of hairs on the middle of the anterior and the posterior margins of the excavation. 


\section{Acroxena nasuta Baly Fig. 66c}

Acroxena nasuta Baly, 1879, Cist. Ent., 2 : 462 (Assam ; BM).-Maulik, 1936, Fauna India, GalerUC. : 565, fig. (India).-Gressitt \& Kimoto, 1963, Pac. Ins. Mon., 1B: 687 (NE India).-Kimoto \& Takizawa, 1972, Kontyâ, Tokyo, 40 (4) : 222 (Nepal).

Acroxena paradoxa Laboissière, 1936, Ann. Soc. Ent. France, 105 : 240 (Tonkin; BRUXELLES). -Gressitt \& Kimoto, 1963, Pac. Ins. Mon., 1B: 687 (Vietnam). New synonym.

Distribution : India, Nepal, Thailand, Vietnam.

Material examined. THAILAND: Khao Yai, 1 ex., 10. iv. 1963, Native collr (BISHOP).

\section{Genus Pseudeustetha Jacoby}

Pseudeustetha Jac., 1899, Stett. Ent. Ztg., 60 : 311 (type : Pseudeustetha quadriplagiata Jacoby ; Sumatra).

Avinasa Maulik, 1936, Fauna India, Galeruc. : 456 (type : Antipha hirsuta Jacoby ; Assam).

\section{Pseudeustetha hirsuta (Jacoby) Fig. 67b}

Antipha hirsuta Jac., 1891, Entomologist, 24 (suppl.) : 32 (Assam; BM).

Anthipha pubescens Jacoby, 1892, Ann. Mus. Civ. Genova, 32 : 971 (Burma :Carin Cheba ; GENOVA). New synonym.

Avinasa pubescens : Maulik, 1936, Fauna India, Galeruc. : 457 (Burma).-Chûjô, 1964, Nature and Life in SE Asia, Kyoto, $3: 288$ (Thailand).

Pseudeustetha hirsuta: Aslam, 1972, J. Nat. Hist., $6: 501$ (genus).

Pseudeustetha pubescens : Aslam, 1972, J. Nat. Hist., $6: 501$ (genus).

Distribution : India, Burma, Thailand, Cambodia, Laos.

Suboval; dorsal surfaces thickly covered by fine hairs ; coloration variable : in most pale colored specimen entirely yellowish to reddish brown and in most dark colored specimen generelly pitchy black with abdomen reddish brown; length 3.8-5.7 mm.

Material examined. THAILAND : Trang Prov., Khaophappha, Kaochang, 200-400 m, 1 ex., 3. i. 1964, 2 exs., 10. i. 1964, 1 ex., 13. i. 1964, G. A. Samuelson ; Chiengmai Prov., Chieng Dao, 450 m, 2 exs., 5-11. iv. 1958, J. L. Gressitt ; Chanthaburi Prov., Prew, 1 ex., 25-30. iv. 1958, T. C. Maa; Banna, 1 ex., 5-10. iv. 1958, T. C. Maa; S. Banna, Nakhon, 108 m, 1 ex., 5-10. v. 1958, T. C. Maa ; Philiu, 1 ex., 23. iv. 1963, Native collr; Khao Yai, 2 exs., 10. iv. 1963, Native collr (BISHOP) ; Chiengmai Prov., Tankeo, Doi Suthep, 800 m, 1 ex., 10. vi. 1965, Y. Miyatake; Khao Yai Nat. Park, 2 exs., 6. vi. 1965, Y. Miyatake, 4 exs., 25. vi. 1965, K. Morimoto \& Y. Miyatake ; Prew, 1 ex., 29. viii. 1973, K. Yano (KU). CAMBODIA: Damrey Phong, 1 ex., 14-16. iv. 1961, N. R. Spencer (BISHOP). LAOS : Vientiane Prov., Phou-kow-kuei, 1 ex., 15. iv. 1965, J. L. Gressitt ; Vientiane Prov., Ban Van Eue, 1 ex., 1-15. ix. 1967, Native collr (BISHOP).

\section{Genus E mathea Baly}

Emathea Baly, 1865, Ent. Monthly Mag., 2 : 147 (type : Emathea aeneipennis Baly ; Sumatra). -Maulik, 1936, Fauna India, Galeruc. : 324. 


\section{K EY TO SPECIES OF Emathea}

1 Elytral humerus distinctly raised

Elytral humerus not distinctly raised ; generally black ; elytron with basal and apical

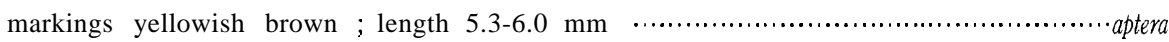

2(1) Elytral punctures finer, interstices of punctures wider than average diameter of punctures; generally reddish brown, elytron with basal and subapical markings violaceous blue, in some specimen elytron entirely bluish; length $5.7-6.5 \mathrm{~mm}$. …....punctata Elytral punctures stronger, interstices of punctures narrower than average diameter of punctures ; generally reddish brown with elytron with purplish sheen in most cases ; length $6.6-7.5 \mathrm{~mm}$ subcaerulea

\section{Emathea aptera n. sp. Fig. $67 \mathrm{c}$}

Apterous. Generally black ; elytron with a basal and an apical markings large, yellowish brown,

Head with vertex smooth, shining, sparsely impressed by minute punctures, interocular space much wider than transverse diameter of single oculus, and interocular transverse impression distinct, frontal tubercle transverse, subtriangular, contiguous, distinctly raised, surface somewhat wrinkled. Antenna slender, nearly as long as body length ; first segment robust, somewhat club-shaped, second

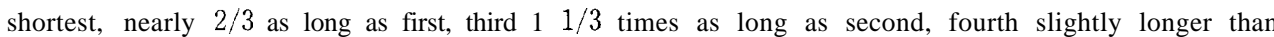
third, fifth subequal to fourth in length and shape, sixth slightly shorter than fifth, and sixth to ninth subequal to each other in length and shape, tenth slightly shorter than ninth in length, eleventh $1 \frac{1 / 3}{3}$ times as long as tenth and its apex pointed. Pronotum transverse, almost twice as broad as long, anterior margin slightly rounded posteriorly, lateral margin distinctly rounded, widest almost at middle, slightly narrowed anteriorly and posteirorly, basal margin distinctly rounded posteriorly ; anterior comer thickened, distinctly produced anteriorly, and posterior comer angulate, each with a setigerous pore ; dorsal surface convex side to side, smooth, shining, sparsely impressed by minute punctures. Scutellum subtriangular, sparsely impressed by minute punctures. Elytron with humerus not raised, lateral margin distinctly rounded, surface smooth, shining, rather closely impressed by distinct punctures.

Length : 5.3-6.0 mm

Holotype (BISHOP) : LAOS : Vientiane Prov., Ban Van Eue, 15. iv. 1965, J. L. Gressitt. Paratypes : LAOS : Vientiane Prov., Ban Van Eue, 1 ex., 15-31. v. 1965, J. A. Rondon; Sayaboury Prov., Sayaboury, 1 ex., 15. iv. 1965, J. L. Gressitt (BISHOP).

This new species somewhat resembles Emathea punctata (Allard), but differs in having elytral humerus not distinctly raised and elytron blackish with a basal and an apical markings yellowish brown.

\section{Emathea punctata (Allard)}

Sphaeroderma punctata All., 1889, Ann. Soc. Ent. France, 1889 : 307 (Annam; PARIS).

Emathea punctata :Laboissière, 1934, Ann. Ass. Nat. Levallois-Perret, $21: 140$ (Tonkin).

Dercetes amoena Weise, 1922, Tijdschr. Ent., 65 : 95 (Tonkin; STOCKHOLM). New synonym.

Emathea moseri Weise, 1922, Tijdschr. Ent., 65 : 98 (Tonkin; STOCKHOLM). New synonym.

Dercetina amoena : Gressitt \& Kimoto, 1963, Pac. Ins. Mon., 1B: 705 (China).

Distribution : Vietnam, China.

Material examined. VIETNAM : Dilinh (Djiring), 1,200 m, 1 ex., 22-28. iv. 1960, S. Quate ; 28 km 


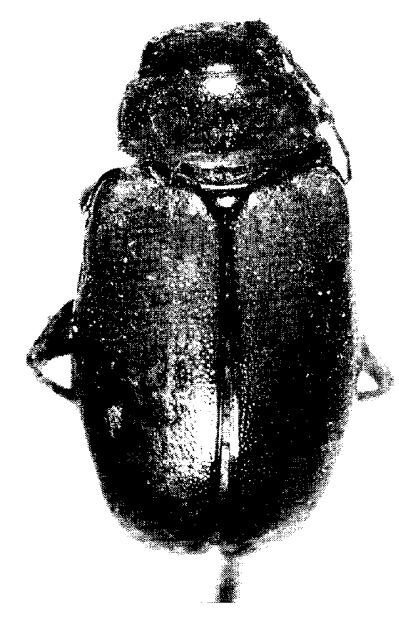

a

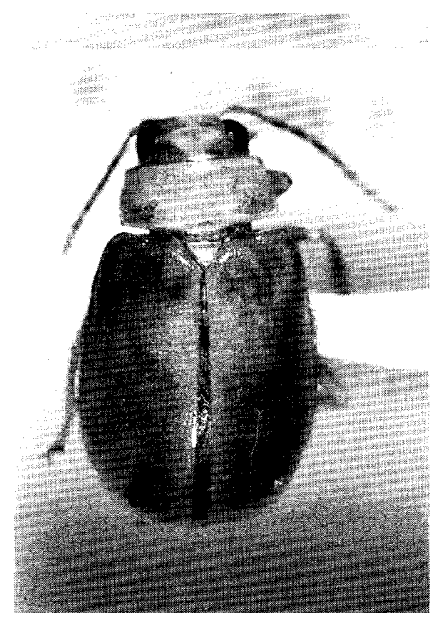

$\mathrm{b}$

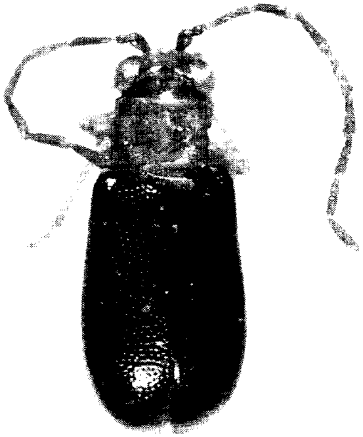

C

Fig. 68. a, Doryidella pallida (Jacoby) ; b, D. minor n. sp.; c, Dercetisoma khonkaenicum n. sp.

N. of Dilinh (Djiring), 1 ex., 22-28. iv. 1960, S. Quate ; SW of Pleiku, 400 m, 1 ex., 12. v. 1960, L. W. Quate ; 20 km N. of Pleiku, 1 ex., 650 m, 9. v. 1960, S. Quate \& L. Quate ; Ban Me Thuot, 500 m, 1 ex., 16-18. v. 1960, L. W. Quate ; 22 km S. of Nha Trang, 2 exs., 20-26. xi. 1960, C. M. Yoshimoto (BISHOP).

\section{Emathea subcaerulea (Jacoby), new combination}

Antipha subcaerulea Jac., 1891, Entomolog., 1891, suppl.: 33 (Assam ; BM).

Anthipha semicaerulea Jacoby var., 1892, Ann. Mus. Civ. Genova, 32 : 998 (Burma) (nomen nudum ; ? error of subcaemlea).

Dercetis subcaemlea : Maulik, 1936, Fauna India, Galeruc. : 364 (India, Burma).

Cyclanthiphaulyssis Laboissière, 1932, Ann. Ass. Nat. Levallois-Perret, 20 : 138 (Tonkin ; HAMBURG). New synonym.

Distribution : India, Burma, Thailand, Vietnam.

Material examined. THAILAND : Chiengmai Prov., Doi Pui, 1,360 m, 1 ex., 2. v. 1958, T. C. Maa (BISHOP) ; Chiengmai Prov., Doi Pui, 1,300 m, 1 ex., 17. vi. 1965, K. Morimoto ; Chiengmai Prov., Fang, 2 exs., 14. vi. 1965, Y. Miyatake (KU).

\section{Genus Doryidella Laboissière}

Doryidella Lab., 1940, Bull. Mus. Hist. Nat. Belg., 16(37): 7 (type : Buphonida pallida Jacoby ; Burma).

$$
\text { KeY to spectes of Doryidella }
$$

1 Pronotum convex from side to side; generally yellowish brown, in some specimen lateral, 
sutural and apical margins blackish in various degrees; antenna pitchy black with two or three basal segments and basal part of each remaining segment brownish in various degrees; length $7.2-9.0 \mathrm{~mm}$.......................................................... pallida

Pronotum with a shallow transverse furrow ; generally yellowish brown; in some specimen lateral, sutural and apical margins blackish in various degrees ; antenna pitchy black with two or three basal segments brown and basal part of each remaining segment brownish in various degrees ; length 5.0-6.0 $\mathrm{mm}$

minor

\section{Doryidella minor n. sp. Fig. $68 \mathrm{~b}$}

Generally yellowish brown; in some specimen lateral, sutural and apical margins blackish in various degrees ; antenna pitchy black with two or three basal segments yellowish brown and basal part of each remaining segment brownish in various degrees.

Head with vertex smooth, shining, sparsely impressed by minute punctures, interocular space much wider than transverse diameter of single oculus, and interocular transverse impression distinct, frontal tubercle oblique, subquadrate, distinctly raised, widely separated to each other by frons, surface smooth, shining, sparsely impressed by minute punctures. Antenna slender, nearly $2 / 3$ as long as body length ; first segment robust, somewhat club-shaped, second shortest, nearly $1 / 3$ as long as first, third $11 / 2$ times as long as second, fourth $13 / 4$ times as long as third, fourth to eighth subequal to each other in length and shape, ninth slightly shorter than eighth, tenth subequal to ninth in length and shape, eleventh subequal to eighth in length but its apex pointed. Pronotum transverse, almost $21 / 3$ times as broad as long, anterior margin slightly rounded posteriorly, lateral margin distinctly rounded, widest almost at middle, slightly narrowed anteriorly and posteriorly, basal margin distinctly rounded posteriorly ; anterior corner thickened, distinctly produced laterally, and posterior corner angulate, each with a setigerous pore, dorsal surface convex, with a shallow transverse furrow subbasally, smooth, shining, rather closely impressed by distinct punctures, Scutellum subtriangular, surface smooth, shining, Elytron with lateral margin rounded, surface smooth, shining, rather closely impressed by distinct punctures.

Length : 5.0-6.0 mm.

Holotype (BISHOP) : LAOS : Borikhane Prov., Pakkading, 23. iv. 1965, J. L. Gressitt. Paratopotypes: 7 exs., same data as the holotype (BISHOP, KIMOTO). Paratypes: THAILAND: "Siam", 4 exs. (BANGKHEN). LAOS: Wapkhamthong Prov., Khong Sedone, 1 ex., 16. v. 1965, Native collr (BISHOP). VIETNAM :M'Drak, E. of Ban Me Thuot, 4-600 m, 1 ex., 8-19. xii. 1960, C. M. Yoshimoto (BISHOP)

This new species is separable from Doryidella pallida (Jacoby), in being the body length shorter, and having pronotum with a transverse furrow, and vertex more strongly separated from frons and frontal tubercle by a distinct transverse furrow.

\section{Doryidella pallida (Jacoby) Fig. 68a}

Buphonida pallida Jac., 1892, Ann. Mus. Civ. Genova, 32 : 959 (Burma ;GENOVA).-Maulik, 1936, Fauna India, Galeruc. : 240 (Burma).

Doryidella pallida:Laboissière, 1940, Bull. Mus. Hist. Nat. Belg., 16(37) : 8 (Burma).

Distribution : Burma, Thailand, Laos, Vietnam.

Material examined. THAILAND: Chiengmai Prov., Fang, $500 \mathrm{~m}, 1$ ex., 13. vi. 1965, P. D. Ashlock (BISHOP). LAOS : Vientiane Prov., Ban Van Eue, 1 ex., 12. iv. 1965, J. L. Gressitt, 11 exs., 15-31. v. 1965, 2 exs., 15. iv. 1966, Native collr ; Khammouane Prov., Phon Tiou, 1 ex., 28. ix. 1965, 
Native collr ; Sayaboury Prov., Sayaboury, 1 ex., 15. iv. 1965, J. L. Gressitt, 1 ex., 17. v. 1965, 1 ex., 30. v. 1965, 2 exs., 21. iv. 1966, Native collr ; Sedone Prov., 5 km E. of Pakse, 1 ex., 13. v. 1965, P. D. Ashlock; Wapikhamthong Prov., Khong Sedone, 1 ex., 30. v. 1965, Native collr ; Muong Sing, NW. of Luang Prabang, 650 m, 2 exs., 6-10. vi. 1960, L. Quate (BISHOP) ; Umgeb. Vientiane, 5 exs., iii-vi. 1963 ; Umgeb. Paklay, 3 exs., 1963 ; Umgeb. Pakse, 1 ex., 1963, 1 ex., 1964 ; Umgeb. Vanky, 1 ex., 1963 (MUNCHEN). VIETNAM : $20 \mathrm{~km} \mathrm{~N}$. of Pleiku, 4 exs., 9. v. 1960, S. Quate \& L. Quate (BISHOP).

\section{Genus Dercetisoma Maulik}

Dercetisoma Maulik, 1936, Fauna India, Galeruc. : 455 (type : Antipha concolor Jacoby ; Burma).

\section{Key to species of Dercetisoma}

Pronotum wider, nearly $12 / 3$ times as wide as long, coloration variable : generally yellowish to reddish brown, in some specimen elytron bluish black; length 3.9-5.3

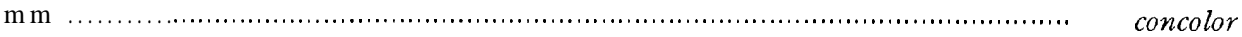

Pronotum narrower, nearly $11 / 3$ times as wide as long, pronotum and elytron more strongly punctate; head and prothorax reddish brown, scutellum black, elytron bluish black, antenna pitchy black, meso- and metathorax black, legs generally reddish brown ; length $3.0-3.3 \mathrm{~mm}$

khonkaenicum

\section{Dercetisoma concolor (Jacoby) Fig. 69a}

Antipha concolor Jac., 1889, Ann. Mus. Civ. Genova, ser. 2, 7 : 222 (Burma ; GENOVA).

Antipha puncticollis Jacoby, 1889, Ann. Mus. Civ. Genova, ser. 2, $7: 222$ (Burma ; GENOVA). New synonym.

Antipha brunnea Allard, 1889, C. R. Soc. Ent. Belg., 33 : 108 (Malacca).-Maulik, 1936, Fauna India, Galeruc. : 455 (= concolor $)$.

Sutrea modesta Allard, 1889, Ann. Soc. Ent. France, 1889 : 306 (Cambodia ; PARIS). New synonym.

Dercetis modesta:Laboissière, 1935, Ann. Ass. Nat. Levallois-Perret, 21 : 140 (Tonkin).

Dercetisoma concolor: Maulik, 1936, Fauna India, Galeruc. : 455 (Burma, Sumata, Java, Mallaca). -Chûjô, 1964, Nature and Life in SE Asia, Kyoto, 3 : 289 (Thailand).

Dercetis flavescens: Châjô, 1961, Nature and Life in SE Asia, Kyoto, 1: 350 (Thailand : Fang).

Dercetisoma puncticollis: Chûjô, 1964, Nature and Life in SE Asia, Kyoto, 3 : 289 (Thailand).

Distribution : Burma, Thailand, Cambodia, Vietnam, Malaya, Sumatra, Java.

As suggested by Châjô (1964), Antipha puncticollis Jacoby is nothing but a infraspecific variation. Also, Sutrea modesta Allard is a synonym of present species.

Material examined. THAILAND : Chiengmai Prov., Chiengmai, 1,100-1,500 m, 7 exs., 11-12. iv. 1966, J. Sedlacek ; Chiengmai Prov., Doi Suthep, 1 ex., 28-31. iii. 1958, T. C. Maa ; Chiengmai Prov., Fang, 2 exs., 12-19. iv. 1958, T. C. Maa ; Chiengmai Prov., Chiengdao, 2 exs., 5-11. iv. 1958, T. C. Maa; Trang Prov., Khaophappha, Khaochang, 200 m, 3 exs., 11-12. i. 1964, G. A. Samuelson ; Chanthaburi Prov., Prew, 2 exs., 25-30. iv. 1958, T. C. Maa; Tak, 1 ex., 10. iv. 1966, J. Sedlacek ; 50 km West of Tak, 900 m, 2 exs., 7-8. iv. 1966, J. Sedlacek ; Praehsi-Saraburi, 20 m, N. of Bangkok, 2 exs., 1. xii. 1957, J. L. Gressitt ; Packchong, 100 m, NE. of Bangkok, 1 ex., 3. xii. 1957, J. L. Gressitt (BISHOP) ; Chiengmai Prov., Chiengmai, 1 ex., 9. vi. 1965, K. Morimoto ; Chiengmai Prov., Botanical Garden, 1 ex., 12. vi. 1965, Y. Miyatake; Chiengmai Prov., Fang, 2 exs., 14. vi. 1965, K. Morimoto \& Y. 

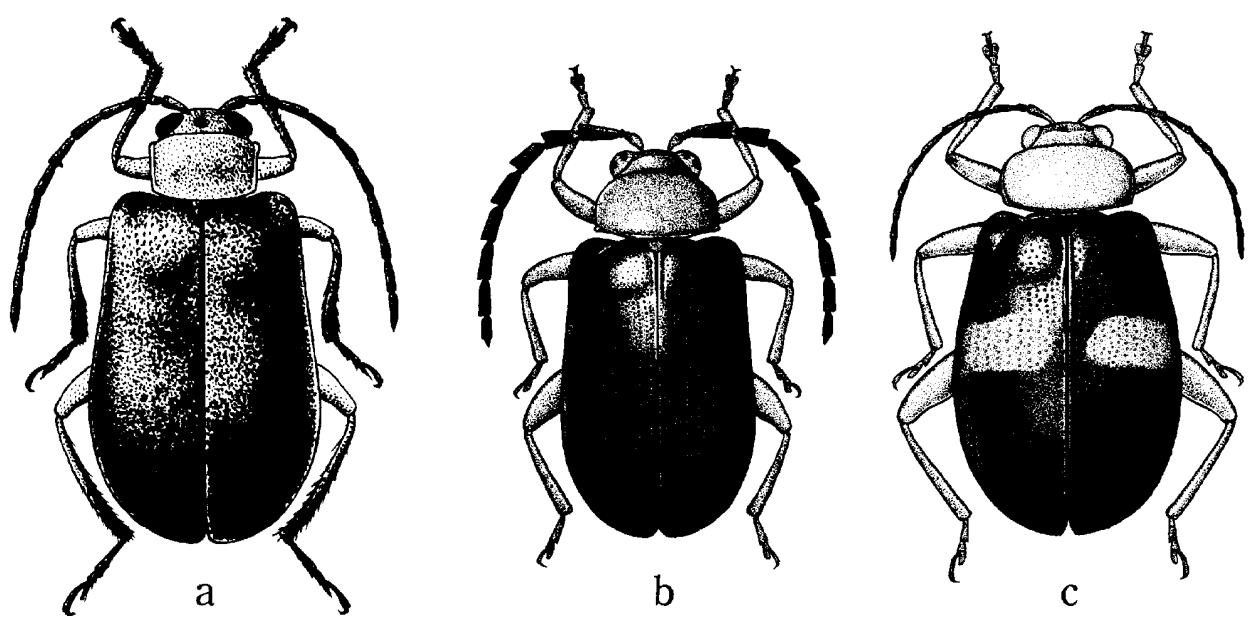

Fig. 69. a, Dercetisoma concolor (Jacoby) ; b, Arthrotus nigripennis (Jacoby) ; c, Dercetina flavocincta (Hope).

Miyatake, 1 ex., 23. iv. 1973, Y. Yoshiyasu ; Chiengmai Prov., Doi Suthep, 1,000 m, 1 ex., 6. vi. 1965, 1 ex., 10. vi. 1965, K. Morimoto ; Chiengmai Prov., Ban Tap Dua, Chieng Dao, 1 ex., 15. viii. 1973, K. Yano ; Mae Sa Water Fall, 1 ex., 16. vi. 1965, K. Morimoto ;Sarapi, nr. Chiengmai, 2 exs., 18. vii. 1970, K. Yano ; Khao Chong, nr. Trung, 2 exs., 26-27. vi. 1965, K. Morimoto ; Khao Yai Nat. Park, 1 ex., 6. vi. 1965, Y. Miyatake ; Mae Klang Water Fall, nr. Chom Thong, 1 ex., 11. vi. 1965, Y. Miyatake ; Muak Lek, 40 km SE of Saraburi, 1 ex., 5. vi. 1965, Y. Miyatake ; Prew, 1 ex., 29. viii. 1973, K. Yano ; Sanpatong, 2 exs., 6. vii. 1970, sweeping of paddy field, K. Yano (KU) ; Khon Kaen, 1 ex., 24. vii. 1980, 1 ex., 8. x. 1981, S. Azuma (RU) ; Khon Kaen, 1 ex., 15. x. 1972, M. Sato ; Fang Spa, 1 ex., 21. xi. 1968, K. Hatta (EHIME) ; Fang, 1 ex., 22. xi. 1968, M. Sato (KIMOTO). LAOS : Vientiane Prov., Vientiane, 1 ex., 29. vii. 1965, 1 ex., 3. viii. 1965, 1 ex., 21. iii. 1966, Native collr ; Vientiane Prov., Ban Van Eue, 2 exs., 13. iv. 1965, J. L. Gressitt, 4 exs., 15-31. v. 1965, Native collr ; Vientiane Prov., Tha Ngone, 1 ex., 30. ix. 1965, 1 ex., 20. xi. 1965, 1 ex., 30. xi. 1965, Native collr ; Vientiane Prov., Gi Sion Vill., de Tha Ngone, 1 ex., 7-21. ii. 1965, 1 ex., 5-19. xii. 1965, 3 exs., 2. i. 1966, 1 ex., 24-31. x. 1966, Native collr ; Sayaboury Prov., Sayaboury, 1 ex., 17. ii. 1965, 5 exs., 6. xii. 1965, 2 exs., 14. xii. 1965, 1 ex., 30. xii. 1965, Native collr ; Borikhane Prov., Pakkading, 2 exs., 31. vii. 1965, 1 ex., 3. viii. 1965, Native collr ; Borikhane Prov., Namkading, nr. Pakkading, 3 exs., 21. iv. 1965, J. A. Rondon, 5 exs., 22. iv. 1965, J. L. Gressitt ; Wapikhamthong Prov., Wapi, 1 ex., 15. x. 1967, Native collr ; Khammouane Prov., Phon Tiou, 1 ex., 17. x. 1965, Native collr ; Nongtevada, 1 ex., 4. xi. 1965, 1 ex., 2. xii. 1965, 1 ex., 22. xii. 1965, Native collr (BISHOP) ; Umgeb. Vientiane, 3 exs., iii-vi. 1963 ; Umgeb. Pakse, 2 exs., 1963, Umgeb. Paklay, 1 ex., 1963 (MUNCHEN). VIETNAM : Fyan, 4 exs., 11. vii.-9. viii. 1961, N. R. Spencer; Dalat, 1,500 m, 1 ex., 29. iv.-4. v. 1960, L. W. Quate ; Dalat, 6 km S., 1,400-1,500 m, 3 exs., 9. vi.-7. vii. 1961, N. R. Spencer ; 6 km SW, Dalat, 1,550 m, 1 ex., 11. ix. 1960, J. L. Gressitt ; Dilinh (Djiring), 1,200 m, 1 ex., 22-28. iv. 1960, S. Quate ; Ap Hung-Lam, 21 km NW of Dilinh, 1,100 m, 3 exs., 29. ix.-5. x. 1960, C. M. Yoshimoto ; Blao (Barao), 500 m, 1 ex., 14-21. x. 1960, C. M. Yoshimoto ; Dai Lanh, N. of Nha Trang, 1 ex., 30. xi.-5. xii. 1960, C. M. Yoshimoto ; 25 km SW. of Pleik, 400 m, 1 ex., 12. v. 1960, L. W. Quate ; Kontum, N. of Pleik, 550 m, 2 exs., 13. v. 1960, L. W. Quate ; Ban Me Thuot, 500 m, 1 ex., 16-18. v. 1960, S. Quate, 1 ex., 20-24. xii. 1960, C. M. Yoshimoto ; 10 km E. of Ban Me Thuot, 570 m, 1 ex., 10. v. 1960, R. E. Leech ;M'Drak, E. of Ban Me Thuot, 4-600 m, 1 ex., 8-19. xii. 
1960, C. M. Yoshimoto (BISHOP)

\section{Dercetisoma khonkaenicum n. sp. Fig. 68c}

Head and prothorax reddish brown, scutellum black, elytron bluish black, antenna pitchy black, meso- and metathorax black, legs reddish brown, with tarsi and apices of tibiae somewhat infuscate.

Head with vertex smooth, shining, sparsely impressed by minute punctures, interocular space much wider than transverse diameter of single oculus, and interocular transverse impression distinct, frontal tubercle transverse, subtriangular, contiguous, distinctly raised, surface smooth, shining, impunctate. Antenna slender, nearly $3 / 4$ as long as body length ; first segment robust, club-shaped, second short, nearly $1 / 3$ as long as first, third slightly shorter than second, fourth nearly $22 / 5$ times as long as the length of second and third combined, fifth slightly shorter than fourth in length, fifth to tenth subequal to each other in length and shape, eleventh subequal to tenth in length but its apex pointed. Pronotum transverse, $11 / 3$ times as broad as long, lateral margin feebly rounded, widest almost at anterior margin, and gradually narrowed posteriorly, basal margin distinctly rounded posteriorly ; dorsal surface convex, with a pair of deep depressions laterally, smooth, shining, rather closely impressed by distinct punctures especially on depression. Scutellum subtriangular, smooth, shining, impunctate. Elytron with lateral margin subparallel-sided, apex rounded, surface strongly and rather closely punctate, and their interstices smooth, shining.

Length : $3.0-3.3 \mathrm{~mm}$.

Holotype (RU) : THAILAND : Khon Kaen, 7. x. 1981, S. Azuma. Paratopotypes : 21 exs., same data as the holotype. Paratypes: THAILAND : Khon Kaen, 8 exs., 8. x. 1981, S. Azuma (RU, KIMOTO).

This new species resembles Dercetisoma concolor(Jacoby), but differs in having the body more elongate, pronotum more narrower, and pronotum and elytron more strongly punctate.

\section{Genus Arthrotus Motschulsky}

Arthrotus Motsch., 1857, Etud. Ent., 6 : 38 (type : Arthrotus niger Motschulsky ;Japan).-Ogloblin, 1936, Fauna USSR, 26, 1 : 328, 376._Gressitt\& Kimoto, 1963, Pac. Ins. Mon., 1B: 691.

Taphinella Jacoby, 1889, Ann. Mus. Civ. Genova, $27: 224$ (type : Taphinella nigripennis Jacoby ; Burma).

\section{Key to SPECIES of Arthrotus}

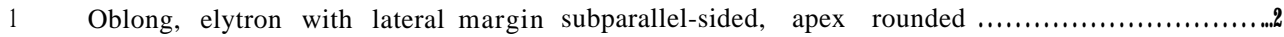
Oval, elytron with lateral margin rounded and widened posteriorly $\ldots \ldots \ldots \ldots \ldots \ldots \ldots \ldots \ldots . \ldots 3$

2(1) Elytron with punctures confusedly impressed ; coloration variable : generally yellowish to reddish brown, in some specimen elytron with basal, lateral, sutural and apical margins blackish in various degrees, and in most dark colored specimen elytron entirely black ; antenna generally black; length 4.5-5.0 $\mathrm{mm} \ldots \ldots \ldots \ldots \ldots \ldots \ldots \ldots$ nigripennis Elytron with puctures arranged in semiregular rows of punctures ; generally blackish

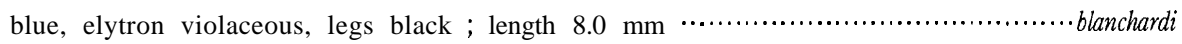

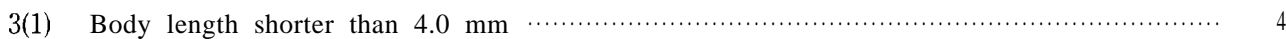
Body length longer ; generally bluish black, abdomen yellowish brown ; length 4.0$4.5 \mathrm{~mm}$ cyanipennis

4(3) Dorsal surfaces in part blackish, at least elytron with margins generally black- 


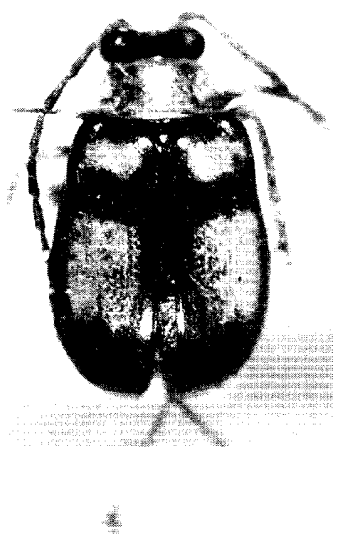

a

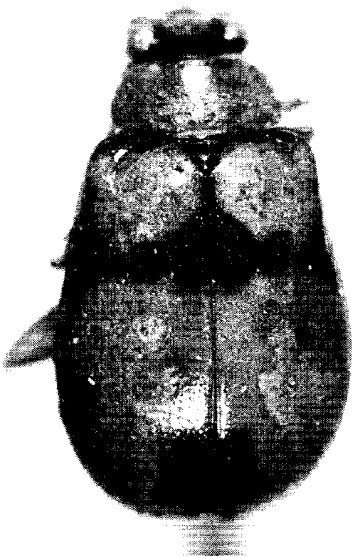

b

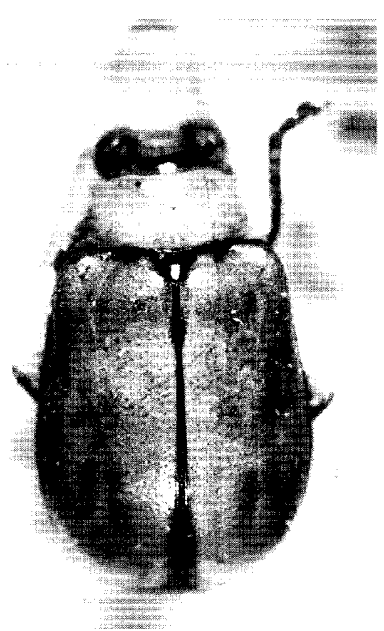

C

Fig. 70. a, b, c, Arthrotus phaseoli Laboissière.

ish

Dorsal surfaces entirely yellowish to reddish brown; antenna generally blackish ; in dark colored specimen head, meso- and metathorax and legs blackish in various degrees ; length 3.0-5.0 mm

-antennalis

Elytrongenerally brownish with several colors of bands

Elytron without many colors of bands

6(5) Elytron reddish brown with basal, postmedian and apical bands blackish ; generally yellowish to reddish brown, meso- and metathorax black; length 3.5-5.0 mm …tonkinensis Elytron with four colors of reddish, blackish, yellowish and blackish bands, of which second band is narrowest ; generally yellowish to reddish brown; head and antenna generally blackish, length $4.3-5.0 \mathrm{~mm}$

7(5) Elytron pitchy black with an apical marking yellowish brown; generally yellowish to reddish brown, meso- and metathorax blackish, antenna blackish, length 3.3-4.5 $\mathrm{m} \mathrm{m}$

Elytral coloration variable, 1) bluish black with basal and postmedian markings yellowish brown, 2) bluish black with postmedian marking yellowish brown, 3) entirely bluish black, 4) basal and postmedian markings enlarged and united, 5) generally yellowish brown with margins blackish in various degrees; generally yellowish to reddish brown ; meso- and metathorax blackish; length $3.3-5.0 \mathrm{~mm}$....................phaseoli

\section{Arthrotus antennalis Laboissière}

Arthrotus antennalis Lab., 1932, Ann. Ass. Nat. Levallois-Perret, 20 : 134 (Tonkin; HAMBURG). Arthrotus pumilus Laboissière, 1932, ibid., 20 : 134 (Tonkin; HAMBURG). New synonym. Arthrotus tarsalis Laboissière, 1932, ibid., 20 : 134 (Tonkin; HAMBURG). New synonym. Arthrotus frontalis Laboissière, 1932, ibid., $20: 133$ (Tonkin; HAMBURG). New synonym. Arthrotus pallidus: Kimoto \& Takizawa, 1983, Bull. Natn. Sci. Mus., Tokyo, ser. A, 9 (2) : 92 
(Nepal).

Distribution : Nepal, Thailand, Vietnam.

This species might be a synonym of Arthrotus phaseoli Laboissière, which is very variable species on the coloration of the dorsal surfaces.

Material examined. THAILAND: Uthaithani, 10 exs., 13. iv. 1963 (BANGKHEN)

\section{Arthrotus blanchardi (Allard), new combination}

Antipha blanchardi Allard, 1891, Nouv. Arch. Mus. Paris, ser. 3, 3 : 234 (Luang-Prabang à Theng) ; 1895, Miss. Pavie Indo-Chine, 3 : 163, fig. (Cambodge).

Distribution : Cambodia, Laos.

Judging from the original description and the figure, this species seems to be a member of Arthrotus. I could not examined the type of this species.

\section{Arthrotus coomani Laboissière}

Arthrotus coomani Lab., 1932, Ann. Ass. Nat. Levallois-Perret, $20: 135$ (Tonkin; HAMBAURG). Distribution : Vietnam.

Material examined. "Indo-China", 1 ex. (BASEL).

\section{Arthrotus cyanipennis (Laboissière), new combination}

Taphinella cyanipennis Lab., 1936, Ann. Soc. Ent. France, 105 :255, figs. (Tonkin ; HAMBURG). Distribution : Vietnam.

Material examined. "Indo-China", 1 ex. (BASEL).

\section{Arthrotus duporti Laboissière Fig. 71b}

Arthrotus duporti Lab., 1932, Ann. Ass. Nat. Levallois-Perret, 20 : 132 (Tonkin; HAMBURG). Distribution : Vietnam.

Material examined. "Indo-China", 1 ex. (BASEL).

\section{Arthrotus nigripennis (Jacoby) Fig. 69b}

Taphinella nigripennis Jac., 1889, Ann. Mus. Civ. Genova, 27 : 224 (Burma ; GENOVA) ; 1900, Mém.Soc. Ent. Belg., 7 : 140 (mentioned) ; 1905, Fac. Malay. App. 2 : 5 (Siam).-Maulik, 1936, Fauna India, Galeruc. : 329 (Burma, Siam).

A rthrotus nigripennis: Gressitt \& Kimoto, 1963, Pac. Ins. Mon., 1B : 699 (China, Tonkin:Hoabinh).

Distribution : Burma, Thailand, Laos, Vietnam, China.

Material examined. THAILAND : Chiengmai Prov., Fang (Agr. Exp. Station), $600 \mathrm{~m}, 1$ ex., 14. vi. 1965, P. D. Ashlock; Chiengmai Prov., Fang, 500 m, 1 ex., 15. iv. 1958, T. C. Maa ; Chiengmai Prov., Chiengdao, 450 m, 2 exs., 5-11. iv. 1958, T. C. Maa ; Chiengmai Prov., Mae Sa, 1 ex., 16. vi. 1965, P. D. Ashlock; Loey Prov., 17 km NW of City, 1 ex., 18. v. 1967, Native collr (BISHOP) ; Chiengmai Prov., Fang, 11 exs., 13-14. vi. 1965, K. Morimoto \& Y. Miyatake, 1 ex., 22. viii. 1973, K. Yano, 1 ex., 22. viii. 1973, S. Nakao ; Chiengmai Prov., Chieng Dao, 9 exs., 15. vi. 1965, K. Morimoto ; Chiengmai Prov., Doi Suthep, 1,000 m, 1 ex., 10. vi. 1965, K. Morimoto ; Chiengmai Prov., Doi Pui, 1,300 m, 1 ex., 8. vi. 


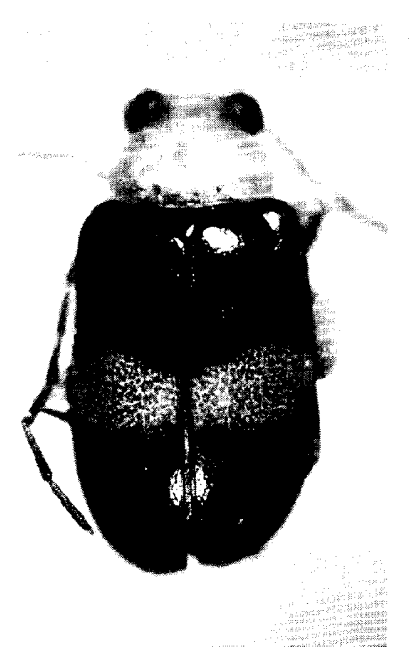

a

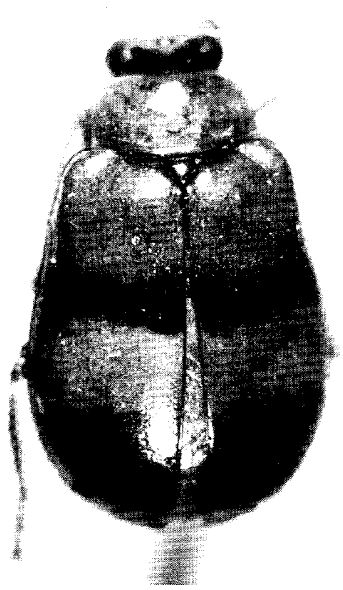

$\mathrm{b}$

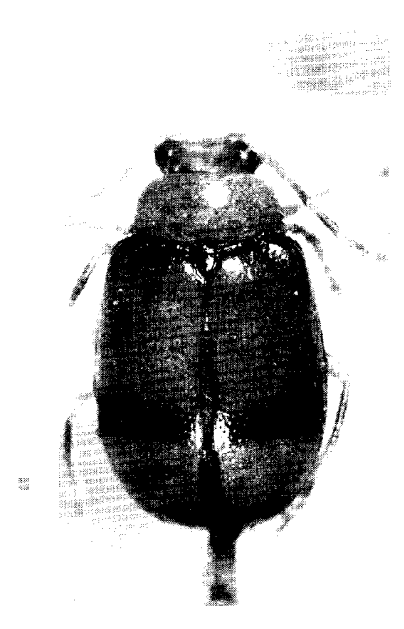

Fig. 71. a, Arthrotus phaseoli Laboissiere ; b, A. duporti Laboissiere ; c, A. tonkinensis Laboissiere.

1965, Y. Miyatake ; Chiengmai Prov., Mae Sa Water Fall, 2 exs., 16. vi. 1965, K. Morimoto \& Y. Miyatake ; Kor Hong, 1 ex., 23. vi. 1965, K. Morimoto (KU). LAOS : Vientiane Prov., Vientiane, 1 ex., 31. vii. 1965, 1 ex., 21. iii. 1966, Native collr ; Vientiane Prov., Ban Van Eue, 4 exs., 31. vii. 1965, Native collr ; Vientiane Prov., Phou Kou Khouei, 3 exs., 10. iv. 1965, J. L. Gressitt ; Sayaboury Prov., Sayaboury, 1 ex., 14. vii. 1965, 1 ex., 3. viii. 1965, 2 exs., 5. v. 1966, 1 ex., 25. viii. 1966, 1 ex., xi. 1966, Native collr ; Khammouane Prov., Phon Tiou, 1 ex., 28. iv. 1965, 2 exs., 30. iv. 1965, 7 exs., 17. v. 1965, 3 exs., 6. vii. 1965, 1 ex., 4. viii. 1965, Native collr ; Khammouane Prov., Bon Nong Sun, 1 ex., 5. vi. 1965, N. Wilson ; Wapikhamthong Prov., Khong Sedone, 1 ex., 17. vii. 1965, Native collr ; Borikhane Prov., Paksane, 1 ex., 7. viii. 1965, Native collr ; Borikhane Prov., Pakkading, 100 m, 1 ex., 23. iv. 1965, J. L. Gressitt ; Houan Khong Prov, Houei Sai, 1 ex., 3. ii. 1965, Native collr ; Sedone Prov., Pakse, 1 ex., 15. v. 1965, P. D. Ashlock; Muong Sing, NW of Luang Prabang, 650 m, 1 ex., 6-10. vi. 1960, S. Quate \& L. Quate (BISHOP) ; Umgeb. Vientiane, 11 exs., iii-vi. 1963 ; Umgeb. Vanky, 4 exs., 1963 ; Umgeb. Pakse, 1 ex., 1963 (MUNCHEN). VIETNAM :Banme Thuot, 500 m, 8 exs., 16-18. v. 1960, L. W. Quate, S. Quate \& R. E. Leech ; 33 km N. of Banme Thuot, 870 m, 1 ex., 18. v. 1960, L. W. Quate ; Fyan, 1,200 m, 2 exs., 11. vii.-9. viii. 1961, N. R. Spencer ; 20 km N. of Pleiku, 2 exs., 650 m, 9. v. 1960, L. W. Quate ; 25 km NW of Pleiku, 400 m, 3 exs., 12. v. 1960, L. W. Quate ; Kontum, N. of Pleik, 550 m, 1 ex., 13. v. 1960, L. W. Quate ; Chute de Babla, 7 km W. of Dilinh (Djiring), 840 m, 27. iv. 1960, R. E. Leech (BISHOP).

\section{Arthrotus phaseoli Laboissière Figs. 70a, b, c, 71a}

Arthrotus phaseoli Lab., 1932, Mém. Mus. Hist. Nat. Belg., 4 : 174, fig. (Sumatra, Java, Tonkin: Cho-Ganh, Hoa-Binh; HAMBURG).

Distribution: Thailand, Laos, Vietnam, Sumatra, Java.

Material examined. THAILAND : Chiengmai Prov., Fang, 500 m, 12. iv. 1958, T. C. Maa; Banna, Chawang, nr. Nabon, 700 m, 1 ex., 4. ix. 1958, J. L. Gressitt (BISHOP) ; Khao Chong, nr. Trung, 2 exs., 26. vi. 1965, K. Morimoto (KU). LAOS : Sedone Prov., Paksong, 2 exs., 18. v. 1965, P. D. 
Ashlock; Vientiane Prov., Gi Sion Vill., de Tha Ngone, 1 ex., 5-19. xii. 1965, Native collr (BISHOP). VIETNAM : Fyan, 900-1,200 m, 2 exs., 11. vii.-9. viii. 1961, N. R. Spencer ; Dalat, 1,500 m, 1 ex., 29. iv.4. v. 1960, S. Quate \& L. Quate ; 6 km SW Dalat, 1,550 m, 1 ex., 11. ix. 1960, J. L. Gressitt ; Ap HungLam, 21 km NW of Dilinh, 1,100 m, 2 exs., 29. ix.-5. x. 1960, C. M. Yoshimoto (BISHOP).

\section{Arthrotus tonkinensis Laboissière Fig. 71c}

Arthrotus tonkinensis Lab., 1932, Ann. Ass. Nat. Levallois- Perret, $20: 136$ (Tonkin ; HAM BURG).-Chûjô, 1964, Nature and Life in SE Asia, Kyoto, $3: 285$ (Thailand).

Distribution : Thailand, Vietnam.

No additional material was examined beside the type series.

\section{Genus Dercetina Gressitt \& Kimoto}

Dercetis Clark, 1865, Ann, Mag. Nat. Hist., ser. 3, 15 : 147 (nec Muenster \& Agassiz, 1834 ; Pisces) (type : Dercetis depressa Clark ; Pulo Penang).-Maulik, 1936, Fauna India, Galeruc. : 348.

Antipka Baly, 1865, Ann. Mag. Nat. Hist., ser. 3, $16: 251$ (nec Antipka Walker, 1855 ; Lepidoptera) (type : Antipha picipes Baly; India ; monobasic).

Dercetes: Jacoby, 1892, Entomologist, 25 : 162 (error for Dercetis Clark).-Hincks, 1949, Ann.

Mag. Nat. Hist., ser. 12, $2: 611$ (not new emendation; not status in nomenclature).

Dercetina Gressitt \& Kimoto, 1963, Pac. Ins. Mon., 1B: 704 (new name for Dercetis Clark).

\section{KEY TO SPECIES OF D ercetina}

1 Elytron with epipleuron narrower subapically

Elytron with epipleuron wider subapcially ; elytron with a deep transverse furrow subbasally ; generally bluish black, abdomen entirely yellowish brown ; length 5.6-6.3

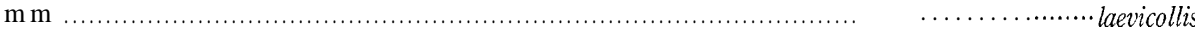

2(1) Elytron with a shallow transverse furrow or without any transverse furrow ; body

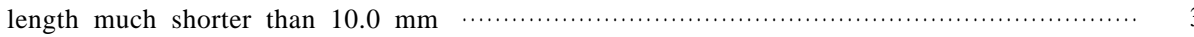
Elytron with a deep transverse furrow subbasally; entirely reddish brown ; length 10.0 $\mathrm{mm}$

3(2) Elytron not combination of basal half yellowish and apical half bluish $\ldots \ldots \ldots \ldots \ldots \ldots \ldots \ldots \ldots$ Elytron yellowish brown with apical half greenish blue; generally yellowish brown ;

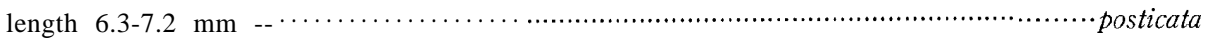

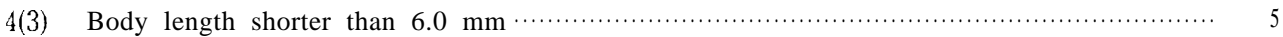
Body length longer; generally yellowish brown; in male elytron entirely bluish black; in female elytron entirely bluish black; in female elytron entirely yellowish brown, in some specimen elytron with basal and subapical markings bluish black;

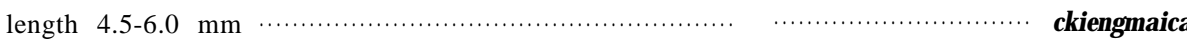

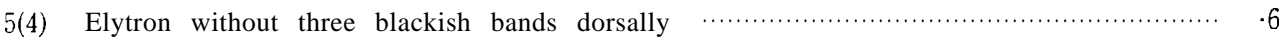
Elytron yellowish brown with basal, postmedian and apical bands black ; generally yellowish brown, head, scutellum, meso- and metathorax, tibiae and tarsi black ;

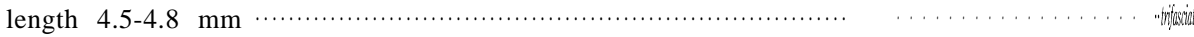

6(5) Elytron bluish black with a broad median transverse band yellowish brown ; in some specimen elytron entirely bluish; pronotum yellowish brown, ventral surfaces black ; legs black with femora yellowish brown; head generally black, in some specimen 
brownish in various degrees ; length 4.2-4.8 $\mathrm{mm}$ flavocincta

Coloration of dorsal surface variable :1) generally yellowish to reddish brown, 2) yellowish to reddish brown, elytron with margins blackish, 3) yellowish to reddish brown with pronotum and elytral margins black, 4) yellowish brown, elytron with base and apical marking bluish black, 5) yellowish brown, elytron entirely black, 6) dorsal surfaces entirely blackish; ventral surface reddish brown, in some specimen blackish in various degrees, and in most dark colored specimen entirely blackish ; legs yellowish brown, in some specimen blackish in various degrees and in most dark colored specimen entirely black ; length 4.5-6.0 $\mathrm{mm}$

bretinghami

\section{Dercetina bretinghami (Baly)}

Antipha bretinghami Baly, 1879, Ann. Mag. Nat. Hist., ser. 5, 4:118 (India ; BM).

Antipha pallida Allard, 1889, C. R. Soc. Ent. Belg., 33 : 108 (Cochinchina ; PARIS). New synonym.

Anthipha inornata Jacoby, 1892, Ann. Mus. Civ. Genova, 32 : 972 (Burma ; GENOVA). New synonym.

Dercetes laetifica Weise, 1922, Tijdschr. Ent., 65 : 97 (Tonkin; STOCKHOLM). New synonym. Arthrotus pallidus Laboissike, 1932, Ann. Ass. Nat. Levallois-Perret, 20 : 133 (Tonkin;HAMBURG).-Chûjô, 1964, Nature and Life in SE Asia, Kyoto, $3: 285$ (Thailand). New synonym. Dercetis bretinghami : Maulik, 1936, Fauna India, Galeruc. : 360 (India).

Dercetis pallida:Châjô, 1959, Sci. Rep. Agr. Exped. Cambodia, Hyogo Univ. Agr. Japan, (2) : 60 (Cambodia) ; 1964. Nature and Life in SE Asia, Kyoto, $3: 285$ (Cambodia).

Dercetina huinana Gressitt \& Kimoto, 1963, Pac. Ins. Mon., 1B: 705, 707 (Hainan ; CAS). -Kimoto \& Takizawa, 1972, Kontyû, Tokyo, 40 (4) : 222, fig. (Nepal). New synonym.

Dercetina bretinghami: Kimoto, 1970, Khumbu Himal, 3 (3) : 417 (Nepal).-Kimoto \& Takizawa, 1972, Kontyû, Tokyo, 40 (4) : 222 (Nepal).

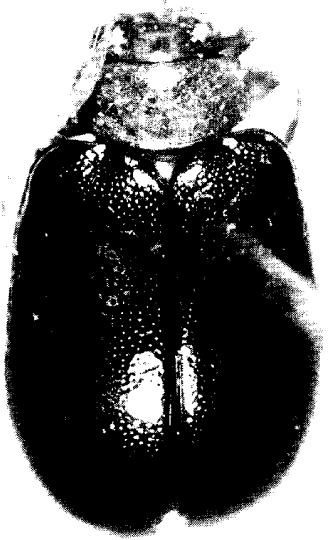

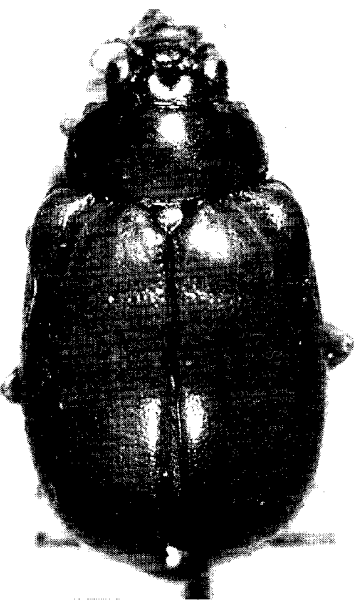

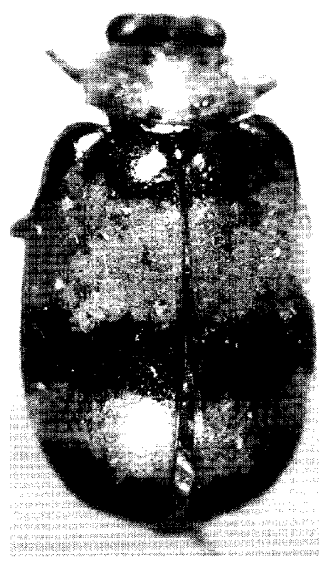

C

Fig. 72. a, Dercetina chiengmuica n. sp. ; b, D. permagna n. sp. ; c, D. trifasciata (Laboissière). 
Distribution : India, Nepal, Burma, Thailand, Cambodia, Laos, Vietnam.

Material examined. THAILAND : Chiengmai Prov., Chiengmai, 2 exs., 9. vi. 1965, P. D. Ashlock; Chiengmai Prov., Chiengdao, 5 exs., 5-11. iv. 1958, T. C. Maa; Mae Sa, '2 exs., 16. vi. 1965, P. D. Ashlock; Chiengmai Prov., Doi Suthep, 1 ex., 14. xi. 1957, J. L. Gressitt, 1 ex., 12. vi. 1965, P. D. Ashlock; Chiengmai Prov., Fang, 2 exs., 12-19. iv. 1958, T. C. Maa; Chiengmai Prov., Ban-ting-doi, 350 m, 1 ex., 13. xi. 1958, J. L. Gressitt ; Bangpra, 25 m, 1 ex., 23. xi. 1957, J. L. Gressitt ; 50 km West of Tak, 900 m, 1 ex., 7-8. iv. 1966, J. \& J. H. Sedlacek; Trang Prov., Khaophappha, Khaochong, 200400 m, 2 exs., 2-3. i. 1964, 1 ex., 10. i. 1964, G. A. Samuelson (BISHOP) ; Chiengmai Prov., Doi Pui, 1 ex., 17. vi. 1965, K. Morimoto ; Chiengmai Prov., Mae Sa Water Fall, 1 ex., 16. vi. 1965, K. Morimoto ; Khao Yai Nat. Park, 1 ex., 6. vi. 1965, Y. Miyatake ; Khao Chong, nr. Trung, 1 ex., 26. vi. 1965, Y. Miyatake (KU) ; Khon Kaen, 1 ex., 4. viii. 1980, 1 ex., 8. x. 1981, S. Azuma (RU) ; Kanchanaburi, 1 ex., 10. viii. 1963 ; Khao Yai, 2 exs., 10. iv. 1963 (BANGKHEN). CAMBODIA : Kiri Rom, 700 m, 1 ex., 31. iii.-7. iv. 1961, N. R. Spencer ; Damrey Phong, 1 ex., 14-16. iv. 1961, N. R. Spencer (BISHOP). LAOS : Vientiane Prov., Vientiane, 1 ex., 22. vii. 1965, Native collr ; Vientiane Prov., Ban Van Eue, 1 ex., 115. v. 1965, J. A. Rondon, 1 ex., 15-31. iv. 1965, 3 exs., 15-31. v. 1965, Native collr, 1 ex., 13. iv. 1965, 2 exs., 16. iv. 1965, J. L. Gressitt, 1 ex., 30. ix. 1965, 2 exs., 30. xi. 1965, 1 ex., 15. iii. 1966, 1 ex., 29. iv. 1966, 1 ex., 15. viii. 1966, Native collr ; Vientiane Prov., Phou-kow-kuei, 9 exs., 15-17. iv. 1965, J. L. Gressitt ; Vientiane Prov., Gi Sion Vill., de Tha Ngone, 1 ex., 10-24. x. 1965, Native collr ; Sayaboury Prov., Sayaboury, 6 exs., 15-16. iv. 1966, J. L. Gressitt, 1 ex., 13. iv. 1966, Native collr ; Borikhane Prov., Pakkading, 4 exs., 21-23. iv. 1965, 2 exs., 13. vii. 1965, 1 ex., 15. ix. 1965, J. L. Gressitt, 1 ex., 1531. viii. 1965, Native collr ; Attopeu Prov., Houei Khong, 1 ex., 16. v. 1965, Native collr ; Phon Tiou, 1 ex., 1-15. iv. 1965, J. L. Gressitt (BISHOP) ; Umgeb. Vientiane, 3 exs., iii-vi. 1963 ; Umgeb. Vanky, 5 exs., 1963 ; Umgeb. Paklay, 2 exs., 1963 ; Umgeb. Pakse, 1 ex., 1963 (MUNCHEN). VIETNAM : Fyan, 900-1,200 m, 12 exs., 11. vii.-9. viii. 1961, N. R. Spencer ; Blao (Balao), 500 m, 13 exs., 14-21. x. 1960, C. M. Yoshimoto ; Dalat, 1,500 m, 2 exs., 29. iv.-4. v. 1960, L. W. Quate \& C. M. Yoshimoto, 1 ex., 26-27. ix. 1960, C. M. Yoshimoto ; Dalat, 6 km S., 1,400-1,500 m, 7 exs., 9. vi.-7. vii. 1961, N. R. Spencer ; Chute de Dourg, 37 km SE of Dalat, 1 ex., 25. iv. 1960, R. E. Leech ; 22 km S. of Nha Trang, 2 exs., 2026. xi. 1960, C. M. Yoshimoto ; 17 km S. of Dilinh, 1,300 m, 1 ex., 6-12. x. 1960, C. M. Yoshimoto ;9 km S. of Dilinh (Djiring), 1 ex., 24. iv. 1960, R. E. Leech ; Ap Hung-Lam, $21 \mathrm{~km} \mathrm{NW}$ of Dilinh, 1,100 m, 6 exs., 29. ix.-3. x. 1960, C. M. Yoshimoto ; Dai Lanh, N. of Nha Trang, 1 ex., 30. xi.-5. xii. 1960, C. M. Yoshimoto ; Kontum, 570 m, 1 ex., 13-14. vi. 1960, R. E. Leech ;M’Drak, E. of Ban Me Thuot, 4-600 m, 2 exs., 8-19. xii. 1960, C. M. Yoshimoto (BISHOP).

\section{Dercetina chiengmaica $n$. sp. Fig. 72a}

Generally yellowish brown ; in male elytron bluish black; in female elytron entirely yellowish brown, and in some specimen elytron with basal and subapical markings bluish black.

Head with vertex smooth, shining, sparsely impressed by minute punctures, interocular space much wider than transverse diameter of single oculus, and interocular transverse impression distinct, slightly depressed at middle, frontal tubercle transverse, subquadrate, slightly separated, distinctly raised, surface smooth, impunctate. Antenna slender, nearly $3 / 4$ as long as body length ; first segment robust, club-shaped, second shrotest, nearly half as long as first, third $11 / 2$ time as long as second, fourth $13 / 4$ time as long as third, fifth slightly shorter than fourth, sixth slightly shorter than fifth, sixth to tenth subequal to each other in length and shape, eleventh slightly longer than tenth and its apex pointed. Pronotum transverse, $23 / 4$ times as broad as long, anterior margin slightly rounded posteriorly, lateral margin feebly rounded, widest almost at middle and slightly narrowed anteriorly and posteriorly, basal margin distinctly rounded posteriorly, dorsal surface covex side to side, smooth, 
shining, sparsely impressed by fine punctures, anterior corner thickened, distinctly produced laterally, basal comer pointed, and each with a setigerous pore. Scutellum subtriangular, convex, smooth, shining, impunctate. Elytron with lateral margin rounded, surface strongly and rather closely punctate and their interstices smooth, shining.

Length : $6.0-7.8 \mathrm{~mm}$.

Holotype (Type No. 2707, Kyushu Univ.) : THAILAND : Chiengmai Prov., Doi Suthep, 1,000 m, 18. vii. 1965, Y. Miyatake. Paratypes : THAILAND : Chiengmai Prov., Doi Suthep, 1,000 m, 1 ex., 10. vi. 1965, 1 ex., 18. vi. 1965, K. Morimoto ; Chiengmai Prov., Mae Sa Water Fall, 1 ex., 16. vi. 1965, K. Morimoto ; Chiengmai Prov., Chieng Dao, 1 ex., 15. vi. 1965, Y. Miyatake ; Chiengmai Prov., Fang, 1 ex., 14. vi. 1965, Y. Miyatake ; Chiengmai Prov., Fang, 1 ex., 14. vi. 1965, K. Morimoto (KU); Kanchanaburi, 1 ex., '21. v. 1962, 3 exs., 31. v. 1962 (BANGKHEN, KIMOTO).

This new species closely resembles Dercetina punctipennis Kimoto, from Bhutan, but differs in having the lateral margin of pronotum strongly rounded.

\section{Dercetina flavocincta (Hope) Fig. 69c}

Galleruca flavocincta Hope, 1831, in Gray, Zool. Misc. : 29 (Nepal ; BM).

Anthipha flavofasciata Baly, 1879, Cist. Ent. 2 : 456 (Assam ; BM).-Maulik, 1936, Fauna India, Galeruc. : 355 (=flavocincta).

Antipha unifasciata Allard, 1889, C. R. Soc. Ent. Belg., 33 : 107 (Cambodge ; PARIS, BRUX ELLES). New synonym.

Arthrotus chinensis Weise, 1889, Horae Soc. Ent. Ross., 23 :626, note (nec Dercetes chinensis Baly, 1879) (China ; ZMB).-Ogloblin, 1936, Fauna USSR, 26,1 :330,331, 438 (Gansu). New synonym. Antipha variipennis Jacoby, 1890, Entomologist, 1890 : 214, fig. (China ; BM).-Ogloblin, 1936, Fauna USSR, 26, $1: 331$ (= chinensis Weise).

Dercetes femoralis Weise, 1922, Tijdschr. Ent., 65 : 97 (Tonkin; STOCKHOLM). New synonym. Monolepta flavocincta : Weise, 1924, Col. Cat. Junk, 78 : 168 (Nepal).

Dercetis flavocincta: Maulik, 1936, Fauna India, Galeruc. : 355 (India, Nepal).

Dercetis taiwana Chajô, 1938, Arb. Morph. Taxon. Ent. Berlin, 5(2): 140 (with var. melania; Formosa ; TARI). New synonym.

Dercetina variipennis : Gressitt \& Kimoto, 1963, Pac. Ins. Mon., 1B:705, fig. (China).

Dercetina flavocincta: Kimoto \& Takizawa, 1972, Kontyâ, Tokyo, 40(4): 222 (Nepal).

Distribution : India, Nepal, Thailand, Cambodia, Laos, Vietnam, China, Taiwan.

Material examined. THAILAND : Trang Prov., Khaophappha, Khaochong, 200-400 m, 1 ex., 3031. vii. 1963, 1 ex., 1. i. 1964, 1 ex., 11. i. 1964, 2 exs., 13. i. 1964, G. A. Samuelson (BISHOP) ; Kanchanaburi, 2 exs., 31. v. 1962 ; Chiengmai, 1 ex., 3. xii. 1962 (BANGKHEN). LAOS : Vientiane Prov., Ban Van Eue, 1 ex., 12. iv. 1965, 1 ex., 16. iv. 1965, J. L. Gressitt, 2 exs., 1-15. v. 1965, J. A. Rondon, 2 exs., 15. viii. 1965, 1 ex., 5. xi. 1965, 2 exs., 15. iii. 1966, 1 ex., 15. xii. 1966, 1 ex., 30. iv. 1967, Native collr; Vientiane Prov., Phou-kow-kuei, 1 ex., 15. iv. 1965, J. L. Gressitt ; Sedone Prov., Paksong, 1 ex., 8. v. 1965, P. D. Ashlock; Sayaboury Prov., Sayaboury, 1 ex., 16. iv. 1965, J. L. Gressitt ; Borikhane Prov., Pakkading, 1 ex., 31. vii. 1965, Native collr ; Attopeu, Houei Kong, 1 ex., 31. v. 1965, Native collr; Muong Sing, NW of Luang Prabang, 650 m, 1 ex., 6-10. vi. 1960, S. Quate \& L. Quate ; Nan Theuong, 18 km NW of Xieng, Khouang, 1,035 m, 1 ex., 10-17. viii. 1960, N. R. Leech (BISHOP). VIETNAM : Fyan, 1,200 m, 1 ex., 11. vii.-9. viii. 1961, N. R. Spencer ; Dalat, 1,500 m, 1 ex., 29. iv.-4. v. 1960, C. M. Yoshimoto; Blao (Balao), 500 m, 1 ex., 14-21. x. 1960, C. M. Yoshimoto ; M' Drak, E. of Ban Me Thuot, 4-600 m, 1 ex., 8-19. xii. 1960, C. M. Yoshimoto (BISHOP). 


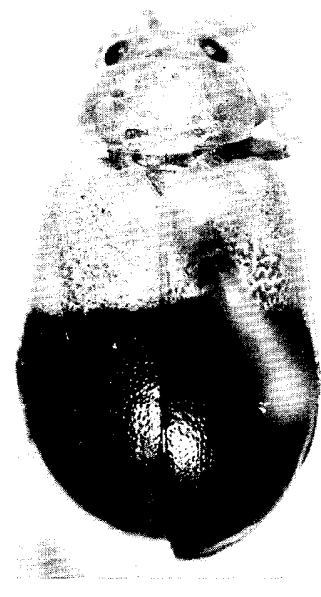

a

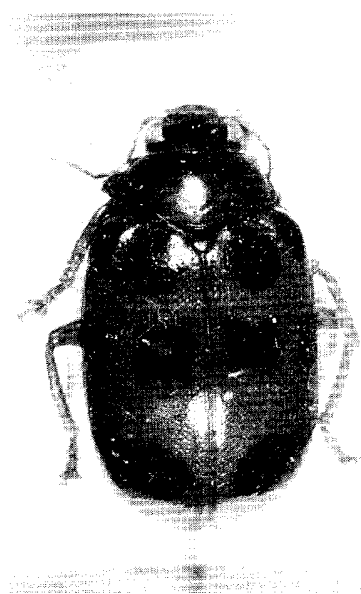

$\mathrm{b}$

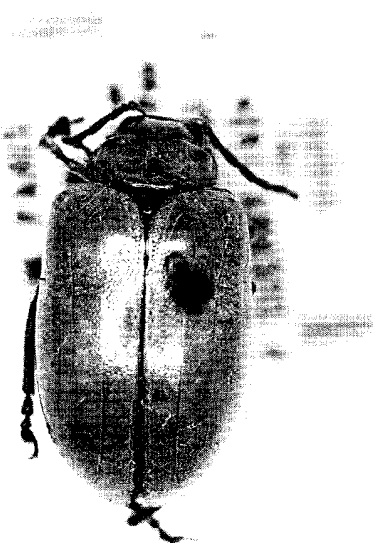

Fig. 73. a, Dercetina posticata (Baly); b, Doryida mouhoti Baly;c,Doryidomorpha souyrisi Laboissière (Y unnan ; type).

\section{Dercetina laevicollis (Jacoby), new combination}

Anthipha laevicollis Jac., 1892, Ann. Mus. Civ. Genova, 32 : 974 (Burma ; GENOVA).

Dercetes metallica Weise, 1922, Tijdschr. Ent., 65 : 96 (Tonkin; STOCKHOLM). New synonym.

Dercetis Zaevicollis : Maulik, 1936, Fauna India, Galeruc. : 360 (Burma).

Distribution : Burma, Thailand, Vietnam.

Material examined. THAILAND : Chiengmai Prov., Doi Suthep, 1,300 m, 2 exs., 8. vi. 1965, P. D. Ashlock, 50 km West of Tak, 900 m, 1 ex., 7-8. iv. 1966, J. Sedlacek (BISHOP) ; Chiengmai Prov., Doi Suthep, 2 exs., 8. vi. 1965, S. Asahina \& K. Morimoto ; Chiengmai Prov., Doi Pui, 1,300 m, 2 exs., 8. vi. 1965, S. Asahina \& K. Morimoto (KU).

\section{Dercetina permagna n. sp. Fig. $72 b$}

Entirely reddish brown.

Head with vertex longitudinally depressed at middle, finely granulate, closely impressed by distinct punctures, interocular space much wider than transverse diameter of single oculus, and interocular transverse impression indistinct, frontal tubercle transverse, subquadrate, distinctly separated by frons, feebly raised, surface impressed by distinct punctures. Antenna slender, nearly $2 / 3$ as long as body length; first segment long, robust, club-shaped, second short, nearly $1 / 3$ as long as first, third $21 / 2$ times as long as second, fourth $11 / 2$ times as long as third, fourth to tenth subequal to each other in length and shape, eleventh subequal to tenth in length but its apex pointed. Pronotum transverse, twice as broad as long, anterior margin distinctly rounded posteriorly, lateral margin distinctly rounded, widest almost at $1 / 3$ from basal margin, strongly narrowed anteriorly and less strongly so posteriorly, basal margin distinctly rounded posteriorly, anterior corner slightly thickened and slightly produced laterally, posterior corner pointed, each with a setigerous pore ; dorsal surface convex side to side, rather closely impressed by distinct punctures and their interstices somewhat wrinkled. Scutelium subtriangular, finely granulate, sparsely impressed by fine punctures. Elytron 
with lateral margin distinctly rounded, surface with a distinct transverse furrow subbasally, strongly and rather closely punctate and their interstices smooth, shining.

Length: $10.0 \mathrm{~mm}$.

Holotype (MUNCHEN) : LAOS: Umgeb. Pakse, 1964. Paratopotype: 1 ex., same data as the holotype (KIMOTO)

This new species somewhat resembles Dercetinabretinghami (Baly), but differs in being the body length longer and having elytron with a transverse subbasal furrow much deeper.

\section{Dercetina posticata (Baly), new combination Fig. 73a}

Antipha posticata Baly, 1879, Cist. Ent., 2 : 455 (Assam ;BM)-_Jacoby, 1889, Ann. Mus. Civ. Genova, $27: 224$ (as postica ; typographical error).

Dercetis posticata : Maulik, 1936, Fauna India, Galeruc. : 354 (India, Burma).

Distribution : India, Burma, Thailand, Laos.

Material examined. THAILAND: Chiengmai Prov., Mae Sa Water Fall, 3 exs., 16. vi. 1965, K. Morimoto (KU) ; "Siam”, 14 exs . , 1938 (BANGKHEN). LAOS : Vientiane Prov., Vientiane, 1 ex., iii. 1966, J. Sedlacek ; Sayaboury Prov., Sayaboury, 1 ex., 30. iv. 1965, 1 ex., 14. xii. 1965, Native collr ; Khammouane Prov., Phon Tiou, 1 ex., 30. iv. 1965, Native collr (BISHOP) ; Umgeb. Vanky, 8 exs., 1963 ; Umgeb. Pakse, 6 exs., 1963 ; Umgeb. Paklay, 5 exs., 1963 ; Umgeb. Vientiane, 2 exs., iii-vi. 1963 (MUNCHEN).

\section{Dercetina trifascia ta (Laboissière), new combination Fig. 72c}

Arthrotus trifasciatus Lab., 1932, Ann. Ass. Nat. Levallois-Perret, $20: 135$ (Tonkin; HAMBURG). Distribution : Thailand, Laos, Vietnam.

Material examined. THAILAND: Khao Chong, nr. Trung, 1 ex., 26. iv. 1967, K. Morimoto ; Nakornrajasima, 1 ex., 25. x. 1970, S. Nakao (KU). LAOS : Vientiane Prov., Vientiane, 1 ex., 21. iv. 1965, J. A. Rondon; Vientiane Prov., Ban Van Eue, 1 ex., 15-31, v. 1965, J. A. Rondon, 1 ex., 15. viii. 1965, 1 ex., 15. xii. 1965, 1 ex., 15. ii. 1966, Native collr ; Sayaboury Prov., Sayaboury, 1 ex., 16. iv. 1965, J. L. Gressitt, 1 ex., 12. xii. 1965, 1 ex., 14. xii. 1965, Native collr ; Borikhane Prov., Paksane, 1 ex., 23. xii. 1965, Native collr (BISHOP). VIETNAM :Fyan, 900-1,200 m, 7 exs., 11. vii.-9. viii. 1961, N. R. Spencer ; Karyu Danar, 200 m, 1 ex., 13-28. ii. 1961, N. R. Spencer ; 20 km S. Dalat, 1,300 m, 1 ex., 12. ix. 1960, J. L. Gressitt ; Ap Hung-Lam, 21 km NW of Dilinh, 1,100 m, 1 ex., 29. ix.-5. x. 1960, C. M. Yoshimoto ; Blao (Balao), 500 m, 6 exs., 14-21. x. 1960, C. M. Yoshimoto (BISHOP).

\section{Genus Doryida Baly}

Doryida Baly, 1865, Ent. Monthly Mag., 2 : 97 (type :Doryida mouhoti Baly ; Siam, Laos). -Chapuis, 1875, Gen. Col., 11 : 237, 239.-Gressitt \& Kimoto, 1963, Pac. Ins. Mon., 1B: 710.

This genus is closely resembles Gallerucida (= Stethidea) but separable in having the middle and the posterior tibiae without any spine apically.

$$
\text { Key to species of Doryida }
$$

1 Ventral surfaces entirely reddish brown ; legs reddish brown; dorsal surfaces and head reddish brown, and with or without blackish markings, in typical specimen pronotum with one meidian and elytron with six discal markings $(2: 2: 2)$, in most pale colored specimen entirely reddish 
brown and in most dark colored specimens entirely pitchy black; length 9.7-11.6 mm $\cdots$ mouhoti Ventral surfaces black; legs black with ventral surfaces of anterior femur and tarsus brownish; head and dorsal surfaces generally pitchy brown ; length $6.3 \mathrm{~mm}$ tarsalis

\section{Doryida mouhoti Baly Fig. $73 \mathrm{~b}$}

Doryida mouhoti Baly, 1865, Ent. Monthly Mag., 2 : 98 (Laos, Siam ;BM).-Jacoby, 1892, Ann. Mus. Civ. Genova, 32 : 990 (Burma).-Laboissière, 1931, Ann. Soc. Ent. France, 100 : 142 (Tonkin ;Hoa-Binh).-Maulik, 1936, Fauna India, Galeruc. : 606 (Siam, India, Burma).-Gressitt \& Kimoto, 1963, Pac. Ins. Mon., 1B: 711 (Tonkin;Hoa-Binh).-Châjô, 1964, Nature and Life in SE Asia, Kyoto, $3: 290$ (Thailand).

Doryida nigripennis Baly, 1890, Ent. Monthly Mag., ser. 2, 1 : 12 (Siam, Laos ; BM). New synonym.

Distribution : India, Burma, Thailand, Laos, Vietnam.

Material examined. LAOS : Vientiane Prov., Ban Van Eue, 1 ex., 31. vii. 1965, 2 exs., 15. vi. 1966, Native collr; Sedone Prov., Pakse, 2 exs., 30. viii. 1965, 1 ex., 15. viii. 1967, Native collr ; Sayaboury Prov., Sayaboury, 1 ex., 15. iv. 1965, J. L. Gressitt (BISHOP) ; Umgeb. Vientiane, 1 ex., iii-vi. 1963, Umgeb. Vanky, 1 ex., 1963 (MUNCHEN).

\section{Doryida tarsalis Baly}

Doryida tarsalis Baly, 1890, Ent. Monthly Mag., ser. 2, 1 : 12 (? Siam ; BM).

Distribution ; ? Thailand.

It is possible that this species is only a color variation of Doryida mouhoti Baly.

\section{Genus Doryidomorpha Laboissière}

Doryidomorpha Lab., 1931, Ann. Soc. Ent. France, 100 : 144 (type :Doryidomorpha souyrisi Labois-
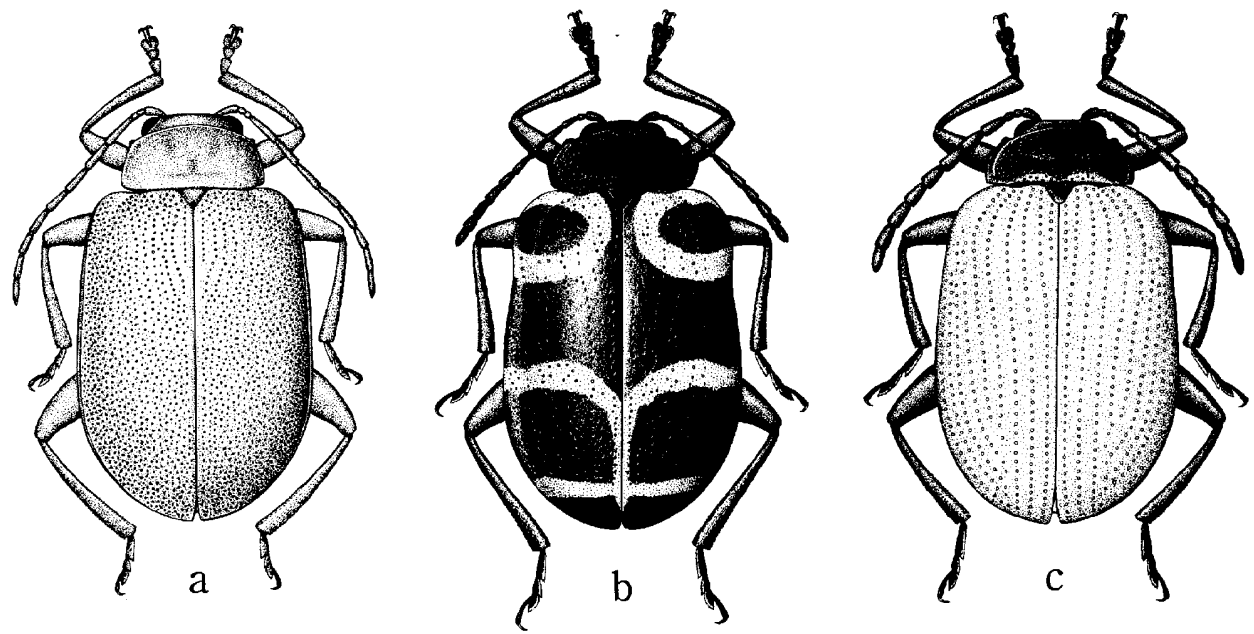

Fig. 74. a, Gallerucidaapicipennis (Duvivier) ; b-c, G. ornatipennis (Duvivier). 
sière ; Yunnan ; Fig. 73c).-Gressitt \& Kimoto, 1963, Pac. Ins. Mon., 1B : 711.

KeY TO SPECIES OF Doryidomorpha

Yellowish brown, antenna blackish with three or four basal segments brownish, legs with tibiae and tarsi blackish; in some specimen antenna and legs entirely brownish ; length

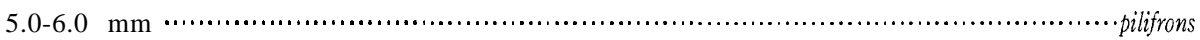

Yellowish brown, with frons, antenna and anterior angle of pronotum, ventral surface of

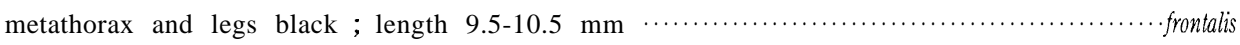

\section{Doryidomorpha frontalis Laboissière}

Doryidomorpha frontalis Lab., 1931, Ann. Soc. Ent. France, 100 : 149 (Tonkin; HAMBURG).

Distribution : Vietnam.

No additional material was examined beside the type series.

\section{Doryidomorpha pilifrons Laboissière}

Doryidomorphapilifrons Lab., 1931, Ann. Soc. Ent. France, 100 : 148 (Tonkin; HAMBURG).

Distribution : Vietnam.

No additional material was examined beside the type series. It is possible that this species is only a color variation of frontalis Laboissière.

\section{Genus Laphris Baly}

Laphris Baly, 1864, Trans. Ent. Soc. London, ser. 3, 2 : 231 (type : Laphris emarginata Baly; China).-Gressitt \& Kimoto, 1963, Pac. Ins. Mon., 1B: 712.

\section{Laphris sexplagiata Laboissière Fig. 4c}

Laphris sexplagiata Lab., 1934, Ann. Ass. Nat. Levallois-Perret, 21: 113 (Tonkin; HAMBURG).

Gallerucida maxima Gressit \& Kimoto, 1963, Pac. Ins. Mon., 1B: 726 (Hainan Is. ; CAS). New synonym.

Distribution : Vietnam, Hainan.

Reddish brown, elytron with five large markings black : one subbasally, two submedianly and one subapically ; antenna pale brown with apical four segments black; legs pale with apices of tarsi black ; sides of pronotum constricted behind middle ; length $11.0 \mathrm{~mm}$.

No additional material was examined beside the type series.

\section{Genus Gallerucida Motschulsky}

Gallerucida Mots., 1860, Etud. Ent., 9 : 24 (type : Gallerucida bifasciata Motschulsky ; Japan). -Maulik, 1936, Fauna India, Galeruc. : 545.-Chûjô, 1962, Phil. J. Sci., 91 (1-2) :150.-Gressitt \& Kimoto, 1963, Pac. Ins. Mon., 1B:174.-Kimoto, 1965, J. Fac. Agr. Kyushu Univ., 13 (3) : 398.

Eustetha Baly, 1861, J. Ent., 1 : 296 (type : Eustetha flaviventris Baly ; N. China).

Melosphila Baly, 1861, ibid. : 297 (type Melosphila nigromaculata Baly ; N. China).

Hylaspes Baly, 1865, Trans. Ent. Soc. London, ser. 3, 2 : 436 (type : Hylaspes longicornis Baly ; 
Himalaya).

Galerucida: Chapuis, 1875, Gen. Col., 11 :224, 227.-Ogloblin, 1936, Fauna USSR, 26, 1 : 346, 441.

Stethidea Baly, 1885, Ent. Monthly Mag., ser. 2, 1 : 13 (type :Doryidabalyi Duvivier ; Malacca).

-Maulik, 1936, Fauna India, Galeruc. : 607. New synonym.

Coptomesa Weise, 1912, Arch. Naturg., ser. A, 7 (2) : 91.

\section{Key то SPECIES of Gallerucida}

1 Lateral margin of pronotum nearly straight, not distinctly narrowed anterior-

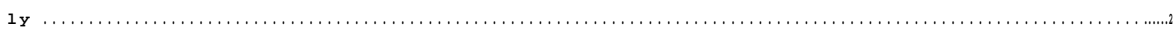

Lateral margin of pronotum distinctly rounded, widest at $1 / 3$ from anterior margin and distinctly narrowed anteriorly and posteriorly

2(1) Dorsal surfaces not entirely violaceous blue

Generally violaceous blue, abdomen entirely ochraceous, antenna ochraceous with

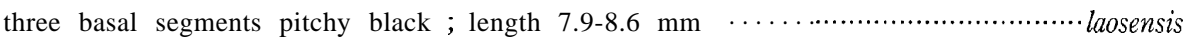

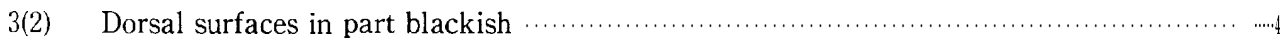

Dorsal surfaces entirely brownish ; antenna, tibiae and tarsi black $\ldots \ldots \ldots \ldots \ldots \ldots \ldots \ldots \ldots . . .5$

4(3) Head, pronotum, metathorax, antenna and legs black ; elytron, scutellum, abdomen, together with basal margin of pronotum reddish brown; length $10.0 \mathrm{~mm} \ldots \ldots . . . \cdots$ nigricollis Elytron, meso- and metathorax, and legs pitchy black ; head, pronotum, abdomen and antenna, together with apex of elytron and anterior femur, reddish brown;

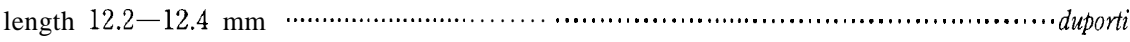

5(3) Elytron with two sizes of punctures, larger ones arranged in subregular rows and

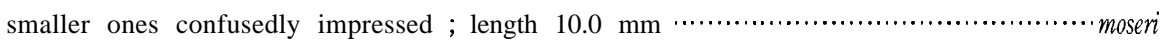

Elytron confusedly impressed by dinstict punctures ; length 10.0-12.0 mm ….... longicornis

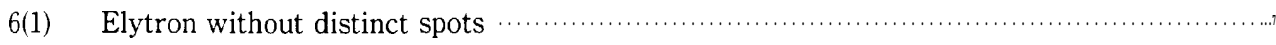

Elytron dark reddish brown with humeral and apical areas yellowish, latter with two or three blackish spots; dark reddish brown, antenna black ; length 7.0-8.5 $\mathrm{mm}$

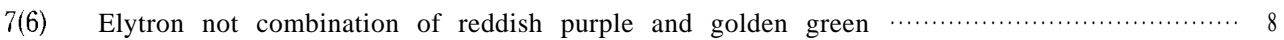
Elytron iridescent reddish purple and golden green ; former dominant subbasally and postmedially, and latter dominant laterally and suturally ; pronotum with former dominant apically and latter basally ; legs purplish ; length $7.5-8.5 \mathrm{~mm} \cdots \cdots \cdots \cdots \cdots \cdot \ldots$........ gloriosa

8(7) Elytron densely and finely punctured ; pronotum without distinct punctures, except

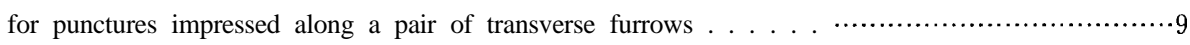

Elytron with two sizes of punctures, larger punctures arranged in subregular rows and smaller ones confused; pronotum finely and sparsely punctured ; violaceous, coloration of elytron variable: 1) subbasal blackish or bluish marking free from basal margin and usually covering humerus; one median and one subapical transverse yellowish band, both rather slender, 2) entirely violaceous blue, 3) entirely yellowish

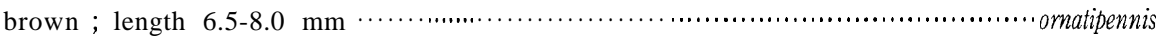

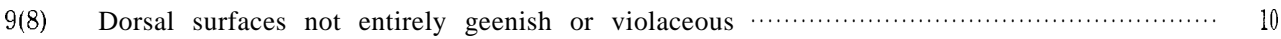
Dorsal surfaces entirely violaceous or greenish blue; abdomen entirely yellowish brown ; length $8.0 \mathrm{~mm}$ -tenuicornis

10(9) Body length shorter, prontoum with a pair of transverse furrows deeper and longer, punctures on elytron stronger ; coloration variable : 1) entirely yellowish to reddish brown, 2) brownish, meso- and metathorax, and legs pitchy black, with tarsi and 
anterior femur brownish ; 3) brownish, elytron black with apex brownish, 4) head, thorax, antenna and legs black, elytron and abdomen reddish brown, 5) brownish, with ventral surfaces, scutellum and a lateral stripe of elytron blackish ; length 7.4$8.3 \mathrm{~mm}$ apicipennis

Body length longer, pronotum with a pair of furrows shallower and shorter, elytron with punctures finer ; coloration variable : 1) entirely reddish brown, 2) brownish, legs blackish in various degrees, 3) brownish, metathorax and middle of each abdominal segment blackish, elytron with blackish markings (2 :2:2:1); length 6.3-7.4 $\mathrm{mm}$ balyi

\section{G allerucida apicipennis (Duvivier) Fig. 74a}

Parastetha? apicipennis Duviv., 1885, Stett. Ent. Ztg., 46 : 395 (Bangkok).

Galemcida fulva Laboissike, 1934, Ann. Ass. Nat. Levallois-Perret, 21 : 129 (nec Stethidea fulva Laboissière, 1931, from Tonkin)(Tonkin; BRUXELLES). New synonym.

Galemcida (Eustetha) apicipennis : Laboissiere, 1940, Bull. Mus. Hist. Nat. Belg., 16 (3) : 25 (Bangkok).

Gallerucida fulva : Gressitt \& Kimoto, 1963, Pac. Ins. Mon., 1B: 724 (Tonkin:Hoa-Binh, Hainan).

Gallerucida apicipennis : Wilcox, 1971, Col. Cat. Suppl., 78 (1) : 201 (Thailand).

Distribution : Thailand, Laos, Vietnam, Hainan.

Material examined. THAILAND : Chiengmai Prov., Chiengmai, 1,100-1,500 m, 2 exs., 1966, J. Sedlacek (BISHOP) ; Chiengmai Prov., Doi Pui, 1,300 m, 1 ex., 17. vi. 1965, K. Morimoto (KU) ; Khon Kaen, 1 ex., 20. xii. 1979, 1 ex., 22. xii. 1979, 3 exs., 24. xii. 1980, S. Azuma (RU). LAOS : Vientiane Prov., Vientiane, 2 exs., 25. iii. 1966, J. Sedlacek ; Khammouane Prov., Phon Tiou, 1 ex., 17. v. 1965, Native collr ; K. Sedone, 1 ex., 13. x. 1965, Native collr ; Ile de Khong, 1 ex., 24. viii. 1965, J. A. Rondon (BISHOP) ; Umgeb. Paklay, 1 ex., 1963 (MUNCHEN). VIETNAM : Ban Me Thoout, 500 m, 1 ex., 2024. xii. 1960, C. M. Yoshimoto ; Fyan, 900-1,000 m, 1 ex., 11. vii.-9. viii. 1961, N. R. Spencer (BISHOP).

\section{Gallerucidabalyi (Duvivier), new combination Fig. $75 \mathrm{~b}$}

Doryida balyi Duviv., 1885, Stett. Ent. Ztg., 46 : 394 (Malacca).

Stethidea fulva Laboissière, 1931, Ann. Soc. Ent. France, 100 : 135 (Tonkin; HAMBURG). New synonym.

Stethidea maculata Laboissiere, 1931, Ann. Soc. Ent. France, 100 : 133 (nec Galerucida maculata Weise, 1912) (Tonkin; HAMBURG). New synonym.

Stethidea ornata Labassière, 1932, Ann. Ass. Nat. Levallois-Perret, 20 : 132 (new name for Stethidea maculata Laboissiere, nec Weise).

Stethidea balyi: 1936, Fauna India, Galeruc. : 608 (India, Burma, Andaman Is., Malacca).

Distribution : India, Burma, Andaman Is., Thailand, Laos, Vietnam, Malaya.

This species closely resembles Doryidamouhoti Baly but is separable in having the middle and the posterior tibiae each with a short but distinct spine apically.

Material exmamined. THAILAND : Chiengmai Prov., Fang, 500 m, 1 ex., 13. vi. 1965, P. D. Ashlock (BISHOP). LAOS : Khammouane Prov., Phon Tiou, 1 ex., 30. iv. 1965, Native collr (BISHOP) ; Umgeb. Vanky, 2 exs., 1963 ; Umgeb. Pakse, 1 ex., 1963 ; Umgeb. Vientiane, 2 exs., iii-vi. 1963 (MUNCHEN). VIETNAM : Fyan, 900-1,000 m, 1 ex., 11. vii.-9. viii. 1961, N. R. Spencer (BISHOP). 


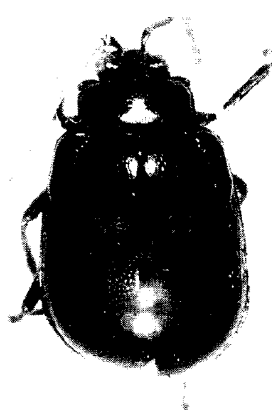

a

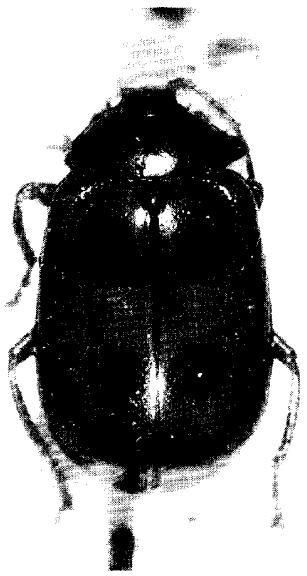

b

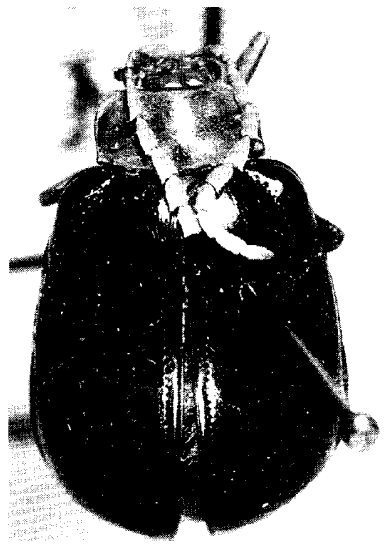

$\mathrm{C}$

Fig. 75. a, Gallerucida laosensis n.sp. ; b, G. balyi (Duvivier) ; c, G. duporti (Laboissière).

\section{Gallerucida duporti (Laboissière), new combination Fig. 75c}

Hylaspes duporti Lab., 1931, Ann. Soc. Ent. France, $100: 139$ (Tonkin; HAMBURG). Distributin : Vietnam.

Material examined. "Indo-China", 2 exs. (BASEL).

\section{Gallerucida gloriosa (Baly)}

Eustetha gloriosa Baly, 1861, J. Ent., 1 : 296 (N. China ; BM).-Fairmaire, 1887, Revue d'Ent., 6 : 334 (Pekin).

Eustetha seriata Fairmaire, 1878, Ann. Soc. Ent. France, $47: 136$ (C. China ;PARIS).-Ogloblin, 1936, Fauna USSR, 26, $1: 365$ (=gloriosa).-Laboissière, 1940, Bull. Mus. Hist. Nat. Belg., 16 (3) : 25 (Pekin).

Galerucida gloriosa : Jacobson, 1911, Käfer Russl., 9 : pl. 59, fig. 1O.-Ogloblin, 1936, Fauna USSR, 26, 1:365, 444 (China).

Gallemcida (Eustetha) gloriosa :Laboissière, 1934, Ann. Ass. Nat. Levallois-Perret, 21 : 128 (Tonkin).

Galerucida jacobsoni Ogloblin, 1936, Fauna USSR, 26, 1: 363,446 (Ussuri, Amur, Korea, Manchuria, Peking, Ganssu).-Laboissière, 1930, Bull. Mus. Hist. Nat. Belg., 16 (3) : 25 (= seriata).

Gallerucida gloriosa : Gressitt \& Kimoto, 1963, Pac. Ins. Men., 1B: 724 (China).

Distribution : China, Korea, E. Siberia, Vietnam.

No additional material was examined.

Gallerucida laosensis n. sp. Fig. 75a

Generally violaceous blue, antenna ochraceous with three basal segments pitchy black, abdomen entirely ochraceous, legs entirely black.

Head with vertex smooth, shining, sparsely impressed by distinct punctures especially on front, 
interocular space distinctly wider than transverse diameter of single oculus, and interocular transverse impression distinct, frontal tubercle oblique, subquadrate, contiguous, distinctly raised, surface smooth, shining. Antenna slightly shorter than long, depressed, in preapical segments nearly $22 / 3$ times as long as wide ; first segment robust, club-shaped, second short, nearly $1 / 5$ as long as first, third as long as second in length, fourth nearly seven times as long as third, fifth slightly shorter than fourth, fifth to ninth subequal to each other in length, tenth slightly shorter than fourth, eleventh $11 / 4$ times as long as tenth and its apex pointed. Pronotum transverse, nearly twice as broad as long, anterior margin distinctly rounded posteriorly, lateral margin almost straight, distinctly narrowed posteriorly, basal margin distinctly rounded posteriorly, anterior corner distinctly produced laterally and posterior corner distinctly angulate, dorsal surface convex, smooth, shining, sparsely impressed by fine puntures, and with a pair of oblique lateral furrows very deep. Scutellum subtriangular, smooth, shining, impunctate. Elytron with lateral margin rounded, slightly widened posteriorly, surface strongly and closely punctate, interstices of punctures smooth, shining.

Length : 7.9-8.6 mm

Holotype (BISHOP) : LAOS : Sayaboury Prov., Sayaboury, 15. iv. 1965, J. L. Gressitt. Paratypes : LAOS : Vientiane Prov., Ban Van Eue, SE of Phou-kow-kuei, 2 exs., 13. iv. 1965, J. L. Gressitt, 1 ex., 1-15. v. 1965, J. A. Rondon (BISHOP, KIMOTO).

This new species resembles Gallerucida abdominalis Gressitt \& Kimoto, from S. China, but differs in having antenna generally ochraceous and the legs entirely blackish.

\section{Gallerucida longicornis (Baly)}

Hylaspes Zongicornis Baly, 1865, Trans. Ent. Soc. London, ser. 3, $2: 436$ (Eastern Himalayas : Mungpu ; BM).-Maulik, 1936, Fauna India, Galeruc. : 602 (India, Burma).-Bryant, 1952, Ann. Mag. Nat. Hist., ser. 12, 5 : 602 (Nepal).-ChQj8, 1966, Mem. Fac. Educ. Kagawa Univ., II, 149 : 14 (Nepal).-Kimoto, 1970, Spec. Bull. Lep. Soc. Japan, 4 : 171 (Nepal).

Hylaspes assamensis Baly, 1879, Cist. Ent., 2 : 460 (Assam ; BM).-Duvivier, 1892, Ann. Soc. Ent. Belg., 36 : 442 (Kurseong, Sikkim).-Maulik, 1936, Fauna India, Galeruc. : 602 (= Zongicornis). Hylaspesdohrni Duvivier, 1885, Stett. Ent. Ztg., 46 : 246 (Birmah) ; 1892, ibid., 36 : 442 (Kurseong). -Allard, 1891, Nouv. Arch. Mus. Paris, ser. 3, $3: 231 ; 1904$, Miss. Pavie Indo-Chine, $3: 160$ (Laos : Luang-Prabang à Theng).-Maulik, 1936, Fauna India, Galeruc. : 602 (= Iongicornis).

Gallerucida longicornis : Kimoto \& Takizawa, 1972, Kontyâ, Tokyo, 40(4) : 223 (Nepal).

Distribution : India, Nepal, Burma, Thailand, Laos.

A specimen taken from Laos is slightly diffrent from the type, in having the legs reddish brown with apices of tibiae and trasi blackish and the antenna brownish in some basal segments.

Material examined. THAILAND : Chiengmai Prov., Chiengdao, 450 m, 3 exs., 5-11. iv. 1958, T. C. Maa (BISHOP). LAOS : Umgeb. Vientinae, 1 ex., iii-vi. 1963 (MUNCHEN).

\section{Gallerucida moseri (W eise)}

Galencida moseri Ws., 1922, Tijdschr. Ent., 65 : 93 (Tonkin ; STOCKHOLM).

Gallerucidamoseri : Gressitt \& Kimoto, 1963, Pac. Ins. Mon., 1B: 727 (China).

Distribution : Vietnam, China.

No additional material was examined. 


\section{Gallerucida nigricollis (Laboissière), new combination}

Hylaspes nigricollis Lab., 1931, Ann. Soc. Ent. France, $100: 140$ (Tonkin; HAMBURG).

Distribution : Vietnam.

No additional material was examined beside the type series.

\section{Gallerucida ornatipennis (Duvivier) Figs. 74b,c}

Hylaspes ? omatipennis Duv., 1885, Stett. Ent. Ztg., 46 : 397 (China).

Eustetha annulipennis Fairmaire, 1889, Ann. Soc. Ent. France, 58 : 79 (China).-Weise, 1922, Tijdschr. Ent., 65 : 93 (= ornatipennis).

Eustetha varians Allard, 1891, Nouv. Arch Mus. Paris, ser. 3, 3: 233 ; 1904, Miss. Pavie IndoChine, 3 : 163, fig. (Cambodge ; PARIS). New synonym.

Galemcida omatipennis :Laboissière, 1934, Ann. Ass. Nat. Levallois-Perret, 21 : 119 (China, Tonkin).-Gressitt \& Kimoto, 1963, Pac. Ins. Mon., 1B: 728 (China).

Galerucida omatipennis varr. decolora, violacea Laboissiere, 1934, Ann. Ass. Nat. Levallois-Perret, $21: 120$ (China).

Distribution : Cambodia, Vietnam, China.

Material examined. VIETNAM : Annam, 1 ex., 1927 (BASEL).

\section{Gallerucida singularis (Harold)}

Galerucida singularis Harold, 1880, Stett. Ent. Ztg., 41 : 145 (Darjeeling, India bor.).-Weise, 1912, Arch. Naturg., 78 : 90 (notes).

Galerucida indica: Allard, 1889, Ann. Soc. Ent. France, ser. 6, $9: 310$ (Saigon, Pnomh-Penh); 1891, Nouv Arch. Mus. Paris, ser. 3, $3: 231$; 1904, Miss. Pavie Indo-Chine, $3: 160$ (no locality cited). -Laboissière, 1925, Encylcl. Ent., ser. B, $1: 53$ (corrected as Galemcida tonkinensis Laboissiere).

Galemcida haroldi Weise, 1912, Arch. Naturg., 78 : 90 (Tonkin). New synonym.

Galerucida gebieni Weise, 1922, Tijdschr. Ent., 65 : 92 (Fukien ; STOCKHOLM).-Gressitt \& Kimoto, 1963, Pac. Ins. Mon., 1B: 733 (=singularis)

Galemcida tonkinensis Laboissiere, 1934, Ann. Ass. Nat. Levallois-Perret, 21 : 120 (Tonkin; PARIS). New synonym.

Gallerucida tonkinensis: Gressitt \& Kimoto, 1963, Pac. Ins. Mon., 1B: 736 (Vietnam).

Gallerucida singularis: Maulik, 1936, Fauna India, Galeruc. : 551 (Mungpu, Assam, Burma, Tonkin).-Chajô, 1962, Phil. J. Sci., 91(1-2) : 150 (Formosa).-Gressitt \& Kimoto, 1963, Pac. Ins. Mon., 1B: 733 (China).

Distribution : India, Burma, Vietnam, China, Taiwan.

The convexity of the anterior process of metastemum of this species is weaker than the other species of this genus. No additional material was examined.

\section{Gallerucida tenuicornis (Weise)}

Galemcida tenuicornis Weise, 1922, Tijdschr. Ent., 65 :91 (Tonkin; STOCKHOLM).

Distribution : Vietnam.

No additional material examined beside the type series. 
The following species was recorded by Châjô (1964), but I could not trace depository of the material treated for this record.

\title{
Anastena nigromaculata (Jacoby)
}

\author{
Astena ? nigromaculata Jac., 1896, Ann. Soc. Ent. Belg., $40: 275$ (Kanara). \\ Anastena nigromaculata: 1936, Fauna India, Galeruc.. 297 (India).-Châjô, 1964, Nature and Life \\ in SE Asia, Kyoto, $3: 284$ (Thailand : Chieng Mai). \\ Distribution : India, Thailand.
}

\section{References}

Allard, M. E. 1888 (1889). Synopsis des Galerucines à corselet silloné transversalement, 1. Ann. Soc. Ent. France, ser. 6, $8: 305-332$.

Allard, M. E. 1889. Contributions à la faune indo-chinoise, 5. Galerucides et Alticides. Ann. Soc. Ent. France, ser. 6, 9: 303-312.

Allard, M. E. 1891. Colêoptères, Phytophages, in Pavie, M., Collection d'Insects formée dans l'IndoChine. Nouv. Arch. Mus. Paris, ser. 3, 3 : 229-234.

Allard, M. E. $\quad$ 1904. Famille de Chrysomelides (suite). Mission Pavie Indo-Chine 1879-1895, 3 : 157164, pl. 9 .

Baly, J. S. 1886. Descriptions of a new genus and of some new species of Galerucinae, also diagnotiac notes on some of the older described species of Aulacophora. J. Linn. Soc., Zool., 20: I-27.

Baly, J. S. 1889. Notes on Aulacophora and allied genera. Trans. Ent. Soc. London, 1889: 297-309.

Chapuis, M. F. 1875. In Lacordaire, Genera des Coléoptères, 11 : 145-250.

Chûjô, M. 1962. A taxonomic study on the Chrysomelidae (Insecta: Coleoptera) from Formosa. Part XI, Galerucinae. Phil. J.Sci., 91(1-2):1-239+iv.

Gressitt, J. L. \& S. Kimoto. 1960. The Chrysomelidae (Coleopt.) of China and Korea. Part 1. Pac. Ins. Mon., 1A : 1-299.

Gressitt, J. L. \& S. Kimoto. 1963. The Chrysomelidae (Coleopt.) of China and Korea. Part 2. Pac. Ins. Mon., 1B : 301-1026.

Gressitt, J. L., J. A. Rondon, \& S. von Breuning. 1970. Cerambycid beetles of Laos. Pac. Ins. Mon., 24: $1-615$.

Jacoby, M. 1892. Descriptions of the new genera and species of the Phytophagous Coleoptera obtained by Sig. L. Fea in Burma. Ann. Mus. Civ. Genova, 32 : 869-999.

Jacoby, M. 1889. List of the Phytophagous Coleoptera obtained by Signor L. Fea at Burmah and Tenasserim, with descriptions of the new species. Ann. Mus. Civ. Genova, ser. 2, $7:$ 147-237.

Laboissière, V. 1927. Contribution à l'étude des Galerucini de l'Indochine et du Yunnan avec description de nouveaux genres et espèces. Ann. Soc. Ent. France, 96 : 37-62.

Laboissière, V. 1929. Nouveaux Galerucini de la faune d'Asie. Rev. Chil. Hist. Nat., 33 : 289-294.

Laboissière, V. 1929-1936. Observations sur les Galerucini asiatiques principlement de Tonkin et du Yunnan, et descriptions de nouveaux genres et espèces, 1-5. Ann. Soc. Ent. France, 98 : 251-288 (1929), 99 : 325-368 (1930), 100 : 133-152 (1931), $102:$ 51-72 (1933), 105 : 239-262 (1936).

Laboissière, V. 1932. Résultats scientifiques du voyage aux Indes orientales neerland. de LL. AA. RR. le Prince et la Princesse Leopold de Belgique. Galerucinae. Mém.Mus. Hist. Nat. Belg., horssérie, 4(4) : 145-184, pls. 2.

Laboissière, V. 1932. Chrysomelidae nouveaux de l'Indo-Chine et de la presque'ile de Malacca. 
Ann. Ass. Nat. Levallois-Perret, $20: 130-142$.

Laboissiere, V. 1933. Descriptions de trois nouveaux Galerucini du Tonkin. Bull. Mus. Hist. Nat. Paris, ser. 2, 5 : 205-208.

Laboissiere, V. 1934. Coléoptères Galerucines nouveaux ou peu connus de la faune indo-malaise. Ann. Ass. Nat. Levallois-Perret, 21: 109-137.

Laboissiere, V. 1935. Rectifications au mémoire de E. Allard. "Contributions à la faune indochinoise". "Galerucides et Alticides". Ann. Ass. Nat. Levallois-Perret, 21 : 139-145.

Laboissière, V. 1939 - 1940. Observations sur les Galerucinae des collections du Musée royal d'Histoire Naturelle de Belgiques et descriptions d'espèces nouvelles, 1-4. Bull. Mus. Hist. Nat. Belg., 15(59) : 1-8 (1939), 16(3) :1-31(1940), 26(25) : 1-16 (1940), 26(37) : 1-41 (1940).

Maulik, S. 1936. The fauna of British India, including Ceylon and Burma. Coleoptera, Chrysomelidae, Galerucinae. 648 pp., 1 pl.

Medvedev, L. N. 1981. A new species of genus Agetocera (Coleoptera, Chrysomelidae) from Vietnam. Zool.Zh., 60(4): 617-620.

Medvedev, L. N. \& Dang Thi Dap. 1981. New genera and species of leafbeetles of the subfamily Galerucinae (Coleoptera, Chrysomelidae) from Vietnam. Ent. Obozr., 60(3) : 629-635.

Ogloblin, D. A. 1936. Fauna USSR, 26, 1 (Galerucinae) : 455 pp.

Vachon, A. 1977. Galerucinae de l'Asie de Sud-Est et de la Nouvelle-Guinée (2 note). Oidini. Nouvelles synonymies. Bull. Soc. Ent. France, 82: 12-14.

Vachon, A. 1980. Les Adorium de Fabricius. Systematique et synonymies. Bull. Soc. Ent. France, 85: $14-19$.

\section{CORRECTION AND ADDITION FOR PARTS I AND III}

Correction for Part I.

Donacia Fabricius, 1775

Prodonacia Chen, 1966.-Borowiec, 1984, Bull. Ent. Pologne, $53: 437$ (=Donacia).

Donaciomima Medvedev, 1973.-Borowiec, 1984, ibid., 437 (= Donacia).

\section{Donacia shishona (Chen, 1966)}

Prodonacia shishona Chen, 1966.

Donacia shishona : Borowiec, 1984, Bull. Ent. Pologne, 53 : 443 (China : Yunnan ; N. Vietnam).

Donacia vietnamensis Kimoto \& Gressitt, 1979, Pac. Ins.-Borowiec, 1984, Bull. Ent. Pologne, 53 : $\mathbf{4 4 3}$ (= shishona).

Distribution : China, Vietnam.

\section{Addition to Part III.}

Scelolanka nigrofemorata Medvedev, p. 136 (CEuyen-Quang, Tonkin, Montes Mauson, H. Fruhstorfer).

Scelolanka phyllanthii Medvedev, p. 135 (Vietnam). 
Correction for Part III.

Page 50, fig. 13. a, Basilepta chiangmaiensis ; b, B. multimaculata; c, B. subcostata.

Page 134, fig. 42, a, Platycorynus pyrospilotus (Baly).

\section{$R$ eferences}

Medvedev, L. N. 1982. New species of Scelolanka (Col., Chrysomelidae, Eumolpinae) of Vietnam. ed. L. N. Medvedev, Animal world of Vietnam. Nauka, Moscow : 134-137.

Borowiec, L. 1984. Zoogeographical study on Donaciinae of the world (Coleoptera, Chrysomelidae). Bull. Ent. Pologne, $53: 433-518$. 